\title{
Eagle Hill: A Late Quaternary Upland Site in Western Lousiana
}

Joel Gunn

Center for Archaeological Research

David O. Brown

Center for Archaeological Research

Follow this and additional works at: https://scholarworks.sfasu.edu/ita

Part of the American Material Culture Commons, Archaeological Anthropology Commons, Environmental Studies Commons, Other American Studies Commons, Other Arts and Humanities Commons, Other History of Art, Architecture, and Archaeology Commons, and the United States History Commons

Tell us how this article helped you.

This Article is brought to you for free and open access by the Center for Regional Heritage Research at SFA ScholarWorks. It has been accepted for inclusion in Index of Texas Archaeology: Open Access Gray Literature from the Lone Star State by an authorized editor of SFA ScholarWorks. For more information, please contact cdsscholarworks@sfasu.edu. 


\section{Eagle Hill: A Late Quaternary Upland Site in Western Lousiana \\ Creative Commons License \\ (c) (1) \& 8}

This work is licensed under a Creative Commons Attribution-NonCommercial 4.0 International License 


\title{
EAGLE HILL: A LATE QUATERNARY UPLAND SITE IN WESTERN LOUISIANA
}

\author{
JOEL GUNN and DAVID O. BROWN
}

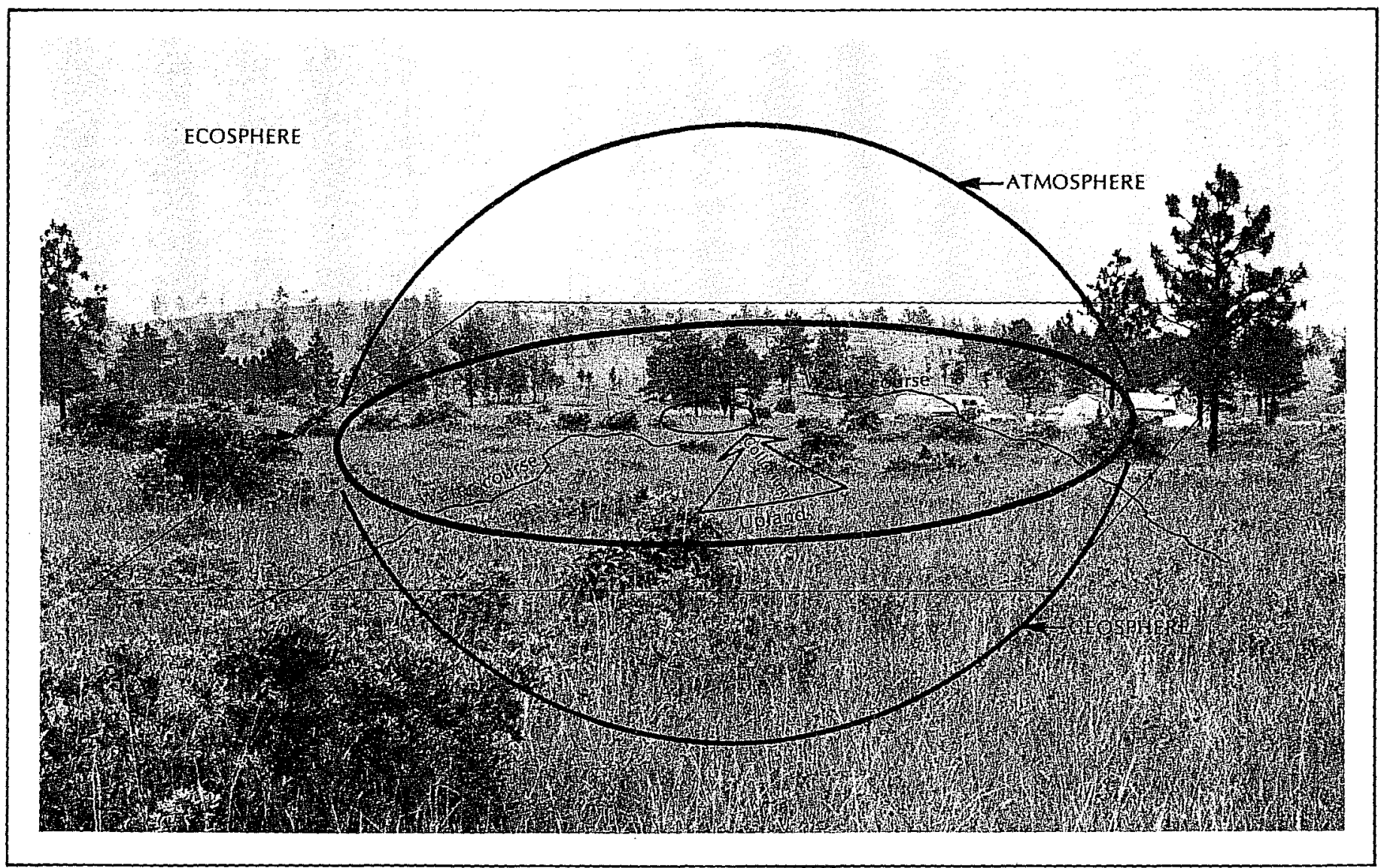

in association with

Eric Gibson, Royce Mahula, Kevin Jolly,

Patricia Wallace, Edward Garner, Mark Sheehan, Joan Sherwood, Margo Lopez, Donald R. Lewis, Beverly Van Note, and Fred Nials

CENTER FOR ARCHAEOLOGICAL RESEARCH

THE UNIVERSITY OF TEXAS AT SAN ANTONIO

SPECIAL REPORT, NO. 12 


\section{EAGLE HILL: A LATE QUATERNARY UPLAND SITE}

IN WESTERN LOUISIANA

Joel Gunn and David 0. Brown

in association with

Eric Gibson, Royce Mahula, Kevin Jol7y, Patricia Wallace, Edward Garner, Mark Sheehan, Joan Sherwood, Margo Lopez, Donald R. Lewis, Beverly Van Note, and Fred Nials

Center for Archaeological Research

The University of Texas at San Antonio

Special Report, No. 12

1982 


\title{
Center for Archaeological Research
}

\author{
The University of Texas at San Antonio
}

78285

Thomas R. Hester, Director

\section{SPECIAL REPORTS}

Publications dealing with the archaeology of Texas and Mesoamerica.

No. 1 (1975) Some Aspects of Late Prehistoric and Protohistoric Archaeology in Southern Texas. By Thomas R. Hester and T. C. Hill, Jr. Photocopy reprints available $\$ 2.28+.13$ tax for Texas residents.

No. 2 (1976) The Texas Archaic: A Symposium. Edited by Thomas R. Hester. Photocopy reprints available. $\$ 6.00+.33$ tax for Texas residents.

No. 3 (1976) Papers on Paleo-Indian Archaeology in Texas. Papers by T. R. Hester and W. W. Bi rmingham. Photocopy reprints available. $\$ 2.16+.12$ tax for Texas residents.

No. 4 (1976) Maya Lithic Studies: Papers from the 1976 Belize Field Symposium. Edited by T. R. Hester and Norman Hammond. Reprinted 1979. $\$ 9.00+.50$ tax for Texas residents.

No. 5 (1977) Hop Hil1: Culture and Climatic Change in Central Texas. By Joel Gunn and Royce MahuTa. Photocopy reprints available. $\$ 18.00+.99$ tax for Texas residents.

No. 6 (1978) Volume 1; Background to the Archaeology of Chaparrosa Ranch, Southern Texas. Studies in the Archaeology of Chaparrosa Ranch. By Thomas R. Hester. $\$ 4.00+.22$ tax for Texas residents.

Volume 2; The Mariposa Site: A Late Prehistoric Site on the Rio Grande Plain of Texas. Studies in the Archaeology of Chaparrosa Ranch. By John Montgomery. $\$ 8.00+.44$ tax for Texas residents.

No. 7

The Study of Biosilica: Reconstructing the Paleoenvironment of the Central Coastal Plain of Texas. By Ralph L. Robinson. Not available.

No. 8 (1979) The Lithic Artifacts of Indians at the Spanish Colonial Missions, San Antonio, Texas. By Daniel E. Fox. $\$ 5.00$ +.28 tax for Texas residents.

No. 9 (1980) Papers on the Prehistory of Northeastern Mexico and Texas. Edited by Jeremiah F. Epstein, Thomas R. Hester, and Carol Graves. $\$ 7.00+.39$ tax for Texas residents.

No. 10 (1980) Excavations at the Alamo Shrine (San Antonio de Valero), 1977. By Jack D. Eaton. $\$ 7.00+.39$ tax for Texas residents.

No. 11 (1980) Papers on the Archaeology of the Texas Coast. Edited by Lynn Highley and Thomas R. Hester. $\$ 6.00+.33$ tax for Texas residents.

No. 12 (1982) Eagle Hi11: A Late Quaternary Upland Site in Western Louisiana. By Joel Gunn and David 0. Brown. Write to the Center for information. 


\section{ABSTRACT}

The Eagle Hill I I site (16 SA 50) is located in a rolling upland area of western Louisiana known as Peason Ridge. Because of its location in a saddle, the locale accumulated colluvial sediments during certain intervals of the late Quaternary; in addition, it served as a habitation area for prehistoric groups. Sediments were preserved from the early and late Holocene, apparently reflecting the relatively cooler and moister conditions of those periods that were conducive to erosion-preventing vegetation.

The site was excavated in a manner to provide both vertical and horizontal information on site occupation at relatively high resolution. A sampling design was used to target critical occupation levels for careful excavation of occupational floors. Floors were stratified based on analysis of lithics from test excavations. On targeted occupation floors, artifacts were provenienced to the centimeter. A battery of information was collected on the sediments to allow definition of fire hearths, activity areas, etc.

The early Holocene levels (10,000-7000 B.P.) began with a Folsom-related occupation and ended with an Early Archaic technology. Analysis of lithic wear patterns, tool morphology, and fire-related attributes clearly defined activity areas. Similar success was achieved with the late Holocene (A.D. 6000-present) ceramic levels.

$X$-ray fluorescence and neutron activation were used to examine lithic source areas and mineral content of the soils in the occupation floors.

The rhythm of occupation at Eagle Hill II can be explained as a product of demographic fluctuations in the adjacent Sabine and Red River valleys, response of those populations to Holocene climatic change, and response of sediments and erosion to the same climatic variations. 
ABSTRACT ........................... . . . ${ }^{i}$

LIST OF FIGURES . . . . . . . . . . . . . . . . . . . . vi vi

LIST OF TABLES ......................... . . . .

LIST OF PLATES . . . . . . . . . . . . . . . . . . . xi

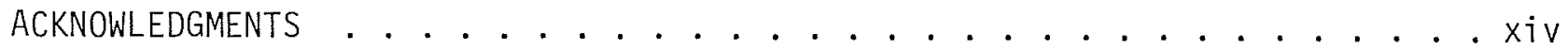

FOREWORD: OVERVIEW OF THE PROJECT AND CONCLUSIONS (Joel GUnn) . . . . . . . 1

I. HiSTORY OF THE HILL ARCHAEOLOGICAL PROJECT (Joet Gunn) . . . . . . . . 7

A. PRELIMINARY INVESTIGATIONS . . . . . . . . . . . . . 13

Preliminary Field Examination of the Site . . . . . . . . 13

B. TESTING . . . . . . . . . . . . . . . . . . . 24

C. OBSERVATIONS ON SITE LOCATION ON PEASON RIDGE (Joel Gunn,

David Brown) . . . . . . . . . . . . . . . . . . . . 24

D. PROJECT METHODOLOGY . . . . . . . . . . . . . . . . 26

Paleo-Indian Site Excavation Design and Method in the

Eastern United States (David Brown) . . . . . . . . . . . . 26

E. EXCAVATION ACTIVITIES AND TECHNIQUES AT EAGLE HILL (Joel Gunn) . . 45

Sequence of Operations . . . . . . . . . . . . . . 45

Analysis of Area A Control Column (Joel Gunn, Royce Mahula) . . 52

Rate of Excavation . . . . . . . . . . . . . . 56

Excavation Procedures . . . . . . . . . . . . . . 58

Screening (Joel Gunn) . . . . . . . . . . . . 62

Data Management . . . . . . . . . . . . . 66

Consultants . . . . . . . . . . . . . . . 69

Laboratory Activities and Procedures . . . . . . . . . . . . . 70

I I. PHYSICAL CONTEXT AND CONTENT . . . . . . . . . . . . . . . . . . . 73

A. THE OPEN-SITE ENIGMA (Joe1 Gunn) . . . . . . . . . . . . . . 73

B. PALEOCLIMATOLOGY OF THE GULF OF MEXICO COASTAL PLAIN

(Joel Gunn) . . . . . . . . . . . . . . . . . 74

Introduction . . . . . . . . . . . . . . . . 74

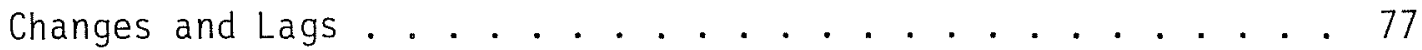

Atmospheric Circulation . . . . . . . . . . . . 77

Lands, GTaciers, and Seas in Eastern North America . . . . . . 86

Geomorphology of the Gulf Coast . . . . . . . . . . . . . 92 
Biological Indicators . . . . . . . . . . . 104

A Methodology for Independent Verification . . . . . . . . . . . 107

Modern Temperature and Salinity of the Gulf of Mexico . . . . . 112

c. UPLAND EROSIONAL EPISODES AND SOUTHEASTERN CLIMATE . . . . . . . 118

D. GEOLOGY AND GEOMORPHOLOGY OF THE EAGLE HILL LOCALE IN THE UPLANDS

OF WEST CENTRAL LOUISIANA (Edward Garner) . . . . . . . . . . . . . 120

Introduction . . . . . . . . . . . . . . 120

Geology .. . . . . . . . . . . . . . . 120

Geologic History . . . . . . . . . . . . . . 122

Post-Pleistocene Physiographic Development . . . . . . 123

Lithic Resources . . . . . . . . . . . . . . 125

Summary . . . . . . . . . . . . . . . . 126

E. GEOMORPHOLOGY AND SOILS (Fred Nials, Joel Gunn) . . . . . . . 126

Immediate Pre-Pleistocene Sediments . . . . . . . . . . . 126

Late Pleistocene-Early Holocene Sediments . . . . . . . . . . 129

Holocene Sediments... . . . . . . . . . . . 129

The Crayfish Problem . . . . . . . . . . . . . 134

Sediments and Soils (David Brown) ............ . 134

Site Catchment and Settlement Pattern (Joel Gunn, Mark Sheehan,

and Edward Garner) . . . . . . . . . . . . . . 140

F. HIGH RESOLUTION ENVIRONMENTAL COLUMN (Joet Gunn) . . . . . . 143

Fine-Grained (Sand) Sediment Analysis (Beverly Van Note) . . . . 145

Course-Grained (Pebble) Sediments (Margo Lopez) . . . . . . 150

G. GEOCHEMICAL ANALYSIS (Beveriy Van Note) . . . . . . . 156

X-Ray Fluorescence Studies, Phase I . . . . . . . . . 156

X-Ray Fluorescence Studies, Phase II (Joel Gunn) . . . . . . 160

H. FLORA AND FAUNA ...................... . . . 161

Flora (Mark Sheehan)............. 161

Fauna (Joel Gunn) . . . . . . . . . . . . . 163

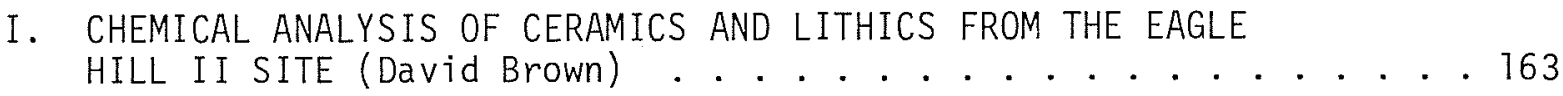

Ceramics . . . . . . . . . . . . . . . 164

Lithics . . . . . . . . . . . . . . . . 174

Conclusions . . . . . . . . . . . . . . . . . . 179 
II. CULTURAL CONTEXT AND FORMAL CONTENT . . . . . . . . . . . . . . 180

A. EAGLE HILL ENVIRONMENT, LITHICS, AND DYNAMIC UTILIZATION

ANALYSIS (Joel Gunn) . . . . . . . . . . . . . . . 180

Introduction . . . . . . . . . . . . . . . . . . . . . 180

The Eagle Hill Literature Search Problem . . . . . . . . . . . . 180

Environmental Control of Peason Ridge Occupation . . . . . . . 181

Environmental Control of Recovered Lithic Technology . . . . . . 183

Lithic Period Background Research . . . . . . . . . . . . . 184

Area Scale Lithic Period Chronology . . . . . . . . . . . . . 186

B. LITHIC PERIOD (Royce Mahula) . . . . . . . . . . . . . . 189

Enterline Tradition ............... . . 189

The Llano Tradition . . . . . . . . . . . . . . . 200

The Cumberland Tradition . . . . . . . . . . . . . 208

Lithic Period Sites in Texas . . . . . . . . . . . . 216

Lithic Period Sites in Louisiana . . . . . . . . . . . 220

Conclusions . . . . . . . . . . . . . . . . 227

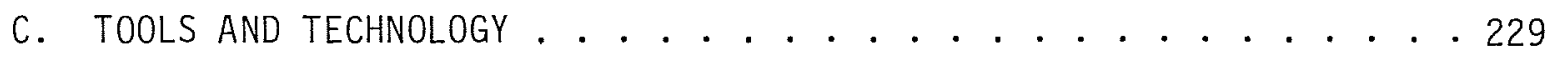

Biface and Core Flake Technology . . . . . . . . . . 238

Frequency of Habitation at Eagle Hill . . . . . . . . . . . 242

Points as a Functional System . . . . . . . . . . . . . . 242

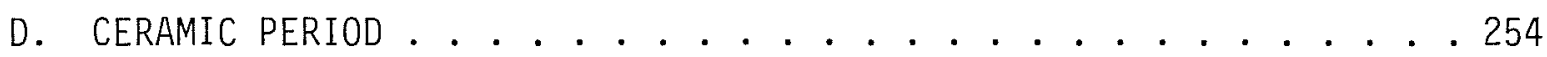

Sedentary Period Analysis (Gunn) . . . . . . . . . . . 254

Global Climate and Culture Chronology in the Lower Mississippi

Valley..................... . 255

Ceramic Analysis (David Brown) . . . . . . . . . . . . 260

IV. BACKGROUND: ANALYSIS OF OCCUPATION PLANES AT ONE-METER RESOLUTION . . 278

A. THE BACKGROUND-FOREGROUND CONCEPT (Joel Gunn) . . . . . . . . 278

B. ANALYSIS OF BACKGROUND DATA . . . . . . . . . . . . 280

Baked Clay Balls (Joan Sherwood) . . . . . . . . . . . 284

Lithics (Kevin Jolly) . . . . . . . . . . . . . . . . 290

General Analysis of Occupation Plane Background Data . . . . . . 301

Principal Components Analysis . . . . . . . . . . . . 303

Conclusions . . . . . . . . . . . . . . . . . 311 
V. FOREGROUND: ANALYSIS OF OCCUPATION PLANES AT ONE-CENTIMETER

RESOLUTION . . . . . . . . . . . . . . . . . . . . 312

A. USE-WEAR ANALYSIS (Eric Gibson, Joel Gunn) . . . . . . . . . 312

Introduction . . . . . . . . . . . . . . . . . . 312

Approaches to Use-Wear Analysis . . . . . . . . . . . . . . . 315

B. INTERPRETATION OF OCCUPATION PLANE PATTERNS (Joel Gunn) . . . . . 316

Introduction . . . . . . . . . . . . . . . . . . . . 316

Occupation Floor Logic . . . . . . . . . . . 317

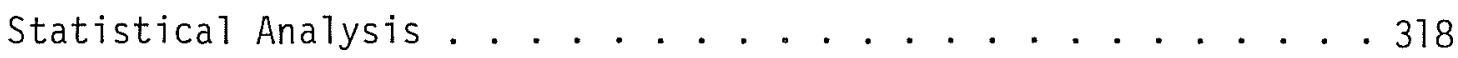

Tool Typology . . . . . . . . . . . . . . . . . . . 321

Vertical Perspective . . . . . . . . . . . . . 321

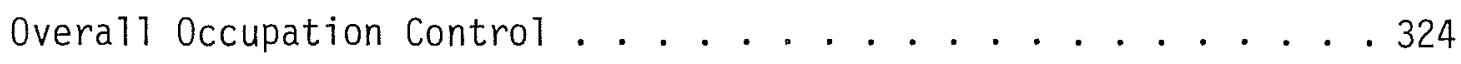

Occupation PTane 1.13 . . . . . . . . . . . . . . . . 324

Occupation Plane 2.13 . . . . . . . . . . . . . . . . . . . . . . . . . . . . . .

Occupation Plane 3.17 . . . . . . . . . . . . . . 330

Occupation P1ane 4.12 . . . . . . . . . . . . . 333

0ccupation Plane 4.17 . . . . . . . . . . . . . 337

Conclusions . . . . . . . . . . . . . . . . . 340

The Net Hypothesis. . . . . . . . . . . . . . . . . . . . . 341

VI. CONCLUSIONS (Joel Gunn) . . . . . . . . . . . . . . . . . 342

A. CONTEXT . . . . . . . . . . . . . . . . . . 342

B. CULTURE HISTORY . . . . . . . . . . . . . . . . . . 343

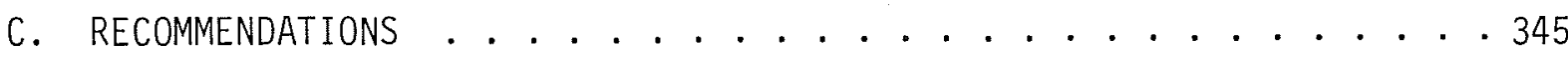

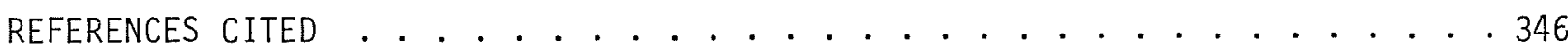

APPENDIX A: pH AND PHOSPHATE TESTING PROCEDURE . . . . . . . . . . 375

APPENDIX B: SITE FORMS . . . . . . . . . . . . . . . . . 379

APPENDIX C: FLAKE CONCENTRATIONS (Joan Sherwood) . . . . . . . . . . 389 


\section{LIST OF FIGURES}

1. Physiographic Map of Central and Western Southeastern United States with the Study Area . . . . . . . . . . . . . . 2

2. Generalized Stratigraphic Sequence and Cultural Chronology . . . 6

3. Contour Map of Eagle Hill II, Areas A and B . . . . . . . . . . . . 9

4. Locations of Bore Holes with Clay Surface . . . . . . . . . . . . 15

5. Isopac Map Showing 10-Meter Grid and Depth of Deposits in Areas A and B............... . . 17

6. Phosphate Spot Tests for Area A Transect . . . . . . . . . . . 19

7. Soi1 Texture Observations for Area A Transects . . . . . . . . . . 19

8. Phosphate Spot Tests for Area B Transect . . . . . . . . . . . . 20

9. Soi1 Texture Observations for Area B Transect . . . . . . . . . . . 20

10. pH Read with Test Strips at the Clay Bedrock Contact . . . . . . . 22

11. Number of FN's Issued per Week During the Excavations . . . . . . . 46

12. Vertical Sampling Strategy ............... 50

13. Factor II (Trend in Disturbance) and Factor III (Deflation) Factor Scores . . . . . . . . . . . . . . . . . 57

14. Excavation Unit with a Defined Substratum Being Exposed . . . . . 60

15. Mean Annual Surface Temperature Changes from Cold to Warm Years . . 76

16. Mean Annual Precipitation Changes from Cold to Warm Years . . . . . 76

17. Schematic Glacial and Vegetative Response to Abrupt Climatic Change .................. . . 78

18. Energy Budget Estimates for Northern Hemisphere . . . . . . . . . . 80

19. Jet Stream and Energy Budget Conditions . . . . . . . . . . . . . 83

20. Estimated Energy Budget for the Last 20k Years . . . . . . . . . 85

21. Selected Simulation Variables ............... 87

22. Sea Level Fluctuations ................ . 89

23. Ice Mass (Meters) and Sea Surface Temperature $\left({ }^{\circ} \mathrm{C}\right)$ at 18,000 B.P. . 91

24. Generalized Geologic Map of the Gulf Coastal Plains and the Principal Hydrographic Features of the Gulf of Mexico . . . . . . 93

25. Physiographic Map of the Southeastern United States Gulf of Mexico Coastal Plain ................ . 94

26. Four Idealized Phases of the Geological History of the Salt

Mine Valley Site (16 IB 23) Showing Stream Cutting and Valley

Filiting.................. . . 100

27. Time Series for Alluvial Chronologies in the Southeast . . . . . . 101

28. Idealized Geologic Section in Vicinity of Natchez, Mississippi,

Showing Sections of Natchez Pelvis Find. . . . . . . . . . . 102 
29. 500-Year Resolution Trajectory for Botanical Indicators, Pollen and Biosilica

30. Causal Model of GTobal-Local Effects . . . . . . . . . . . . . 109

31. Range of Temperature (Energy Budget) Movements . . . . . . . . . 111

32. Temperature and Salinity in the Western Gulf of Mexico . . . . . . 113

33. Paleo-Palmer Index for East Texas and East Central Mississippi . . . . 115

34. Holocene Level History of the Pomme de Terre River in Southern

Missouri . . . . . . . . . . . . . . . . 119

35. Measured Section of Bedrock Material Exposed in the Vicinity of

Eagle Hil1 . . . . . . . . . . . . . . . . . . 121

36. Geomorphic Map, Eagle Hill Area, Louisiana . . . . . . . . . . . . . . 124

37. Bedrock Stratigraphy in the Peason Ridge Area . . . . . . . . . . 126

38. Geomorphical Origins of Eagle Hil1 . . . . . . . . . . . . . . 128

39. North Wa17 Profile Along the N1002 Grid Line . . . . . . . . . . . . 130

40. Profile View of Clay Surface Transect . . . . . . . . . . . . . 131

41. Geologic History of the Eagle Hill Site . . . . . . . . . . . . . . 133

42. Eagle Hi11 Catchment (1-5 km rings) and Colluvium-Clay Interface

(Heavy Lines) .. . . . . . . . . . . . . . . . . . 141

43. Percentage of Original Weight According to Fraction . . . . . . . 146

44. Percentage of Material Type According to Level . . . . . . . . . . . 149

45. Percentage of Subangular Particles . . . . . . . . . . . . . 151

46. Concentrations of Pebbles by Count . . . . . . . . . . . . 153

47. Horizontal Distribution of Pebbles in Excavation . . . . . . . 154

48. Occurrence of Smooth (Round) Pebbles per Occupation Plane . . . . 157

49. Quantities of Trace Elements Found by Substrata . . . . . . . 159

50. X-Ray Fluorescence Cluster Tree . . . . . . . . . . . . . . . . . 170

51. Ceramic and Clay Source Cluster Tree . . . . . . . . . . . 170

52. Lithic Cluster Tree . . . . . . . . . . . . . . . . . 178

53. Stages of Lithic Tool Manufacture and Maintenance Relative to a

Satel1ite Camp such as Eagle Hil1 . . . . . . . . . . . . . 184

54. Dynamic Potential Lithic Tool Utilization Series Model . . . . . 185

55. North American Areas Relevant to the Literature Search . . . . . . . 187

56. Sites in the Enterline Area . . . . . . . . . . . . . . . . 191

57. Geographic Distribution of the Paleo-Indian Llano Tradition . . . . 201

58. Vegetation Regions of the Southeast . . . . . . . . . . 211

59. Lithic Period Sites in Texas and Louisiana . . . . . . . . . . . 218 
60. Relationship between Demography, Climate, and Cultural Change in the Louisiana Area Based on Point Frequencies and Patterns of Manufacture . . . . . . . . . . . . . . . . . 226

61. Paleo-Indian Lithics from OP 4.17 . . . . . . . . . . . 231

62. Tools, Resharpening Flake, and Bipolar Cores from OP 3.11,

A.D. 820 . . . . . . . . . . . . . . . . . 234

63. Late Holocene Artifacts . . . . . . . . . . . . . . . . . . . . 235

64. Progressive Bifacialization of Flakes in the Biface Producing Process .................. . . 240

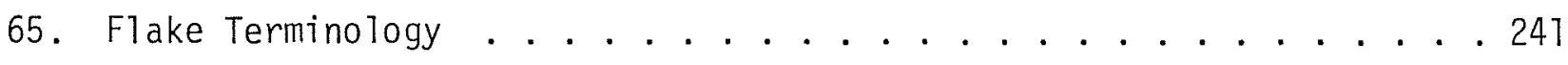

66. Bifacing Loci on the Occupation Planes . . . . . . . . . . . 243

67. Range of Variation in the Point Morphology Universe . . . . . . . 245

68. Through Time, the Darl-Scallorn-Perdiz Sequence Marked by a Genera1 Reduction in Size . . . . . . . . . . . . . . 247

69. Attribute Measurements on Three Types . . . . . . . . . . . . 250

70. Factor Score Plot for the Mid-Southern Point Morphology Sample . . . 253

71. Vertical Distribution of Ceramics at 16 SA 50 .......... 261

72. Eagle Hill Ceramics . . . . . . . . . . . . . . . . . . . . . . . . 264

73. Distribution of Ceramics on 0ccupation Planes . . . . . . . . . 274

74. Background-Foreground Site Evaluation Strategy . . . . . . . . . 281

75. Clay Ba11 Distribution . . . . . . . . . . . . . . . . 285

76. Clay Balls Found at Eagle Hill . . . . . . . . . . . . . . 287

77. Clay Ba17 Plots Found with Charcoal . . . . . . . . . . . . 288

78. In Theoretical Space, Use Spheres are Round and Concentric . . . . . . 296

79. In Practice, Use Spheres may be Distorted by Local Topography and Resource Distribution ... . . . . . . . . . . . 296

80. Relative Mean Frequencies of Hypothesized Local Materials . . . . . 298

81. Relative Mean Frequency of Hypothesized Local Materials . . . . . . 298

82. Presence and/or Absence of Hypothesized Exotic Materials . . . . . . . 298

83. Lithic Material Distribution on the Occupation Planes at Eagle

Hill . . . . . . . . . . . . . . . . . . . 299

84. Factor Scores for Five Components on Five Occupation Planes . . . . 306

85. Clusters 1-12 and Deviant (D) Units . . . . . . . . . . 308

86. Map of Occupation P1ane 1.13 .............. . . 325

87. Map of Occupation Plane 2.13 . . . . . . . . . . . . . 328

88. Map of Occupation Plane 3.11 . . . . . . . . . . . . . . 331

89. Map of 0ccupation Plane 4.12 . . . . . . . . . . . . . . . . 334 
90. Map of Occupation P1ane 4.17 . . . . . . . . . . . . . . 338

91. Physical Unit Record . . . . . . . . . . . . . . . 380

92. Culture Unit Record . . . . . . . . . . . . . . . . . 381

93. Unit Mapping Record . . . . . . . . . . . . . . . . . 382

94. Soil Chemistry Record . . . . . . . . . . . . . . 383

95. Transit Shot Record . . . . . . . . . . . . . . . . . 384

96. Substratum Plan Map . . . . . . . . . . . . . . . 385

97. FN Assignment Inventory . . . . . . . . . . . . . 386

98. Field Notes . . . . . . . . . . . . . . . 387 


\section{LIST OF TABLES}

1. Site Reports Examined in This Report . . . . . . . . . . . . 26

2. Varimax Rotated Factor Matrix for Occupation Intensity/Disturbance Indexing . . . . . . . . . . . . . . . . . 54

3. Occupation Intensity Index (Factor I Scores) for the Substrata, Occupation Factor . . . . . . . . . . . . . . 56

4. Key to Record Numbers . . . . . . . . . . . . . . . . . . . 69

5. Primary Sources for Alluvial Chronologies Cited by Various Reviewers . . . . . . . . . . . . . . . . . 96

6. Late Quaternary A7luvial Chronology for the Colorado River on the Blackland Prairie below Austin, Texas . . . . . . . . . . . . . 98

7. Radiocarbon Ages of Vicksburg, Mississippi, and Tunaca Bayou Loess Deposits ................... . . 103

8. Synchronous Alluvial Down-Cutting Episodes for the Northern Hemisphere ................... . 118

9. Geomorphic Map Explanation . . . . . . . . . . . . . . 123

10. Particle Size Distribution in Area A . . . . . . . . . . . . . 137

11. Surface Sediment Types in the Eagle Hill Catchment by One Kilometer Concentric Rings . . . . . . . . . . . . . . . . 142

12. Particle Size Scale . . . . . . . . . . . . . . . . 148

13. Particle Settling Times ................... . . 148

14. Pebble Counts by Occupation Plane . . . . . . . . . . . . . . 152

15. Pebble Frequency at Eagle Hill . . . . . . . . . . . . . . . 155

16. Angularity of Pebbles at Eagle Hill . . . . . . . . . . . . . 156

17. Correlations of Elements from XRF Data . . . . . . . . . . . . 160

18. Eagle Hill (HREC) Microfossils, Selected Levels . . . . . . . . . 163

19. Clay Source Provenience Data . . . . . . . . . . . . . . . . 165

20. XRF Clay Sample Peaks . . . . . . . . . . . . . . 167

21. Ceramic Sample Provenience . . . . . . . . . . . . . 169

22. Discriminant Analysis Scores . . . . . . . . . . . . 173

23. Lithic Sample Provenience Data . . . . . . . . . . . . . . . 176

24. Area Scale, Lithic Period Chronology . . . . . . . . . . . . . 186

25. Comparison of Artifact Types and Lithic Techniques in Some North American Fluted Point Industries . . . . . . . . . . . . 197

26. Plains Chronology for the Paleo-Indian Period . . . . . . . . . . 204

27. Primary Paleo-Indian Point Types in Louisiana . . . . . . . . . . . 224

28. FNs of Tools Recovered from Occupation Planes at 16 SA 50 . . . . 230 
29. Flake to Tool Ratios . . . . . . . . . . . . . . 232

30. Frequencies of Platformed Bifacing to Nonbifacing Flakes by Occupation Planes.................. . . 239

31. Unrotated Principle Components Analysis of Mid-Southern Projectile Points . . . . . . . . . . . . . . . 251

32. Values Used to Calculate Factor Scores Plotted in Figure 68 . . . . . 252

33. Energy Budget Levels for the Late Holocene . . . . . . . . . . . . . 257

34. Lower Mississippi Valley Cultural Chronology . . . . . . . . . . . . 259

35. Distribution of Ceramic Types . . . . . . . . . . . . . . 266

36. Texture by Substratum . . . . . . . . . . . . . . . 268

37. Technological Correlations . . . . . . . . . . . . . 269

38. Sand Temper by Combined Substrata . . . . . . . . . . . . . . . 270

39. Grit Temper by Combined Substrata . . . . . . . . . . . . . . . . . 270

40. Variation in Finish Characteristics with Depth . . . . . . . . . . 272

41. Exterior Color and Core Color . . . . . . . . . . . . . . 272

42. Ceramic Distribution in $\mathrm{OP} 2.21 \ldots . . . . . . . . . .275$

43. Ceramic Distribution in $\mathrm{OP} 2.31$............. . . 275

44. One-Meter Resolution Data Set . . . . . . . . . . . . . . . 282

45. Tabulation of Clay Balls, Weight, Average Size, and Percentages of Total . . . . . . . . . . . . . . . . . . . 284

46. Material Type; Descriptions . . . . . . . . . . . . . . . 291

47. Correlation Between Selected Chert Types . . . . . . . . . . 293

48. Regression Analysis ...... . . . . . . . . . . . . . 293

49. Frequencies of Lithic Material Categories for Eagle Hill Occupation Planes . . . . . . . . . . . . . . . . 294

50. Material Categories by Spatial Model . . . . . . . . . . . . 300

51. Variables Used in the Background Analysis . . . . . . . . . . . . . . 302

52. Primary Loadings and Variables for Principal Components Analys is of

Eagle Hill Background Data . . . . . . . . . . . . . . . . . 304

53. Variables Observed on Eagle Hill Lithics . . . . . . . . . . . 313

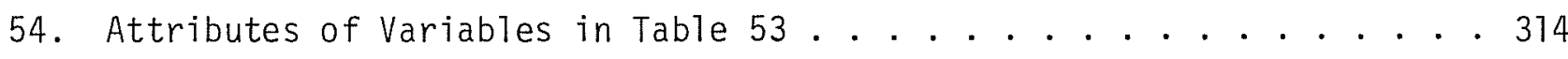

55. Eagle Hill Tool-Wear Pattern Distributions . . . . . . . . . . . . 320

56. Eagle Hill Tool-Wear Pattern Distributions . . . . . . . . . . . 320

57. Eagle Hi11 Tool-Wear Pattern Distributions . . . . . . . . . . 320

58. Eagle Hill Tool-Wear Pattern Distributions . . . . . . . . . . 320

59. Eagle Hill Tool-Wear Pattern Distributions . . . . . . . . . . . . 320

60. Formal Tool Types Found at Eagle Hill and Symbols Used in Maps . . . . 321 
61. Use Wear at Eagle Hill . . . . . . . . . . . . . . . . . . 322

62. Types of Wear Suggested by Hard and Soft States . . . . . . . . . . 323

63. Hard and Soft Use Through the Occupation PTanes . . . . . . . . . 323

64. Frequencies of Wear Categories in OP 1.13 by Concentration . . . . . 326

65. Frequencies of Wear Categories in OP 2.13 by Concentration . . . . . . 330

66. Frequencies of Wear Categories in OP 3.11 by Concentration . . . . . 332

67. Frequencies of Wear Categories by Concentrations on OP 4.12 . . . . 335

68. Occurrence of Soft Cutting in Sets . . . . . . . . . . . . 335

69. Distances between Sets of Soft Cutting Implements . . . . . . . . . 336

70. Frequencies of Wear Categories by Concentrations on OP 4.17 . . . . 339

71. Number of Concentrations by Occupation Plane . . . . . . . . . . . 390 


\section{LIST OF PLATES}

1. End of Excavation, Facing Northeast . . . . . . . . . . . . 48

2. End of Excavation, Facing Southeast ................ 48

3. Excavation of Units at One-Centimeter Resolution and One-Meter

Resolution .................. 50

4. Control Column (E1000-N300) ............... 60 60

5. Water Conservation Screening ................ 64

6. Dry Screening for Sandier Sediments . . . . . . . . . . . . . . . 64

7. Excavation of the High Resolution Environmental Column (HREC) . . . . . 144

8. Paleo-Indian Lanceolate (Folsom-7ike) from OP 4.17 . . . . . . . . . 236

9. Late Holocene Artifacts . . . . . . . . . . . . . . . . . . . . . . . 237 


\section{ACKNOWLEDGMENTS}

A project as involved as the Eagle Hill II excavation leaves innumberable persons to be thanked. Those persons directly involved in the project are cited in the text either as authors or as contributers in other capacities.

Deserving of special thanks are the employees from The University of Texas at San Antonio administration who facilitated the project and from the employees of the Center for Archaeological Research. Mary Lou Ell is, Patricia Wallace, and Ann Young typed the manuscript, and Sharon Quirk was the editor.

Vic Carbone and Ed Hession of the former Interagency Archeological Services, Atlanta, deserve special recognition for their council relative to the design of the project. Dr. Stephanie Rodeffer of the National Park Service, Santa Fe, New Mexico, managed the later stages of the project because of an administrative reorganization and is to be thanked for her understanding.

Persons from other universities, Mark Sheehan of the University of Indiana, Ed Garner of The University of Texas at Austin, Fred Nials of Eastern New Mexico, the Nuclear Reactor Laboratories at Texas A\&M, and many colleagues were most helpful and free with their council and advice.

Certainly the project would have been less productive and less possible had it not been for the support and assistance of Fort Polk personnel. Special thanks goes to Ron Tomas and James Grafton of the Environmental office and John Guy and his family of Annacoco, Louisiana. Also, the Air Force Personnel of the bomb targeting facility on top of Eagle Hill contributed immensely to the quality of life during the field work.

Final1y, the many U.T. San Antonio and U.T. Austin students who devoted their hours to the project deserve special recognition and respect. They not only learned, but created knowledge. First among these was David Brown whose careful scholarship and ceaseless argumentation contributed materially to the design and final form of the project. Eric Gibson is also to be thanked for rushing to the rescue with appropriate lithic wear analysis techniques. 


\title{
FOREWORD: OVERVIEW OF THE PROJECT AND CONCLUSIONS
}

\author{
Joel Gunn
}

Proof of assertions spelled out in such a manner that it can be observed by others is the substance of modern scientific method. Unfortunately, as important as it is, scientific evidence is often tedious reading even to the interested specialist and the bane of the casual reader. A remedy for the tedium is a middle ground of writing that attempts to interpret the works of science into a more generally readable format. The growing interest of the public in matters of science and growing desire on the part of scientists themselves to cross disciplinary bounds and see what is on the other side of the academic fences has led to the successful publication of several magazines in the last few years. While this foreword is unlikely to appear on the newsstands of airports, it is intended to serve as an extensive introduction to the report which follows. Those who are skeptical of the assertions made in the foreword are encouraged to examine the detailed reports that follow and which outline in great detail the supporting arguments, methods, and data.

Excavations at the Eagle Hill locality (Fig. 1) were suggested by a survey of the Peason Ridge area of Fort Polk, Louisiana, during 1976 by Frank Servello of Southwest Louisiana University. Subsurface testing of several sites indicated considerable evidence of prehistoric occupation during both the ceramic and preceramic periods. One site designated in the Servello survey as Eagle Hill II (16 SA 50) appeared to have Paleo-Indian artifacts and was in danger of being eroded. It is this site to which this report pertains. The Eagle Hill site is located $500 \mathrm{~m}$ southwest of a peculiar topographic prominence of the same name.

A Request for Proposals to excavate the Eagle Hill locality was issued in 1979 by Interagency Archeological Services (IAS) in Atlanta. The proposals were examined during the early months of 1980 and the contract granted to the Center for Archaeological Research. The University of Texas at San Antonio (CAR-UTSA), in Apri1 1980.

The excavation and analysis of artifacts progressed through six stages during the remainder of 1980 and the early half of 1981 .

(1) Late in April 1980 the site was examined in a preliminary mapping and coring expedition. (2) In May preparation for the UTSA Summer Field School were made, the results of the coring expedition examined, and plans laid for extensive excavations. (3) Excavation began in mid-May with a sma11, seasoned crew that mastered the record-keeping procedure and carefully removed a one meter square of soil to the bottom of the site's approximately one meter depth. Nearly surgical excavation revealed occupation floors frequented by prehistoric inhabitants of the region. (4) The full crew consisting mainly of UTSA Archaeological Field School students arrived on the site the first of June. Excavation began on a large block in a manner designed to recover evidence of camp patterns as well as the usual pottery and stone artifact inventories. This intensive excavation process lasted through the summer until the first week in August. A $5 \times 6 \mathrm{~m}$ block was excavated to the underlying, archaeologically sterile deposits. (5) Laboratory operations designed to gather interpretable data from the summer's excavated artifacts were conducted from August to December. Project staff and interested students measured, weighed, 
5.

5.

HIf

(1)

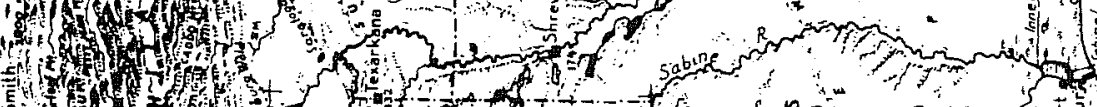

ond

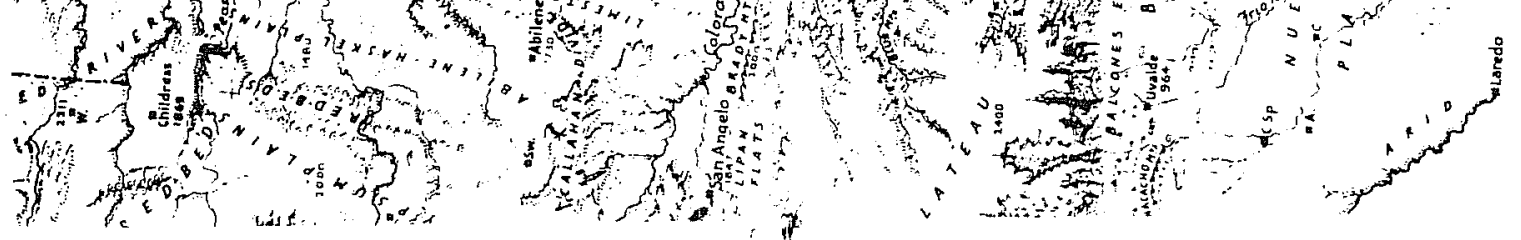


and coded thousands of pieces of relevant evidence and wrote preliminary reports. (6) The spring of 1981 was devoted to final analysis of the data, report writing, and production.

The first question addressed by the April expedition was why there should be a site of such great age on the top of a hill. Two geologists, Fred Nials and Ed Garner, examined the deposits on Peason Ridge and, thanks to their thorough knowledge of soils and the geologic past of the area, an answer soon emerged. Several million years ago the area, which is now on top of Peason Ridge, was on the bottom of a lake. The Gulf of Mexico was closer then, and the lake was probably somewhat Tike present-day Lake Pontchartrain near New Orleans, Louisiana.

The sediments deposited in the bottom of the lake contained a substantial amount of sand and herein lies the key to survival of Peason Ridge through millions of years of erosion. Ordinarily, sand is thought of as an easily moved and eroded sediment. However, if a sand deposit is deep enough to absorb all of the precipitation which falls on it, the sand will not move. The water simply seeps through the sand without disturbing the soil. Thus, the sand acts as a protective layer shielding Peason Ridge from erosion as long as precipitation is smal1.

An additional clue to the survival of the Eagle Hill site is that prehistoric inhabitants of the Ridge chose to locate in a saddle at the base of the two gentle slopes, one from the northeast and one from the southeast. They were probably attracted by running water at the base of the slope. When there was sufficient precipitation to move sediments down the hill, they piled up at the very location where prehistoric men lived. Thus, through time, alternating layers of sediment and artifacts built up over the site.

Close inspection of the deposits in the site showed that there were two separate periods of deposition at the site. The top zone was separated from the bottom by a period of erosion. We can therefore assume that for some reason the sands of Peason Ridge were not always the effective erosional shield they are now. The lower zone contained no ceramics. The upper zone revealed ceramics and considerable evidence of human occupation.

Having resolved some of the problem of the origin of the site, we turned next to its surroundings and their attraction to early man. Peason Ridge is about $120 \mathrm{~m}$ (400 feet) above sea level. These elevations are relatively recent developments according to geological time. Thirty million years ago Peason Ridge was at sea level. Since then, however, rivers such as the nearby Sabine and Red have dumped great quantities of sediment into the Gulf of Mexico. There have been two results. First, the shore of the Gulf has moved away to the south about $180 \mathrm{~km}(170 \mathrm{miles})$. Second, the weight of the sediments has forced areas off the coast downward. Surfaces inland from the coast have responded in a seesaw fashion by rising about $140 \mathrm{~m}$ (450 feet). Naturally, the movements have been so slow, that conditions have been much as they are now during the last few thousand years and during the time of human occupation in Louisiana.

An aerial photo survey of the area within a six kilometer radius of the site shows that the top of Peason Ridge has probably been relatively stable for some time, 
excepting the recent traumatization of the ridge top by logging and stumping operations in this century. The land is relatively flat, and the range of the habitats available for prehistoric people to exploit is consequently narrow. Flat, unbroken countryside suggests the hunting of larger animals.

A survey of the literature of the archaeology of the lower Mississippi Valley suggests that, there may have been periods during the last 10,000 years when people would have been more likely to explore and utilize upland locations such as Peason Ridge. Clovis hunters (11,500-10,500 years ago) may or may not have been interested in the Eagle Hill area. In the lower Mississippi Valley, they seem to have confined themselves to the river bottomlands. However, western Louisiana is near the Plains margin, which was inhabited by a more open-ground, herd-hunting people.

During the subsequent Dalton (10,500-9900 years ago; for dating of Dalton see Goodyear 1982) and Early Archaic (9900-8000 years ago) periods, there is considerable evidence that humans inhabited not only the river valleys, but also the uplands all over the southeastern United States. The reasons are not clearly understood. It may have been related to overpopulation. In any event, Peason Ridge was frequented during the time interval 10,500-8000 years ago. A Late Paleo-Indian point and a tool kit of scrapers, knives, and so forth were found in the lowest level.

The Middle Archaic is $i 11$ defined in the lower Mississippi Valley. It probably falls between 5000 and 8000 years ago. Sherwood Gagliano, who has a longstanding familiarity with the problem, thinks that upland cultures were probably stagnant during this time. River bottoms were accumulating sediment, which suggests that ridge tops were being eroded. Peason Ridge was probably a dry and unpleasant place during these times.

After 5000 years ago, populations began to grow in the alluvial floodplains of the Southeast. During the periods of Coles Creek (A.D. 900-1150) and Plaquemine (A.D. 1150-1250) interest in the uplands was rekindled, perhaps by overpopulation of the productive lowlands (Griffin 1978:56).

On a purely speculative basis, then, surges of activity on Peason Ridge might be expected relatively early and late in the human history of the Southeast with intervening episodes of disinterest or, at least, reduced human activity.

Additional search of the literature indicated that lithic tools should resemble those of the Paleo-Indians to the end of the Early Archaic with the exception of projectile points. After that time, we expected to find fewer formal tools, such as scrapers, and more use of less well-prepared tools, such as utilized flakes.

Naturally, when we turned our attention to the excavation and analysis of artifacts from Eagle Hill, we hoped that we would see clues as to what transpired there by studying cultural chronologies from surrounding areas. However, many nearby excavations have produced meaningful, through-time collections of archaeological materials. Our ambitions for this particular excavation went far beyond a collection of temporal or vertical indicators. We hoped, as we11, to examine the horizontal evidence of primitive man's lifeways stored at the Eagle Hill 
site. By recovering remains of campfires and tools measured to their exact location on living floors, we hoped to treat the whole site as a big artifact of human camp activity.

The procedure adopted to achieve this goal also had to take into account the limited amount of time available to us. Our late May excavations showed that there were about 20 discernible levels of flakes in the $100 \mathrm{~cm}$ depth of the site. Excavating a 1120 would have been impossible if we intended to excavate enough area to recover a meaningful segment of the camp space at each level. It was apparent that we would have to select the most important levels and excavate them with the greatest of care. Intervening levels would be removed in one meter squares by shovels. Seven levels were selected for excavation by trowel with all artifacts plotted on maps or provenienced by exact measurements. Measurements were recorded in such a way that they could be analyzed by computer. Figure 2 shows the numbering of the levels and their relative positions in the site.

In the laboratory, various types of material, such as baked clay, charcoal, and chert were analyzed first for vertical changes. Attention was then turned to five of the $6 \times 5 \mathrm{~m}$ horizontal floors recovered during the summer.

There was a great deal of charcoal in the upper levels. Radiocarbon dates were run on charcoal from the upper four floors for two reasons. The obvious reason was to acquire dates meaningful to the time of occupation of the floors. In addition, we were not sure whether the increase of charcoal in the upper levels was due to increased occupation or just the burning of roots during the modern deforestation process. This was very important since charcoal was instrumental in defining fireplaces, the presumed center of camp activities.

Baked clay also increased substantially in the later floors. Clay potsherds appeared in the upper zone of occupation (Zone I). Another, somewhat mystifying series of clay objects consisted of small clay balls normally from one to three centimeters in diameter. They appeared in a 11 levels, al though much more frequently in the upper zone associated with pottery. We could only surmise that they came from humans building fires on the remains of crayfish castles. Crayfish castles are often high in clay, because they burrow down to the Miocene clays under the archaeological layers of the site.

Lithic pieces appear in great numbers throughout al1 levels of the site. A close examination of the types of material being brought into the site indicates that the greatest numbers of exotic materials, perhaps carried from very far away, were brought in during occupation of Occupation Plane (OP) 3.11 . During this time, the trade and/or exchange of lithic materials seems to have been at a maximum. David Brown undertook the task of determining the source of both the lithic and ceramic materials. He used neutron activation to study trace elements in the materials and compared them to sources in Texas and Arkansas.

Examination of flakes for use on hard (bone and wood) or soft (meat, skins, and soft plants) materials shows that there was a marked increase of soft wear during the OP 2.13 ceramic period. This observation coupled with a notable increase in the amount of occupation debris suggests that Eagle Hill probably assumed a much more important status during this time than it did before or 


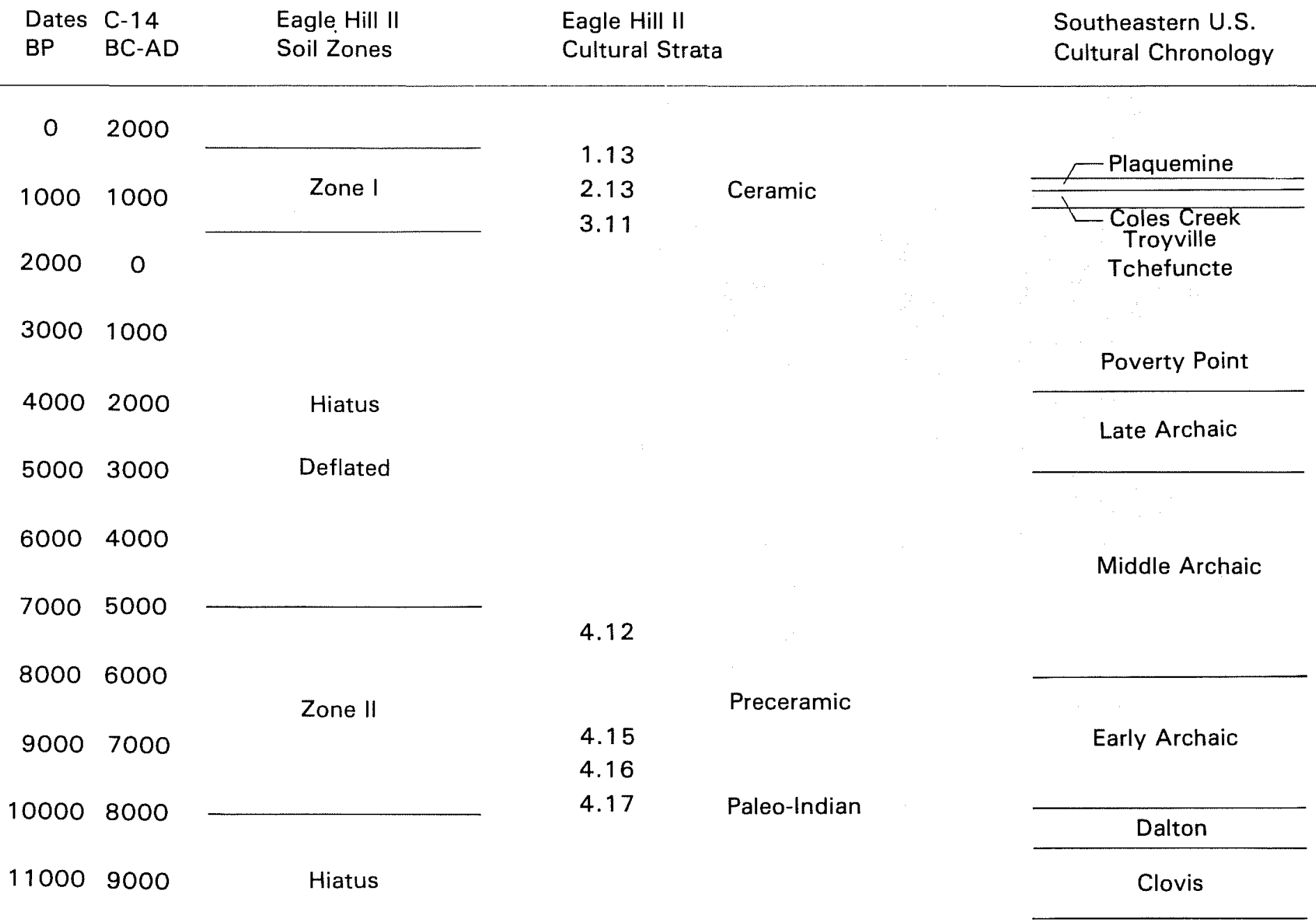

1200010000

Zone III

Figure 2. Generalized Stratigraphic Sequence and Cultural chronology. 
after. It may be that during OP 2.13 times, the site was visited by whole families. At other times, it was only occupied by men on hunting trips.

Close examination of the patterns of worked flakes around fireplaces indicates that some areas were used to work on hard materials, while others were devoted to soft substances. Interestingly enough, soft cutting is usually next to a fire, while hard cutting and scraping occurs both by the fire and in isolated areas away from fires. This may mark the use of flakes next to fires to cut soft, cooked foods, while some harder tasks, such as the manufacture or refurbishment of weapons, were relegated to more out-of-the-way spots.

When occupation at Peason Ridge is studied in the context of the climate of the greater southeastern United States, it seems likely that the ridge attracted human occupation during moderately cool and moist periods. It would have been during such intervals that water would have been available for a reasonably long period during the year. Also, the vegetation would have supported game and provided nuts and other vegetable foods in greatest abundance. The appearance of pine forests on high sandy ridges, known as the "pine barrens," during the last 500 years appears to have discouraged occupation as affectively as earlier, excessively dry intervals.

\section{HISTORY OF THE EAGLE HILL ARCHAEOLOGICAL PROJECT (Gunn)}

The Eagle Hill II site (Servello n.d.) is located at the top of a northeastsouthwest trending sand ridge in west central Louisiana. As subsequent discussions will verify, the site is one of those points in the landscape that, thanks to its geomorphological situation, has preserved the remnants of sediments as old as 10,000 years. The site is an erosional remnant which at present appears as a one-meter deep mound. The deposits are approximately equally divided between an eroded late Pleistocene/early Holocene paleosol and a late Holocene cap. The cap is at least partly of aeolian origin.

Efforts to obtain the 16 SA 50 contract were begun by CAR personnel in the fall of 1979. The principal investigator, having had experience with such sites in other parts of North America and Europe, was attracted by the prospect of Pleistocene age deposits. Such a site would not only be of considerable scientific interest, but attract students to the field school in the summer of 1980 as well.

Early in April 1980, Joe Watkins notified the CAR of receipt of the 16 SA 50 contract. However, more specific notification was delayed until mid-April by a bid protest. Formal notification was forthcoming by telegram on April 14 and the contract received April 24.

A five-day field expedition was launched almost immediately upon receipt of the telegram. At midnight on April 15, the principal investigator and a crew of three left for Fort Polk to explore and auger the site and meet Fort Polk personnel. We arrived at midday on Apri1 16, surprised at the length of the trip, but pleased to be met by Ron Tomas, James Grafton, John and Billie Guy, and others. We were soon apprised of the folk history of Peason Ridge. On the night of April 17, rain and wet roads forced a move to the site, where we stayed for the duration of the exercise. Fortunately, both weather and roads improved consistently over the next few days. 
Our geomorphologist, Fred Nials, arrived on April 17; his efforts and those of the auger crew rapidly revealed the geomorphological and sedimentological nature and setting of the site. An incipient fragipan within the walls of Servel10's (n.d.) test pits precluded the possibility that the site was disturbed by recent military traffic. A preliminary report was prepared in the field. Also, transit shots were taken to generate a contour map of the area (Fig. 3 ).

Upon our return to UTSA on April 21, chemical analyses of soils for pH and phosphates were initiated. During the last 10 days of the month, laboratory analyses proceeded in conjunction with administrative and material preparation for the field season.

A literature search of the archaeology of the Louisiana/east Texas/Arkansas area and of lithic tool kits in that and surrounding areas (the Plains and eastern United States) was launched. To give these researches a common underlying theme, we incorporated the development of a nonprojectile point tool typology into the more general background. Properly managed and quantified, such a typology would serve as a vehicle to test the tool kit hypothesis posed in our original proposal.

During the first 10 days of May, gathering supplies, chemical analys is of soils, and recruiting and organizing the field school occupied Gunn, Scruggs, and Sims. Colorimeter tests of phosphates were delayed by acquisition of chemicals and proved too slow for extensive use in the time remaining. However, they did provide a useful backdrop for the more extensively used spot test. Results showed that there were accumulative layers of phosphate in Area $A$, and that phosphate concentrations were generally higher in Area $B$. Our subsurface testing also showed that the areas with respectable deposits were substantially smaller than anticipated.

Thanks to the cooperation of the UTSA purchasing department and Mary Lou E1 I is of the CAR, acquisition of supplies and organization of project personnel were almost completed by the tenth of the month. Supplies were purchased in San Antonio when possible, but most were purchased in the Florien/Many, Louisiana, area so that supplies would not have to be transported across 500 intervening miles. Field school and later excavation personnel came from several Texas educational institutions and England.

Gunn, Scruggs, and Sims loaded equipment on May 11 and journeyed to the site to set up camp and begin excavation. Brown and Sullivan arrived at the end of the week. Huber joined the crew after the beginning of the next week. It rained the first six days at the site, so we took advantage of this enforced leisure to examine the results of our chemical tests, auger soils data, and plan the excavation.

Complementing this effort was a visit by Victor Carbone, Edwin Hession, and Frank Servello. Through them we were able to broaden our knowledge of previous excavation at the site and clarify our understanding of the government's project expectations. Talks on May 15 and 16 led to an agreement to concentrate on questions raised by Carbone relative to geomorphology, soils, and cultural chronology of the site and to meet again at the site during the second week of June. This meeting would include Fred Nials and Ed Garner of our staff and a soils morphologist commissioned by the government. The goal was to resolve unanswered questions of geomorphic and pedogenic development at the site. 


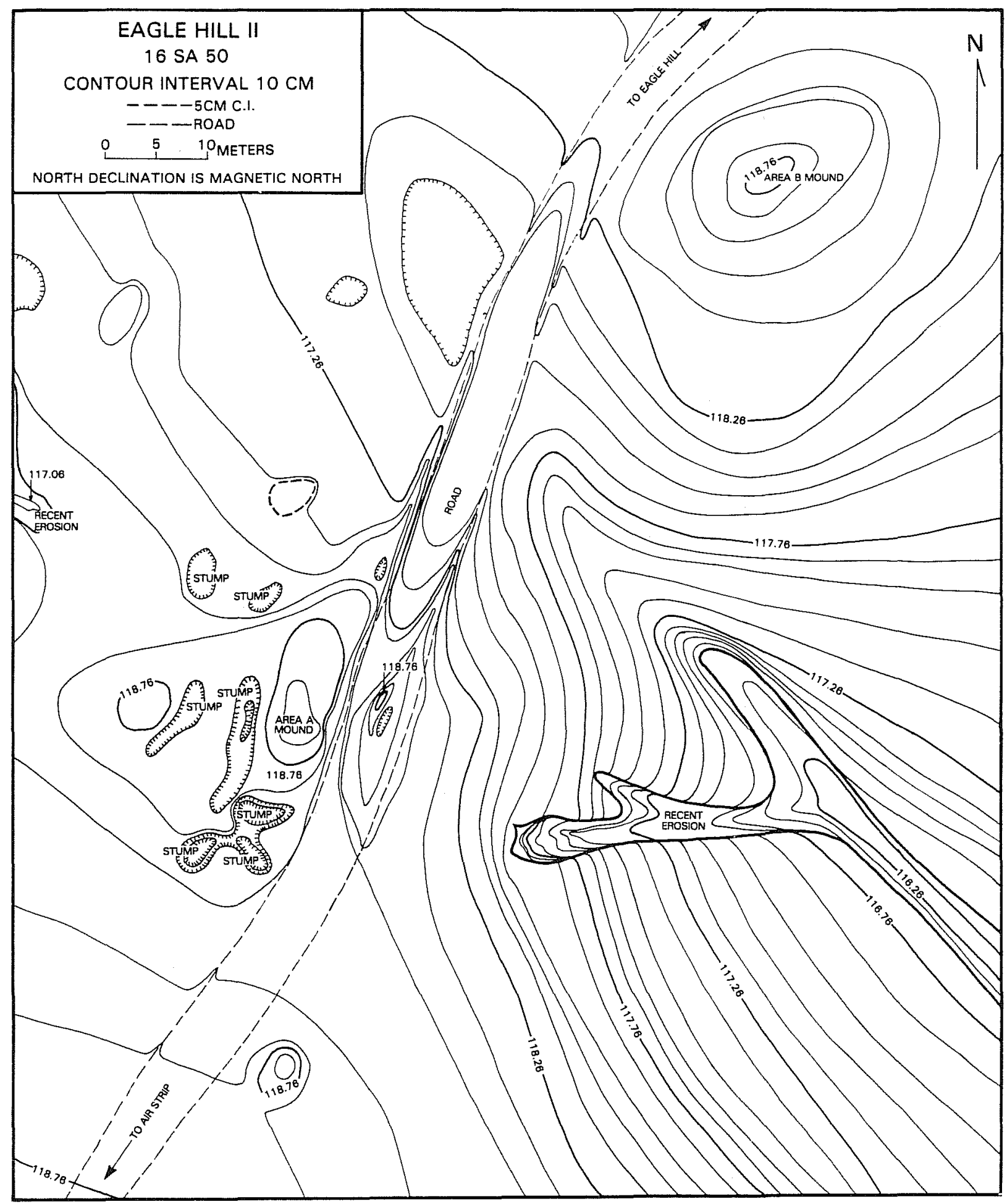

Figure 3. Contour Map of Eagle Hill II, Areas A and B. 
As with the earlier field excursion, excavation began again by opening Servel10's test pits. Since we were not equipped to work in the rain, this operation was hampered. Our most judicious move upon arrival was to leap out of our trucks into the rain and cover the site with polyethylene tarp to save it from excessive water. If this had not been done, the subsequent six days would have been most unproductive. Re-excavation was begun on May 13 and ended simultaneously with the beginnings of excavations on May 16. By then, we had learned to use our tarps to facilitate excavation during light rain. During the third week of May, we concentrated on excavating a $1-\mathrm{m}^{2}$ block of Servel1o's checkerboard pattern to clarify our stratigraphic problems and some of the cultural/chronological problems Carbone had raised. We also planned to define strata and substrata for reference during subsequent planar excavations and to develop our excavation, recording, screening, and other techniques. We found water screening to be quite efficient. The principal investigator returned to San Antonio on May 22. Excavation efforts continued at a more modest rate, while camp facilities were built.

Our excavation techniques were quite slow compared to the more customary approaches. We were, however, recovering substantial information on artifacts and cultural substrata. While each item of that battery of information provides some data on the cultural and stratigraphic situation, it may or may not be indispensible, depending upon the kind of information that is ultimately desired. Our approach was state-of-the-art relative to occupation floor data recovery. We did, on occasion, streamline those techniques, as long as they agreed with our research goals; the integrity of the data set was maintained so that it would be respectable and serviceable to future generations of archaeologists.

Pottery was recovered from Soil Horizon A2, and two Archaic points were recovered from the lower Soil Horizon A2 and the upper Soil Horizon IIB. The two points were in undisturbed context and contradicted Servel10's position that there was a Clovis occupation in the sediments of the lower A2/upper IIB soil zones. While cleaning the test pits, we did recover flakes in the gray clay interface (lower IIB) that could be of Paleo-Indian workmanship.

During June, the UTSA Archaeological Field School participated in the Eagle Hill excavations. Good weather permitted us to excavate a $5-\mathrm{m}^{2}$ block of Area $A$ from the surface to the IIB/IIIB interface. Early in the excavation it became apparent that we could not excavate an adequate area using the most precise excavation technology, so a vertical sampling scheme with a mixed excavation strategy was developed (this will be discussed in detail in a later section). Cultural strata judged to be more important were excavated with trowels. Other zones that appeared to be less important (disturbed or deflated) were removed with shovels.

Ed Hession, Victor Carbone, John Foss (a soil scientist consultant for Interagency Archeological Services--Atlanta), and Fred Nials and Ed Garner of our staff visited the site to clarify the pedological and geomorphic situation. The consensus was that, the site was as intact as an open site could be expected, and that excavation should continue.

Aside from the Area A controlled excavation, all other activities were initiated in June. With a mind to further excavation, a control column was opened in 
Area $B$ to determine the nature of the occupation floors. With student help, the laboratory analyses at UTSA continued throughout June, primarily directed toward analysis of artifacts from the Area A control column.

During July, crew morale sagged as excavators confronted the hard realities of the lower levels and the daily routine of professional archaeology. From the laboratory came our first detailed view of the materiais; from kind neighbors on Peason Ridge came some insight into the probable prehistoric hydrology of the area--a picture which suggests a much more favorable situation than can be observed today. Finally, our thoughts turned to profiling, backfilling, and calling in some second opinions for what we had found in the field and were likely to find in the laboratory.

August saw the end of the field season and the beginning of the laboratory season. Due to complications of IAS reorganization, a visit to the site by IAS personnel did not materialize. However, consultant Albert $C$. Goodyear did visit the site and provided insights from a pan-southeast perspective. By the end of the month, the bulk of curation was completed, as well as keypunching of the summer's data store. In September, data generation and analyses reached a detailed planning stage. Laboratory analyses of Eagle Hill materials was begun:

Lithic Analysis--Quantities and types of material were coded at one-meter and one-centimeter precision levels.

Wear Analysis--Eric Gibson, who had attended one of Lawrence Keeley's workshops on lithic use-wear analysis, developed a coding scheme for the provenienced artifacts from the five targeted occupation planes. Since there were over 1500 such artifacts, it was necessary to devise a rapid coding format. We identified the general types of material on which artifacts were used, i.e., hard or soft substances, and the type of technological activity indicated in a square.

Material Analysis--David Brown proceeded with plans to perform neutron activation analysis on ceramic and lithic materials.

Clay Artifacts--David Brown also organized the ceramics. Joan Sherwood, a graduate student at UTSA, worked on the clay balls.

Literature Search--Royce Mahula was assigned to unravel the literature search problem. The lack of formal tools recovered during excavation forced us to develop a new scheme--bracketing Louisiana with materials from surrounding states in the context of presumed ecotonal shifts across the region.

Granules and Pebbles--Margo Lopez, an undergraduate student at UTSA, undertook analys is of granules and pebbles.

Charcoal--Pat Wallace, a graduate student of UTSA, analyzed the carbonized plant macrofossils, in addition to her duties as data manager. The materials were weighed and mapped in preparation for selecting radiocarbon samples. We received a post-1950 date on the carbon from the soil profile $100 \mathrm{~m}$ north of the site and assumed that its colluvial/fluvial sediments were in large part a product of stumping activity of the 1950s. 
Geomorphology/Pedology--Both Ed Garner and Fred Nials continued work on their individual contributions.

During 0ctober, most of the laboratory effort was directed at collection of data on lithics, clay balls, charcoal, pebbles, and flake concentrations. A wear analysis was performed on 1500 flakes from five targeted occupation floors and preliminary analyses conducted.

Neutron activation studies proceeded in a most encouraging manner. Brown prechecked clay and lithic specimens through X-ray fluorescence to avoid unnecessary expense. In addition, when he visited Texas A\&M University, the staff of the Nuclear Research Laboratory provided several thousand dollars in matching funds.

Pollen samples were sent to Mark Sheehan for analysis. Careful examination of carbon samples for radiocarbon dates revealed that some of the carbon was the result of burned roots.

In November, the Eagle $\mathrm{Hi} 11$ Project moved from the data collection stage to the preliminary data analysis stage. The bulk of the data was coded, keypunched, and proofed. Students and staff were busy analyzing their data, primarily for statistical patterns in the vertical aspect of the site.

Analysis of the horizontal aspect of the site also commenced. Maps of occupation planes were prepared showing various types of material. Additionally, analysis of wear pattern data and plotting the distribution of 300 flakes, which showed evidence of use, was completed.

Various specialized tests continued to yield data into January. Among these was neutron activation of cherts and clays from Louisiana, Texas, and Arkansas. Charcoal had been sent to the Center for Applied Isotope Studies at the University of Georgia at Athens for radiocarbon assay. Seven constant volume samples (CVS) were sent to Jerry Hoffer at The University of Texas at El Paso for a series of X-ray fluorescence tests. These samples were taken from the High Resolution Environmental Column (HREC) from levels targeted for analysis. Principal components analysis suggested a great deal of free silicon in the samples; this provided us no useful information. We were more interested in clay particles, etc., that might bind trace elements concentrated by human activity. Therefore, we instituted a procedure to settle particles larger than fine silt. A most encouraging letter from Sheehan indicated that there was pollen in the HREC with reasonable botanical assemblages. This seemed almost too good to be true; Sheehan tried a new technique that concentrates pollen grains to increase frequencies and variety of species, but the results were disappointing.

In December, a preliminary report was issued that suggested that the remaining minimal horizontal deposits could add to existing horizontal information. However, a more than adequate vertical sample was recovered, and it seemed likely that the primary archaeological value of the site had been recovered.

January through March 1981 were devoted to data analyses and report preparation. Some analytical activities were continued in student papers. Radiocarbon assays arrived, and lack of dates for the IIB soil zone encouraged an 
attempt to date fire-burned 1ithics through thermoluminescence (TL). The results of TL dating provided a chronological context for the lower soil zone.

\section{A. PRELIMINARY INVESTIGATIONS}

Preliminary Field Examination of the Site

The preliminary field excursion was made between Apri1 16 and 21, 1980. After spending the afternoon looking over the site, we returned to John Guy's home for the night and received a substantial introduction to the local archaeological lore. Throughout the remainder of the field program, John Guy was a consistent supporter of our efforts, as well as Environmental Office personnel.

During the week before our arrival at Fort Polk, it rained in Louisiana and promised more. Given the difficult status of the roads and the brevity of our planned stay, we stocked up on food and moved to the site for the duration of the period. This was done to avoid spending time extracting our vehicle and its trailer from forbidding mud holes, which were all too frequently encountered on the northern reaches of the Fort Polk Reservation. During the first two days of grid setting and augering, we had to retreat rapidly to the vehicle more than once to avoid sudden showers. However, the weather improved steadily thereafter, and the last days were most pleasant.

Trapped as we were, we were not distracted from our tasks and logged a substantial list of accomplishments in those four working days. The crew consisted of Joel Gunn, principal investigator; David Brown, field supervisor; Lang Scruggs, data and logistics manager; and Darrell Sims, aspirant archaeology student. Fred Nials, geomorphology consultant, arrived Thursday, April 17.

The Fort Polk Connection--The Environmental office at Fort Polk provided us with much needed maps of the area, introductions to area residents, and guidance in finding our way about, which saved immense amounts of our shortest commodity--time.

The Community Connection--We were able to locate relevant roads, grocery stores, Tumber yards, service stations, cafes, and motels necessary for the successful maintenance of an archaeological crew.

History of the Site--Jim Grafton provided us with an oral history of the site, which included the location of Frank Serve110's test pits and explanations for the devastated appearance of Peason Ridge. Thanks to his efforts, we learned that the holes that pockmarked the ridge were left by the bulldozing of tree stumps. We could very well have wasted time and effort testing and puzzling over their genesis.

Condition of the Site--The rapidity of the headward erosion of the two gullies encroaching upon the site was apparent, as were the efforts of the army to prevent or at least slow the destructive effects. The most threatening erosion from the north was already flanking the culture-bearing deposits on the west and eroding rapidly into the bedrock clay.

The present site is a remnant of a larger site that existed before deforestation. As will be discussed later. Area B is probably equivalent to the upper 
soil zone of Area $A$; this suggests that Areas $A$ and $B$, intermediate zones, and adjacent zones were once a contiguous site.

Gridding the Site--A grid, aligned with magnetic north, was established over the site (Fig. 4); pin flags were placed at 10-m intervals and other permanent data established. Grid point E3020 N1000 was located at the highest point on the Area $A$ mound. It was also conformant with a yellow stake in the grid system established by Serve110. Our grid system was $12.5^{\circ}$ out of al.ignment with Serve170's grid. Over 200 transit shots were taken. These included elevations of all bore holes, Servello's test pits, and prominent topographic features.

Geomorphology and Geology of the Site--Environs were geomorphically analyzed, and a preliminary report prepared. To check the correspondence between our observations and Servello's, we reopened his test pits in Area $A$ and mapped the profiles. Nials' observations disagree with those of Servel1o. One notable exception was an incipient fragipan in Stratum IIB2. Such a soil structure insures that the site was not disturbed by the recent military traffic, since it takes hundreds or thousands of years for a fragipan to develop. Area $A$ is al so marked by less rilling and gullying than reported by Servello, and we observed no vertical "tongues" as Servel10 had indicated. There was heavy mottling in the levels as he had indicated, but mottling develops with the aging of the soil and implies no disturbance.

Groundwater--Upon our arrival, we were able to observe the groundwater at the site, thanks to their sodden conditions. The groundwater might have hampered excavation, but our excavation efforts in Servel10's test pits lowered the water table in the adjacent bore holes.

Crayfish Problem--Thanks to the assistance of an amenable crayfish, we gained some insight into the hazards of the site. No doubt, wet-season crayfish activity accounts for some disruption of the soil, but there is no reason to believe that the problem is any more acute at Eagle Hill than similar rodent and crustacean problems elsewhere.

Paleosol--In a gully north of the site, we observed what we thought to be a paleosol. A radiocarbon assay of charcoal in this horizon proved to be younger than 1950 (UGa-2531) and reflects the extent of the damage perpetrated by the l950s stumping operations.

Site Location Hypothesis--During our stay on Peason Ridge, a preliminary sitelocation hypothesis was developed (see Settlement Pattern section, page 140).

Where To Excavate: Bore Hole Analysis (Gunn)

One always approaches the testing of a Pleistocene age site with mixed feelings-for fear of spoiling some rare trophy or pattern. Normally, such sites are excavated in repeated seasons over long periods of time. F. Bordes has been returning to his favorite sites since 1948. For decades, each season has revealed a part of the structure of the site, and no part of the site is sacrificed as a means of understanding other parts of the site. 


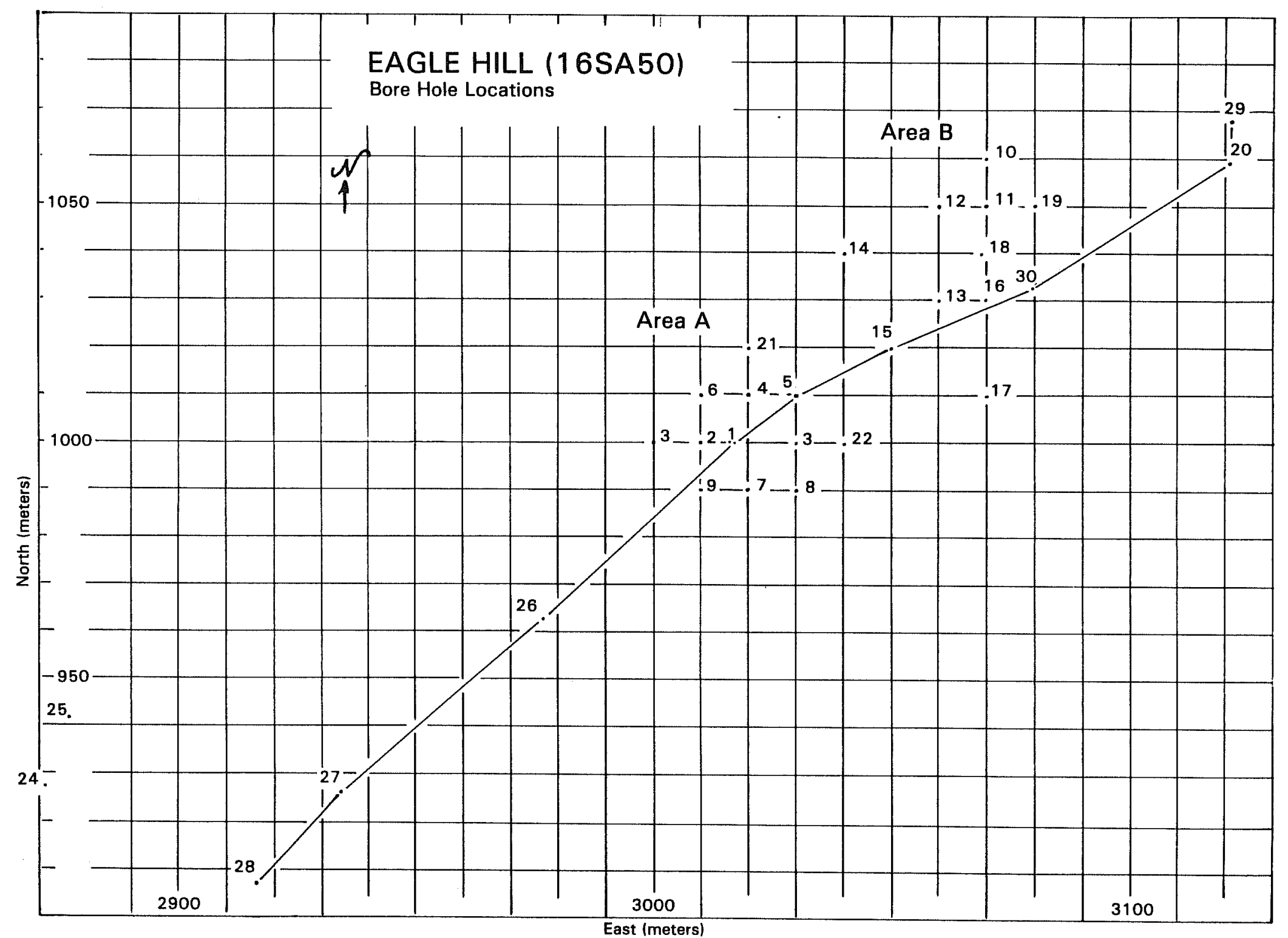

Figure 4. Locations of Bore Holes with Clay Surface. 
Time limitations at Eagle Hill, however precluded such a time-consuming and laborious process. It was necessary to know what to expect and how to manage excavation problems expeditiously. Fortunately, coring has proven to be a minimally destructive mode of data recovery. We located core holes precisely and proceeded to auger in approximately $5-\mathrm{cm}$ increments to the underlying Miocene clay (Fig. 4). The depth-to-bottom of each increment was measured. Thus, a 3-inch auger hole provided exact provenience on any artifact recovered, so that if the area was excavated, the artifacts could be mapped onto occupation floors with nearly accurate precision. Servello also found that $5-\mathrm{cm}$ increments were sufficiently refined to define multiple occupation levels. The only real damage posed by augering is edge damage. Since it is unlikely that the whole edge of an important artifact could be reworked by the auger, we felt that the risk was acceptable.

Chemical analysis of soils and inspection of the site served to locate the best area for excavation. Figure 5 is an isopac map of the site. Contours show the depths of the soils above the bedrock clay. Augering showed that the area within which deposits of appreciable depth remain was relatively small. In Area A, for instance, the deposits are about $90 \mathrm{~cm}$ deep at the top of the mound. Because of a road through the site and the surrounding erosion, the area from which we could retrieve the full temporal spectrum represented at the site was small in comparison to the overall size of the site mounds.

\section{Core Series}

Three core series were taken: inside occupation areas, around occupation areas, and a transect of occupation areas and the surrounding terrain. In a11, 30 cores were recovered. The first (Fig. 4; bore holes 1-15) consisted of 15 cores taken at 10-m intervals within areas of known prehistoric habitation and at 20-m intervals within areas of unknown potential. Twenty auger holes were originally scheduled for this. However, we found that the space between Areas A and B was eroded into the Miocene clay bedrock. Except for two bore holes augered within the gully bed, cores were confined to the erosional remnants in Areas $A$ and $B$.

The spacing of holes was based on the density necessary to detect family occupation space (Yellen 1977). Yellen's examination of camping patterns among the Bushmen of the Kalihari was used as a model. The camping patterns of the Bushmen indicated that family units usually occupied circles 5 to $10 \mathrm{~m}$ in diameter. Since he studied nuclear familes, we can expect a 10-m sampling interval to detect family-sized occupation and work areas. Within Areas A and B, tests were located approximately $10 \mathrm{~m}$ apart. Nine cores were placed in Area A and four in Area B. The numbers are commensurate with a reasonable coverage of each area on the "10- $\mathrm{m}$ " principle. Seven augers were placed in the intermediate area at approximately $20-\mathrm{m}$ intervals. Twenty meters generally correspond to minimal social units; i.e., the band.

Mode of Operation

The mode of operation of an auger team is important to its results and to the transition to the second core hole series. In addition to the person who turns the auger, at least two other persons are necessary to observe the soils and 


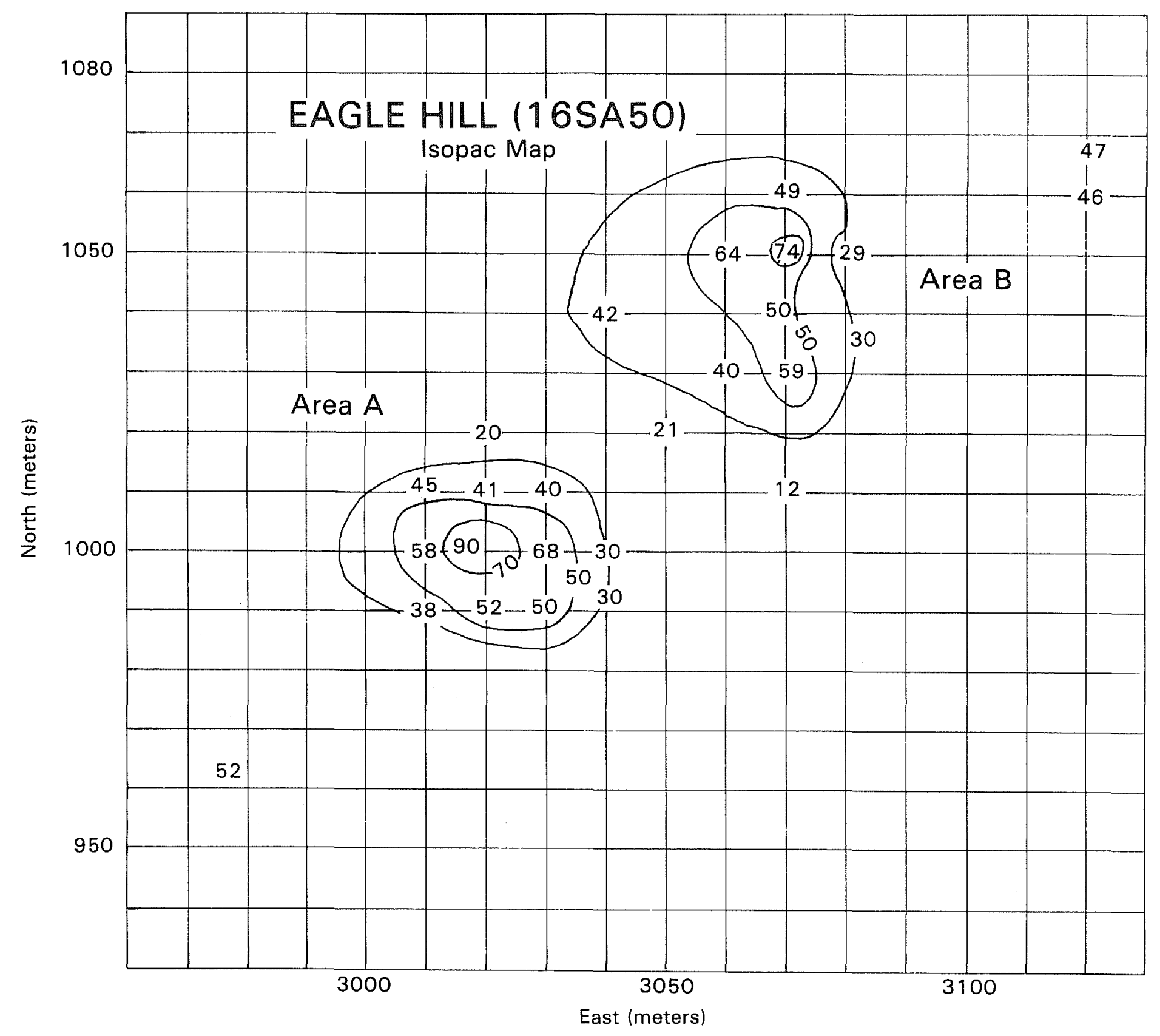

Figure 5. Isopac Map Showing 10-Meter Grid and Depth of Deposits in Areas A and B. 
keep records. The observer determines the grain size, texture, and color of the soil and decides when interfaces between lithological units are crossed. The recorder enters information on computerized data-coding forms and written notes. Samples are bagged in ziplock plastic bags.

Synthesis of information is very important. Through conversation, a team consciousness of the subsurface characteristics of the area being tested is developed. During the augering exercise, the team offers comments on what is being encountered in terms of discontinuities, soil color, etc., and their observations are duly entered in the log by the recorder. As the work progresses, a mental picture develops in the minds of the auger team members as to the subsurface trends in frequency of artifacts, and at what depths. In most cases, these trends lead to further questions that can only be answered with judgmentally placed auger holes. For instance, the team may have noticed that the concentration of flakes is thinning to the west and, judging by the rate of the decreasing frequencies which they have kept in mind, suspect that the margin of a lithic workshop area in the second stratum down is near point $X$, so many meters to the west. If this hypothesis and test sequence work out, an important boundary has been established. In the bore hole sequence, units 1625 are such judgmentally placed holes.

To determine the depth of the bedrock clay, the final series (Fig. 4; bore holes 26-30) was placed on a transect parallel to Peason Ridge, $150 \mathrm{~m}$ southwest of Area $A$ and $100 \mathrm{~m}$ northeast of Area B.

\section{Analysis of Core Samples}

Upon returning to UTSA, the coding of a number of criteria proven useful in previous analyses (Muto and Gunn 1982), such as color, texture, and the presence of artifacts and charcoal, was immediately ready for analysis following completion of data entry. These observations allowed us to map artifact concentrations and 1ithologic characteristics at various levels and thus, define cultural'areas. Increment depths, sediment texture, and artifact locations are shown for downhill transects of Areas A and B in Figures 6-9.

We were very much interested in the extent of human activity in the locality. For some time, phosphate and $\mathrm{pH}$ have been regarded as indicators of human activity and may define to some extent the nature of that activity. Yellen (1977), for instance, found that hide scraping was done in the area marginal to the camp's hut complex. One might expect phosphate residues to be associated with hide processing. Accumulated and decayed plant remains associated with bedding, huts, etc., would leave a higher $\mathrm{pH}$.

To retrieve this information, samples were processed for $\mathrm{pH}$ and phosphate and the information added to the data set. Samples from all cores from all soil zones were analyzed for chemical constituency. On the Tombigbee River, phosphate was found to be prone to downward migration in the soil (Muto and Gunn 1982), while pH seemed to stay in place. By examining the phosphate concentrations at the bottom of the section, we expected to locate areas where humans had chemically altered the soil. In those areas, we proceeded to analyze up the column to locate the relevant strata, presumably with $\mathrm{pH}$ anomalies that should pinpoint appropriate levels. 


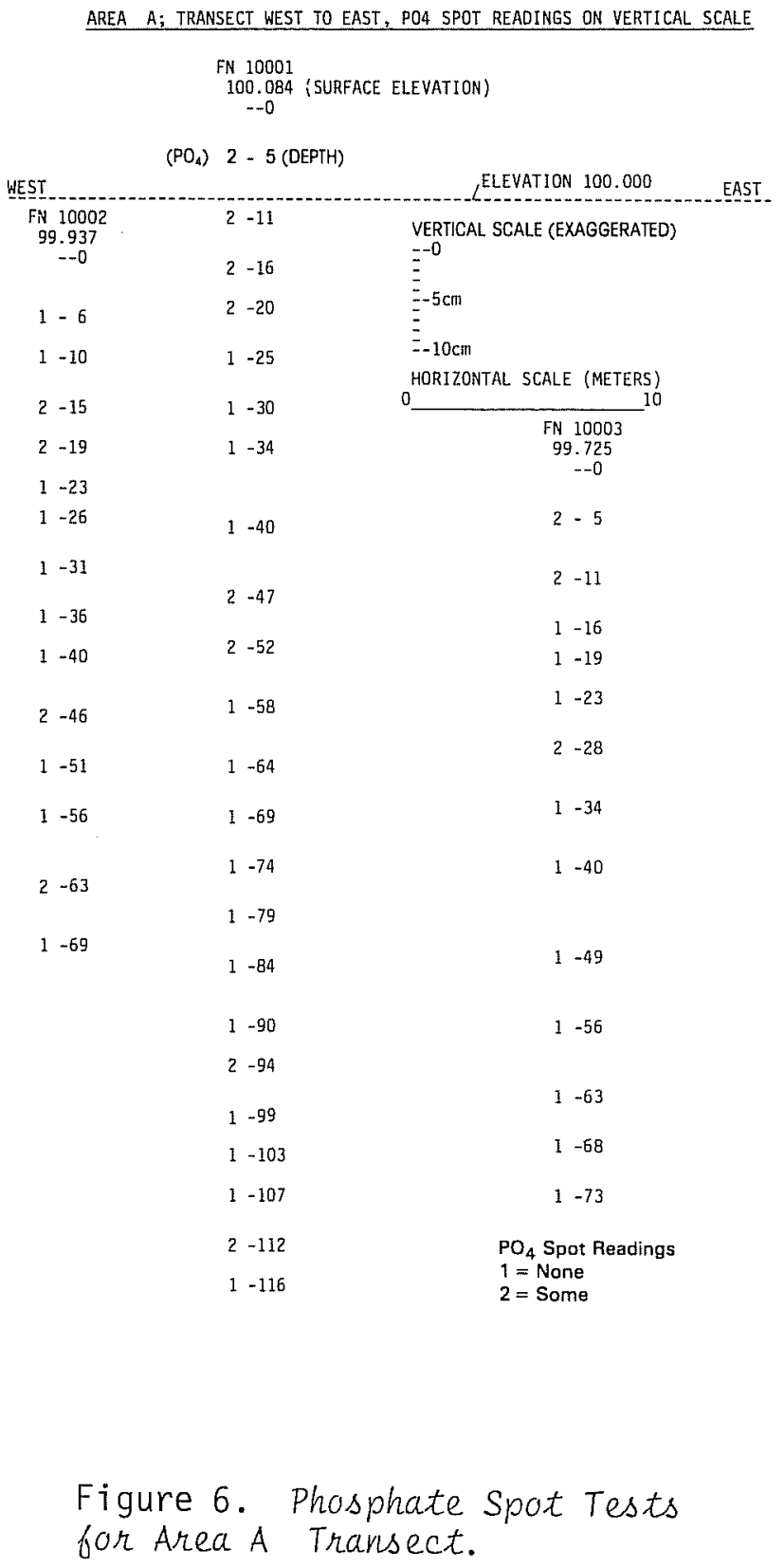

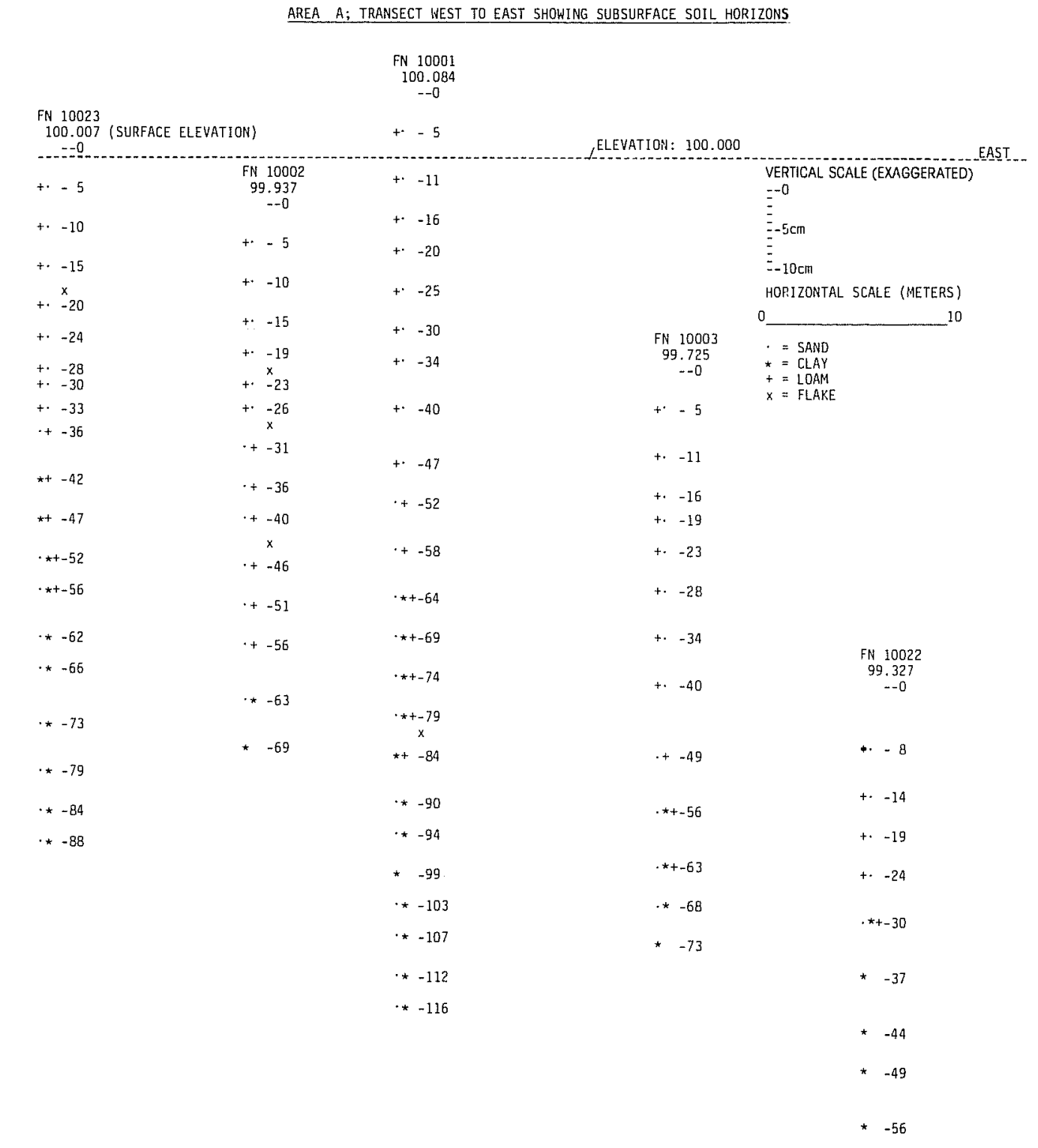

Figure 7. Soil Texture observations for Area A Transects. 
AREA B; TRANSECT SOUTH TO NORTH, PO SPOT READINGS ON VERTICAL COLUMNS
SOUTH

VERTICAL SCALE (EXAGGERATED)

$=-0$

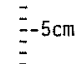

$=-10 \mathrm{~cm}$

HORIZONTAL SCALE (METERS)

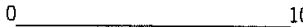

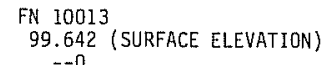

$2-5$

$2-10$

$2-15$

$2-19$

$2-25$

$2-3$

$1-4$

$1-45$

$1-70$
$1-74$

$\mathrm{PO}_{4}$ Spot Readings
$1=$ None
$2=$ Some

Figure 8. Phosphate Spot Tests for Area $B$ Transect.
AREA B; TRANSECT NORTH TO SOUTH SHOWING SUBSURFACE SOIL HORIZONS
NOBTH

FN 10011
99.866
--0

$\left(\mathrm{PO}_{4}\right) 1 \cdot 5$ (DEPTH)

$$
2-11
$$$$
2-16
$$

$2-22$

$2-27$

$1-31$

$2-38$

$2-44$

$2-51$

$1-60$
NORTH.

$\begin{array}{ccc}\text { FN } & 10010 \\ 99.812 & -0 \\ 1 & - & 6 \\ 2 & -11 \\ 2 & -15 \\ 1 & -19 \\ 1 & -24 \\ 2 & -30 \\ 1 & -40 \\ 1 & -46 \\ 1 & -49 \\ 1 & -55\end{array}$

HORIZONTAL SCALE
ELEVATION 100.000 VERTICAL SCALE

FN 10011
99.865 (ELEVATION)
--0

FN 10010
99.812
--0

+. -5

$+-6$

$+\cdot-11$

+. -15

+. -19

+. -24

+. -30

+. -30

+. -40

+. -46

$+-49$

* -55

$\cdot-11$
$x$

+. -16

$x$

$+-22$

+. -27

+. -31

+. -38

+. -44

.+-51

$+-60$

$\cdot *+-70$

$\cdot \star+-74$ --0 (EXAGGERATED) $\vdots-5 \mathrm{~cm}$

$\vdots-10 \mathrm{~cm}$ (METERS)

$$
0
$$

99.64

- = SAND

$x=$ FLAKE

$+\cdot-7$

+. -11

+. -16

$+\cdot-19$

$+\cdot-24$

+. -29

$+\cdot-36$

+. -42

$*+\dagger-50$
$* \quad-52$

$\begin{array}{ll}\text { FN } 10013 & +=\text { LDAM } \\ 99.64 & =\text { SAND }\end{array}$

FN 10016

9.511

$+\cdot-8$

$+\cdot-13$

+. -18

$+\cdot-23$

$+-29$

$+\cdot-35$

$-42$

$\star+-51$

$\star+-59$

* -67

* -73

$-81$
Figure 9. Soil Texture observations for Area B Transect. 
Without knowing the nature of the geochemical system of the sites, the exact nature of anomalies or signatures is hard to define. All constituents of that system (which is dependent upon the environment) must be in equilibrium. For instance, phosphate levels are dependent upon the acidity of the soils. If the soils are very acid, a minute trace of phosphate might be very important. To assist in this analysis, the chemical nature of offsite soils was examined. Also, the last two millenia have seen a great deal of climatic variation, and it is likely that Soil Horizon $A 2$ is a reasonable estimate of the surface soils of the past. In a sense, we would like to subtract the effect of time on the lower horizons by examining the difference between SoiT Horizons A2 and IIB.

Selected results of the chemical tests are illustrated for discussion. Figure 10 shows the results of the bottom of hole pH tests. Figures 6 and 8 are downhill transects of phosphate determinations. The methods used for these determinations are discussed in Appendix A.

Phosphate Ion Distribution in Soils (Gunn, Lewis)

As is often the case, anticipated and actual results diverge and suggest reformulations of plans and models. Surface sediments in the Eagle Hill locality were much more porous than we had envisioned in our planning and the bedrock much more impermeable. This situation led to a re-examination of our soil chemistry research design. Because of the $\mathrm{pH}$ and porosity of these surface sediments, we came to expect significant downward migration of the phosphates. However, there was some chance that the phosphates would be bound in place (either by iron or by the clays at the bottom of the section) and therefore not migrate laterally, once they had descended from the surface sediments.

The prospect and character of phosphate binding is discussed in the literature. Other researchers have found that the native phosphate distribution in soil profiles depends upon the degree of development and the composition of the soil (Smeck 1973; Sjoberg, Smeck, and Runge 1971). The extent and rate of phosphate elevation depends upon the $\mathrm{pH}$ (soil acidity) and the Eh (oxidation-reduction potential) of the soil (Patrick and Khalid 1974). In systems containing iron, aluminum, and calcium, phosphate ions are most soluble at pH 7. For archaeological studies, the pH must be greater than 5.5 (Sjoberg 1976). For anaerobic, low Eh conditions, phosphate will be mobile at somewhat lower pH values.

The formation of an alluvial phosphate concentration at the soil C-horizon (equivalent to our clay bedrock contact) is commonly observed. Whether or not this is modified, when associated with anthrosols (such as occur in archaeological sites), has not been established. The relative rates of phosphate dissolution from various soils have been studied by 01 sen (1975). The interaction of clay minerals with phosphate in soils and sediments has been studied by Nriagu (1976) and Viellard, Tardy, and Nahon (1979).

The basic questions at Eagle Hill relate to the rate of movement of phosphate in soil in the time frame of the anthropogenic modification of soil phosphates. Eidt (1977) was able to show that phosphate concentrations in the upper $50 \mathrm{~cm}$ of soil, which reflected anthropogenic activities to 700 B.C., were preserved. 
EAGLE HILL (16SA50)

$\mathrm{pH}$ at the Bedrock Contact

\begin{tabular}{|c|c|c|}
\hline \multirow[t]{2}{*}{ Area B } & & 4.9 \\
\hline & 4.9 & 5.2 \\
\hline \multirow[t]{2}{*}{5.6} & & 4.9 \\
\hline & 5.1 & 4.7 \\
\hline \multicolumn{3}{|c|}{5.6} \\
\hline & & 5.1 \\
\hline 4.9 & & \\
\hline
\end{tabular}

5.2

Figure 10. pH Read with Test Strips at the Clay Bedrock Contact. 
The evidence from this work strongly suggests that man's influence will be most evident in the near-surface soils or in those now buried that were directly affected by man's activities. Cultivation of plants can reduce the phosphate concentration; burials, human and animal wastes, and food processing can increase the phosphate concentration.

To incorporate this information into the research design, the highty migratory properties of phosphate suggested first analyzing the bottoms of bore samples. Where high phosphate concentrations are found, we might readily expect significant sites above the bore hole bottom.

Given the probable seasonality and brief span of camps on top of Peason Ridge, the sandiness of the soil suggested that $\mathrm{pH}$ would not be a particularly useful indicator. On the other hand, pH was expected to provide us with some interesting information on phosphates. Phosphate is most soluble at pHs of 5.5 and 6.5. If the pH was in this range, we could expect phosphate to migrate down the section. If the soil was more alkaline, perhaps greater than 6.7, then the phosphate could be locked in the section and indicate the levels in which occupation would be found. As Figure 7 shows, $\mathrm{pH}$ in most parts of the site falls within the range at which phosphate is highly soluble. There appears to be no significant lateral differences in the distribution of $\mathrm{pH}$.

Since the $\mathrm{pH}$ indications seemed to be rather indeterminate with respect to the research design, we undertook phosphate tests in several columns that seemed to be most critical. As Figure 6 shows, there are concentrations of phosphate in the Area A section. An expectable concentration occurs in the undisturbed humic zone on top of the mound. To the west in the stump hole, this zone is understandably truncated. A comparison of Figures 6 and 7 indicates a concentration of phosphates near the bottom of the sandy loam. This horizon eventually proved to be our densest occupation at the site. It is also about 1000 years old, apparently within the staying duration of the phosphates.

Figures 8 and 9 show a similar set of data for Area B. Phosphate readings were generally higher in Area B as compared to Area A. Interestingly enough, the high readings are toward the surface with no concentration at the bottom. As in Area $A$, the high readings are associated with the upper layer of sandy loam. We take this to support the geomorphologist's belief that Area B is a very recent development and equivalent to the sandy loam on top of the Area $A$ mound.

The two bore holes (to the left in Fig. 10) suggest a concentration of phosphates at the contact between the colluvium and the Miocene lake sediments. This is the horizon from which the Paleo-Indian occupation was excavated. Whether the phosphates are there because of the Paleo-Indians or because of downward migration of phosphates is unclear. Using phosphate fractionation, a more detailed study, might determine the source.

The highest point of the mound is located over the deepest part of an apparent basin in the Miocene lake sediments/Pleistocene weathered Soil Horizon IIIB. Taken together with the impermeability of the lower sediments, the basin may have formed a tiny aquifer that fostered surface vegetation and accounted for the existence of the Eagle Hill II mound. 


\section{Conclusions}

The bore hole operation familiarized us with the subsurface sediments of Eagle Hi11. It showed that there were concentrations of phosphates, which eventually proved to contain important occupation horizons. Peason Ridge had been eroded to the Pleistocene soils and pockmarked by erosional remnants, some of which contained cultural material. These were Areas A and B of Eagle Hill II.

\section{B. TESTING}

In the original proposal an extensive testing program was scheduled for the end of the summer. However, our augering operation revealed that most of the area on the ridge between erosional remnants was eroded to the Miocene clay and therefore devoid of cultural material. We augered some of the erosional remnants in the vicinity of the site to determine their character. The soil was generally very sandy and bright yellow, in contrast to the darker colors of the soil horizon in the site. This, coupled with their less advantageous positions relative to the southward flowing gully, which reportedly carries water from seasonal springs, suggested that occupation in the more distant erosional remnants was uni ikely.

Additionally, the 50-cm test pits that Servello used for his subsurface survey were found on virtually every prominence on the ridge for miles around. It seemed that the resources of the project could be better spent excavating the Area A mound rather than duplicating Servello's testing operation. IAS officials agreed.

Given this situation, testing was Timited to Area $A$ and the environs of Area $B$. The excavation of $4 \mathrm{~m}^{2}$ in Area $B$ amounted to little more than a test. Area $B$ was determined to be geomorphically equivalent in age to the upper soil horizon in Area A. A control column similar to the one in Area A was excavated (see Project Methodology). Its artifacts were examined, but none were diagnostic and the occurrence of flakes low. Three more 1- $\mathrm{m}^{2}$ units were excavated in arbitrary levels. No more spectacular materials appeared in this control column, and the effort was abandoned with IAS approval. The information to be obtained from the Area A mound was again judged more valuable.

\section{OBSERVATIONS ON SITE LOCATION ON PEASON RIDGE (Gunn, Brown)}

Our efforts were concentrated on exploring the Eagle Hill II site. However, living and working in the area through the summer provoked some thoughts on the location and preservation of sites on Peason Ridge. The model is not very sophisticated and certainly not very well tested. However, given the availability of Titerature concerning the region, it seemed advisable to make these observations a matter of record so that they stand as testable hypotheses. We will deal with five basic concepts ranging from specific locational phenomena to general site patterning on Peason Ridge. These concepts constitute the beginnings of a site-location model that explains the locations of sites and the reason for their existence by means of geomorphic and/or cultural causes. This model is elaborated in the section on site catchment. 
Erosion is a key element in locating sites. Several aspects of erosion are pertinent. As discussed in the section on geomorphology and soils, natural erosive forces were well under way before the advent of Europeans. However, deforestation during this century appears to have accounted for the bulk of the erosion now evident. In combination, natural and man-made erosion accounts for the destruction of most of the colluvial land surfaces that once probably contained evidence of human occupation. The result is a relatively unbroken underlying Miocene clay surface upon which rest erosional remnants of colluvium, some located in culturally favored areas and some not.

The confinement of archaeological remains to prominent erosional remnants appears to be quite concise. For instance, because surface sediments have eroded into the Miocene clays, the zone between Area A and Area B in the Eagle Hill locality is devoid of artifactual material. In areas not marked by erosional remnants, most of our tests showed only a thin veneer of recent sands over the Miocene clay.

In addition to normal erosional processes, Peason Ridge was, and sti11 is, plagued by a peculiar variety of soil disturbances generated by the removal of large tree stumps. According to J. Grafton, the virgin pine forests were logged during the early part of this century. After World War II, the stumps left by these earlier logging operations were bulldozed to recover gums, resins, etc. These operations left gaping holes in the landscape that are still being healed by the movement of nearby sediments into the voids. Such scars exist within the perimeter of Areas $A$ and $B$ and contribute to the diminished size of the sites.

Given that Peason Ridge is dotted with erosional remnants, there appear to be areas favored for occupation and others not. The proximity of the Eagle Hill locality to a possible seep spring is of interest. We tested a few of the mounds proximate to the known archaeological sites and found no archaeological materials. Servello (n.d.) appears to have followed a similar procedure with 50-cm test pits. When his data become available, it will be interesting to see if a similar lack of occupation is found in the mounds adjacent to the site. Our auger test also showed that there were no buried soils or soil structures in the extraneous mounds, and that these sands were a bright yellow color not found in the remnants evidencing human occupation.

While augering various mounds, we noticed that water stood in the bottom of the auger holes placed in erosional remnants, but not in holes adjacent to mounds. Several possible explanations can be made: (1) capillary action in the mounds draws water up to a higher level in the remnants; (2) the Miocene clay is sandier and more porous under the spots where the mounds are located; or (3) the mounds are located in basins in the Miocene clay. Whatever the reason, it seems quite likely that the erosional remnants can be accounted for, at least in part, by favorable conditions for erosion-preventing vegetation. There appears to be some support for the latter two arguments, because of the enhanced status of vegetation on remnants. In any event, the possibility exists that the location of archaeological sites on erosional remnants is independent of the existence of the remnants, and it is only by good fortune that prehistoric 
remains appear in occasional remnants. In other words, the remnants are not occupation mounds, but mounds which happen to contain pieces of once continuous living surfaces.

Given the elevation of sites, it seems likely that the force controlling the location of archaeological remains in this milieu is the presence of water. Sites should, therefore, be located in erosional remnants near existing or relict streams or springs.

\section{PROJECT METHODOLOGY}

Paleo-Indian Site Excavation Design and Method in the Eastern United States (Brown)

Introduction

In the fall of 1979, it was decided to pursue the matter of selecting a PaleoIndian site for excavation during the 1980 UTSA Archaeological Summer Field School. Our intentions were to be as innovative as possible in our excavation methodology. However, we were also interested in the compatibility of data. A study of previous methods of excavation was undertaken. Twenty Paleo-Indian and Early Archaic excavations (Table 1) spanning the last four decades and more than two thousand miles from eastern Texas to central Nova Scotia were examined from a technical point of view to ask the question "Is there a better way?"

\section{TABLE 1. SITE REPORTS EXAMINED IN THIS REPORT}

Rodgers Shelter, Missouri

Rose Island, Tennessee

Thunderbird, Virginia

Brand, Arkansas

We 115 Creek, Tennessee

John Pearce, Louisiana

St. Albans, West Virginia

Hatchery West, 111 inois

Habron, Virginia

Debert, Nova Scotia

Holcombe Beach, Michigan

Hardaway, North Carolina

Wolfshead, Texas

Eva, Tennessee

Jake Martin, Texas

Quad, Alabama

Silver Springs, Florida

Brohm, Ontario

Starved Rock Archaic, I1linois

Parrish Village, Kentucky
(MCMillan 1976)

(Chapman 1975)

(Gardner 1974)

(Goodyear 1974)

(Dragoo 1973)

(Webb, Shiner, and Roberts 1971)

(Broyles 1971)

(Binford 1970)

(Rodgers 1968)

(MacDonald 1968)

(Fitting, DeVisscher, and Wahla 1966)

(Coe 1964)

(Duffield 1963)

(Lewis and Lewis 1961)

(Davis and Davis 1960)

(Cambron and Hulse 1960)

(Nei11 1958)

(MacNeish 1952)

(Mayer-Oakes 1951)

(Webb 1951) 
For many years, American archaeology has nurtured the association between the early Paleo-Indian hunters and the western United States. Two principal factors have supported this association. The first is simply the early and dramatic discoveries of the first recognized Paleo-Indian remains on the fringes of the High Plains. This first and oldest mystique has penetrated the literature to the degree that it has returned in a kind of circular defense for western Paleo-Indian origins. The Paleo-Indian pattern of 1 ife is more than a few spear points and dead elephants. It is a projection of these cultural associations into the kind of environment where we have found them, the High Plains. Yet Gordon Willey (1966:480) argues for a High Plains origin for Clovis peoples because their ". . . pattern of life seemed best adapted to the kind of environment which has been reconstructed for the High Plains in the Late Pleistocene. . . ."

The second important factor is the correlation between the earliest we11documented Amerindian remains and their supposed route of migration. This makes a very nice theoretical picture, but the correlation is not strong and may be even less significant. Not only is the concept of an ice-free corridor questioned (Fladmark 1979), but there are an increasing number of claims for pre-Clovis sites (Stalker 1977; Adovasio et al. 1978; MacNeish 1973; Reagan et al. 1978).

The presence of Paleo-Indian artifacts in the eastern United States has long been recognized (Roberts 1938), not just as occasional occurrences, but in quantities that far outnumber finds of western specimens (Willey 1966:48). One eastern archaeologist has gone so far as to postulate an eastern origin for the Clovis tradition (Dragoo 1976). Whether one accepts such a speculative hypothesis or not, it is clear that interest in the eastern Paleo-Indian tradition is growing (Newman and SaTwen 1977).

The growing interest in the eastern Paleo-Indian is paralleled by a growing interest in eastern Paleo-Indian sites and the special problems they present. Some of the more perplexing problems include the vast majority of projectile points as surface finds, very few radiocarbon dates associated with these finds, almost no faunal evidence (many of these sites are shallow and not all of the excavated artifacts from good subsurface contexts), a greater degree of variability in eastern fluted point styles, and fluted point styles persistina longer in the East, perhaps into the Archaic. Clearly, these problems are not unique to eastern Paleo-Indian sites; they plague archaeologists working everywhere in every period. Ultimately, practical solutions that arise from Paleo-Indian research will find a much wider application.

Research Design

When dealing with the eastern Paleo-Indian question, the serious need for relevant data is generaliy justification enough for excavation of the sites reviewed below. To excavate a site "because it is there" may be a somewhat questionable methodological approach in later and better known periods, but there is at least some support for this approach to rare and rapidly vanishing Paleo-Indian sites. On the other hand, the lack of a clear picture of what kind of data is needed or desired from a particular site may result in the inefficient and even possibly inordinately destructive collection of data. 
This is perhaps a harsh judgment and one certainly biased toward more recent excavations where explicit research design and problem orientation is becoming standard. It is tempting to state that this is a problem in excavation techniques rather than research design, and that more careful techniques are requisite, so that no data are lost. Certainly the more careful the excavation the better, but perfection is impossible. A number of constraints are present, such as time and money, as well as excavator experience and popular excavation methodology. In the recovery of certain selected or preferred data, it is invariably the case that other data are lost or destroyed.

Extreme examples of selected data recovery are not terribly uncommon. At the St. Albans site (a deep, stratified site in West Virginia), Broyles (1971:11) implicitly commits herself to the relatively common "deeper is better" research orientation when she bulldozes 30,000 cubic feet of Middle Archaic deposits in order to begin the third season of excavation closer to the Paleo-Indian paydirt. No justification is offered, although the site is being gradually destroyed by erosion from the dammed Kanawha River.

These violent examples are the tip of the iceberg of selective data recovery. Almost every archaeological recovery technique involves a trade-off of some sort. Such common practices as the use of $1 / 4-i n c h$ screen instead of $1 / 8$-inch or window screen or perhaps just the use of dry screen techniques in place of water screening can greatiy influence the amount and kind of data recovered. It is clear that within such 1 imitations, every excavation is guided by at least an implicit interest in the collection of certain kinds of data before other kinds. This implicit interest can be derived from an analysis of the excavation procedures, but the careful statement of an explicit research design often makes this derivation unnecessary.

\section{Hypothesis Testing}

It would be most inappropriate for any discussion of archaeological research design to skip over hypothesis testing and the new archaeology. If, over the past two decades, the concept of research design and the new archaeology have become almost synonymous in the minds of a few archaeologists, the technique of formal hypothesis testing is even more so. At no Paleo-Indian site in the East is this concept more closely approximated than in the report of excavations at the Brand site in Poinsett County, northeastern Arkansas (Goodyear 1974).

In his research design, Goodyear (1974:6) states that he intends to go beyond the usual approach to Dalton tools as indicators of the temporally and spatially bounded Dalton culture. Following the excellent discussion of prior research related to the Dalton culture and the problems therewith, Goodyear (ibid.) details his intentions at the Brand site:

The immediate problem to be investigated at the Brand site was an exploration into Dalton Tithic technology designed to pursue not only customary form and style of stone tools, but also to reconstruct manufacturing systems and the specific functions of Dalton tools. It was assumed that by regarding lithic technology as a sub-member of a larger energy-extracting system other 
aspects of prehistoric behavior would also be illuminated. At a more hypothetical level the field and laboratory research design was intended (1) to test certain hypotheses presented in the settlement model constructed by Morse (1971), which provided certain filed expectations to be verified, and (2) to generate other hypotheses that could be tested once certain formats of data were recovered, namely activity loci.

Goodyear's (1974:19-33) reconstruction of the manufacturing systems and specific functions of Dalton tools results in one of the most informative sections on 7 ithic analysis in a group of papers that is heavily oriented to lithic analysis. The manufacturing strategy involved in producing Dalton points is examined in detail, and a theory proposed by Dan Morse outiining the steps produced by resharpening is analyzed statistically. His conclusion may be essentially correct, but his discussion is hampered by his awkward mathematical attempts to divide an approximately continuous set of points into three discrete categories, and then to statistically prove the existence of the categories. Although there is no proof that his categories are incorrect, it appears that what his analysis of variance has done is prove that the low ends of a normal distribution bell curve are statistically different from the center, rather than prove the existence of three separate groups, as he had wanted.

His attempts at defining the functional framework of the Dalton tool kit are nicely done. It is one of the best examples of Paleo-Indian stone tool wear analyses. The sole difficulty is the heavy reliance either on speculation of function or on Semenov's (1964) classic text, either directly or indirectly through Wilmsen (1970) and MacDonald (1968). This has to be overlooked, however, since Semenov's work was the only comprehensive treatise on wear analysis available at the time.

In regard to certain hypotheses formed from Morse's settlement model, one finds that only one prior settlement hypothesis was actually tested. This hypothesis states that the site was utilized exclusively as a temporary hunting-butchering station (Goodyear 1974:104-107). The hypotheses generated during the excavation were restricted to three competing explanations of the relationships between the living floors and activity areas excavated at the site. Emphasizing the central theme of the tested hypothesis, Goodyear and the new archaeology are able to give dramatic proof of site function (a temporary hunting camp) on the basis of functional classes of artifacts (scrapers and projectile points).

The latter point is a grossly unfair simplification and ignores the many strengths of the report, but it does point to one common flaw in the hypothesistesting technique--a tendency toward oversimplification. This tendency is probabiy nowhere better illustrated than in the report of the first season of the Palmetto Bend Reservoir test excavations (McGuff 1978). For the central Texas coastal region, where ethnohistoric documentation and a relatively clear artifactual sequence indicate a long tradition of hunting and gathering, McGuff generates test expectations to prove that the precontact peoples were hunters and gatherers. 


\section{Limited Excavation Strategies}

At the opposite end from the explicitly stated research design are various implicit research designs. The most common example is probably the limited excavation strategy in which the excavator clearly approaches the site with the intent of recovering only that subset of data which is of particular interest to him. More often than not this design is implicit (as in the case of Broyles's bulldozed Middle Archaic zone), but it can be more or less explicitly stated. Goodyear (1974), for example, states his particular interest in the subtitle of his report (A Techno-Functional Study of a Dalton Site in Northeast Arkansas), and that interest is maintained throughout. Despite the fact that Dalton materials are most numerous, there is some indication of a rather long sequence at the site, including at least 37 Late Archaic projectile points and a Late Woodland house floor. The space allotted to these later occupations is equivalent to one page in a 111-page report.

Goodyear's excavation strategy can best be described as horizontal. He was interested in intrasite patterning and clearly succeeded in obtaining exactly what he wanted--a plan view of a Dalton campsite. His intense concentration on the Dalton floor makes this an extreme example, but in any case, the shallow nature of deposits at the site $(\mathrm{ca} .50 \mathrm{~cm})$ might make a stratigraphic orientation impractical.

Impractical or not, the vertically oriented excavation of shallow sites is not uncommon. In many respects, the Wolfshead site in the McGee Bend Reservoir of Texas (Duffield 1963) is like the Brand site in northeastern Arkansas. Like the Brand site, the Wolfshead site is a shallow site overlying a basal clay soil. In both the upper third or more of the profile is a plow zone, and in both, some artifacts have worked down into the lower "sterile" clay stratum. The most important differences are the interval of eleven years and a wholly different approach to understanding the site.

The orientation of the excavations at the wolfshead site is antithetical to the "new archaeology" approach used at the Brand site. Whereas Goodyear's primary concern was horizontal patterning, Duffield's was vertical patterning. Culture chronology and the projectile point sequence are the emphasis at the Wolfshead site. Given the inherent limitations of the depth (2-1/2 feet) and its plowed and sandy nature, this was certainly a valid, although difficult, goal. Not that Duffield ignores horizontal patterning. He is quick to point out the significant differences between two major areas of the site (Duffield 1963:9). His concern for the horizontal interpretation, however, is similar to Goodyear's concern for the upper zones of the Brand site. It is hard to say if the approach taken toward horizontal excavation of the Brand site would have been useful in the mixed strata of the wolfshead site, but the similarities between the sites make for interesting speculation.

At the Wolfshead site, a lack of depth at the site and the definition of cultural zones with arbitrary strata account for the mixing of cultural materials. Where natural strata are nonexistent or difficult to follow, it may be necessary to devise other means. At the Late Archaic Berger Bluff site in Goliad 
County, Texas, for example, the definition of cultural strata was assisted by the calculation of depths below a previously recorded set of surface elevations using a standard three-dimensional provenience (Brown 1983).

In certain cases, the definition of cultural strata might be carried to ridiculous extremes. At the Silver Springs site (a deep Paleo-Indian site in Marion County, Florida), the horizontal provenience of artifacts was recorded only by their respective $5 \times 5$ or $10 \times 10$ foot excavation unit, yet for every artifact and flint chip found, a depth from surface was recorded (Nei11 1958: 36). Not surprisingly, the artifacts from the site form a series of discrete cultural strata.

Deep sites lend themselves beautifully to the eliciation of cultural strata and nicely drawn projectile point sequence charts. It would be impossible to ignore the potential for vertically orjented data recovery provided by such sites as St. Albans (Broyles 1971). In fact, for the St. Albans site, which has 40 feet of beautifully defined natural strata, there are two projectile point charts and an appendix of point types, which is a miniature version of the type descriptions in the Handbook of Texas Archeology (Suhm and Jelks 1962).

Horizontally and vertically oriented strategies are not mutually exclusive. Ideally, one should consider both in a thorough site analysis. Usually the data from most archaeological sites are presented in one or the other format. Deep sites are always presented as vertical records of cultural change through time; shallow sites are depicted as campsites of whatever phase or age found just as it had been left.

The break between these two types of sites occurs at about a meter's depth, although there are a few exceptions. One is the Wolfshead site, where Duffield (1963:5) searched for vertically oriented data in less than two feet of culture-bearing sediment. Undoubtedly, the most successful is the Hardaway site in North Carolina, where Coe (1964:59, 81-83) found four distinct Early Archaic or Late Paleo-Indian phases in 2-1/2 feet of sediment. More than 10 years before either author had puzzled over their tightly compacted vertical stratigraphic problems, the ultimate in shallow-site vertical orientation had been demonstrated. At the Parrish Village site in Kentucky, Webb (1951:24) found no culturally relevant stratigraphy and no significant differences between the artifact assemblages in his levels. Undaunted, he separated Archaic artifacts using his experience at other Archaic sites within the area and called the rest Paleo-Indian.

The two exceptions to the unidimensional excavation strategy include the Eva site in western Tennessee (Lewis and Lewis 1961) and the Thunderbird site in Virginia (Gardner 1974). The latter site, with just over a meter of cultural deposits ( 39 to 42 inches), falls predictably and heavily into the vertical category. Gardner (1974:13-17, 36-41) discussed eastern PaleoIndian phases and the transition into the Early Archaic as they are reflected in the stratigraphy. Gardner (1974:20) also described and i1lustrated what he believed to be a Paleo-Indian structure, indicated by post molds and a quantity of typical Paleo-Indian jasper chipping debris. In a later article within the same volume, Gross (1974) discusses clusters of chipping 
debris found on apparent occupation floors. At the Eva site, with a maximum of approximately two meters of cultural deposits, the orientation is clearly vertical. Nevertheless, a plan map of each stratum with the location of burials, features, and concentrations of animal bone is also included (Lewis and Lewis 1961:6-14).

\section{Multidisciplinary Approaches}

Another approach to the problem of what to do with a site is the compendium of multidisciplinary studies. This approach is relatively recent and not yet common. In the present group of 20 studies, ranging in date of publication from 1951 to 1976, only four studies could be classified as truly multidisciplinary. All were published after 1965 and make up more than a third of the studies published during this latter half of the span.

The earliest multidisciplinary study of an eastern Paleo-Indian site was pub1 ished in 1966 as a special issue of the journal Quaternaria and was devoted to the Debert site in Novia Scotia. In this volume, four different specialists present data on the Debert site from their particular perspective (Byers 1966). This attempt is unique in that the actual site report was published two years later in a monograph series by a single author (MacDonald 1968). Because of this approach, MacDonald does not have to synthesize contrasting views or edit cumbersome multiauthored volumes for consistency.

Other multidisciplinary approaches included those employed at the St. Albans site (Broyles 1971), the Thunderbird site (Gardner 1974), and the Rodgers Shelter-Pomme de Terre site (Wood and McMillan 1976). The latter is by far the finest, presenting a well-organized series of distinct studies that generally follow a logical order and are synthesized at different levels of analysis. The other two suffer from problems of presentation and synthesis.

Broyles's (1971) own report takes up half of the volume in the St. Albans report. It is balanced by five appendices, one of which (another one-fourth of the volume) is her own. Although there is some confusion resulting from discussion of changes in methodology (discussed below), there is a relatively coherent and detailed description of how the site was dug. Gardner's (1974) report leaves much of this to the imagination. In addition, his synthesis takes up one-third of the edited volume, yet does not adequately describe the work that has been attempted. A critical difference between the reports is that Broyles summarizes the lithic tools herself, while in Gardner's report, the summary is spread among four different authors with four different approaches and no real synthesis.

The Thunderbird site report exhibits one important aspect that is not a part of the other multidisciplinary studies reviewed here. The data on this important early site are presented in a small-scale regional framework. Included in the report are data on the excavations of two other sites, as well as a consideration of local jasper sources and regional environmental records. Although this attempt is perhaps a bit too ambitious for the single flint Run volume, the importance of putting a site in its proper culturalgeographical context should not be minimized. One other nonmultidisciplinary study takes a similar approach. In the same volume with a report on the Hardaway site, Coe (1964) integrates a multisite excavation design into a description of regional culture change. 


\section{Excavation Methodology}

Whatever approach to excavation planning and design or incorporation of external studies one adopts, actual excavation eventually becomes a reality. At this point, the significant difference between studies with a carefully stated research design and those without is a set of explicit guidelines that assure that the data collected will be applicable to a particular set of research problems. The use of any prior design does not, however, guarantee excavation quality. This must be judged independentry.

\section{Unit Location}

The first, and perhaps the most important, question in the excavation of an eastern Paleo-Indian site (as in any site) is where to dig. This is, of course, not much of a problem in a total excavation. Realistically, however, even moderate-sized sites must be sampled. Although locating this sample has involved everything from random numbers tables to psychic advice, the most common method has been, and still is, the visual examination of the site surface. Excavation units can be located on the basis of highest artifact density, as at the Brohm site in the Thunder Bay District of Ontario (MacNeish 1952), or on the basis of surface topographic features, as at the Brand site (Goodyear 1974:15), where the initial units were located on the highest point of the sma11 hillock that contained the site.

Both of these can work well for shallow sites. Artifact density estimation can be adapted to a quantitative measure of density which satisfies even the most statistically minded of new archaeologists. This technique has onty recently been applied to eastern Paleo-Indian sites and is still not common. At the We11s Creek site in Steward County, Tennessee, Dragoo (1973) reports a systematic surface collection of all cultural material. As a result of this collection, he was able to isolate "hot spots," which he believed were foci of aboriginal habitation (Dragoo 1973:7). Systematic surface collections were also used at three sites reported in the volume on the archaeology of the Flint Run area: the Thunderbird site (Gardner 1974), the Fifty site (Carr 1974), and the Rudacil site (Walker 1974).

Although it does contain a preceramic (Archaic?) component, the Hatchery West site on the Kaskaskia River in Illinois is not a Paleo-Indian site (Binford 1970:72-73). It is included here because of the emphasis placed on the use of surface collection at the site. This site, which was situated in a cultivated field, was freshly plowed prior to collection. The site was then gridded into $6-\mathrm{m}^{2}$ units, and after a short period of exposure aided by rain, the artifacts in the gridded units were collected. After collection, the plow zone was stripped using a roadgrader and bulldozer; then excavation and recording of subsurface features began. Data from the surface collection and from the excavations showed excellent correlations. The surface collection proved to be not only a valuable indicator of subsurface features, but a valuable asset to the general picture of occupation type and density at the site.

In its application to shallow or wholly surficial components at eastern PaleoIndian sites, controlled surface collection is considered to be of potentially major importance. It ties in closely with orientation of horizontal-versus- 
vertical excavation. Although there is a clear bias in surface data toward showing the upper portion of a vertical section, the bias may be useful in delineating horizontally distinct artifact concentrations at shallow sites.

Controlled surface collection is the only means of obtaining quantitative delineation of components and activity areas at surficial sites such as the Shoop site in Pennsylvania, which is so thin that ". . If it were any thinner it would not be a site" (Witthoft 1952:467); the Reagan site in Vermont (Ritchie 1953); or the Bu11 Brook site in Massachusetts (Byers 1954).

In the excavation of deep sites, surface collections may be limited and even misleading. Brown (1975) discusses some of the problems inherent in sampling deeply buried components. In the relatively rare, deep eastern Paleo-Indian and Early Archaic sites, the approach has been varied. At the Rose Island site in Monroe County, Tennessee, Chapman (1975:17-18) used a backhoe to trench and remove overburden (at least some of which must have contained Woodland materials) from his Early Archaic horizons. At the Rodgers Shelter in the western Ozark Highlands of Missouri, McMillan (1976) combined a large block excavation and a checkerboard pattern of smaller sondages in order to effectively sample this deep and rich alluvial site.

One of the more effective methods of dealing with the problems of deep site sampling has been used at one of the earliest and deepest Early Archaic (Late Paleo-Indian?) sites excavated in the East--core drilling. During the initial season of investigation at the St. Albans site, a geological core-drilling rig was used to take samples from the terraces of the Kanawha River in the vicinity of the site (Broyles 1971:3-6). Using data from these and subsequent cores, the geomorphology of the terraces has been partially reconstructed. This technique was so successful that it was possible to correlate the cultural zones in the excavation unit and the strata observed in core profiles.

\section{Excavation}

Despite similarites in orientation and the kind of data sought, there are few similarities in actual excavation technique. Simply put, there are many ways to dig a site. An estimate of the number of ways is the product of the number of archaeological sites multiplied by the number of archaeologists. Fortunately, there is not always a clearly right or wrong way, and many approaches are equally valid. Most of the sites examined have basically sound excavation techniques with only occasional minor problems. Most of these problems may ultimately be due to lack of time or money.

One of the most popular initial excavation strategies is the trench. At least nine of the 20 sites examined use some form of trenching as a prelude to major areal excavation or in its initial stages. Most of the remaining sites had no consistent initial strategy and depended on opening a number of small units or more larger units. Interestingly enough, although trenching is believed to be an old-fashioned means of attacking a site, the mean excavation date of those sites using trenching is 1965.5 or slightly greater than the 1965.25 mean excavation date of the whole population of sites under consideration here. 
The Eva site in eastern Tennessee is an excellent example of the use of crossed trenches in large-scale excavations to delimit the area of concentrated occupation (Lewis and Lewis 1961). At two more recently excavated sites, the Rodgers Shelter (McMillan 1976) and the Rose Island site (Chapman 1975), trenches are used to initial advantage. At the Parrish Village site in Kentucky (Webb 1951), crossed parallel trenches were used to block out a number of units for excavation. The most unusual use of trenching was at the Wells Creek site in Tennessee (Dragoo 1973), where the last excavation unit was a 200-foot variable-width trench across the top of the hill on which this extremely shallow site was located.

An extremely important detail of excavation is the method of dividing up the site for excavation. Obviously one large unit would yield data radically different from data from many small ones. This is partially dependent upon the site and the topography. Thus, because of modern disturbances and the discrete nature of the units, it would have been impractical, if not impossible, to dig the Debert site (MacDonald 1968) as one large excavation unit.

There is a slight tendency for the sites that use only one or two large excavation blocks to show an early excavation date. The eight sites, which report this technique, have a mean excavation date of 1961. Two sites, Parrish Village (Webb 1951) and Eva (Lewis and Lewis 1961) use single large-scale excavation blocks. The publication data on the Eva site is misleading, since it, Tike the Parrish Village site, was a WPA excavation project dug in 1940 (MacDonald 1968:v). The trend away from the single large block is best shown by later sites: the Thunderbird site (Gardner 1974), the Rose Island site (Chapman 1975), and the Rodgers Shelter site (McMilian 1976). All use multiple area excavation strategies.

Although there seems to be a trend away from the use of a single large excavation block, it is recognized that the size and number of excavation units reflect a particular interest in more than just the amount of data collected. As pointed out above, large-scale, contiguous-area excavations yield a different type of data than does an equivalent amount of disjunct smaller units. With less than complete excavation, the smaller units can be successfully utilized to determine the extent of the site and the intrasite trends in spatial variability. The large-scale units uncover microareal patterning, as might be found in a family dwelling or a small-group campsite. An excellent example of the latter kind of microareal variability occurs at the Debert site (MacDonald 1968), where each of the 11 excavation units is believed to contain a spatially bounded cluster of artifacts. A similar situation occurs at the Brand site, where seven distinct artifact clusters are isolated (Goodyear 1974).

Data on these small activity area or campsite features do not always indicate an understanding of the entire site, as at the Brand site where there could be major differences between the defined clusters and unexcavated portions of the site. One solution is the excavation of the entire site or a large portion of it, as Binford (1970) did at the Hatchery West site in 111 inois. His approach enabled him not only to define intrasite features, but their larger relationships as we11. Another less expensive and less destructive approach is to coordinate large-scale excavations with smaller test units, as at Rodgers Shelter. 


\section{Stratigraphy}

Perhaps the most important part of the success of an excavation depends upon the presence or absence and the use or abuse of stratigraphy. This is a complex subject and can only be touched upon here in a general way.

At the simplest level, this subject involves the choice between digging in arbitrary or natural levels. Most archaeologists would probably agree that, where possible, the use of natural stratigraphy for excavation control is preferred. This is, of course, not an unequivocal choice. The use of pedogenically derived soil strata, or those soil strata, which may have formed in situ after the deposition of the cultural material, may be quite misleading. As a rule, however, depositional strata can be used successfully in culturalhorizon separation.

Postoccupational pedogenic development is displayed in the formation of the Podzol (Spodosol) soil formed at the Debert site. Podzols typically have very distinct horizon separation between the bleached, white Soil Horizon A2 and the reddish brown Soil Horizon B2, yet the estimated time of formation (between 2000 and 4000 years) clearly postdates the 10,600-year-old date of occupation at the site (MacDonald 1968:17). The separation of the artifactual material by such criteria could result in erroneous separations concerning the occupation of the site.

Nine of the sites studied in this report used natural strata as the primary, or one of the more important, cultural-level markers in excavation. In most of the others, natural stratigraphy is recognized and used as an interpretive tool in understanding material from arbitrary levels. If soil stratigraphy is uninterpreted, the potential for error in the interpretation of cultural data increases. For this reason, it is critical to present as much data as possible on the nature and formation of strata when using natural stratigraphy in excavation.

Although sites with clear natural stratigraphy can occur in almost any kind of terrain and in almost any age soil deposit, the best stratified and most easily interpreted of all open sites are deep alluvial sites. As noted earlier, these sites are ideal for the elicitation of temporally or vertically patterned data. Eight of the sites studied in this paper have more than a meter of deposits; six of these are clearly alluvial. These are Rodgers Shelter, Rose Island, Thunderbird, St. Albans, Eva, and the Habron site in Virginia (Rodgers 1968). The nonalluvial deep sites are Silver Springs and the Starved Rock site in northern I11 inois (Mayer-0akes 1951).

The finest example of an alluvial site in the sample is the extremely important St. Albans site in West Virginia. In the main excavation block, a we11separated sequence of 41 alluvial strata containing 18 occupation zones has been delineated. Each occupation zone is separated by one or more strata of sterile clay or sand. A date of 9850 B.P. has been obtained on Zone 36 at a depth of about 16 feet below the surface. Core samples indicate that cultural deposits may extend to more than twice this depth (Broyles 1971:1).

Excavation at the St. Albans site has taken full advantage of this excellent natural stratigraphy. During the first season of major excavations a stratum 
block was isolated and excavated in natural levels. In subsequent seasons, the stratum block technique was abandoned, but excavation by natural levels was still maintained.

The lack of cultural stratigraphy is a common problem at shallow sites. Mixing by bioturbative processes can totally destroy whatever vertical separation might have existed between components. If separation does exist, it is often impossible to understand vertical relationships in terms of large arbitrary levels. The deposits at the Wolfshead site, for example, show only a vague stratigraphy of projectile points within the five 6-inch arbitrary levels (Duffield 1963:577). Yet these vaguely defined relationships, with Early Archaic San Patrice projectile points most common at the base of the sequence and Late Prehistoric arrow points common at the top, seem to be essentially correct.

Shallow sites can be stratified, however, although it may not always be obvious at first. The initial excavation units at the Brand site (Goodyear 1974:15) were placed in the highest and most disturbed portion of the hill where the site is located. Although some Dalton points were recovered, no stratigraphic relationships were recognized. Later, test units placed on the sides of the small hill were able to discern the natural stratigraphy used throughout the remainder of the excavations and were critical in separating the material identified as Tying on the Dalton occupation floor.

At the John Pearce site in northwestern Louisiana, the decision to switch from arbitrary to natural vertical units was a simpler one. After only two small units had been excavated the natural stratigraphy was recognized, and natural levels were adopted (Webb, Shiner, and Roberts 1971:4). At other sites, this decision may not come so easily. After 23 five-foot-square levels failed to reveal any cultural stratigraphy at the Hardaway site in North Carolina, excavation was shifted to natural stratigraphy. Coe $(1964: 60)$ had concluded that ". . . any further excavation by arbitrary levels and in single isolated units was a waste of time and a destruction of potential data."

\section{Reporting}

The most important and most ignored aspect of excavation methodology is accurate reporting of the actual methods and techniques used. Without some data on the techniques of excavation, it is often impossible to assess the validity of cultural interpretations at a given site. This seems especially true in the case of the eastern Paleo-Indian sites where there are sti11 large gaps in our understanding of the cultural relationships. Rather than being tested against a coherent, previously formulated cultural model (which is implicit in many excavations), eastern Paleo-Indian studies appear to be only now falling into a unified pattern. Most of the reports considered were involved in the formulation of that model in their particular region, and thus, our knowledge of the eastern Paleo-Indian is almost totally dependent upon the quality of excavation techniques utilized in these particular excavations.

This is not a consoling thought, because if there is any problem which is common to these diverse sites, it is the inadequate or only barely adequate reporting of excavation methodology. Few reports fail to expound on their particular theory 
of the origins and developments of eastern Paleo-Indian culture, but fortunately, almost all provide some information on the actual excavations. Few actualiy provide the data necessary for judging their interpretations.

There are several important variables that influence the quality of reporting. Perhaps the most important is the organ of publication. Invariably, reports published as monographs are of higher quality than those in journals. This is largely because of severe space limitations placed on journal articles, compounded by the fact that more detailed excavations are rarely published as journal articles (at least not in primary site report format). Four of the six journal articles reviewed here were authored by amateurs.

This latter distinction is not a universal rule, however. The John Pearce site in Caddo Parish, Louisiana, is a fine exception to the amateur authorship. This report, authored by two amateur archaeologists and one professional, was published in the annual Bulletin of the Texas Archeological Society, and, offers an adequate, if somewhat condensed, description of procedures (Webb, Shiner, and Roberts 1971:4-6). When comparing between the professional and amateur journal articles reviewed here, it is apparent that perhaps the worst of the lot, the Wells Creek site excavation, is authored by a well-known professional (Dragoo 1973).

Dragoo's article runs counter to another major trend in reporting excavation details. It seems that, in general, the more recent articles are more conscientious about reporting the fine details on excavation and are more likely to provide supporting physical data, such as soils and geomorphology. The Wells Creek article does contain a very interesting section on the geology of the Wells Creek crater area. The site is located near the center of a hypothesized ancient-meteor impact crater (Dragoo 1973:1-5), a unique situation for archaeological sites in any area. However, that the impact occurred during the 1ate Mississippian geologic period, some 315 mil1ion years ago, detracts from its application to Paleo-Indian cultures in the area.

The excavation of the Wells Creek site met with limited success, since no artifacts were found below the plow zone. This limits the potential for vertically oriented interpretations at the site, but it is no excuse for the complete lack of data on excavation method. No information is provided on the manner of excavation or on any screening techniques that might have been used. Only the barest of verbal descriptions is given of the soil matrix, in contrast to another mixed plow zone site (the Plenge site in New Jersey) reported in the same issue of the Archaeology of Eastern North America where a tabular reporting of quantitative soils data by a soil scientist is provided (Kraft 1973).

Leaving aside the obvious lack of vertical data, Dragoo (1973:7-8) himself reports the potential of horizontal patterning at the site, yet no explanation of the actual method of defining these patterns is given, and no data on the patterns are provided. No maps of the test unit locations are given, and the verbal description of the areas is insufficient to locate them. No information on individual units is provided, and no proveniences are given for artifacts.

The other professional site report reviewed here is also one of the earliest published articles in the sample. This article (Mayer-Oakes 1951) on the Starved Rock Archaic site in Illinois contains a section on methodology, which 
is equivalent to most of the amateur articles and is perhaps less detailed than the John Pearce report. Most of the other early sites lack specific excavation details. The Parrish site (Webb 1951) in Kentucky is a good example of this.

At the Parrish site, the description of the methods employed at this sevenmonth excavation is three-fourths of a page (Webb 1951:410). A verbal description of the layout of the excavation grid is not supported by corresponding maps or photos. It is literaily impossible to figure out where the excavation was conducted with respect to the general topography of the site, since no map of the site area is included. A plan view of the greater part of the excavated area shows features and burials, but these are not tied into the site. No estimation can be made of the amount of the site excavated.

Other more modern sites also suffer from problems in reporting excavation techniques. The extremely important and often-quoted Debert site (MacDonald 1968) is a case in point. Where one might defend the wells creek report because of its magazine format, the Debert report is published as a long monograph. Yet no section on techniques is contained in the report. The reader must piece together the excavation strategy from short statements throughout the report.

Although excavation was obviously careful, there are no data on screening techniques or excavation control. No data are provided on the total amount of area excavated. A11 excavation data are given in terms of the 11 areas, which are interpreted as discretely bounded cultural units. Apparently no excavations were conducted in unproductive areas, and all of the remaining site was recovered (a large portion of the site had been disturbed by leveling).

These few examples are only a selection of the more dramatic reporting errors, but they are not unique. As mentioned above, most of the reports are flawed. It should be sufficient to point out that the problem exists, and although, the trend may be toward more detailed reports, there are still problems.

\section{Interpretation}

If the reconstruction of prehistoric cultural systems and the formulation of theories of cultural change were not dependent upon data and those data not ultimately dependent upon the quality of data recovery at archaeological sites, this paper would be meaningless. Fortunately, or unfortunately for the profession of archaeology, data form the basis for most valid interpretations. The following section takes a look at interpretation in the light of various forms of data collection.

Obviously, the quality of data collection influences the quality of interpretation. Certain archaeological data-recovery techniques can be measurably improved, and examples of this are seen in the 30-year span of eastern PaleoIndian studies. An example of a change to a horizontal provenience, such as recorded at the Brand site (Goodyear 1974) in Arkansas, can be more useful in understanding camp and village patterns than provenience to a 10-foot unit, as at the Silver Springs site in Florida (Neil1 1958).

Yet there is a 1 imit to qualitative improvement of data recovery. How much more would be gained by digging a site with no natural stratigraphy in 
one-centimeter levels rather than five-centimeter levels? Or how much more data could be gained by screening all sites through window screen? In certain cases, these might be absolutely necessary procedures, but are the increased time and effort worth the increase in data for every site? The answer to this may lie in site-specific recovery techniques and data-dependent interpretation. Thus, just as it is ridiculous to compute a six-decimal place mean from data collected to one decimal place, it is equally ridiculous to overestimate the interpretive powers of certain recovery techniques.

\section{Vertical Patterning}

Vertical interpretations are the ultimate tool of cultural history--the reconstruction of cultural chronologies. As such, they have served a long and useful role in understanding archaeological cultures. More than half of the sites reviewed are directly concerned with the interpretation of vertically patterned data, and the remainder are concerned with the placement of their particular data set within a temporal sequence previously synthesized from vertically patterned data.

A critical question in vertical data recovery is how the data got to be vertical in the first place. This is patently obvious in alluvial terrace sites such as St. Albans or Rodgers Shelter, but less so in many upland sites. Even in alluvial sites, the "deeper is older" hypothesis may be difficult to sustain in some ordinary terrace sequences. The sequence of terrace formation at the Thunderbird site (Gardner 1974:28-33) illustrates this problem. Although the ground surface is essentially level across the terrace sequence, Early and Middle Archaic materials on the lower terrace are much deeper than the Clovis materials on the upper terrace.

In nonalluvial sites, the question of depositional processes should be an important one. In other words, how much of the site depth can be attributed to depositional episodes and how much to bioturbational and pedoturbational processes. In many sites, the lowest occupation is just above weathered bedrock or Pleistocene clays or gravels. A casual reader trying to put all of this data together might come to several interesting conclusions, i.e., that the world is from two to 10 feet thicker than it used to be, and the Paleo-Indians were fond of living on clay and gravels. To some degree this might be true. Those areas where we find buried sites are in continually aggrading areas (at least since the end of the Pleistocene), and there have been major climatic shifts since the end of the Pleistocene which caused soil to form in areas where there had been nothing but gravels for thousands of years. Two other active processes are not always considered as alternatives. One is the continual cycles of erosion and deposition that occur in many areas. The other is the possibility that artifacts might be moved downward by bioturbational or pedoturbational processes.

At the Hardaway site in North Carolina, Coe (1964:57) states that his earliest occupants (Hardaway phase peoples) were living on a two- to three-inch layer of humic soil, which overlies a clay residuum weathered from the greenstone bedrock. It is interesting to note that in very gradually aggrading soil on top of a landform essentially unchanged since the Triassic, two to three inches of soil had developed prior to the Hardaway culture and almost two feet developed 
subsequent to their departure. A simple calculation shows that the Hardaway culture must date to Jurassic times or approximately 150 million years ago. The Hardaway culture is well bracketed by cross dating to the Holocene, so other possibilities must be considered. Coe suggests that the recent soil buildup is due to human activity. This is possible, but not supported by any analysis of the sediments. Other possibilities are cycles of erosion and deposition, or that the older materials have worked their way down further into the soil. In this particular case, the natural levels used in stratigraphic separation at the site need to be carefully described and documented, and some data on their potential means of formation gathered.

Even in sites where the origin of the sediments is understood, the origin of the vertical patterning in the artifacts might not be clear. At the Silver Springs site in Florida, Neill (1958) outlines a very clear verbal stratigraphic sequence from Middle Woodland period ceramics down to Late Paleo-Indian or Early Archaic Suwannee points. The troublesome part of his interpretation is in his identification of the formation in which the artifacts were found: ". . . dunelike, and composed of homogeneous, seemingly windblown sand" (Nei11 1958:34). Any archaeologist who has ever dug in pure sand deposits has to be somewhat amazed at the excellent cultural stratigraphy preserved at the Silver Springs site. Some of the problems with mixing in windblown sand sites are outlined in a discussion of the misleading artifactual stratigraphy at the Meer II site in Belgium (Van Noten, Cahen, and Keeley 1980) and the artifact mixing at the Debert site in Nova Scotia (MacDonald 1968:16-20). At both of these sites, identified as single component sites by various methods, artifacts of a single time period had been mixed throughout a half-meter section of the profile. Several deep sandy sites tested in the Coleto Creek Reservoir in the inland coastal region of Texas were also found to have lost any stratigraphic separation of artifacts due to intense bioturbation (Fox, Black, and James 1979:19-24).

One measure of the importance of an undisturbed site is the frequency of statements by archaeologists that their site is undisturbed even in the face of considerable evidence against it. In reality, there are no undisturbed sites, with artifacts lying just as the Indians left them ten thousand years ago. Postdepositional disturbances are a factor to be contended with or at least carefully considered at al1 sites.

Many deep alluvial sites are only minimally disturbed by postdepositional processes, but one wonders at the potential violence of the actual depositional processes in these cases. At any rate, even they are subject to some postdepositional disturbances. In alluding to the undisturbed importance of the St. Albans site, Broyles (1971:1) points out that, throughout the site, ". . only one type of projectile point is found in a zone. "This kind of data is reassuring when dealing with disturbances, but upon closer reading one finds that she had failed to read her own report. Perhaps she considers the two Charleston Corner Notched points and one Kessel Side Notched point found on top of the same hearth as the same basic point type (Broyles 1971:10). And, of course, this statement does not apply to the upper zone where a St. Albans, a Kirk, and a Kanawha Stemmed were found together in Zone 4 (Broyles 1971:24, 47), nor does it apply to Kirk and Lecroy Bifurcated-Base points found together in Leve1 8, or to Kirk and St. Albans Side-Natched points found in Zone 11 . 
The St. Albans site is still unquestionably an immensely valuable site with thousands of years of clearly bracketed cultural sequence. On the other hand, one can imagine the horror of an archaeologist who has just excavated a 20-foot deep alluvial site with beautiful stratigraphy and recovered nearly 200 identifiable projectile points, all of them exactly the same type. If such a situation is impossible in deep sites, it is just as nearly so in shallow sites. There is almost always the question of mixing of cultural groups. A very important exception is the shallow and highly bioturbated Debert site, which yielded 168 projectile points and fragments, all belonging to a single type (MacDonald 1968:70; Hans Mueller-Beck, personal communication). Such is not the case at most shallow sites, however, and many different approaches to separation are taken. In general, they boil down to a single basic method: ignore what you do not want to study and call it intrusive or the product of a transient occupation. In many cases, this may be justified, but the data are confusing. Contrasted to the more than 100 Paleo-Indian projectile points from the Holcombe Beach site in Michigan, there were eight non-Paleo-Indian projectile points found in the surface or in rodent burrows (Fitting, DeVisscher, and Wahla 1966:36, 41). Based on this, Fitting assigns all other flint artifacts to the Paleo-Indian occupation. Fitting is, in effect, ignoring the possibility that the later transient occupations have contributed significantly to the nonprojectile point artifacts. This may be the case, and it is fairly clear that at least 102 basal fragments are from fluted or lanceolate Paleo-Indian point styles. From another point of view, the statistics may be misleading. Only six Paleo-Indian points from the site are substantially complete, and only one of these is "typical" (Fitting, DeVisscher, and Wahla 1966:43), yet at least five of the eight non-Paleo-Indian points are complete. Could there be additional biface fragments assignable to the later projectile point? In addition, the majority were from surface collections by an amateur archaeologist apparently interested in Paleo-Indian remains. The easy dismissal of the eight Archaic projectile points suddenly becomes more questionable.

At the Thunderbird site in Virginia, there is more evidence from the intrusiveness of Late Archaic stemmed points. A series of post molds are assigned to the middle Paleo-Indian occupation, because of the quantity of jasper debitage and two (presumably jasper) middle Paleo-Indian points (Gardner 1974:20). Four Late Archaic points from the same area are of quartzite and rhyolite.

\section{Horizontal Patterning}

The concept of horizontal patterning is not a new one. In his report on the excavation of the Parrish Village site in Kentucky, Webb (1951:411)includes a plan map of all features in the excavation area. Yet he makes no attempt to explain it or to search for regularities within it. Less than 10 years later, Davis and Davis (1960:13-14) are actively searching for the horizontal patterning in the artifactual material from the Jake Martin site in east Texas. One suspects that this search, which proved essentially fruitless, was all the more important to them, because of the lack of vertical stratigraphy. A year after the publication of the Jake Martin report, the Eva site report was published (Lewis and Lewis 1961). The plan map presented for each stratum shows not only the distribution of burials, but of features and animal bone concentrations. A glance at these maps shows that intrasite variability was present in most, if not a 11, of the components. Yet there is no real discussion of horizontal patterning in the text of the report. 
One type of horizontal patterning, which was actually observed rather early in the surface sites assigned to the eastern Paleo-Indian period, is the presence of discrete artifact clusters or "hot spots" at sites such as Bull Brook (Byers 1954) and Shoop (Witthoft 1952). This same kind of patterning was observed in the excavated areas at the Debert site.

At Debert, the excavated evidence was gathered to find the solution to a problem in horizontal patterning, which had a long history of speculation. Witthoft (1952:468), in discussing the artifact clusters at the Shoop site in Pennsylvania, suggested that they might be individual camps or hearths within a campsite. MacDonald (1968:134) finally concludes that they are likely to be seasonal campsites or groups not exceeding 30 to 40 individuals utilizing the site over the span of a few decades. This is indeed a strong possibility. It should be noted that he rejects the possibility of a single large campsite, with family or smaller band group hearths, with no adequate reason offered for the rejection.

Actually the evidence is ambiguous. Eight of the campsite areas are located close to one another in the "nuclear area," and all are discrete, separate sections with approximately the same number of artifacts and hearths. The exception is one area almost twice the size of the rest and interpreted as a re-occupation (MacDonald 1968:21-23). Three other areas are set apart and are interpreted as functionally different. Two of the functionally different areas, involved with the production or maintenance of stone tools and crossmends of broken tools, were made between one of these areas and several of the campsite areas.

While such a situation is easily imagined for the same group returning year after year, it makes as much or more sense for a large group to contain functionally specific intergroup work areas. In any case, the verification of the reality of MacDonald's hypothesis is dependent to some degree upon the intersectioned areas and his criteria for definition of these sections. It appears that he tested only in areas where there was a camp. Perhaps this was because he had prior information from some surface data, but the reason for placement of his pits is not stated.

The same problem in interpretation of the function of artifact clusters is encountered again at the Brand site. Here, however, the clusters are much smaller and dubiously separated. While one can visualize a band returning to the same hillside every summer for a decade and camping in the same place twice during only one of those seasons, it is immensely more difficult to imagine the same band of hunters returning to the same small mound (which may not be much larger than the largest artifact cluster at Debert) to butcher a white-tailed deer without the least bit of overlap with any of the 4-10 $\mathrm{m}^{2}$ butchering areas from previous years (Goodyear 1974:110). On a large hi11 with widely scattered butchering areas, the chance of overlap in a number of years would be very sma11; at the Brand site, the actual clusters are considered discrete, but are separated by no more than a meter in any direction from the other clusters. The recognition of these clusters is highly questionable, and to imply function to the clusters must be done with caution. 


\section{Conclusions}

Each of the subdivisions in this section has pointed out some obvious failing in methodological procedures commonly used on eastern Paleo-Indian sites. As already shown, the problems of eastern Paleo-Indian sites are not necessarily unique. Conclusions drawn here should be applicable to similar sites anywhere. In this last section, some of the earlier conclusions are reiterated in a cookbook format.

The two fundamental rules for the excavation of any site should be to understand the reasons for every methodological choice made in the excavation of a site and to make certain that the choice and the reasons for making it are reported. An explicit research design may facilitate some of the minor choices, but it should never be allowed to limit the research to the point where there is no longer any feedback from the collected data. Research designs should always exist in a state of fluid homeostasis with continual input from completed goals and collected data.

Often, in multiseason investigations, techniques are subject to some degree of evolution. Changes in excavation techniques at the St. Albans site are a good example of this progressive evolution (Broyles 1971:6-14). In this particular case, however, there is a lack of clarity in the description, which makes reader interpretation of the various excavation strategies difficult. This difficulty serves to emphasize the necessity for careful documentation of all techniques and all alterations in those techniques.

Site documentation should not only include reporting of what has been done. It should also include, depending upon the scope of investigations, reports of supporting studies that are written according to the professional standards of that particular discipline. For example, soil profiles, especially when they are used to guide naturally stratified excavations, should not be described as "brown soil" or "yellow sand," but rather in terminology that is acceptable to soil scientists for the description of a profile. Geomorphological investigations should be presented likewise. This is not easy, especially for archaeologists who have no training in these fields or who cannot hire specialists, but is nevertheless essential.

Excavations should be geared to the elicitation of not only temporal, but also spatial data. Vertically patterned data should be interpreted carefully with an eye to possible disturbances and the possibility of faulty strata identification. Wherever usable strata exist, they should be used, but their means of formation should be investigated. The recording of exact proveniences within a given stratum of occupation floor is essential, and the search for potential campsite patterns is extremely important. Chapman (1975:200) indicates an important direction for future horizontal studies in the area of computer graphic displays of artifacts and three-dimensional distribution studies of materials from the Rose Island site.

An obvious trend in increasing quality of data collection and supporting techniques has been observed in the eastern Paleo-Indian studies. This clearly suggests that future excavation techniques will be increasingly better and more data will be recovered. The theme of site preservation is strongly supported here. It is hoped that the recent increases in the number of archaeologists do 
not actualiy result in an enormous increase in the number of Paleo-Indian sites so that there will be none left for the next generation of sophisticated methodologies to investigate. However, there are probably enough Paleo-Indian and Early Archaic sites destroyed each month in the United States by various state, federal, and private construction and energy-extraction projects to satisfy the needs of most researchers for many years. It is to those endangered sites that we should concentrate our resources.

\section{E. EXCAVATION ACTIVITIES AND TECHNIQUES AT EAGLE HILL (Gunn)}

Sequence of Operations

As was discussed in the section, Preliminary Investigations, one of the primary objectives of the project was to determine the most advantageous locations to excavate. The problem was resolved when it was found that only a $50-\mathrm{m}^{2} \mathrm{tri-}$ angle contained the full depth of the section. Apparently the rest had been eroded, graded away for the road which interdicts the site or ripped out by stumping operations to the west. In accord with these findings we centered our operational grid on the highest point on the remnant and began to excavate.

The project proposal called for the excavation of Area B followed by the presumed more difficult excavation of Area A. The complexity of Area A disappeared when we examined the profiles in the field and found that the contacts posed none of the problems suggested by Serve110's field drawings. In Area B our subsurface testing showed that the area of consistent depth was relatively large and the phosphate readings consistently high. For geomorphical and strategic reasons, which were explained earlier, excavation of Area B was deferred.

During May, the core crew opened excavations, organized the recording system, and excavated a $1-\mathrm{m}^{2}$ control column in the deepest part of Area $A$. These events foreshadowed ful1-scale excavation during the month of June. Fourteen persons supplemented the core crew during June. They were sponsored by the UTSA Summer Field School in archaeology provided by the Division of Social Sciences. During this month, the participants mastered a complex excavation technology and, in the process, excavated a $5 \times 5 \mathrm{~m}$ area in the Area A erosional remnant. The effort was abetted by nearly perfect weather. Only a couple of hours of one day were lost due to a rain shower. With few exceptions, the crew performed as near to professional standards as any novice crew it has been my privilege to observe. Their morale was high, their dedication admirable, and their accomplishments significant.

The trainee crew achieved its optimum pace relatively early in the month in terms of units per day closed. However, it was two weeks into excavation before they reached full proficiency locating and recording artifacts. Most of the time was spent in the upper three cultural levels of the site, horizons which were probably well selected for training since there is some evidence of disturbance. One visitor to the site, John Guy, suggested that the Eagle Hill erosional remnant was an ideal location for an observation-oriented military foxhole. There appeared, however, to be limited disturbance below the humic zone 9 . 
Figure 11 shows the rate of work over the course of the excavations. The number of field numbers (FNs) issued per week represents a combination of all units opened, artifacts recorded, features excavated, etc. These show healthy increases during the second and third weeks of the field school, level out during the third, and drop during the fourth, because of more difficult excavation in the lower levels. The relatively low projection during early july is a result of several factors: increased rainfall, difficulty in excavation and screening, and profiling and excavation of a geologic environmental soil sample column (see section on High Resolution Environmental Column, HREC). By the end of the field season, a $5 \times 6-\mathrm{m}$ block had been excavated in Area $A$ and a $2 \times 2-m$ unit in Area $B$.

In addition to the block excavation of Area $A$, three test pits were excavated around Area $A$ to determine the nature and extent of the erosional remnant. The results suggest that there is little beyond the $50-\mathrm{m}^{2}$ triangle. There is limited potential to excavate northward from the present block since the lower sediments appear to be deep and intact.

$$
\vec{\circ} \quad \tilde{\circ} \text { : }
$$

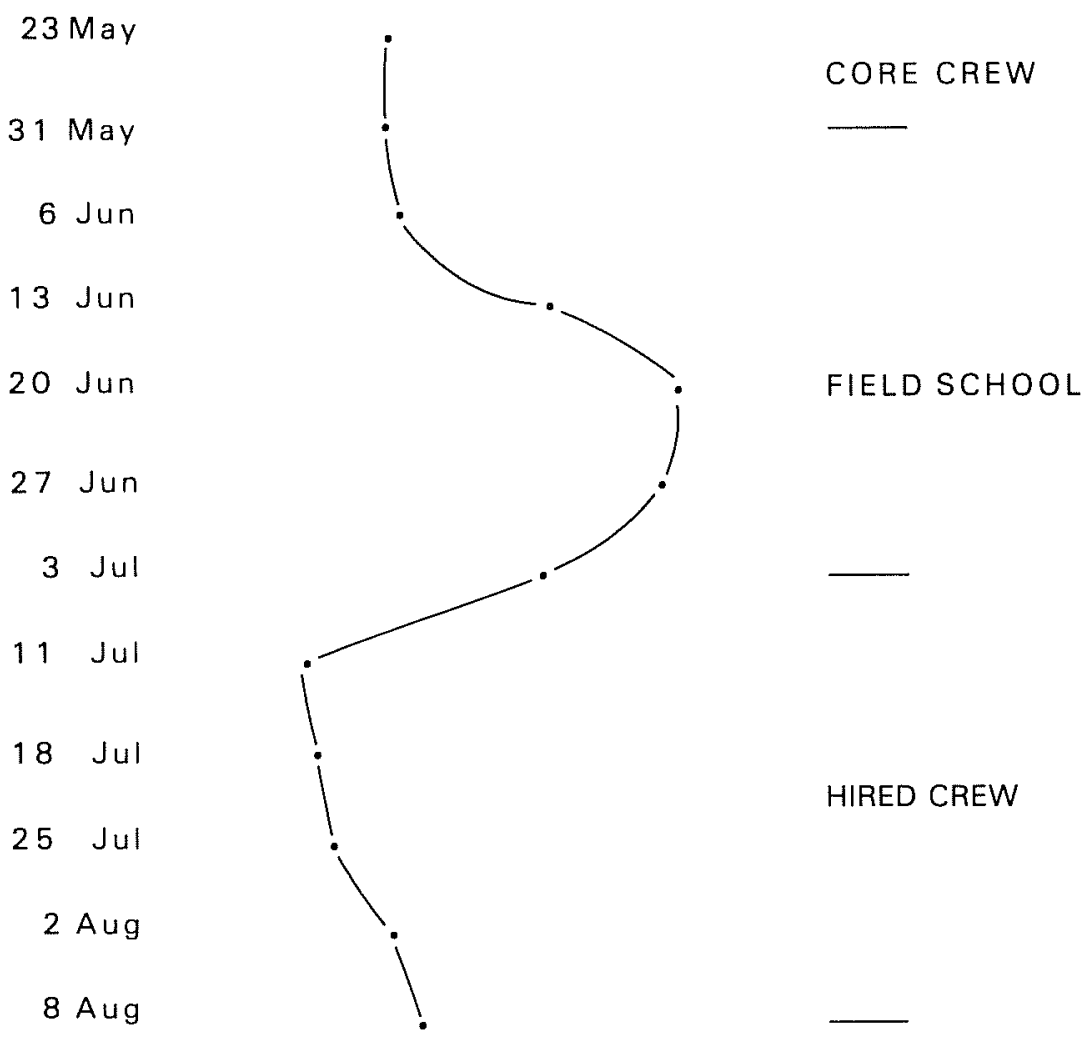

Figure 11. Number of FN's Issued per week During the Excavations. 
Geology, geomorphology, and soil development were clarified and expanded by a four day visit from Nials and Ed Garner. This knowledge along with some information gleaned from Servel10's testing operation and analysis was combined to develop a vertical sampling procedure, which governed the progress of excavation during the second half of the month.

In July, after the field school, the remaining crew personnel were hired for the duration of the summer. They were forced to deal with the difficult excavation of Soil Horizon IIB23, rains which soaked the lower strata, and increasing humidity, and high temperatures. Temperatures rose consistently above $100^{\circ} \mathrm{F}$ during the middle of July. In order to give the excavators some relief from the tougher sediments, we opened a 1-m trench along the north side of the $5 \times 5-m$ unit nearly completed in June. This expanded our excavation block to $5 \times 6 \mathrm{~m}$ and allowed the excavators to alternate between easy and hard digging. Water screening also became a more complex problem. The IIB23 sediments had to be water screened with high water pressure. The Air Force Observation Station came to our rescue by allowing us to process buckets of dirt in their front yard.

The Area B control column was completed in time for a visit from Hession and Carbone on June 17. Examination of the contents of the column suggested that Area B probably was intact as pertains to stratified cultural levels. We had reason to believe, however, that the Area $B$ sediments were equivalent in age to the modern soil horizon in Area A. It seemed to us, and Hession and Carbone, that it would be more profitable to continue to concentrate most of our efforts in Area A. We could expect the same information from Area A upper levels as from Area $B$ in terms of changes in material types and technological and camping habits. By recovering this information from Area $A$, however, we would expose more of the older sediments in Area A. It was suggested by the government that we open some more squares in Area B with shovels to search for chronological diagnostics, which would support the geomorphological arguments for Area $B$ being relatively young. The opening of three $1-\mathrm{m}$ units revealed no diagnostic artifacts and few flakes.

During the first week of August, removal of the occupation floors immediately above the Miocene clays was finally completed. Difficult excavation and frequent rains during July had considerably slowed the work in the lower levels and caused us to concentrate most of our effort on the sixth meter north in Area $A$ and on Area B. A break in the weather in the first week of August allowed us to complete the excavation. As a result an unfluted lanceolate point and several blades similar to the scraper found earlier were associated with a Paleo-Indian occupation. A High Resolution Environmental Column (HREC) was removed during the latter part of July and the first week of August, which resulted in 94 soil samples $(1 \times 50 \times 100 \mathrm{~cm})$, and a series of 94 pollen/biosilica samples were removed in one-centimeter arbitrary levels. Brown stayed in the Peason area for a week after the close of the field season to collect clay and lithic samples for experimentation and to supervise backfilling the site. The Air Force compound provided a front-end loader and an operator for the backfilling operation.

The Area $A$ excavation was completed as a $5 \times 6-m$ rectangle in all levels except a $20-\mathrm{cm}$ ledge along the north side of the three northeastern units (Plates 1 and 2). The ledge consisted of substrata OP 4.15-4.17. The excavation was lined with plastic tarp before backfilling. 


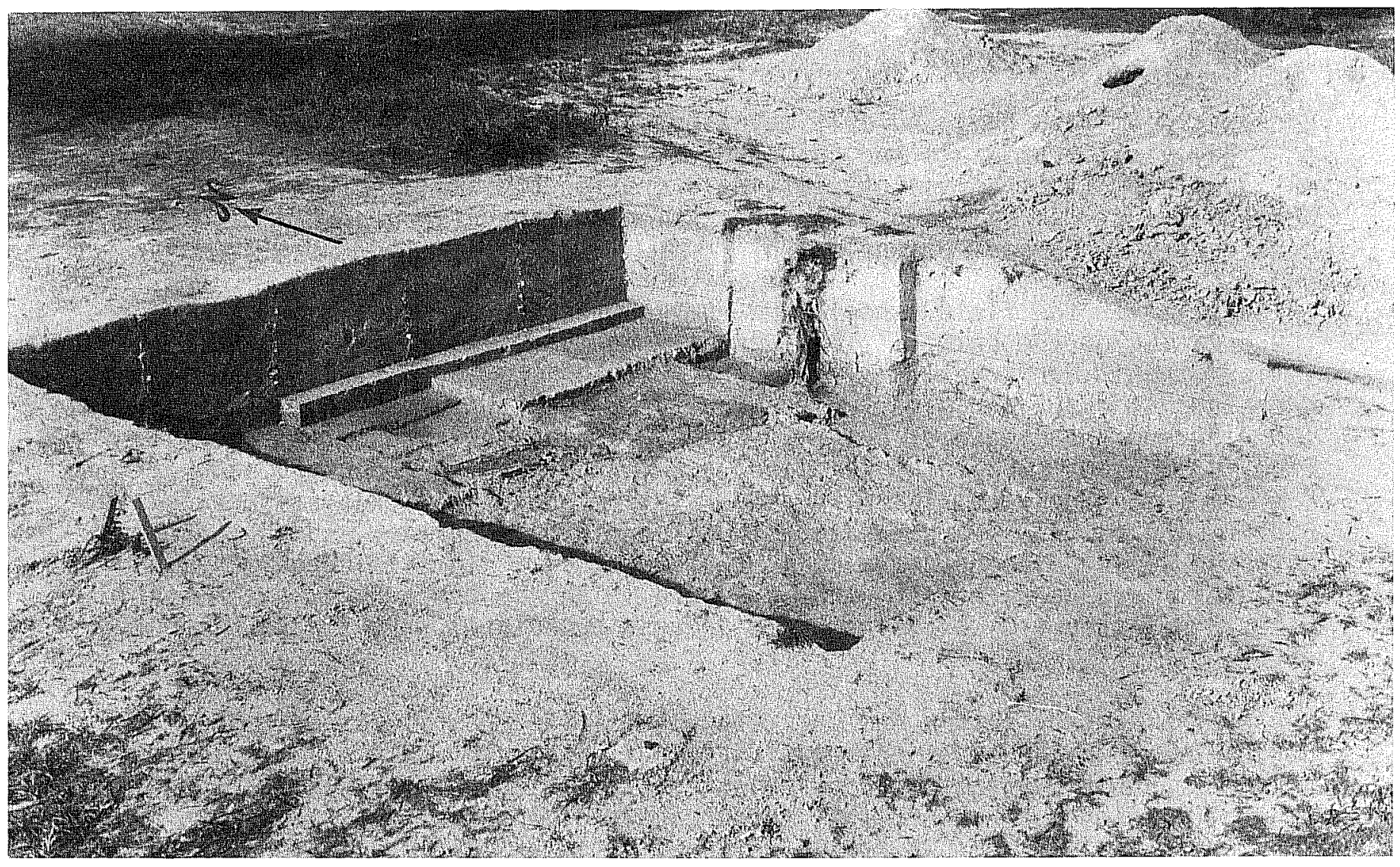

Plate 1. End of Excavation, Facing Northeast.

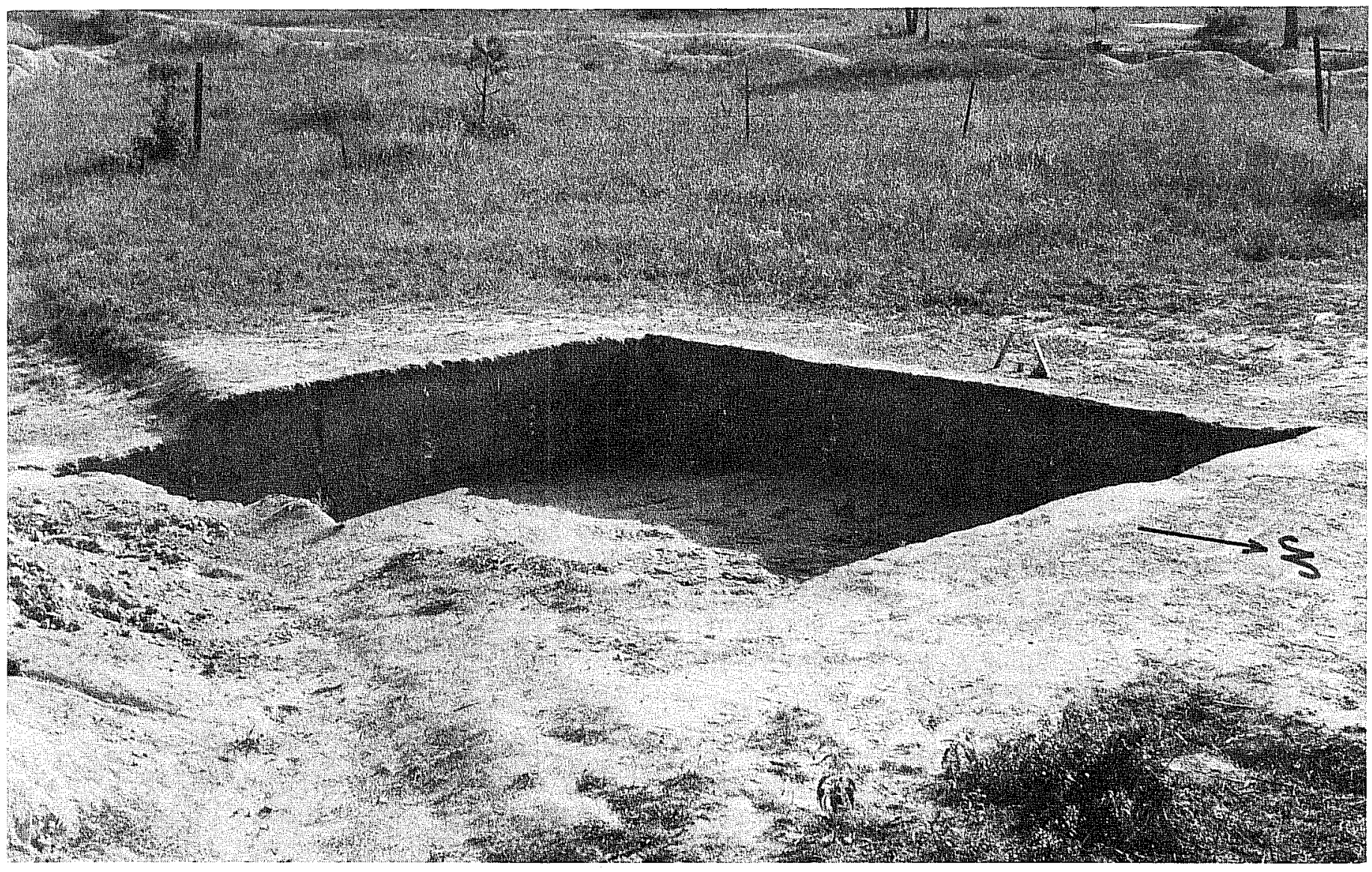

Plate 2. End of Excavation, Facing Southeast. 


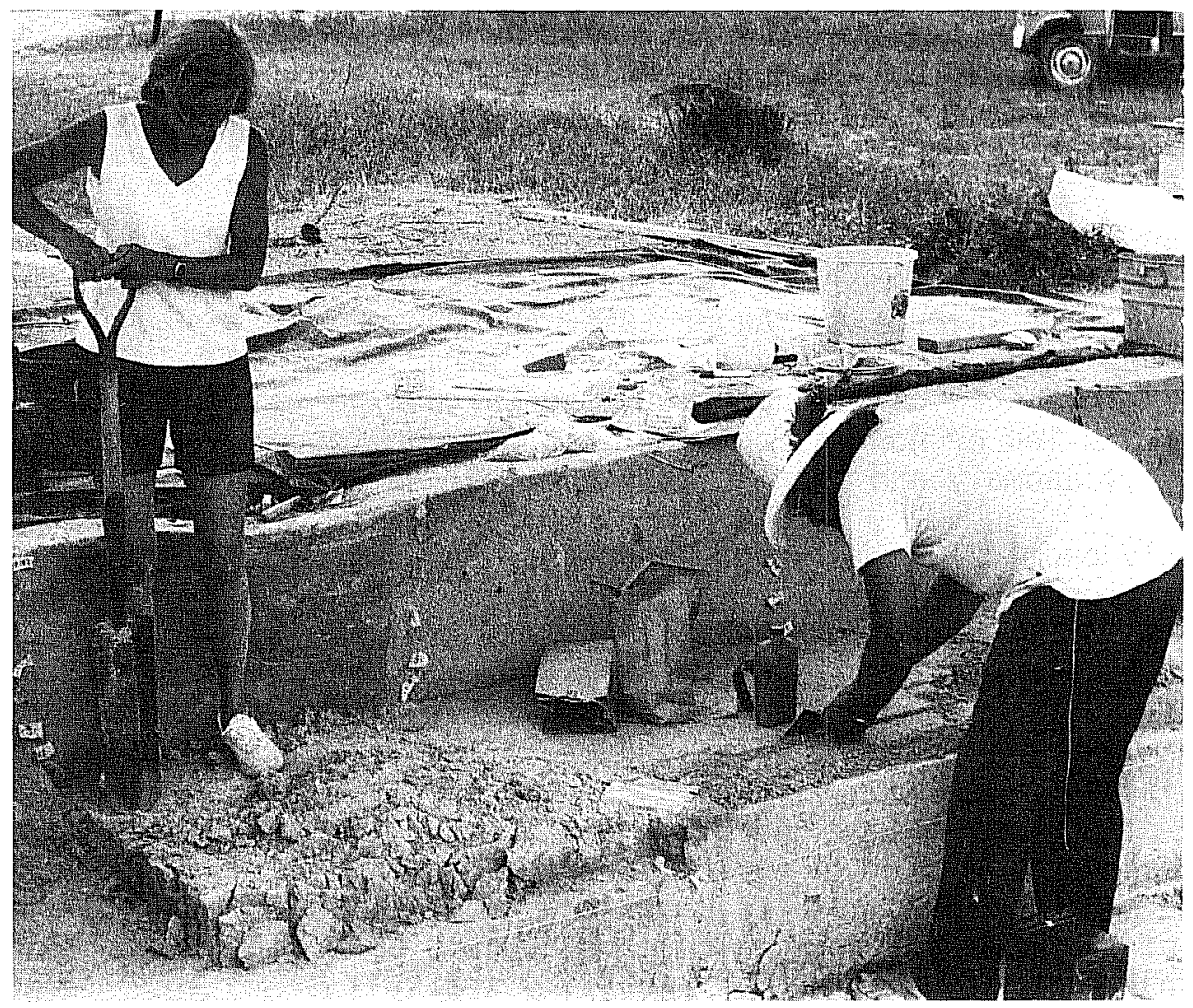

Plate 3. Excavation of Units at One-Centimeter Resolution and One-Meter Resolution. Right, High Resolution Environmental Column (HREC) and Left, Intermediate Level.

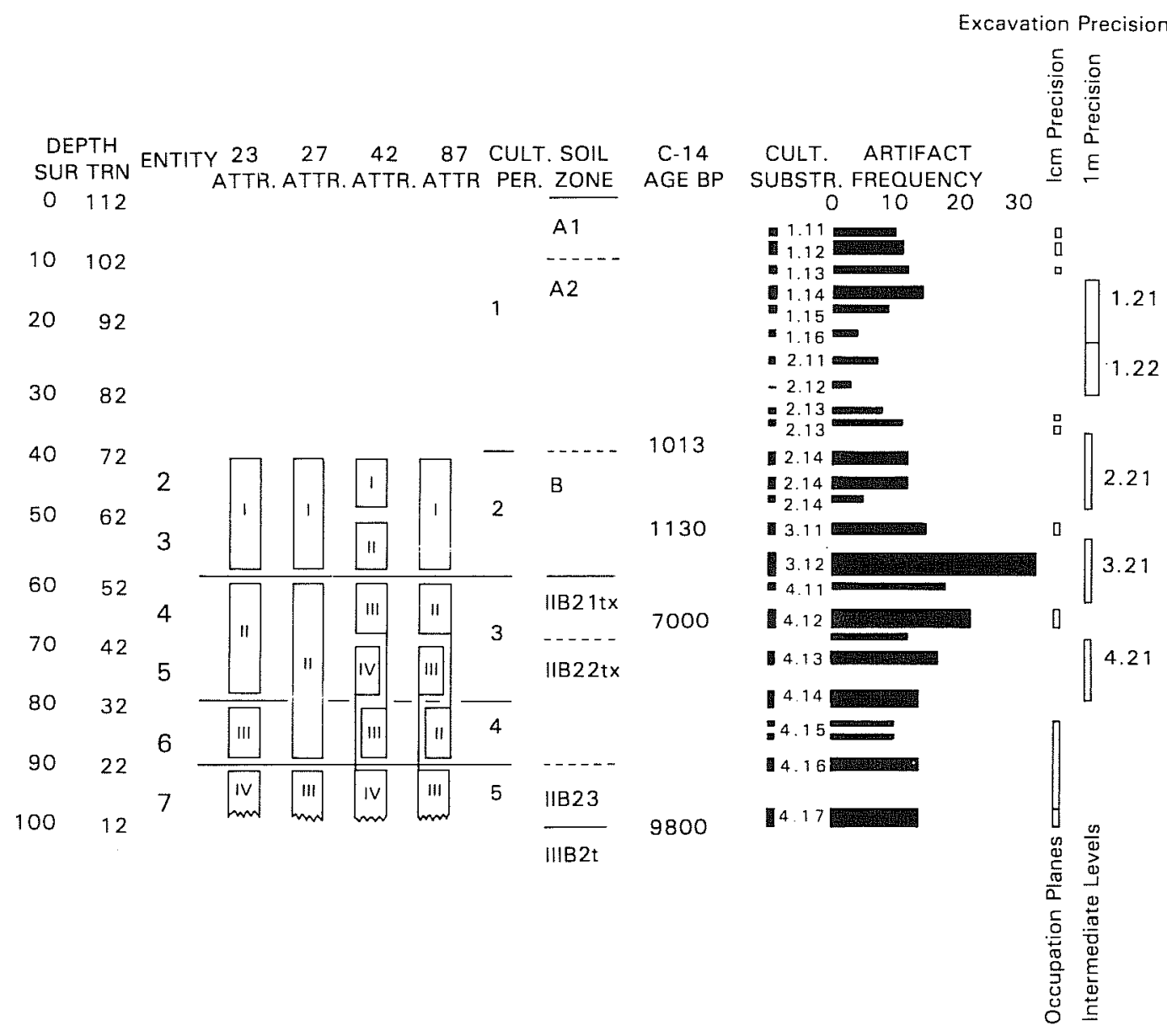

Figure 12. Vertical Sampling Strategy. 
Horizontal Sampling Procedure

In addition to the practical and geological aspects of the block excavation location problems mentioned earlier, there were logical reasons to concentrate excavation effort in Area A. Auger tests and subsequent geochemical analyses defined three kinds of activity areas. The first two are those with artifacts with and without chemical signatures. The third type is areas with chemical signatures and low or nonexistent artifact frequencies. The first type of site is a cultural anomaly and the latter a chemical anomaly.

The strictly chemical anomaly represents an activity involving only perishable tools and materials or, at least few, if any, discarded nonperishable items. The possibility of studying chemical anomalies is very interesting. However, we felt that it would require probes sensitive to the appropriate chemicals attached to an infield computer, which would collect the data and analyze it on site with maximum efficiency. The process of collecting soil samples and analyzing them in the lab would be prohibitively expensive for a number of reasons. Also, much of our understanding of prehistoric cultures is bound up in lithics and ceramics. In the beginning, such studies would be fruitful only in conjunction with studies of surrounding artifact-bearing deposits. The function of the chemically anomalous areas could be studied as complementary relationships with the rest of the activity areas.

Following this line of reasoning, we decided to concentrate on Area $A$ with its concentrated artifact-bearing levels with and without chemical signatures.

\section{Vertical Sampling Procedure}

Excavation of the $1 \times 1-\mathrm{m}$ column in Area A during May indicated that there were over 20 relatively discrete, vertical concentrations of cultural debris. Since our excavation methods and recording at full precision (accurate to one-centimeter) were progressing too slowly to recover an acceptable amount of cultural material in the time allotted, we decided to develop a vertical sampling scheme. Selected cultural substrata, "occupation planes," would be excavated at full precision, while "intermediate levels" would be removed at one-meter precision (Plate 3).

Occupation levels recovered at one-centimeter precision were excavated with trowels, with all artifacts recovered in situ, and important artifact locations measured to the centimeter. One-meter precision units were excavated with shovels and then screened, so that the locations of artifacts were noted to within a meter.

Substrata to be excavated at one-centimeter precision were selected by a twostage decision-making process. For the first stage, we turned to Servel10's analyses of lithic debris from the Area A pits. Servello ran a series of analyses, which showed that there were recognizable changes through time in the lithic technology being practiced at the site. These analyses show that there are about five technological periods at Eagle Hill. The second stage was to select an occupation floor to excavate at one-centimeter precision (from each of the five technological strata). 
Vertical Sampling of the Technological Strata

Once it was decided that sampling the cultural strata would be necessary, our first concern became one of determining what cultures were represented at Eagle $\mathrm{Hi} 11$, and where these cultures were represented in the strata. For information we turned to the test excavation analyses of Servello (n.d.). In an attempt to define an Eagle Hill complex, Servello performed an attribute analysis of flakes from levels of various sites on Peason Ridge. These included six levels from 16 SA 50. The results of these analyses are illustrated in Figure 12, along with depths (from surface = SUR, transit depth = TRN), soil horizons, and cultural periods. Servello ran four cluster analyses illustrated under the $23,27,42$, and 87 attributes columns (ATTR). The rectangles in the ATTR columns span one or more $10-\mathrm{cm}$ levels indicated in the column on the far left (SUR). The rectangles indicate which of the $10-\mathrm{cm}$ levels or "entities," as Servello calls them, are most similar to each other as determined by average linkage cluster analysis of lithic data. For instance, the 23-attribute analysis shows that levels 40-50 and 50-60 cluster together. We have designated this cluster I. Cluster II is levels 60-70 and 70-80, etc. The 27-attribute analysis is different from the 23-attribute analysis only in the combination of level 80-90 into Cluster II. In general, the 42 and 87 attribute analyses confirm the results of the less sophisticated runs.

The single most consistent characteristic of all four analyses is the break in clusters between levels 50-60 and 60-70. The difference between flakes above and below $60 \mathrm{~cm}$ is the single most characteristic pattern in the data set and corresponds to the erosional surface between the modern soil zone ( $A$ and $B$ ) and Soil Horizon IIB. The correspondence between geological and cultural unconformities is strong and lends a certain amount of credence to the analysis. It was eventually determined that the erosional surface represents a 6000-year time gap. During that time the Eagle Hill area saw a substantial change in 1ithic technology and possibly a corresponding change in cultures and populations.

If we proceed, assuming that each of Servello's clusters represents a technologically distinct period and a corresponding cultural episode, we can safely derive five cultural periods from the analysis (CULT. PER. in Fig. 12). These five cultural periods are as follows.

Cultural Period 1--Since Servello did not present an analysis of the upper levels, we must assume that they represent one cultural period. We know that the upper horizons contained ceramics. Stratigraphical1y, the period extends down to $40 \mathrm{~cm}$ from the ground surface and contains 10 cultural substrata, substrata 1.11 to 2.13 , as is indicated in Figure 12 .

Cultural Period 2--The second cultural period is defined by Servel10's Cluster I (23 ATTR) and corresponds to Soil Horizon IB. It contains two cultural substrata, 2.14 and 3.11 , and the deflation surface 3.12 . Artifacts at the bottom of the level are probably on a deflation surface, so it is somewhat surprising that the $50-60 \mathrm{~cm}$ level did not cluster with the unit below. The high frequency of artifacts in cultural substratum 3.12 probably represents a concentration of artifacts from the deflated Soil Horizon IIA.

Cultural Period 3--This unit is defined by Cluster II and is pedologically located in the fragipan of Soil Horizon IIB. It contains four cultural substrata, 4.11 through 4.14 . It is between 60 and $80 \mathrm{~cm}$ below the surface. 
Cultural Period 4--A single 10-cm interval in the bottom of the fragipan appears from other analyses to be related to the levels above. We decided to separate and sample it, because it is near the bottom of the cultural deposits. It contains two cultural substrata, 4.15 and 4.16 , and resides between 80 and 90 $\mathrm{cm}$ below the surface.

Cultural Period 5--The last cluster contains the IIIB deflation surface, 4.17. Soil Horizon IIIB is thought to be late Pleistocene in age. Several formal tools of Paleo-Indian vintage were found in substratum 4.17 between 90 and 100 $\mathrm{cm}$ below the surface.

\section{Sampling of Cultural Substrata within Cultural Periods}

Once the cultural substrata were stratified on the basis of Servello's technological studies, determining the substrata to be excavated with one-centimeter precision became a matter of selecting occupation planes within the cultural horizons. Since our control column was the only information available at a high enough level of resolution, occupation floors to be excavated with onecentimeter precision were selected on two criteria. Those selected were thought to be least likely disturbed and showed the highest frequency of artifacts; this is illustrated in Figure 12 (ARTIFACT FREQUENCY). Thus, cultural substratum 3.12 was removed as an intermediate level with substratum 4.11 , since it was thought to be the IIB deflation surface, and additionally, substratum 4.11 was suspected as downwardly mobile artifacts from the destroyed levels due to the presence of occasional ceramics. The lower levels were removed with high precision in hopes of recovering early occupation floors.

The substrata excavated with one-centimeter precision were removed with trowels and processed by the screening methods outlined below. Intermediate levels were removed with shovels and screened through 1/4-inch screens.

\section{Analysis of Area A Control Column (Gunn, Mahula)}

Later in the field season a more sophisticated analysis of the control column became available as the laboratory staff completed quantification of the recovered materials. During excavation, 19 occupation planes were identified in the control column. Each plane was coded for 17 attributes. These are 1 isted below.

1. Substratum--the substratum number was recorded to indicate the relative depth of the unit. Numbers range from 1.11 to 4.17 (see Figure 12 for complete sequence).

2. Potlidding--frequencies of actual potlids or flakes with potlids were counted to indicate the likelihood of fire.

3. Total Specimens--total number of platformed and nonplatformed flakes from the screens.

4. Pottery--sherd count. 
5. Charcoal--weight in decigrams.

6. Mussel and Snail She11--fragment count.

7. Clay Balls--count.

8. Pebbles--count.

9. Ferric Concretions--count.

10. Charred Resin--count.

11. Seeds--count.

12. One-Centimeter Provenienced Flakes--counts of platformed flakes and mapped chips. Chips (shatter without platforms) were mapped and bagged by the unit.

13. Average Size of One-Meter Provenienced Lithics with Platforms--all flakes were classified into seven sizes using a graded series of parallel lines. The method is discussed in Katz (1976) and Gunn and Mahula (1977). It is a means of characterizing assemblages as to lithic stage reduction. In other words, it classifies the assemblage into workshop, quarry, base camp, or satellite camp, if one is willing to accept flake size as a criterion. For this study, the size categories were reduced to averages per substratum. Averages were figured by assigning each flake the value of the midpoint of the class in which it fell. The average flake size per unit was calculated as the class midpoint multiplied by the number of flakes in the class, summed for all classes, and divided by the total number of flakes.

14. Average Size of One-Centimeter Provenienced Flakes with Platforms--computation same as above.

15. Average Size of Mapped Chips--computation same as above.

16. Average Direction--each artifact provenienced to one centimeter was observed to point in a direction measured in degrees east of north. See the discussion of the Cultural Unit form for further details. The downslope direction was taken as an indicator of disturbance and floor slope. Values were averaged per unit.

17. Average Slope--Each artifact observed to one-centimeter provenience was given a value for the slope on which it was resting. Values ranged from $0-90^{\circ}$ below horizontal. Slope per unit was given as the average for the unit.

A principal components analysis was performed on the 19 substrata by the 17 variable data set to index occupation intensity and determine disturbed levels. Table 2 shows the varimax rotated principle components matrix (Nie et al. 1975). Principle components are clusters of attributes that tend to covary and, therefore, as a group characterize the substrata. In order to avoid confusion with the traditional archaeological connotation of "component," I will refer to the components as "factors." 
TABLE 2. VARIMAX ROTATED FACTOR MATRIX FOR OCCUPATION INTENSITY/DISTURBANCE INDEXING

$\begin{array}{cccccc}\text { FACTOR I } & \begin{array}{c}\text { FACTOR II } \\ \text { Trend in } \\ \text { Disturbance }\end{array} & \begin{array}{c}\text { FACTOR III FACTOR IV } \\ \text { Deflation }\end{array} \text { FACTOR V } & \begin{array}{c}\text { FACTOR VI } \\ \text { Charcoal and } \\ \text { Flake Size }\end{array} & \begin{array}{c}\text { Resin and } \\ \text { Shell }\end{array}\end{array}$

\begin{tabular}{|c|c|c|c|c|c|c|}
\hline Substratum & 0.014 & $0.901 *$ & 0.076 & 0.188 & -0.110 & 0.166 \\
\hline Potlidding & -0.150 & -0.060 & -0.144 & $0.854^{*}$ & -0.047 & -0.043 \\
\hline Total Specimens & $0.726^{*}$ & -0.060 & $0.461 *$ & -0.188 & 0.070 & -0.260 \\
\hline Pottery & $0.900^{\star}$ & 0.006 & -0.138 & -0.002 & -0.073 & 0.065 \\
\hline Charcoal & $0.644 *$ & -0.082 & 0.065 & -0.279 & $0.482 *$ & 0.156 \\
\hline $\begin{array}{l}\text { Mussel and Snail } \\
\text { Shell }\end{array}$ & 0.199 & 0.190 & -0.090 & -0.076 & 0.067 & 0.781 * \\
\hline Clay Balls & $0.819 *$ & -0.083 & -0.143 & -0.249 & 0.222 & 0.074 \\
\hline Pebbles & 0.152 & $0.427 *$ & $0.803^{*}$ & 0.030 & 0.148 & -0.068 \\
\hline $\begin{array}{l}\text { Ferric } \\
\text { Concentrations }\end{array}$ & -0.166 & -0.331 & $0.658^{*}$ & -0.287 & 0.018 & 0.126 \\
\hline Charred Resin & 0.300 & $0.460 *$ & -0.004 & -0.333 & 0.211 & $-0.556 *$ \\
\hline Seeds & -0.016 & 0.064 & $0.876^{*}$ & 0.029 & -0.009 & -0.079 \\
\hline $\begin{array}{l}\text { 1-cm Provenienced } \\
\text { Flakes }\end{array}$ & $0.890 *$ & 0.234 & 0.072 & 0.029 & 0.132 & -0.054 \\
\hline $\begin{array}{l}\text { Average Size } \\
\text { of } 1-m \\
\text { Provenienced } \\
\text { Lithics with } \\
\text { Platforms }\end{array}$ & -0.004 & 0.136 & 0.038 & $0.784^{*}$ & $0.418^{*}$ & 0.077 \\
\hline $\begin{array}{l}\text { Average Size } \\
\text { of } 1-\mathrm{cm} \\
\text { Provenienced } \\
\text { Flakes with } \\
\text { Platforms }\end{array}$ & -0.010 & $0.942 *$ & -0.076 & -0.050 & -0.146 & -0.102 \\
\hline $\begin{array}{l}\text { Average Size of } \\
\text { Mapped Chips }\end{array}$ & $0.763^{*}$ & 0.136 & 0.060 & 0.283 & -0.304 & $0.382^{*}$ \\
\hline Average Direction & 0.094 & -0.129 & 0.083 & 0.217 & $0.829 *$ & -0.021 \\
\hline Average Slope & -0.049 & $-0.624 \star$ & -0.360 & 0.101 & -0.134 & -0.370 \\
\hline
\end{tabular}

*High loadings 
Factor I--Occupation. The positive co-occurrence of most of the cultural indicators on the factor suggest that it is an index of the intensity of occupation. It shows that the screened flakes, pottery, clay balls, charcoal, provenienced platformed flakes, and mapped chips appear in corresponding, greater, or lesser numbers depending on the substratum. A11 of these are clearly cultural phenomena except for the questions raised earlier about the clay balls. The cooccurrence of clay balls with other cultural indicators supports their human origin. Pebbles do not appear on this factor suggesting that they are related to other sources, most likely of geological or avian origin.

Factor II--Trend in Disturbance. The presence of the substratum number on this factor indicates that it measures those phenomena that change with time. Pebbles and charred resins become more frequent toward the bottom of the site. Platformed flakes become larger. The negative sign on Average Slope indicates that the upper levels have more tilted artifacts in them than do lower levels. As will be shown presently, it is the uppermost substrata that have the most tilted artifacts, and it seems most likely that the tilted flakes represent bioturbation and perhaps disruption of the upper levels by recent military and/or logging activity.

Factor III--Deflation. Factor III is most notable for the association of pebbles and ferric concretions. There is also a high frequency of screened lithics and some indication of tilting of artifacts. The overall picture is one of deflation/disturbance. As will be shown later, the level best characterized by this set of attributes is immediately above the B-IIB soil interface and is surely a deflated surface.

Factor IV--Fire. Factor IV suggests that fire-related activities, potlids, etc., are associated with larger flakes. It is probably a secondary indicator of occupation intensity, especially domestic occupation.

Factor V--Charcoal and Flake Size. Large flakes also tend to be associated with charcoal on occasion.

Factor VI--Resin and Shel1. Charred resin and shell have a complementary vertical distribution, except the resins are lower in the section. The shells, no doubt due to poor preservation, are found only in the uppermost levels.

Factor scores were calculated by multiplying the factor matrix by the original data matrix. The resulting numbers are indicative of the relative strength of the occupation planes on the component. For instance, Table 3 shows the component scores for the levels on Factor I, the occupation factor. The units with the highest score are indicated to be heavily occupied. Those with low numbers are sparsely occupied. The scores are an effective, overall occupation index. 
TABLE 3. OCCUPATION INTENSITY INDEX (FACTOR I SCORES) FOR THE SUBSTRATA, OCCUPATION FACTOR

Substrata

2.13

2.14

4.12

3.11

4.14

1.15

4.15

4.11

2.12

3.12

1.13

1.12

4.16

2.11

1.11

1.74

1.76

4.17

4.13
Occupation Intensity

2.54

2.05

1.47

0.34

0.04

$-0.04$

$-0.08$

$-0.11$

$-0.15$

$-0.15$

$-0.19$

$-0.32$

$-0.37$

$-0.44$

$-0.46$

$-0.91$

$-0.94$

$-1.01$

$-1.26$

As will become evident as the report progresses, the principle components analysis of the control column correctly indicated the second cultural period, especially substrata 2.13 and 2.14 as being intensively occupied. This is the Coles Creek interval. The Paleo-Indian 4.17 substratum rates low in occupation intensity at the control column. The Paleo-Indian occupation centers elsewhere in the site. Substratum 3.12, the deflation surface, has a relatively large amount of occupation debris. It, however, suffers from disturbance and so appears on the factors that register disruption.

Figure 13 is a plot of the factor scores for Factor II (Trend in Disturbance) and Factor III (Deflation). Substratum 3.12 clearly is demonstrated to be a deflation level. The substrata in the box at the lower right of the illustration are the sites that are low in disturbance as measured by tilting of artifacts and low in deflation characteristics. All of the substrata targeted for careful excavation fall in this desirable range except substratum 2.13. Substratum 2.13 is clearly not a deflation surface, but it does bear the mark of disturbance. Its proximity to the surface probably exposed it to root action. The analysis suggests that due caution should be used during interpretation of substratum 2.13 and vertically adjacent levels.

\section{Rate of Excavation}

In addition to selecting substrata, which would be desirable to excavate based on cultural criteria, we also had to work within a specified time frame. In a project confined to a certain time interval, as the Eagle Hill endeavor 


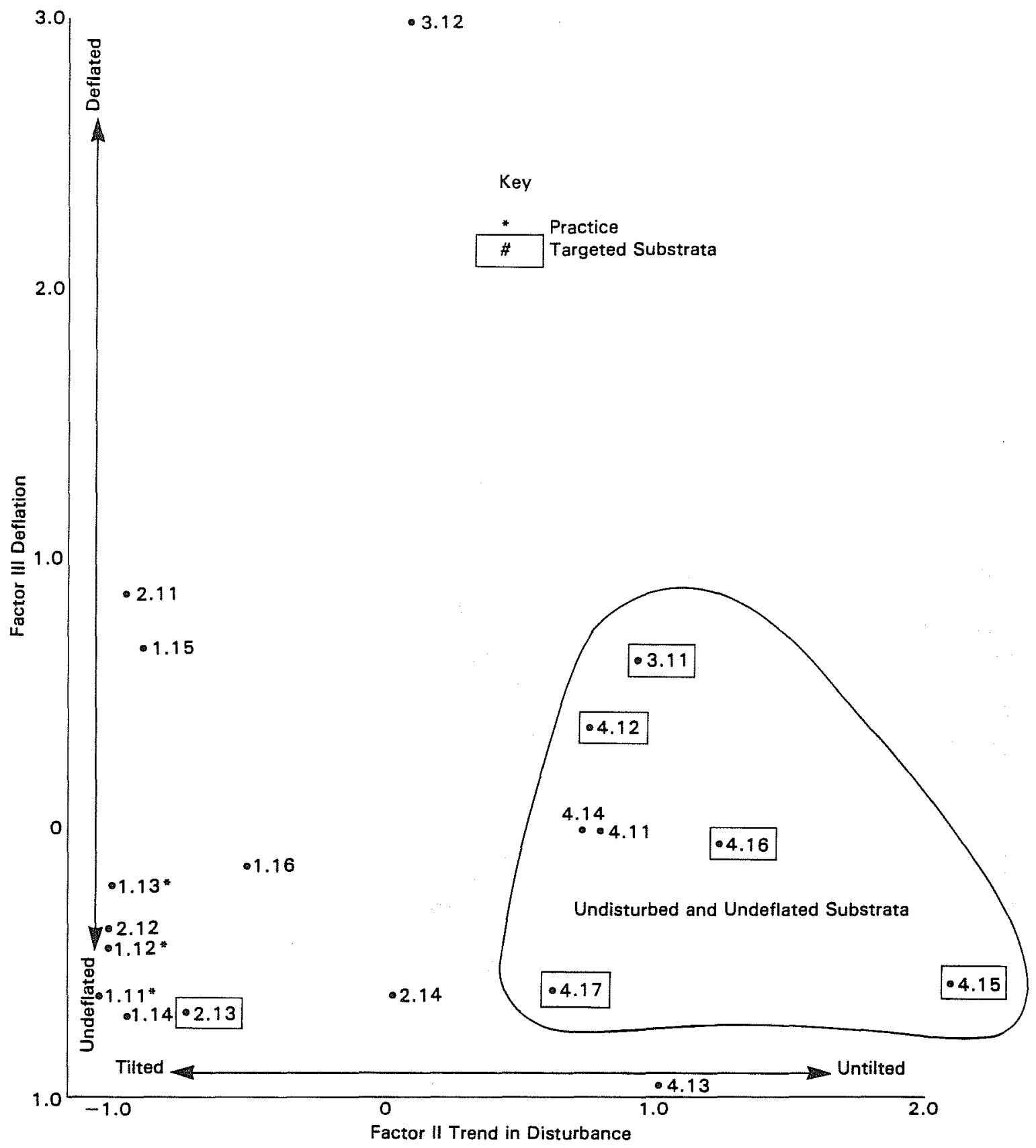

Figure 13. Factor II (Trend in Disturbance) and Factor III (Deflation) Factor Scores. 
was, the speed of excavation was a matter of some concern to all involved. Natural7y, there are a number of factors that must be taken into account when determining the desired rate of excavation. These include the amount of information to be recovered from a given excavation unit, the amount of area that must be excavated to recover a meaningful sample of cultural remains, and the amount of time within which these tasks must be accomplished.

The amount of information to be recovered is integrally tied to the excavation procedures and recording methods. In theory there are two basic approaches to excavation. The first is to remove sediments from predetermined units, usually in arbitrary levels of some specified depth, and recover artifacts from the matrix by screening. Although some larger artifacts and features may be recovered in situ, most of the artifactual material comes from the screens. There is no specific provenience to a resolution less than the size of the square, and no information recovered on the attitude of artifacts in the ground. Most information on vertical concentrations of artifacts and on the physical strata in which they reside is recovered in retrospect by profiling walls and by laboratory examination of vertical concentrations.

The second method involves following natural strata or cultural levels during excavation. Artifacts of specified importance are provenienced by Cartesian coordinates when found, and care is taken to find as many artifacts in situ as possible.

The first approach can be termed the "retrospective" excavation and the latter "anticipatory" excavation. Naturally any given archaeologist probably combines some aspects of both approaches, although there do seem to be two rather we11defined camps of thought. While it is always dangerous to generalize on such matters, retrospective excavation techniques have clearly dominated American archaeology. Some early professionals such as Alfred Kidder followed the anticipatory approach, but North Americans have generally preferred the retrospective approach, because it provides an element of efficiency in terms of "dirt moved." Except in cases of very low artifact densities, information is sacrificed for speed.

European archaeology, on the other hand, has developed more along the lines of the anticipatory model. This tradition was probably encouraged by the frequency of subtle cave and rockshelter deposits in karstic Western Europe and by an older and more mature archaeological perspective, which went through its "efficiency" period in the latter part of the 19th and first quarter of the 20th centuries.

The literature search recounted earlier suggests the desirability of a combined horizontal-vertical strategy. To this end, calculations were made with respect to person days remaining in the field season. A goal of a $5 \times 5$-m unit was set, and a schedule developed to assure its accomplishment. This, along with the vertical sampling strategy, guided the progress of the data recovery.

\section{Excavation Procedures}

While we came to the site with a basic outline of the techniques we expected to use for excavation, one can always expect a gap between theory and reality. Excavation procedures must be tailored to each site and to each group of 
excavators. Our first days of excavation were intended primarily to develop a consensus among core crew members as to approaches to be taken, the nature of the sediments to be excavated, the location of culture-bearing substrata within those sediments, how to record and package the artifacts found during excavation, and screening.

Excavation procedure was organized around eight concepts, each intended to define our means of control over some critical aspect of data recovery.

Control Face--This most basic concept literally dictates trowel movements in the hands of excavators. All excavation, whether it be following a strata contact, an occupation floor, or removing a rodent disturbance, is done against a vertical control face. The upper sediments at Eagle $\mathrm{Hill}$ are very sandy and lend themselves to easy excavation by troweling. The lower sediments proved more difficult, due to high clay content. A control face (Fig. 14) is composed of a vertical cut, the material being removed; a horizontal surface, the material being left; and the contact, the perpendicular juncture between the cut and the surface. An excavator normally works against a two-to-five-centimeter deep control face, which extends across his square. Moving his trowel conformant with the strata, the excavator slices off a defined amount of the control face with each pass, systematically moving across the meter unit. Each slice moves the contact back a few millimeters exposing more of the surface and removing more of the cut. In addition to giving the excavator a clear perception of the materials to be removed and those to be left, the control face allows supervisors to readily check the accuracy of the excavator's efforts.

Control Front--As with the trowel, the movement of the crew needs to be coordinated in a systematic manner. A control front is composed of a line of control faces crosscutting adjoining excavation units. Excavators aligned in this manner are encouraged to pay close and constant attention to the progress of excavation by their flanking comrades. The spirit of cooperation engendered by the excavators on the control front not only spurs efficiency, but also leads to constant communication on matters of density of artifacts, vertical location of artifacts, facies changes in lithology and pedogenic development, and field analyses of interesting distribution patterns. Such discussions insure cross referencing of unit excavation notes and help to avoid problems of after-thefact correlation of occupation floors and lithologic contacts from square to square.

Planing--In spite of determined efforts and optimistic attitudes, we were not able to determine lithologic substrata or microstrata within the grosser sedimentary units at Eagle Hi11. The strata were quite homogenous. We assumed that excavation could not proceed on pedogenic criteria, but had to be judged as part of the original sedimentation processes, which bear cultural units. Our only remaining option was to establish excavation strata on cultural criteria. "Planing" is the technique by which this is done. The first step in planing is to shave a profile leaving artifacts on pedestals until a battery of artifacts is exposed along the face. If good fortune is with the excavator, the artifacts will define a linear pattern across that face (Plate 4). This alignment of materials is taken to mark an occupation floor. At this point, the excavator establishes a control face with the contact two centimeters below the line of flakes, completes a Physical Unit Form (Appendix B, Fig. 91) on the substratum, and moves across the square pursuing the vertical concentration of cultural debris. 


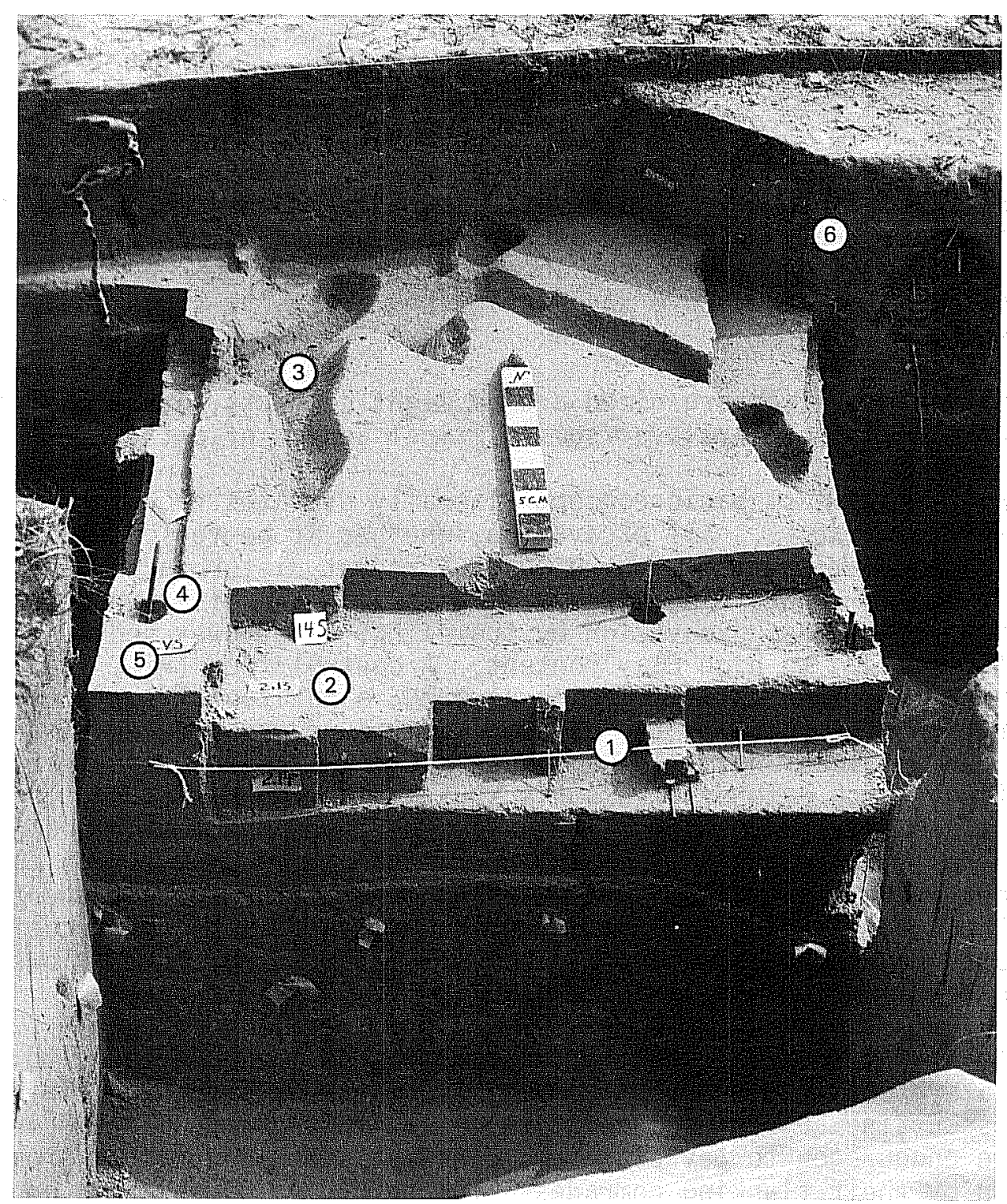

Plate 4. Contral Column (E1000-N300).

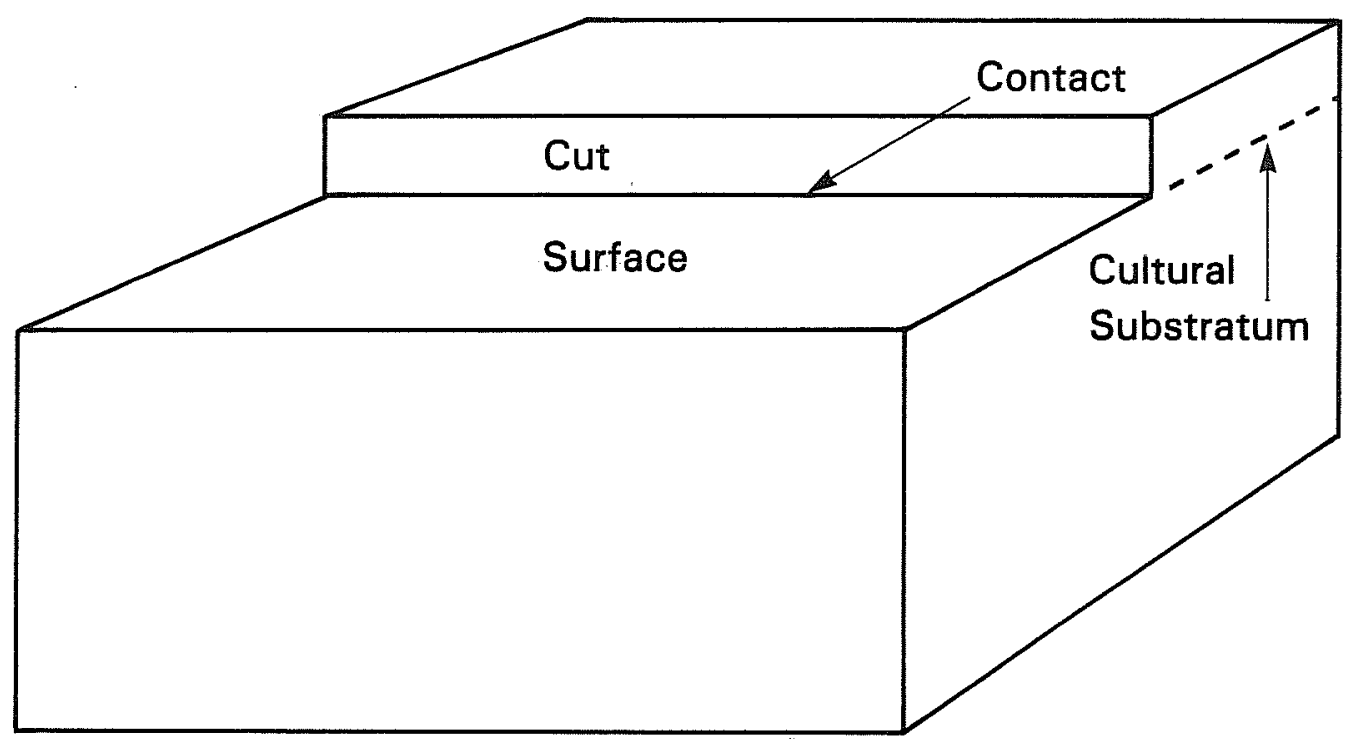

Figure 14. Excavation Unit with a Defined Substratum Being Exposed. 
The surface exposed at the contact must be understood in a very special sense. It is not the bottom of an excavation unit as would be the case in an arbitrary unit, but a plane which estimates the location of an occupation floor. Therefore, artifacts found immediately above and below the plane are considered to be a part of that occupation floor. It is the responsibility of the excavator in consultation with the supervisor, to decide whether an artifact above or below the plane is a part of the targeted occupation floor. Normal procedure was to consider artifacts two centimeters above or below the floor to be a part of that floor. If artifacts appeared outside what the excavator felt to be the normal distribution of the floor, the artifact was tagged with a "+" for above the floor or a "-" for below the floor. If it was found that a pattern of tags existed in a quadrant of the unit upon its completion, one would suspect that the excavator was undershooting or overshooting the real occupation floor, a fact which was compensated for when tagging the wall with the substratum unit number.

Provenience--Artifacts that are considered to be important for one reason or another are provenienced by Cartesian coordinates. Horizontal coordinates are taken from the boundaries of the unit so as to conform with the site grid system. The vertical dimension is determined by transit. This information and description of the artifact, its orientation, slope, and volume are on the Culture Unit Record, Appendix B, Figure 92.

Provenienced items include the following: tools such as points, other shaped lithic items, and edge-altered flakes. Large flake fragments were provenienced, since there was reason to believe they were tools as well.

Platformed flakes were also provenienced. The reasons are as follows. Platformed flakes represent a single act of lithic reduction as opposed to the accompanying shatter. The frequency of shatter is variable and only roughly proportional to the amount of 1 ithic reduction activity at the site. In addition, platformed flakes occur in about the right frequencies to provide opportunities for locating elevation points on occupation floors without overdoing the transit work.

Features such as caches and fire pits were also provenienced. The Culture Unit Form is designed to accommodate virtually any item of cultural origin. Naturally, more extensive cultural phenomena required more than just recording on a computer-oriented form. These items required such devices as plan and profile maps, verbal descriptions, etc.

Mapping--One-meter unit maps were kept for each floor in each unit. The Unit Mapping Record (Appendix B, Fig. 93) is designed so that it can be directly placed under a large sheet of graph paper and copied onto a plan map of the entire occupation floor. We customarily mapped nonplatformed chipping debris, charcoal greater than one centimeter in diameter, disturbances, and any item pertinent to the eventual understanding of the unit.

Bagging--A \#4 paper bag, labeled for each substratum unit, was kept with the forms on a clipboard for that substratum until the floor was excavated.

Excavation, of course, includes recovery of artifactual material from above and below the plane of estimation. A small plastic bag is marked with the FN of the unit/substratum and a "p" for items plotted on maps. The excavator retains 
the plotted items, such as pebbles recovered from within the floor, etc., in this bag. Items recovered from the screens are kept in a bag marked "S." Material from the " $S$ " bag is somewhat problematic, because we do not know for sure if it is associated with the occupation floor above or below. In practice, however, occupation floors contain widely varying frequencies of flakes. In the event that important items are missed, they can be assigned with some certainty to one or the other floors. Plotted charcoal wrapped in al uminum foil is also kept in the unit bag. Provenienced items are placed in separate plastic bags with their unique FN noted on the outside and retained in the unit bag.

Constant Volume Sample (CVS)-A block of soil is left in the southwest corner of the unit until it has been excavated. At that point, when the excavator has become as familiar with the location of the occupation as he will ever be, a 2000- $\mathrm{cm}^{3}$ sample is taken from the block.

We generally try to take this slice out of the block vertically centered on the occupation floor. Special care is taken to locate and remove disturbances from the CVS.

Plate 4 is one of those curious moments when several things happen together allowing rather economical illustration of several points. The following discussion is keyed to the numbers in the photo.

The six penny nails along the front edge of the square (1) point to a line of flakes across the square. The 1 ine of flakes and the decision to excavate them as substratum 2.14 are marked by the stringed 1 ine. Substratum 2.13 (2) was defined in a similar manner, and the control face of that unit has been followed about 1/4 of the way across the square. FN 145 is a cache of flakes, pottery, and charcoal fragments. A rodent disturbance (3) was noted on substratum 2.12 and removed before proceeding with the excavation of substratum 2.13. Also, two bug balls are visible (4) in the photo. One is on the CVS block. The other is at the substratum 2.13 contact in the right half of the unit. Bug balls were confined to the control column and probably permitted by the removal of surrounding dirt for a length of time which allowed bugs to nest. A CVS block (5) has been left in the southwest corner of the square awaiting removal of the rest of the square. A crayfish disturbance (6) is clearly visible as a vertical column in the wall. There are two such disturbances in the square.

\section{Screening (Gunn)}

Screening of the excavated sediments proved to be more of a necessity than originally anticipated. We found that we could not maintain full precision and excavate the volume of sediments stipulated by the contract or dictated by logic to provide a reasonable view of prehistoric camp life at Eagle Hill. Speeding up the excavators, however, insured that some possibly important artifacts would be missed in the excavation process, since the assemblage was composed of generally smaller artifacts than expected. Rather than the lithic workshop we had originally expected, the site was a satellite campsite with characteristic diminutive chipping debris from maintenance activities such as resharpening and reshaping of stone tools. To effect total recovery, it was apparent we would 
need a screening program. Wet screening is virtually the only efficient means of recovering such chipping debris.

Ideally lithic debris of the type found at Eagle Hill should be screened through 1/8-inch screen. A study by Gunn, Mahula, and Sol1berger (1976) showed that 1/8inch screen recovers about $80 \%$ of the most useful chipping debris. Our plans for screening were complicated by a scarcity of water on top of Peason Ridge but, thanks to numerous favorable circumstances, we were able to proceed with rather normal screening techniques early in June. The army's erosion prevention efforts provided us with a pond about $50 \mathrm{~m}$ southwest of Area $A$, and a wet spring season insured that the pond was full to the brim. Also, the upper sediments, those of the modern soil horizon, are very sandy and present virtually no obstacle to water washing. We therefore screened for the first ten days of June in the shadow of good fortune. As the initial phase of excavation passed, however, the pond dried, and the sediments became more clayey. Good fortune had to be replaced by ingenuity.

With our natural water supply gone, the number one priority became water conservation. The personnel of the Air Force observation post on Eagle Hill supplied us with a 500-gallon tank trailer known in military parlance as a "water buffalo" and the means to transport it. Even so, we could not launch a massive water screening effort. We were pleasantly surprised to find, however, that reasonable volumes of sediment could be satisfactorily managed through a multistage screening process that required very little water and was essentially as effective as more typical water screening techniques. Because of the increasing clay content of the matrix to be processed, the strategy had to be varied, sometimes from level to level. Essentially two processes were used. The first is utilized on sandier sediments, the second on those of higher clay content.

\section{Water Conservative Screening of Sandy Sediments}

Once removed from the excavation units, the sediments are dumped onto a suspended $1 / 8$-inch screen and shaken until the majority of the matrix is gone (Plate 5). The use of trowels was held to a minimum to avoid damaging delicate pressure flakes. If the sediments had to be broken a wooden stake was used.

The residues of this operation were removed from the swinging screen and placed in the basket made of $1 / 8$-inch screen. The wire basket was water screened in a washtub perched on the top of a 55-gallon barrel. Once washed, the residue was placed on a plank to dry and then bagged. The washtub and barrel served as a water recycling unit. Sediments washed from the basket settle, for the most part, to the bottom of the tub while the water flows over the edge of the tub and down into the barrel where it is retained. When the tub becomes too full to function as a screening basin, it is set aside for a time to allow the settling process to progress to an advanced stage. The water, so freed, is poured off and recycled through the system, while the sediments are removed to the backdirt pile. Water from the tub is normally recycled from the barrel, although a certain amount of water is acquired new from the water buffalo at each recharge. The process was quite efficient and required two screeners to keep up with eight to ten excavators. 


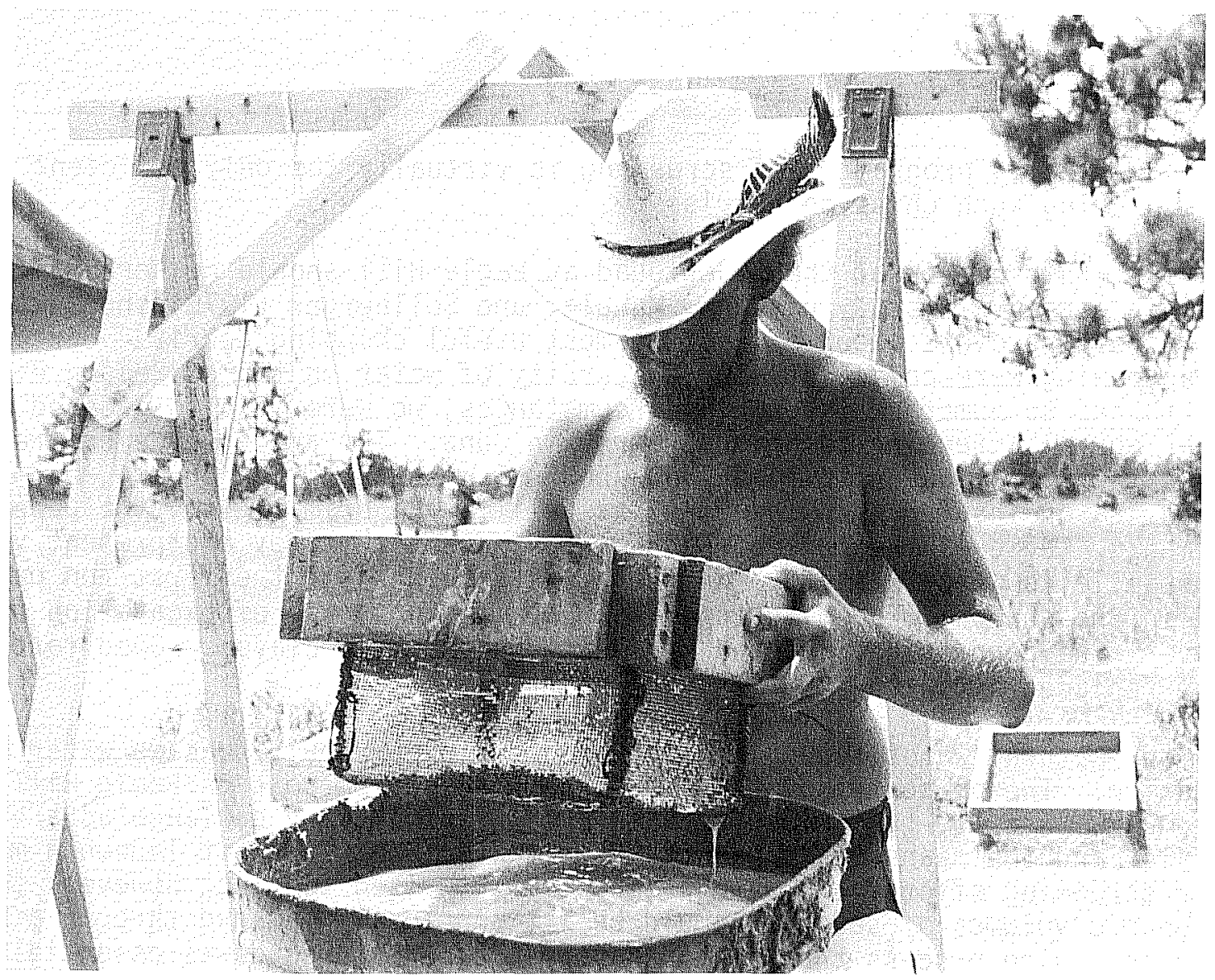

Plate 5. Water Conservation Screening.

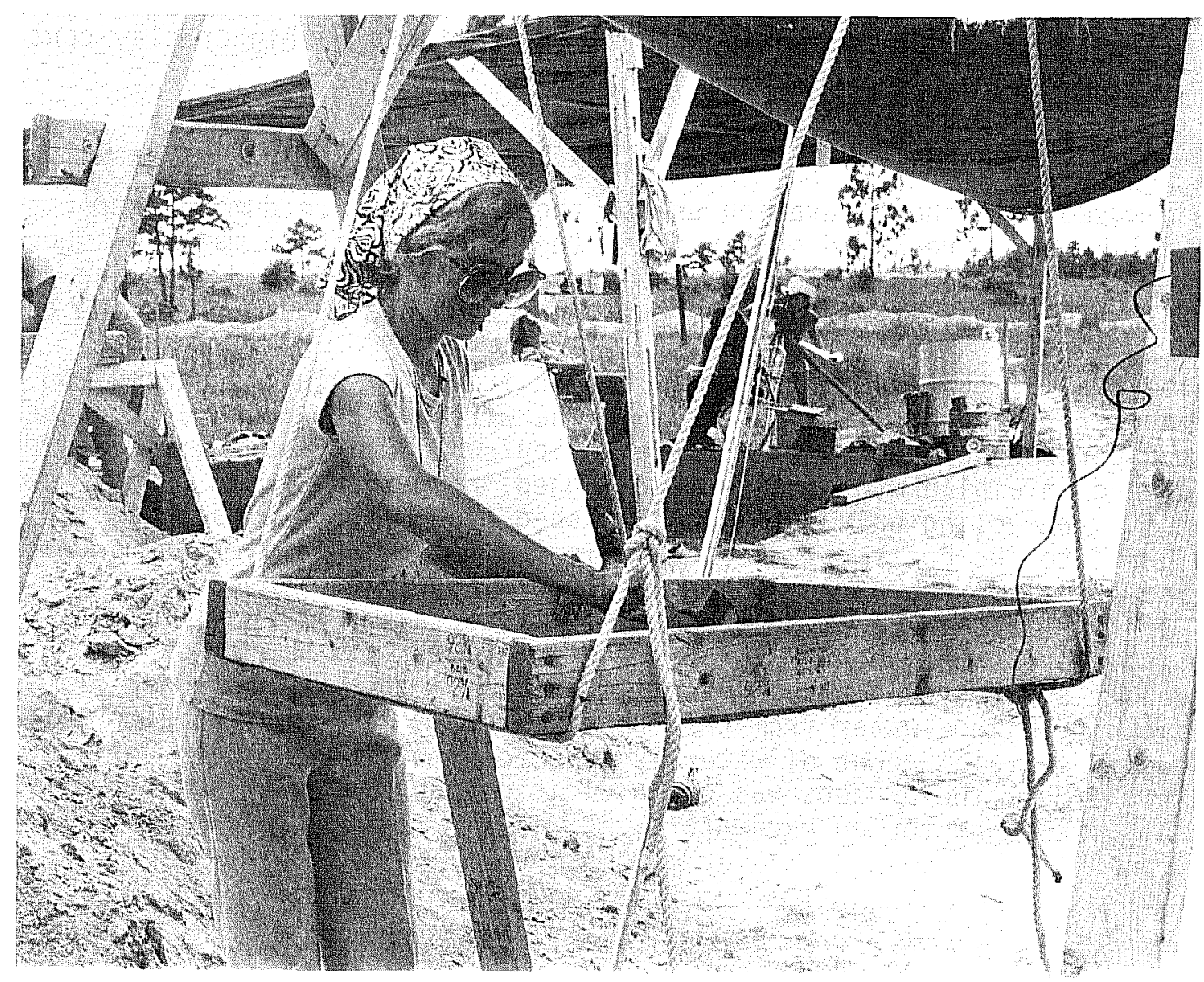

Plate 6. Dry Screening for Sandier Sediments. 


\section{Water Conservative Screening of Clay Sediments}

Screening of Soil Horizon IIB with a higher clay content required a noticeably more complex process, although the flow of motion was essentially the same. There were extra steps added to cope with the aggregating tendencies of clay. As before, the first step was screening on a 1/8-inch suspended screen. This process was quite slow and frequently bottlenecked the operation. We used two suspended screens normally with one or two persons per screen depending upon how difficult the operation was.

Once reduced as far as could be in a reasonable amount of time, the residue of this operation was moved to a plastic, gallon milk container with the top cut off to sit in water for a few minutes. The longer the wait the better, so containers were allowed to back up several deep. With the very difficult finegrained clays toward the bottom of the section, ammonium hydroxide was added immediately after dry screening since the chemical must penetrate the clays from the first. Pre-wetting only slowed the penetration process. The wetted sediment residue was poured into the 1/8-inch basket and water screened in the tub-barrel water recycling unit.

If resistent clay globules remained after the water screening, the residue of this operation was allowed to sit in a $1: 2$ solution of $58 \%$ ammonium hydroxide until the clay was disaggregated. This solution was again screened in the 1/8inch basket allowing the ammonium hydroxide to enter the water recycling system and encourage disaggregation of clay in the first water screening step. We found it most efficient to open an ammonium hydroxide dissolving-container with a unit, and process the disaggregated clays later after the unit was closed, and the ammonium hydroxide had time to do its work. This allowed the excavation and disaggregation processes to proceed together.

The measures described above are conservative of scarce water and are environmentally sound anywhere. The water recycling process insures that water screening does not add particulate matter to natural or constructed waterways. The use of ammonium hydroxide as a disaggregant only slightly fertilizes the soil, but avoids contamination of sediments with harmful chemicals. On the other hand, ammonium hydroxide is not the most effective disaggregant available, and more difficult clay matrices may require more effective chemicals such as hydrogen peroxide or trisodium phosphate.

Late in the summer in the deepest, clayiest sediments (IIB23; Fig. 13), yet another screening method had to be implemented. The clay content was high enough that we could not reduce the volume of the material to be water screened. The only solution seemed to be filling the buckets with water as soon as they were excavated. Then when a large number of buckets had accumulated, they were hauled to the Eagle Hill Air Force observation post and screened under water pressure.

Procedure for Chemical Screening

Place sediment on 1/8-inch suspended screen and screen out as much sand as possible. 
Water screen the residue in a $1 / 8$-inch screen basket. If any clay remains in residue place remainder in container of one cup ammonium hydroxide.

Add subsequent material from 1/8-inch screen as excavation proceeds. Let set for approximately one hour after last bucket from unit is screened. Do not screen until unit is closed--all material from a unit goes into this one container.

Pour ammonium hydroxide and residue through 1/8-inch screen basket over water screening bucket.

Dry and bag.

Be careful using the ammonium hydroxide--if any bodily contact occurs, wash affected area IMMEDIATELY.

Data Management

Present day excavation methods produce prodigious amounts of numerical data. Sometimes the numbers involved are staggering, even to those directly involved in the effort. For instance, there-were 1519 objects provenienced to the centimeter during the field season at Eagle Hill. For each of these objects, 19 observations were made in the field and 32 in the laboratory. Observations ranged from the coordinates of the object, to the material from which it was made, to microscopic observation of wear patterns. Nearly 80,000 observations were made on this data set alone. Each $1 \mathrm{~m}^{2}$ in the site received 106 observations ranging from soil color and texture to excavator number to $X$-ray fluorescence determinations. About 10,000 observations were made for the targeted occupation planes.

It goes without saying that the human mind is not well equipped to wrestle meaning from such masses of figures. However, masses of numbers properly collected in the field and examined with appropriate computer-assisted numerical analyses are often the key to unravelling the messages left by prehistoric peoples.

The problems of deciphering prehistoric "messages" are complicated for a number of reasons. Naturally the patterning of implements and living floors is obscure in itself, because we do not easily see the cultural norms that dictate these patterns. Also, nature has further complicated the issues by encoding her own patterns amidst the cultural remains. Thus, numerical methods are often as important in factoring out, or controlling for extraneous messages from nature, as they are in providing interpretable patterns from among the cultural data.

Numerous studies were conducted on materials from Eagle $\mathrm{Hill}$ in addition to the one-meter and one-centimeter resolution data sets mentioned above. Each individual type of material (charcoal, clay balls, etc.,) was studied for distributions both horizontally and vertically in the site. Neutron activation determinations were made on lithics and clay to determine similarities and differences of source materials.

The plan for controlling these massive flows of data has become rather standardized over the last decade or so, but each excavation has its own special 
problems, which requires tailoring data collection and management procedures. Also, the changing field of archaeological study often suggests innovations that better the potential for analysis and require modification of old procedures. The Eagle Hill procedures were developed out of several years of experimentation by personnel of the CAR. Data collection methods used at Eagle Hill were by far the most satisfactory we have developed to date. Naturally we have improved methods with each past excavation. However, the order initiated with the Hop Hill excavations (Gunn and Mahula 1977) was finally brought to a fully integrated and satisfactory system at Eagle Hill.

\section{Coding Forms}

The coding forms used in the project are in Appendix B. Field forms serve several purposes. In addition to data collection, they serve as prompting devices that insure not only that data are collected systematically from each square, but also when those data are collected. They also insure consistent performance of tasks such as collection of constant volume samples, tagging the wall of a finished unit with the occupation plane number, and observing the presence of various uncollectables such as charcoal flecks. Field forms are prepared with room for written remarks as to relationships between features and artifacts, hunches about stratigraphy, etc. Field forms are also as nearly self-explanatory as possible. Lists of artifact types, soil texture, presence and absence codes, etc., appear on the form so that no field time was lost looking up vital coding information.

Laboratory forms were constructed in a more compact format. The Soil chemistry Record (Appendix B, Figure 92) for collecting $\mathrm{pH}$ and phosphate observations on soil samples is a good example. In the lab, verbal explanations are usually not so important. Explanations of coding procedures are not as likely to be lost as they are in the field. Therefore, the forms are usually designed to save paper and reduce storage space.

Any form, field or laboratory, has to be arranged to minimize confusion on the part of the coders and data-entry personne1. Open formats with carefully marked, meaningfully spaced columns assist in the constant battle against data-transfer errors.

It is also often helpful to know who is collecting the data. This allows for spotting of systematic errors and correcting personnel who are incorrectly implementing procedures. Even if the person who has committed a systematic error is no longer with the project, knowing often provides a key to unravelling problems. To this end, each excavator and lab person was assigned a number:

01 Joel Gunn

02 Fred Nials

03 David Brown

04 Lang Scruggs

05 Darrel1 Sims

06 W. L. Sultivan

07 Bil1 Huber
08 Patricia Wallace

09 Julia Baker

10 Elizabeth Nethery

11 Penn Jenkins

12 Mike Perez

13 Dianne Detrio

14 Beverly Marshal1

15 Burma Hyde
16 Joan Sherwood

17 Eve Mathis

18 Luis Ramirez

19 Isabelle Ruben

20 Ian Shaw

21 Kevin Jolly

22 Robert Guy 
In sites such as Eagle Hill, which have long periods of time contained in a relatively thin veneer of sediments, maintaining vertical control was often the most critical and difficult task relative to provenience. Given the proportions of depth and time at Eagle Hill, it was immediately apparent that line levels would not provide the necessary grade of depth measurements, so we determined to make all depth measurements with a transit. Therefore, we devised a transit form (Appendix B, Fig. 95). Our original intention was only to code the transit shot foresight and backsight in the field and let the computer calculate the depths to avoid problems with miscalculations.

There were problems with this scheme, however. Part of the "control front" excavation program discussed in the excavation techniques section was to keep the excavators constantly aware of the depths of prominent concentrations of artifacts. This necessitated many calculations in the field and resulted in having those acquainted with the transit spend more time than was desirable with matters pertaining to depths.

In future efforts, it would be well worth investing in one of the small computers now on the market to calculate depths, making them immediately available to the excavators and storing the results for future transmission to the ultimate data storage computer. Computers now available would even speak the resultant depths to excavators thus relieving transit personnel of al1 responsibility after entry of the foresight. It is also possible that the transit person could speak the foresight to the computer.

Three other forms were used in the field, which were not directly related to the data collection effort, but did lend some organizational stability to the program. The Substratum Plan Map (Appendix B, Fig. 94) was filled out with to-be-assigned field numbers when a new substratum or occupation plane was opened. The person in control of recording and assigning FNs (Field Numbers) could then make immediate reference to the FNs assigned to various locations without undue time loss.

Similarly, the FN Assignment Inventory sheets (Appendix B, Fig. 97) were used to keep track of blocks of FN numbers assigned to various phases of the excavation. Constant reference to the FN Assignment Inventory sheet during the field season prevented duplicate assignments of FNs in all but a half dozen cases during the whole field season.

Finally, field notes were kept on a form (Appendix B, Fig. 98) that allowed for entry of the time of day and the name of the person entering the notes. Each day was covered in a series of pages marked in the " $n$ of $x$ " pages convention. This assured proper closing and securing of the notes at the end of the day. Notes were kept in ACCO strap binders in duplicate. Duplicates were removed week $7 y$ and returned to UTSA.

The numbers assigned to the formats during the project are listed in Table 4. 
TABLE 4. KEY TO RECORD NUMBERS

Record \#

01

02

03

04

05

06

07

08

09

10

11

12

13

18
Data

Transit Shot Data

Physical Unit Record

Cultural Unit Record

Pebble/Granule Data

One Meter Resolution Study

Wear Pattern Analys is

Clay Ball Analysis

$X$-Ray Fluorescence Data

Core Field Record

Soil Chemistry Record

Lithic Material Type Count

HREC (High Resolution Environmental Column) Analyses

Sherd Data (preliminary ceramic data counts, etc.,)

Unit Mapping Record

\section{Consultants}

The Eagle Hill archaeological project developed its multidisciplinary breadth and archaeological depth through the assistance of several able consultants. Fred Nials and Ed Garner began their examination of the geomorphology and pedology of the 1ocale immediately upon receipt of the contract in Apri1 1980. Their efforts set the context for the definition of excavation procedures, choice of location for excavation, etc. David Brown did final preparation of the geomorphically related reports.

Immediately before the end of the excavation season, Albert Goodyear visited the site, inspected the geomorphical situation, and examined the lithics from the site. His first-hand knowledge of lithics in Arkansas and the Southeast in general provided many pertinent insights into our literature search problems, and he suggested several interesting directions that our lithic analysis could take. Of particular interest to our problem of developing a nonprojectile point tool typology is the fact that Goodyear had just toured a good part of the Southeast examining the so-called Albany spokeshave. He has identified the same tool, in several areas, named differently at different times by different people. Goodyear is of the opinion that the literature on lithic technology in the Southeast does not lend itself to wide-ranging generalizations about tool types. Virtually the only way that such a tool typology can be built is to visit collectors and archaeologists, examine their collections, and determine the nature and distributions of specific tool morphologies.

During Taboratory analysis, Don Lewis of The University of Texas at San Antonio provided us with assistance in the interpretation of several difficult geochemical problems including the analysis of the X-ray fluorescence (XRF) data. 
Various laboratories also assisted us. Jerry Hoffer of The University of Texas at El Paso ran XRF samples. The Center for Applied Isotope Studies at the University of Georgia ran radiocarbon dates. Ralph Rowlette of the University of Missouri at Columbia made the thermoluminescence determinations on fireburned 1ithics. Mark Sheehan of the University of Indiana examined sediments for evidence of pollen.

\section{Laboratory Activities and Procedures}

Thanks to the efforts of Royce Mahula and a small staff of students and "workstudies," the Area A control column and the bulk of the one-meter precision excavated material was curated and some analysis done by the end of the field season. Upon returning from the field the crew organized the summer's records, keypunched the data collected, and began work on the one-centimeter precisioned materials. By the end of August the general provenience materials (plotted artifacts and materials from the screens) were nearly curated. The approximately 1500 provenienced artifacts were curated and studied in the fa11 of 1980 with sediment samples, tools, etc.

Processing of materials from Eagle Hill began following the preliminary test excavation of the Area A control column. Procedures for processing materials from this column served as the basic laboratory method for all subsequent materials. The purpose of the Area $A$ analysis was to assist in vertical sampling decisions.

The following is a summary of the personnel involved and the procedures used in the study of Eagle Hill materials.

Preliminary processing of the control column was performed by Royce Mahula assisted by Betty Neumann (a student doing independent study in Tithic identification) and Debra Jenkins (a work-study student). A11 work was double checked by Mahula. The laboratory procedures for the Area A control column were basically the same as for the rest of the project. Their detailed description will serve as a model for the entire operation. Discrepancies between control column and noncontrol column procedures will be noted in a following section.

\section{Provenienced Materials}

Artifacts provenienced to one centimeter (FNs) were removed from the level bag, inspected to verify field classification, recorded in a FN Log noting coordinates and artifact type, stapled to a $3 \times 5$-inch index card with the FN recorded in the upper right hand corner, and filed in a $5 \times 12$-inch file box by FN sequence for future material, technology, and wear analysis.

The more generally provenienced mapped chips (flakes without platforms that were plotted on substratum maps and then bagged as a unit) were checked and rechecked, counted, and refiled in the same manner as the more specifically provenienced lithic materials. 
Bags of one-meter provenienced materials were washed and contents separated. Categories of materials include Tithics, pebbles, clay balls, charcoal, charred resin, she11, and hematite/limonite concretions. All categories were quantified and recorded on coding forms.

Screen-recovered 1 ithics were separated into those with platforms and those without platforms. Counts of each type were made and recorded.

Small polished quartzite pebbles were saved in the hopes of illuminating depositional situations. Pebbles were separated, weighed, recorded, and bagged.

Charcoal fragments were separated, weighed together, recorded, and bagged.

Implications of charred resin could be important. Therefore, clumps of charred resin were identified, weighed together, recorded, and bagged.

Although sparse, some specimens of mussel shell were recovered. Pieces of she 11 were counted, recorded, and bagged.

Nodules of 1 imonite and, less frequently, hematite were in evidence. These were weighed, recorded, and bagged.

Potlids were isolated and bagged separately from other lithics. Counts were made and recorded. Evidence of firecrazing and potlidding on other lithics was noted and recorded.

In addition to the above weights and counts (i.e., FN artifacts, plotted chips), al1 lithics and one-meter provenienced flakes were sized according to Katz (1976).

Following processing and recording, all materials except FN artifacts were returned to the level bags and refiled by square and level coordinates. Data were then keypunched and analyzed.

After the commencement of the formal excavation season, materials from the field were returned to the 1 ab at somewhat irregular intervals. Although handled in a slightly different manner, the basic categories and procedures implemented during analysis of the control column were utilized for the new material with the following exception. Control column materials were excavated at one-centimeter resolution, i.e., at the level of the occupation floor. Therefore, al] levels contained precisely provenienced materials that were already classified, bagged, and labeled separately. During the course of the excavation when the general character of the various occupation floors became clearer and the importance of each more easily assessed, several levels were excavated at one-meter resolution. The dirt from these levels was removed in bulk from the unit, screened through 1/4-inch mesh and bagged. As a result, these levels did not contain previously identified and labeled artifacts (FNs). Consequently, while the incoming one-centimeter precision levels were treated in basically the same manner as the control column (materials were only washed, separated, and bagged, but not quantified); the level bags from the one-meter levels were processed differently. 
Since the materials in the field were screened only, all materials were washed in the lab. Material was then sorted into the categories outlined above. All tools were identified, labeled (on card), and bagged separately. 0ther lithics were separated into platformed and nonplatformed flakes, counted, and bagged separately. Since all noncontrol column material was going to be further processed and quantified by a laboratory analysis class in the fall, all classes of materials including tools were returned to the level bags and refiled, rather than filed in card boxes.

\section{Geo-environmental Samples}

During the summer, incoming constant volume samples (CVS), soil, phytolith/ pollen; and HREC samples were shelved by unit for later analysis.

Radiocarbon samples were sent to Dr. Barbara Brandau at the Center for Applied Isotope Studies, University of Georgia, Athens, Georgia.

After the field season and with an increase in available laboratory personnel, processing was concentrated on completing the remaining one-centimeter provenienced levels. Following the washing and sorting process, quantification of materials began.

Washing and Storing--Lithic artifact curation involved three classes of artifacts. Shaped tools such as points, scrapers and burins, edge-modified flakes, and platformed flakes were individually bagged in small plastic bags. These were not washed because the dirt on the artifacts was considered important. A second portion of the artifacts were cleaned and labeled in standard archaeological fashion. These were mainly whole flakes with platforms that were extensively analyzed by measurements, etc., and therefore needed to be individually distinguishable for technological studies. The third set included the bulk of the chipping debris, chunks, chips, shatter, broken flakes without platforms, etc. These were washed in a calgon or mild hydrogen peroxide solution, weighed, and studied in other ways en masse. It would have been an extremely time-consuming task to label these artifacts individually, and there would have been no particular benefit to come from it. They were stored in small plastic bags, stapled to $3 \times 5$-inch index cards and labeled as to their provenience.

This plan was discussed with Duke Revet of the Louisiana Division of Archaeology and Historic Preservation. He indicated that his institution is in the process of re-examining their cataloging procedures, and that the procedures we proposed were in accord with those they plan to implement. 


\section{PHYSICAL CONTEXT AND CONTENT}

\section{A. THE OPEN-SITE ENIGMA}

The archaeologist comes to a site as an applied stratigrapher and through the clever and correct interpretation of sediments decodes the events of prehistory. There are, however, considerable variations in the complexity and subtlety of the interpretations to be made. Schiffer (1975) has discussed some of the natural and cultural transformations that alter site sediments after deposition. Logically we could suppose that stratigraphy in caves and rockshelters is potentially least complicated because of partial to complete protection from the elements. This simplicity is often unraveled by cultural perturbations associated with intensive occupation. At any rate, the natural forces are minimized in the effort to interpret the stratigraphy and passage of time.

Open sites, on the other hand, are subjected to a battery of altering processes following their stratigraphic deposition. The initial making of a site is primarily the product of geomorphic and cultural processes, which are often interrelated in their mutual affects on each other. For instance, a site on a gentle slope is geomorphically subjected to an influx of sediments from up slope. Whether those sediments remain is determined by numerous factors which may include the verdure of the vegetation fostered by the enriching effects of human activity. Such interaction often results in a steady accumulation of deposits that assist in the separation of units of time.

Ideally, open sites are composed of layers that can be followed during the excavation process. Subsequent to geomorphic deposition, forces are active that rework the colors, redistribute grain sizes of soil particles, and alter soil chemistry and the original geomorphic structures marking the archaeological horizons. In other words, the soils, or pedogenic processes, can mask the interpretive clues upon which the archaeologist depends to insure the integrity of archaeological components. The problems presented by pedogenic structures increase through time. Sites as old as Eagle Hill II represent thousands of years of alteration of the original geomorphic-cultural complex. To avoid errors of interpretation, it becomes necessary to understand the geomorphic development of the site and subsequent pedogenic developments that obsure the culturaliy related phenomena.

In this section a number of sources on paleoenvironmental information pointing to substantial climatic changes during the late Quaternary in the Southeast are reviewed. As will become evident in this and succeeding sections, sedimentation and human occupation on Peason Ridge are strongly related to climatic change in the general Southeast. Once the climatic stage is set, the geomorphic details of the Eagle Hill locale and its sedimentology are recreated by various means ranging from on-site observation to detailed laboratory analysis. 


\section{B. PALEOCLIMATOLOGY OF THE GULF OF MEXICO COASTAL PLAIN}

Introduction

For a number of important reasons, ranging from oil to presumed isostatic stability, the Gulf of Mexico has been the focus of considerable paleoenvironmental study during the post-World War II period. Landward, the Gulf of Mexico Coastal Plain is a recent arrival to environmental interests. There, too, however, is a growing body of knowledge relative to past climatic variation. While much of these data are products of purely environmental research, part are results of archaeologically related activities aimed at the discovery of the ecological conditions to which human inhabitants of the American Southeast were compelled to adapt.

While early efforts to determine climatic variability in the Gulf Coastal Plain have, to a11 appearances, roughed out the nature of past climatic change, few were conceived and/or executed on an interregional or interdisciplinary basis. Notable exceptions are the efforts of H. Delcourt (1979), P. Delcourt (1980), Delcourt and Delcourt (1980), Delcourt et al. (1980), Gagliano (1977), and Muto and Gunn (1982). Most existing models of climatic change in the Southeast are, therefore, verified by the same data from which they were drawn. While such reasoning may be satisfactory during the early and basically empirical stages of a problem, those models that are derived through processes involving circular reasoning must eventually stand the scrutiny of interdisciplinary and interregional crosschecking, or in other words, independent verification (see, for instance, step 3 of CLIMAP [1976:1134] methodology).

Given the apparent need for interdisciplinary cooperation on the matter of prehistoric climatic change, the author participated in a series of projects beginning in 1976 under the auspices of the CAR-UTSA. The CAR undertook these projects either alone or in cooperation with other institutions, notably Texas A\&M University, the Benham Group, and the University of Indiana. Funding at various times was provided by the Ewing Halsell Foundation of San Antonio, Bureau of Land Management, Corps of Engineers, Texas Parks and Wildlife, The University of Texas at San Antonio, and Fort Polk Louisiana. While each of these projects served various immediate goals, the ultimate objective was to advance the integration of modern and prehistoric data into a conceptual whole, which could act as a model of Gulf Coastal Plain climatic process and progress. These ends were particularly abetted by the Tennessee-Tombigbee Early Man and Quaternary Environments project of which Victor Carbone was instrumental in designing and administrating.

This section is intended, in part, as a review of the progress of paleoclimatic studies on the Gulf Coastal Plain and adjacent related areas such as the Gulf of Mexico and continental areas to the west and north. While I have attempted to be as exhaustive as space and time would allow, I can only imagine that there are gaps in the review. For these I can only apologize to the neglected researchers and ask their indulgence until such time as their efforts can be incorporated. 
A matter which might be profitably discussed at the beginning of this venture is the benefits that can be expected. This article is directed toward three methodological issues. There is the matter of scientific soundness of an independently verified model of climatic change for the Gulf Coastal Plain that was mentioned earlier. Second, it is also possible that a clarification of interregional and interdisciplinary consistencies could avoid a great deal of useless quibbling such as has occurred in other areas of North America. I am thinking specifically of the greater southwestern United States. During the 1950s and 1960s, seemingly contradictory empirical evidence from various regions led to a great deal of argument over whether the Altithermal interval was dry or wet. This argument spread to other areas, but was ultimately resolved by an understanding of atmospheric air streams, which suggest that al1 of the combatants were correct in their respective regions. In other words, a spatially broader and more encompassing interdisciplinary and interregional view of the problem rendered two decades of seemingly important issues unimportant. Much of what follows is specifically intended to provide a broad global and continental backdrop for paleoclimatic studies in the Southeast.

I think there is evidence that the Gulf coast is susceptible to interregional climatic variations which could lead to similar misunderstandings. For instance, Wigley, Jones, and Kelly (1980) conducted a study of the five coldest and five warmest years on record between 1925 and 1974. Relative to the Southeast, their results showed the global warming causes increased moisture in the lower Mississippi Valley as is illustrated in Figures 15 and 16 . The remainder of the area, in contrast, experienced drying associated with warmer global temperatures. If we assume that the five warm years are analogous to the Hypsithermal (45007500 B.P.) and the five cold years like a mildly glaciated Little Ice Age climate (A.D. 1450-1850), the analysis would imply that part of the Southeast would experience increased precipitation in the Hypsithermal and part would be drier. Without an interregional model of moisture response to global climatic change, needless arguments could very well result.

A third issue is most fundamental and overriding to this article. Virtually all meteorological work on climatic change and, in a sense, most paleoenvironmental investigations have been structured around a predominantly spatial fabric. Even those most oriented toward diachronic reconstruction are methodologically a series of spatial "snapshots" set end to end, notably Bernabo and Webb's (1977) synthesis of northeastern pollen data, Lamb and Woodroffe's (1970) circulation analysis, and CLIMAP's (1976) reconstruction of Pleistocene global surfaces. In this section an effort will be made to develop a time series oriented methodology that will be more useful to diachronically involved disciplines. Toward this end theoretical atmospheric, geomorphic, alluvial, botanical, zoological, and cultural chronologies have been set to 500-year resolution trajectories. If nothing else, this procedure shows the relative strengths of the various indicators of climatic change. In addition, the data are set to a format that facilitates analysis, as will be demonstrated on a subset of the data. 


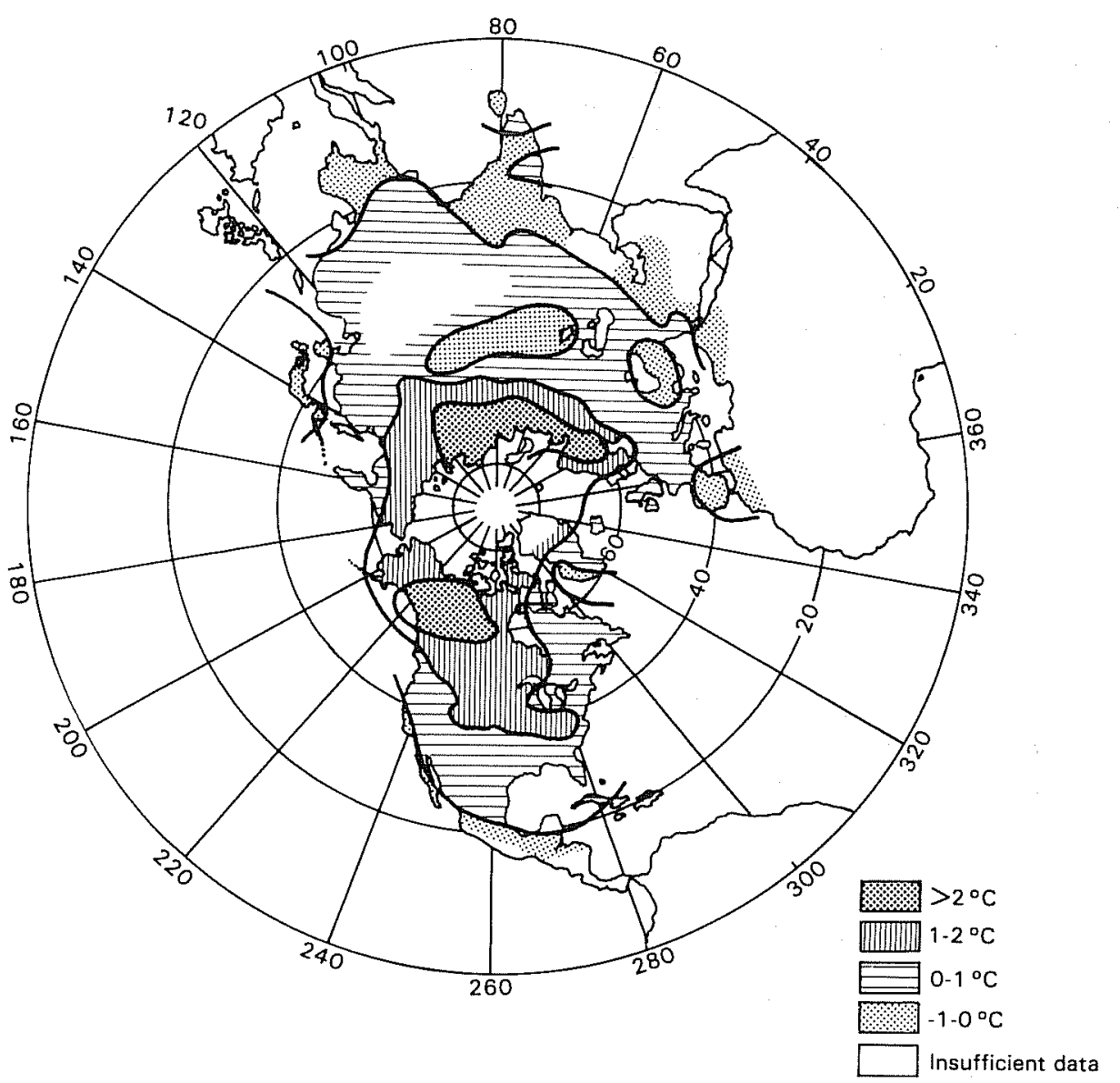

Figure 15. Mean Annual Surface Temperature Changes from cold to warm years. The corresponding change in the hemispheric mean temperature is $0.6^{\circ} \mathrm{C}$. For reference, the expected change in global mean temperature due to a doubling of atmospheric $\mathrm{CO}_{2}$ concentration is $\sim 2^{\circ} \mathrm{C}$.

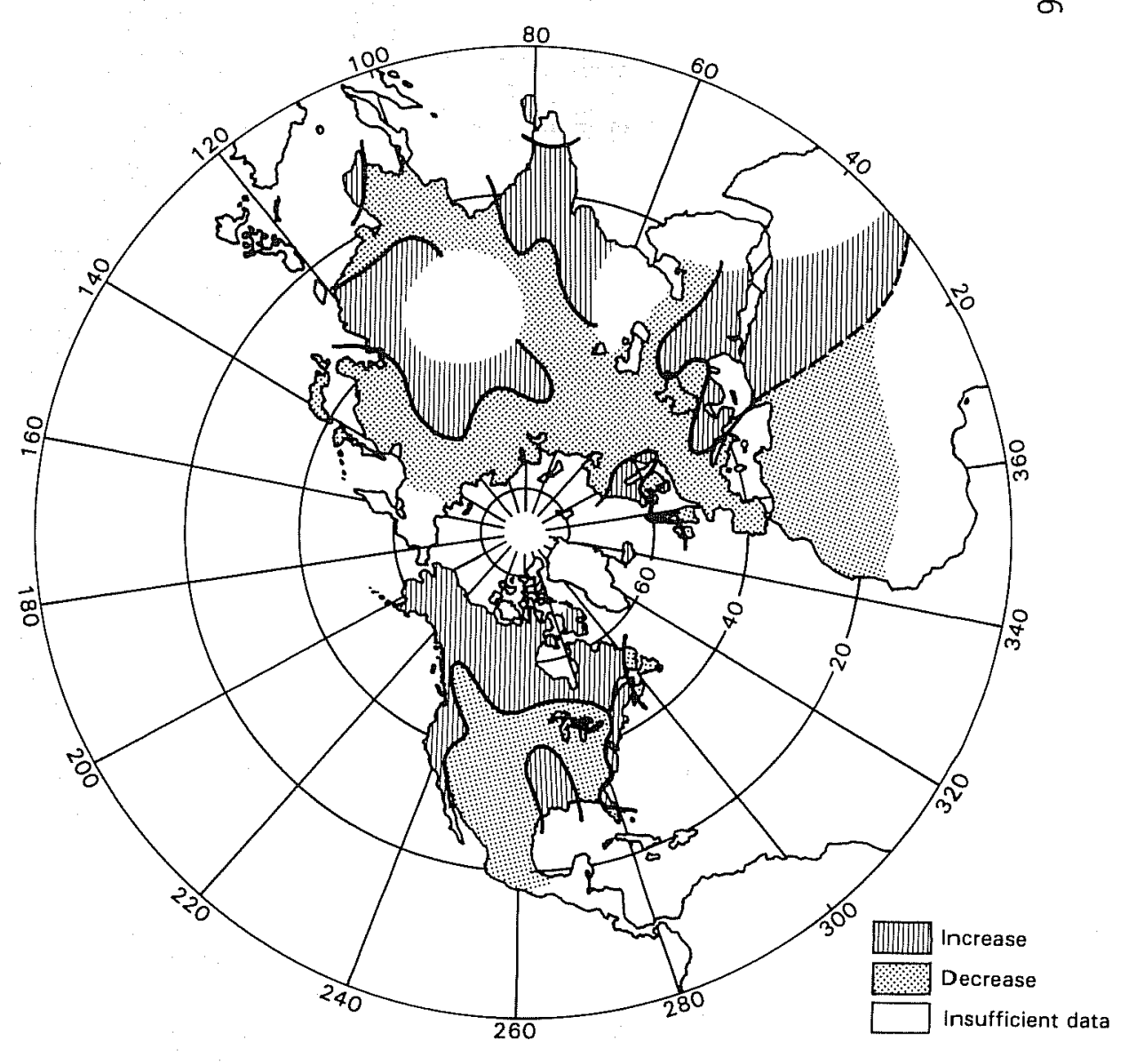

Figure 16. Mean Annual Precipitation Changes from cold to warm years. 
Changes and Lags

Since we will be discussing numerous data bases in a basically temporal context, it will be necessary to establish an understanding of the relative behavior of the variables involved. In this, I have largely followed Bryson and Wendland's (1967) lead as illustrated in Figure 17. Naturally, sea levels will follow glaciers instantaneously. Climate is defined by global forcing variables, such as variation in solar output (Eddy 1977a, 1977b; Mitche11, Stockton, and Meko 1979); variations in solar input to the atmosphere and hydrosphere, as controlled by orbital configurations (Kukla 1975); and atmospheric aerosols (Bryson and Goodman 1980). Surface albedo is taken to be a dependent variable controlled by orbital configuration (Kukla 1975) and by vegetational response to climate (see CLIMAP 1976 for a discussion of various surface a Tbedos).

Important lag factors reside in some components of the marine energy reservoir (CLIMAP 1976) and the vegetational migration (Whitehead 1982), which may result in lags of hundreds or thousands of years.

Atmospheric Circulation

It is the belief of the author that local data bases such as have been generated by previous single discipline efforts can be best reconciled through an atmospheric model. Atmospheric circulation models have the potential to engage independent forcing variables, such as variation in solar output, to encompass the thermodynamics of the Earth's energy system, readily span interregional space, and dispense with lag times unimportant at the scale of interest, which is seasonal.

At this point, I hasten to add that an "atmospheric model" as used in this paper differs from the normal meteorological application of the term. Meteorologists are concerned with a great deal of space--the globe, over a very short period of time--the present. By contrast, the paleoclimatologist and archaeologist are concerned with great lengths of time or "time series." In the inevitable trade-offs that must be resolved to apply meteorological principles to prehistory, some of the spatial detail has to be sacrificed for a clearer understanding of climatic process through time. By necessity, then, much of what follows is a matter of selecting from what is available on the literature of modern climate and adapting it so that it can be applied to long temporal spans in the past.

Bryson and his associates have been particularly successful at constructing generalized atmospheric models which explain interregional climatic variation under varying global energy budgets (Bryson 1966; Bryson and Wendland 1967; Bryson and Murray 1977; Bryson, Baerreis, and Wendland 1968). Bryson's reconstructions generally involve application of knowledge of the behavior of the jet stream and related fronts as global mean temperatures vary. An understanding of jet stream behavior is basic to any serviceable atmospheric model (Reiter 1961). There are numerous jet streams at various latitudes and flowing in different directions a 71 depending on the thermodynamic demands of the atmosphere and the effects of the Earth's rotation. Of most concern to the 


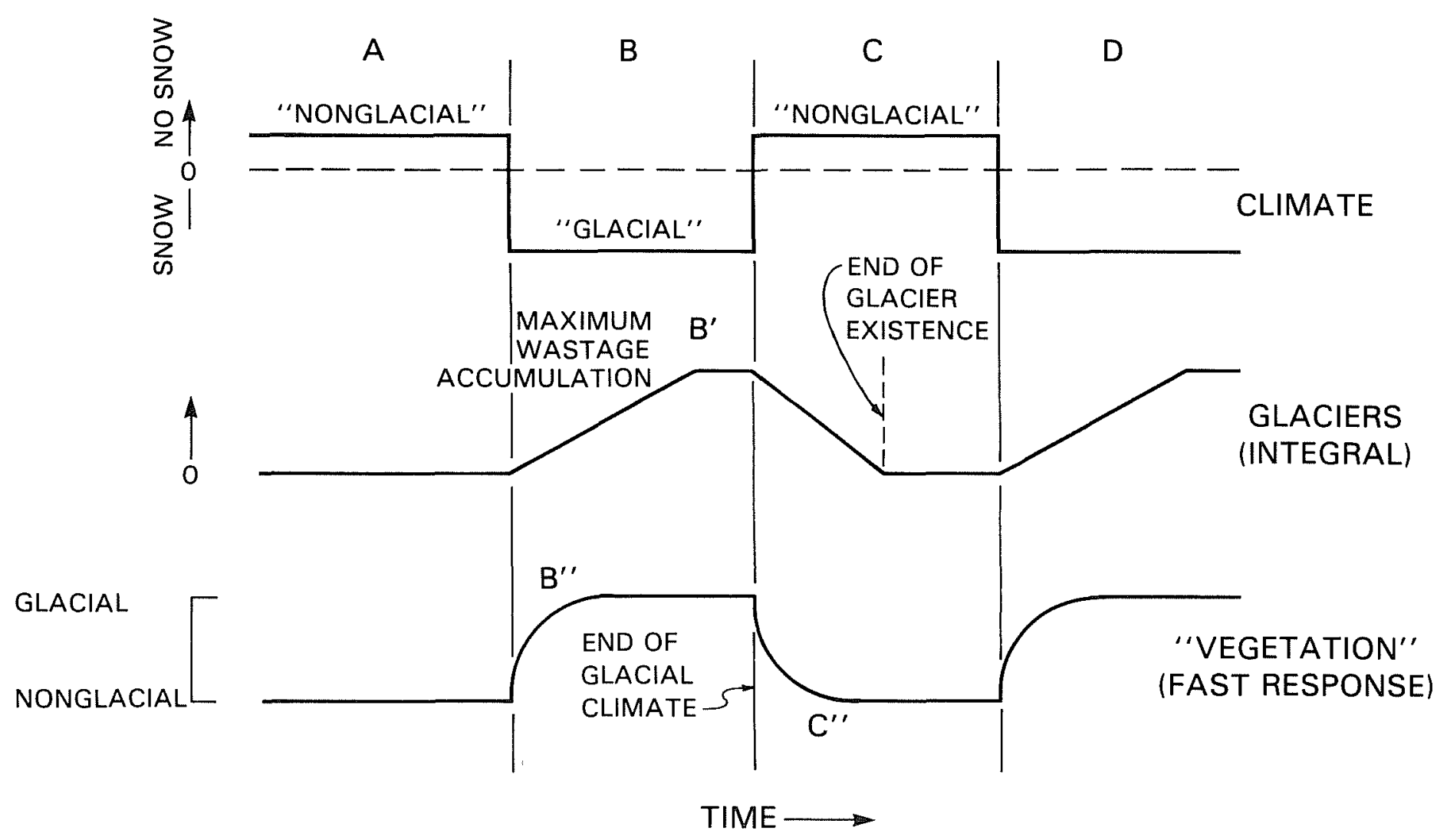

Figure 17. Schematic Glacial and Vegetative Response to Abrupt Climatic Change. From Bryson and Wend 1 and 1967. 
climate of North America is the midlatitude jet stream and its tributary, the subtropical flow. The jet stream flows generally from west to east at about $10 \mathrm{~km}$ altitude and is accompanied at the surface by westerlies and westerly moving weather systems. The rate and latitude of flow is dependent upon the status of the global energy budget, the summed energy reservoir in the atmosphere, hydrosphere, and land mass (Budyko 1974). If the energy budget is high, as during the summer or a warm climatic interval, it flows relatively slow at a more northerly latitude (Sanchez and Kutzbach 1974). During winter or cold intervals, the jet stream speeds are faster and at a more southerly latitude. The fastest winds observed in the midlatitude jet stream are over $200 \mathrm{mph}$.

The details of temperature and precipitation patterns in an area such as the Southeast are dependent on the paths the jet stream takes under varying energy budget conditions. These paths may follow a more or less straight west to east pattern called "zonal flow," or they may undulate across the continent giving an element of north to south flow for surface winds, a pattern termed "meridional flow."

The undulations in the flow of the jet stream have a normal tract for various energy budget levels. These tracts are called "standing waves." The forces controlling the standing waves are of considerable interest since they ultimately control local climate. There appear to be two schools of thought as to what causes the standing waves. Whether the two modes of control are exclusive or complementary seems to be an unresolved question in the climatological literature. However, it appears to me that both may have their place in the paleoclimatic record.

Namias (1976) has shown that the pattern of winter severity over North America is related to summer water temperatures in the northern Pacific through a rather complex chain of causal factors. If the waters remain cool throughout the summer, winds flow unobstructed across the northern Pacific in a zonal pattern. If, on the other hand, the northern Pacific waters warm in the summer, there is enough energy given up in the fall to sponsor a cyclonic low over the Gulf of Alaska. The lows experienced in the Gulf of Alaska sponsor cold fronts and cold winters in eastern North America. Western North America under a high, enjoys warmer, although drier conditions.

This model supports the normal meteorological point of view that the Gulf of Alaska is the maker of weather in the United States. There are some interesting sidelights to the mode1. Figure 18 illustrates data drawn from a publication by Angell and Korshover (1978). The data are radiosonde temperature readings, averaged from the surface to the $100 \mathrm{mb}$ level of the atmosphere over all the world, and a good estimate of the atmospheric energy budget. The winter of 1976-1977 was clearly a cold one. It is also the example par excellence of a winter controlled by high summer water temperatures in the northern Pacific. Thus, we have the interesting situation of a low energy budget year and warm northern Pacific waters generating the coldest winter on record in the East. It would also be interesting to know what the mechanisms producing warm water in low energy budget years are. Namias mentions the effect of cloud cover, although he offers no further explanation. Perhaps low energy budget years produce fewer clouds allowing sunlight to reach the waters of the northern Pacific. 


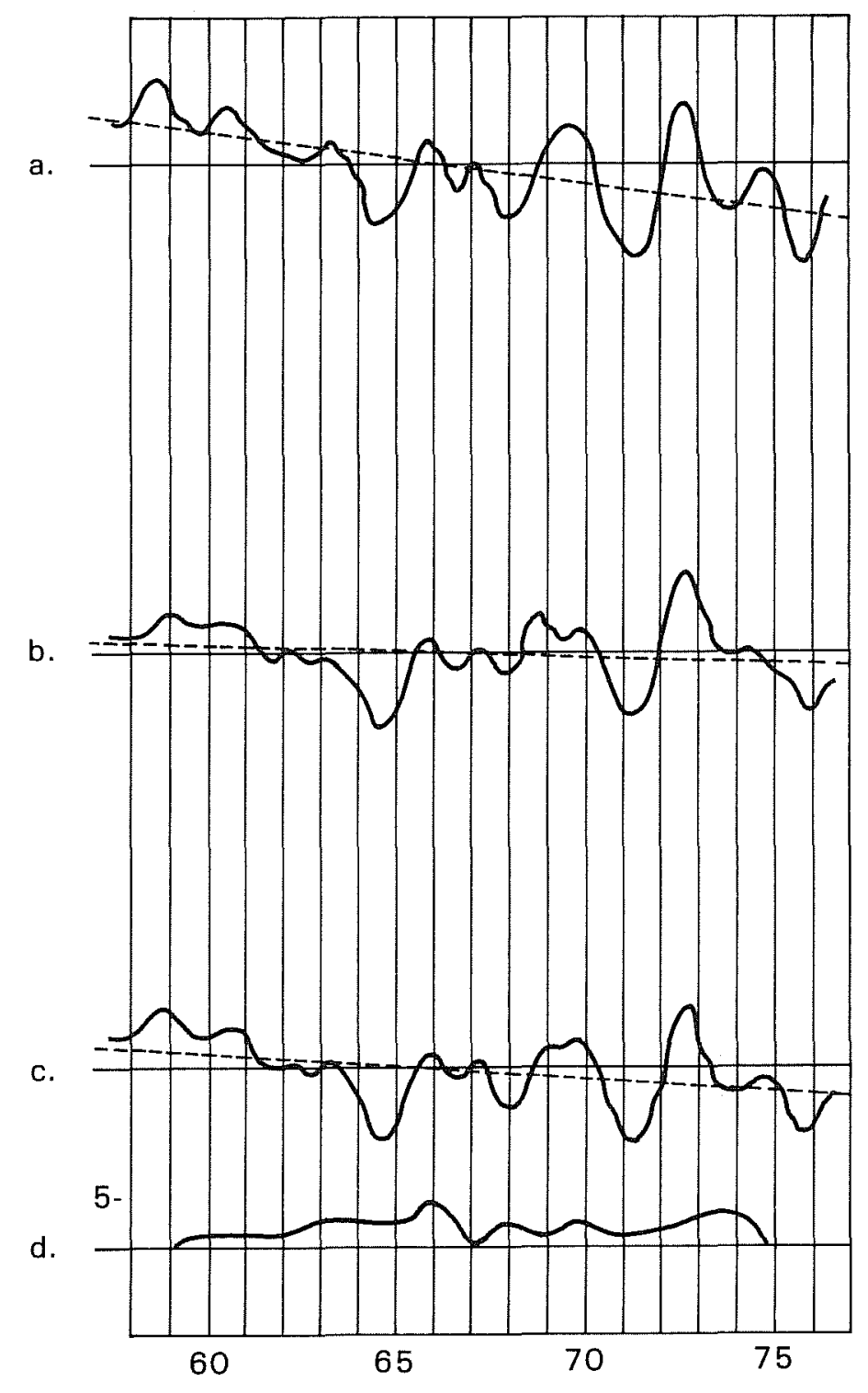

Figure 18. Energy Budget Estimates for Northern Hemisphere. $a$, southern hemisphere; b, the world; $c$, as determined by Angell and Korshover (1978:761) from radiosonde observations at 63 globally distributed stations. Slashed lines are regression lines between temperature observations and annual sequence of observation. Observations are seasonal; $d$, frequency of major volcanic eruptions. 
In our search for an effective paleoclimatic (paleometeorological) model, we need to ask whether this winter bears elements of an ice age winter. We will return to this question later.

First, let us examine an alternative explanation of the cause of cold winters in eastern North America. The idea that weather is controlled by lows and highs in the lower troposphere was developed in the 1930s by Reiter (1961) before high-flying B-29s discovered the jet stream during World War II. Reiter heralded the discovery of the jet stream as a more effective means of explaining weather behavior and a new age for meteorology.

From the point of view of jet stream meteorologists, an explanation for standing waves is as follows. The behavior of the jet stream over a particular land mass depends on the topography of the 1 and mass. In the case of North America, the high north-south trending mountains along the western edge of the continent are an influential surface feature. When the energy budget is high, the jet stream flows easily across the Sierra Nevadas and Rockies. Presumably, for reasons we will discuss later, the higher the energy budget the stronger the influence of the jet stream on North America east of the Rockies. This influence was clearly demonstrated in the 1930s when the midlatitude jet stream and its accompanying dry westerlies acted to keep moisture out of the Central Plains. Mitchel1, Stockton, and Meko (1979) have demonstrated convincingly the sun's 22-year Hale cycle. Paleoclimatic evidence indicates that the dry prairies trailed the jet stream all the way to western Pennsylvania during the Hypsithermal creating the so-called "Prairie Peninsula" phenomenon. This influence would tend to shrink precipitation on the western and northern periphery of the Southeast.

To this point, we have examined a warm weather climate scenario. It appears that the higher the energy budget the greater the influence of the jet stream across the central United States. Before extending the discussion to cold and glacial conditions, we need to examine the underlying controlling force behind the route the jet stream takes across the continent. The central issue seems to be one of turbulence caused by the western mountains. When the jet stream is moving faster, the mountains generate turbulence in a general westerly air flow as do rocks in a stream of water. The resulting areas of high pressure tend to disperse the jet stream route to the north and south. The slower the jet stream the less the turbulence and the more regular the west to east flow.

The principle of turbulence control can be extended toward the cold end of the temperature spectrum as we11. As the speed of the jet stream increases, more turbulence and high pressure are created over western North America. At maximum speeds, the resistance to the passage of the jet stream is so great that it is forced to split into two tributaries and flow around the western mountains as mentioned before.

Judging by what evidence is available, it seems quite possible that both the turbulence and sea surface temperature mechanisms are effective, but operating under different global energy budget levels. Namias's (1976) analysis of 1973 air flow patterns and an examination of Monthey Weather Review weather and circulation reviews for the winter of 1976-1977 indicate that the Aleutian Low 
causes extreme northward deflections of the jet stream (Fig. 19,b), so that it coursed over Alaska and then down through Canada and the eastern United States. G. L. Wells demonstrated through an analysis of orientations of Pleistocene aeolian features that the prevailing winds were from a northwesterly direction, but not nearly so exaggerated as the Aleutian Low cases (Fig. 19, C). The course indicated is through the lower and narrower mountain ranges of the American Northwest and southern Canada, but skirting the broader Sierras Basin and Range and the Colorado Rockies. An alternative confirmation is to be found in the southwesterly features in the lower southwestern United States. CLIMAP (1976) reconstructed the northern Pacific climate as being cold in August at 18,000 B.P., another bit of evidence suggesting that the turbulence factor was the dominant weather maker during the extremely low energy budget, full glacial period.

With turbulence defined as the weather maker in low energy budget periods, the Aleutian Low is probably active in moderately cool intervals below conditions which produce clouds, but above conditions which preclude summer warming of the northern Pacific. Such periods might be expected during intervals such as the Little Ice Age, the Subboreal and Preboreal.

One bit of possible contradictory evidence needs to be reconciled. The highest ridge of the ice mass delineated for the CLIMAP reconstructed climate suggests that the Laurentide ice sheet was fed by the exaggerated arc of the Aleutian Low's deflected jet stream. If the ridge is reconstructed from independent evidence it may suggest the intervention of some sea warming mechanisms to feed the glacier during its maximum stand (review Adam 1975).

The effects suggested for the Southeast are as follows. A full glacial, low energy budget indicates a moderately meridional flow as strong westerlies force their way across the continent. The increased strength of the westerlies (Saltzman and Vernekar 1975) probably acted to shield the Southeast from the harsh effects of the ice-chilled air mass to the north. Note that in this model atmospheric flow is taken to be causally prior to the ice stand. By the same token, the westerlies shielded the glacier from the ameliorating effects of southerly air movements.

A moderately low energy budget, which allowed for sufficient heating of the northern Pacific to sustain a low throughout the declining segment of the year, would result in a much more turbulent weather pattern in the Southeast. Once the jet stream arcs through Alaska, the accompanying westerlies are markedly chilled. Such strong arctic air masses would sponsor the movement of arctic fronts into the Southeast. At frequent intervals such fronts preclude the landward movement of moist air of the Gulf (Orton 1964). Namias, however, found a net increase in mojsture in the Southeast during 1973, a year of the Aleutian Low.

Such turbulent meridional circulation replaced the persistent system of zonal flow that maintained the glacier and, probably explains, once established, the apparently abrupt disappearance of the bulk of the ice mass. This abrupt turn is noted both in Andrews and Miller's (1978) research on the ice mass itself and in Gagliano's (1977) examination of submerged features in the Gulf of Mexico. 

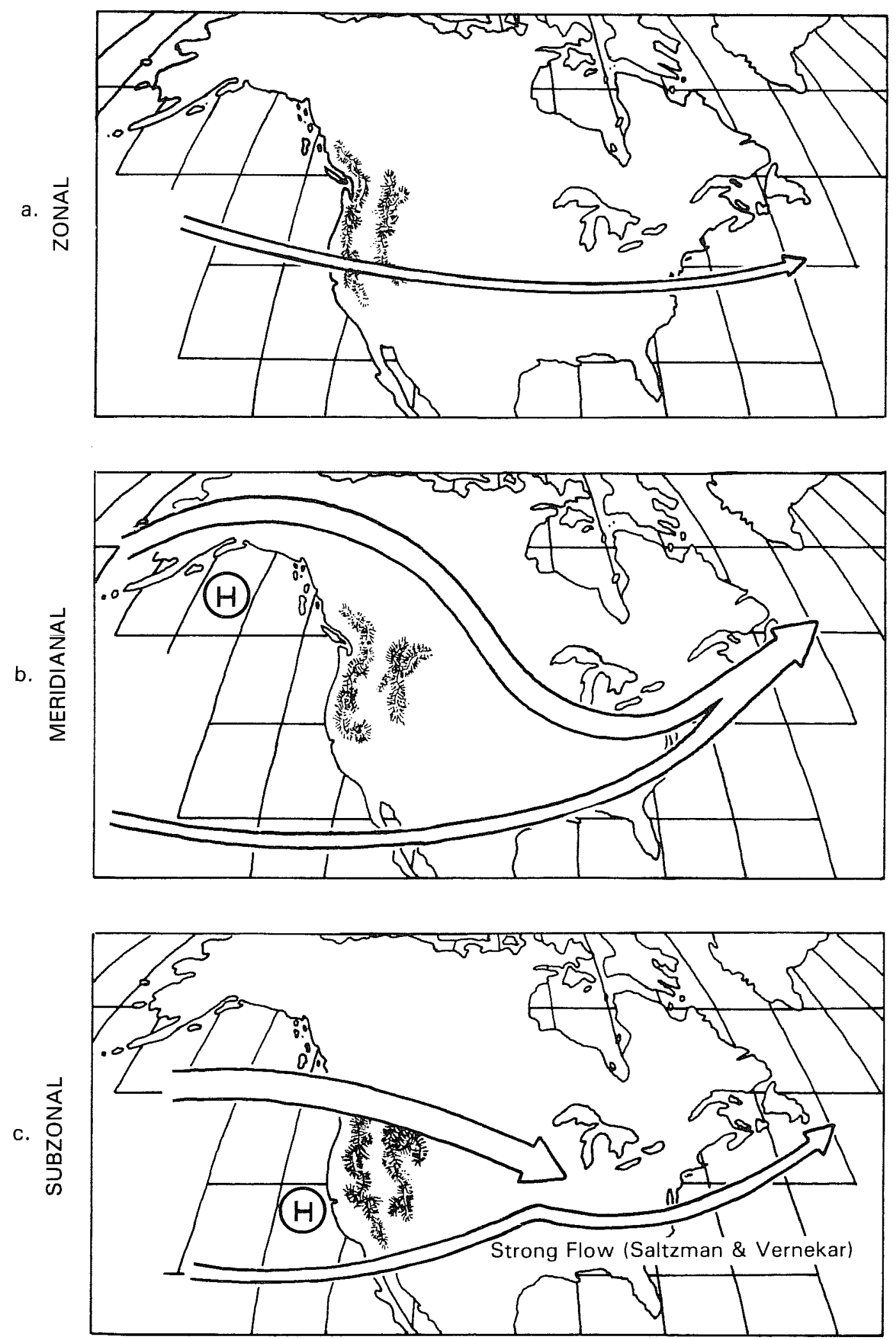

Thermodynamics (Saltzman and Vernekar 1975) + Standing Wave $\rightarrow$ Very High Winds

Figure 19. Jet Stream and Energy Budget Conditions. a, high; $b$, medium; $c$, low. 
In the final analysis, there are three extreme atmospheric states. At high energy budget levels, constant zonal flow dries the northern reaches of the Gulf Coastal Plain, while a northerly displaced subtropical high wets the coastal West and dries the coastal East. Under moderately low energy budget conditions, marked meridional circulation cools and dampens the area. Under low energy budget conditions, a modified zonal flow shields the Southeast from extremes, but probably also dries it.

Generating a time series from this model required a detailed understanding of the global energy budget. Figure 20 is an attempt (Gunn 1982c) to create an energy budget curve from orbital and volcanic data. It is based on two cycles. The larger cycle is 23,000 years and is drawn from Kukla's 1975 findings that the annual albedo and, ultimately, the energy budget are heavily influenced by October temperatures. KukTa, therefore, reasons that glaciers should occur in periods when the earth's orbital precision dictates low temperatures in the northern hemisphere during October. The second cycle superimposed on the first is based on data published by Bryson and Goodman (1980) on frequencies of volcanic eruption in the Holocene.

The time series illustrated in Figure 19 shows expected circulation patterns for different time periods: zone ( $Z$. for warm periods, subzonal (S) for very cold intervals, and meridional (M) for intermediate periods.

There is some literature relative to assessing the validity of the time series. However, most previous work is short of the detail necessary for comprehensive evaluation. The range of approaches runs the gamut of scientific enterprise from very theoretical to very empirical, from very detailed to very general.

Full glacial circulation is most difficult to reconstruct. The theoretical efforts include simulations of atmospheric processes such as those by Saltzman and Vernekar (1975), Manabe and Hahn (1977), and Gates (1976). Presumably the latter two models should be more serviceable, because they are based on three-dimensional representations of atmosphere. However, their utility seems to be limited to the tropics for which they were directly intended. Their projections of North American full glacial climate are not borne out by the paleoenvironmental record. The Saltzman and Vernekar model on the other hand, provides a great deal of serviceable information on atmospheric thermodynamics in spite of rather obvious limitations, such as two-dimensional representation of the atmospheric, symmetrical air flow relationships to the poles, and lack of standing waves caused by physiographic features such as mountains.

The meteorologists' concern with short periods of time is clearly illustrated by attempts to apply simulation models to past climates. Since simulating the atmospheric processes is such a massive undertaking spatially, pairing them with thousands of years under varying energy budget levels, such as would be most useful for archaeologists, is a financial and practical impossibility. For this reason, such efforts have been limited to the simulation of glacial maximum conditions around 18,000 B.P. While this is unfortunate in a way, it is fortunate that simulators have chosen the glacial maximum to devote their efforts. As will be discussed later, less glaciated conditions can be approached through other means. Glacial maximum, however, is completely foreign to our present experience and can only be estimated in theory. 


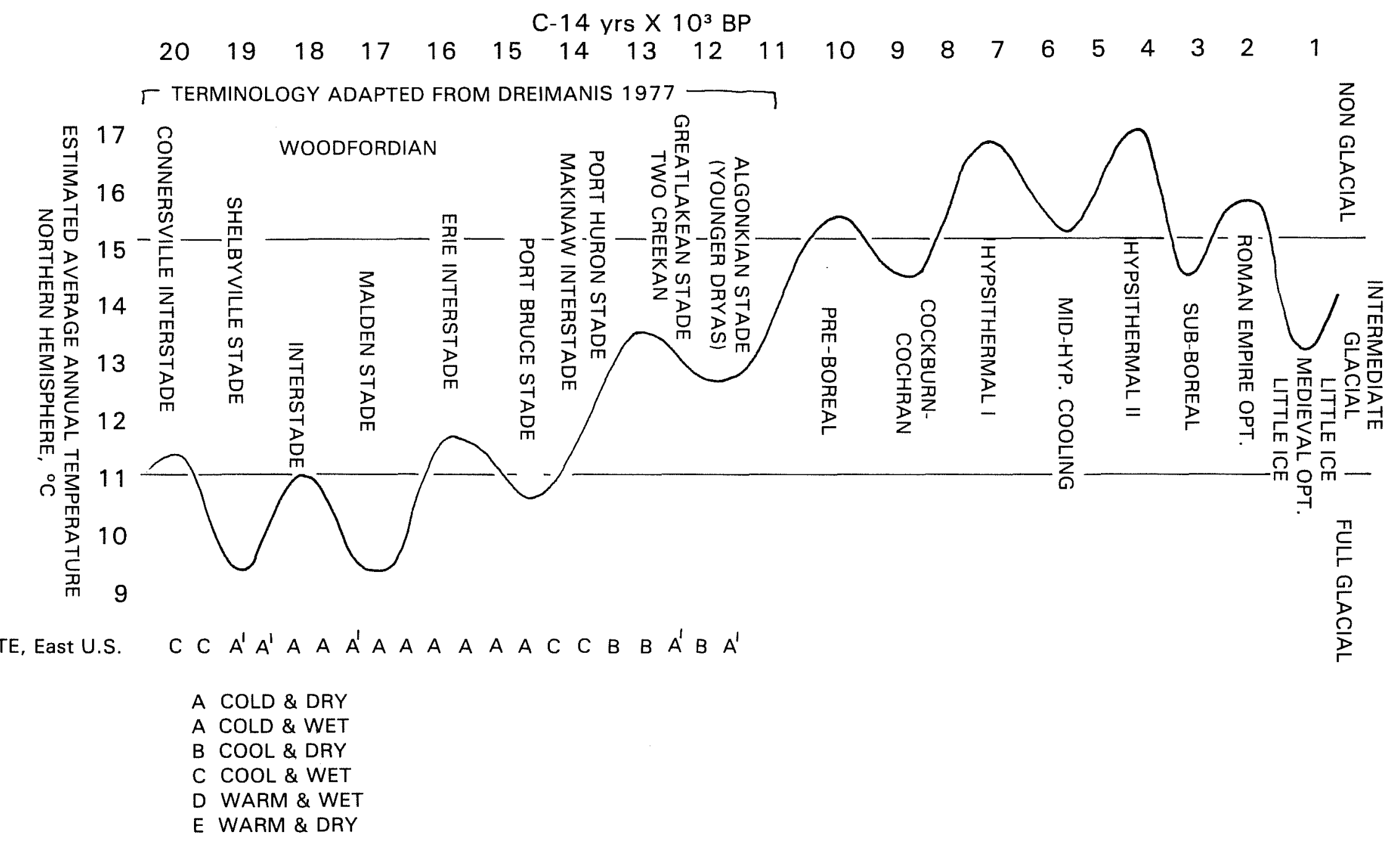

Figure 20. Estimated Energy Budget for the Last 20K years. From Gunn (1982c). Glacial terminology from Dreimanis (1977). Most confusion between estimates and empirical evidence occurs when the energy budget is at about $13^{\circ} \mathrm{C}$. Pleistocene-Holocene boundary is where energy budget permanently crosses $13^{\circ} \mathrm{C} .13^{\circ} \mathrm{C}$ may be system threshold between glacial and nonglacial climate. 
Figure 21 illustrates three of many helpful graphs presented by Saltzman and Vernekar (1975). The solid line represents present temperature gradients at various degrees of latitude north and south of the equator. The dashed lines represent simulated conditions at 18,000 B.P. Notably, there are major increases in temperature gradients over the ice between 50 and 70 degrees north latitude. Areas as far south as the Gulf Coast, however, experience much smaller drops in temperature, perhaps as 1ittle as two or three degrees Kelvin (Fig. 21,b) in the midlatitude. Saltzman and Vernekar suggest that the strong zonal winds, or subzonal as we have determined them for North America because of the mountains, were instrumental in maintaining the sharp temperature gradient which in turn sponsored the ice sheets. The Southeast must have been windier and cooler if not nearly so deviant from modern conditions. As is illustrated (Fig. 21,C), the Saltzman-Vernekar model correctly infers a drier full glacial at the latitude of the Southeast.

Intermediate glacial and Holocene circulation can be approached more directly. There are several empirical studies designed to mark the air flow patterns during the relatively warm present century and the moderately glaciated Little Ice Age of the last century. Sanchez and Kutzbach (1974) utilized the cooling trend of the last quarter century to demonstrate southward movement of belts of rainfall and temperature. Blasing's (1975) analysis of tree ring data and modern pressure data indicates a strong meridional or north-south component to colder winters (Blasing's Type 4 Winter). Both Dzerdzeevskij's (1968) analysis of 20th-century atmospheric flow and Namias's (1976) study on the Aleutian Low, and numerous analyses of the very cold winter of 1976-1977, support a general chilling of the eastern United States during periods of global cooling. This is in contrast to the western United States, which warms as the world cools.

Virtualiy all of the literature supports a strong zonal flow for high energy budget intervals. The meridional circulation pattern, however, is much less visible and is, in my opinion, an important blind spot in our understanding of moderately cold periods.

Lands, Glaciers, and Seas in Eastern North America

The late Quaternary conditions of eastern North America were regulated by the atmospherically coordinated ebb and flow of glaciers and seas. Along the Gulf coast, lowered sea levels exposed a low latitude plain apparently rich in fauna and flora and possibly a refugium for many of the plant species now extant over much of eastern North America. To the north, glaciers advanced and retreated over higher latitude landscapes. In a sense, eastern North America was a shifting corridor of land whose position was controlled by the ratio of water and ice. Within the corridor the habitat was equally unreliable. As we have seen, colder times saw a strong westerly flow, which shielded the ice sheets from inroads by warmer southern air. At the same time equal protection from arctic air was afforded the South, so temperatures were only moderately colder on the Gulf Coastal Plain. Moderately cold climate exhibited wetter conditions as meridional flow led to the collision of cold and warm air masses. During warm periods the Gulf watered the Southeast saving it from the desert climate that one would ordinarily expect at latitudes equal to that of the Sahara 
a.

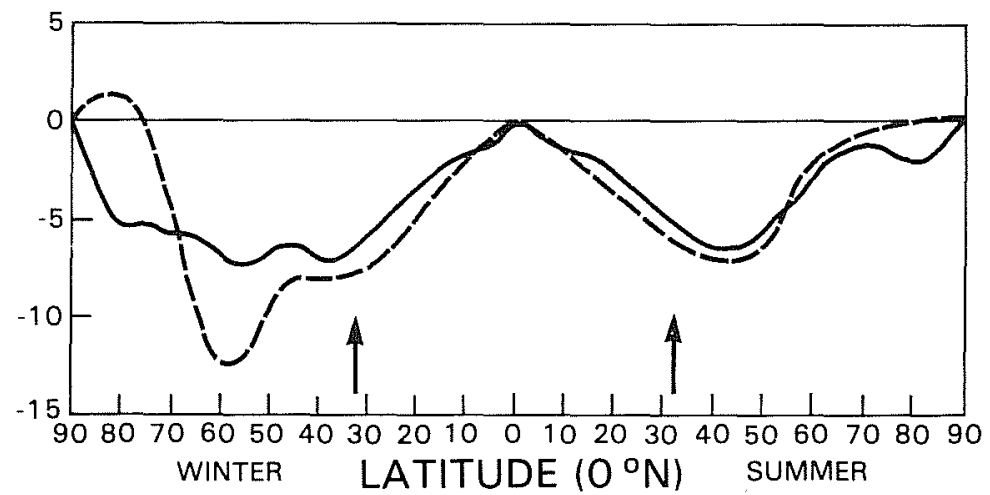

Mean surface temperature gradient $\partial \mathrm{O}_{\mathrm{os}}$ a $\partial \phi$ for present (full) and $18,000 \mathrm{BP}$ (dashed).

b.

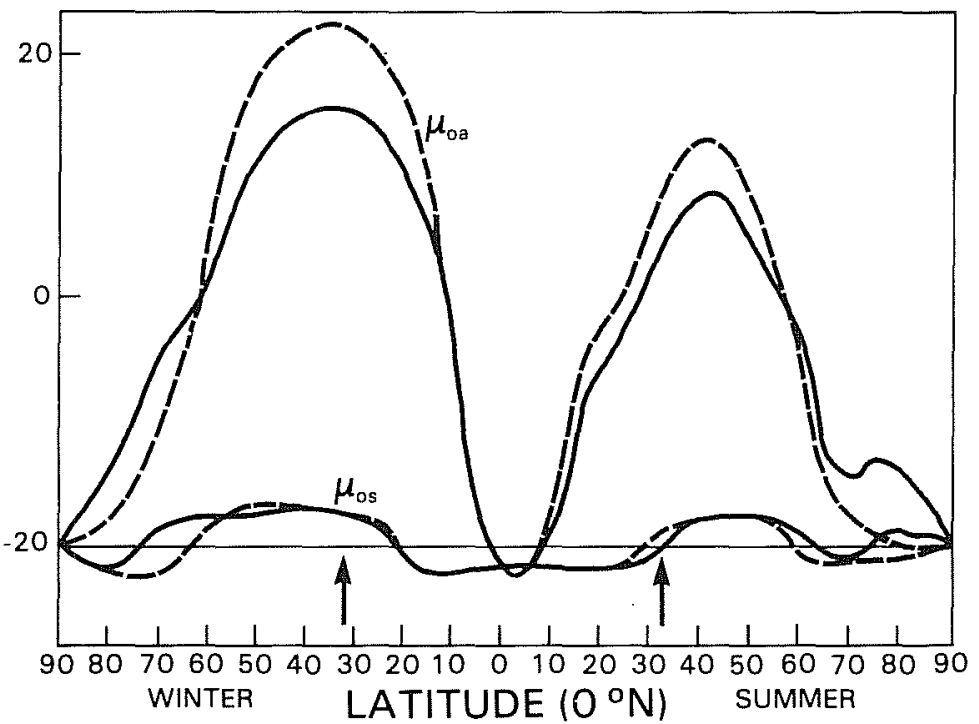

Mean surface zonal wind speed $\mu_{0 s}$ (lower curves) and vertically averaged zonal wind speed $\mu_{0 a}$ (upper curves) for present (full) and 18,000 BP (dashed).

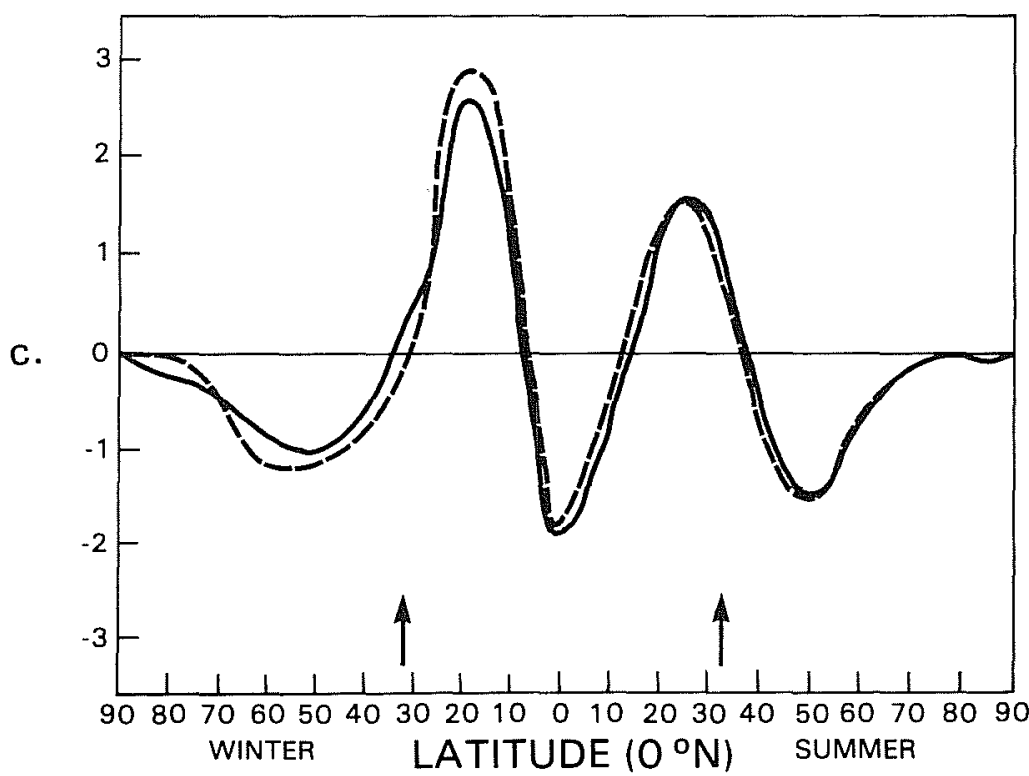

Mean difference between evaporation and precipitation $\left(E_{0}-P_{0}\right)$, for present (full) and 18.000 BP (dashed).

Figure 21. Selected Simulation Variables. From Saltzman and Vernekar 1975. 
and Sonora Deserts. In the following two sections the implications of sea levels and glaciers for life in the Gulf Coastal Plain will be examined.

\section{Sea Levels}

Sea levels in the Gulf of Mexico have been of considerable interest because of the relative isostatic stability of the Gulf Coast. Mahula (1982a) has reviewed the research. Beginning in the 1950s several curves were generated by various researchers in attempts to describe the ups and downs of Gulf sea levels during the late Quaternary. Utilizing the methods of bathymetry, submerged landforms were studied and dated by radiocarbon assays of shel1 and peat deposits bored from submerged beaches.

A discussion of two of these curves will facilitate our examination of the Mexico Gulf Coast climate. The first is by Curray $(1960,1965)$ and the second by Stapor and Tanner (1977). The Curray curve is the most general1y accepted curve for the late Quaternary spanning the time period from about 20,000 B.P. to the present. The Stapor and Tanner curve is not from the Gulf of Mexico, but from St. Vincent's Island off the coast of Florida. However, it is sensitive to the more subtle sea level fluctuations of the last 5000 years. Figure 22 is drawn from the Curray and Stapor and Tanner evidence. It is plotted on a logarithmic scale to exaggerate the importance of the Holocene sea level fluctuations.

Curray's work shows six periods in Tate Quaternary sea level changes:

1. 18,000-20,000 B.P., Full Glacial. The sea surface is about $120 \mathrm{~m}$ below present mean sea level. Winds, currents, and drifts are much like they are now. Shores are over the edge of the continental shelf, where slopes are about 600 feet per mile, so shallows rich in marine fauna and flora are scarce or lacking. Gagliano's (1977) examination of continental shelf bathymetry indicates that the $120-\mathrm{m}$ stand was brief, and that the sea rose rapidly to the $82-m$ stand (see section on geomorphology for details).

2. 16,000-18,000 B.P., decline of Tazewel1. Sea level rose from $120 \mathrm{~m}$ to $80 \mathrm{~m}$. Winds, currents, and drifts are similar to the present.

3. 12,000-16,000 B.P., stand or reversa1. Sea level is at $82 \mathrm{~m}$ and may have dropped back to $88 \mathrm{~m}$ at some time during the period.

4. 11,000-12,000 B.P., corresponds to the Two Creeks and A1leröd interstadia1. During the 64-m stand winds were strong and southwesterly, the northward longshore current along the Rio Grande coast was intensified, and the westbound currents along the upper Texas coast were reversed. After the cold episode, waters warmed as indicated by foraminifera, and currents returned to their present condition.

5. 10,000-11,000 B.P., Mankato glacial advance. Sea level is at $40 \mathrm{~m}$ at the beginning of the period and recedes to $64 \mathrm{~m}$. There was a $46-\mathrm{m}$ stand early in the period. 
$\begin{array}{lllllllllllllllllllll}20 & 19 & 18 & 17 & 16 & 15 & 14 & 13 & 12 & 11 & 10 & 9 & 8 & 7 & 6 & 5 & 4 & 3 & 2 & 1 & 0\end{array}$

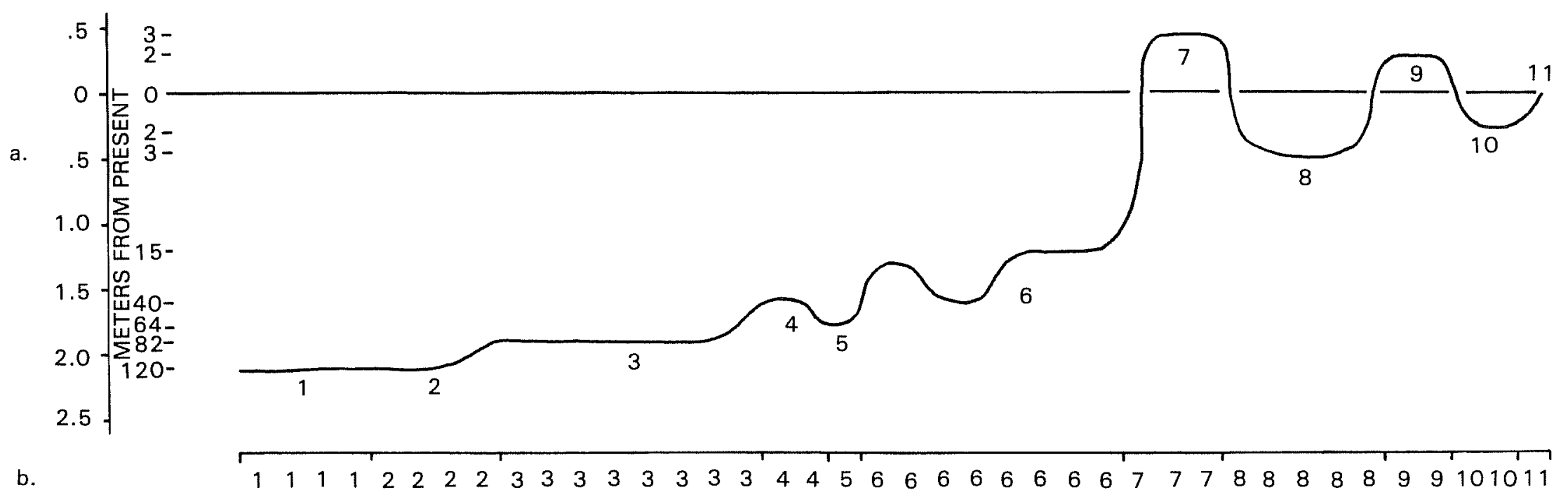

Figure 22. Sea Level Fluctuations. a, sea levels for the last 20,000 years; b, time series for sea 1evels. 
6. 7000-10,000 B.P., early Holocene. Sea level was at $18 \mathrm{~m}$ and then regressed to $38 \mathrm{~m}$. Winds and currents responded as in a cold period. Then sea level rose to a stand at $15 \mathrm{~m}$ below present sea level.

Sea levels show a slow, but continuous rise from 7000 B.P. to the present in Curray's graph. Untike the Curray curve, the Stapor and Tanner curve shows subtle variations in sea level during the last 7000 years. They drew evidence from a number of sources including prehistoric sites, beach ridges, and shell mounds. They define five periods (the names are mine). The period numbers continue from the Curray curve.

7. 4900-6200 B.P., Hypsitherma1. Sea level was higher than it is now, perhaps as much as two meters.

8. 2600-4900 B.P., Subboreal. Sea level was lower than present, probably somewhere between two and three meters.

9. 1400-2600 B.P., Roman Empire Climatic Optimum. Sea leve1 was above present levels. Exact figures are questionable, but it is estimated to be between one and two meters.

10. 1400 B.P. to present, Little Ice Age. Sea leve1s were below those of the present, probably at the order of minus two meters.

11. Present, warm century.

It is of interest to note that both the Curray and Stapor and Tanner curves correlate well with the Denton and Karlén (1973) tree line data.

Figure $22, b$ illustrates a time series for sea level changes.

\section{Ice Levels}

The ice mass at full glacial was reconstructed by the CLIMAP (1976) task group to show the extent and elevation of permanent ice as is illustrated in Figure 23. As was indicated earlier, glaciers waxed behind a shield of strong slightly subzonal westerlies.

Andrews and Miller (1978:175) have suggested that the glaciers in "North America were substantially reduced after about 16,000 B.P. The hypothesis is supported by a marked sea level rise in the Gulf of Mexico universally dated to the same time period (Ballard and Uchupi 1970; McFarlan 1961; Curray 1960; Poag 1973). This suggests that the energy budget conditions, which sustained the cold zonal westerlies, were altered probably by an increasing energy budget sponsored by precession of the earth's orbit (Kukla 1975).

The atmospheric flow patterns somewhat typical of the 19th and 20th centuries resumed at this time. The late glacial would have resembled the brusque climate of the 19th century, while the various Holocene climatic optima paralleled the very warm mid-20th century. 


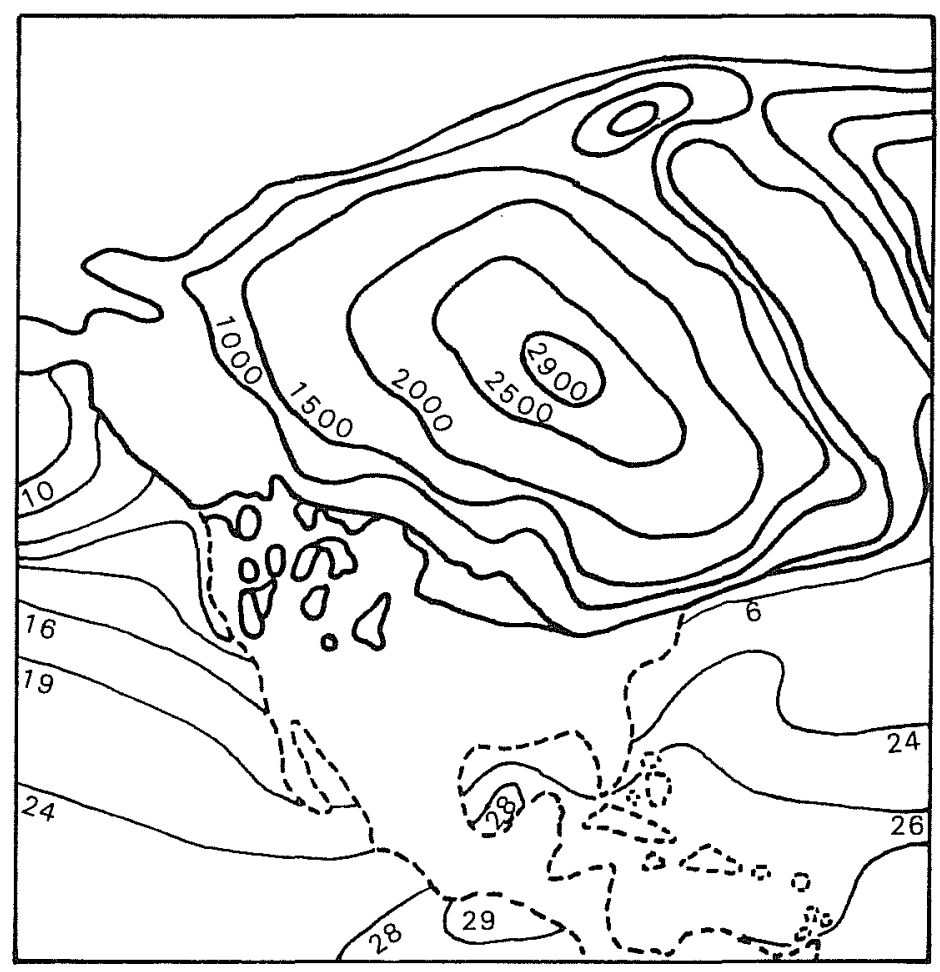

Figure 23. Ice Mass (Meters) and Sea Surface Temperature $\left({ }^{\circ} \mathrm{C}\right.$ ) at 18,000 B.P. From CLIMAP 1976. Wendland (1977) indicates that sea surface temperatures in the Atlantic indicate no tropical storm activity is likely from the East. Sea surface temperatures in the northern Pacific fall far below the $<25^{\circ} \mathrm{C}$ necessary to effect an Aleutian Low (Namias 1976). 
The model being used in this paper, which takes the behavior of the atmosphere to be causally prior to that of the ice, suggests very little direct influence of the glaciers on the Southeast during glacial maximum. However, we must note water and sediment outflow in the Arkansas and Mississippi Rivers (Saucier 1974) and the increased land area along the coast (Gagliano 1977). On the other hand, increased meridional flow during the late glacial probably chilled outbreaks of arctic air below temperatures experienced during the present or last century.

Geomorphology of the Gulf Coast

When Bernard and LeBlanc (1965) wrote theip general description of the northwest coastal plains of the Gulf of Mexico, over 350 publications had been prepared on the geology of the Gulf of Mexico, the Atlantic Coastal Plains, and the adjoining continental shelf. The Gulf of Mexico Coastal Plains from northeastern Mexico to the panhandle of Florida (Fig. 24) are characterized by landward uplift and seaward subsidence. The process is driven by the loading of sediments onto the continental shelf by nine major river systems (Fig. 25). The juncture between subsistence and uplift, or "hinge line," is generaliy just landward of the present coast except near the mouth of the Mississippi River where heavy sedimentation has deposited a delta nearly to the edge of the continental shelf. The extended loading has moved the hinge line nearly 30 miles seaward.

The continental shelf is composed of sediments from the river systems, which are smoothed by high water stands, such as that of the late Holocene. It is relatively flat and extends seaward $200 \mathrm{~km}$ at the Sabine River. From there it narrows in both directions until it reaches the Florida and Yucatan peninsulas. The continental shelf is considered to extend to the $200 \mathrm{~m}$ contour. The slope is very gradual to the $100 \mathrm{~m}$ contour, between 0.2 and $1.7 \mathrm{~m} \mathrm{per} \mathrm{km}$ depending upon location. Slope increases and becomes more variable beyond, five meters or more per kilometer.

Beyond the continental shelf, the continental slope grades rapidly down to the Sigsbee Abyssal Plain, which is $3500-4000 \mathrm{~m}$ below the surface. Down to $130 \mathrm{~m}$ the topography is very rough and shows signs of instability such as landslide scars. These are probably the sediments that were built up during the low stands of sea level during the Pleistocene. Below, on the continental slope are more stable sediments.

Coastal Environments, Inc., under the supervision of Gagliano (1977) produced an extensive examination of sea level variation and near-shore environments and their cultural implications for the late Quaternary. The study concentrates on phenomena seaward of the hinge line.

Coastal Environments' study of continental shelf bathymetry adds numerous interesting details to the record of glacio-marine interaction. For instance, close examination of contours at the $120-230 \mathrm{~m}$ depth of the mouth of the Rio Grande indicates that the river stood only a brief period of time before sea levels rose rapidly to $80 \mathrm{~m}$ to stand for a considerable interval. The long stand is witnessed by a large delta, shoreline features, etc. Similar features appear at $54,44,28$, and $18 \mathrm{~m}$. The equivalent of the $44-\mathrm{m}$ stand is 


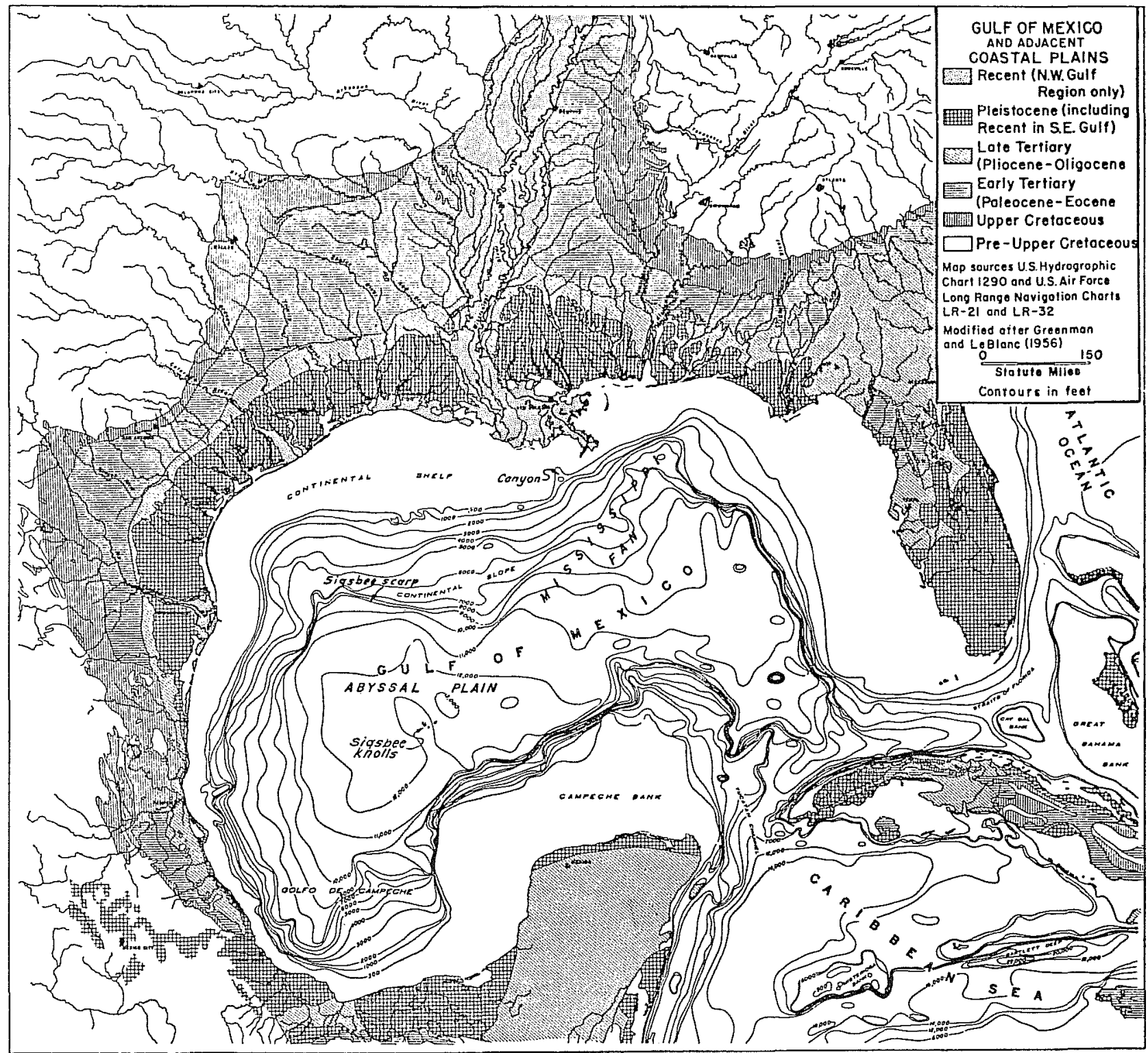

Figure 24. Generalized Geologic Map of the Gulf Coastal Plains and the Principal Hydrographic Features of the Gulf of Mexico. Modified after Greenman and LeBlanc (1956) and Ewing, Ericson, and Heezen (1958). 


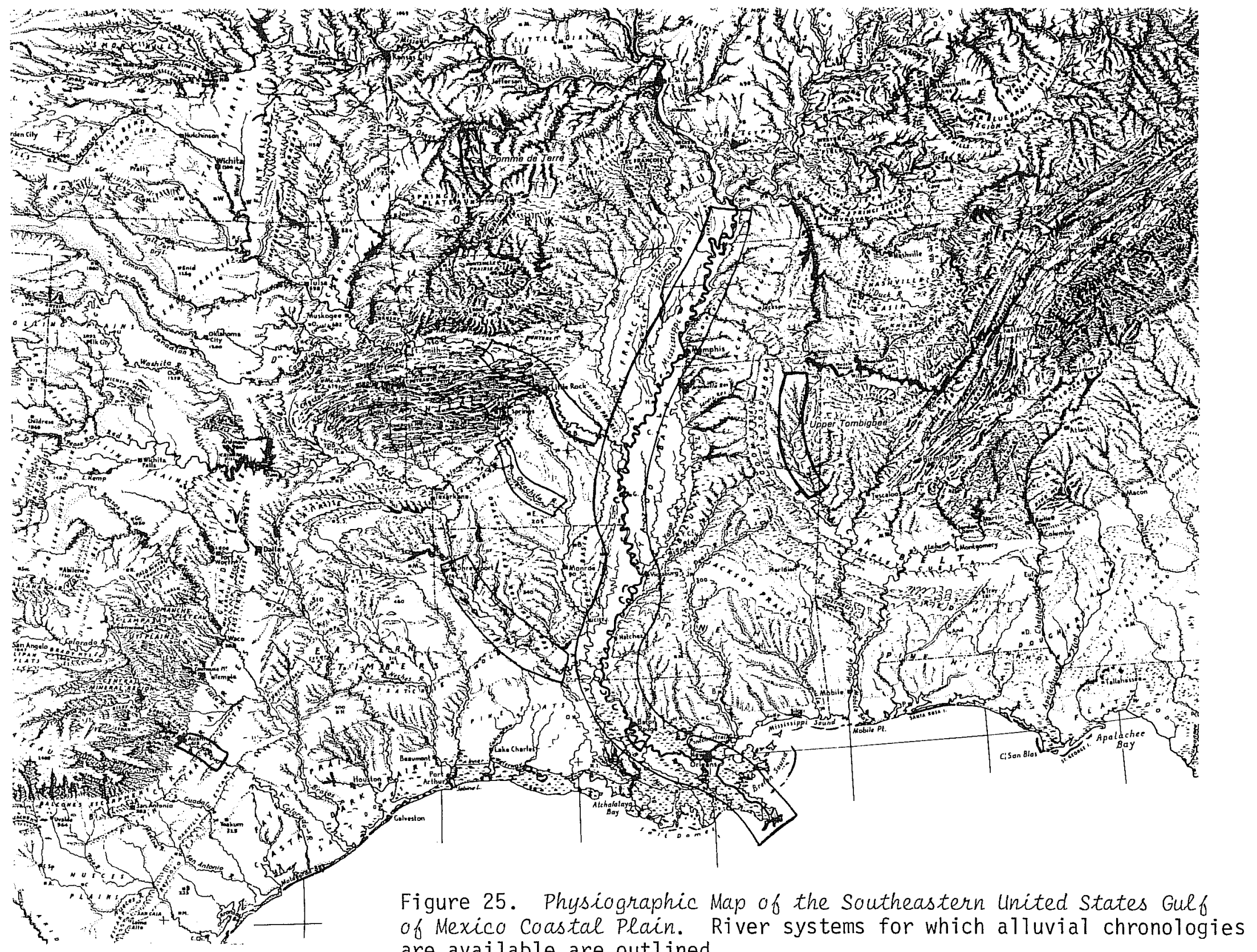
of Mexico Coastal plain. River systems for which alluvial chronologies are available are outlined. 
associated with freshwater forms and extinct megafauna on the central Texas coast. Such features appear around the bulk of the Gulf Coast, at or near the same depth. Along the Texas and Louisiana coasts, there is evidence that the Colorado, Brazos, Trinity, and Sabine Rivers with adjoining lesser systems met at a point on the then exposed continental shelf to form one large river.

Salt domes have forced Tertiary sediments upward in areas of the Louisiana and east Texas coasts. These submerged islands, or "banks," may have been appealing shelters to human occupants of an otherwise windswept and relatively dry plain (Gagliano 1977:86).

Before 17,000 B.P. the Mississippi River is thought to have emptied into the Mississippi trough, a canyon in the continental shelf off the Louisiana coast. Subsequently the river began to develop deltas. Gagliano (1977) details a sequence of 10 delta complexes and lobes that marked the activities of the river to the present. The rich deltaic environments attracted habitation during and since Paleo-Indian times, and each delta lobe was found to contain a distinctive array of archaeological sites indicating its date of activity. The delta chronology suggests a relatively stable sea level between 8500 and 12,000 B.P. The sea level may have been nearer that of today rather than 20 to $45 \mathrm{~m}$ lower suggested by other researchers. Since a sea level stand at $58 \mathrm{~m}$ is well dated at 12,900 B.P. (Neumann 1958), the possibility of a rapid rise or rapid fluctuations around 13,000 B.P. seems to be indicated. An additional note of interest is that the size of the delta lobe of the Brazos-Colorado River at $60 \mathrm{~m}$ may suggest a lengthy stand, perhaps indicating relatively stable sea levels in the period 17,000 to 13,000 B.P. (Gagliano 1977:90).

The delta complex dates range between 6000 and 8500 B.P. and is associated with the Early Archaic. During this interval sea levels fall, reaching its lowest point about 7000 B.P. Because of falling sea levels, swamps in the shore zone and delta were drained, suggesting a somewhat resource-reduced environment. Archaic sites are associated with a complex of deltas between 4000 and 6000 B.P. Poverty Point and Tchefuncte are found on yet another lobe, which was active between 2000 and 4000 B.P.

Landward from the hinge line are a series of narrow plains paralleling the coast. Landward each plain represents a successively older period and is slightly more tilted toward the sea. In the region of the Sabine-Neches Rivers, early Pleistocene deposits have been uplifted by this process as much as $180 \mathrm{~m}$. Uplift decreases along the plains to the east and west to about $120 \mathrm{~m}$.

Rivers approach the coast at a much lower gradient than the tilted plains. The oldest plains grade as much as $3-4 \mathrm{~m}$ per $\mathrm{km}$. The rivers generally are entrenched with a gradient of about $0.25 \mathrm{~m}$ per $\mathrm{km}$. At the hinge line gradients slack, and stream deposits change from alluvial to deltaic.

\section{Alluvial Chronology}

Rivers aggrade or degrade alluvial floodplains and change their mode of sediment transport from meandering to braided depending on the amount of sediment and water they carry. The changes are ultimately related to climate, although 
the relationships are often complex and not entirely transparent to analysis (Schumm 1965).

Numerous river systems in the Southeast have been subjected to analysis (Table 5) in such a manner as to outline the history of changes over the last few millenia. A survey of these "alluvial chronologies" will serve not only to direct the purpose of outlining the effect of climatic change recorded in the river valleys, but it will also serve as a basis for the study of uplands. In streams whose water originates within the environmental context of the Southeast, sediments are derived from the uplands so alluvial aggradation is an obverse history of the upland erosion.

TABLE 5. PRIMARY SOURCES FOR ALLUVIAL CHRONOLOGIES CITED BY VARIOUS REVIEWERS

\begin{tabular}{|c|c|}
\hline Arkansas River & Saucier 1967* \\
\hline Big Black River & Saucier 1967* \\
\hline Red River & $\begin{array}{l}\text { Gagliano and Thom 1967* } \\
\text { Frye and Leonard 1963; Frye 1961+ }\end{array}$ \\
\hline Sabine River & Gagliano and Thom 1967* \\
\hline Pearl River & Gagliano and Thom 1967* \\
\hline Ouachita River & $\begin{array}{l}\text { Saucier and Fleetwood 1970* } \\
\text { Fleetwood 1969* }\end{array}$ \\
\hline
\end{tabular}

* from Saucier 1974

+ from Schumm 1965

While understanding alluvial sequences is basic to any climatic context, Brakenridge (1980) has suggested a scheme that has broader implications, e.g., that of alluvial dating. The Pomme de Terre River in southern Missouri (see Fig. 25 north of Ozark Plateau) has been extensively studied in relation to the Truman Reservior project. Brakenridge noted that the cut and fill sequence defined for the Pomme de Terre River corresponded with synchronous events in other areas of the northern hemisphere. The Pomme de Terre River is wholly contained within the southern woodlands, although securely related to the prairie-forest ecotone. As with most southeastern rivers, sediments begin relatively low in the late Pleistocene and aggrade through the Hypsitherma1. Post Hypsithermal events result in a steady degradation, although the process is systematically stepped downward. Brakenridge notes that the periods of downcutting are systematically related to the periods of glacial advance defined by Denton and Karlén (1973).

Neither water or sediments of the Mississippi River originate within the Southeast in any significant proportion. However, there are aspects of the 
behavior of the Mississippi River that seem to reflect local climatic influences. Also, a review of the Mississippi River alluvial history will serve as a broader backdrop for local river systems.

The Mississippi River is the focal drainage of central North America and as such bears the marks of the complications that implies. Saucier (1974) has updated earlier efforts by H. N. Fisk to define an alluvial chronology for the lower Mississippi Valley. During the advancing phase of the final Wisconsin stadial, 20,000 to 25,000 B.P., the Mississippi River downcut its valley as did its tributaries and nearby rivers. Entrenchment was 75-80 feet below Baton Rouge and 20-25 feet between Baton Rouge and Vicksburg. A combination of increased precipitation and the laying down of the last major loess sheets to the east of the Mississippi River suggest to Saucier substantial, warm-season precipitation, while the cold season was dry and windy.

Glacial outwash sediments increased significantly between 18,000 and 20,000 B.P. A braided stream regime resulted, which marked the declining stage of the glaciation. The Arkansas River, which was also carrying glacial outwash from the Rockies, developed a braided channel. The Red River without glacial outwash maintained a meandering mode throughout the late Pleistocene and Holocene.

The Mississippi River changed suddenly to a meandering stream about 12,000 B.P. below Baton Rouge probably in response to rising sea levels during the Two Creeks interstadia1. The Arkansas River also changed to a meandering regime. By 9000 B.P. the glaciers withdrew north of the Great Lakes and so ceased to contribute a heavy sediment load of glacial outwash. From that time the stream changed to a meandering system progressively northward reaching Memphis by 6000 B.P.

The last 9000 years are a complicated history of changes from one meander belt to another. The Mississippi River alluvial floodplain is very wide; as a result there is little bedrock structural control of $i$ ts course. Where the meander belt is located could very well be as much a response to climatically regulated discharge and sediment load of tributaries to factors internal to the Mississippi River system itself. While Saucier (1974) offers no climatic explanation for changes in the meander belt, it is worth noting that there were major changes at $7500,6000,4700,3500,2800$, and 1200 B.P. Streams, whose origin is within the Southeast, can serve as a more direct entree to climatic effect on stream behavior.

The upper Tombigbee River was studied by Nials (1982). Previous to about 16,000 B.P. the valley was largely scoured out. After 16,000 B.P. the valley began to aggrade, and a braided stream regime was established. The increase in sediments implied episodic precipitation, perhaps falling mostly in the summer. The unevenness of the rainfall reduced vegetation and increased erosion of the uplands.

After 8000 B.P. the regime changed to one of a meandering stream which implies a moderated bed load in conjunction with increased and more evenly spaced precipitation relative to the braided stream. 
Baker and Penteado-Orellano (1977) conducted an alluvial study of the Colorado River in Texas from the edge of the Balcones Escarpment, the edge of the Gulf Coastal Plain, to about $100 \mathrm{~km}$ downstream. The central Texas area is subject today to highly variable annual precipitation as is typical of the prairie-forest ecotone. The study is ideally situated to mark the western terminus of this survey and of the southeastern woodlands of the United States.

Studies were made of the variable grain size and sinuosity of the river's relict meander scars. Dry conditions were assumed to be associated with low sinuosity and coarse-grained sediments. Wet periods are marked by high sinuosity and fine sediments. The transition from wet to dry resulted in the scouring of fine-grained sediments from the previous period followed by depositions of coarse-grained sediments at the top of the unit. Aggradation seems to be slower in the mesic periods.

The sequence of events is shown in Table 6. Unfortunately, the dating prior to 700 B.P. is insufficient to define with any certainty the temporal placement of the critical late Pleistocene-early Holocene events. One might suspect that the incision before 7000 B.P. is the beginning of the Hypsithermal and the previous wet period, the Preboreal. This would suggest a dry mid-Wisconsin interstadial and final stadial. Confirmation must await a more refined radiocarbon chronology.

TABLE 6. LATE QUATERNARY ALLUVIAL CHRONOLOGY FOR THE COLORADO RIVER ON THE BLACKLAND PRAIRIE BELOW AUSTIN, TEXAS
Channe]
Phase
Climate
Indicators
Radiocarbon
Date B.P.

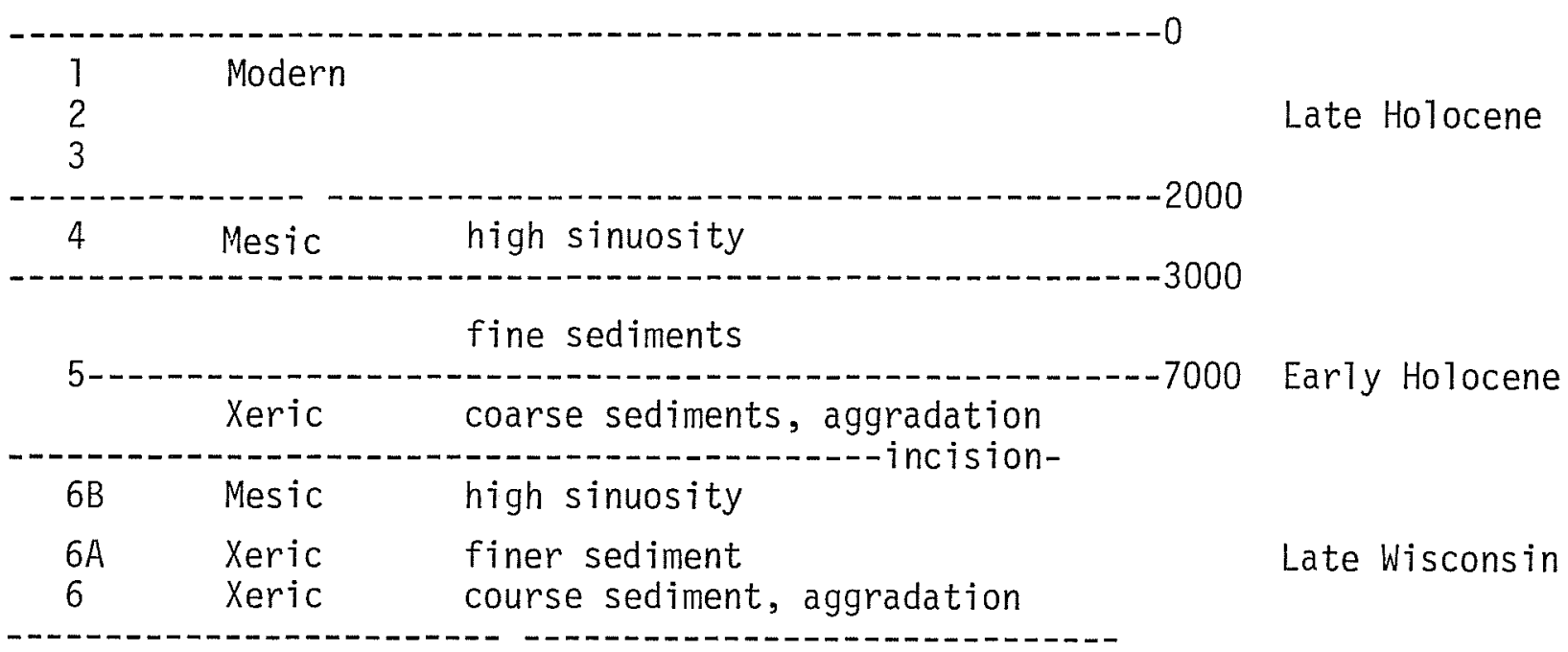

SOURCE: Adopted from Baker and Penteado-Orellano 1977. 
At a nearly microenvironmental scale is the alluvial stream sequence delineated by Gagliano (1980) for the Salt Mine Valley site on Avery Island. The sequence (Fig. 26) shows Holocene filling of a downcut Pleistocene valley with some erosion of the fill in the mid-Holocene.

Figure 27 illustrates the alluvial trajectories of several of the rivers and streams reviewed. The various water courses are marked by 500 -year intervals as to their status, "A" for aggradation and "D" for degradation. If the valley is aggrading it is superscripted by an " $m$ " for meandering and a "b" for braided, where the information is available and pertinent. A subscripted " $c$ " indicates a change of meander belt.

Certain of the alluvial chronologies are of higher resolution than the others. Brakenridge (1980) points out on the Pomme de Terre River that there are synchronous degradation episodes in many northern hemisphere streams (Fig. 27). There appears to be some concordance after 8000 B.P., which is marked by degradation in several streams. It is clear that the late Quaternary is generally a period of aggradation. Braided streams earlier in the sequence point to the aridity during intervals of glacial outwash sediment loads. Meandering streams during the Holocene suggest moister conditions and/or less sediment load. When other rivers in the Southeast are as well understood as the Pomme de Terre, the timing of aggradation and degradation will be of considerable interest to archaeological climatology. It will be especially helpful if the sequences are synchronous. Present information only supports broad and modestly useful generalizations.

\section{Uplands}

Uplands in the Gulf Coastal Plain show evidence of strong wind action during full glacial and the last Wisconsin interstadial. There are loess deposits on the uplands east of the Mississippi River as illustrated in Figure 28, the related dates are shown on Table 7 (Snowden and Priddy 1968; 0tvos 1975; Gagliano 1977:214-218; Saucier 1978; Saucier and Fleetwood 1970). The east Mississippi loesses are correlated with the Peorian Loess in Illinois (Gagliano 1977:218).

Saucier (1978) has reported on dune fields in the Mississippi River valley. They seem to have been active during glacial maxima and perhaps during the Hypsithermal. While not strictly within the scope of this survey, Thom (1967) has reported dune fields in the last stadial of the Wisconsin in South Carolina, and Whitehead (1973, 1982:203) has examined other similar features in the same area. It would be of some interest if active dune fields were found on the continental shelf clearly separable from shoreline developments. West of the Mississippi River, Gagliano (1977) reports numerous sand-related features on the continental shelf. However, none are clearly identified as being climatically related rather than as a product of normal shore processes. In addition, sandy features are nearly ubiquitous on the northwestern shores of the Gulf. However, there does seem to be some stabilization of the south Texas sand sheet after the Pleistocene (Gagliano 1977:151). 


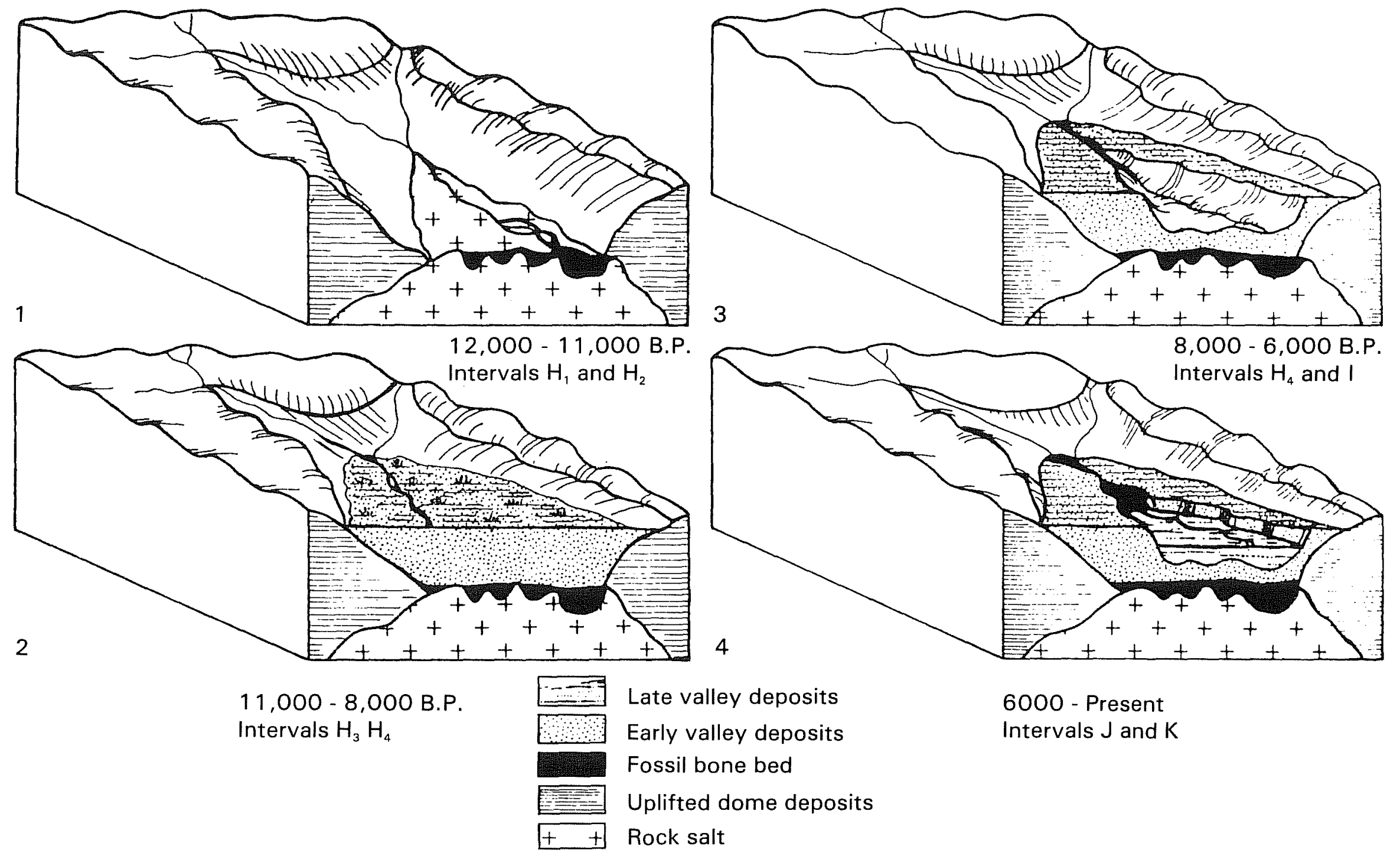

Figure 26. Four Idealized Phases of the Geological History of the Salt Mine Valley Site 116 IB 231 Showing Stream cutting and Valley Filling. After Gagliano 1970. 
RADIOCARBON YEARS B.P.

$\begin{array}{llllllllllllllllllllll}20 & 19 & 18 & 17 & 16 & 15 & 14 & 13 & 12 & 11 & 10 & 9 & 8 & 7 & 6 & 5 & 4 & 3 & 2 & 1 & 0\end{array}$

RIO GRANDE

COLORADO

D? A A A A A A A A A A A A

BRAZOS

TRINITY

NECHES

SABINE

POMME DE TERRE

RED

A A A A A A D A A A A D A A A A D A A D A $A^{M} A_{A} A_{A} M_{A} M_{A} A_{A} A^{M} A_{A}^{M} M_{A} M_{A} M_{A} M_{A} M_{A} M_{A} M_{A} M_{A} M_{A} M_{A} M_{A} M_{A} M_{A} M_{A} M_{A} M_{A} M_{A} M_{A} M_{A} M_{A} A_{A} M_{A} M_{A} M_{A} M_{A} M_{A} M_{A} M_{A} M_{A} M^{M}$

OUACHITA

ARKANSAS

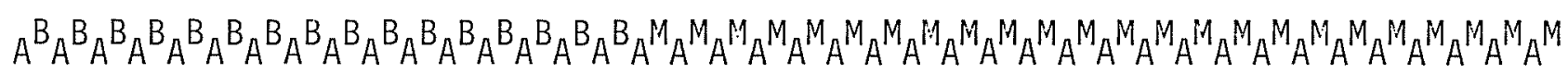
MISSISSIPPI

SALT MINE

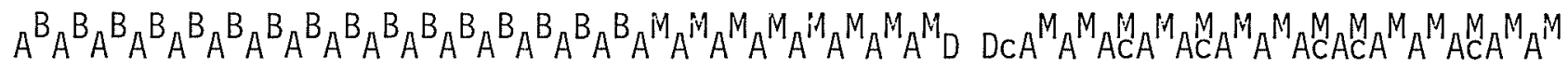

PEARL

$D D A A A A A A D D D D A A A A A A A A A A A A$

TOMBIGBEE

D D D D D D D D A $A^{B} A_{A} A_{A} B_{A} B_{A} B_{A} A_{A} B_{A} B_{A} B_{A} B_{A} B_{A} A_{A} A^{M} A^{M} A^{M} A_{A} A_{A} A_{A} A^{M} A_{A} A^{M} A_{A} A^{M} A^{M} A_{A}^{M}$

BLACK WARRIOR

ALABAMA

CHATAHOOCHEE

FLINT

Figure 27. Time Series for Alluvial Chronologies in the Southeast. 


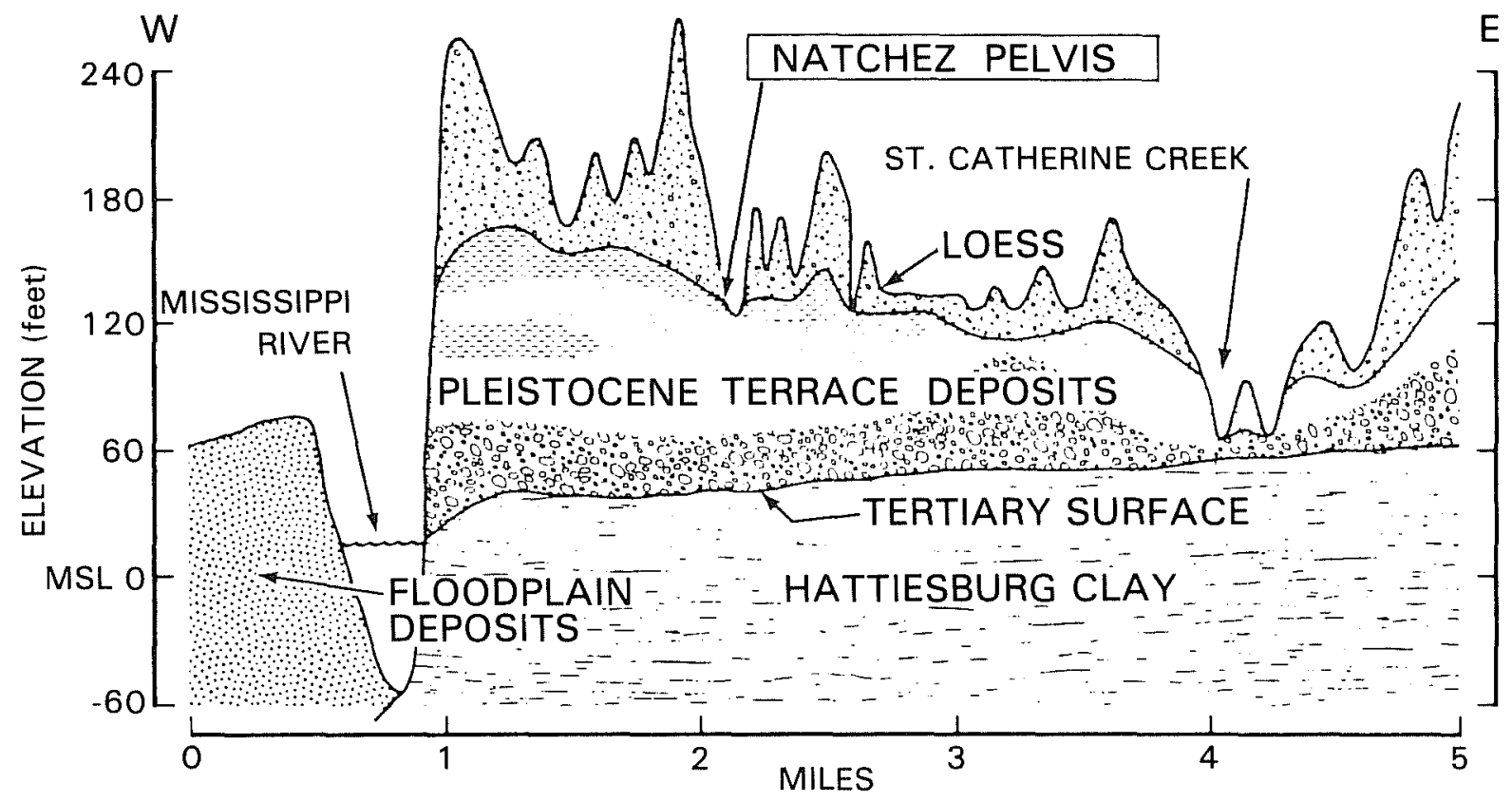

Figure 28. Idealized Geologic Section in Vicinity of Natchez, Mississippi, Showing Sections of Natchez Pelvis Find. Geology modified from Saucier $(1967)$. 
TABLE 7. RADIOCARBON AGES OF VICKSBURG, MISSISSIPPI, AND TUNACA BAYOU LOESS DEPOSITS

$$
\text { Radiocarbon Age (Yrs. B.P.) }
$$

\begin{tabular}{|c|c|c|}
\hline \multirow{8}{*}{$\begin{array}{l}\text { WOODFORDIAN LOESS } \\
\text { (INTERVAL "F") }\end{array}$} & Tunaca Bayou & Vicksburg, Mississippi \\
\hline & & $17,850 \pm 380 *$ \\
\hline & & $18,200 \pm 500 *$ \\
\hline & & $18,640 \pm 380^{*}$ \\
\hline & & $19,200 \pm 420 *$ \\
\hline & & $19,250 \pm 350 *$ \\
\hline & $20,690 \pm 250$ & $20,500 \pm 600^{*}$ \\
\hline & & $21,270 \pm 440^{*}$ \\
\hline \multicolumn{3}{|l|}{ WOODFORDIAN LOESS } \\
\hline \multirow[t]{3}{*}{ (INTERVAL "E") } & $21,750 \pm 310$ & $2], 800 \pm 500 *$ \\
\hline & & $22,600 \pm 700 *$ \\
\hline & & $22,600 \pm 800^{*}$ \\
\hline FARMDALE LOESS & & $23,550 \pm 750^{\star}$ \\
\hline (INTERVAL "D") & & $23,550 \pm 1000^{*}$ \\
\hline FARMDALE SOIL & & $25,600+1000 \Delta$ \\
\hline
\end{tabular}

SOURCE: Snowden and Priddy 1968

Otvos 1975

* Fossil pulmonate gastropod she11s

$\triangle$ Fossil Wood 
East of the Mississippi River the modern coast appears not to be sand oriented. Even so, there is an extensive dune field at a depth of 36 to $42 \mathrm{~m}$ off Bonsecour Bay (Gagliano 1977:115) on the Alabama-Florida coast. In the event that this field could be interpreted as an indication of dessication, it would be of interest to know the age of the dune fields.

A study of paleosols along the Colorado River by Sorenson, Mandel, and Wa1lis (1976) provides some insight into terrace surfaces in the East and east central Texas regions. They noted that paleosols of the type found under relict pine populations in east central Texas appeared in the area of present-day Austin, considerably west of any present-day pine stands. Chemical and mechanical examination of those soils confirmed the similarity. Sorenson, Mandel, and Wallis (1976) suggest that, based on paleosol evidence, pines migrated westward along the river terrace during ameliorated conditions during the Pleistocene. Since such soils appear to be limited to river terraces, they concluded that the botanical regime was galleria in general outline.

\section{Biological Indicators}

Climate and physiography provide the environment in which biological organisms and communities flourish. As if climatic change and alteration of the landscape were not complicated enough, the organisms have their own complicated means of adapting to environment and, as we have seen, with erosion, can have major feedback effects on the environment, particularly physiography. The botanical picture is clouded by lag times in colonization. Zoological organisms often are so mobile as to be impervious to short term climatic change. Perhaps the most complicated subset of the biological milieu is human culture, which is not only constantly adapting, but also displays a tendency toward qualitative evolutionary jumps during the time interval with which we are concerned.

\section{Vegetation}

Climatic variation in the Southeast has been marked enough over the last 20,000 years to produce considerable restructuring of botanical communities. Whitehead (1973, 1982) proposed an azonal, southward displacement of boreal species of about $1000 \mathrm{~km}$ at full glacial. More recent analyses from the Gulf Coastal Plain by various investigators (notably P. Delcourt, H. Delcourt, Watts, Whitehead, Sheehan, and Robinson) indicate that the vegetational response to late Quaternary warming was spatially complex. P. Delcourt and H. Delcourt (personal communication) are in the process of publishing a series of maps showing vegetational changes in the declining stages of the Wisconsin and Holocene.

Recently a physiographically based model of botanical response east of the Mississippi has emerged (Delcourt and Delcourt 1979; Delcourt et al. 1980). The model suggests relatively brief lag times for vegetational response to climatic change east of the Mississippi and adds some much needed regional character to a previously bland theoretical landscape. Central to the model, the "Bluffland's Hypothesis," is the highly dissected bluffland east of the 
Mississippi River. The bluffs are composed of the loesses discussed earlier; they range from Tennessee to Louisiana. During colder intervals, ravines act as microrefugia for more temperate species.

During warmer times the bluffs orographically generate fog from the moist air of the river, which cools the blufflands. The river further contributes to the system by moving northern species rapidly southward at the beginning of cold intervals.

For the following discussion I have selected botanical sites along two transects. The blufflands hypothesis is valuable for two reasons relative to the selection of climatically sensitive localities. First, it indicates that sites in the blufflands should be relatively insensitive to climate, because of the refugia. Second, it indicates that sites to the east of the blufflands' effects reduces lags in vegetational response time to the east. Transect A-A' is located east of the Mississippi River and out of the blufflands. It is within the area almost wholly dominated by maritime air masses at present (Bryson 1966). Transect $B-B^{\prime}$ is west of the Mississippi River and traverses coastal and east Texas and then on to southern Missouri. Transect B-B' is on or subject to continental air masses and is loosely related to the prairieforest ecotone.

Transect A-A' is composed of three sites. Anderson Pond, Tennessee ( $H$. Delcourt 1979), spans the entire interval of study and is probably the most highly resolved and completely analyzed sequence in the series. B. L. Bigbee (Whitehead and Sheehan 1982) is in northern Mississippi on the Tombigbee River. It spans only the 1ast 11,000 years. Goshen Springs, Alabama (Delcourt 1980), is located on an upland interfluve in southern Alabama. I have only secondary sources on this site. On appearances it seems to span the entire study interval, but the sedimentation rates must have been very low during glacials.

Transect B-B' begins with the biosilica site of Coleto Creek (41 GD 21) east of Victoria, Texas (Robinson 1978). Boriac Bog (Bryant 1977) is in east central Texas, and the 01d Field Swamp northeast of the Ozarks (King and Allen 1977) completes the series.

\section{Transect $A-A^{\prime}$}

At full glacial the foot of the Cumberland Plateau in Tennessee was marked by a boreal pine/spruce forest, while coastal southern Alabama is thought to have had a xeric oak/pine regime. Little change is observable in the coastal pollen record until the end of the Hypsithermal about 5000 B.P. Tennessee, on the other hand, shows substantial changes. Between 16,300 and 12,500 B.P. jackpine/spruce pollen gives way to deciduous elements. Between 8000 and 4500 B.P. there is evidence for a warm, dry Hypsithermal indicating the effect of the Prairie Peninsula Phenomenon.

Northern Mississippi shows evidence for reduction of mesic elements from the lowlands during the period 3500 B.P. to 7300 B.P. in favor of more xeric oak and hickory. Late in the Holocene, after 3500 B.P., moister conditions return. 
Moisture indicators show a peak at 2300 B.P., and there appears to be some drying in the final segment of the record. This could very well be a product of a more meridional air flow pattern during the Little Ice Age, which blocked a portion of formerly available Gulf moisture. The last 2500 years have seen a general increase in pine on the Gulf Coastal Plain. Pine increases began in southern Alabama as early as 5000 B.P.

There is some conflict between the pollen and alluvial indicators for the upper Tombigbee River during the Hypsithermal (Whitehead and Sheehan 1982). The stream appears to retain a meandering mode through the Hypsithermal indicating a relatively well-watered situation. Analysis of modern discharge data, however, indicates that the Fall Line Hills at the north end of the drainage orographically collect more precipitation during warmer years, while the midvalley turns dry (see Modern Temperature and Salinity of the Gulf of Mexico section, p. 112). Thus, the Hypsithermal may well have had dry midvalley conditions, while the hills acted as rain collectors to water the river.

\section{Transect $B-B^{\prime}$}

A study of biosilica from an archaeological site on the Texas coast (Robinson 1978) indicated a high biomass, mesic forest before 8000 B.P. Subsequently, the forest largely disappeared to be replaced by a seasaw battle between tal1 and short grass prairies. Phytoliths from mesic tall grasses appeared with evidence of human occupation and radiocarbon datable materials at 5000 B.P. and 3000 B.P., the dates correspond to Denton and Karlen's (1973) periods of glacial advance and lowered tree lines. A study of unirrigated sorghum yields (Jones 1979) showed that effective moisture moves westward in Texas with global cooling. It seems probable that the intervening dry periods, dominated by short prairie grasses, correspond to periods of warmer global climate. Bryant (1977) examined pollen from Boriac Bog in central Texas. Full glacial vegetation is marked by an open deciduous woodland with some conifers. The period $8000-12,000$ B.P. shows a reduction in cooler indicators, and by the end of the interval the modern post-oak savanna is established.

North of the Ozarks a considerable amount of work has been devoted to southern Missouri (King and Allen 1977). Spruce persisted in the Ozark uplands until 12,000 B.P. Between 7000 and 12,000 B.P. oak and hickory forests dominated the region. Analysis of a pollen core from the 01d Field Swamp in extreme southeastern Missouri, about $75 \mathrm{~km}$ north of the Tennessee border, showed vegetational changes reflecting a more prairielike environment after 8700 B.P. The change appears to have been more related to a drop in precipitation than a rise in temperatures. The dryness peaked at 7000 B.P. Around 6500 B.P. there was an abrupt turn toward moister conditions, although climate was basically xeric until after 5000 B.P.

King and Allen (1977) argue that the 0ld Field Swamp marks the southern boundary of the Prairie Peninsula. Given H. Delcourt's findings in Tennessee, however, the question of where the border is must be raised, if it exists. It seems possible, given present evidence, that the Southeast generally experienced drier conditions during the Hypsithermal. These conditions may have been 
moderated near orographic uplifts (Gunn 1982d) and in the lower Mississippi Valley (Wigley, Jones, and Kelly 1980). The evidence, however, remains to be reported.

Figure 29 displays the 500-year resolution trajectory for the botanical indicators. Mid-Holocene vegetation indicates virtual1y universal drying. The upper Tombigbee core (B. L. Bigbee) is the most anomalous. The variance with other cores may be due to dating (Whitehead and Sheehan 1982) or to the Fall Line Hills into which the Tombigbee River heads providing enough of an orographic uplift to 1ocally delay drying (Gunn 1982d).

Based on less universal evidence, the declining stages of the last Wisconsin Glacial seem to be discernible. Full glacial cool and dry is replaced by intermediate glacial cool and wet about 16,000 B.P. Around 12,000 B.P. to 13,000 B.P. an apparently unstable transitional glacial Holocene period sets in with mixed readings. Contradictory readings may be due to varying levels of resolution from core to core. Anderson Pond records a highly detailed succession, while Goshen Springs and Boriac Bog seem to be less sensitive. Goshen Springs and Boriac Bog suggest cool, dry conditions, while Anderson Pond indicates warm and wet followed by $\operatorname{cool}$ and wet. Anderson Pond may have been moistened by orographic rainfall provided by the perimeter of the Cumberland Plateau.

\section{Fauna}

I have not systematically examined the faunal evidence. However, Lundelius (1974) and Carbone (1976) have undertaken such reviews. It appears that the fauna provide some fascinating insights into seasonality of glacial climate. A combination of milder winters and cooler summers is suggested, which allowed the mixing of creatures now only able to survive in geographically diverse regions. There seems to be, however, very little time resolution to paleontological data. A very important question for paleontologists to answer in the future will be when the so-called mozaic environments of the Pleistocene gave way to Holocene zonation.

The Mississippi State Museum at Jacksonville recently engaged the services of a paleontologist who has already extended the list of cold species known to have existed in the past in that state (Frazier 1982). Lundelius has studied the Pleistocene fauna of the Texas coast; these include some late Pleistocene fauna from the continental shelf.

While these studies are not highly time resolved, they may be useful through some sort of calibration process, which will allow a closer definition of time series by other methods.

\section{A Methodology for Independent Verification}

The five time series defined in the previous discussions represent the opinions of the respective disciplines as to the progress of climatic change over the last 20,000 or so years. Earlier, I expressed a desire to test the regional models internal to the Southeast against an independent model of climatic change. 
For several reasons, the deep sea core work by Kennett and Huddleston (1972) in the western Gulf of Mexico seemed to offer the most promising source for an external, but relevant set of data. First, the deep sea core data offer a long, sequent, and datable time series, which reflects, in large part, the conditions in the seasonally responsive, upper few meters of sea water. Second, thanks to the relative homogeneity of the sea surface as compared to land, the climatic record of deep sea cores will be less susceptible to microclimates. Third, the Gulf of Mexico is strongly related to the southeastern United States, relative to moisture conditions. The relationship is such that most climatic conditions in the Gulf, whether they relate to temperature or moisture, appear as either direct or inverse analogues of conditions on land.

Radiocarbon years B.P. $\times 10^{3}$ $\begin{array}{lllll}2 & 1 & 1 & & \\ 0 & 5 & 0 & 5 & 0\end{array}$ Anderson Pond, Tennessee ccbbbbbbccccccddccccddddeeeeeedddddddddd

B. L. Bigbee, Mississippi

cccccceeeeeecccccb

Goshen Springs, Alabama

bbbbbbbbbbbbbbbbbbbbbbbeeeeeeecccccccccc

Boriac Bog, Texas

cccccbbbbbbbbecceeeeeeeeeeeeeee

01d Field Swamp, Missouri

ceeeedddcccc

41 GD 21, Texas

ccceeeeedebccccccc

Symbols

Sources (in order as above)

a. Cold and Dry
b. Cool and Dry
c. Cool and Wet
d. Warm and Wet
e. Warm and Dry

H. Delcourt 1979

Whitehead and Sheehan 1982

P. Delcourt and H. Delcourt 1979

Bryant 1977

King and Allen 1977

Each symbol $=500$ years

Robinson 1978

Figure 29. 500-Year Resolution Trajectory for Botanical Indicators, Pollen, and Biosilica. 
Naturally, any analysis which involves the complicated set of variables outlined earlier, extraterrestrial, global, and local phenomena, must include a set of simplifying assumptions in a model which explains their interactions. The most important simplifying assumptions are concerned with the fact that, aside from the extraterrestrial variables, global climate is an interacting system with complex feedback loops which make analysis of the data difficult in terms of standard regression techniques. In the jargon of the causal analysts, the global climatic milieu is a "nonrecursive" system with complex feedback loops. It is desirable, from the standpoint of economy, to examine the system as a "recursive" system; that is to say without undue complications from feedback. In practice, I was able to develop a concept which allows the management of the data analysis in an uncomplicated analytical framework. The model is illustrated in Figure 30 and might be called a Global-Local Climatic model.

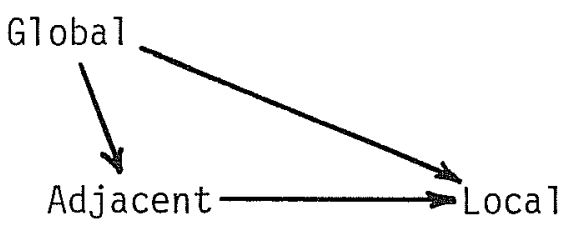

Figure 30. Causal Model of Global-Local Effects. Arrows represent the direction of causality.

Global variables include extraterrestrial forces such as solar output and "solar input," that is those variables that modulate solar output such as orbital configuration as defined by Milankovitch (1930) and modified by Kukla (1975), and aerosols in the upper atmosphere such as volcanic dust (Bryson and Goodman 1980). Adjacent variables are those that represent conditions in regions adjacent to the area of targeted interest, the local region. In the example I will use here, the upper Tombigbee River is our region of interest. The Gulf of Mexico is a strongly relevant adjacent region, because of the high frequency of air flow off the Gulf and into the Tombigbee as regulated by the clockwise circulation around the subtropical high. The model qualifies as a recursive model in that there is little feedback from the local region (it being only a few thousand square kilometers) to the global variables, because of the sheer magnitude of surface and volume of air involved. There is 1ittle feedback to the adjacent regions because it is "up" the air stream, from the targeted region. Naturally, there is some feedback. Temperature of the surface in the Gulf Coastal Plain has to have some affect on air temperature the next time it circulates around the world, and the temperature of Gulf waters is influenced by discharge from 1and. Since the variables are not completely independent of feedback it might be advisable to cal1 the model a predominantly recursive system.

In a regression equation of the system,

$$
y=x_{1}+x_{2}+(u, f, e),
$$


$Y$ represents local variables, let us say moisture discharge and temperature indicators on the Gulf Coastal Plain, while $X_{1}$ and $X_{2}$ are the global and adjacent environmental indicators. Error in the predominantly recursive system represents regressional residual which contains, in part, measurement error (e), spurious correlation due to feedback in the system ( $f$ ), and correlation due to unidentified variables in the system $(u)$. The $f$ component of the system is not necessarily harmful as long as it is reasonably low as measured by the Durbin-Watson (Johnston 1972:251-252) statistic. In most of our analyses, serial correlation of the residuals as measured by the Durbin-Watson statistic did not appear as a problem. In fact, much of the nebulousness that one usually sees in numerical analyses of global climatic data (low $\mathrm{R}^{2}$, diffuse eigenvalues) disappears with the inclusion of a set of local variables.

This suggests to me that perhaps linear models are better adapted to analysis of the model than to the more general models.

Examining the Mode1: 1956-1978 Data

The most comprehensive records of weather phenomena in the Gulf Coast date from February 1956, when radiosonde stations were established. Monthly upper air data collected by the radiosonde probes, Palmer soil moisture indices, and discharge volumes from rivers and tributaries can be used to measure climate in local regions (Gunn 1982d). The adjacent Gulf of Mexico was quantified as to water temperature and salinity measures at 10 and $100 \mathrm{~m}$ depths from three, two-degree squares stratigically located to represent different phases of Gulf waters: the western Gulf, which is relatively isolated from the Atlantic waters; the eastern Gulf, which is heavily influenced by the Gulf stream; and the northern Gulf near the mouth of the Tombigbee River, which was included to control for the affects of discharge interaction between the Gulf and the local study area. Global variables were represented as solar flux, optical depth of the atmosphere, atmospheric $\mathrm{CO}_{2}$, season of the year, and frequency of global circulation type for the month.

A principal components analysis of this data set showed clearly that the global variables were active in the adjacent and local weather phenomena. Component VIII, for instance, indicated higher solar flux values (lagged one year) are associated with (1) higher water temperatures in the western Gulf of Mexico, (2) increased discharge and moisture in the rugged upper reaches of the Tombigbee River in northern Mississippi, but (3) less moisture and lower discharge rates in the Gulf Coastal Plain further downstream.

\section{Extending the Mode1: Prehistoric Environments}

As a product of several months of working with the modern data from Texas and Mississippi, I developed some insight into the weather processes that conspire to produce climate during the 20 th century. The next concern was to extend this complex of principles into the past, and from it derive a set of conditions that would describe the climate with which humans of past eras were required to cope. There are certain limitations to the data. For instance, the period 1956 to 1978 (Fig. 31) comfortably spans the transition 


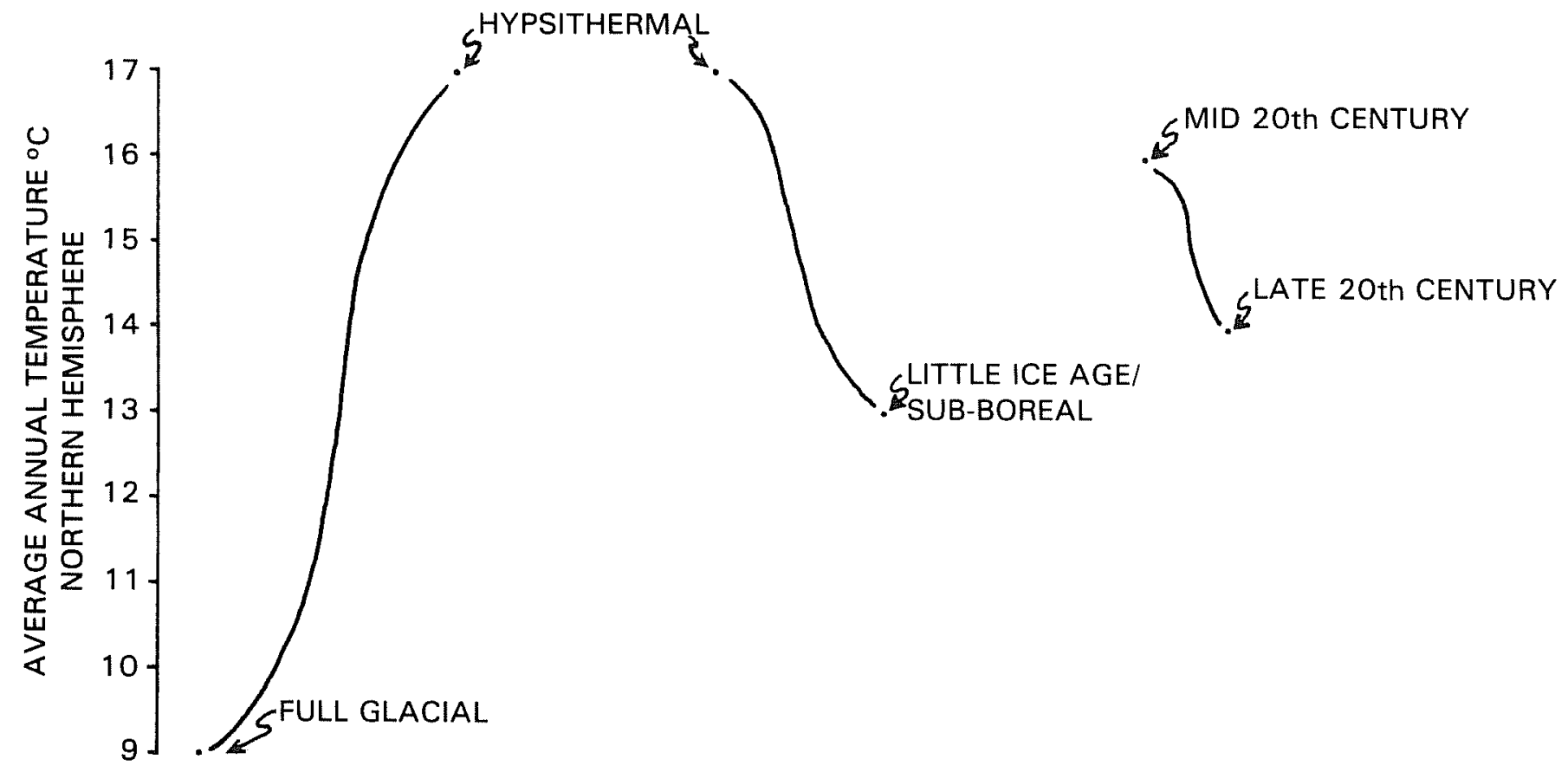

Figure 31. Range of Temperature (Energy Budget) Movements. 
from what might be called the mid-20th century climatic optimum of the more characteristically Little Ice Age conditions of the 1960s and 1970s (Sanchez and Kutzbach 1974). We can therefore surmise with some confidence the affects on local conditions when global parameters move from those of climatic optima to the milder varieties of glacial advance. On the other hand, projecting into the times of the more serious ice advances demands more caution.

\section{Deep Sea Cores}

Kennett and Huddleston (1972) studied many deep sea cores from all over the Gulf of Mexico and found that those in the western Gulf contained enough foraminifera to determine communities of organisms representative of water temperature by principal components analys is (Malmgren and Kennett 1976).

I thought that it would be worthwhile to conduct our own analysis of the data from the deep sea cores, because most of Kennett's applications were concerned with relatively long periods, approximately the last 100,000 years and with water temperatures only. Our concerns for archaeological reconstruction, however, were limited to the last 20,000 years. We were also interested in salinity since it affects evaporation. The core, which appeared to have the most highly resolved record in the requisite time period, was selected and Kennett very kindly provided us with the data. The analysis (Mahula 1982b) showed there to be six components, or communities, of foraminifera. The first component, as is normal for such analysis, was demonstrably related to water temperature. Component scores are plotted in Figure 32. This time the scale is corrected for depositional rates and converted to radiocarbon years (Gunn 1982c; Gunn and Stuckenrath 1982).

In addition to the water temperature, we thought it might be of some interest to examine sea salinity as well. Salinity lowers vapor pressure and specific heat. Examination of the remaining five components showed Component II to be loading inversely for Globigerinoides sacculifera, which according to Berger (1969) prefers high salinities, and Globigerina bulloides; which prefers waters of low salinity (Berger 1969). The component scores for component II are plotted in Figure 32 and reflect a pattern variable from that of the temperature curve and apparently heavily inflected by episodes of glacial melt water in the declining stages of the Pleistocene.

Modern Temperature and Salinity of the Gulf of Mexico

Namias (1976) demonstrated, through a rather long series of causal relationships, that the Aleutian Low exerts control on the rigor of North American winters. When the sun heats the waters to above average values off the south coast of Alaska and the Aleutians during summer, cyclonic activity is sustained over that area through the fall as the ocean gives up its heat to the atmosphere. The lows so generated move landward fostering a pressure ridge over the western United States and troughs over the east. The end result is temperature and precipitation in a relatively standard and recognizable pattern. This pattern includes a predictable pattern of temperature and precipitation over the southeastern United States. 


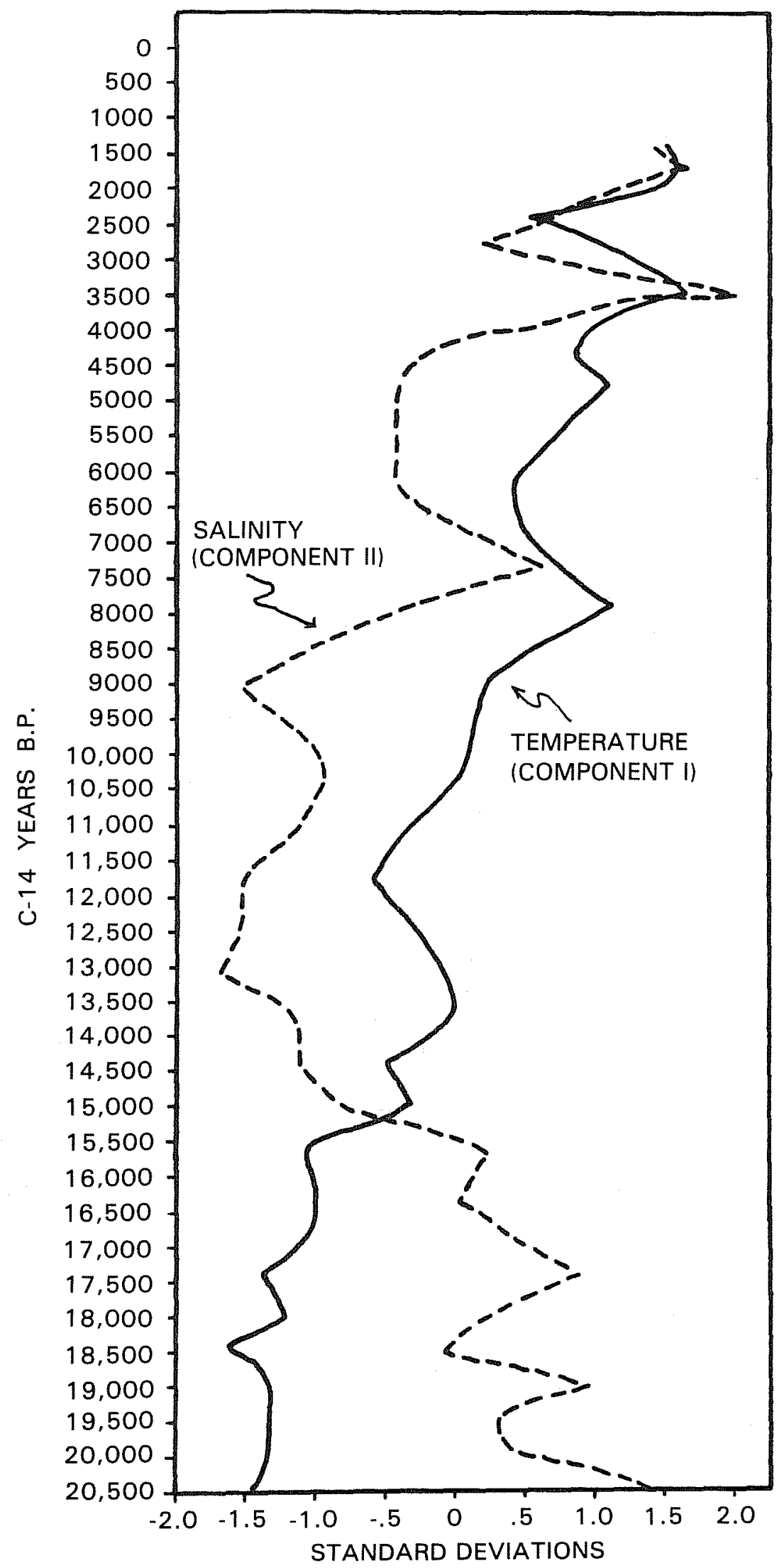

Figure 32. Temperature and Salinity in the Western Gulf of Mexico. From Gunn (1982e). 
I have attempted to apply a somewhat similar methodology to the problem of independent verification of paleoclimatic data from the Gulf of Mexico and the southeastern United States (Gunn 1982b). As we have seen, paleoclimatic data are available from deep sea cores in the Gulf of Mexico and from the various regions of the Gulf Coast. There are also modern sea temperature and salinity data from the Gulf and various indicators of moisture and temperature on 1 and.

The methodology for independent verification consisted of the following. A series of regression coefficients describing the relationship between modern sea temperature, salinity, and landward moisture were generated. These regression coefficients were then multiplied by the sea temperature and salinity trajectories described above. The curves resulting from these calculations were posed as models to be verified by the biological and physiographical data. Curves for east central Mississippi and east Texas are shown in Figure 33.

Previous efforts have concentrated on the Mississippi and Alabama regions. For the study a Paleo-drought-index was calculated for east Texas, the nearest point to Eagle Hill for which data were available. The results of these calculations are shown in Figure 33. Note that the Paleo-Indian period deposits date from the period of highest moisture. The end of early period sediments about 7000 B.P. radiocarbon corresponds to a relatively dry interval. Unfortunately the deep sea core record terminated before the A.D. 800-900 occupations. However, the later interval is notable for high volcanic activity as is shown in Figure 20, very much like the early period.

Pollen Analysis Test, East Central Mississippi

The B. L. Bigbee-2 core discussed earlier (Whitehead and Sheehan 1982) provided the most secure test information to date on prehistoric vegetation in the Tombigbee area. The core appears to span the last 10,000 to 12,000 years and is interpreted to indicate moist conditions between 7300-9800 B.P., dry conditions from 3500-7300 B.P., and a return to more mesic conditions after about 3500 B.P. The 8000-10,000 B.P. mesic period accords well with the climate projected from the deep sea core during Dalton times. However, due to problems with pollen preservation and resolution of the dating in the column, we cannot say at this point whether the two subsequent suspected moist periods can be supported or rejected by the pollen record.

\section{Biosilica Analysis Test, East Central Mississippi}

Eleven samples removed from archaeological sites were analyzed for microscopic opals that develop in the cells of many plants and some aquatic animals. In contrast to pollen analysis, biosilica analysis provides a very localized view of vegetation as well as some indication of local hydrological conditions as indicated by sponge spicules. A more detailed examination of the biosilica record than can be discussed here shows that as nearly as can be determined, the biosilicas support the projected climatic sequence in about the same fashion as the pollen. However, the biosilicas indicate a mesic climate during the Clovis period (about 11,000-12,000 B.P.). The east central Mississippian curve indicates the $11,000-12,000$ B.P. interval to be the first late Quaternary mesic surge. 


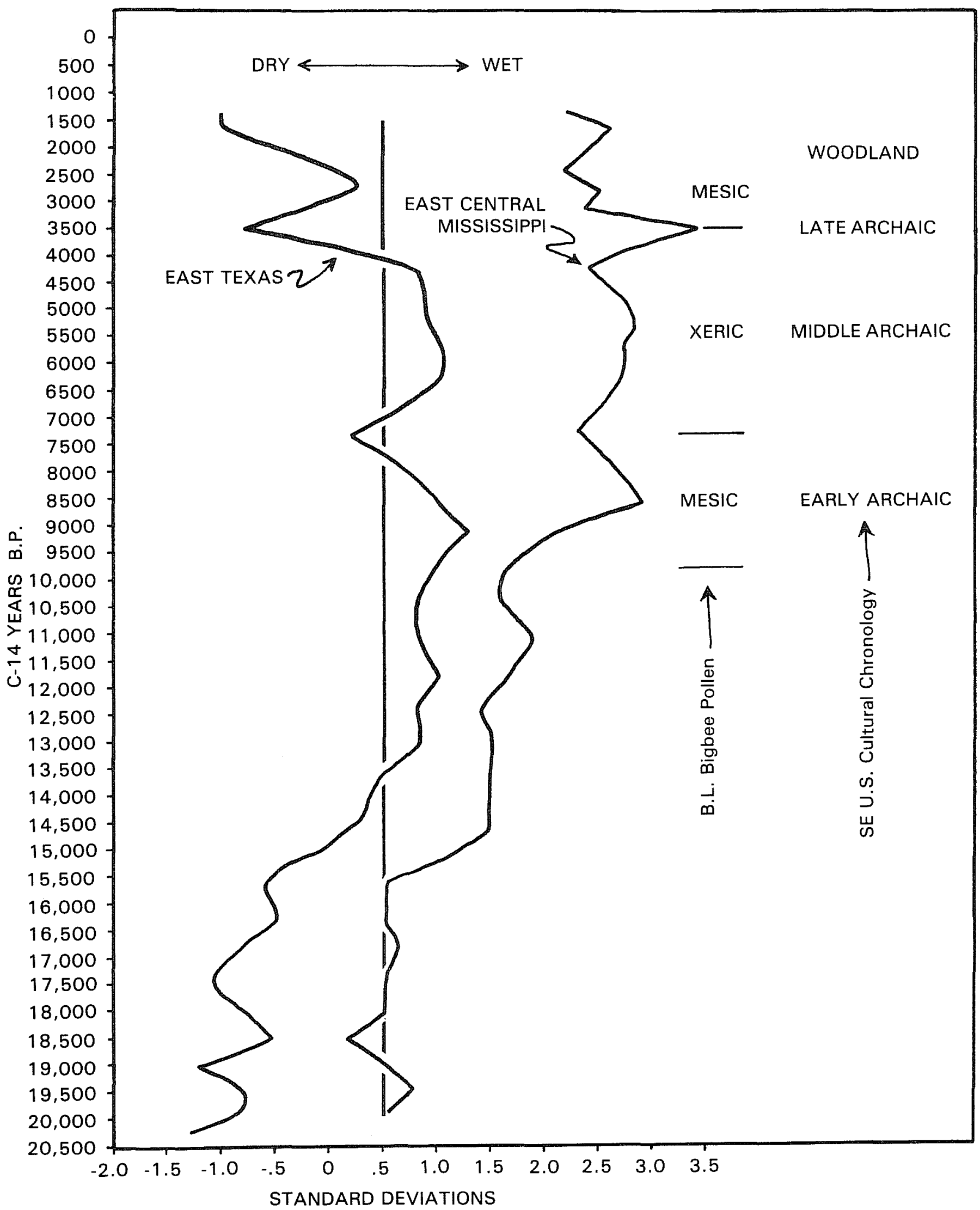

Figure 33. Paleo-Palmer Index for East Texas and East Central Mississippi. 


\section{Cultural Test}

A considerable amount of archaeological work has been done on the Tombigbee River relative to the Tennessee-Tombigbee Waterway project. With the exception of some reports by Blakeman (1975a, 1975b) and Bense (1982), much of the information gleaned from the project remains to be synthesized. However, using Blakeman, Bense, and other sources, a tentative demographic chronology for the Tombigbee can be reconstructed. Evidence for early Paleo-Indian (Clovis) is very scarce. The Tombigbee Valley joins with most of the rest of the Southeast in a rather spectacular display of Dalton-aged archaeology suggesting a developed population.

The Early and Middle Archaic periods appear to be marked by low populations that camped at ecologically diverse ecotones and exploited a wide range of food resources. Late Archaic sites are numerous, thick middens that indicate a large population. A major economic shift occurred between Late Archaic and Early Woodland. Plant foods appear to have been emphasized, and hunting changed from generalized to specialized hunting of the most productive game (Blakeman 1975a:92). Uplands appear to have been inhabited and exploited.

During the Late Woodland, Mississippian peoples inhabited the valley, but the population appears to have been relatively low (Blakeman 1975b).

I suggest the following relationships between the projected climate in Figure 33 and the cultural chronology. During Clovis times the Tombigbee was dry and unappealing in contrast to the stable and rich Tennessee Valley to the north. Since the population was probably rather sparse anyway, most of the Clovis-aged activity was confined to the Tennessee Valley area.

During Late Paleo-Indian/Early Archaic the climate is projected as being moister, a condition supported by the pollen and biosilica data. There is also evidence of significant human activity during this time period. It would seem that the combination of more favorable conditions, and a probable natural upward trend in population conspired to encourage considerable human activity in the valley, notably at the Hester site on the middle upper Tombigbee River.

The Early Archaic/Middle Archaic period is projected as being somewhat drier, a surmise fully supported by the pollen. The deep sea projection indicates a subtle movement toward drought. The population seems to have dropped to correspondingly lower levels.

A resurgence of moist climate about 4000 B.P. apparently encouraged a large Late Archaic population, an event probably supported by the globally recognizable Subboreal cooling caused by massive volcanic activity. We lose the deep sea core at this point and have to turn to standard climatic chronologies for the late Holocene.

The Roman Empire Climatic Optimum corresponds to the Early Woodland. Warmer global conditions probably turned the Tombigbee drier. Speculation is large populations developed during the Late Archaic and were forced to turn to intensive food production and collecting in order to survive. Thus, the vigor of the food production and specialized hunting effort. 
picture. North American and Mesoamerican cultural chronologies are marked by developments and catastrophies, which correspond in time with the 2600year glacial cycle (Gunn and Adams 1981; Folan et al. 1982; Dahlin 1980). The coastal plain appears to be no exception to this pattern. Eagle Hill II depositional episodes clearly correspond to mesic periods. The critical controlling variable appears to be the amount of global volcanic activity.

\section{UPLAND EROSIONAL EPISODES AND SOUTHEASTERN CLIMATE}

Brakenridge (1980) pointed out the possibility of synchronized alluvial cut and fill sequences over a large portion of the northern hemisphere (Table 8). It is of more than passing interest that Brakenridge's globally synchronous, alluvial sequence shows virtually perfect correlation with Denton and Karlén's (1973) high-latitude glacial and tree-line sequence. Periods of alluvial cutting occur coincident with glacial advance and increased meridional air movements.

TABLE 8. SYNCHRONOUS ALLUVIAL DOWN-CUTTING EPISODES FOR THE NORTHERN HEMISPHERE

$$
\begin{gathered}
\text { Radiocarbon Years B.P. } \\
400-500 \\
1500-1600 \\
2500-2900 \\
4900-5000 \\
7700-7900
\end{gathered}
$$$$
\text { Episode }
$$

Main Little Ice Age

Early Little Ice Age

Subborea]

Mid-Hypsithermal Cooling

Cockburn-Cochrane

SOURCE: Brakenridge 1980

Naturally, one might suspect that erosion on Peason Ridge is related to this sequence in a systematic manner. If warm intervals are marked by alluvial filling, then ridge tops must be yielding sediments. With a Paleo-Indian component marking the beginning of deposition in the saddle of Peason Ridge, one might suspect that the overlying erosional event corresponds to the 7800 B.P. erosion on the Pomme de Terre River (Brakenridge 1980).

Figure 34 is taken from Brakenridge and illustrates the Holocene sequence of the Pomme de Terre River in southern Missouri, the point of departure for his comments on synchronized alluvial chronologies. The Pomme de Terre River is $900 \mathrm{~km}$ north of Eagle $\mathrm{Hill}$ and in basically the same climatic system in respect to the prairie forest ecotone and circulation of moisture of the Gulf of Mexico. 
This Mississippian period is reported by Blakeman to be low in population. However, the time interval contains several important global climatic changes. It may be that the local Mississippian culture eventually participated in the general population decline observed by sequential reductions in stockade perimeters over most of the south (Haag 1965). However, it seems unlikely that there was no fluorescence during the Late Woodland, and it is probably best to await the results of excavations in progress before venturing a judgment of the interaction of Mississippian culture and climatic change.

\section{Summary}

This survey of modern and prehistoric climate, geomorphology, alluvial chronology, upland erosion and deposition, vegetation, fauna, and culture has at the level of evidence available revealed some things about regional climatic variation in the Southeast and shown that there are other questions which remain to be answered.

It would appear at this time that the idea of a wet Hypsithermal in the lower Mississippi Valley is largely unsupported. A mid-Holocene erosional episode on Avery Island indicates that there was an element of instability in the climate at that time. If it was wetter it was probably at a season of the year inappropriate to vegetation. The last few years have been relatively dry ones in the Southeast, and they have been dry in the spring and wet in the summer due to tropical storms. There is, in fact an element of shift from winter to summer moisture implied by the components plotted in Figure 33. Dry springs could very easily assume a causal role in alluvial erosions. Perhaps the one great gap in lower Mississippi paleoclimatology at this point in time is the lack of botanical studies in the coastal delta and delta plain. Dry springs and wet summers could also provide upland erosion in the Louisjana-Mississippi area delineated by Wigley, Jones, and Kelly (1980). Some of the answers to these problems may reside in Saucier's meander belt shifts in the Mississippi River which should be intensively interpreted for paleoclimatic information.

If the lower Mississippi does not provide ameliorated conditions during hot times, it appears that certain other regions do. The blufflands east of the Mississippi River and uplifts facing the air streams in the eastern coastal region appear to be providing shelter to plant species and discharging water to river valleys. Uplifts to the west of the Mississippi River may also provide islands of precipitation. Whether these are limited to late summer during the tropical storm season or support vegetation during the spring is a very important question to the study of upland sites. The uplifted coastal plain and the older features of the Ouchita Mountains and Balcones Escarpment are important in this category.

There seem to be at least two transitional phases to the last glacial period. Before 12,000 B.P. there is a true intermediate glacial situation with meridional circulation and dry climate. After 12,000 B.P. the situation is dominated by Holocene conditions with resurgencies of glaciation and glacial climate, probably related to Denton and Karlén's (1973) 2600-year glacial cycle of, as yet, undetermined cause. These cold surges, which continue on into the Holocene, appear to be the prime movers in the cultural change 


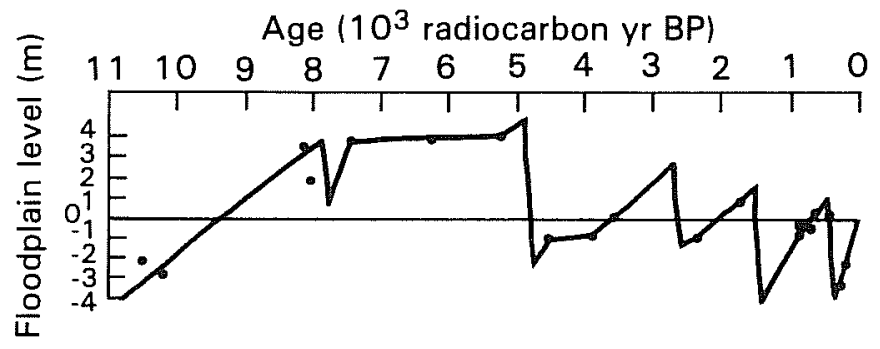

Figure 34. Holocene Level History of the Pomme de Terre River in Southern Missouri. Elevations are in meters above or below the modern floodplain, set at zero. 
Since our evidence indicates that the upper colluvium is relatively late, after 1500 B.P., we might presume from the Pomme de Terre River sequence that the sedimentary contribution of Peason Ridge to stream loads was rather heavy throughout the middle Holocene. The return of moister, late Holocene conditions served to stabilize the sediments and retain the latter part of the record. Whitehead and Sheehan (1982) found a marked increase in pine in the Tombigbee River area in the late Holocene record. Since Peason Ridge was apparently a verdant pine habitat up to the present century, the evidence suggests that the stabilization may have been affected by pine forests.

\section{GEOLOGY AND GEOMORPHOLOGY OF THE EAGLE HILL LOCALE IN THE UPLANDS OF WEST CENTRAL LOUISIANA (Garner)}

Introduction

A geologic/geomorphic study was conducted in the vicinity of Eagle Hill, Louisiana, including part of Sabine, Vernon, and Natchitoches Parishes, and in the northern reaches of the Fort Polk Military Reservation, Peason Ridge Artillery Range, and adjacent areas. This study was conducted by UTSA (Purchase Order No. 0-05399) in support of the Eagle Hill (16 SA 50) project for Interagency Archeological Services-Atlanta, Heritage Conservation and Recreation Service. The modern geomorphic/geologic relationships have been studied in an effort to assess the physiographic and geologic setting that was present when early man lived in the region.

Mapping was accomplished by field examination and photogeomorphic interpretation utilizing color infrared photographs (scale approximately $1: 30,000$ ) provided by the U.S. Army. The final map is presented on a topographic base prepared from the U.S. Geological Survey (USGS) 7.5 minute quadrangle maps (scale $1: 24,000)$.

\section{Geology}

The geologic units mapped during this investigation include bedrock sediments of Miocene and Plio-Pleistocene age, alluvial materials of Pleistocene-Holocene age, and colluvial materials of Holocene age. Anderson (1960) described a sequence of deposits in an exposure about seven kilometers north of Eagle Hill. The units described in this measured section (Fig. 35) are typical of Miocene and Plio-Pleistocene units recognized in this investigation and differentiated on the geomorphic map.

\section{Description of Units}

The Catahoula Formation (Miocene) in this study area has a total thickness of about $18 \mathrm{~m}$ (55 feet) and consists of two members, the Cassel Hill and Chalk Hills equivalents (Fig. 35). The Cassel $\mathrm{Hill}$ is composed primarily of buff to light reddish brown, fine- to medium-grained quartz sand, which is fairly well sorted and round. It is commonly cross-bedded and contains a localized thin zone of siliceous pebbles ( 0.3 to $0.6 \mathrm{~cm}$ in diameter) near the base. The Chalk Hill equivalent is the uppermost unit in the Catahoula Formation (Fig. 35). 
CATAHOULA FORMATION

Sec. $28 \&$ Sec. 33, T.6N., R. 9W

SABINE PARISH, LOUISIANA

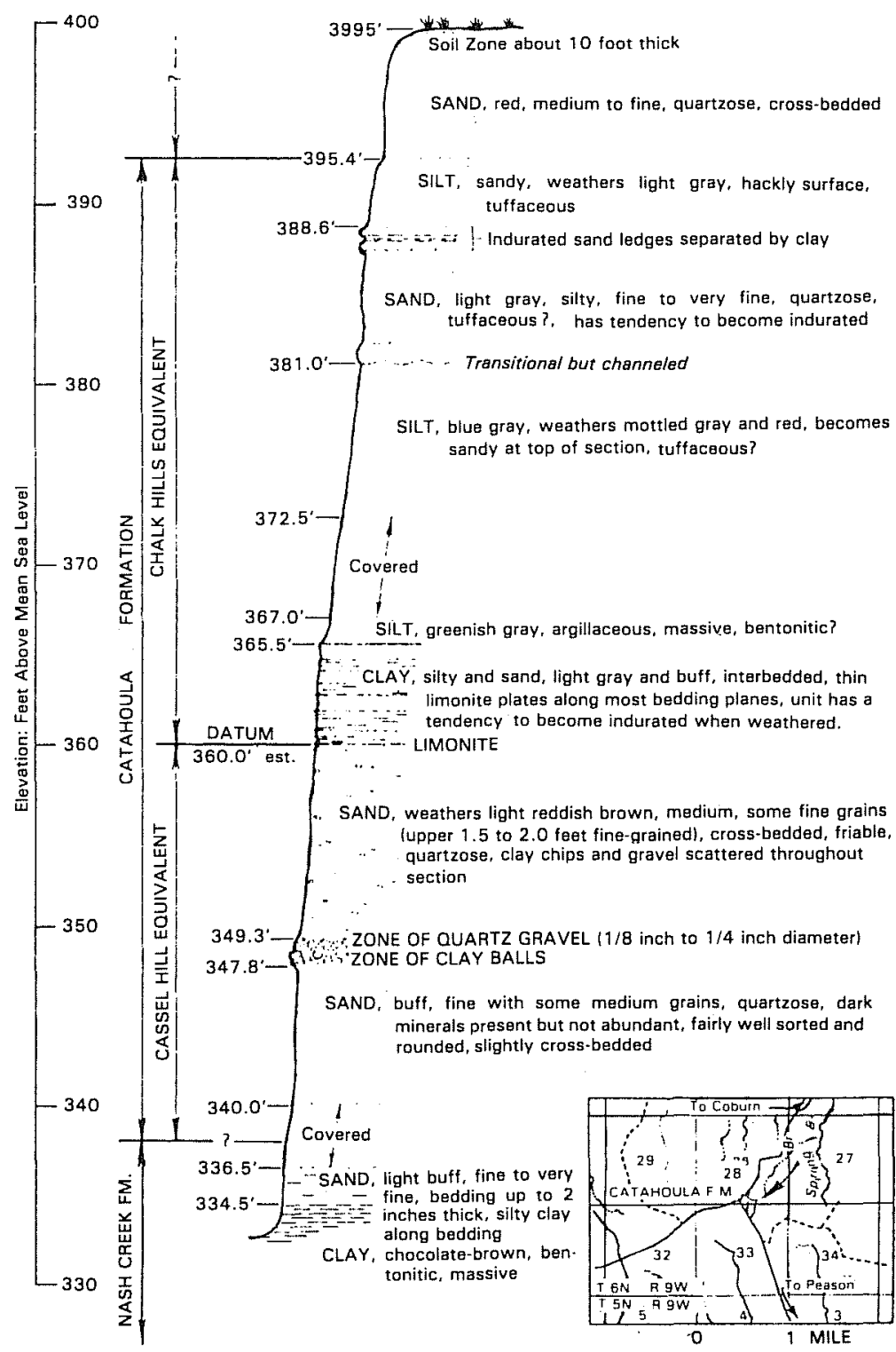

Figure 35. Measured Section of Bedrock Material Exposed in the Vicinity of Eagle Hill. After Anderson (1960). 
It consists primarily of light gray to blue gray tuffaceous clays, silts, and sands. The basal part of this unit is characterized by thin-interbedded sands, silts, and clays with limonite partings, whereas the upper units are texturally more massive and have thicker beds. An indurated sandstone ledge occurs near the top of this unit in many areas. This sandstone ledge was observed in the bank of the small stream just north of the Eagle Hill II excavation site.

The Catahoula Formation is locally overlain by a reddish tan, medium- to coarsegrained, cross-bedded quartz sandstone. Siliceous pebbles of quartz and jasper ranging in diameter from about one to five centimeters are commonly found distributed throughout this unit. Concentrations of these pebbles occur in a few local areas. One such concentration has been reported at Eagle Hill (Woodward and Gueno 1940) where this unit caps the hi11. However, only scattered gravel was observed at the surface (no borings or excavations were made). The exact age of this unit is unknown. Levert (1959) and Welch (1942) considered this unit to be of Pliocene or Pleistocene age and possibly correlated to the Citronell or Williana Formation.

Streams in this area are the uppermost reaches of large fluvial systems and are primarily erosional in nature. Since these streams are characterized by headward erosion and bedrock dissection, they have not established a sequence of fluvial deposits in the local area, but are actively transporting eroded materials during high flow conditions to downstream areas rather than accumulating them. Stream deposits occurring within the project area consist of accumulations of clayey and silty sands and sandy muds. These deposits are derived entirely from the local bedrock deposits described above and reflect the composition of these materials.

Colluvial materials occur along the margins of the alluvium and consist primarily of sheetwash from bedrock materials that overlap alluvial deposits. These deposits were mapped with the alluvium, because dense vegetation prohibited differentiation on photographs in most areas. In addition, these colluvial materials do not differ significantly from the alluvium.

In many areas, colluvial materials represent erosional remnants of P1ioPleistocene deposits. These colluvial deposits occur along many ridge tops and on hills that lie downslope from the Plio-Pleistocene sands. Colluvium ranges from a few centimeters to a meter or more in thickness and probably covers most of the Miocene bedrock deposits. Generally, deposits less than 1/2 meter thick were not mapped. These deposits are significant, because they are the result of erosional processes that have been active since the last Pleistocene deposition. This erosive episode has produced the present landscape.

\section{Geologic History}

The oldest deposits that occur in this area are of Miocene age. During the Miocene this area was very similar to many parts of today's Gulf Coastal Plain. Processes active in this area reflect a complex of fluvial and lacustrine systems. Galloway (1977) indicates that fluvial systems were relatively small in extreme eastern Texas, and environments were probably dominated by a series of coastal lakes as indicated by the type of sediment, texture, bedding, and depositional sequence. 
As sedimentation proceeded, materials gradually filled in the margin of the Gulf Basin and the coastline shifted southward. At that time (Plio-Pleistocene) fluvial processes gradually became dominant. Bernard and LeBlanc (1965) and many other authors have demonstrated the dominance of fluvial systems in the Gulf Coastal Plain during the Pleistocene and Holocene. Plio-Pleistocene deposits mapped in this area probably represent channel facies of fluvial deposits. The general lack of course gravel materials in Plio-Pleistocene fluvial deposits in this area is the result of the low gradients of streams typical of coastal plain streams. The physiographic character of this area at the end of the Pleistocene was one of a low relief coastal plain similar to the modern Gulf Coastal Plain.

Post-Pleistocene Physiographic Development

Since late Pleistocene, the landscape in this area has been dominated by erosional processes. Therefore, the total relief of the area is essentially the result of processes that are not active. The principal process active during this period has been the dissection of the low-relief topography of headwardly eroding coastal plain streams. Dissection has been accomplished by sheetwash and downslope movement of disaggregated bedrock sediments, erosion of bedrock along active stream channels, and the transport of eroded sediments to downstream segments of the fluvial systems. Three small fluvial complexes converge in the vicinity of Eagle Hi11. These include Mill Creek and Dawden Creek, which are part of the Sabine River system and Kisatche Bayou, which is part of the Red River system. The dissected topography and the colluvial and alluvial deposits mapped on Figure 36 illustrate the main components of the physiographic development. Table 9 explains the notation used on the map.

TABLE 9. GEOMORPHIC MAP EXPLANATION

C Colluvium - $\begin{array}{r}\text { Medium- to coarse-grained quartz with minor } \\ \text { amounts of gravel }\end{array}$
A Alluvium - $\begin{array}{r}\text { Quartz sands and muddy-quartz sands occurring } \\ \text { along modern streams }\end{array}$
M Plio-Pleistocene Sand - Medium- to coarse-grained cross-
bedded sand, locally contained minor
amounts of small gravel

S-1 Measured section locality (Anderson 1960)

A-1 Archaeological site (16 SA 50). 


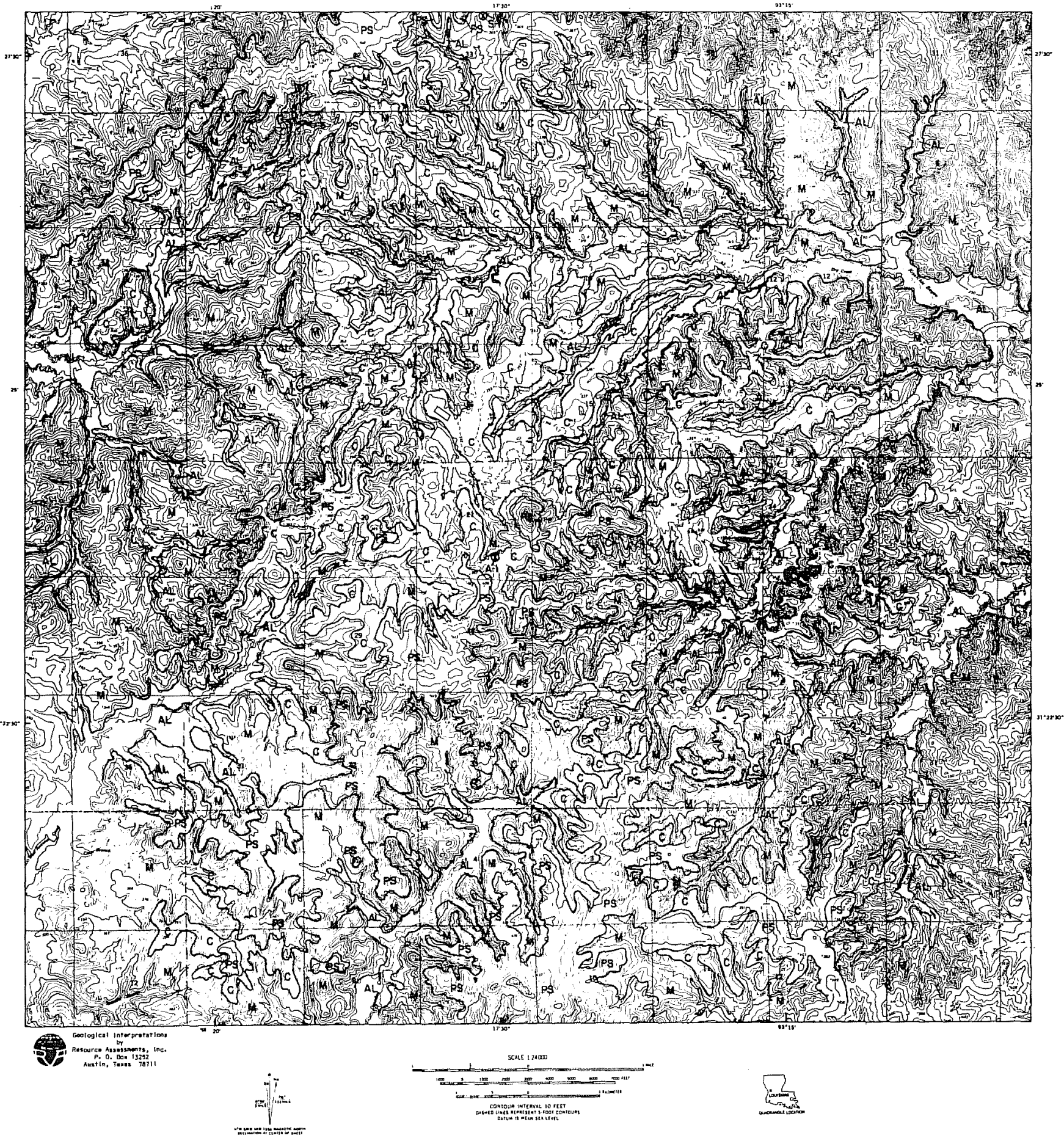

Figure 36. Geomorphic Map, Eagle Hill Area, Louisiana. See Table 9 for symbol explanation. 
Eagle $\mathrm{Hill}$ is one of the highest points along the divide between the Sabine River and Red River. Since this area is relatively distant from the primary fluvial systems, it was probably one of the last parts of the terrain to be affected by the headwardly eroding tributaries.

No Pleistocene or Holocene chronology can be developed independent of archaeology based on fluvial or colluvial sediments of the Eagle Hill area. While it is obvious from pedogenic relationships that colluvial activity has interacted with development of the archaeological site A-1 (see following section), no chronologically significant geologic materials were found in the colluvium. The domination by erosional processes has almost eliminated the potential for long-term preservation.

Regional chronology should be based on studies of the Red River and Sabine River. In any case, the correlation of specific chronology with local upstream areas will be extremely difficult.

As previously stated, the present landscape is the net result of erosional processes since the late Pleistocene. Progressive dissection of the area has probably rendered a more rugged topography with higher relief than was present during the occupation by early man. The elevation of the early man site $(A-1)$ excavated during the course of this project is approximately $115 \mathrm{~m}$ (380 feet). The lowest elevation occurring within the map area of Figure 36 is $73 \mathrm{~m}$ (240 feet). Assuming a minimum Paleo-Indian age of about 7000 B.P. for the Eagle Hill site, the maximum amount of erosion would be $43 \mathrm{~m}$ (140 feet). This erosion would require a rate of only $6 \mathrm{~mm}(0.02$ feet) per year. It is unlikely that the original site was located at the lowest elevation on the landscape because of its relationship with colluvial deposits (Fig. 35). Therefore, the net erosion is probably significantly less than $43 \mathrm{~m}$ maximum.

\section{Lithic Resources}

Lithic materials available in the vicinity of Eagle Hill were probably obtained primarily from siliceous pebbles occurring in the Plio-Pleistocene deposits (Fig. 36). Pebble zones occur near the base of this unit in many areas. Woodward and Gueno (1940) noted the occurrence of siliceous gravels of Eagle Hi11. However, they did not provide a description of the materials. Although the size of available pebbles is small (one to five centimeters), this deposit was probably the most significant occurrence in the area.

A second type of lithic material was found in the project area (David Brown, personal communication). Examination of a part of this sample revealed that the material is primarily opaline-cemented siltstone. The opaline silica, which is found in the locally cemented porous beds of the Miocene deposits, was probably derived from Miocene volcanic materials (tuff) incorporated in the deposits. Amorphous silica in tuffaceous deposits is readily dissolved by groundwater and redeposited in porous beds. Opaline cement is common to deposits in the Catahoula Formation in Texas and Louisiana. 
Summary

Bedrock materials in the vicinity of Eagle Hill are composed primarily of loosely consolidated sandstone of the Catahoula Formation (Miocene age). The Catahoula Formation is locally overlain by discontinuous sand deposits that are probably equivalent to the Williana Formation (Plio-Pleistocene age).

The modern environment is dominated by erosional processes that have acted continuously since late Pleistocene. Headwardly eroding streams and colluviation have been the dominant processes in sculpturing the landscape. The relatively slow rates of erosion have not significantly modified the Eagle Hill area since the advent of early man.

Two basic lithic resources are available in the area that can provide satisfactory material for tool manufacture: (a) opaline-cemented siltstone and fine sandstones related to alteration of volcanic parent material occurring in the Catahoula Formation and (b) siliceous gravels that occur in the Plio-Pleistocene deposits. It is likely that the siliceous gravels have been the largest local source of lithic material, but very little direct evidence is available.

\section{E. GEOMORPHOLOGY AND SOILS (NiaTs, Gunn) \\ Immediate Pre-Pleistocene Sediments}

The physical strata below the surface layers on Peason Ridge is a clay level (Fig. 37). The clay is gray, although it has oxidized to yellow and red near the uneroded surface. The modified spots appear as mottles. The clay is reported to be the Miocene Catahoula Formation by Anderson (1960). Evidence for the relative great age of the deposits is to be found in the fact that the clay contains lenses of sandstone less than a meter down in the section and that most of the weathering is of groundwater origin rather than pedogenic. Further investigations into the age and origin of the subsurface clays sought by microscopic examination of the sediments showed no ostrocodes, foraminifera, etc. The clays are of terrestrial, near-shore origin and date to the pre-

Pleistocene epoch.

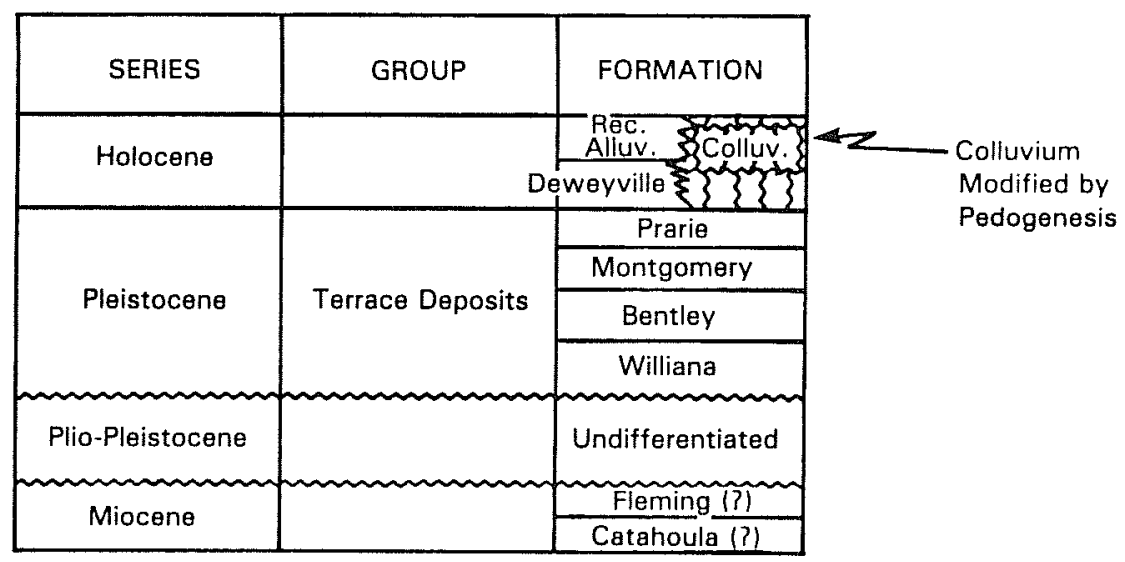

Figure 37. Bedrock Stratigraphy in the Peason Ridge Area. Geologic structurebedrock dips slightly to the south as a result of uplift along the Sabine uplift. 
The clays are topped by a rather regular lag deposit composed mainly of red jasper pebbles from one to five centimeters in diameter. The high frequency of the pebbles is attested by the fact that almost every three-inch bore hole produced a pebble at the interface with the clay. Archaeologically it is helpful to observe that wherever such stones are observed on the surface, the deposits have been deflated below the level of the pre-Pleistocene clays; and any artifacts found in such places will be deflated and/or laterally disturbed. This principle constitutes part of the support for some of the conclusions that follow concerning the condition of the Pleistocene and post-Pleistocene surfaces at the Eagle Hill site and environs.

Within the locality the clay bedrock is overlaid by sandy clay, sandy clay loams, and sandy soils, which range from about a meter in thickness on erosional remnants to $50 \mathrm{~cm}$ or less in more eroded areas.

Eagle Hill itself stands prominently above the surrounding landscape so as to constitute a geological curiosity. It is about $600 \mathrm{~m}$ northeast of the site and stands about 70 feet above the surrounding surface of the ridge. Before it was bul1dozed off to prepare for an Air Force observation post, the top of the hill is reported to have been composed of several feet of sand (John Guy, personal communication 1980). Apparently there were substantial numbers of artifacts in the sand pushed off the hill by the bulldozing operation (Robert Guy, personal communication 1980). The sand on Eagle Hill suggests that it is a product of the same geological process that shaped the various knobs of lesser size which dot Peason Ridge and include the four prominences of the Eagle Hill locality. Given the tendency of primitive people to visit such prominences during ceremonial seasons and the fact that the knobs, as we sha11 see, represent our most likely source of archaeological information, their genesis bear discussion (Fig. 38).

Examination of the USGS $7.5^{\prime}$ Peason Quadrangle shows that Eagle Hill, although peculiar for its abruptness and the fact that it lies at the divide of three drainages (Sabine River, Red River, and Kisatche River), is one of several 450-feet high prominences in the southern Sabine and northern Vernon Parish areas. This suggests to us that the landscape prior to deposition of PlioPleistocene sands and clays was once relatively flat, at least a few tenths of feet higher than it is now relative to the surrounding landscape (Fig. 38,a). Subsequent erosion generally lowered the surface, al though erosional remnants of the former surface in drainage areas are common (Fig. 38,b,d). Anderson (1970) notes that most hills in Sabine Parish are underlain by sands. At least part of the explanation for the presence of these remnants can be posed in terms of the factors controlling erosion.

Under certain conditions sand can present an inhibition to erosion. Precipitation will infiltrate into sand more readily than fine-grained sediments, thus reducing the amount of runoff. Runoff will occur only after the sand becomes saturated or if the rate of precipitation exceeds the rate of infiltration. At the point of saturation, runoff occurs with consequent erosion. Thus, thicker sand deposits have relatively reduced amounts of runoff and erosion. As long as the ability of a layer to absorb moisture is not exceeded by precipitation, the sand will serve as a resistant sedimentary unit. Erosional remnants in the Peason Ridge area can therefore be accounted for by 
$\mathbf{a}$

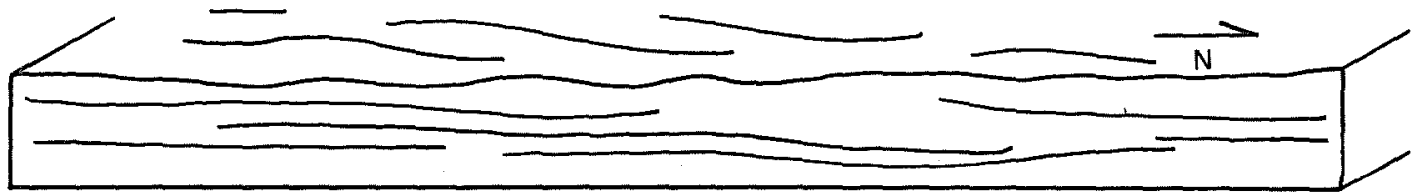

DEPOSITION AND EROSION OF MIOCENE DEPOSITS

b

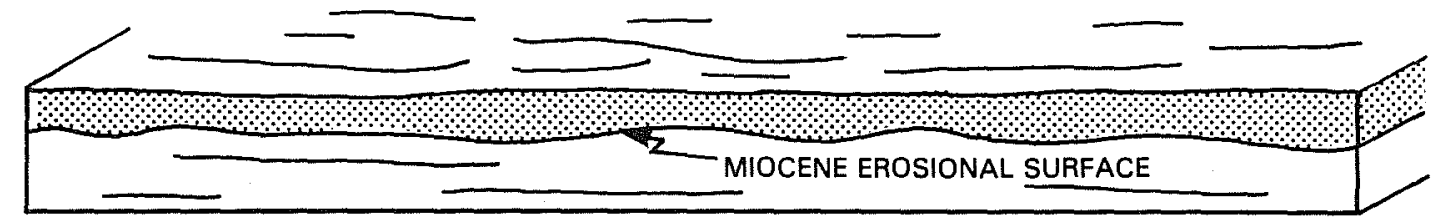

DEPOSITION OF PLIO-PLEISTOCENE SANDS

c

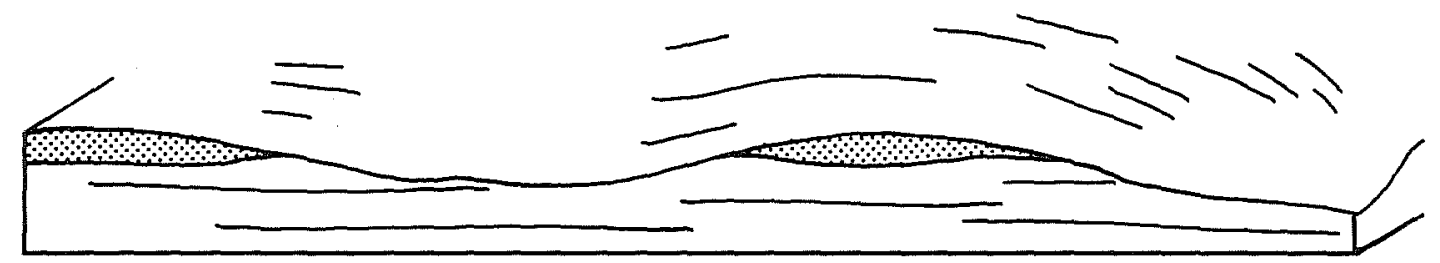

EROSION

d

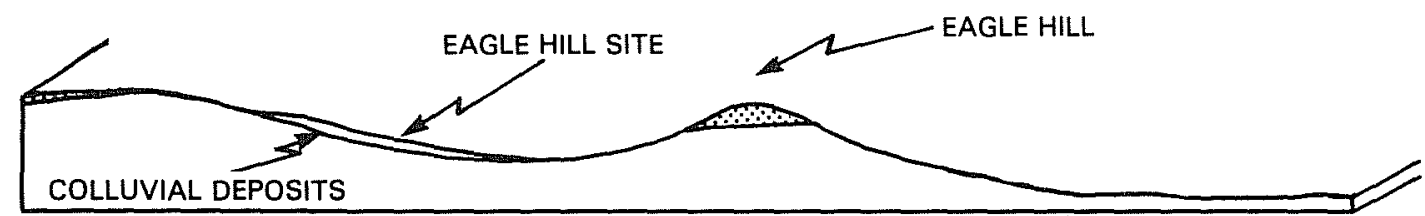

FURTHER EROSION

Figure 38. Geomorphical Origins of Eagle Hill. a, deposition of erosion of Miocene deposits; b, deposition of Plio-Pleistocene sands; $c$, erosion; d, further erosion. 
the presence of sandy surface deposits. The source of the sand is weathering of Miocene sandy clay deposits and Plio-Pleistocene sandy deposits in the area. Wherever sands accumulate for one reason or another to depths whose capacity exceeds the precipitation minus evaporation budget, an erosional remnant can be expected.

The reasons for differential concentration of sands resulting in erosional remnants are more than likely numerous. It is likely that there is some unevenness in the distribution of sands in the substrate, which results in variances on exposure. However, once exposed, geomorphological forces redistribute the sandy sediments with occasional effects toward concentration. In addition, vegetation exerts considerable stabilizing effects on sand erosion resulting from water and wind. Dense vegetation can occur in thickets either due to natural or cultural causes resulting in concentrations of sands and increased ability to absorb water before erosion begins.

The implications of archaeological prospecting are evident. Some of the erosional remnants on Peason Ridge are likely to contain cultural remains. In fact, some may have been caused by cultural activity. It seems likely that these remnants most likely to contain cultural materials would be near sources of water in such a high and dry area. The potential for a seep spring in the area (Servello n.d.) of the sandstone exposed on the south slope of the ridge near the Eagle Hill locality may have provided an appealing spot for at least seasonal camps during the Prehistoric period.

\section{Late Pleistocene-Early Holocene Sediments}

Various lines of evidence suggest that the Miocene bedrock had been eroded to a relatively smooth surface in the vicinity of the Eagle Hill site during the late Pleistocene or early Holocene, and that the surface had been modified by a thin argillic soil. Colluviation during this time is assumed to be relatively inactive. Pedogenic modification of these sediments indicates that relatively stable conditions existed for a considerable length of time, at least 100,000 years.

The developed soils at the bottom of the profile are dated archaeologically to the late Pleistocene and suggest a flat-lying surface. Note that the underlying soil profile (Fig. 39) is flat-lying and does not follow the contour of the present-day surface. This independence of contour suggests a different, probably flat-lying profile for the earlier surface.

We sought further support for this idea by boring for the top of the clay surface on a 250-m transect centered on the Eagle Hill locality and paralleling the trend of Peason Ridge. A plan map of the transect is shown in Figure 4, Section I, page 15. The profile of the transect is illustrated in Figure 40. As can be seen, the surface of the clay bedrock and the present surface do behave as expected, particularly under Area A.

\section{Holocene Sediments}

During the Holocene there appears to have been a marked increase in colluvial activity as indicated by the general suspension of pedogenic development. 


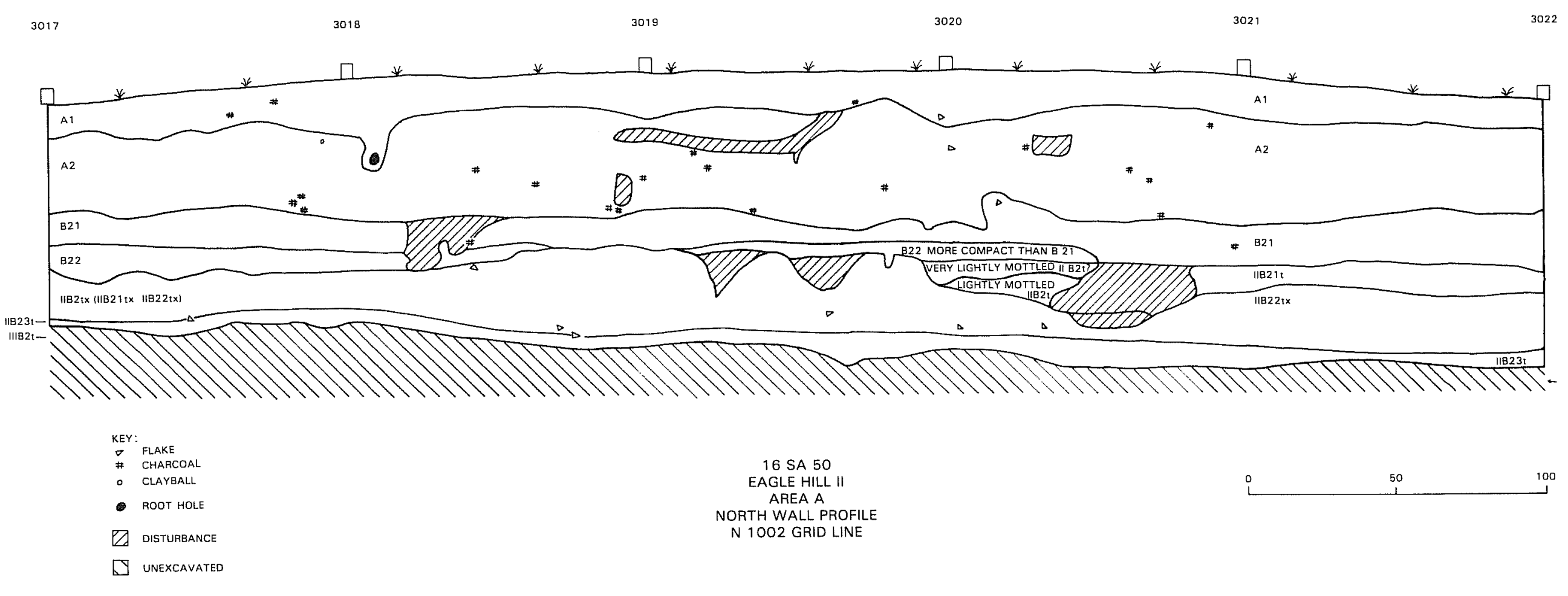

Figure 39. North wall Profile Along the N1002 Grid Line. 
EAGLE HILL (16 SA 50)

SOUTHWEST

24

28

27

BORE HOLE TRANSECT PARALLEL TO PEASON RIDGE

26

1

15

$\varsigma^{\text {WEST REMNANT }}$

101.0

(10)

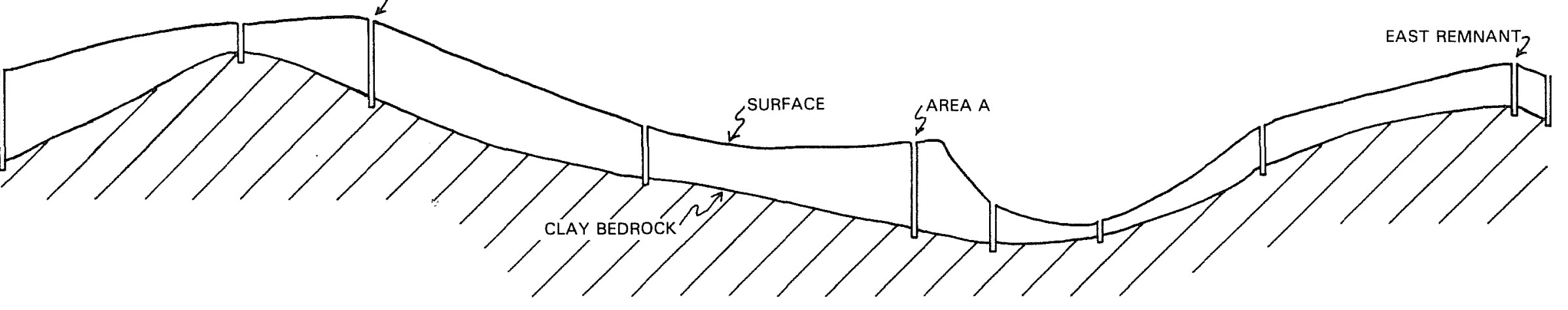

$99.0-$

Figure 40. Profile View of Clay Surface Transect. 
Movement of sediments appears to have been, at least in part, a product of fluvial processes, and the period is punctuated by erosional surfaces within the section. Such a change in the depositional regime was probably fostered by a change in the precipitation pattern. The exact nature of the change is indeterminant. A decrease in vegetational cover caused by a shift to wet winters and dry summers, or intense thunderstorms following periods of dryness are likely prospects for the cause.

Colluvial movement of materials down slopes is generally modeled as follows. A slope can be divided into an upper and lower segment. The ratio of upper to lower area is dependent on the degree and regularity of the slope, amount of precipitation, nature of the sediments, length of the slope, etc. Highly vegetated (Schumm 1965) and relatively flat slopes are inefficient at moving sediments downslope. Efficiency increases with less vegetation and as the slope is organized into systems of ri11s, gullies, and streams.

Holocene development of sediments at the Eagle Hill locality appear to be a classic case of development of efficiency (Fig. 41,a-e). At the end of the Pleistocene a planar clay surface with a thin soil (Fig. 4l,a) began to be subjected to relatively rapid colluvial action (Fig. 41,b). The upper segment of the slopes to the northeast and to the southwest contributed sandy clay sediments toward the gentle swale or saddle at the locality. Our transect, Figure 40, confirms a decrease in surface sediment depth toward higher ground. Apparently the very slick condition of the air strip at the top of the hill to the southwest is attributable to the lack of surface sands to buffer the clays.

In addition to the colluvial dumping of sediments into the saddle, the drainage systems apparently began to organize to more efficiently evacuate sediments from the hill (Fig. 41). Gullies cut into the plain from the north and the south. Some erosional activity is evident in the profile in Figure 39 . By our estimates, however, there does not appear to be decisive surface alterations as would be evidenced by the appearance of prominent rills or gullies in the section within Area $A$.

The reason for the lack of prominent erosional forms may be shown in the present-day geomorphic forms. To the southeast of the site is a resistant siltstone outcrop with a very old gully emptying onto it. It is about three to five meters below Area $A$ in elevation. The age of the gully is attested by the oxidation of the clay bedrock in gentle swales visible in the cutbank. Apparently the relative flatness of the locality was maintained by an underlying caprock, until extreme disruptions such as logging, stump removal, and frequent firing characteristic of this century took place.

To the north of the locality about $100-150 \mathrm{~m}$ is a cutbank that contains a buried soil. It is about $60 \mathrm{~cm}$ above sandstone bedrock and is topped by another $60 \mathrm{~cm}$ of high energy fluvial deposits. We found a piece of sawn timber between the soil and the fluvial deposits, and therefore assume that it is an example of north slope soils before deforestation during the first quarter of this century. As was mentioned earlier, we have found evidence of pedogenesis on the south slopes in the clay. While it is too early to generalize from so few observations, the possibility needs to be examined that the 
a

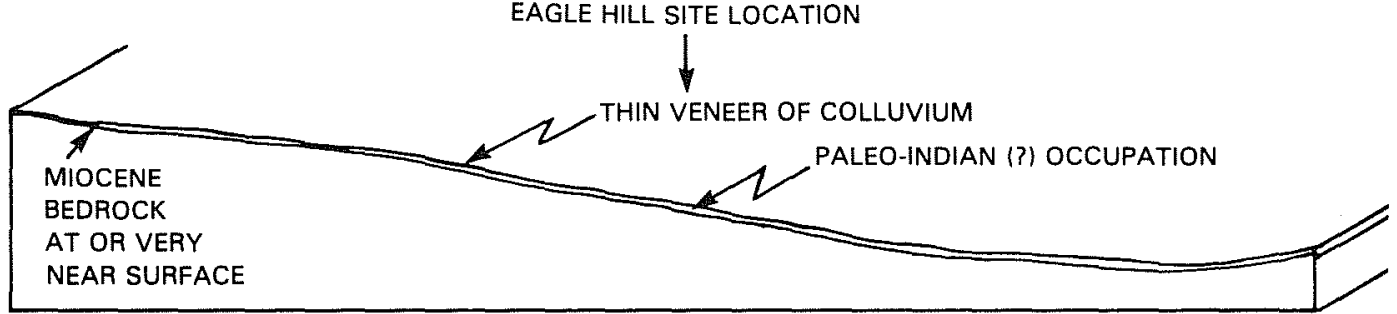

TERMINAL PLEISTOCENE CONDITION

b

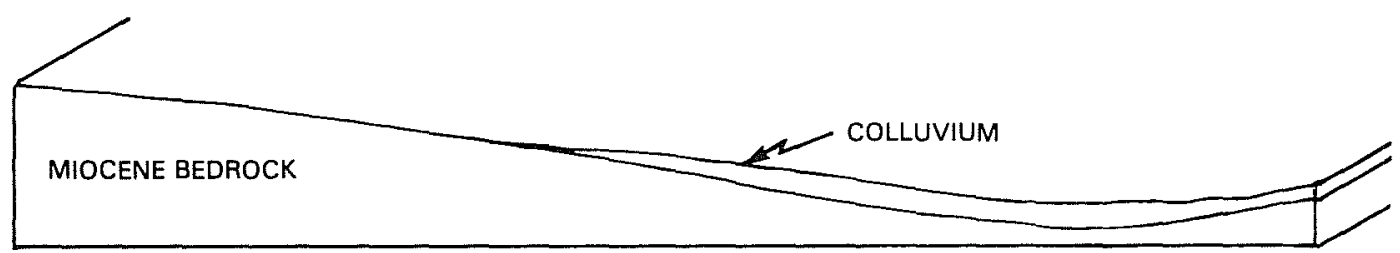

EARLY HOLOCENE - WEATHERING AND COLLUVIATION RESULT IN THICK COLLUVIAL MANTLE IN LOWER PORTIONS OF SLOPES

C

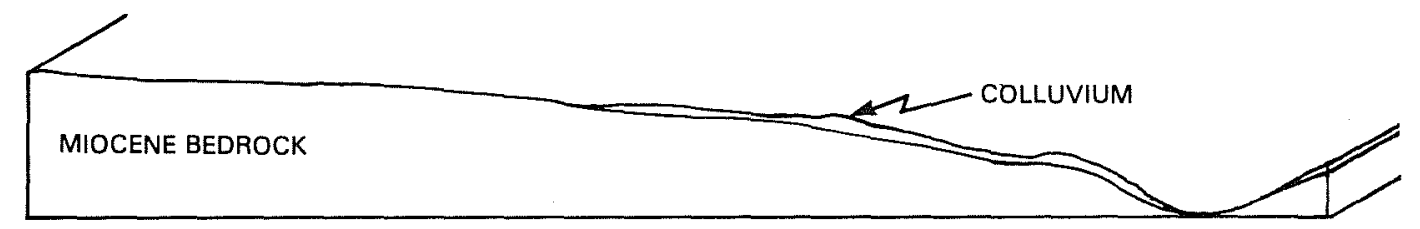

MID(?) - HOLOCENE - EROSION REMOVES MUCH OF COLLUVIAL MANTLE

d

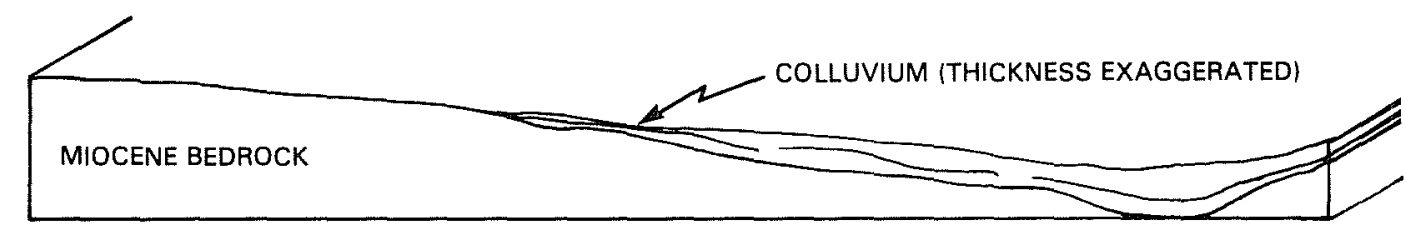

LATE HOLOCENE - INCREASED COLLUVIATION RE-COVERS HILLSIDE AND VALLEY BOTTOM WITH COLLUVIUM

$\boldsymbol{\theta}$

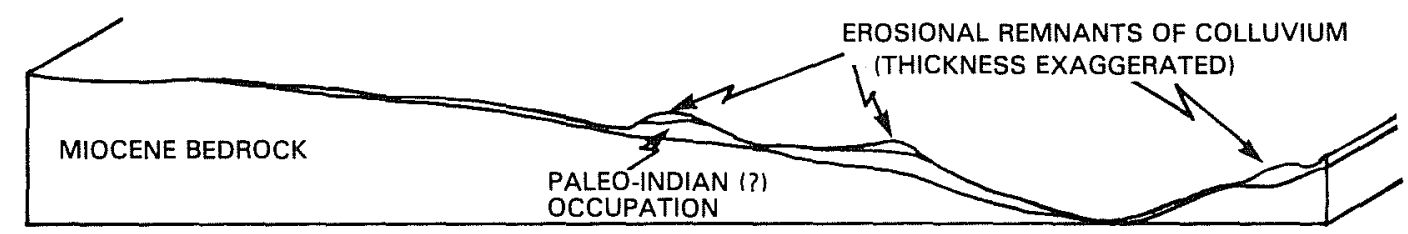

LATE HOLOCENE - EROSION REMOVES MOST OF COLLUVIUM

Figure 41. Geologic History of the Eagle Hill Site. 
north slopes were supporting a robust soil development, while the south slope was more prone to periods of intensive erosion. It would also be of interest to examine the pollen and biosilica profiles of the north and south slopes to determine the characteristics of the pre-European vegetation. Such studies could well throw light on the erosional characteristics of the vegetation regimes extant during the Prehistoric period. It is the judgement of Nials that the soil is less than 2500 years old and is therefore probably postHypsithermal.

The Crayfish Problem

While we were on the site from Apri1 16-21, 1980, we noticed that there were numerous crayfish (Orconectes limosus) castles on the site. Crayfish can cause considerable disruption to archaeological deposits, and Nials (personal communication) in another study of a site in southeastern Oklahoma found between five and 38 holes per square meter. A small biface was found to have been moved downward 45 to $60 \mathrm{~cm}$ in the section. While we were re-excavating Servello's test pits, we found one hole parallel to the wall all the way to the clay. When encountered, the crayfish was retreating into the clay bedrock.

The crayfish problem suggested an element of archaeological caution. For one, we should be cautious of far-reaching conclusions based on a few artifacts, since they could be vertically disturbed by crayfish. Since there are no flakes of any size on the surface, we might conclude that the crayfish do not create upward disturbance. We have some evidence that they move materials downward. Also, since the observed creature was moving presistently into the clay, there may be a reservoir of artifacts in the clay.

Sediments and Soils (Brown)

The soils at 16 SA 50 are indicative of a relatively complex pedologic development, one that is of critical importance to the archaeological remains buried at the site. The interaction of in situ pedogenic activity with cycles of erosion and deposition necessitated a preliminary evaluation of the archaeological significance of the strata recognized at the site. As pointed out in earlier sections, this consideration resulted in the development of an excavation technique, which combined natural, arbitrary, and cultural strata into a system that aided rapid and efficient data recovery from the site. The following section discusses soil development at 16 SA 50 and its relevance to aboriginal occupations at the site.

As noted in previous sections, the depositional history of the soils around the Eagle Hill II site centers around the movement of colluvial materials from higher positions on the ridge to more stable lower slopes, forming a surface layer of varying depth above the underlying Miocene clays and silt/sandstones. In addition to the fact that colluvial rates must have been quite variable through time, the soil picture is complicated by evidence that indicates major periods of erosion as we11, when unknown amounts of material were removed from the surface of previously formed soils. At least three and possibly four or 
more erosional events have truncated the main area of the site since the late Pleistocene, leaving remnants of the lower portions of previously developed soils.

The main focus of this discussion is the soil found in the central portion of Area A, tentatively classified as a Typic Fragiudult. A brief physical description of the typical soil profile is below. This is followed by a general discussion of the significance of these horizons and their distribution.

Soil Horizon A1, 0-12 cm. Grayish brown (10 YR 5/2), very fine sandy loam. Dark grayish brown (10 YR 4/2) moist, massive, soft, very friable. Few fine grass roots. Lower boundary generally clear, wavy, marked by many fine and medium faint to distinct mottles of material from the zone below, apparently as a result of root activity. This zone is marked by a slight humic accumulation. The color of this zone becomes gradually lighter from top to bottom (ca. 10 YR $4.5 / 2$ to 10 YR 5.5/2).

Soil Horizon A2, 12-44 cm. Very pale brown (10 YR 7/3), very fine sandy loam, (10 YR 7/4) moist, massive, slightly hard, very friable; common very fine and few fine roots. Moderate amount of smal1 (1-5 cm) manganese concretions; few very small strong brown iron oxide stains; some small root and rodent burrows $(2-10 \mathrm{~cm})$. Lower boundary clear, smooth, marked by many distinct fine and medium mottles of material from stratum below. Because of its light color and otherwise apparently unaltered nature, this zone is regarded as an A2 or albic horizon, although there is no incontrovertible evidence that it has been leached; it could possibly have been formed by colluvial deposition so rapid as to inhibit the addition of humus. As with the stratum above, there is a slight tendency to lighten in color with depth.

Soil Horizon B2, 44-60 cm. Brownish yellow (10 YR 6/6) very fine sandy loam, yellowish brown (10 YR 5/6) moist; weak medium crumb structure; slightly hard, very friable, nonsticky, not plastic; few very fine roots; rare tiny manganese concretions and iron stains; lower boundary gradual, wavy. The lower few centimeters of this stratum are transitional in nature with some mottling from the lower zone and a slight tendency to brittleness of the peds. These fragipanlike characteristics are contrasted to a marked increase in fine roots at the bottom, where they have turned horizontally in response to the more developed fragipan of the stratum below. In Figure 39 this horizon is divided into two subunits, B21 and B22. The lower of these two subunits is discontinuous and is marked primarily by a slightly higher clay content than in B21.

Soil Horizon IIB27tx, $62-70 \mathrm{~cm}$. Yellow (10 YR 7/6) very fine sandy loam, light yellowish brown (10 YR 6/4) moist; mottled with few, prominent fine red $(2.5$ YR $5 / 6$ ) iron stain mottles (and many very fine [0.5-2 mm] iron stain mottles) as well as common distinct medium mottles of light gray (10 YR 7/2) sand; weak, medium subangular blocky structure; slightly hard, very friable, nonplastic, nonsticky; very clear sand skins on some ped faces and in some smal1 vertical cracks; common, very fine roots following filled cracks; lower boundary clear, generally smooth. This stratum is not continuous across the area. Where it is recognized, it appears as transitional between Soil Horizon B2 and Soi1 Horizon IIB22tx below; where it does not occur, the transition between these two strata is more abrupt. 
Soil Horizon IIB22tx, 70-80 cm. Yellowish brown (10 YR 5/8) and light gray (10 YR 7/2) very fine sandy loam, dark yellowish brown $(10$ YR 4/6) and brown (10 YR 5/3) moist, mottled with red iron oxide (2.5 YR 4/8); moderate, medium to coarse subangular blocky structure; slightly hard, friable, very brittle, nonplastic, nonsticky; sand skins on ped faces and cracks; mottling is generally vertically oriented suggesting cracking or vertical piping; coarse hexagonal patterning shown is horizontal section, with sandier material lining hexagon boundaries; lower boundary clear, smooth. This zone is noticeably clayier than those above. Fragipan characteristics are best developed in this stratum. Mottling is more intense in this stratum than any other.

Soil Horizon IIB23t, $80-90 \mathrm{~cm}$. Light brownish gray (10 YR 6/2) sandy clay loam, same when moist, mottled with yellowish brown (10 YR 5/8) and red (2.5 YR 4/8); moderate coarse to very coarse subangular blocky structure; hard, firm, slightly plastic, slightly sticky; very few sand grains present on some ped faces, but no brittleness or other fragipan characteristics. Lower boundary clear to abrupt, slightly wavy, and marked by occasional smal1 jasper pebbles present as lag gravels on the erosional surface.

Soil Horizon IIIB2t, 90-102 cm. Light brownish gray (10 YR 6/2) clay, loam grayish brown (10 YR 5/2) moist, mottled with red $(2.5$ YR 4/6) medium to coarse prominent iron oxide mottles; strong, very coarse subangular blocky structure; very hard, very firm, plastic, sticky; lower boundary gradual, smooth.

Soil Horizon IIIC, $102 \mathrm{~cm}$ and below. Light brownish gray (10 YR 6/2) sandy clay loam, grayish brown (10 YR 6/2) moist; moderate, very coarse subangular blocky structure; very hard, very firm, plastic, sticky.

The lowest recognizable depositional unit at 16 SA 50 is a grayish clayey sediment (which includes both IIIC and IIIB2t) thought to date to the Miocene. Technically, a sandy clay loam (cf. Table 10), this sediment has a very silty clay feel, because of the near absence of coarse sand and the predominance of very fine sand. The upper 15 to $20 \mathrm{~cm}$ of this sediment (IIIB2t) has been pedogenically altered by partial oxidation (resulting in a deep red mottling) and the introduction of translocated clays (cf. Table 10) and coarse sand into the original Miocene matrix. This stratum is considered a moderately developed argillic B horizon.

At the top of this stratum is an erosional surface marked by jasper lag gravels and a distinctive change in the nature of the sand fraction. While pedogenic alteration of this unit may still be occurring, the lack of a clear transition and quite distinctive patterns of mottling suggest that the IIIB2t alteration was initiated prior to the truncation, which resulted in the erosional surface. The depth of this original soil or the time of its development is unknown.

During excavation this stratum was quite moist and plastic (though not easy to dig), but upon exposure to the air, it rapidly dried and began to show large peds (ca. $10 \mathrm{~cm}$ ) with moderate size cracks (up to $1 \mathrm{~cm}$ in width) between. This high shrink-swell potential could have potentially displaced artifacts under conditions of extreme drought, but little indication of such displacement was encountered during excavation. 
TABLE 10. PARTICLE SIZE DISTRIBUTION IN AREA A

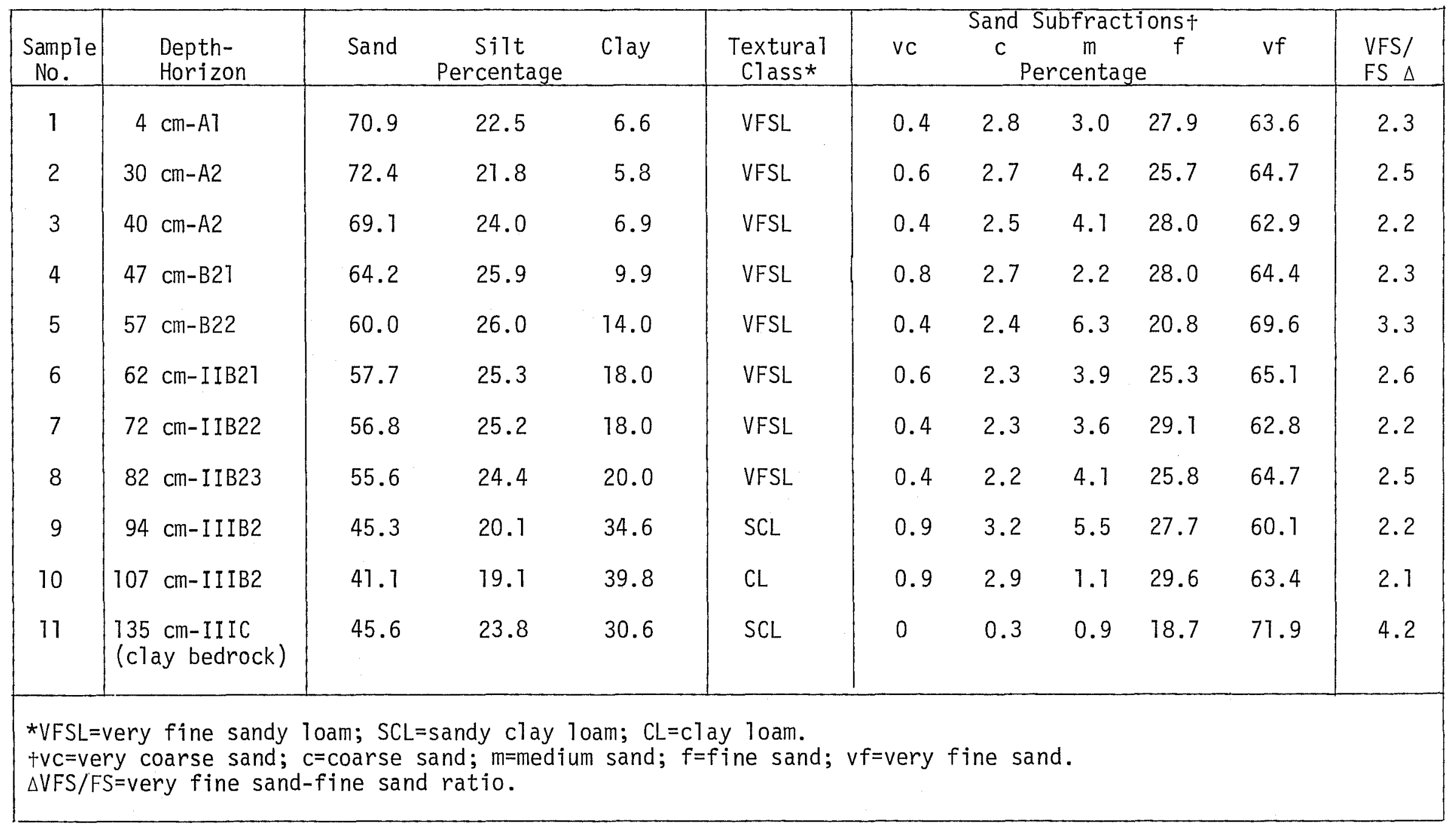


In addition to its vertic tendencies, this unit does have considerable cultural significance in that it probably was at or very close to the surface during the Paleo-Indian occupation of the site. This may have significantly altered groundwater hydrology during that time, causing relatively rapid runoff, but allowing some standing wet areas where depressions in the truncated clay held water at or just below the surface. As pointed out earlier, such a depression may have been responsible for the formation of the low rise that marks Area A.

Of some interest is the areal extent of the argillic modification of this Miocene clay. It is only found in the general area of the Area A mound. In other places Soil Horizon IIB2 directly overlies unaltered (or minimally altered) clay bedrock. This indicates either a localized soil development within the depression or extensive erosion of the surrounding areas. In either case, since the surface of the altered clay under the mound is lower than the surrounding unaltered clay, it suggests a slightly different drainage pattern at the time of Paleo-Indian occupation than at present.

While the Miocene sediments underlying the site probably resulted from a low energy fluvial or lacustrine depositional environment, all of the overlying depositional units seem to be of colluvial origin. The unit which immediately overlies the Miocene clay (which includes IIB23t, IIB22tx, and IIB21tx from bottom to top) shows the strongest pedogenic alteration of any unit, with intense multicolored mottling, translocated clay and sand, and fragipan development. As noted previously, the boundary between these two depositional units is an erosional surface.

Soil Horizon IIB2t $(x)$ contains the oldest in situ cultural material found at the site and is divided into three subunits based on texture and structure. The differences between these units are primarily pedogenic rather than depositional. Taken as a whole the unit is regarded as argillic, with marked structural development and the apparent presence of translocated clay. The lowest unit (IIB23t) contains the clay maxima and exhibits no fragipan development. Both of the upper two units show some fragipan development and generally differ only in the pattern and color of mottling. The uppermost stratum (IIB21tx) shows transitional characteristics and is partially discontinuous.

One of the most important questions at the site is the history of postdepositional disturbances to this critical strata. The upper portion of this unit is marked by the horizontal polygonal network commonly seen in fragipans. It seems likely that these are vertic cracks filled at an early stage in the development of the fragipan. The possibility of vertical displacement of artifacts within these cracks was recognized and carefully watched for during the excavation. Artifactual materials from this stratum were recovered almost exclusively from within the prisms.

In contrast to this potential disturbance, the presence of the fragipan itself indicates very little soil disturbance, since its development. The structure of the fragipan inhibits vertic movement and limits root penetration, except along prism faces. In this case, the few penetrations of the fragipan by large taproots were quite distinct and easily separated from the intact soil. 
The core series and soil tests conducted at the site indicate that this soil unit is more widely distributed at the site than the pedogenically altered bedrock. Despite this, its thickest and most highly developed segment is beneath the mound at Area $A$. In addition to its wider distribution, the erosional surface contours at its top more closely approach the modern surface contours, suggesting that microrelief began to approximate that of the present at some time prior to the end of the major erosional period, which resulted in its truncation.

The erosional surface that separates this unit from the overlying one is less distinct than the one above the Miocene clay, but is indicated by a number of lines of evidence, including both soils and cultural data. Soil evidence includes slight changes in the mineralogic composition of the sand fraction and a relatively high percentage of coarse sand and small pebbles near what is thought to be the erosional surface (cf. Lopez, page 150). Archaeological evidence includes the dense cultural substratum 3.12, which mixes apparent Archaic dart points with ceramic materials, and the wide ranging radiocarbon dates to either side of the surface.

Overlying the truncated argillic horizon (IIB2t[x]) is a relatively thick depositional unit that contains all of the ceramic remains at the site. Diagnostic soil horizons within this depositional unit include a thin, slightly humic Soil Horizon Al (note there is no plow zone) overlying a bleached Soil Horizon A2 or albic horizon. Together these form an ochric epipedon that overlies an altered subsurface horizon (B2), which might be best termed as cambic rather than argillic. Although there is a slightly higher clay content within Soil Horizon B2, the most distinctive feature is its deep gold color. This color appears to be the result of the liberation of limonitic iron minerals that have stained the very fine sandy matrix. The relatively low clay content within this stratum has apparently precluded the development of any strong structure; the reported crumb structure is so ephemeral that it could almost be regarded as massive.

Although the thicknesses of the individual units vary considerably, all of the horizons within this upper unit are widely distributed in the area surrounding the site, and the pedogenic alterations of these units may be much later than their colluvial deposition. The loosely compacted nature of this soil horizon and the absence of a strong structure make it easily penetrable by roots and rodents, as was observed during the excavation of the site. Fortunately, the distinctive colors of these three upper horizons make such bioturbation highly visible. The amount of bioturbation may be even greater than that observed, however, due to the possible late development of the pedogenic horizons. In general, some artifactual mixing must be expected from the upper levels.

There are slight indications of a possible truncation surface between Soit Horizons $A 2$ and $A 1$, based again on sand grain composition and the nature of the boundary itself. This is not clear, however, and because of its thinness, the $A 1$ zone has not been assigned to a different depositional unit even though it was treated separately during excavation. In any case, because of its nearness to the surface, care should be taken in the interpretation of artifactual materials from this zone. 
Site Catchment and Settlement Pattern (Gunn, Sheehan, and Garner)

The overwhelming topographic fact about the location of the Eagle Hill II site is that it is located on a relatively flat segment of Peason Ridge, a distintively upland habitat. Most of our inferences concerning the utility of the site must be drawn from reasonable assumptions about the purposes such a habitat can serve.

In spite of some severe difficulties, we thought that an effort should be made to familiarize ourselves with variation in the terrain and its possible implication toward resource availability. To do this, we chose to generate a photogammetric, geomorphical map (Fig. 36). Naturally the relative economy and speed of production were a factor, especially given the fact that no detailed Soil Conservation Service (SCS) soil maps were available. Also, the site is on the edge of a bombing and artillery impact range, and no access was permitted for ground observations. As can be seen in the map (Fig. 36), detail is quite precise, and we feel it provided a more than adequate analysis of exploitable microhabitats in the locale.

Thomas and Campbe11 (1978:66-95) conducted an extensive catchment analysis of the Whatley site (16 LA 37) about $100 \mathrm{~km}$ east of Eagle Hill. The following analysis generally conforms to the Whatley site design to provide comparable data on the two locations. Usually a site catchment analysis is conducted with the assumption that the site centered in the study area is a base from which resources are gathered. Given the remote location and apparent nonpermanent nature of the Eagle Hill I I site, the site catchment analys is can be alternatively viewed as a search for base areas from which individuals were likely to come.

The geomorphic mapping identified four major surface sediments/features in a five kilometer radius around the site (Fig. 42). The highest locations are topped by $\mathrm{Pleistocene} \mathrm{sands} \mathrm{(PS);} \mathrm{sand} \mathrm{bodies} \mathrm{that} \mathrm{are} \mathrm{relatively} \mathrm{erosion}$ resistent and which act as aquifers for the occasional springs at surprisingly high elevations along the ridge. Military vegetation maps (Fort Polk Terrain Analysis, Map G) indicate that these areas are marked by short grasses and sparse stands of evergreens. Historically, they were covered by large longleaf pines. It is not unlikely that during periods of high regional aboriginal population density, the area may have been as burned-off and relatively treeless as it is now. During dry summers, fires start easily by natural and human causes. We were privileged to fight one such fire during the time of our excavations.

Below the sands are fields of colluvial sediments. Compositionally they are not unlike the Pleistocene sands, since the higher sandy areas are, in large part, their source. Soil development supplies some clays. The colluvia accumulate in locales that are prone to deposition, but are sedimentologically active. The Eagle Hill site exists due to such an accumulation. The varied Holocene record of deposition and erosion at the site bears ample evidence of this sedimentological restlessness.

Miocene lake bed clays (M) are exposed at the surface of steeper slopes and generally the active erosional surface facies. The modern vegetation appears 


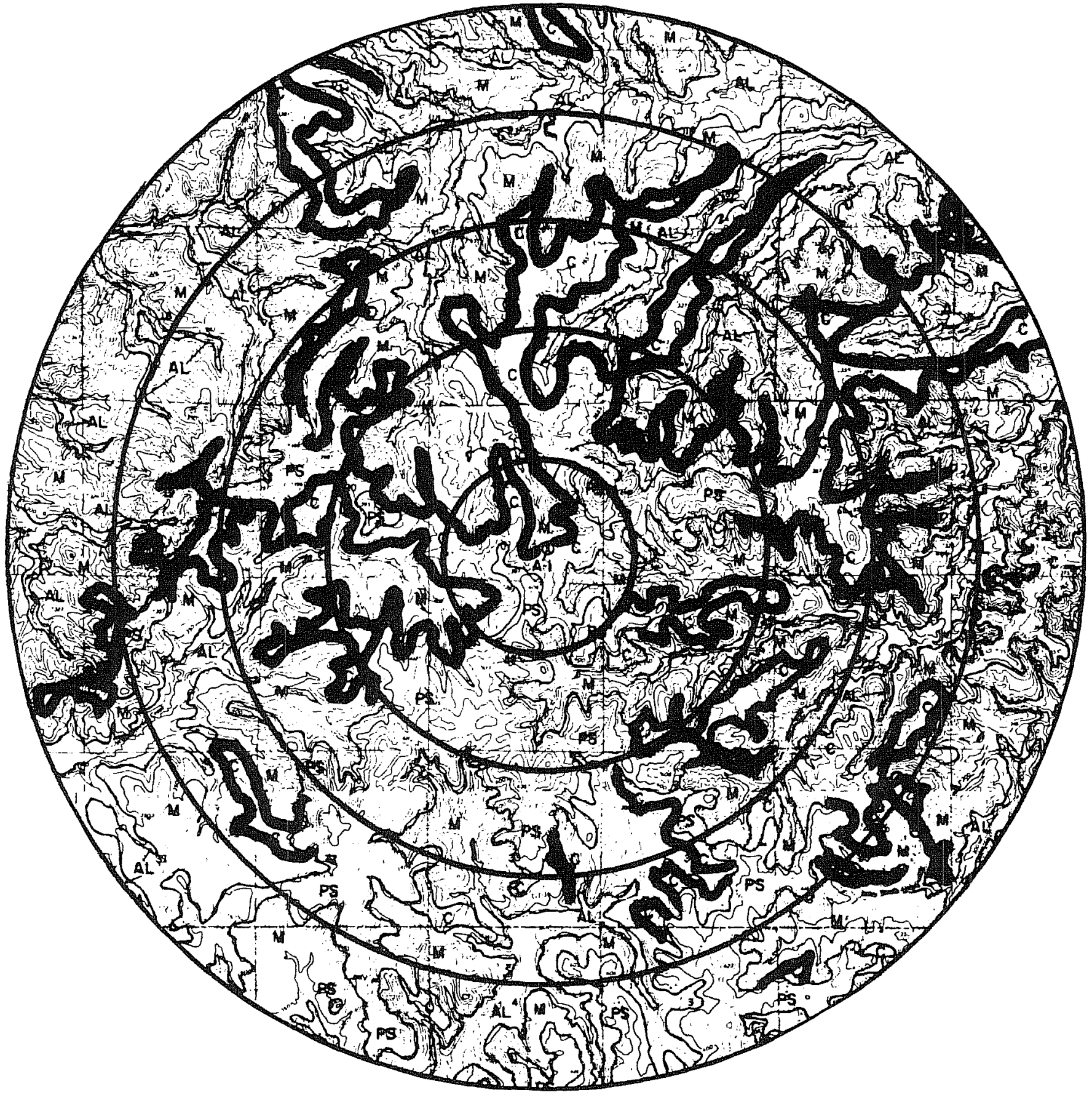

Figure 42. Eagle Hill Catchment (1-5 km Rings) and Colluvium-Clay Interface (Heavy Lines). 
to be represented by sparse to medium densities of evergreens and open grasslands in we11-drained areas. In less we11-drained areas, a deciduous component asserts itself. Finally, the alluvium is dominated by deciduous varieties.

Table 11 summarizes the surface sediment regime within five kilometers of the site. Little of the catchment is in Pleistocene sands (14\%) or alluvium (13\%). There is no alluvium in the immediate one kilometer of the site and what is there only reaches $20 \%$ of the landscape in the most distant concentric circle. Colluvium represents over half the land surface near the site, while Miocene clays appear a distant second at $26 \%$. The bottom totals for the row percentages are the expected values for the outward progression from ring to ring. Colluvium and sands are clearly overrepresented near the site. Clays occupy half of the catchment as a whole.

TABLE 11. SURFACE SEDIMENT TYPES IN THE EAGLE HILL CATCHMENT BY ONE KILOMETER CONCENTRIC RINGS

Sediment Type

Catchment Rings $(\mathrm{km})$

\begin{tabular}{lrrrrrr} 
& \multicolumn{7}{c}{1} & 2 & 3 & 4 & 5 & Total \\
\hline Area (mil1ions of square & meters) & & & & \\
PS & .62 & 2.27 & 2.84 & 2.36 & 2.83 & 10.91 \\
C & 1.69 & 2.88 & 4.85 & 4.69 & 2.84 & 16.95 \\
M & .83 & 4.15 & 7.09 & 11.56 & 16.79 & 40.42 \\
AL & .00 & .13 & .97 & 3.38 & 5.83 & 10.37
\end{tabular}

Totals
Row percentages

\begin{tabular}{lllllll} 
PS & .06 & .21 & .26 & .22 & .26 & 1.00 \\
M & .10 & .17 & .29 & .28 & .17 & 1.00 \\
AL & .02 & .10 & .18 & .29 & .42 & 1.00 \\
\hline Totals & .00 & .01 & .09 & .33 & .57 & 1.00 \\
\hline Column percentages & .04 & .12 & .20 & .28 & .36 & 1.00 \\
PS & .20 & .24 & .18 & .11 & .10 & .14 \\
C & .54 & .31 & .31 & .21 & .10 & .22 \\
M & .26 & .44 & .45 & .53 & .59 & .51 \\
AL & .00 & .01 & .06 & .15 & .21 & .13 \\
\hline Totals & 1.00 & 1.00 & 1.00 & 1.00 & 1.00 & 1.00 \\
\hline
\end{tabular}

Without archaeological information, the attraction of prehistoric peoples to Peason Ridge remains a matter of conjecture. However, the specific microhabitat of the site offers some possible explanations. The site is located midway 
between two large Pleistocene sand bodies and on the margin of the colluvium next to the Miocene clays. From the perspective of arboreal food sources, this location has several potential advantages.

The location between the sands is probably relevant to the nonproductive nature of the sandy areas. They would have been vegetated by longleaf pine, slash pine, shortleaf pine, turkey oak, and blackjack oak. The pines provide no foodstuffs, and the two oaks are of the black oak family, which has small, bitter acorns. Leaching their acorns for consumption would require quantities of running water.

The colluvium, however, probably provided a more interesting dietary selection. Although the upper reaches of the colluvium would have differed little from the sands at the lower edge of the colluvium, water would have seeped along the clays and surfaced at the colluvium-clay interface. We were able to observe this process in the early phase of the project during the spring rains. The colluvium-clay interface would have provided, in addition to moisture, the higher nutrient content of the clays. This ideal situation would have fostered post oak, which bear sma11, but very good acorns, hickories, and hickory nuts, and probably a rich understory of berries. Conditions of the clays would have been less favorable, because they do not act as an aquifer. The land would have been susceptible to extreme seasonal drying.

The Eagle Hill site, therefore, offers the optimal location for harvesting along the colluvium-clay interface between the sands. The vegetation-colluvium-clay system also provides a ready explanation for the apparent limiting of occupation activity to moist sedimentation periods. During long dry summers, the sand and colluvial aquifers would dry up and restrain the productivity of the colluviumclay interface.

This hypothesis could best be tested by determining if sites located on Peason Ridge are frequently associated with similar situations. The highest potential areas are marked by a heavy line in Figure 42.

\section{F. HIGH RESOLUTION ENVIRONMENTAL COLUMN (Gunn)}

Constant volume sediment samples (2000 cc taken from the southwest corner of each one-meter square in each occupation plane) were designed to sample the horizontal distribution of phenomena on occupation floors. However, since our excavation design called for removal of only selected occupation floors at one= centimeter precision, the constant volume samples do not constitute a continuous vertical sample of soils from top to bottom of the site.

In order to insure a column of samples from top to bottom, we collected what we call a High Resolution Environmental Column (HREC). The HREC column was collected in arbitrary one-centimeter levels from the surface down to the Miocene clay (P1ate 7). On occasion when arbitrary levels crosscut obvious natural levels, the sediments from the two levels were bagged separately. The HREC column was collected from the south half, $50 \times 100 \mathrm{~cm}$, of excavation unit E3019 N7002 and, therefore, consisted of about $5000 \mathrm{cc}$ per sample. This figure is reduced somewhat by the removal of a $1 \times 10 \times 10 \mathrm{~cm}$ sample from the southwest corner of each unit for pollen/biosilica analysis. Since the site is about one meter deep, the HREC column produced 94 such samples. 


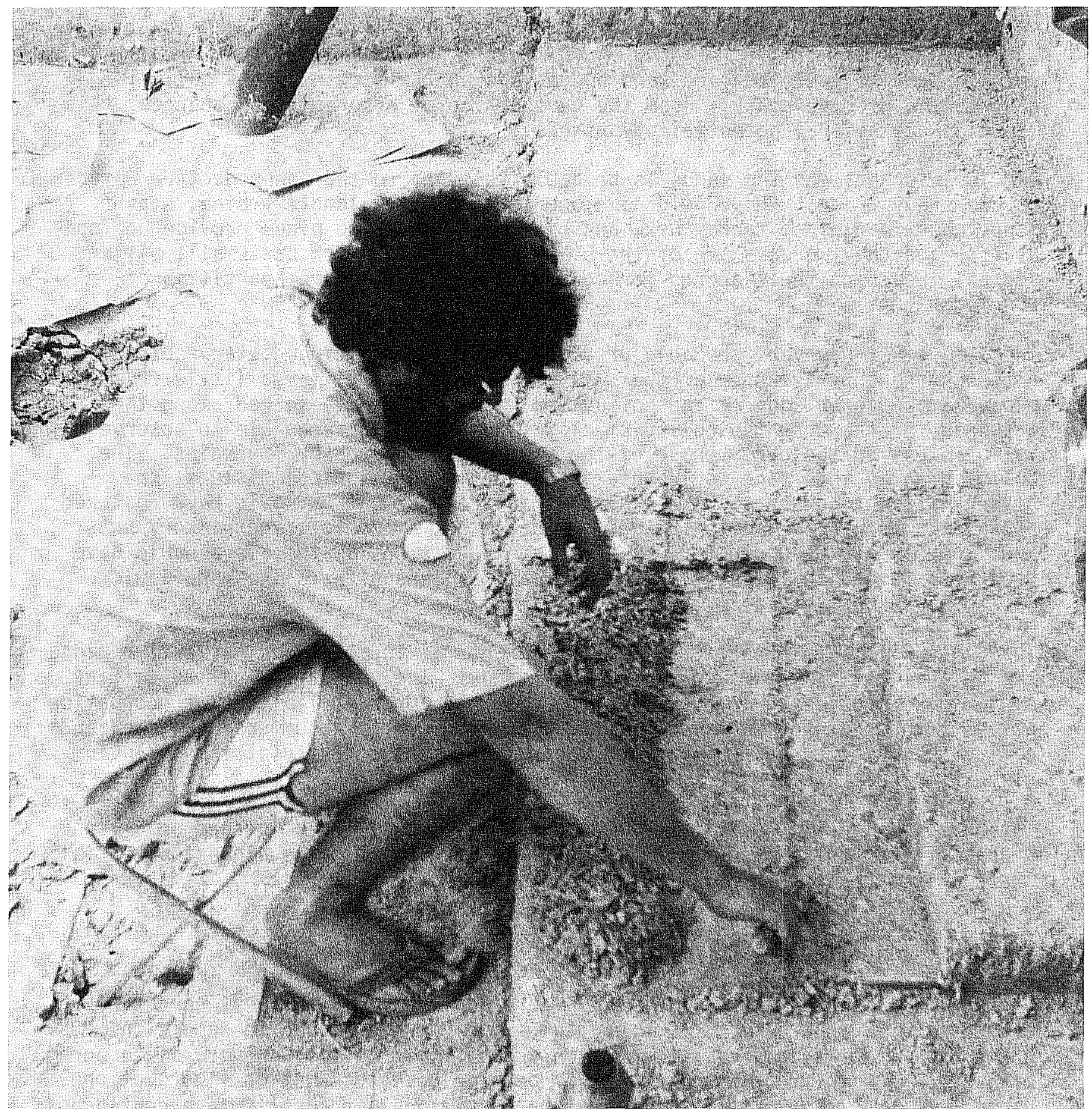

Plate 7. Excavation of the High Resolution Environmental Column (HREC). 
The HREC provided a sample large enough for the study of microfaunal remains, grain size analysis, geochemical analysis, pollen analysis, biosilica analysis, etc. The logic behind collecting the samples in one centimeter arbitrary levels was to collect samples at such a refined level that once in the laboratory, data from the samples could be combined at whatever level of resolution desired.

\section{Fine-Grained (Sand) Sediment Analysis (Van Note)}

Examination of HREC column sediments in the Eagle Hill site was undertaken to discover information about depositional environment (soil composition, types and possible sources of parent materials, and distance and means of transportation to the site). The geological situation of the site indicates that sediments may have been eroded from hills to the southwest and northeast of the site, or deposited by wind. These sediments have been subsequently altered by plant, animal, human, and pedogenic activity. Analysis consisted of studies of size, material type, and angularity.

\section{Particle Size Analysis}

Seven sediment samples from the HREC column corresponding to substrata 1.13, $2.13,3.114 .12,4.15,4.16$, and 4.17 were processed through three screens to separate particles according to size and to facilitate material type and angularity studies of the fraction most likely to provide specified environmental evidence.

Procedure--Each sample was weighed, put into $500 \mathrm{ml}$ of water, and poured through a stack of three screens $(1.70 \mathrm{~mm}$ mesh, $0.50 \mathrm{~mm}$ mesh, and $0.15 \mathrm{~mm}$ mesh). An additional $500 \mathrm{ml}$ of water was added. The material in each screen was then washed by recycling the water until clean, tapped off the screen, dried, and bagged. Particles remaining in the water were allowed to settle ten to fifteen minutes and placed in an evaporating dish. Each fraction was subsequently weighed.

Results--No information on level 1.13 is available, because the original weight of the sample was not recorded. However, there appears to be only slight variance with substratum in the percentage of the original weight of the sample which accumulated in each fraction (Fig. 43). The large fraction (all materials larger than $1.7 \mathrm{~mm}$ in diameter) occupies on $1 \mathrm{y} 0.1 \%$ to $0.2 \%$ of the original weight, with the exception of substratum 4.16 where a pebble was noted. The 0.50 fraction (particles between $0.50 \mathrm{~mm}$ and $1.70 \mathrm{~mm}$ in diameter) remains fairly constant, varying from $1 \%$ in levels 4.12 and 4.15 to $1.6 \%$ in level 2.13 . There is less than $5 \%$ difference in weights with respect to the 0.15 fraction (particles between $0.15 \mathrm{~mm}$ and $0.50 \mathrm{~mm}$ ) with the largest percentages found in the two lowest levels. The percentage of weight that accumulated in the evaporating dishes, the small fraction, is fairly constant in the three uppermost levels, occupying between $74 \%$ and $76 \%$ of the total weight, but decreases in the three lower levels where on $1 y 56 \%$ to $63 \%$ of the total weight appears. 

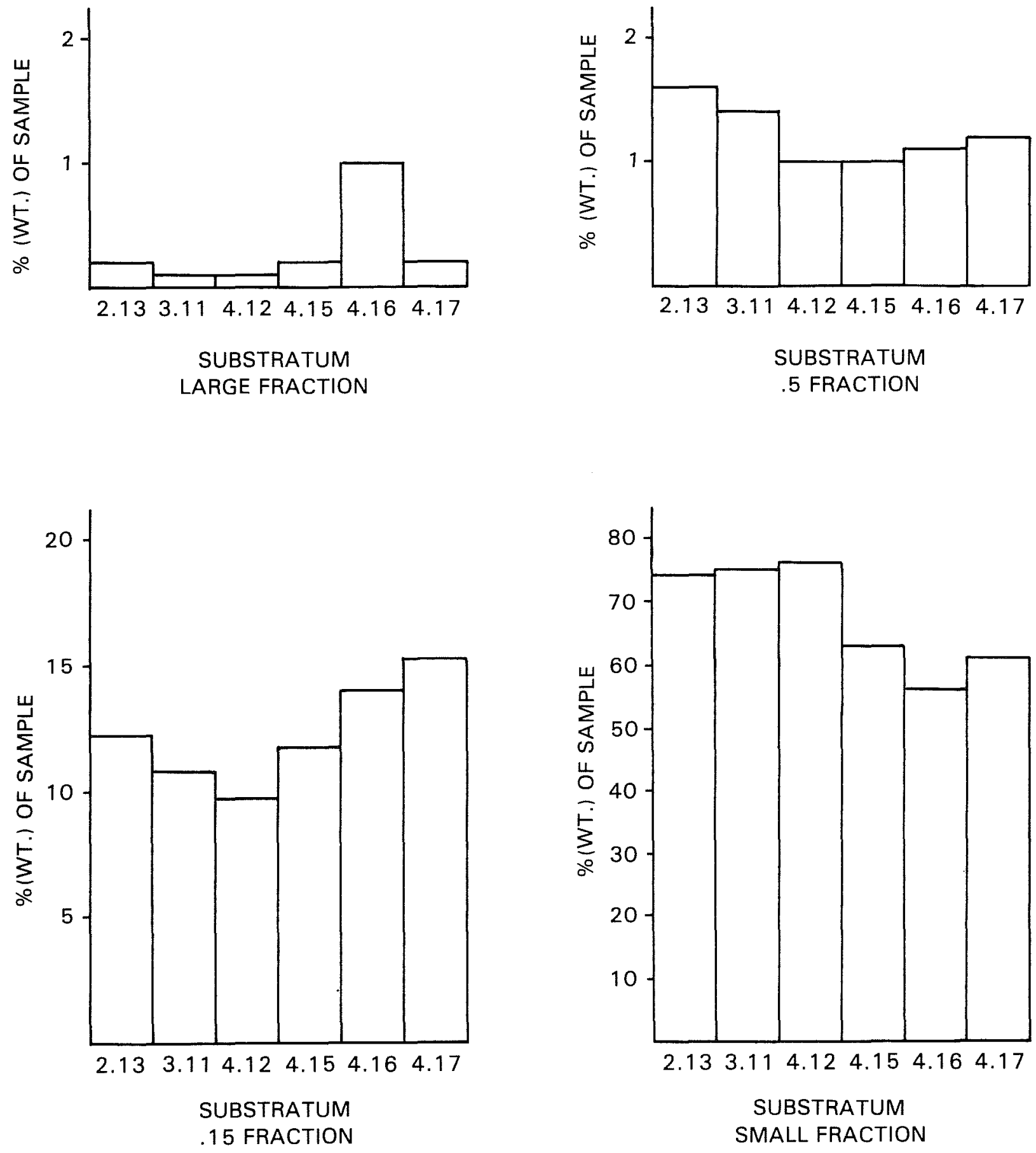

Figure 43. Percentage of Original weight According to Fraction. 
Observations--The most significant differences appear in the small fraction. A comparison of Krumbein and Sloss' (1963) scale of particle sizes with the screen sizes used shows which types of particles accumulated in each fraction (Table 12). The large fraction is composed of very coarse sands and very small pebbles. The 0.50 fraction is made up of medium to coarse sands while the 0.15 fraction encompasses the very fine to fine sand particles. The small fraction would contain some very fine sand as well as medium- to coarse-sized silts. The clay-sized particles were discarded with the water since the time was short (Table 13). The settling time that was allowed during the screening process would have allowed most of the silts to settle, but the finer silts were lost as well as the coarse clay that would have required several hours of settling time.

The variance between upper and lower substrata of weights of the sma11 or siltsized fraction may be an important environmental indicator. Cornwal1 (1958) indicates that sediments composed of wind-blown dust are made up largely of silt particles (he suggests $70 \%$ as typical) between $0.002 \mathrm{~mm}$ and $0.06 \mathrm{~mm}$ in diameter. Cornwall further indicates that clay-sized particles (less than $0.002 \mathrm{~mm}$ in diameter) are widely dispersed by winds and lost as an entity, but silts are heavy enough to settle fairly rapidly and thus are important as a wind-blown sediment. This rapid settling would be important to the depositionvegetation mechanism which is suggested to be the cause of the Area $A$ mound. The fact that the fourth fraction of the three upper levels is composed of $10 \%$ to $20 \%$ more of the total weight may indicate that wind-blown sediments were a more important factor in deposition in the uppermost levels than in the lower substrata. The rapid deposition rates during the OP 3.11-OP 2.13 interval were no doubt assisted by an open forest parkland (see section $\mathrm{H}$ ), which allowed aeolian movement of silt-sized particles during certain seasons of the year.

\section{Material Type and Angularity}

Particles from the 0.50 fraction $(0.50 \mathrm{~mm}$ to $1.70 \mathrm{~mm}$ in diameter) were examined microscopically in order to study material types and angularity. This particular particle size was chosen for study, because it might yield further environmental indicators, such as rodent teeth.

Procedure--The second fraction of each sample was subjected to a 100-grain count analysis. Each grain was classified by material type and angularity. Quartz was classified as clear, white, yellow, rose or smoky. Other classifications include charcoal, hematite concretions, limonite concretions, quartzite, conglomerates of calcite and quartz, chalcedony, burned resin, seeds, snails, and teeth. Angle types were recorded as angular or subangular. Data were recorded and subjected to principal components analysis.

Results and Observations--Quartz was the dominant material type (Fig. 44) with the clear and yellow varieties most common. Rose quartz was more common in the lower levels, possibly indicating that the source rocks were greatly eroded by the time of occupation of stratum 2.13. This might be confirmed by examination 
TABLE 12. PARTICLE SIZE SCALE

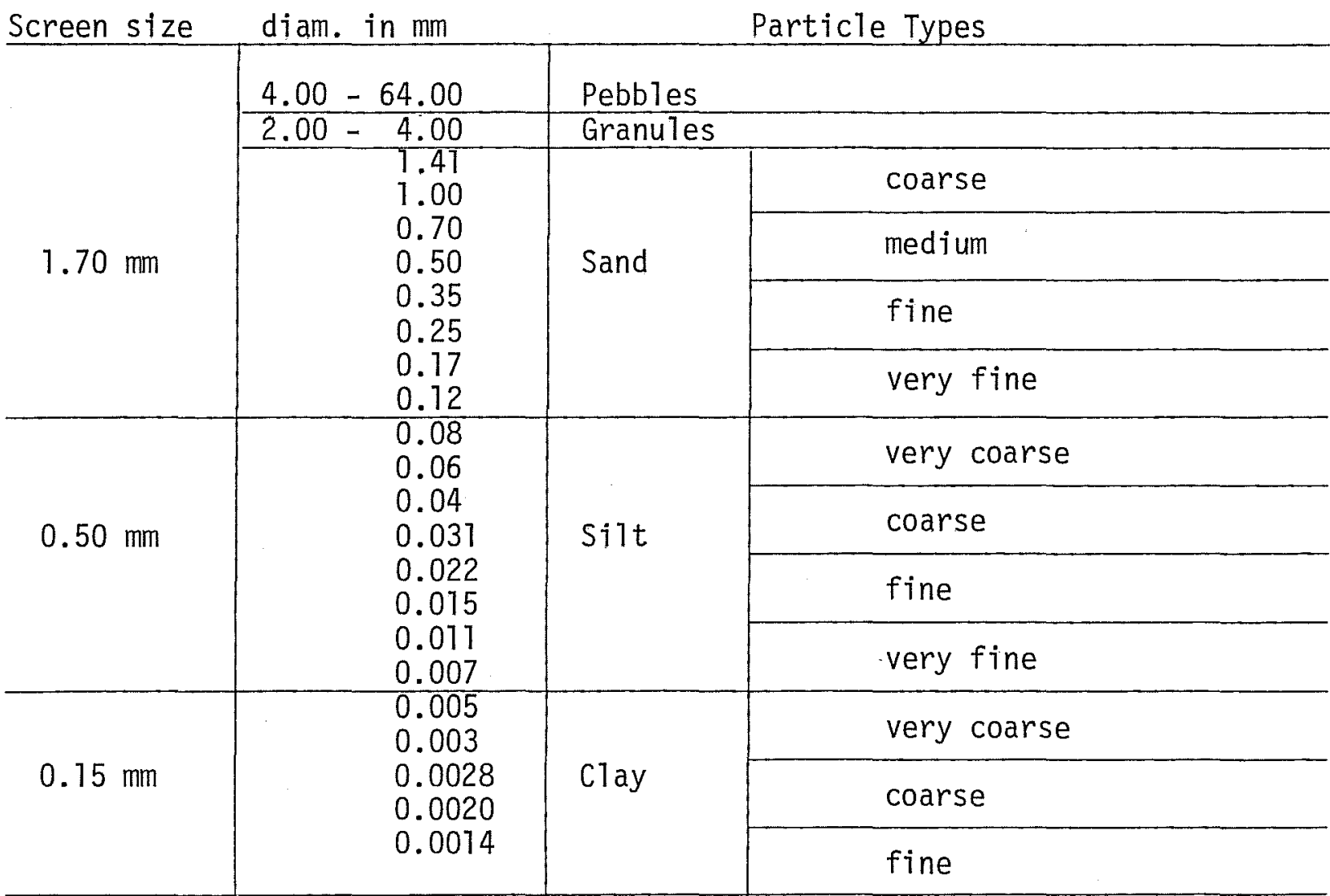

NOTE: A comparison of screen sizes used and Krumbein's scales as presented in Shackley (1975).

TABLE 13. PARTICLE SETTLING TIMES

Particle size (microns)

62.5

44.2

31.2

22.1

15.6

11.0

7.8

5.5

3.9

2.8

1.95

1.40

0.98
Withdrawal depth (cm)

20

20

10

10

10

10

10

10

10

10

10

10

10
Time hours minutes seconds

58

56

56

52

42

7

15

31

1

4

8

16

32

0

0

0

0

0

0

0 

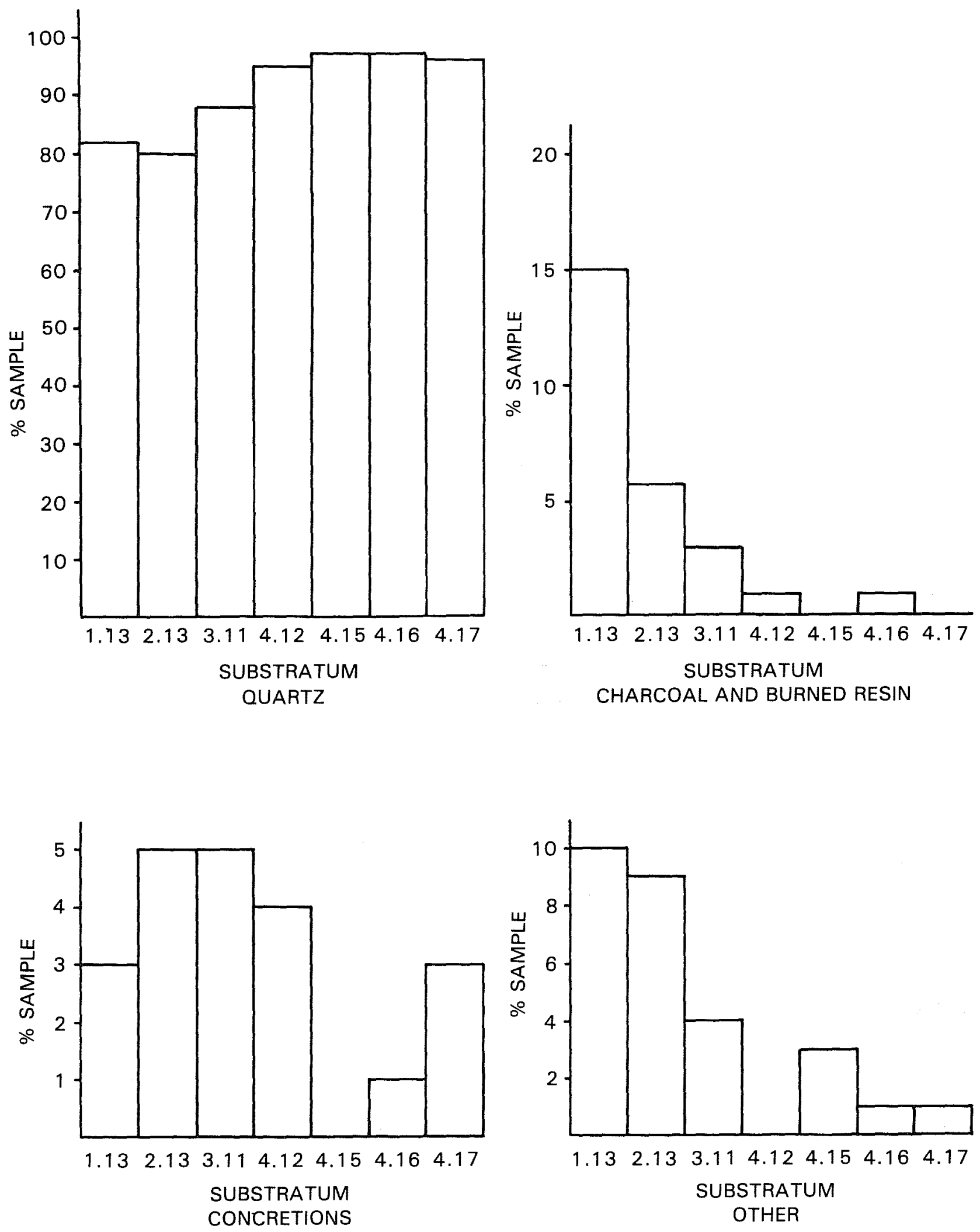

Figure 44. Percentage of Material Type According to Level. 
of the hillside cores taken near the site. Charcoal is quite common in the uppermost levels, but becomes almost nonexistent in the lower levels. This is probably due to destruction by soil action and by highly acid water percolating through the soil. Concretions were present throughout the column, but hematite concretions disappear in the lower levels. In fact, the lower the substratum, the stronger the tendency for the fraction to be composed entirely of quartz. Seeds, although not always present in the grain count, were found in a11 but the lowest levels and were separated from the matrix. One snail was found in OP 1.13. There was a possible tooth fragment in OP 3.11 .

Particles were found to be predominantly subangular (Fig. 45), but certain levels, notably OP 2.13 and 4.12 , showed a marked increase in instances of angularity. It may indicate the close proximity of certain source rocks or more rapid burial of sediments, thus allowing a shorter time span for transportation and/or weathering of particles.

Principal components analysis confirmed some of these observations. An examination of correlation coefficients by substratum reveals a strong positive correlation with subangular clear quartz, subangular yellow quartz, and both angular and subangular rose quartz, indicating the tendency of these components to increase toward the bottom of the column. On the other hand, a tendency to decrease in number toward the bottom of the column is shown by the strong negative correlations of angular clear quartz, both types of white quartz, smoky quartz, charcoal, hematite, quartzite, conglomerates, chalcedony, burned resin, and seeds. The hardness of quartz and its tendency to weather extremely slowly, compared to the other components of the fraction, would explain this market decrease in all components other than quartz.

\section{Coarse-Grained (Pebble) Sediments (Lopez)}

Coarse-grained sediments generally in the pebble range $(4.0 \mathrm{~mm}$ to $64 \mathrm{~mm}$ in diameter) were recovered from 1/8-inch screens. Determinations were made of material types, angularity, and size frequency of pebbles from Occupation Planes $1.13,2.13,3.11,4.12,4.15,4.16$, and 4.17 . The analysis was intended to discover changes in source area from stratum to stratum, locate deflation surfaces, and discover stones that may have been brought to the site by human occupants.

Two analyses were performed. In the first, a column of squares was taken from E3017 N999 and analyzed in detail. The second analysis consists of weighing pebbles from all units of occupation.

Table 14 shows the frequencies of pebbles from the column. An increase in size and range of sizes is evident in $O P 3.11$ and below: The predominant sizes range from $5 \mathrm{~mm}$ to 8 or $10 \mathrm{~mm}$ in all levels. However, there is a marked increase in the number of sizes in the 6 to $10 \mathrm{~mm}$ range in levels 3.11 through 4.17 . This supports the presumption that materials were transported to the site from different source areas and by different means. These are geomorphical (i.e., sheet erosion, gully erosion, etc.,) and by animals (especially birds, gizzard stones). The consistent presence of sizes 5 to $8 \mathrm{~m}$ argues for land movements, soil accretion, and hydrolic activities being the responsible agents. Inconsistencies 


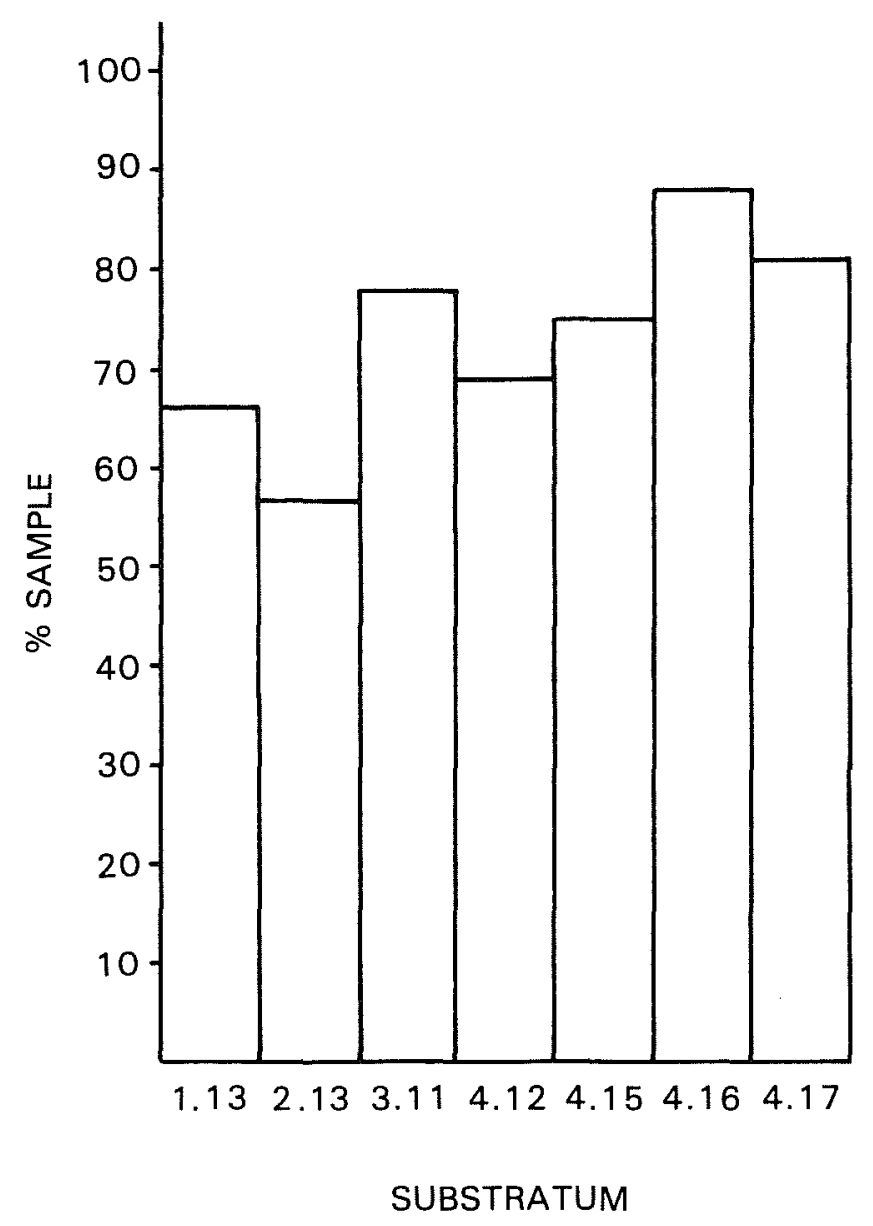

Figure 45. Percentage of Subangular particles. 
in other categories suggested other native agents of transport from upslope. The fact that Eagle Hill is in a saddle allows colluvial deposition from either direction upslope. Pedological evidence suggests that the site was originally located at or near the bottom of the slope on the east side of the saddle. Eventually, colluvium from the other slope overrode the lower levels. Consequently, pebbles larger than $5-8 \mathrm{~m}$ in the lower levels may have had their source to the east. Unfortunately, the source sediments by which this hypothesis could be tested have since been eroded.

TABLE 14. PEBBLE COUNTS BY OCCUPATION PLANE

\begin{tabular}{|c|c|c|c|c|c|c|c|c|}
\hline Size $(\mathrm{m})$ & $\frac{\text { Occup }}{4.17}$ & $\frac{i o n}{4.16}$ & 4.15 & 4.12 & 3.17 & 2.13 & 7.13 & Totals \\
\hline $\begin{array}{r}4.0-4.9 \\
5.0-5.9 \\
6.0-7.9 \\
8.0-9.9 \\
10.0-11.9 \\
12.0-13.9 \\
14.0-17.9 \\
18.0-21.9 \\
22.0-25.9 \\
26.0\end{array}$ & $\begin{array}{l}5 \\
54 \\
74 * \\
27 \\
19 \\
10 \\
7 \\
2 \\
1 \\
0\end{array}$ & $\begin{array}{l}20 \\
53^{*} \\
36 \\
29 \\
19 \\
9 \\
5 \\
1 \\
0 \\
0\end{array}$ & $\begin{array}{c}7 \\
47^{*} \\
48^{*} \\
5 \\
9 * \\
4 \\
3 \\
0 \\
1 \\
0\end{array}$ & $\begin{array}{l}10 \\
18^{*} \\
17^{*} \\
6 \\
9 * \\
6 \\
6 \\
1 \\
0 \\
0\end{array}$ & $\begin{array}{l}18 \\
44 \\
51^{*} \\
13 \\
23^{\star} \\
7 \\
10 \\
13^{*} \\
1 \\
3^{*}\end{array}$ & $\begin{array}{c}5 \\
20^{*} \\
19 * \\
4 \\
5 \\
3 \\
4 \\
0 \\
0 \\
0\end{array}$ & $\begin{array}{c}0 \\
14 \\
26^{\star} \\
9 \\
11 \\
3 \\
0 \\
0 \\
0 \\
0\end{array}$ & $\begin{array}{r}65 \\
250 \\
271 \\
93 \\
95 \\
42 \\
35 \\
17 \\
3 \\
3\end{array}$ \\
\hline Totals & 199 & 172 & 124 & 73 & 183 & 60 & 63 & 874 \\
\hline
\end{tabular}

Note that pebble sizes greater than 18 microns are nearly absent from all levels except OP 3.11 , possibly indicating human transport to the site. OP 3.11 has the greatest frequency of cultural material in the strata except OP 2.13.

There are differences in the frequency of pebbles from level to level (Fig. 46). By far, level 4.17 has the most pebbles per unit area. OP 3.11 ranks a notable second, especially given the trend throughout the section. Both of these levels were identified in the field as being on or near erosion surfaces. Increased frequency of pebbles may well be due to deflation.

Figure 47 summarizes pebble weights for each occupation plane by one meter squares. Weight ranges are approximate and reflect the average occupation area per level. The weights are recorded in grams, and ranges are set at 0-10, 10$25,25-50$, and over 50 grams. The weights recorded show a remarkable differentiation in weight distribution over each occupation plane. Pebble weight is indicative of at least two factors: those pebbles brought in by humans and the different natural source areas. In theory, a careful study of weight distributions may also indicate how far source areas were from the site.

Table 15 describes the pebble materials in each level. These materials include rose quartz, smoky quartz, clear quartz, white quartz, yellow quartz, 


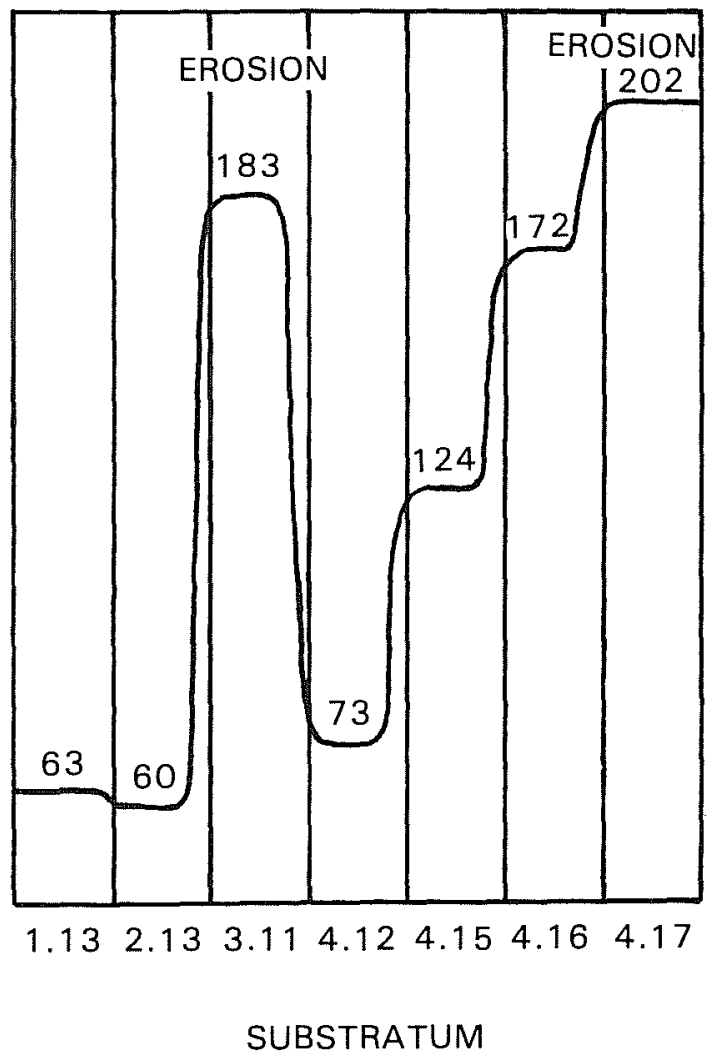

Figure 46. Concentrations of Pebbles by count. 


\section{EAGLE HILL (16SA50)}

Occupation Plane Analysis - 1m Study

TOPIC: PEBBLES

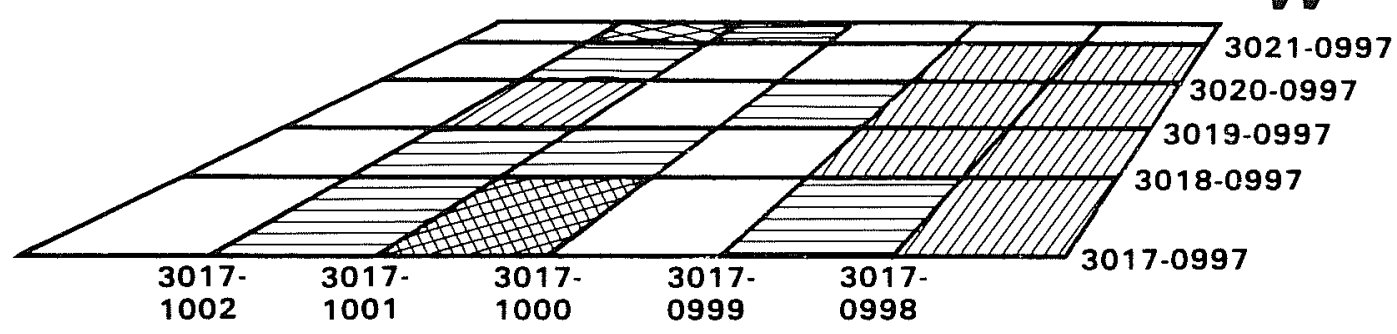

1.13

cupation

Plane

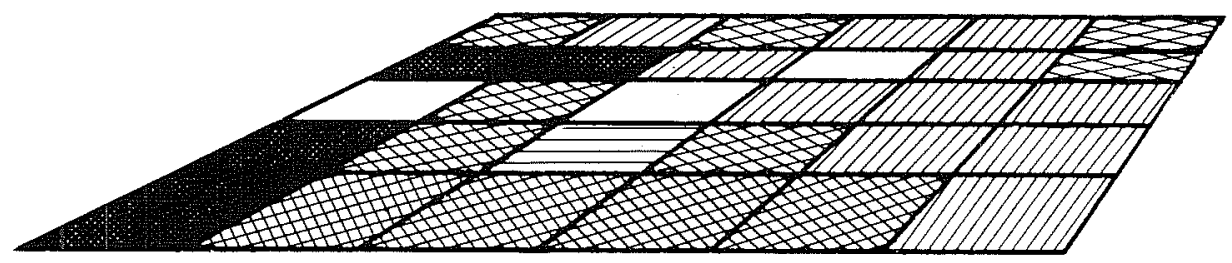

3.11

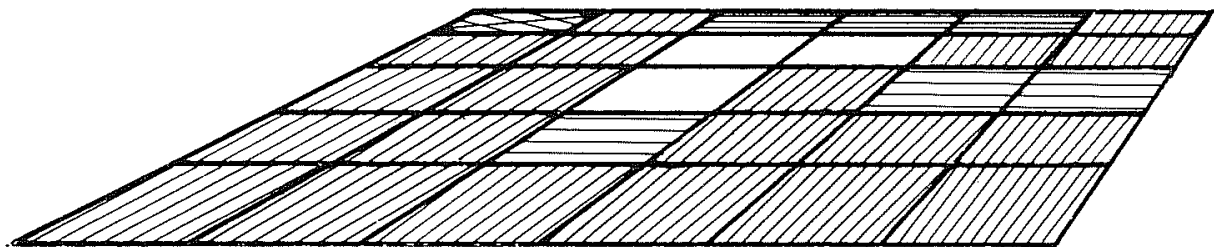

4.13

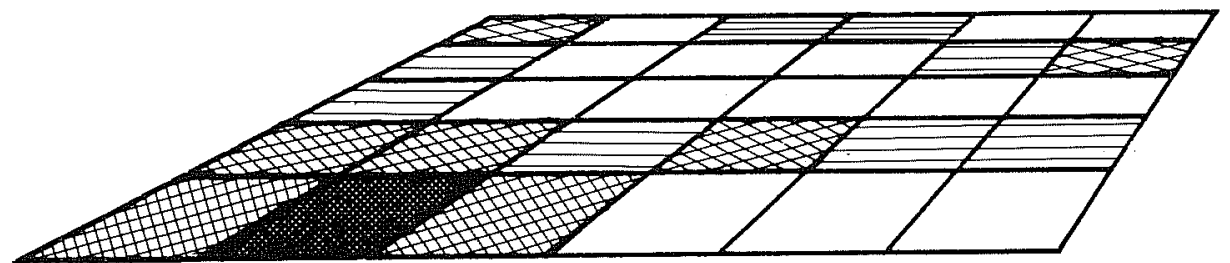

4.15

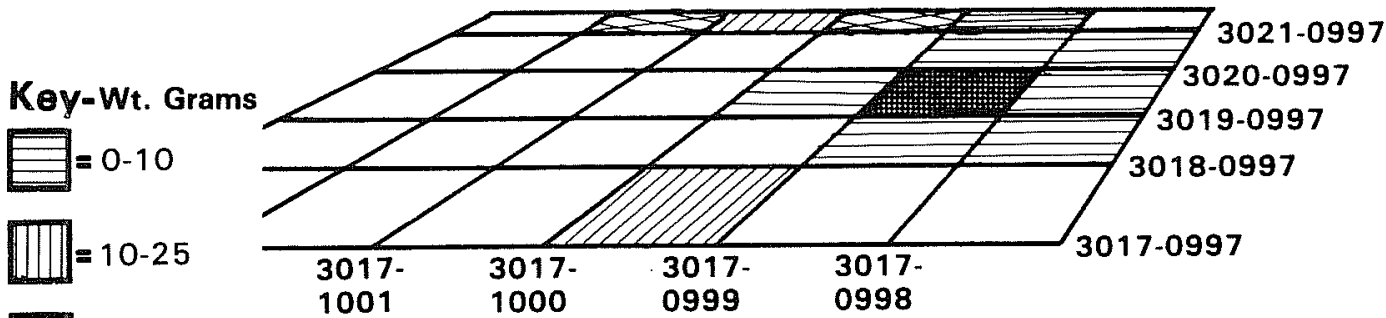

4.16

$=25-50$

= OVER 50

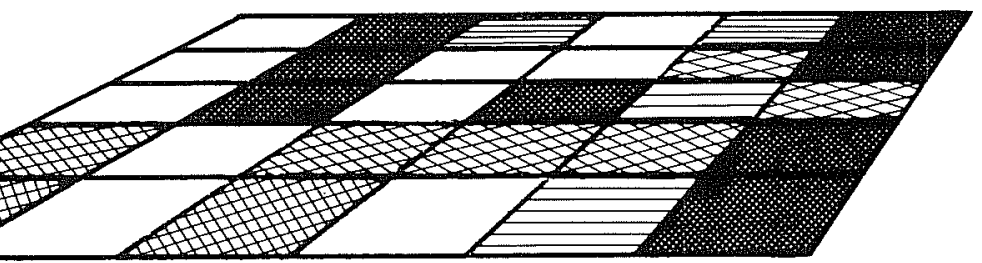

Figure 47. Horizontal Distribution of Pebbles in Excavation. 
limestone, sandstone, flint, concretions, shale, and chert. There was a high frequency of rose quartz, limestone, and stones that are noted as shale (smooth, shiny, fine-grained pebbles usually red, gray, and black). The evidence suggests that the rose quartz, the limestone (most abundant), and the shales are the most common pebbles found at the site. Limestone consists primarily of calcite. Some of the sediment on the Miocene lake bed may have been in an early stage of formation (Flint and Skinner 1974:A-32). The predominant texture of the limestone in our samples was coarse-grained through crystallization of the calcium carbonate elements or because they consisted largely of detrital shell fragments (Flint and Skinner 1974).

Table 15 also shows the frequency of material types in the total column. The deeper levels contain more types of pebble material. Apparently the earlier source area contained more variety of material.

\section{TABLE 15. PEBBLE FREQUENCY AT EAGLE HILL}

\begin{tabular}{|c|c|c|c|c|c|c|c|c|}
\hline Material & $\begin{array}{c}\text { Substrat } \\
4.17\end{array}$ & 4.16 & 4.15 & 4.12 & 3.11 & 2.13 & 1.13 & Total \\
\hline $\begin{array}{l}\text { Rose Quartz } \\
\text { Smoky Quartz } \\
\text { Clear Quartz } \\
\text { White Quartz } \\
\text { Yellow Quartz } \\
\text { Limestone } \\
\text { Sandstone } \\
\text { Flint } \\
\text { Concretions } \\
\text { Shales } \\
\text { Chert }\end{array}$ & $\begin{array}{r}21.8 \% \\
9.4 \% \\
2.0 \% \\
18.3 \% \\
9.9 \% \\
11.4 \% \\
0 \% \\
0 \% \\
6.9 \% \\
20.3 \% \\
0 \%\end{array}$ & $\begin{array}{r}9.9 \% \\
6.4 \% \\
2.3 \% \\
7.6 \% \\
7.6 \% \\
24.4 \% \\
0 \% \\
.6 \% \\
18.0 \% \\
23.3 \% \\
0 \%\end{array}$ & $\begin{array}{r}15.3 \% \\
5.6 \% \\
4.0 \% \\
5.6 \% \\
28.2 \% \\
16.9 \% \\
1.6 \% \\
0 \% \\
5.6 \% \\
13.7 \% \\
3.2 \%\end{array}$ & $\begin{array}{r}10.9 \% \\
4.1 \% \\
1.4 \% \\
5.5 \% \\
6.8 \% \\
20.5 \% \\
6.8 \% \\
8.2 \% \\
1.4 \% \\
21.9 \% \\
12.3 \%\end{array}$ & $\begin{array}{r}3.8 \% \\
1.0 \% \\
.5 \% \\
1.6 \% \\
2.7 \% \\
37.7 \% \\
1.0 \% \\
2.1 \% \\
17.4 \% \\
31.7 \% \\
0 \%\end{array}$ & $\begin{array}{r}13.3 \% \\
3.3 \% \\
1.7 \% \\
8.3 \% \\
5.0 \% \\
41.7 \% \\
0 \% \\
0 \% \\
3.3 \% \\
23.3 \% \\
0 \%\end{array}$ & $\begin{array}{r}30.2 \% \\
19.0 \% \\
3.2 \% \\
14.3 \% \\
9.5 \% \\
12.7 \% \\
0 \% \\
0 \% \% \\
0 \% \\
11.1 \% \\
0 \%\end{array}$ & $\begin{array}{r}122 \\
56 \\
18 \\
78 \\
87 \\
203 \\
9 \\
11 \\
87 \\
193 \\
13\end{array}$ \\
\hline TOTAL $(n)$ & 202 & 172 & 124 & 73 & 183 & 60 & 63 & 877 \\
\hline TOTAL $(\%$ co 1 & $23.0 \%$ & $19.6 \%$ & $14.1 \%$ & $8.3 \%$ & $27.0 \%$ & $6.8 \%$ & $7.2 \%$ & \\
\hline
\end{tabular}

Table 16 summarizes the angularity of pebbles per level. The predominance of sharp pebbles indicates a relatively static erosive environment at Eagle Hill. There are few smooth pebbles in all of the seven substrata. Stream and riverine activity can account for uniform wearing away; however, given the sparse numbers (23) of smooth pebbles, it seems likely that the stones were worn smooth in the gizzards of birds. Some of the smoothed stones are unusually large and may have been imported by humans.

The results of this analysis indicate that colluvial deposition was active in the period of OP 3.11 and OP 4.17. As Figure 46 shows, there has been a general decline in the ability of the transporting agents to bring quantities of heavy materials into the site through time. This general trend is punctuated by a reversal during the OP 3.11 period. It is not entirely clear, but human 
activity may have played some part in the OP 3.11 pebble concentration. However, the nature of the evidence suggests a large natural component to the OP 3.11 reversal and pertains to the erosion surface immediately below OP 3.12 . Apparently OP 3.11 was directly on the erosion surface or at least our excavators dipped into the OP 3.11-3.12 intermediate area enough to pick up some of the deflated materials. While the numbers are not impressive, there is some indication of stronger erosive agents during OP 4.16 and OP 3.11 in the form of smoothed stones (Fig. 48).

TABLE 16. ANGULARITY OF PEBBLES AT EAGLE HILL

\begin{tabular}{|c|c|c|c|c|c|c|c|c|}
\hline Angularity & $\begin{array}{c}\text { Substrata } \\
4.17 \\
\end{array}$ & 4.16 & 4.15 & 4.12 & 3.11 & 2.13 & 1.13 & Total \\
\hline $\begin{array}{l}\text { Sharp } \\
\text { Semi-sharp } \\
\text { Subangular } \\
\text { Smooth }\end{array}$ & $\begin{array}{r}47.0 \% \\
28.2 \% \\
23.3 \% \\
1.5 \%\end{array}$ & $\begin{array}{r}21.5 \% \\
27.3 \% \\
47.7 \% \\
4.1 \%\end{array}$ & $\begin{array}{r}50.8 \% \\
25.8 \% \\
21.8 \% \\
1.6 \%\end{array}$ & $\begin{array}{r}42.5 \% \\
28.8 \% \\
24.7 \% \\
4.7 \%\end{array}$ & $\begin{array}{r}45.4 \% \\
24.6 \% \\
26.2 \% \\
3.8 \%\end{array}$ & $\begin{array}{r}48.3 \% \\
.3 \% \\
27.7 \% \\
0 \quad \%\end{array}$ & $\begin{array}{r}33.3 \% \\
36.5 \% \\
28.6 \% \\
1.6 \%\end{array}$ & $\begin{array}{r}359 \\
243 \\
252 \\
23\end{array}$ \\
\hline Total (n) & 202 & 172 & 124 & 73 & 183 & 60 & 63 & 877 \\
\hline Total $(\% \operatorname{col})$. & $23.0 \%$ & $19.6 \%$ & $14.1 \%$ & $8.3 \%$ & $21.0 \%$ & $6.8 \%$ & $7.2 \%$ & \\
\hline
\end{tabular}

G. GEOCHEMICAL ANALYSIS (Van Note)

X-Raj: Fluorescence Studies, Phase I

Soil samples from the HREC column levels that underwent screening and grain analys is were sent to Dr. Jerry Hoffer of The University of Texas at El Paso, Department of Geology, for chemical analysis by energy dispersive X-ray fluorescence (XRF)

\section{Procedure}

XRF method detects major, minor, and trace elements ranging from sodium (atomic number 10) to uranium (atomic number 92) in concentrations of parts per million. It also determines the relative intensities of the elements from sample to sample.

The following elements appeared in the analysis: Major--aluminum, potassium, calcium, titanium, iron; Minor--magnesium, phosphorus, chlorine, manganese; Trace--scandium, chromium, nickel, copper, germanium, rubidium, strontium, tungsten.

The data were coded and subjected to principal components analysis. 


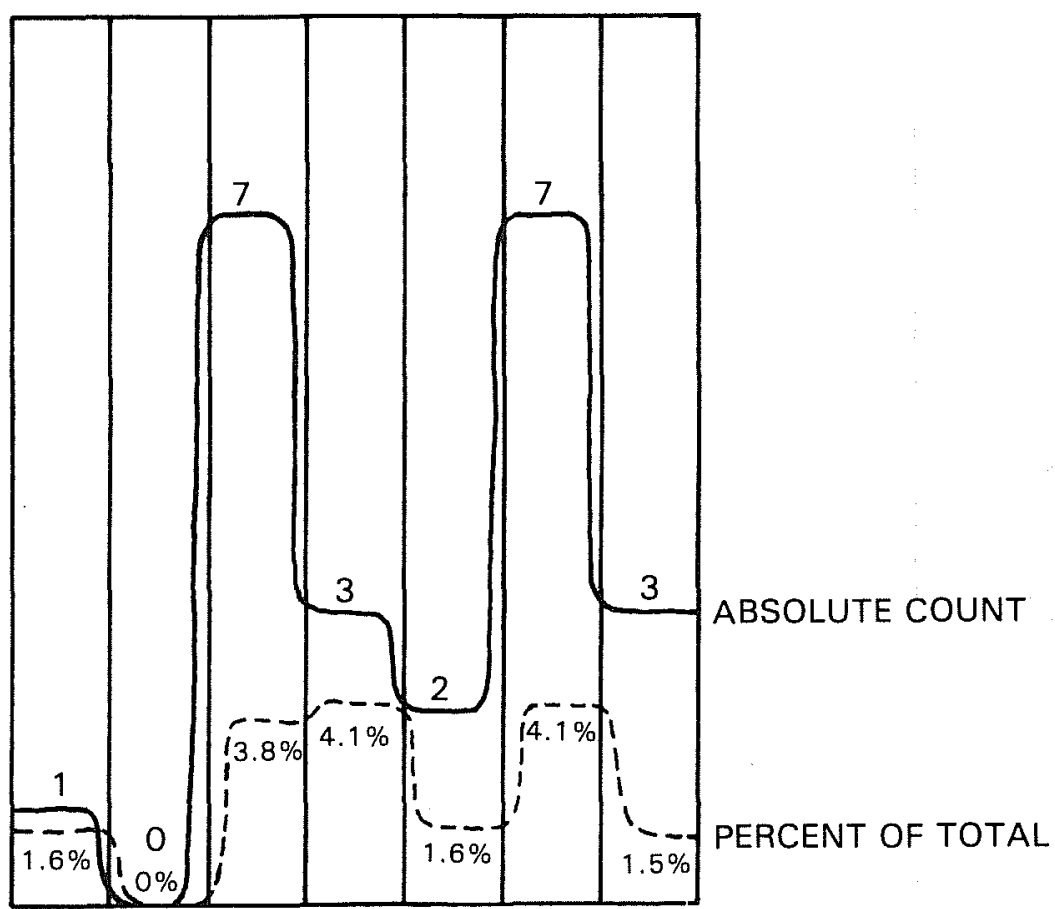

$\begin{array}{llllllll}1.13 & 2.13 & 3.11 & 4.12 & 4.15 & 4.16 & 4.17\end{array}$

SUBSTRATUM

Figure 48. Occurrence of Smooth (Round) Pebbles per Occupation Plane. Numbers correspond to the number of pebbles found. 


\section{Results}

Analysis confirmed field observation on the nature of the sediments. The sandy upper levels proved to have a higher silicon content (Fig. 49,a). The high iron content of the lower substrata (Fig. $49, \mathrm{~b}$ ) is a consistent characteristic of the site and apparently is a product of downward leaching of iron. Aluminum is also present in greater quantities in the lower levels reflecting a higher clay content.

Major Elements--Aluminum and iron were found to have a strong positive correlation with substratum indicating a tendency to increase toward the bottom of the column. Silicon, on the other hand, exhibited a strong negative correlation with substratum. Potassium and titanium remained fairly constant through time, but calcium showed a marked increase in OP 3.11 and OP 4.17 with twice the amounts of calcium in the other occupation planes. Since these are on or near deflation surfaces, there must be some tendency for calcium to accumulate at those surfaces in spite of intense leaching.

Minor Elements--Magnesium was reported in fairly constant amounts in a11 strata, except OP 2.13 and OP 3.11 where none was reported. Phosphorus was also present in fairly constant amounts, except in OP 2.13 and OP 4.16 where none was reported. Chlorine was detected only in substrata 1.13, 4.15, and 4.16. Manganese was present in all levels and showed a negative correlation with substratum revealing its tendency to decrease toward the top of the column.

Trace Elements--Scandium was present in small amounts in substrata 2.13, 4.12, and 4.17. Chromium was present in fairly constant, although small amounts in a11 substrata, except $O P 1.13$ and $O P 3.11$ where none was recorded. Nickel was found in substrata 1.13,4.15, and 4.16. Copper was found only in OP 4.12 and OP 4.17. Germanium was present in a11 but the two lowest levels, OP 4.16 and OP 4.17, where none was detected and thus, was found to be negatively correlated to substratum. Rubidium was found in all levels and al though it appeared to be unrelated to substratum, it showed strong positive correlations to aluminum and titanium. Strontium and tungsten were also detected in all levels. Strontium showed a positive correlation to substratum, while tungsten showed a negative correlation.

\section{Observations}

Several groupings of factors with strong positive and negative correlations were determined from the varimax rotated factor matrix (Table 17). These seem to indicate compounds present and so is a possible alternative to X-ray diffraction. Groupings with a high substratum correlation probably indicate vertical translocation of elements after deposition or changes through time in the depositional environment.

The negative correlation of aluminum and iron with silicon would normally be positive and indicates the presence of free silicon in the samples (Don Lewis, personal communication). Rubidium (present in this analyses as a trace element) is always present in nature in compound form but, with this type of analysis and the limited number of cases, it is difficult here to tell with what other elements rubidium is combining. XRF also did not detect elements of lower atomic weights such as sodium which limits the analysis. 

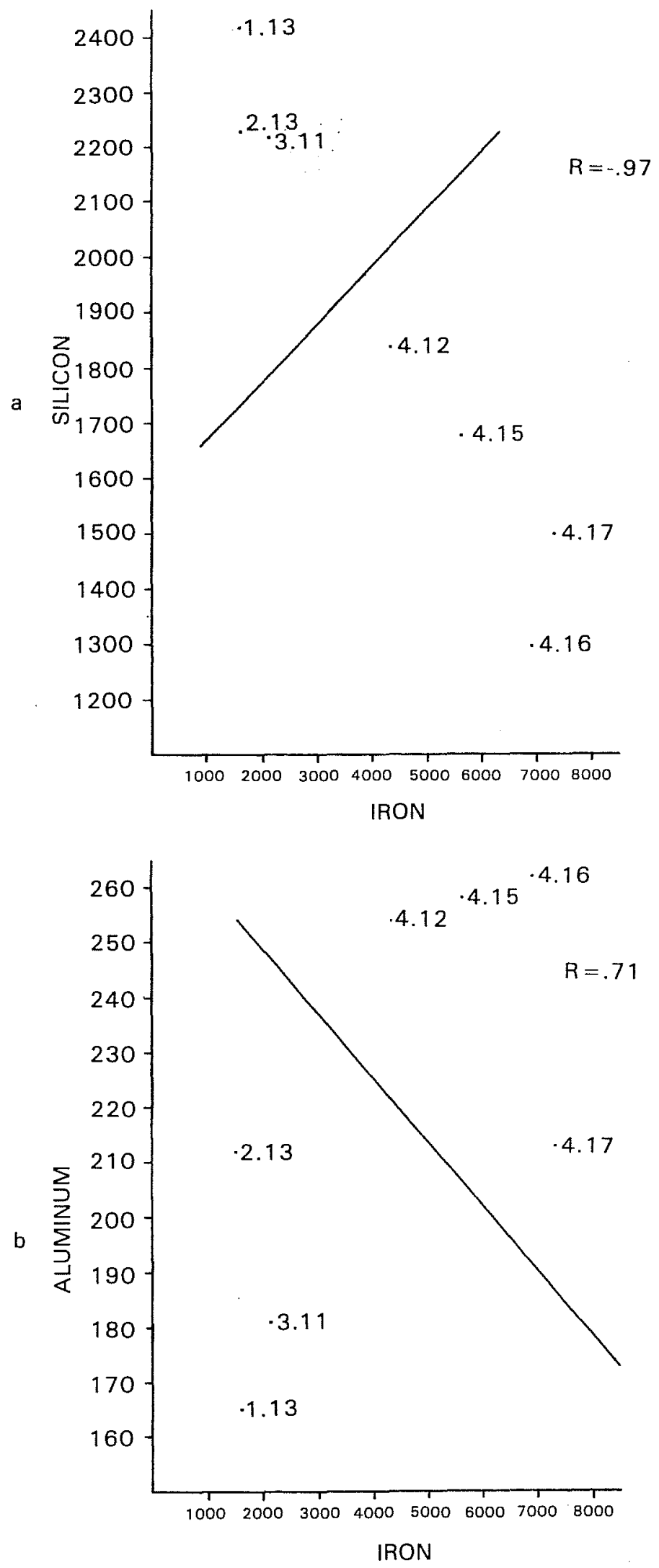

Figure 49. Quantities of Trace Elements Found by Substrata. $a$, iron and silicon; b, a Tuminum and iron. 
TABLE 17. CORRELATIONS OF ELEMENTS FROM XRF DATA

Positively correlated elements

$\begin{array}{lllll}\text { Factor I } & \text { Factor II } & \text { Factor III } & \text { Factor IV } & \text { Factor V } \\ \text { Aluminum* } & \text { Iron* } & \text { Chiorine } & \text { Calcium* } & \text { Potassium } \\ \text { Iron* } & \text { Magnesium } & \text { Nicke1 } & \text { Copper } & \text { Phosphorus } \\ \text { Chromium* } & \text { Strontium* } & \text { Rubidium } & & \text { Manganese } \\ \text { Rubidium* } & & & \text { Germanium }\end{array}$

Negatively correlated elements

$\begin{array}{lllll}\text { Factor I } & \text { Factor II } & \text { Factor III } & \text { Factor IV } & \text { Factor V } \\ \text { Silicon* } & \text { Silicon* } & \text { Scandium } & \text { Titanium* } & \\ \text { Manganese* } & \text { Germanium* } & \text { Zinc } & & \end{array}$

*high substratum correlation

X-Ray Fluorescence Studies, Phase II (Gunn)

Following Don Lewis's suggestions, it was decided to send only the silt and clay-sized particles for further $X$-ray fluorescence analysis. Samples were processed from occupation plane constant volume samples and sent to Dr. Jerry Hoffer for analysis.

\section{Procedure}

Twenty grams of soil from each sample were placed in a clean plastic cup and soaked in $250 \mathrm{ml}$ of distilled water for 24 hours. Each sample was stirred thoroughly with a glass rod and transferred into a graduated cylinder filled with distilled water to the $500 \mathrm{ml}$ level. The cylinder was then vigorously shaken for 25 seconds sideways and 5 seconds upside down, and allowed to settle for 25 seconds. This gave particles larger than 100 microns time to settle. The top $300 \mathrm{ml}$ of this mixture was decanted into a clean unused plastic cup and allowed to settle for another 24 hours. The water was then removed by pipette and the slurry transferred to a labeled glass vial for shipment. The coarse fraction was retained and transferred to an evaporating dish.

Results

As expected, the removal of the larger particle sizes from the samples substantially reduced the silicon component and revealed the less abundant elements. The resulting data were analyzed in conjunction with other environmental and cultural indicators in section IV. 
H. FLORA AND FAUNA

Flora (Sheehan)

Vegetation

The Eagle Hill site is situated between two widely recognized vegetation regions. To the north is the Gulf Slope section of the Oak-Pine forest (Braun 1950) or Oak-Hickory-Pine forest (Kuchler 1964). To the south is the westernmost extension of the Southeastern Evergreen forest (Braun 1950) or Southern Mixed forest (Kuchler 1964). The conspicuous distinction between these two forest types are controlled primarily by precipitation, $1140 \mathrm{~mm}$ per year in the 0ak-Pine forest (Brown 1972).

On upland surfaces the Oak-Pine forest is characterized by open stands of shortleaf (yellow) pine (Pinus echinata) and several species of oak especially post oak (Quercus stellata), blackjack oak (2. marilandica), and southern red oak (2. falcata). Hickories (Carya spp.) are important as we11. Loblolly pine (Pinus taeda) is now common in this forest region, but in the aboriginal forests it occurred infrequentiy (Brown 1972).

Bottomland forests are characterized by trees common to alluvial soils throughout the South. Important among these are sweet gum (Liquidambar styraciflua), oak (2uercus lyrata, 2. phellos, 2. prinus), dogwood (Cornus spp.), redbud (Cercis canadensis), basswood (Tilia americana), and hackberry (Celtis laevigata).

Poorly drained sites support bald cypress (Taxodium distichum), willow oak (Quercus laurifolia), overcup oak (2. lyrata), and water elm (Planera aquatica).

The Southeastern Evergreen forest in west central Louisiana is distinguished from the Oak-Pine forest by the presence of longleaf pine (Pinus palustris) and, in moister soils, by the presence of broad-leaved evergreen trees and shrubs. In many parts of the Southeastern Evergreen forest the longleaf pine of the aboriginal forest has been replaced by loblolly pine. This is usually attribuuted to the loblolly's more vigorous colonization of disturbed areas.

Associates of longleaf or loblolly pine on hills and in well-drained flatwoods are slash pine (Pinus elliotii), shortleaf pine, spruce pine (Pinus glabra), and turkey oak (Quercus laevis). In moister soils, longleaf and loblolly pine are associated with several oaks, hickories, sweet gum, sour gum (Nyssa sylvatica), red maple (Acer rubrum), ash (Fraxinus spp.), and hol1y (Ilex opaca) (Braun 1950).

In this forest region along sloughs occur swamp black gum (Nyssa sylvatica var. biflora), sweet bay (Magnolia virginiana), water oak (Quercus laurifolia), blackjack oak, gallberry (Ilex glabra), silverling (Baccharis halimijolia), and several shrubs of the heath family (Brown 1972). Swamps are often dominated by pond cypress (Taxodium ascendens).

Pollen Anatysis

As mentioned previously, the Eagle Hill site is located in a zone of transition between two distinct vegetation types where rainfall is thought to be the 
controlling factor. Because slight changes in climate will affect the position of the boundary ("tension zone") between two climatically controlled forest zones, a record of shifts in regional forests can serve as a record of regional shifts in climate. This situation is of particular interest in the reconstruction of vegetational and climatic history. Accordingly, samples of sediment from the High Resolution Environmental Column (HREC) from Eagle Hill were submitted to the palynological laboratory at the University of Indiana.

An initial series of seven samples from the HREC were selected for pollen analysis on the basis of sediment texture and color. Silty or clayey samples were chosen in preference to sandy ones and samples of gray or brown color selected in preference to those of red or yellow (Table 18).

A11 seven samples (five cubic centimeters each) were processed by the following method (modified from Faegri and Iversen 1974).

1. Carbonates were removed with cold $10 \% \mathrm{HCl}$.

2. Samples were screened to remove particles larger than 250 micrometers.

3. Samples were repeatedly water-rinsed, swirled, and decanted to remove heavy particles.

4. Remaining silicates were removed with hot $40 \% \mathrm{HF}$.

5. "Soft" organic matter was dissolved by acetolysis (10:1, acetic anhydride: conc. $\mathrm{H}_{2} \mathrm{SO}_{4}$ ).

6. Samples were dehydrated, stained, and then suspended in tert-butyl alcohol. Drops of the pollen suspension were mounted in silicon oil on glass slides.

Counts were made at 500 diameter magnification.

Very little pollen was recovered from the samples (Table 18). A second technique was used on samples $3.049,3.052$, and 4.088 similar to the first technique. This technique employed $5 \%$ sodium pyrophosphate to remove clay particles and a 7-micrometer-mesh monofilament nylon screen to eliminate small particles remaining after the chemical treatments (Cwynar, Burden, and McAndrews 1979). No additional pollen was extracted with this technique.

It is impossible to reconstruct a credible vegetational or climatic history for the Eagle Hill area using the sparse pollen data recovered. The quantity and variety of pollen in the individual counts is too small to allow confident speculation on the nature of past vegetation assemblages.

Nonetheless, a vague trend exists in the data. From sample 4.091 to 3.052 there is a decreasing importance of birch and an increasing importance of pine and herbs. From 3.052 to 1.031 there is a continued trend toward increasing pine values. In the Southeast, birch is a streamside tree. Pines are ubiquitous, but most common on well-drained sites. High proportions of herbs suggest an open type of forest. Thus, if the pollen data from the HREC were representative of changes in forest composition, the trend seen in them would suggest (7) a riverine environment contemporaneous with level 4.091, (2) an open, probably dry, pine woods contemporaneous with level 3.052 , and (3) possibly a less open pine forest contemporaneous with leve1 1.031. 
Future analyses of more suitable sediments from the Eagle $\mathrm{Hill}$ region will be necessary before the trend suggested by this study can be verified.

TABLE 18. EAGLE HILL (HREC) MICROFOSSILS, SELECTED LEVELS

\begin{tabular}{|c|c|c|c|c|c|c|c|c|c|c|c|}
\hline Sample Number & 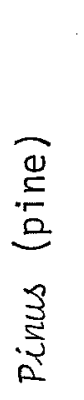 & 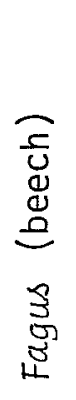 & 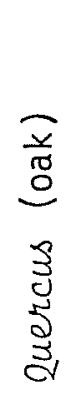 & 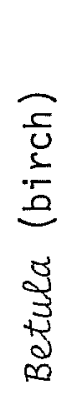 & 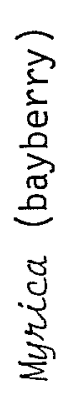 & 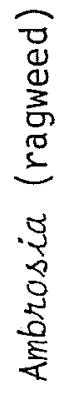 & 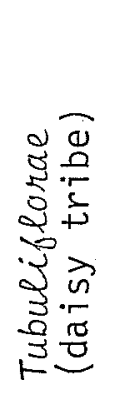 & 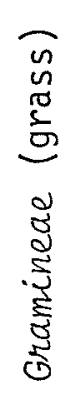 & 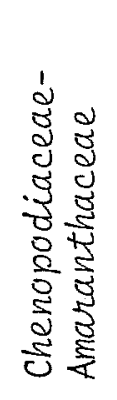 & 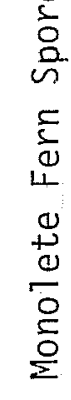 & 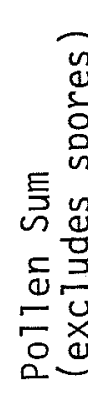 \\
\hline SS 1.031 & 6 & - & - & - & - & - & 1 & - & - & - & 7 \\
\hline SS 1.046 & - & - & - & - & - & - & - & - & - & 3 & 0 \\
\hline SS 3.049 & 2 & 1 & - & - & - & - & 2 & - & - & - & 5 \\
\hline SS 3.052 & 3 & - & - & - & 1 & 1 & 19 & 3 & - & - & 27 \\
\hline SS 4.085 & - & 1 & 1 & - & 1 & - & - & - & - & - & 3 \\
\hline SS 4.088 & - & - & - & 1 & - & - & - & - & 1 & - & 2 \\
\hline SS 4.091 & 1 & - & - & 6 & - & - & - & - & - & - & 7 \\
\hline
\end{tabular}

Fauna (Gunn)

We were aware from the beginning of the project that the likelihood of finding faunal remains were slight. Only occasionally, minute fragments of aquatic shells were found in the uppermost levels of the site. Our one hope was that rodent teeth would survive the acid soils. Also, humans might act as agents to concentrate teeth in recoverable quantities. Rodents can be identified by their teeth, and they are a good climatic indicator. Careful examination of several samples revealed no teeth, and the effort was abandoned.

\section{CHEMICAL ANALYSIS OF CERAMICS AND LITHICS FROM THE EAGLE HILL II SITE (Brown)}

While a large part of traditional archaeological research has been aimed at the site and its contents as a discrete spatial unit for comparison to other such units, many recent studies have attempted to view the site as a node of activity within a regional framework. Settlement pattern, regional ecology, and siudies such as the catchment area analysis in this report have stressed the dynamic relationship of the inhabitants of the site with their environment. Perhaps the earliest and most common example of the interest in external activity patterns has been the analysis of introduced materials, such as trade goods 
from known areas. It was also recognized quite early that certain kinds of raw materials, most notably cherts, could be identified, and grade or movement patterns hypothesized.

The use of raw materials as indicators of trade routes and group movements has expanded almost exponentially within the last several decades, coinciding with the increased availability of, and improved techniques in materials analysis. New data have been gathered to help answer old problems, and new kinds of questions have begun to be asked.

In planning for the analysis of materials from the Eagle Hill site, it was thought that material provenience studies might have particular relevance, because of apparent changes through time in various raw materials in parts of Louisiana. In particular, the early Paleo-Indian period seems to be represented by a disproportionate number of projectile points manufactured from exotic cherts (Gagliano and Gregory 1965), many of which are from sources easily identified on the basis of macroscopic characteristics.

Unfortunately, excavation at the Eagle Hill site yielded no macroscopically obvious diagnostic indicators of known raw material apparent in basic raw material types. Certain kinds of cherts, represented primarily as finished artifacts, were rare and quite distinct from other types present. Other siliceous stone artifacts were similar to opals occurring in sediments of the immediate area. Between these two extremes was a wide range of colors, textures, and qualities of stone with little clear indication of whether the vast majority of lithic debitage is local, regional, or extra-regional. The article by Jolly in this report approaches this question from the point of view of observable characteristics and frequencies; the following section explores these hypotheses further using data from chemical and mineralogical analys is of lithic materials from the site.

Although the site consists primarily of lithic debitage, within which are apparent differences in procurement options through time, the ceramics are also represented in the upper substrata at the site. Equally significant questions can be asked of the ceramic raw material data and their relationship to aboriginal procurement behaviors. Although the presence of local or exotic ceramic raw materials cannot be hypothesized on the basis of visual characteristics, there is a wide range of variation in ceramic pastes at the site. Some of this variation is easily explicable in terms of technological differences, but certainly some of it may be due to differences in clay sources.

The basic data for this section consists of mineralogical and chemical analyses of samples of lithic and ceramic materials, discussed in separated sections below. The mineralogical analyses were primarily conducted at the Geology Department at The University of Texas at Austin, while the chemical analys is was conducted both in Austin and at the Nuclear Science Center at Texas A\&M University, aided by Melinda Urbantke. The Nuclear Science Center provided additional funding for part of this study.

\section{Ceramics}

As mentioned in the chapter on ceramic analysis, the sherds collected at the site exhibit a considerable range of technological variation. Such variation could clearly mask otherwise visible differences in raw material usage. In some cases, what is apparently technological variation might not be due to 
clay preparation, but to initial differences in raw material sources. The numerous sandy paste sherds are a good example. Are they naturally sandy clays or do they represent added aplastic materials?

The numerous sandy clays of this upland area suggest that many of these ceramics may have been made at the site or in the nearby area. Short of an analysis of the ceramic paste materials, it would be very difficult to approach such a question in any but the most cursory fashion. One observation made in the field was relevant to this question; beneath the site was a good source of plastic clay, which might be an excellent ceramic raw material. In addition, the possibility could not be ignored that the great number of fired clay balls found in the ceramic levels might be waste from the ceramic manufacturing process.

One of the more preliminary kinds of questions which can be asked is the nature of variation present in local clays. Are they totally homogeneous throughout the area, do they vary randomly from one meter to the next, or is there some consistent gradient of variation? In order to attempt an answer to this question, a number of local clay samples were collected from in and around the site and from clay exposures at some distance from the site. Table 19 gives a brief description of these samples and their general location in relation to the site.

TABLE 19. CLAY SOURCE PROVENIENCE DATA

Sample

$\mathrm{C} 1$

C2

C3

C4

C5

C6

C7

C8

C9

C10

$\mathrm{C} 12$

$\mathrm{C} 13$

C15

$\mathrm{C} 17$

C19
Material Type

Gray Sandy Clay

Gray Sandy Clay

Orange Mottled Brown Sandy Clay

Dark Red Mottled Gray Clay

Gray Clay w/Some Red Mottles

Gray Clay

Grayish Brown Fine Sand

Light Gray Sandy $\mathrm{Cl}$ ay

Light Gray Sandy Clay

Loose Brownish Gray Sand

Brown Sandy $\mathrm{Cl}$ ay

Light Gray Sandy $\mathrm{Cl}$ ay

Whitish Gray Sandy Clay

Red Clayey Sand

Light Gray Clay
Location

Upper profile, gully $50 \mathrm{mE}$ of Area $A$

Upper profile, gully $50 \mathrm{mE}$ of Area $A$

Lower IIB2t, Area A

Upper IIIB2t, Area A

Mid IIIB2t, Area $A$

Lower II IB2t, Area A

Mixed I and II soil from backdirt

Upper profile, gully west of Area A

Lower profile, gully west of Area A

Eagle Hil1 I, $0.5 \mathrm{~km} \mathrm{~N}$ of 16 SA 50

Perimeter Rd Bridge, $1.5 \mathrm{~km}$ NW of 16 SA 50

Perimeter Rd Bridge, $1.5 \mathrm{~km}$ NW of 16 SA 50

Eagle Hill III, $0.7 \mathrm{~km} \mathrm{NW}$ of 16 SA 50 just below lithic sample IIIW- 1

Eagle Hi11 I, $0.5 \mathrm{~km} \mathrm{~N}$ of 16 SA 50

Lowest point in gu71y $100 \mathrm{~m}$ NNW of Area A 
Rather than immediately submit all of the source clays and a number of ceramic samples to neutron activation analysis, a somewhat expensive technique; the potential source clays were first analyzed by $X$-ray diffraction and $X$-ray fluorescence to make a preliminary assessment of the variation. Utilizing the methods available at The University of Texas at Austin, these techniques are not as powerful discriminators as neutron activation analysis nor are they absolutely quantitative. Only relative amounts of various easily recognizable minerals and elements can be determined. In addition, these methods are both destructive and require a relatively large sample. For X-ray fluorescence, for example, the four gram minimum sample size would eliminate all but a few of the largest sherds from the analysis and necessitate the destruction of large portions of each of those for the analysis. For this reason, only the source clays were included in the initial analysis.

The first step in this analysis was the X-ray diffraction analysis of a sample of these clays in order to determine their basic mineralogy. Five samples from the site $(\mathrm{C} 1, \mathrm{C} 2, \mathrm{C} 3, \mathrm{C} 4$, and $\mathrm{C5})$ were selected for this analysis; five grams of each of these were dissolved in dejonized water and a pipette sample collected from near the surface after twenty minutes settling time. This allows for the settling-out of sands and other large mineral fragments, which might obscure the clay mineral peaks. The pipette samples were then placed on a clean glass slide and allowed to dry. The dried sample was run through the general clay range on an X-ray diffractometer and the resulting mineral peaks identified. These samples all proved to be primarily montmorillonite with a sma11 amount of kaolinite present. The relationship between these two clay mineral groups was very consistent among the samples, the major variation being the total amount of clay present in the different sediments.

At this time a number of clay samples were selected for $X$-ray fluorescence analysis. Preparation for this analysis included grinding the sample and mixing a carefully weighed amount with a small amount of Bakelite. This mixture was pressed into a small pellet under pressure and cured at approximately 110 to $120^{\circ} \mathrm{C}$. In a 11 , thirteen of the clay samples collected were analyzed by this technique. These were $\mathrm{C} 1, \mathrm{C} 2, \mathrm{C} 3, \mathrm{C} 4, \mathrm{C} 5, \mathrm{C} 6, \mathrm{C} 8, \mathrm{C}, \mathrm{C} 10, \mathrm{C13}, \mathrm{C} 75, \mathrm{C} 17$, and $\mathrm{C19}$. The relative amounts of eight elements were recorded using this procedure. These elements are Titanium, Barium, Vanadium, Chromium, Samarium, Manganese, Iron, and Zirconium. Table 20 shows some of the approximate relationships of the elements identified. Values shown are simply chart divisions with no absolute basis, but given the similar matrices and similar clusters of elements, they could be considered quantitative in a relative sense.

Several conclusions are indicated by the results of the $X$-ray fluorescence study. In the first place, there are quite recognizable differences between the clays of the area. But most important, these differences are not random, but are related to the spatial separation of the various strata. In general, those clays which are farther apart show the greatest differences. Figure 50 illustrates a cluster tree diagram for the sampled source clays based on these XRF peaks. This cluster analysis, which uses the Biomedical Computer Program BMDP2M (Dixon and Brown 1977), groups those samples which have the closest values for all elements (based on an Euclidean distance measure of normalized data) into clusters two at a time, treating each newly formed cluster as a single case for further clusters. 
TABLE 20. XRF CLAY SAMPLE PEAKS

$\begin{array}{lllllllll}\text { Sample } & \text { TI } & \text { BA } & V & \text { CR } & \text { SM } & \text { MN } & \text { FE } & \text { ZR } \\ \text { C1 } & 28 & 18 & 18 & 27 & 24 & 31 & 56 & 47 \\ \text { C2 } & 28 & 19 & 18 & 27 & 23 & 35 & 80 & 40 \\ \text { C3 } & 29 & 18 & 18 & 28 & 23 & 31 & 74 & 47 \\ \text { C4 } & 29 & 18 & 19 & 30 & 24 & 32 & 69 & 49 \\ \text { C5 } & 29 & 17 & 19 & 29 & 21 & 31 & 94 & 46 \\ \text { C6 } & 27 & 19 & 18 & 26 & 22 & 34 & 56 & 43 \\ \text { C8 } & 29 & 18 & 19 & 30 & 24 & 30 & 71 & 43 \\ \text { C9 } & 31 & 30 & 20 & 24 & 24 & 32 & 71 & 44 \\ \text { C10 } & 16 & 18 & 15 & 29 & 24 & 29 & 10 & 40 \\ \text { C13 } & 26 & 18 & 19 & 25 & 21 & 33 & 85 & 37 \\ \text { C15 } & 17 & 19 & 19 & 25 & 21 & 33 & 94 & 38 \\ \text { C17 } & 18 & 16 & 17 & 28 & 27 & 29 & 77 & 33 \\ \text { C19 } & 29 & 16 & 18 & 26 & 21 & 42 & 100 & 36\end{array}$

The first two and therefore the strongest groups in this analysis are formed between the upper strata of clays from the site. Samples $\mathrm{C} 4$ and $\mathrm{Cl}$ and samples C8 and C3 first form two separate clusters and one step removed from one single cluster, which includes all of the samples from the upper 30 to $50 \mathrm{~cm}$ of Miocene clay surrounding the site. Two lower samples, C6 and C2, which are 50 or more meters apart, but stratigraphically equivalent, group at the third cluster step. Sample C5 which is intermediate to C6 and C4 stratigraphically, behaves as one would expect a transitional sample to behave, joining the cluster at an intermediate step.

One of the more interesting clays from the site is C9, a gray sandy clay that is the most chemically distinct of all of the samples and which joins the cluster tree last of all. As is obvious from the data, it has extreme values for both barium and chromium. This sample, although apparently stratigraphically equivalent to $\mathrm{C} 6$ and $\mathrm{C} 2$, appears somewhat different and its distinctiveness may indicate the presence of another strata. Sample C19, at a depth of several meters below C9, shows more similarities to the rest of the clays from the site than to C9. It is somewhat different, however, and samples $\mathrm{Cl} 3$ and C15, which are both more than a half kilometer distant (from the site and from each other), join the upper site clay cluster first. These two, which are texturally similar and probably also stratigraphically equivalent to one another, form a cluster with each at the fifth cluster step. Samples $\mathrm{Cl7}$ and C10, both from the top of Eagle Hill, are quite distinct (one is a red clayey sand while the other is a loose brown sand) and group at such a low level that it is probably not significant.

Five of these clay source samples were selected for more detailed study in neutron activation analysis. These included two from beneath the site ( $\mathrm{C} 4$ and C6), one from the guily to the east of the site (C2), one from the deepest part of the gully to the west of the site (C19), and another (C12) from a series of eroded gullies near the perimeter road, one kilometer northwest of the site. One of these samples, C19, was drilled twice to examine the amount of variability present in a single clay source; the two samples were taken less than 
a foot apart at the bottom of the gully. These five clays were thought to potentially represent a large portion of the variation present in the clays of the immediate surrounding area.

In addition to these five clay samples, several other sediment samples were included in the analysis. Two soil samples from the constant volume sample and from the High Resolution Environmental Column in E3019 N1002 were included, one from substratum 2.13 and the other from substratum 4.12. These were primarily included as a test of the origin of the site's clay balls, two of which (one each from substrata 2.13 and 4.12) were also analyzed. These sediment samples also provided a check on the possible effects that burial in these sediments might have on trace element chemistry. One further sample, collected from the excavation backdirt (C7), is a mixed fine sand that probably approximates the Plio-Pleistocene colluvium that covers the area and which may have served as an aboriginal source of tempering material. This sample should approximate the samples from both 2.13 and 4.12 , but not precisely, being a mixture of the sediments from several different substrata.

Contrasted to these local sediment samples were fifteen ceramic samples thought to represent the major technological types present at the site. These samples were selected subjectively; all of the sherds were spread out on a table and divided into groups based on visible characteristics such as color, thickness, apparent temper, etc. One of the larger and more representative sherds of each of these groups was then selected for analysis. Two sherds were sampled twice to assess the variability in trace elements from one end of the sherd to the other. The proveniences of the sampled sherds are shown in Table 21.

Sampling of al1 of the ceramic and clay sources was done by drilling a smal1 amount from one edge after first cleaning the surface with a burring tool. In order to minimize drill contamination, drill bits and burrs were of tungsten carbide; in any case, tungsten was ignored in the analysis because of this. Large inclusions of organic material or obvious tempering were avoided in drilling, but in the case of the sherds, the samples were too large and the temper too fine and evenly distributed to avoid the inclusion of some tempering material.

Samples were then weighed and placed in a plastic container which was then sealed and subjected to neutron irradiation at the Nuclear Science Center at Texas A\&M University for three different time periods: two minutes, two hours, and 14 hours. Fifty milligram samples were used in the two minute run, while 100 milligram samples were used for both the two hour and 14 hour runs. Collect times for the various runs were five minutes for the two minute run, 15 minutes for the two hour run, and 20 minutes for the 14 hour run. Gamma ray emissions during this period were counted and analyzed by the computer at the Nuclear. Science Center, which produced a printout of the various elements recognized in parts per million. These data were subsequently keypunched for analys is by The University of Texas at Austin.

In tota 7 , more than 60 elements were recognized in some or all of the ceramic samples in amounts varying from fractions of a part per million for rare earths like lanthanum or europium to several percent of the total composition for more common elements like iron or magnesium. In order to reduce the data set to a more useful and workable size, the printouts were scanned for possible sources 
of error in peak recognition. Elements with extremely high recorded errors or which had possible unrecorded peaks were excluded from the initial analysis. This eliminated the possibility that an element present in a given sample might have simply been missed either in counting or in computer peak analysis. Additionally, elements such as tungsten, from the drill bits, and argon present in the air in the sample container, were eliminated from the analysis. The remaining 11 elements were present in some quantity in every one of the 28 samples (a 29th sample, an empty vial, was also run, but, other than argon and less than 30 PPM sodium from salt, almost nothing was present, and this sample was not included in further analysis).

TABLE 21. CERAMIC SAMPLE PROVENIENCE

\begin{tabular}{|c|c|c|c|c|c|}
\hline Sample & North & East & Substratum & Temper & Comments \\
\hline 8 & 3018 & 1001 & 3.11 & grit & \\
\hline 12 & 3019 & 1001 & 2.13 & grit & \\
\hline 16 & 3020 & 1002 & 2.13 & grit & not in situ, decorated \\
\hline 20 & 3018 & 1002 & 3.11 & grit & \\
\hline 25 & 3020 & 1002 & 3.11 & grit & \\
\hline 27 & 3021 & 1000 & 2.21 & grit & coarse tempered \\
\hline 28 & 3020 & 1002 & 3.11 & grit/sand & 2 samples drilled \\
\hline 40 & 3018 & 1002 & 1.12 & none visible & \\
\hline 53 & 3018 & 999 & 2.27 & sand & \\
\hline 55 & 3017 & 999 & 3.11 & grit & \\
\hline 64 & 3019 & 1000 & 2.31 & grit & 2 samples drilled \\
\hline 76 & 3019 & 999 & 2.31 & grit & \\
\hline 79 & 3017 & 1000 & 2.31 & grit & \\
\hline 90 & 3021 & 1002 & 2.31 & sand & \\
\hline 97 & 3020 & 1000 & 2.13 & grit & decorated \\
\hline
\end{tabular}

Even with only 11 elements left in the analysis, the attempt to make sense of the data by hand yielded little information. This attempt did seem to indicate that there were a number of differences between the clay sources and most of the ceramics, and that one small group of sherds seemed to differ considerably from the rest. To verify these preliminary observations, the data were subjected to a cluster analysis, using the same BMDP2M procedure as above. This cluster, presented in Figure 51, clearly separates these hypothesized groupings.

In this analysis, the clay bal1 from substratum 2.13 (CB21) clusters first with the soil sample from substratum 4.12. Significantly, the next two samples to group together (64-1 and 64-2) are two separate drillings from the same sherd. The other double drilling (28-1 and 28-2) does not cluster as near the top of the analysis, but these two samples also group with one another before they are grouped with any other sherd. Other early and strong groupings occur between sherds 64 and 79 and between 8 and 20. A good group also forms between the various soil samples and the clay ball from substratum 2.13. The clay bal1 from substratum 4.12 eventual1y joins this group, but not until there is a considerable increase in cluster distance. 


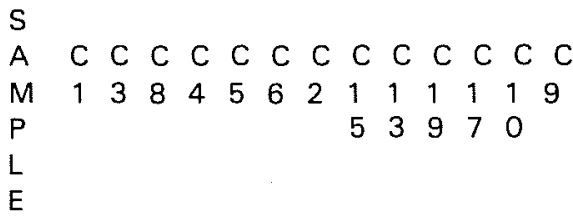

AMALG.

DISTANCE

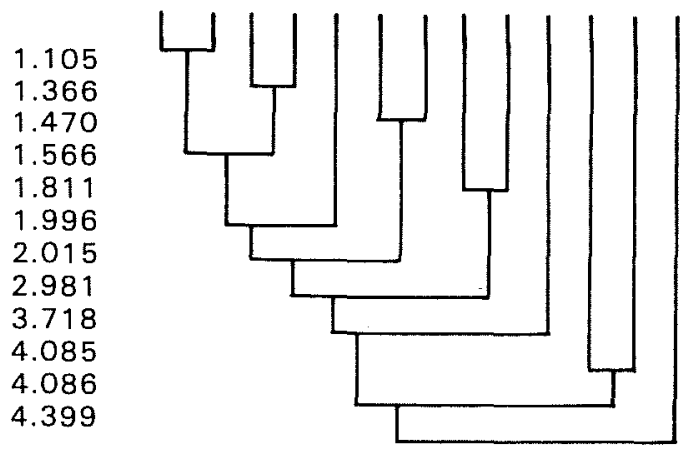

Figure 50. X-Ray Fluorescence Cluster Tree.

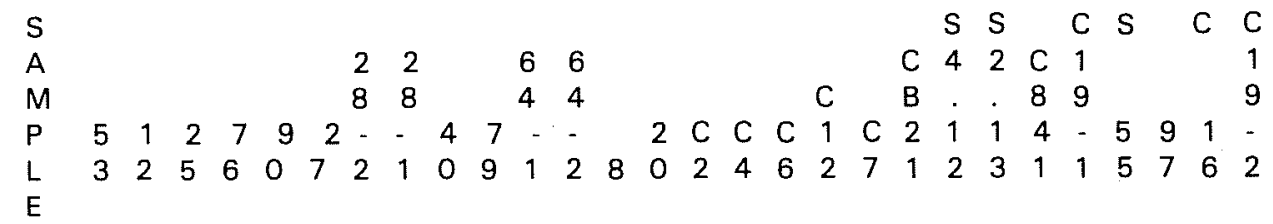

AMALG.

DISTANCE
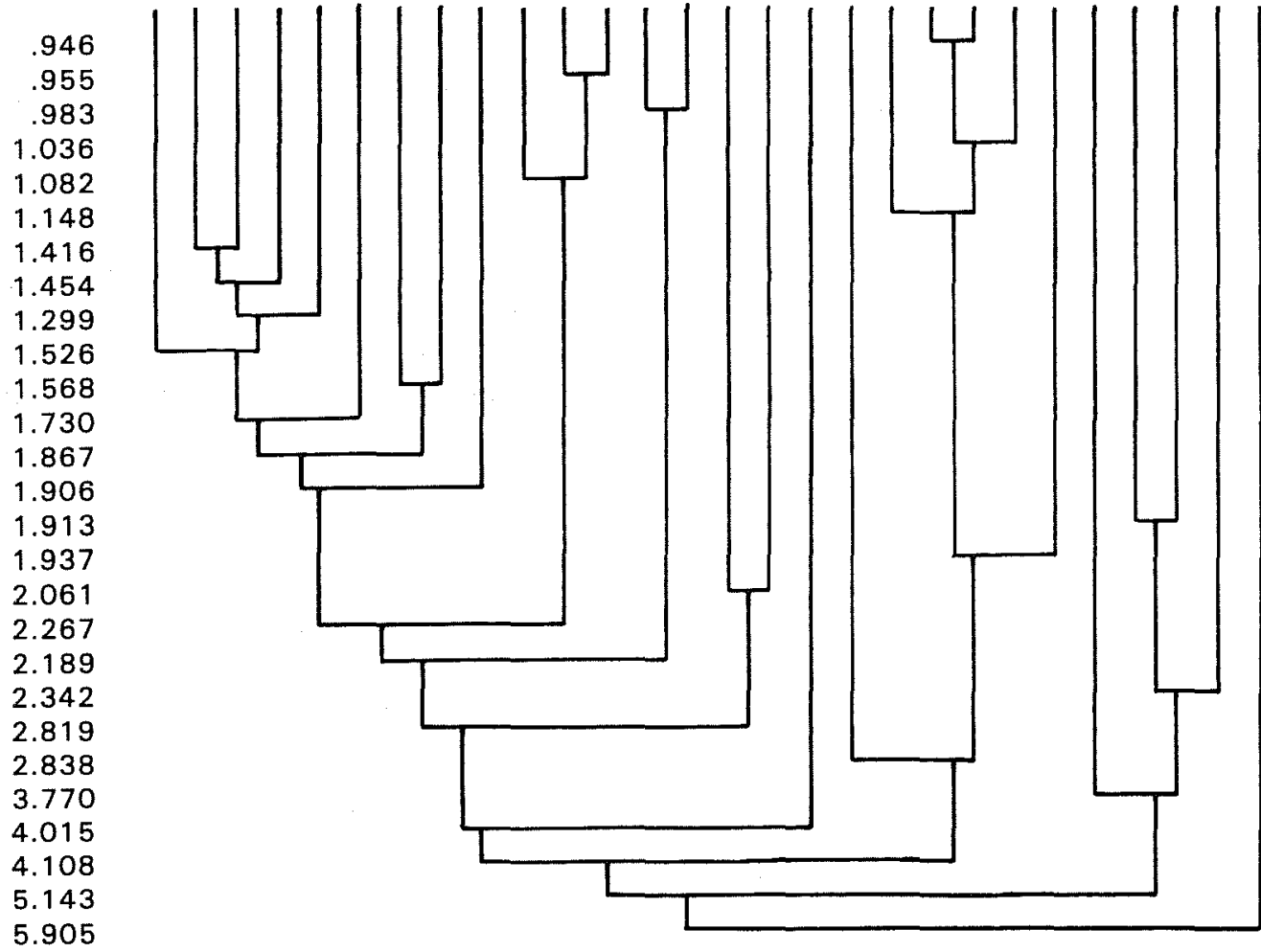

Figure 51. Ceramic and Clay Source Cluster Tree. 
On the left side of this diagram, a cluster is formed that includes most of the sherds from the site, with the exclusion of the two small groups already mentioned and three sherds which do not join the main group of sherds until after almost everything in the analysis has joined. These three sherds, which do not form a very distinct cluster in themselves, are clearly distinct from everything else at the site. Two of these three are decorated, the only decorated sherds included in the neutron activation analysis. These two (16 and 97) both have incised horizontal lines and are included in the coles creek ceramic classification discussed in a later chapter of this report. These two are quite distinct from one another and are not clearly related to many other sherds from the site. Number 16 has a red paste with numerous large inclusicns that appear to be clay grit temper. Most of the horizontal parallel incised sherds from the site are of this paste, but there are no undecorated sherds that have a paste similar to this.

The other incised sherd is the single unique example of a somewhat irregularly incised sherd found in a rodent burrow in substratum 2.13; it cannot, therefore, be clearly tied to any vertical provenience. Upon close inspection, however, it does have a number of similarities in paste characteristics with sample 55 , with which it clusters first. Although they are probably not the same vessel, it seems likely that they may have been made from a similar clay source and perhaps have even been made by the same potter. The reason for suspecting that they are not the same vessel is the size and color distribution of clay grit particles in the exterior vessel wall; on the other hand, sample 55, which seems to be related to two other sherds from the site, is also somewhat distinct in paste and temper characteristics from most of the other sherds at the site.

It appears that there are four distinct groups of sherds present in the sample, which in turn fall into two larger groups distinguishing between the three unique sherds discussed above and al1 the other specimens. The main group of eight sherds contains a number of visually distinct sherds that could not possibly have come from any less than five or six different vessels. For the most part these sherds have a sandy paste, although they vary in grain size and amount. Thickness of these specimens varies considerably, also, ranging from $4.4 \mathrm{~mm}$ in 53 to $11.8 \mathrm{~mm}$ in 27 (the thick, coarsely tempered sherd discussed on page 265). Sherds 64 and 79 are quite similar specimens of a thin high-fired ware and could potentially be from the same vessel; they may also be related to the crosshatch incised specimen discussed on page 265. The other group, composed of 8 and 20 is not particularly visually distinct; they do not appear to have come from the same vesse1, but this possibility cannot be ruled out.

Although one major group of sediment samples and fired clay balls is formed in the early stages, these do not in turn group well with any of the nearby clay samples. Samples C7, S2.13, and \$4.12 group with each other, but do not group at al1 with $\mathrm{C4}$, the clay sample from the top of stratum 5 , on $1 \mathrm{y} 30 \mathrm{~cm}$ below substratum 4.12. In fact, the only clay sample that joins these sediment samples at all is $\mathrm{C12}$ (from one kilometer distant) which joins so low in the tree as to not form a very significant bond. The implications of this for the clay balls are not totally clear, but they do not appear to be related to the Miocene clays of the lower strata of the site. Actually, they appear to be closely related to the sediment samples that contain relatively small clay fractions. If they are indeed formed from fired argillic clays, the implication 
is that they were exposed by erosional truncation or bioturbative movements if they were fired when near the surface.

While none of the clay source samples form very strong groups, samples C4 and C2 are the best approximation of a cluster. These two clays, suggested as different units in the XRF anaTysis and separated by almost a $100 \mathrm{~m}$ horizontal distance, may be part of the same original depositional unit, a unit subsequently altered by soil-forming processes. Sample $\mathrm{C} 6$, located only some $30 \mathrm{~cm}$ below $\mathrm{C} 4$, does not cluster with anything much less with its closest neighbor. A scan of the original data set shows that $\mathrm{C} 6$ and $\mathrm{C} 4$ are actually very close in all of their trace element compositions, with the exception of sodium, where the difference is so extreme as to prevent their forming a cluster. In fact, sample C6, with 8774 PPM sodium (compared to 3373 PPM for C4), has the highest sodium content of any ceramic or clay sample in the analysis. This difference seems to be due to downward leaching of sodium through the sediment.

Perhaps most disappointing of the clay sample groupings is that of the two separate drillings from C19, the clay from the lowest part of the gully to the west of the site. This clay, located three or more meters below the other clay samples in the neutron activation analysis, would not necessarily be expected to group with other samples from the site. Unfortunately, the two samples do not really even come close to grouping with one another. In this case, an analysis of the actual data shows again that many of the values are nearly identical, with two significant exceptions (titanium and magnesium), which are divergent enough to prevent a cluster.

The most obvious conclusion from this cluster pattern is that the ceramics found at the site were not made from any of the clays collected in the site area. Although it might be that clay preparation procedures have totally disguised these clays, it is unlikely because the main cluster of ceramics represents a technologically very diverse group that appears to represent a variety of added materials. Yet, despite this diversity of added materials, sherds from the main group cluster with each other before clustering with any of the clays.

One further analytical approach was taken to examine the ceramic groupings. Discriminant analysis was used to study the strength and direction of the groupings. This technique evaluates the discriminating power of variables for cases placed into known groups and attempts reclassification based on discriminant functions created from those variables (Nie et al. 1975). For this ana 1ysis, a11 of the sherds were placed in one group and all of the clays in another. Table 22 shows the various samples and the probability of their being as far from the group centroid (assuming normal distribution) as they actually are. Although a number of samples have probabilities below $30 \%$ or $40 \%$, this includes samples from both extremes. The discriminant scores for each sample are included so that those which lie outside the range of either group can be distinguished from those which lie between. The two samples which lie closest to the middle ground between the groups are $\mathrm{C} 4$ and 20 , but also in the middle are samples 8, 25, 53, and 64-2. No other clay lies between. This suggests that perhaps the two smaller groups, especially the one containing 8 and 20 may have been made from clays similar to the local ones.

Although none of the sherds could be clearly identified with clay samples from the area, it is suggested that four distinct clay sources are represented, none 
of which came from the immediate area around the site. The sma11 group containing 8 and 20 may represent a clay source near the site, however. The larger group and the smal1 group containing numbers 64 and 79 do show some similarities to the area clays; it may be that these sherds were all manufactured from similar, but unsampled upland clays from the general area. The small cluster including one plain and two decorated sherds, because of its distance from the local clays, may actually have been imported from some distance. The tentative hypothes is that these sherds come from floodplain ceremonial centers is offered here.

\section{TABLE 22. DISCRIMINANT ANALYSIS SCORES}

\begin{tabular}{lcr} 
& Pample & Discriminant Score \\
\cline { 2 - 3 } 53 & .2714 & -1.7660 \\
$28-1$ & .4278 & -2.0728 \\
C6 & .8268 & 4.2107 \\
C4 & .2481 & 3.2739 \\
16 & .5233 & -3.5040 \\
20 & .2028 & -1.5922 \\
$64-2$ & .2415 & -1.6946 \\
90 & .8262 & -2.6461 \\
12 & .1072 & -4.4765 \\
C2 & .4209 & 5.2338 \\
27 & .0660 & -4.7045 \\
55 & .1072 & -4.4768 \\
CB41 & .8209 & 4.2026 \\
79 & .4186 & -3.6746 \\
40 & .7352 & -2.5275 \\
C7 & .2789 & 5.5117 \\
C19-2 & .0612 & 6.3007 \\
76 & .9797 & -2.8403 \\
$28-2$ & .6911 & -3.2631 \\
C12 & .6046 & 3.9110 \\
8 & .2164 & -1.6296 \\
S2.73 & .8280 & 4.6462 \\
CB21 & .6986 & 4.0417 \\
97 & .6241 & -3.3558 \\
C19-1 & .5823 & 3.8788 \\
$64-1$ & .8198 & -2.6380 \\
S4.12 & .3569 & 3.5076 \\
25 & .3123 & -1.8554 \\
& & \\
C1ay Group Discriminant Function & \\
Ceramic Group Discriminant Function & -2.86576 & \\
& & \\
& &
\end{tabular}

\section{Lithics}

The amount of obvious variation present in the excavated lithics from the site was enormously greater than that found in the ceramics. Stone tools and the 
debitage from tool maintenance (and perhaps manufacture) ranged from translucent, very fine-grained cryptocrystalline cherts to grainy quartzites. That some of this material was imported into the site was suggested by the high percentage of formal tools made on extremely rare types. Yet, as mentioned above, much of the debitage at the site bears some resemblance to the locally occurring opaline silicates. The probability that the aboriginal groups inhabiting the site selected for high quality cryptocrystalline cherts for many of their tools cannot be doubted. Yet, at the same time, tools with perhaps equal functional utility were manufactured from materials not as easily manipulated. In fact, neither of the two most obvious early diagnostic indicators, the spurred end scraper or the lanceolate projectile point found in the lowest level, is made from what could be termed a high quality chert.

The section by Jolly (page 290) detailed the variation through time in the usage of various visually distinct lithic types from the site. He hypothesizes that the changes in the frequencies of occurrence of various raw materials should reflect changes in the subsistence pattern of the site's occupants. Given that chert sources do not generally move around, this hypothesis seems obvious enough. Several problems remain, however. Without some idea of the actual direction and distance to the chert sources, it is difficult to equate a change in range with a change in subsistence patterns. Some changes in range may actually reflect differential preferences in procurement areas for identical resources between different bands utilizing the same site as a temporary encampment. Conversely, since many similar lithic sources are correlated with eastwest trending geological formations that can contain different subsistence resources depending on elevation and precipitation (which also tends to occur on an east-west gradient), there is the possibility that identical lithic resources could be obtained from different areas following substantial changes in range and subsistence strategies.

The following section was undertaken primarily as a test of the hypothesis that the aboriginal inhabitants of the Eagle Hill area utilized the widely distributed local opaline silicate resources, perhaps even selecting this particular locality over others because of the presence of serviceable stone in a region that does not have extensive natural chert deposits. At the same time, it can be considered a partial test of some of the categories of stone described and utilized by Jolly. Although no attempt will be made to verify or negate that hypothesis here, it is hoped that some of the data presented will be useful in operationalizing the model so that it can be tested.

The characterization of the upland area of west central Louisiana as a chertless region is not strictly true. Extensive gravel deposits occur $40 \mathrm{~km}$ to the south in the Quaternary Williana Formation, and gravels also occur in the Pleistocene terraces of both the Sabine and Red Rivers (Welch 1942). In the central upland areas to the north of the Williana contact, however, there are few concentrated sources of siliceous stone. Petrified wood and opals are the primary naturally occurring stones, varying considerably in their serviceability as lithic tool raw materials.

The distance to usable stone is not so great that it would be out of any given group's ordinary range. A source of adequate stone could hardly be more than a couple of days walk from any spot in the Eagle Hill area. But the possibility should be considered that a long trek undertaken solely for tool 
manufacture might be less attractive to an aboriginal uplander than the difficulties involved in finding and working locally available stones. As is implied in Jolly's hypothesis, the aboriginal perception of these difficulties is probably strongly correlated with their ordinary subsistence range.

In order to determine the possibility of the presence of local stone types at the site, a chemical analysis of debitage samples from the site and some locally collected opals was undertaken. For comparison, debitage samples from several adjacent prehistoric sites and chert source samples from several distant areas were also included. The debitage from the site was part of the type collection identified by Joliy. Dr. Frank Serve110, of the University of Southwestern Louisiana, pointed out the location of several nearby opal sources used in the analysis and provided the debitage samples from surrounding sites. An additional sample, from Dowden Creek, was furnished by John Guy, a local avocational archaeologist. The samples from central Texas were collected by the author at various times while the samples from Arkansas were provided by Mark A. Mathis of the North Carolina Department of Cultural Resources. Table 23 gives the provenience of all of the samples used in the analysis.

Because of the small amounts of many trace elements contained in cherts, X-ray fluorescence is not generally sensitive to chemical variation in lithic samples (G. Karl Hoops, The University of Texas at Austin Geology Department, personal communication). Therefore, in contrast to the ceramic samples, no preliminary chemical analyses were conducted on the lithics from the Eagle Hill site. Thin section analyses, however, confirmed that the locally collected materials were indeed opals (E. Garner, personal communication). Lithic samples selected for neutron activation analysis underwent much the same procedures as for the ceramic samples with the following exceptions. Because of the hardness of the lithic samples, they were not drilled; a small bit of each sample was placed in a heavy plastic bag and crushed to a fine powder. Three separate runs, equivalent in length to the ceramic runs were conducted, each with a 100 mi11igram sample of crushed stone. Count time for the shortest run (five minutes for the Tithics) was 10 minutes; for the two hour run count time was 30 minutes; while for the 14 hour run count time was 20 minutes.

Cursory examination of the data set from the neutron activation analysis showed that only a few of the 60 elements contained values for most of the samples examined, and none of the elements were recorded present in every sample. However, comparison of the zero values showed that the presence or absence of certain elements seemed to be correlated with some of the more obvious known groups of samples. For example, the local samples contained small amounts of certain elements not present in the other samples. It appears that the presence or absence of certain elements within the lithic sample may be a useful diagnostic indicator. Therefore, the first analytical method chosen was a discriminant analysis of the known groups within the lithic sample in order to determine the best discriminating elements which could then be used for further analysis.

In the primary discriminant analysis, the debitage from 16 SA 50 and surrounding sites was compared to the locally collected source materials, with the exotic materials left ungrouped. None of the samples were misclassified, and there was little if any ambiguity between the groups. Only four samples (DTM-2, W0-1, IWO, and $\mathrm{GO}_{\mathrm{O}} \mathrm{-1}$ ) had discriminant scores that placed them between the two groups, 
TABLE 23. LITHIC SAMPLE PROVENIENCE DATA

16 SA 50 DEBITAGE

\begin{tabular}{|c|c|c|c|c|}
\hline Sample & Material Type & East & North & Substratum \\
\hline BLM-1 & Black Mottled & 3078 & 998 & 2.13 \\
\hline $\mathrm{BrO}-1$ & Brown Opaque & 3020 & 998 & 4.12 \\
\hline $\mathrm{CH}-1$ & Chalcedony & 3017 & 1001 & 2.13 \\
\hline DT-1 & Dark Tan & 3020 & 998 & 4.12 \\
\hline DT -2 & Dark Tan & 3020 & 997 & 4.17 \\
\hline DTM- 1 & Dark Tan Mottled & 3020 & 997 & 4.17 \\
\hline DTM-2 & Dark Tan Mottled & 3017 & 1001 & 2.13 \\
\hline GO-1 & Gray Opaque & 3020 & 998 & 4.12 \\
\hline LTM- 1 & Light Tan Mottled & 3017 & 1001 & 2.13 \\
\hline LTM-2 & Light Tan Mottled & 3018 & 998 & 2.13 \\
\hline W0-1 & White Opaque & 3020 & 998 & 4.12 \\
\hline wo-2 & White Opaque & 3017 & 997 & 4.17 \\
\hline \multicolumn{5}{|c|}{ COMPARATIVE DEBITAGE SAMPLES } \\
\hline Sample & Material Type & \multicolumn{3}{|c|}{ Location } \\
\hline $\begin{array}{l}\text { IBLM } \\
\text { IGO } \\
\text { IIIBL } \\
\text { IIILTM }\end{array}$ & $\begin{array}{l}\text { Black Mottled } \\
\text { Gray Opaque } \\
\text { Black Mottled } \\
\text { Light Tan Mottled }\end{array}$ & \multicolumn{3}{|c|}{$\begin{array}{lll}\text { Eagle Hi11 } & \text { I, } 0.5 \mathrm{~km} \mathrm{~N} \text { of } 16 \mathrm{SA} 50 \\
\text { Eagle Hi11 } & \mathrm{I}, 0.5 \mathrm{~km} \mathrm{~N} \text { of } 16 \mathrm{SA} 50 \\
\text { Eagle Hi11 III, } 0.5 \mathrm{~km} \text { NW of } 16 \mathrm{SA} 50 \\
\text { Eagle Hi11 III, } 0.5 \mathrm{~km} \text { NW of } 16 \mathrm{SA} 50\end{array}$} \\
\hline
\end{tabular}

\section{COMPARATIVE SOURCE SAMPLES}

\begin{tabular}{|c|c|}
\hline Sample & Material Type \\
\hline IIIE- $]$ & Opal, Light Tan Mott \\
\hline IIIW-1 & Opal, Black Mottled \\
\hline II IW-2 & Opal, Black Mottled \\
\hline PR-1 & Opal, Black Mottled \\
\hline$D C-1$ & Opal, Black Mottled \\
\hline$D C-2$ & Opal, Black Mottled \\
\hline $\begin{array}{l}\text { PW-1 } \\
\text { NF79-1 }\end{array}$ & $\begin{array}{l}\text { Petrified Wood } \\
\text { Gray Opaque }\end{array}$ \\
\hline NF79-2 & Gray Opaque \\
\hline NF80-1 & Gray Opaque \\
\hline NF80-2 & Gray Opaque \\
\hline$P F-1$ & Gray Opaque \\
\hline PITKIN & Black Opaque \\
\hline EVRTON & Brown Mottled \\
\hline
\end{tabular}

Location

Eagle Hill III

Eagle Hill III

Eagle Hill III, not same cobble as II IW-1

Perimeter Road Bridge, $1 \mathrm{~km}$ NW of 16 SA 50

Dowden Creek, $7.5 \mathrm{~km} \mathrm{SSW}$ of $16 \mathrm{SA} 50$

Dowden Creek, $7.5 \mathrm{~km} \mathrm{SSW}$ of $16 \mathrm{SA} 50$, same cobble as $\mathrm{DC}-1$

Mi11 Creek, $9 \mathrm{~km}$ W of $16 \mathrm{SA} 50$

Andice Road, near Georgetown, central Texas

Andice Road, near Georgetown, central Texas, same cobble as NF79-1

Andice Road, central Texas, $5 \mathrm{~km} \mathrm{SE}$ of NF79

Andice Road, central Texas, $5 \mathrm{~km} \mathrm{SE}$ of NF79, not same cobble as NF80-1

Pedernales Falls, near Johnson City, central Texas

Pitkin Chert, north central Arkansas Everton Chert Breccia, north central Arkansas 
but the actual distance from the group centroid was relatively small; only two of these, w0-1, with a probability of distance from the centroid of 0.0413 and DTM-2, with a probability of distance from the centroid of 0.1346 , were below 0.35 probability. Most interesting, however, was the fact that all of the ungrouped exotic chert samples grouped with the debitage from the site. Two of these, NF80-2 and PITKIN had discriminant scores between the two groups, but still much closer to the debitage than the local materials.

Discriminant analyses were conducted using several of the known chert groupings as bases. The best discriminating variables from all of these analyses were then subsequently used in a cluster analys is of the lithic samples. The resulting cluster tree is shown in Figure 52. As is apparent from this tree, the local samples do not cluster well with the debitage samples, and no separate clusters are formed between individual local samples. The closest of these local materials to the main sample clusters are the two Dowden Creek samples, one of which actually enters the larger cluster immediately before the last of the debitage samples to cluster; the black mottled sample from Eagle Hill III joins the cluster. The rest enter at a considerable distance.

The debitage from 16 SA 50 forms two main clusters, one of which (on the left side of the tree) contains most of the debitage samples from the site. This cluster also contains most of the higher order (stronger) clusters formed; the first five samples to join are in this cluster. The other centrally located cluster mixes central Texas source samples with debitage from the site and surrounding areas. Actually, only two debitage samples from the site, $\mathrm{CH}-1$ and $w 0-2$, are included in the higher orders of this cluster; DT-2 does not join until much lower down in the analysis. The other two debitage samples included here are from the surrounding sites, IWO from the top of Eagle Hill and IIILTM from the site surrounding the gully where three of the opal samples were collected.

The strongest cluster in the mixed central Texas and debitage cluster is between $\mathrm{CH}-1$ and one of the Georgetown samples, NF80-1. Although this particular debitage sample is excellent quality chert, certainly worth importing, it not only does not closely resemble the central Texas samples, but it is one of the most prevalent debitage types at the site (see Jo11y, page 290). Thus it is difficult to argue that this group of samples is imported into the site from central Texas. The other 16 SA 50 sample in this cluster, w0-2, is linked first to a visually similar sample, IWO, and then, at a lower level, into the main group.

Little can be said with respect to the meaning of the links formed within the other main cluster. The two distinct dark tan mottled samples, DTM- 1 and DTN -2 , group with one another before joining to any other samples, although the two light tan mottled specimens, which join at the next lower level, are each grouped with another sample first. On the whole, however, there is no obvious grouping in terms of either visual types, local-area-region designations, or excavation substrata.

Since the majority of the debitage samples identified and tested are potentially regional (either regional, local/regional or regional/area), it is somewhat difficult to postulate significant groupings along these lines. It 


$$
\begin{aligned}
& \begin{array}{llllllllllll}
S & \mathrm{~N} & \mathrm{~N} & 1 & \mathrm{~N} & \mathrm{~N} & 1 & \mathrm{E} & 1 & 1 & \mathrm{P} & 1
\end{array}
\end{aligned}
$$

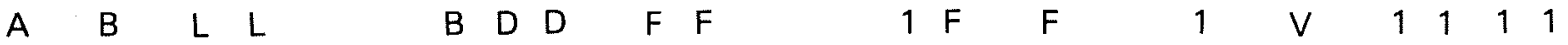

$$
\begin{aligned}
& \begin{array}{llllllllllllllllllllllllllllllll}
M & \mathrm{~L} & \mathrm{D} & \mathrm{T} & \mathrm{T} & \mathrm{G} & \mathrm{W} & \mathrm{r} & \mathrm{T} & \mathrm{T} & \mathrm{D} & 7 & 8 & \mathrm{P} & \mathrm{W} & 1 & 7 & \mathrm{C} & 8 & 1 & \mathrm{D} & 1 & \mathrm{D} & \mathrm{R} & \mathrm{P} & 1 & 1 & \mathrm{~T} & 1 & \mathrm{P}
\end{array} \\
& \text { P M T M M O } 10 \text { O } 0 \text { M M T } 9 \text { O }
\end{aligned}
$$

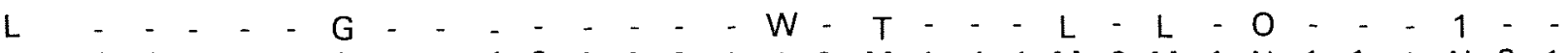

$$
\begin{aligned}
& \text { E } \quad \begin{array}{lllllllllllllllllllllllllllllll}
1 & 1 & 1 & 2 & 1 & 0 & 1 & 1 & 1 & 2 & 2 & 2 & 2 & 1 & 0 & 2 & M & 1 & 1 & 1 & M & 2 & M & 1 & N & 1 & 1 & 1 & N & 2 & 1
\end{array}
\end{aligned}
$$

AMALG.

DISTANCE

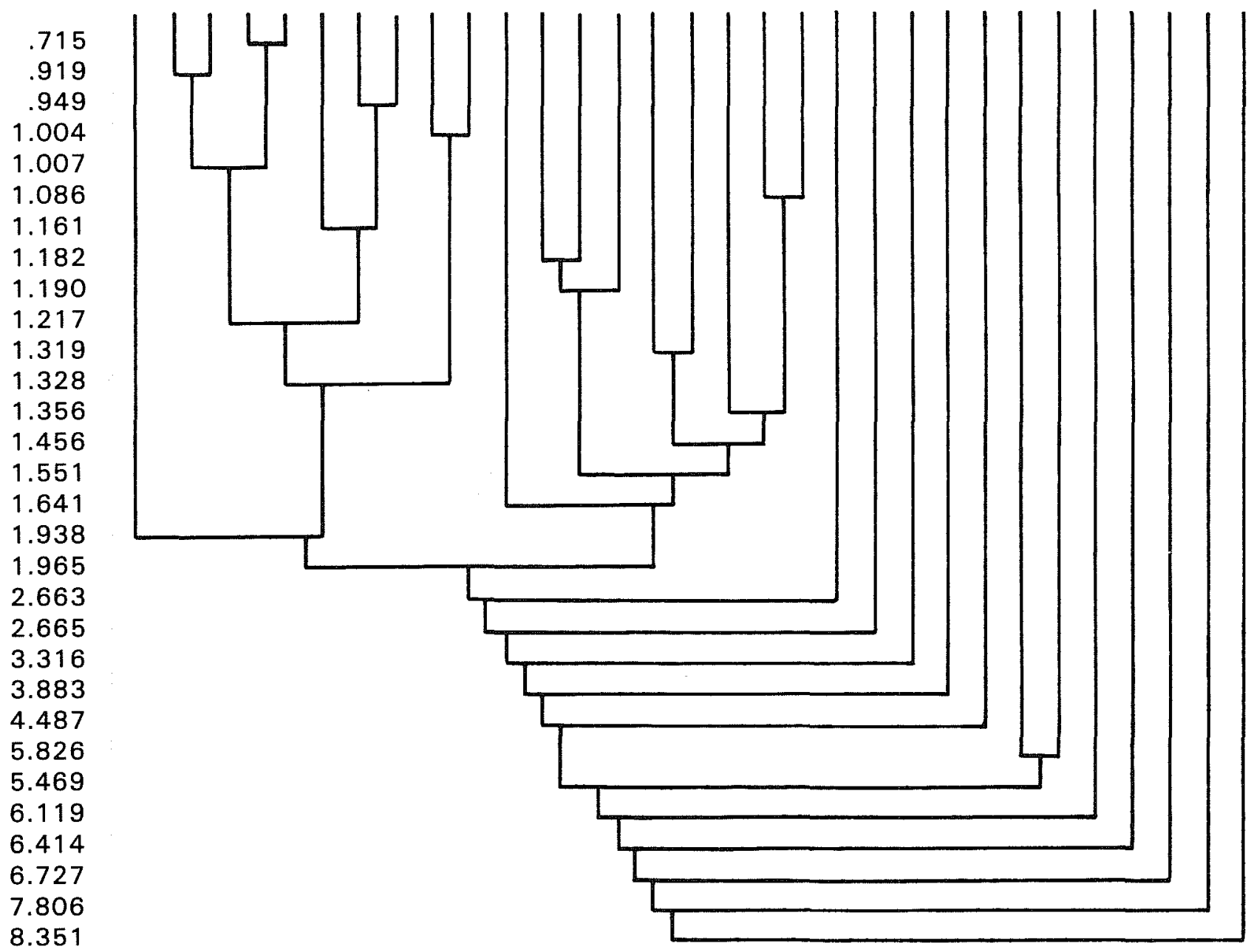

Figure 52. Lithic Cluster Tree. 
is interesting to note, however, that both of the local samples tested, $\mathrm{CH}-1$ and DT-2, group with the mixed central Texas-local debitage cluster. On the other hand, two regional samples are also included in this cluster.

More specific conclusions regarding the relation of the local debitage to area chert resources will have to await further analysis of the local chert gravel deposits. One conclusion seems warranted, though: none of the debitage samples studied shows any relationship to the local opal samples. Although visually similar in some cases, they are chemically quite distinct from these local source materials. It is perhaps possible that either the IIIBLM or IBLM samples, which do not group well with the main debitage clusters, might be local opals; but none of the site debitage shows any evidence of being such. In addition, it is possible that there are at least two major chert sources represented at the site, one which corresponds to the central Texas debitage group and the other corresponding to the main debitage group. The similarities of the former group to central Texas cherts is noted, but there is insufficient evidence as yet to understand the possible relationship.

\section{Conclusions}

An analysis to understand some of the potential relationships existing between artifacts excavated from 16 SA 50 and their possible sources through chemical analyses has been presented. Although no unquestionable source has been identified, the similarities between one of the small group of sherds ( 8 and 20) suggest that they may have been made in the general area of the site. The failure to identify other discrete source areas is unfortunate, but general areas of origin can be hypothesized for further testing with a more extensive collection of source samples. The larger group of sherds, for example, may come from a Miocene upland area similar to the environment of Eagle Hill, while the smaller unusual group may come from sites on the Sabine or Red River floodplain.

Difficulties with the analysis of the chert sample trace elements were anticipated because of the extreme variability in their chemistry, even within the same cobble. The scattered nature of the chert outcrops in this part of Louisiana naturally makes the identification of a discrete source difficult. The fact that there are two distinct groups of chert from the site, one of which clusters with central Texas cherts suggests possibilities for further analysis. And, in itself, the evidence against the utilization of local silicates is significant and somewhat surprising. Analysis of a larger sample of material from the site should provide more data on this. 


\section{CULTURAL CONTEXT AND FORMAL CONTENT}

A. EAGLE HILL ENVIRONMENT, LITHICS, AND DYNAMIC UTILIZATION ANALYSIS (Gunn)

\section{Introduction}

The study of Eagle Hill fell naturally and comfortably into two parts, because of a 6000-year hiatus between the upper and lower strata in the site. The missing interval is significant not only stratigraphically, but also environmentally and culturally. A Paleo-Indian or lithic period is associated with the lower stratum, while the upper stratum is clearly inhabited by ceramic period people. The following sections are organized accordingly. The site is on a ridge, exposed and highly vulnerable to the elements. Marginal habitats, such as Peason Ridge, are sensitive barometers to climate, demography, and probably many other data. As we shall see, properly treated lithics are no exception.

\section{The Eagle Hill Literature Search Problem}

The assemblage of artifacts recovered by excavation from the Eagle Hill site was substantially different from what had been anticipated. The report of the test excavations (Servello n.d.) and previously described sites from the region, such as the John Pearce site (Webb, Shiner, and Roberts 1971) and the Whatley site (Thomas and Campbel1 1978), strongly foretold a Paleo-Indian occupation with large numbers of formal tools, such as points, scrapers, burins, and dril1s. Our careful excavation of $30 \mathrm{~m}^{3}$ of sediments resulted in only enough tools to confirm a Paleo-Indian component at the bottom of the site and a long chronology ranging from the lithic period nearly to the present. Of the thousands of artifacts recovered, over two dozen could conclusively be identified as formal tools. The remainder were flakes and ceramic fragments.

This turn of events altered the literature search from a rather routine examination on the background of regional lithic and ceramic development to an in-depth examination of the literature from a completely different point of view. Our initial tactic would have been to compare tools recovered from Eagle Hill with those found at other sites, determine proportions of tool kits, and infer functions in a standard manner. Full realization of the type of assemblage we were dealing with required a reworking of the original plan. The questions to be resolved became a matter of why the atypical assemblage was at the site and how to make use of the nonstandard data to interpret prehistoric lifeways on top of Peason Ridge. The composition of the assemblage can be explained as a combination of the habitat in which the site is located and the resources available at the location.

The assemblage of flakes and how to make use of it for cultural interpretations complicated the literature review process. We assumed that the flakes we recovered were products of refabricating the types of tools found at other regional Paleo-Indian sites. Our analys is is, therefore, one step removed from the normal examination of formal tools, and our literature search had to be more demanding in order to show the genesis, development, and utilization of 
the envisaged tools from which they came. This combination of circumstances, expecting formal tools and finding only flakes, forced a reorganization of our view of flakes, which we feel may be quite productive. The study of formal tools is, in fact, a study of end products of utilization, the part that was no longer useful to the prehistoric artisan. On the other hand, the study of flakes removed from the still useful tool is an examination of the tool use system in its dynamic aspect. We will return to the idea of "dynamic utilization analysis" presently.

\section{Environmental Control of Peason Ridge Occupation}

It seems that the predominant flake composition of the Eagle Hill assemblage is a product of its location in the regional environment. The site is high and sometimes dry on Peason Ridge. Periods of dryness are a part of both the seasonal cycle and the long term climatic cycles. During seasons or periods of high precipitation and/or low evapotranspiration, the sands of Peason Ridge act as an aquifer. The sands portion out water through springs and streams until it is expended. During periods of low precipitation/high evapotranspiration springs and streams are absent and, no doubt, the ridge is quite dry. Human occupation will be encouraged or discouraged by the availability of water. Sites would only be able to assume permanent status if water was available year round. There is no evidence of permanent occupation. It seems likely that water was never permanent.

In support of this position, local, lang term, periodic dryness can be inferred from the 16 SA 50 sediments. The Eagle Hill site is a peculiar sediment trap, which appears to have partially resisted erosion for at least 100,000 years. A few meters in any direction, however, the beds are eroded down to the Miocene lake deposits. The timing of the erosive periods can be determined from the depositional/erosional sequence in the site. There is a developed Pleistocene soil with the A horizon removed (Soil Horizon III), an early Holocene soil with the A horizon removed (Soil Horizon IIB), and a late Holocene soil (Soil Horizon I) with an A and B horizon.

The rhythm of Holocene climatic change on the Gulf Coastal Plain was extensively researched in section II, and the results correlate logically with the Eagle Hill upland erosional/depositional sequence. The pivotal interval is the Hypsithermal (4500 to 7500 B.P.). The middle Holocene is characterized by net aggradation in the river bottoms of the Southeast. Sedimentation in one part of the system implies erosion in another. Given the biotic evidence for the middle Holocene in the Southeast, it seems reasonable to assume that the dry and probably unstable climate resulted in impoverished upland vegetation and degradation of upland soils, including that on Eagle Hill.

By contrast, the early and late Holocene periods before and after 4500 to 7500 B.P. were generally cooler and wetter, although there were exceptional intervals. We would expect stable, verdant vegetation during these periods in the uplands and net aggradation in topography subject to colluviation. This is, in fact, the situation at Eagle Hill. 
The late Holocene sequence is of special interest relative to the sensitivity of the interaction between the cultural and climatic sequences. Existing deposits start during the cool interval between 1000 and 1300 years ago (A.D. 700-900). This period is marked by a heavy occupation of the site, which supports the inference of a well-watered environment. There is, however, a great concentration of artifacts on So il Horizon I/I I interface, nearly twice as many as any other substructure. One might expect that these artifacts were a result of early Holocene occupation and were deflated from the now eroded Soil Horizon IIA. An intensive analysis of debitage from the test excavation (Servel10 n.d.), however, indicated that the flakes were more closely related to the late Holocene specimens than to the early Holocene assemblage. If we follow the reasoning that Peason Ridge was occupied during moist periods, the period 3400-3900 B.P. is not represented in extant and radiometrically dated horizons at Eagle Hi11. This time period variously termed "Late Archaic" in the Southeast and "Middle Archaic" in Texas, is normally we11 represented in archaeological sites. It seems probable, therefore, that artifacts deflated onto the I/II interface are the remains of a Middle Archaic Eagle Hill occupation. There are two diagnostics to support this contention. Sedimentologically, the inference must be made that there was an erosional episode within the late Holocene.

The 1iterature supports a rather consistent set of cultural-demographic-climatic relationships across the Southeast for contrastive wet and dry periods. The middle Holocene/Middle Archaic is dry and so low-profile culturally that it is practically untraceable in most regions. The dry and unstable climate apparently precluded large populations. The general lack of cultural development in spheres other than subsistence-oriented activities suggest a nomadic lifeway probably restrained by scarce resources. The exceptions which prove the rule are persistently stable "resource oasis" such as the Tennessee Valley (Lewis and Lewis 1961) and the climatically exempted coastal regions (Gagliano 1977). In the immediate region of Eagle $\mathrm{Hill}$, the Whatley site with its ideal microhabitat, natural weir, etc., may be such a place.

Cooler and/or wetter times appear to support populations characterized by growth, progressive stability, and increasing cultural complexity. This phenomenon can be read in the archaeological record as occupation of the uplands, presumably forced by overutilization of lowland habitats. The occupation of upland habitats during the late Holocene is documented in Florida/Georgia (Brose and Percy 1978), the upper Tombigbee River (Blakeman 1975a), and in the lower Mississippi Valley (Gagliano 1977; Haag 1965; Webb 1951). A similar trend appears to be developing in central and south Texas. The environment of Texas is quite sensitive to climatic change (Gunn et al. 1982), and the research by Gerstle, Kelly, and Assad (1978) shows there was a retreat to the lowlands during the inferred hot period between 2000 and 3400 years ago (1400 B.C. to A.D. 1). Peason Ridge would have been only slightly less affected by this trend because of its more easterly location.

The early Holocene appears to have experienced a similar set of cultural/ climatic levels. Clovis populations were sparse either because they were a pioneer culture or because they lived during the relatively warm Two Creek interval. The presence of mammoth in Arizona suggests the former. In contrast, the Dalton occupation of the Southeast, appears to have experienced a 
population explosion. Nearly 300 Dalton localities were reported in the eastern Arkansas region as early as 1973 (Schiffer 1975). The high frequency of Dalton period sites is accompanied by pioneer occupation of the uplands, so that the earliest artifact-bearing levels in most upland sites, such as StanfieldWorley Rockshelter are of Dalton age (Goodyear 1982).

Should this very manageable relationship between climate, population, and occupation of upland habitats stand the test of future research, we can expect to infer that occupation and climate on Peason Ridge are very much in concert, and that we are probably able to account for all of the significant periods of habitation at Eagle $\mathrm{Hill}$ by climatic change. Low occupation frequencies during the middle Holocene would be matched by equally low sediment rates or erosion. There would be no sediment record and no cultural record to be made. Moister early and late Holocene occupations would be duly recorded by sediments.

The 1 ikelihood that there was no middle Holocene occupation on Peason Ridge is supported by the lack of ground stone on the I/II deflated interface. Ground stone is the hallmark of the Middle Archaic. A metate was found in the ceramic age occupation, but there were no ground stone artifacts in the deflated horizon below.

\section{Environmental Control of Recovered Lithic Technology}

In a somewhat different sense, environment is also likely to be the reason for the lack of formal tools at the site. Three factors seem to be relevant. First, the site is removed from sources of high quality cryptocrystallines. Second, it is likely that there was a locally available, low grade source of siliceous materials. The likely product of this combination of circumstances, according to current efforts at a logistical theory for hunters and gatherers, is careful curation and refabrication of prized materials obtained from a distance (Goodyear 1982; Binford 1978). Less valued local materials, used for whatever purposes, were treated in a cavalier fashion. The net result for Eagle Hill is an assemblage in which local, discarded materials are frequent and only flakes remain of the more valued materials that were used, resharpened, and carried on to the next camp.

The fact that no valued material was ever cached at Eagle Hill can further be interpreted to mean that when hunters and/or collectors came to Peason Ridge they had no destination in mind as a part of a regular annual subsistence cycle. Under such a set of assumptions, items so cached would most likely never be retrieved and therefore would be wasted.

It is interesting to note that the assemblage is of a relatively consistent local/exotic composition over a period of ten millenia. Therefore, the site served the same basic function whether the operational base camp was a hunting or an agricultural village. This interesting prospect allows for a certain continuity in our analysis, which would be lost in a more ideally suited locality where function changes with the subsistence base and cultural evolution. In other words, the environmental situation acts as an information filter which allows us to examine adaptive solutions of people with vastly different world views under relatively controlled conditions. It is a good laboratory for the study of culture process rather than culture change. 


\section{Lithic Period Background Research}

As a result of the surprise assemblage recovered at Eagle $\mathrm{Hill}$, we found ourselves returning from the field with a great deal of carefully collected information on platformed flakes, but few tools. We were unwilling, however, to give up the effort to achieve a meaningful distributional analysis of activities on our painstakingly excavated occupation horizons. This led to a re-examination of the problem of lithic use and wear analysis and resulted in a somewhat altered model and approach to the problem.

The concept to which we turned can perhaps best be termed "Dynamic Series Utilization Analysis." As is illustrated in Figure 53, the manufacture of artifacts can be divided into four basic stages. This is the commonly accepted generalized scheme for the use of lithics and accounts for most of the contingencies we might expect at Eagle Hi1l. Naturally, exceptions are likely to occur, but we can assume with some safety that Eagle Hill is a satellite camp because of its environmental situation. Lithic activity will consist mostly of refabrication, and one would expect discard. The reason for the expected lack of discard was discussed earlier as a probable effect of being unsure of destinations when on Peason Ridge. Essential tools were apparently brought to the ridge in a youthful, good condition and virtually always survived the activities so that only debitage remains. It is worth noting that when tools were found they were always solitary items, losses not caches. This conservative attitude toward lithics may have been encouraged by limited access to good lithics in Louisiana.

\begin{tabular}{|c|c|c|c|}
\hline \multicolumn{3}{|l|}{ Location } & Activity \\
\hline \multicolumn{3}{|c|}{ Enter System } & \\
\hline Quarry & \multicolumn{2}{|c|}{ Prefabrication } & $\begin{array}{l}\text { Testing and } \\
\text { Preforming }\end{array}$ \\
\hline Base, Satellite & \multicolumn{2}{|c|}{ 2. Fabrication } & Shaping \\
\hline Satellite, Base, & Field & 3. Refabrication & $\begin{array}{l}\text { Resharpening and } \\
\text { Reshaping }\end{array}$ \\
\hline Satellite, Field & Base & 4. Discard & $\begin{array}{l}\text { Failure to } \\
\text { Refabricate }\end{array}$ \\
\hline
\end{tabular}

Exit System

Figure 53. Stages of Lithic Tool Manufacture and Maintenance Relative to a Satellite Camp such as Eagle Hill.

As a result of this strategy, we will be examining a stage in the 1 ife of a tool, which is obscured by the standard reduction models. This stage is best illustrated in Figure 54 . The fabrication stage of an artifact is a product of some 
sort of norm which accounts for the fact that a tool, if it is to have a long and useful 1ife, must have within its structure the potential for a certain amount of refabrication. This "dynamic potential" falls within the acceptable range of variation for the tool.

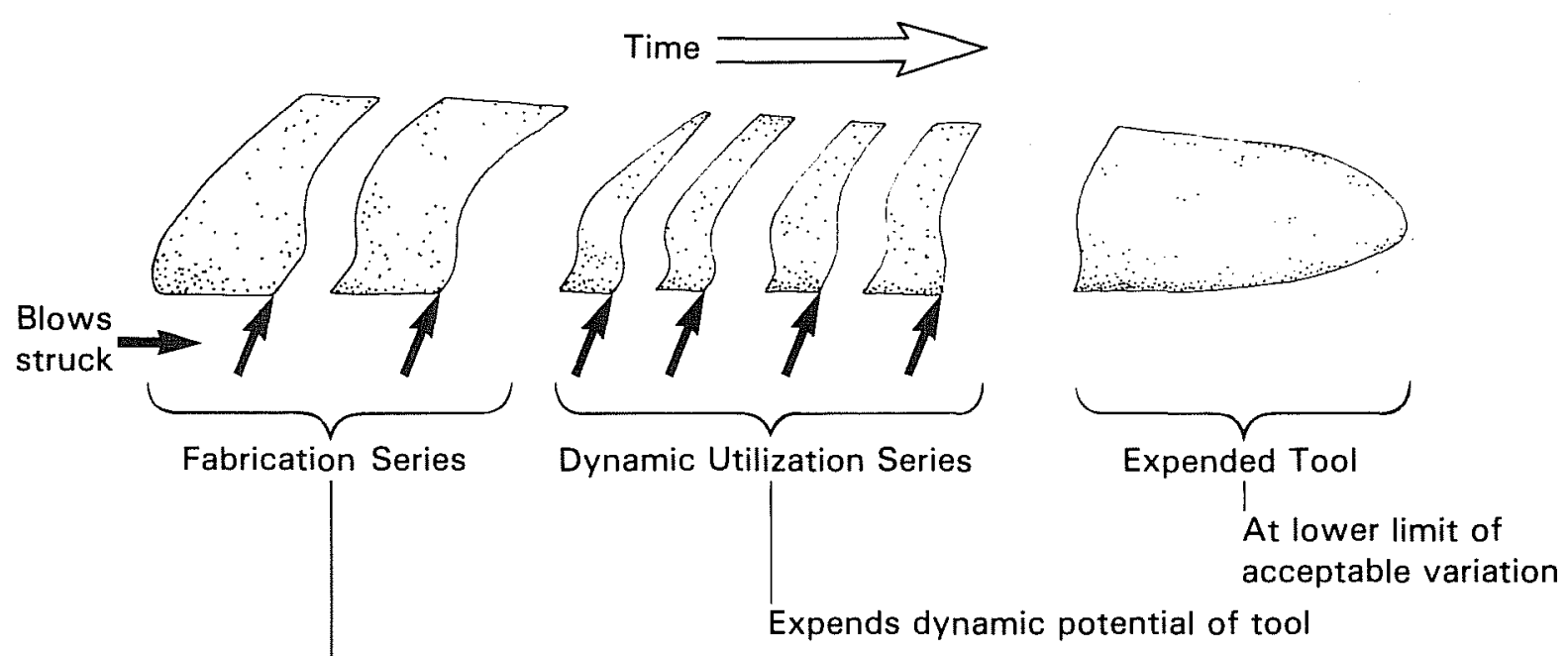

Reduces tool to acceptable range of variation.

Figure 54. Dynamic Potential Lithic Tool Utilization Series Model.

In this model, the refabrication stage becomes a long period in terms of number of steps during which the dynamic potential of the artifact is used up. This dynamic utilization series consists of a number of flakes upon which the evidence of utilization appears. It can appear on the platform edge, the spines, or wherever else the tool contacts the subject material during utilization.

Eventually the tool is refabricated to the point that it no longer exists within the acceptable range of variation for its form. It can no longer be refabricated or it no longer offers the necessary purchase for holding or hafting. At this point, it has to be discarded or recycled to another tool form.

Given the circumstances of an apparent Paleo-Indian site whose neighbors possess sophisticated formal tools, it seems that the flakes recovered from Eagle Hill represent, at least in part, the dynamic utilization series component of the tool life series. The use of this model implied two actions on our part. First, we should do a more thorough than usual examination of the origin and evolution of formal tools in North America, especially relative to environments, in order to understand the likely genesis of our flakes. Second, it implied an attempt to use some of the new 1ithic use-wear analysis techniques on the flakes to verify that this system of Jithic function was operative at Eagle Hill. 
Area Scale Lithic Period Chronology

In the following sections, literature relevant to the lithic period stratum at Eagle Hill will be reviewed. In order to focus this discussion directly on the problem it seemed advisable to formulate a chronology and spatial structure for the relevant areas, regions, and time periods of North America. Table 24 shows this general schema. North America is divided into three areal traditions (Fig. 55) defined by well-known general characteristics of the area. Phases are assigned within each tradition. The terms Pre-Clovis and Proto-Clovis are suggested to account for some new information that is appearing in the archaeological literature.

TABLE 24. AREA SCALE, LITHIC PERIOD CHRONOLOGY

Area/Tradition Llano Enterline Cumberland

\begin{tabular}{|c|c|c|c|c|c|c|}
\hline Period & & Date & & & & \\
\hline Sedento & $\operatorname{ary}$ & 4500-500 B.P. & & & & \\
\hline $\begin{array}{l}\text { Ground } \\
\text { Stone }\end{array}$ & & 7500-4500 B.P. & & $\begin{array}{l}\text { Middle } \\
\text { Archaic }\end{array}$ & $\begin{array}{l}\text { Middle } \\
\text { Archaic }\end{array}$ & \\
\hline \multirow{3}{*}{ Lithic } & \multicolumn{2}{|r|}{ 11,500-7500 B.P. } & $\begin{array}{l}\text { Plano } \\
\text { Folsom } \\
\text { Clovis }\end{array}$ & $\begin{array}{l}\text { Kirk } \\
\text { Late Paleo } \\
\text { Mid Paleo } \\
\text { Clovis }\end{array}$ & $\begin{array}{l}\text { Kirk } \\
\text { Dalton } \\
\text { Clovis }\end{array}$ & $\begin{array}{l}\text { Serrated points } \\
\text { Serrated points } \\
\text { Fluted bifaces }\end{array}$ \\
\hline & \multicolumn{2}{|c|}{$15,000-12,000$ B.P. } & $\begin{array}{l}\text { Proto- } \\
\text { Clovis }\end{array}$ & $\begin{array}{l}\text { Proto- } \\
\text { Clovis }\end{array}$ & ----- & $\begin{array}{l}\text { Mousterioid } \\
\text { industry }\end{array}$ \\
\hline & \multicolumn{2}{|c|}{ ??????-15,000 B.P. } & $\begin{array}{l}\text { Pre- } \\
\text { Clovis }\end{array}$ & & $\begin{array}{l}\text { Pre- } \\
\text { Clovis }\end{array}$ & $\begin{array}{l}\text { Various } \\
\text { possibilities }\end{array}$ \\
\hline
\end{tabular}

Enterline Tradition--Areally the Enterline tradition extends over the present northeastern United States. It is intended to mark those regions usualiy accepted as being periglacial and park tundra during the declining stages of the last glaciation. Naturally there is an element of the Enterline fluting technique and associated tool types intended. However, the Enterline tradition encompasses all apparently Clovis-related cultural manifestations in the area including antecedents.

Llano Tradition--The Llano tradition is conceived of as a similar broad scale concept which encompasses Clovis-related manifestations on the High Plains. Adjacent regions of the Southwest are also a part of the Llano tradition. It again includes antecedents and descendents. The Llano tradition seems to be characteristically big-game oriented, and its demise seems to be rather abruptly marked by the Hypsithermal. The northwestern Louisiana uplands appear to be a 


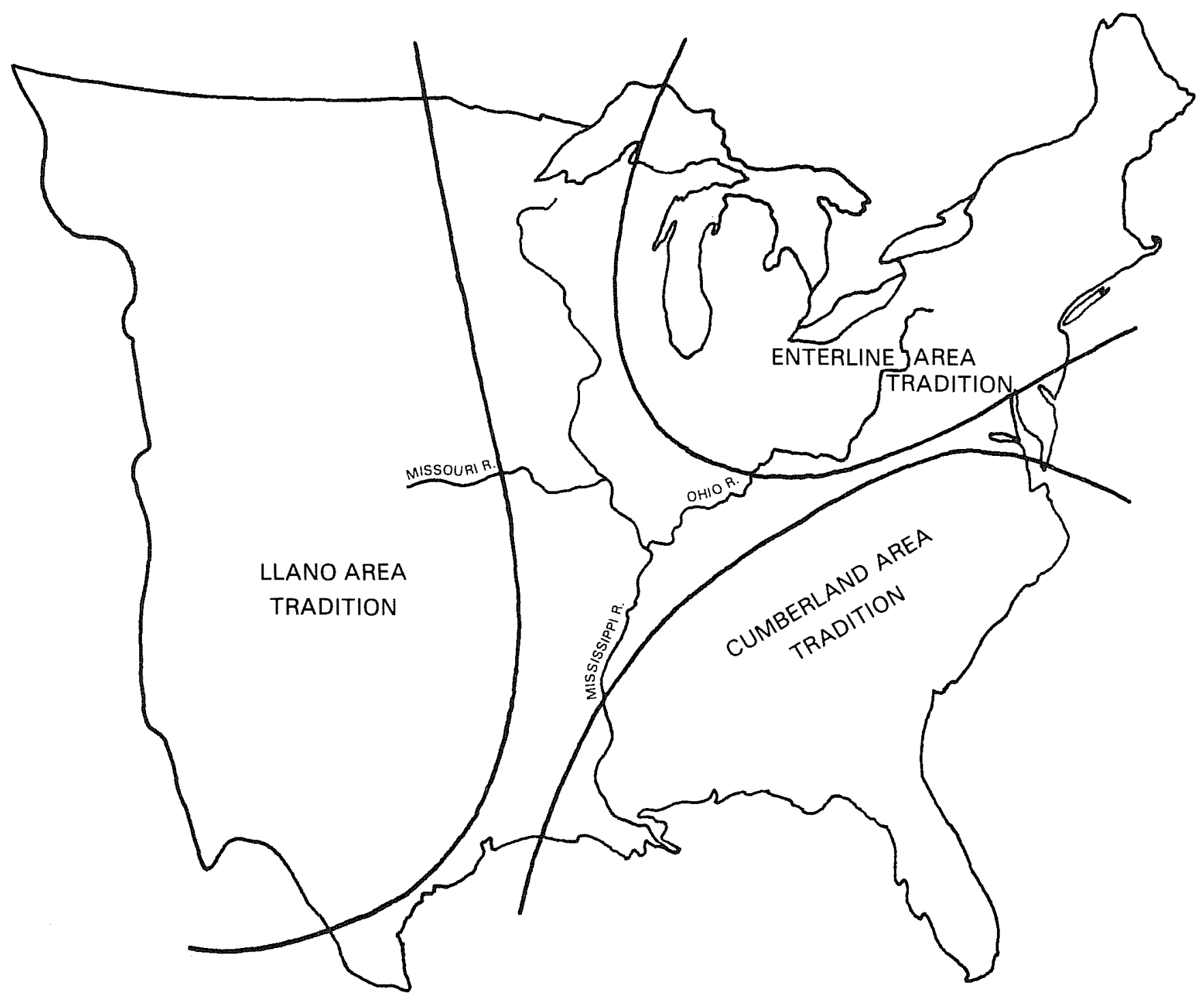

Figure 55. North American Areas Relevant to the Literature Search. 
part of the Llano tradition during some periods (Gagliano and Gregory 1965; Haag 1965).

Cumberland Tradition--The Cumberland tradition appears to have been the first secure riverine adaptation by Clovis pioneers (Muller 1978). It is perhaps the first securely post-Pleistocene tradition in the New World (Fitting 1974). The Cumberland tradition is taken to include the Early Archaic of the Southeast, because of the technological continuity with the Paleo-Indian period. The northwestern Louisiana uplands appear at times to have also been a part of this tradition.

Pre-Clovis--The term is used to imply cultures before and not related to Clovis. So little is understood of Pre-clovis culture that it hardly warrants mentioning. However, a growing body of evidence suggests that it does exist. In fact, Gagliano (1977) has made a convincing argument for a very ancient inhabitant of Mississippi. We are quite sure that whatever Pre-Clovis was, it is not represented at Eagle Hill. A Clovis-Llano tradition tool kit was discovered in the lowest stratum immediately above the culturally sterile Miocene lake sediments at Eagle Hill.

Proto-Clovis--Proto-Clovis is taken to mean antecedent and related to Clovis. Again there is firm evidence that the lowest stratum at Eagle Hill postdates Proto-Clovis. There is a growing and convincing body of evidence that is beginning to define Proto-Clovis culture. Two sites have been discovered in recent years, which do not contain lanceolate projectile points, but predate Clovis. Interestingly enough, they are located in glacial-margin areas of the northern United States. The Shriver site (Reagan et al. 1978) in northwest Missouri and Meadowcroft Rockshelter, western Pennsylvania (Adovasio et al. 1978), contain culture bearing levels below Clovis-related materials. Comparable tool kits containing trianguloid flakes (Mungai knives), bladelets, bifacing flakes, etc., suggest a Mousterioid industry and a logical antecedent to the fluted point tradition. Both of the sites date to about 15,000-13,000 B.P. and are within 200 miles of the ice front. Recent glaciological models of the Tife history of the Wisconsin ice sheet suggest an ice free corridor would have been present at that time. A rapid rise in sea levels about 16,000 B.P. supports this argument (Gagliano 1977).

Clovis--The Clovis phase is well dated and clearly identified in the Plains ca. 11,000 years ago. To date, evidence indicates a dedicated mammoth-hunting culture in the Llano area. The standardized tool kit, especially fluted points, suggests that fluting was probably experimentally developed somewhere in the periglacial homeland and adapted to the Plains environment at an advanced state of application. Fitting's (1974) classic study of cultural adaptation in eastern North America traces a torturous and probable long period of development of fluted points in the East, a position which is supported by the great diversity of points (Kraft 1973) at the Plenge site. Radiometric dates are unfortunately not available to support such a process, and chronological control is not very good in the East.

Hester and Grady (1977) found little evidence for territoriality during Clovis times on the Llano Estacado, instead there was considerable evidence for Clovis hunters of the Plains leading a spectacular wandering existence. Elephants are thought to have migrated from the Gulf Coastal Plain to the glacial fronts on an 
annual round (Churcher 1978). Even if this migratory cycle was 1 imited by half, as is indicated by two variants of mammoth (Eileen Johnson, personnal communication), the migratory movements were still formidable, when compared to later Plains inhabitants. Not until the reintroduction of the horse in historic times were movements again so extensive. As evidence, consider that clovis points made from material collected in the Texas Panhandle have been found washing out of the beaches near Beaumont, Texas (Long 1977). Such transport is not unusual for Clovis materials and suggests that Plains bearers of Clovis culture were indeed well traveled. This wandering seems to have ceased with Folsom people. Hester and Grady (1977) found rather definite evidence for territoriality on the Llano Estacado during the Folsom occupation.

Fitting (1974) thinks that Clovis period people settled rapidly into a more vegetable-oriented existence in the Cumberland area. His analysis stems from the proportions of hunting to gathering tools in respective northern and southern assemblages. It is supported by an apparent preference for islands of rich riverine resources. Clovis-related artifacts are frequently reported from the Tennessee Valley, eastern Arkansas-Mississippi Valley (Morse and Goodyear 1973), and the Mississippi Delta (Gagliano 1977).

During subsequent phases and subphases the contrast between Llano and Cumberland area lifeways became more pronounced. Naturally, the location of Eagle Hill on the border between two highly contrastive culture areas makes it of utmost interest to the archaeological community.

\section{B. LITHIC PERIOD (Mahula) \\ Enterline Tradition}

\section{Introduction}

In this discussion, the Northeast includes all lands east of the Mississippi River and north of Maryland, Virginia, West Virginia, and Kentucky. The northern boundary will tentatively be considered the southern boundary of the Valders ice advance. It must be pointed out, however, that the southern boundary is extremely tenuous since cultural influences from the Southeast blur distinctions between archaeological assemblages in the Northeast and Southeast. The southern margin of the Valders ice is considered (Willey 1966) to have run from southern Maine, across New York and southwestern Ontario, dipping southward around Lake Michigan, and turning northward again through central Wisconsin and southern Minnesota at approximately 11,000 years ago. With the discovery of the Debert site (MacDonald 1968) in Nova Scotia, well dated at approximately 10,600 years ago (Stuckenrath 1965), and the Holcombe Beach site (Fitting, DeVisscher, and Walha 1966), however, some rethinking of glacial chronology and retreat ensued. Borns (1966) proposes, after analysis of the chronological and geological position of the Debert site, that all of the continental ice had dissipated from northern Nova Scotia at the time of occupation. He thinks the margin of the Valders age ice cap was less than 60 miles to the southwest with continuous snow as little as five miles to the north in the Cobequid Mountains. The site is underlain by a layer of aeolian sand deposited by southwestern winds under periglacial conditions from the surface of the wasting continental ice in the Debert River valley. All artifacts were either on or just within the 
aeolian sand suggesting that occupation followed the beginning of the modification process. Debert inhabitants existed in an extremely cold, periglacial environment. Relative sea levels in the area, considering geologic uplifting and eustatic changes, were 100-200 feet lower than at present which would have considerably widened the land corridor from New Brunswick into Nova Scotia. This fact could prove significant to possible migrations of PaleoIndians and perhaps offer a clue as to why they camped at Debert (Byers 1966: 53-55). In addition, there had been a minor oscillation from warmer to colder climate just prior to the occupation of Debert resulting in a tundra condition of continual permafrost. This climate lasted only a very short time, however, and with continual wasting of the ice masses and concomitant elevations in sea level, the character of the locality was soon altered. Distributions of major known early hunter sites in the Northeast illustrate known cultural limits of the region (Fig. 56). The foregoing notwithstanding, however, a pre-Valders occupation of more northerly regions is not an impossibility (Willey 1966; MacDonald 1971; Byers 1966).

In discussing man's earliest entry into the eastern regions of the continent, Fitting (1969, 1974) proposes that early man entering the New World would have had several options open to him. To the northeast, the ice front was bordered by a periglacial zone of treeless to park tundra. It consisted of a ground cover of grasses, sedges, lichens, and willows in the open areas interspersed with scattered clumps of spruce, fir, larch, and birch. Tree stands were densest along waterways and low drainage areas. The entire zone would have been characterized by poorly drained soils. Permafrost was possibly more than $300 \mathrm{~m}$ thick with seasonal thaws reaching only 10-60 cm (Ritchie and Funk 1973; Strahler 1969:251-269). An environment of this sort would have been suited to grazing mammals, such as the barren ground caribou and mammoth, and would have supported a small population of hunters (Ritchie 1969:212; Ritchie and Funk 1973:6-7).

Interdigitating to the south with the park tundra environment would have been a taiga or open cold woodland biome dominated by spruce, fir, jack pine, white pine, and a few deciduous species which would have been the haven for browsing mammals such as the mastodon, woodland caribou, woodland muskox, moose-elk, and some still extant species like deer, elk, moose, bear, and wolf. This environment would have had a high carrying capacity for game and was probably the principal milieu of the early Paleo-Indian hunter over most of the Northeast (Butzer 1964:138; Ritchie and Funk 1973:6-7).

Fringing this bountiful woodland to the south was a needleleaf (or boreal) forest composed of relatively few species of conifers including spruce fir and pine. This type of closed canopy evergreen forest has a low carrying capacity for game and would have been a rather inhospitable and a less productive environment for hunters.

Further to the south, grading into the southeast region, existed a temperate summergreen deciduous forest, characteristic of a humid continental climate, with a southward progression of a predominantly oak forest grading into richer forests of oak, beech, hickory, chestnut, birch, walnut, maple, basswood, elm, ash, and others (Strahler 1969:267; Ritchie 1969:213-214; Ritchie and Funk 1973:8). This area, laden with mast foods, would have held the highest carrying capacity for animals and man. 


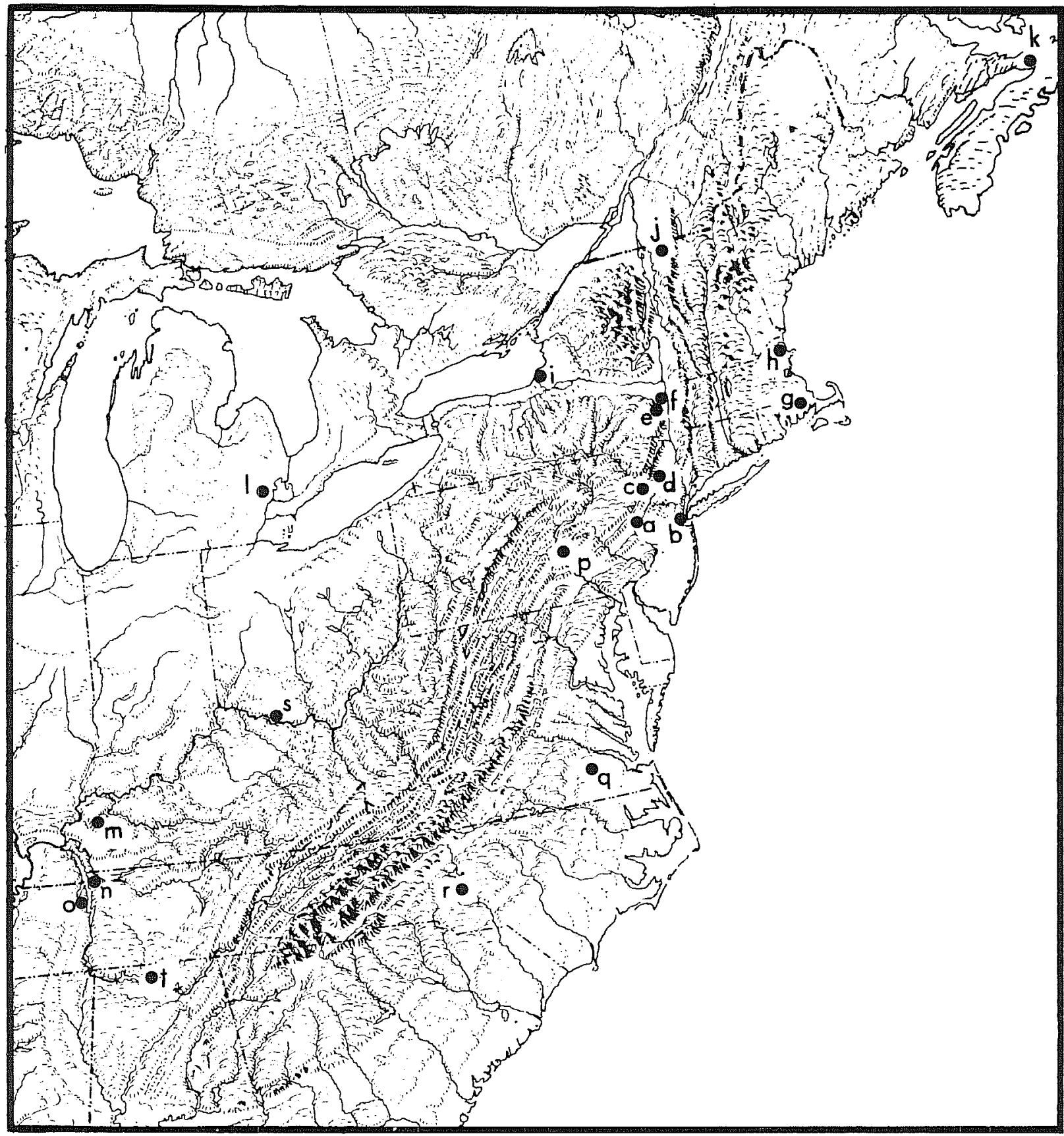

a. Plenge (NJ)

b. Port Mobil (NY)

c. Zierdt (NJ)

d. Dutchess Quarry Cave (NY)

e. West Athens Hills (NY)

f. Kings Road (NY)

g. Wapanucket 8 (MA) h. Bull Brook (MA)

i. Potts (NY)

j. Reagen (VT)

$k$. Debert (Nova Scotia)

1. Holcombe Beach (MI)

m. Parrish (KY)

n. Wells Crater (TN) o. Nuckolls (TN)

p. Shoop (PA)

q. Williamson (VA)

r. Hardaway (NC)

s. Adams Co. $(\mathrm{OH})$

t. Quad (AL)

Figure 56. Sites in the Enterline Area. From Kraft (1973:58). 
The most likely choice for early man from these options would have been to follow the periglacial front eastward along the edge of the retreating ice front (Fitting 1974; Dragoo 1976). The Shriver site and Meadowcroft Rockshelter would be examples of early settlements.

Many no doubt did choose the periglacial environment--as distributions of fluted point sites across the Northeast indicate. Although extremely cold and moist, this familiar environment apparently attracted the ice age hunters who probably wandered in small bands upward along the major river valleys, where they kept principally to the elevated terraces, probably because of higher water levels at this time (Byers 1966; Jennings 1978).

Although the periglacial environment would have probably been a first choice for new explorers, its supportive character would have soon begun to alter. As the ice masses retreated, early hunters would have been forced to continually follow the glacial front northward. The periglacial zone would soon have deteriorated, due to changes in drainage systems with attendant changes in plant and animal associations, and the accompanying increase in periods of darkness with increases in latitude (Strahler 1969:256).

In general, deglaciation of the Northeast followed the autogenic succession patterns observed in the evolution of present day biomes. Although Davis (1969) has noted that the generally accepted progression of boreal forests northward following the maximum glaciation meets with some disconfirmation in the pollen record, most experts agree that, at least in general, the Northeast underwent just such a displacement of biotic zones. This, of course, still allows for considerable local variation due to highly specific combinations of drainage patterns, underlying geomorphic and resulting edaphic components, and local climatic patterns.

The pattern of autogenic succession in the Northeast following deglaciation is as follows. As the glacial masses dissipated leaving poorly drained depressions, bog succession invaded lowlands and shrinking lakes with a progression of sedges, rushes, and peat deposits followed by hygrophytic trees, such as spruce, followed in climax stages by mesophytes such as pine and fir. Most mountainous elevations and plateaus are characterized by closed canopy needleTeaf forests of mesophytes such as fir, pine, and mountain ash, which are dependent upon orographic rainfall, high humidities, and relatively flat temperature curves.

A northward movement of summergreen deciduous forests following glaciation would be dependent upon climatic change fostering humid continental climate which at the same time receives adequate precipitation in all months, and where there is a strong annual temperature cycle with a cold winter season and warm summer. As climatic regimes altered following deglaciation, probably by Middle Archaic times in the Northeast, the richer deciduous forest would have pushed northward fostering the expansion of the Archaic forest adaptation begun earlier in the Southeast. Temperate deciduous forests with a predomination of oak became established in the southern New England area around 8000 B.P. Later additions of beech, hickory, and chestnut with their attendant mast foods around 5000 B.P. set the stage for extensive occupation of the Northeast by Archaic peoples (Davis 1969; Ritchie and Funk 1973:37-39). 
Following occupation of the periglacial region the attention of a hunting economy would have turned most naturally to the Plains and then to the parklike woodlands south of the boreal forests. Fitting (1968) proposes a wave of emmigrants through this great belt of deciduous forest. In addition to being resource "rich," this area was not subject to great climatic fluctuations during the late Pleistocene and Holocene as was the Northeast. In fact, although the early hunter fluted point complexes were first discovered and defined in the Plains region, if sheer numbers of fluted points and accompanying tool types are any gauge, the heaviest Paleo-Indian occupation would have been in the East rather than the Great Plains.

\section{Paleo-Indian Complexes}

Early man complexes in the Northeast are characterized by the fluted points discovered over the entire area and the general similarity in tool assemblages/ types indicating a single, broad pattern of culture prevailing over the area. Some temporal and regional variations in the details. of the typologies as a result of microenvironmental and lithic resource availability do, of course, exist. Byers (1966:36) characterizes this early fluted point tradition as belonging to the LTano complex as designated where first identified in the Great Plains. This complex is represented by a consistent group of unifacial tools composed of standardized end scrapers (most often spurred or "eared" which are almost a diagnostic trait); side scrapers, many also with graver spurs; bifacial and unifacial knives; single and multiple gravers or perforating tools; and lanceolate projectile points with straight to concave bases usually trimmed by the removal of one or more longitudinal flakes from one or both faces. Characteristic types include Clovis and Folsom points with numerous subtypes in each category. Spokeshaves and side scrapers with one or more notched areas (also "strangulated" blades), probably used for processing wood or bone dart shafts, and flint wedges or piéces esquillées are also most consistently found in these early tool kits. Projectile points of similar general lanceolate form (such as Plainview) and lacking flutes are only associated with uniface artifacts similar to those of the Llano complex, so they must be considered as part of another tradition--probably a Late Paleo-Indian adaptation in the Northeast.

Piéces esquillées, as first recognized in North American assemblages at Debert, are described by MacDonald (1968) as being flakes (not necessarily blades or bladelike flakes) which evidence battering on opposite edges probably as a by-product of use as wedges for splintering bone, antler, or ivory. Experimental studies comparing flakes detached by bipolar flaking with those utilized as wedges for splitting wood demonstrated that piéces esquillées "can be as easily replicated by slotting and chiselling functions as they can by the bipolar technique of manufacture" (Cable and Most 1979:10). It is, of course, possible that piéces esquillées were bipolar cores and tools as we11. MacDonald (1968:64) also suggests they could have served the dual purpose of wedge and burin, noting that in 01d World sites as the incidence of piéces esquillées increases, the incidence of burins declines. It has been suggested that piéces esquillées are exhausted polyhedral cores. In many cases the discarded flake resembles (or is mistaken for) an exhausted polyhedral core suggesting an extensive blade industry. 
Early Paleo-Indian assemblages are flake and not primarily blade industries (Byers 1966; Ritchie and Funk 1973). Tools are manufactured on lamellar flakes or reduced from bifacial preforms depending upon resource availability. It is primarily a uniface technology and a conservative one.

Lithic Materials--Another hallmark of Paleo-Indian industries is the use of exotic materials (usually heat treated) in the manufacture of tools. Heat treatment of stone, in order to improve workability, may well be another fundamental Paleo-Indian trait (Crabtree and Butler 1964). As these materials had to be curated from great distances they were generally used in an extremely economical fashion. Many theories have been offered to explain the cultural choices of these exotic materials. These range from purely functional to aesthetic and symbolic hypotheses. Goodyear (1979) hypothesizes that the preference for fine-grained cryptocrystalline materials was the result of a mobile adaptive pattern necessitating a portable and a flexible technology which could be instantly worked, reworked, and refashioned to meet the contingencies of quickly fluctuating hunting resources. High quality, easily flaked material could be more easily converted into new tools when broken. Traveling hunters forced to be prepared for any contingency would need dependable and portable "gear." The importance of being prepared for any eventuality is demonstrated by the Nunamiut Eskimo practice of caching tools at known hunting and lookout spots in order to avoid being caught without necessary equipage (Binford 1978:174).

Exotics are found to some degree in nearly every recorded Paleo-Indian assemblage. High quality cryptocrystalline materials in the Northeast include blue black flint from upper Mercer County, Ohio, chalcedony from Licking County, Ohio, and jasper from southern Pennsylvania.

Prey Species--Due to poor preservation conditions (acid soils, etc.,) there has been little success in gaining evidence on faunal species exploited by early man in the Northeast. At Dutchess Quarry Cave (Funk, Walters, and Ehlers 1969), six caribou bones were found in a basal stratum with a cumberland fluted point and remains of modern fauna. Collagen from the caribou bones was radiocarbon dated to 12,530 B.P. However, the extreme age of the site (older than mammoth kill sites on the Plains) and the relatively late Paleo-Indian fluted point type, casts some doubt on the reliability of the association. Since sites such as Debert, Holcombe Beach, and Bull Brook (Byers 1954) were apparently reoccupied on a seasonal basis for long periods of time, caribou would have probably been the only species which would have been available in enough quantity to support settlements of this size and concentration. Also, utilizing animals with well-known migratory habits would allow the establishment of a central hunting camp, as opposed to many scattered kill sites. Remains of mammoth and mastodon, its forest browsing cousin, have been found in the Northeast and would have been available in some numbers for exploitation by early man.

The first incontrovertible association of mastodon and Clovis-type 1ithics recentiy appeared in the literature (Graham et al. 1981). Although the mastodon may have been the primary motivators for movements of people, other available animals were probably utilized as well. In fact, the "idea of elephant hunters based on the western United States model is probably a gross exaggeration" (Ritchie 1969:103). It is more likely they would have followed a balanced hunting economy adjusted to local conditions. 
Site Types--Ritchie and Funk (1973) describe three basic types of sites for the Paleo-Indian phase in the Northeast: major recurrent seasonal occupation camps, small temporary hunting camps such as in rockshelters in caves, and quarry workshops. Debert, Holcombe Beach, and Bull Brook are representative of the first type and point strongly to the recurrent occupation of a favorable locale for the purpose of caribou hunting. Binford (1978:169-178) describes the Nunamuit patterns of spring campsites where numerous families come together seasonally at a strategically placed locale for the purpose of intercepting large herds of caribou during spring migrations. The caribou move in herds of 100-1000 through migration routes at intervals of twenty minutes up to one day. Groups are able to move up from the base camp to dispatch the animals as they pass through the restricted area. Aside from choosing a strategic position along the migration route, camps are placed in or near a good stand of willows near a source of reliable water, ice, or spring overflow and protected to some degree from the wind. Binford (1978:169) also describes many smal1 or solitary scouting campsites from which individual hunters scout and predict herd movements and harvest early meat. This type of camp corresponds to such sites as Dutchess Quarry Cave and the numerous small fluted point finds scattered over the area, often in rockshelters and caves. Quarry workshops are self-explanatory and are evidenced by sites such as Williamson (McCary 1951) and West Athens Hill (Ritchie and Funk 1973).

Representative Sites and Tool Kits

Debert--Lithic industries evidenced at Debert (MacDonald 1968, 1971) exhibit extreme economy of material. A notable aspect of technology is the use of irregular flakes with only minor modification by retouch. Extreme conservatism has probably precluded the preservation of complete bifacial cores. No blades are found on the site: $80 \%$ of the assemblage are unifacial, predominantly end scrapers (1600), most of them spurred. Six types of end scrapers were identified. The most common was a peculiar "eared" variety limited in the Northeast to Paleo-Indian assemblages. Other types included round bit, end of flake (no blades at Debert), and humped-back. Side scrapers occurred in myriad forms. One percent of the assemblage was spokeshaves. Bifaces included two types of bifacial dri11: a basally fluted twist drill for hafting, and a wide-based, hand-held variety. Other tools in the bifacial category were piéces esquillées (15\%), bifacial knives, and two large thin bifaces.

Plenge--The Plenge site (Kraft 1973) is the first extensive Paleo-Indian site to be discovered in New Jersey. It is located in an area of extremely rich lithic resources in the form of cobbles of shale, flint, jasper, and chalcedony deposited by terminal moraines. There was a long, intensive occupation of the site from Paleo-Indian to Early Archaic, probably due to the quantity of quality material. Numbers of fluted artifacts were found: knives, points, and preforms. Of the 49 points, $59.2 \%$ had a single flute on each side; $40.8 \%$ showed multiple flutes on each side. Three sma11, hafted knives similar to those from Debert were recovered; two were reworked from points, while one was made on a flake. Of the total inventory, $10.4 \%$ were bifacial knives of two different types--blunted knives on flakes and crude biface knives. Unifacial artifacts included side scrapers of varying design, with end scrapers making up the bulk of the assemblage. Of the end scrapers, $32.6 \%$ had graving spurs and probably many more were worn away. Thumbnail and steeply beveled 
"keeled" or hump-backed scrapers were present along with end-of-blade scrapers and hinge fracture scrapers. Spokeshaves (concave scrapers) and "strangulated blades" unifacially chipped, predominantly with a single concavity, accounted for $5.5 \%$ of the total inventory. Other tools included burins, gravers (many hafted), beaks, denticulates, thick biface drills, perforators, and piéces esquillées. Although the latter are rare--Kraft (1973) suggests possibly they are not found as frequently at sites where lithic materials are abundant. Table 25 compares artifact types and 1ithic techniques at Plenge with other northeastern and some Plains Paleo-Indian sites.

Bull Brook--Fluted points recovered from Bull Brook resemble clovis points and are similar to those found at Williamson and Shoop. They are believed to be representatives of the Enterline Chert industry. Finely chipped, needlelike uniface gravers, some with more than one point are also found, along with examples of a bifacial "twist" drill and one "spokeshave shaped" instrument. Snub-nosed end scrapers with the bulb of percussion removed are characteristic. Other tools include side scrapers with graver spur on heels, thin side scrapers made from retouched blades, retouched blades, and Enterline side scrapers. Byers (1954) points to the similarity of the Bull Brook site assemblage with that of the Lindenmeier site. Again, others suggest these assemblages are flake rather than true blade industries. No polyhedral cores were recovered.

West Athens Hill--West Athens Hill (Ritchie and Funk 1973) is a quarry-campsite on the Hudson River. Most of the material at the site is Normanskill flint with small amounts of Pennsylvania jasper. Occupation probably dates back to early Anathermal with vegetation sparse along the rocky hill upon which the site rests. Large biface cores are absent. Bifaces recovered were grouped into four sets, each representing some stage in the production of projectile points and knives. Thirteen fluted points were excavated, all conforming to the clovis fluted type. Unlike many other Paleo-Indian sites, side scrapers outnumber end scrapers. Side scrapers were made from almost any convenient flake, blank, or core, whatever its size or shape. Most conform to the "ear-shaped" category found at many eastern Paleo-Indian sites. Others were of a "turtleback" form. Unique was a massive, fist-sized "pulping plane" heavy tool with a roughly retouched edge. The great majority of end scrapers are of the classic trianguloid or trapezoidal form with the bulbur face unmodified. A small proportion are of irregular form. Two are double ended. Some have sharp or right-angled corners which, like spurs, could have been used for graving. Only one graver and only five examples of piéces esquillées were found in the collection. This lack of piéces esquillées at a site where lithic material is in abundance conforms to the pattern at the Plenge site. Piéces esquillées may well be a product of an extremely conservative lithic industry.

Shoop--Shoop is the type site of Witthoft's (1952) Enterline Chert industry. He proposes this to be the oldest cultural manifestation in America and describes it as a blade industry without microliths (see disagreement with this proposition in earlier section). Tools include pointed side scrapers with the tip or bit generally at the bulb end of the "blade," end scrapers, bladelets, flake knives, and uniface gravers based on flakes. Tools are predominantly manufactured of Onodaga chert from an area 200 miles from the site. 
TABLE 25. COMPARISON OF ARTIFACT TYPES AND LITHIC TECHNIQUES IN SOME NORTH AMERICAN FLUTED POINT INDUSTRIES

\begin{tabular}{|c|c|c|c|c|c|c|c|c|c|c|c|c|c|c|c|}
\hline \multirow[b]{2}{*}{ Artifact Types } & \multirow[b]{2}{*}{ Selected Attributes } & \multirow[b]{2}{*}{$\begin{array}{l}\frac{d}{+} \\
\frac{1}{n} \\
0 \\
\frac{5}{0} \\
\frac{c}{2}\end{array}$} & \multicolumn{11}{|c|}{$\begin{array}{c}\text { Northeastern } \\
\text { Sites }\end{array}$} & \multicolumn{2}{|c|}{$\begin{array}{c}\text { Western } \\
\text { Sites }\end{array}$} \\
\hline & & & 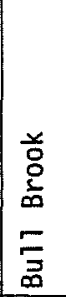 & $\begin{array}{l}+ \\
\frac{1}{d} \\
0 \\
0 \\
0\end{array}$ & $\begin{array}{l}\stackrel{.}{~} \\
\frac{0}{0} \\
\frac{0}{0}\end{array}$ & 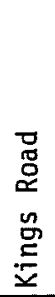 & 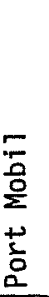 & $\stackrel{\stackrel{n}{+}}{\stackrel{0}{0}}$ & 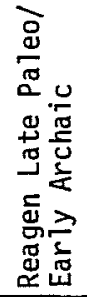 & $\frac{2}{\frac{0}{0}}$ & 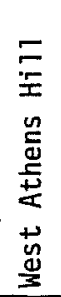 & 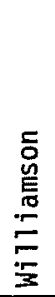 & 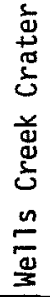 & 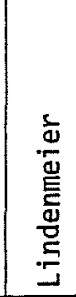 & 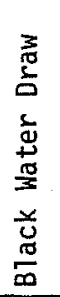 \\
\hline Fluted points & $\begin{array}{l}\text { Debert } \\
\text { Clovis-like } \\
\text { Folsom-like } \\
\text { Stubby } \\
\text { Lanceolate } \\
\text { Plano-like } \\
\text { Unfluted, Paleo }\end{array}$ & $\begin{array}{l}\mathrm{x} \\
\mathrm{x} \\
\mathrm{x} \\
\mathrm{x} \\
\mathrm{x} \\
\mathrm{x} \\
\mathrm{x}\end{array}$ & $\begin{array}{l}- \\
x \\
x \\
- \\
- \\
- \\
-\end{array}$ & $\begin{array}{l}X \\
- \\
- \\
- \\
- \\
- \\
-\end{array}$ & $\begin{array}{l}- \\
x \\
- \\
\bar{x} \\
- \\
-\end{array}$ & $\begin{array}{l}- \\
x \\
- \\
- \\
- \\
- \\
-\end{array}$ & $\begin{array}{l}- \\
x \\
- \\
x \\
- \\
- \\
-\end{array}$ & $\begin{array}{l}- \\
x \\
- \\
- \\
- \\
- \\
-\end{array}$ & $\begin{array}{l}- \\
x \\
- \\
x \\
x \\
- \\
x\end{array}$ & $\begin{array}{l}- \\
x \\
- \\
- \\
- \\
-\end{array}$ & $\begin{array}{l}- \\
x \\
- \\
- \\
- \\
-\end{array}$ & $\begin{array}{l}- \\
\bar{x} \\
\bar{x} \\
x \\
- \\
-\end{array}$ & $\begin{array}{l}\bar{z} \\
\bar{x} \\
\overline{-} \\
\overline{-} \\
\overline{-}\end{array}$ & $\begin{array}{l}- \\
- \\
x \\
- \\
- \\
-\end{array}$ & $\begin{array}{l}- \\
x \\
- \\
- \\
- \\
-\end{array}$ \\
\hline Bifacial Knives & $\begin{array}{l}\text { Large (over } 10 \mathrm{~cm} \text { ) } \\
\text { Smail } \\
\text { Concave based eared } \\
\text { Fluted complete }\end{array}$ & $\begin{array}{l}x \\
x \\
x\end{array}$ & $\mathrm{x}$ & $\begin{array}{l}x \\
x \\
x\end{array}$ & $x$ & $x$ & & $\begin{array}{l}\mathrm{x} \\
\mathrm{x}\end{array}$ & $\begin{array}{l}x \\
x\end{array}$ & & $\begin{array}{l}x \\
x\end{array}$ & & $\begin{array}{l}x \\
x\end{array}$ & $\begin{array}{l}x \\
x \\
x\end{array}$ & $x$ \\
\hline Drills & $\begin{array}{l}\text { Bifacial chipping } \\
\text { Unfluted on flake } \\
\text { Unfluted expanding } \\
\text { base } \\
\text { "Twist" drills }\end{array}$ & $\begin{array}{l}x \\
x\end{array}$ & $\begin{array}{l}x \\
- \\
- \\
x\end{array}$ & $\begin{array}{l}x \\
x \\
- \\
-\end{array}$ & $\begin{array}{l}\overline{-} \\
\overline{-} \\
\overline{-}\end{array}$ & & - & $\begin{array}{l}- \\
- \\
? \\
-\end{array}$ & $\begin{array}{l}- \\
- \\
-\end{array}$ & $\begin{array}{l}- \\
- \\
-\end{array}$ & $\begin{array}{l}- \\
- \\
-\end{array}$ & $\begin{array}{l}x \\
- \\
x \\
x\end{array}$ & $=$ & $\begin{array}{l}- \\
- \\
x \\
-\end{array}$ & $\begin{array}{l}- \\
- \\
-\end{array}$ \\
\hline Piéces esquillees & & $x$ & & $x$ & $x$ & & & - & - & $x$ & & $?$ & $=$ & $x$ & - \\
\hline Enid scrapers & $\begin{array}{l}\text { Spurred } \\
\text { Rounded bit } \\
\text { Hump back } \\
\text { "End of blade" }\end{array}$ & $\begin{array}{l}x \\
x \\
x \\
x\end{array}$ & $\begin{array}{l}x \\
x \\
- \\
x\end{array}$ & $\begin{array}{l}x \\
x \\
x \\
x\end{array}$ & $\begin{array}{l}x \\
x \\
- \\
-\end{array}$ & $\begin{array}{l}x \\
x \\
x\end{array}$ & $x$ & $\begin{array}{l}x \\
x \\
x \\
x\end{array}$ & $\begin{array}{l}x \\
x \\
x \\
x\end{array}$ & $\begin{array}{l}\mathrm{x} \\
\mathrm{x} \\
?\end{array}$ & $\begin{array}{l}x \\
x\end{array}$ & $\begin{array}{l}x \\
x \\
- \\
-\end{array}$ & $\begin{array}{l}x \\
x \\
- \\
-\end{array}$ & $\begin{array}{l}\mathrm{x} \\
\mathrm{x} \\
\mathrm{x} \\
?\end{array}$ & $\begin{array}{l}? \\
\mathrm{x} \\
- \\
-\end{array}$ \\
\hline Side scrapers & $\begin{array}{l}\text { Subtriangular } \\
\text { Bulb at tip } \\
\text { Ridge-backed } \\
\text { Form with graver spur }\end{array}$ & \begin{tabular}{l|}
$x$ \\
$x$ \\
$x$ \\
$x$
\end{tabular} & $\begin{array}{l}x \\
x \\
x \\
\end{array}$ & $\begin{array}{l}\mathrm{x} \\
\overline{-} \\
\mathrm{x}\end{array}$ & $\begin{array}{l}\mathrm{X} \\
- \\
- \\
-\end{array}$ & $x$ & & $\begin{array}{l}x \\
x \\
x \\
x\end{array}$ & $\begin{array}{l}x \\
x \\
x \\
x \\
\end{array}$ & $\begin{array}{l}\mathrm{x} \\
- \\
\mathrm{x} \\
\mathrm{x}\end{array}$ & $\begin{array}{l}x \\
x \\
x\end{array}$ & $\begin{array}{l}x \\
- \\
- \\
-\end{array}$ & $\begin{array}{l}? \\
- \\
- \\
-\end{array}$ & $\begin{array}{l}x \\
- \\
x \\
- \\
\end{array}$ & $\begin{array}{l}? \\
\bar{x} \\
-\end{array}$ \\
\hline \multicolumn{2}{|c|}{$\begin{array}{l}\text { Flake perforators } \\
\text { Stone Awls } \\
\text { Gravers } \\
\text { Chisel gravers } \\
\text { Spokeshaves } \\
\text { Scraping planes } \\
\text { Choppers } \\
\text { Hammerstones } \\
\text { Bone artifacts } \\
\text { Pigment }\end{array}$} & \begin{tabular}{l|}
$\mathrm{x}$ \\
$?$ \\
$\mathrm{x}$ \\
- \\
$\mathrm{x}$ \\
$?$ \\
$\mathrm{x}$ \\
$\mathrm{x}$ \\
- \\
-
\end{tabular} & $\begin{array}{l}? \\
\mathrm{x} \\
\mathrm{x} \\
- \\
\mathrm{x} \\
- \\
- \\
\mathrm{x} \\
- \\
\mathrm{x}\end{array}$ & $\begin{array}{l}x \\
x \\
x \\
- \\
x \\
x \\
x \\
x \\
- \\
x\end{array}$ & $\begin{array}{l}\mathrm{x} \\
- \\
\mathrm{x} \\
- \\
\mathrm{x} \\
? \\
? \\
\mathrm{x} \\
- \\
-\end{array}$ & $\begin{array}{l}- \\
- \\
x \\
x \\
- \\
-\end{array}$ & $\begin{array}{l}x \\
-\end{array}$ & $\begin{array}{l}\overline{-} \\
\bar{x} \\
\bar{x} \\
- \\
- \\
- \\
- \\
-\end{array}$ & $\begin{array}{l}\overline{-} \\
\bar{x} \\
\bar{x} \\
\overline{-} \\
\overline{-} \\
\overline{-} \\
-\end{array}$ & $\begin{array}{l}x \\
x \\
x \\
- \\
- \\
- \\
- \\
- \\
-\end{array}$ & $\begin{array}{l}x \\
- \\
- \\
\end{array}$ & $\begin{array}{l}- \\
- \\
- \\
- \\
- \\
- \\
x \\
-\end{array}$ & $\begin{array}{l}\text { ? } \\
- \\
- \\
- \\
\bar{x} \\
? \\
\bar{x} \\
- \\
-\end{array}$ & $\begin{array}{l}? \\
- \\
x \\
x \\
? \\
x \\
x \\
x \\
x \\
x\end{array}$ & $\begin{array}{l}- \\
- \\
- \\
- \\
- \\
- \\
- \\
- \\
x \\
-\end{array}$ \\
\hline \multicolumn{2}{|c|}{$\begin{array}{l}\text { Bifacial cores } \\
\text { Flakes with bifacial striking platforms } \\
\text { Polyhedral cores } \\
\text { Elongated flakes } \\
\text { Ground striking platforms } \\
\text { Ground point margins }\end{array}$} & $\begin{array}{l}\mathrm{x} \\
? \\
- \\
\mathrm{x} \\
\mathrm{x} \\
\mathrm{x}\end{array}$ & $\begin{array}{l}- \\
? \\
\bar{x} \\
x \\
x\end{array}$ & $\begin{array}{l}- \\
x \\
- \\
x \\
x \\
x\end{array}$ & $\begin{array}{l}\mathrm{x} \\
? \\
\overline{\mathrm{x}} \\
\mathrm{x} \\
\mathrm{x}\end{array}$ & - & - & $\begin{array}{l}\bar{?} \\
\bar{x} \\
\mathrm{x} \\
\mathrm{x}\end{array}$ & $\begin{array}{l}\overline{-} \\
? \\
\bar{x} \\
? \\
\mathrm{x}\end{array}$ & $\begin{array}{l}- \\
? \\
\bar{x} \\
x \\
x\end{array}$ & $\begin{array}{l}- \\
-\end{array}$ & $\begin{array}{l}- \\
- \\
? \\
? \\
\bar{x}\end{array}$ & $\begin{array}{l}\mathrm{x} \\
\mathrm{x} \\
\mathrm{x} \\
\mathrm{x} \\
\mathrm{x} \\
\mathrm{x}\end{array}$ & $\begin{array}{l} \\
x \\
x \\
x \\
x \\
x \\
\end{array}$ & $\begin{array}{l}\mathrm{x} \\
\mathrm{X} \\
? \\
\mathrm{x}\end{array}$ \\
\hline \multicolumn{2}{|l|}{ Sample size } & $\frac{1}{5}$ & \& & & & $\stackrel{+}{\stackrel{m}{m}}$ & & $\widetilde{\sigma}$ & $\stackrel{\infty}{=}$ & 总 & 志 & 㺼 & 志 & $\sim$ & œ. \\
\hline
\end{tabular}

SOURCE: Modified from MacDonald 1968:144 and Kraft 1973. 
Late Paleo-Indian Developments

Traces of the Late Paleo-Indian period in the Northeast are very rare and sporadic and are probably best represented by sites such as the Reagan site (Ritchie 1953) in northwestern Vermont, the Brohm site (MacNeish 1952) on Thunder Bay on Lake Superior, and the Renier site (Mason and Irwin 1960) in northeastern Wisconsin. Stray finds of Plano points have been discovered as far north as New Brunswick in Canada. They are rare in the central Northeast, but are found frequently in the Ohio and Tennessee Valleys. Further to the south and east they appear to diminish, while Dalton points, a Late PaleoIndian type from the Southeast, take over in complementary distribution (MacDonald 1971:37). A cultural trade-off seems to exist between the Plains and the Southeast during the Late Paleo-Indian period, with Plano points filtering slightly southeastward, and Dalton influences moving into the Plains, as evidenced by the presence of the Meserve point on the southern Plains.

The Reagan site in Vermont documents the end of the fluting tradition in the Northeast. Lanceolate projectile forms with parallel flaking are found in a typical Enterline Chert industry assemblage, although possibly a "less pure" expression (Byers 1954). One form of projectile point, a fluted lanceolate pentagonoid form, resembles the fluted lanceolate pentaganoid group from the Williamson site and is also classified as belonging to the Enterline Chert industry (Witthoft 1952). The rest of the assemblage is composed of typical eared and spurred end scrapers, side scrapers, flake knives, gravers, and combinations thereof. A sandia-reminiscent, weak-shouldered knife (?) was also recovered. The too $1 \mathrm{kit}$ is in $\mathrm{a} 11$ other respects representative of those of the fluted point tradition. The Reagan site may actually be considered a Tate Middle to Late Paleo-Indian transition.

The Brohm site and the Renier sites are representative of a Plano occupation a long the Northern Great Lakes between 10,000 and 8000 years ago. Apparentiy groups of Plains-adapted groups moved northeastward during warmer times and occupied lakeside environments in this area. Both sites were seemingly occupied for a limited time and not reoccupied again. There is no evidence of a Late Paleo-Indian to Archaic transition on the Great Lakes. The Plano occupation is represented at Renier by ripple-flaked, lanceolate points, Eden and Scottsblufs points, ripple-flaked scrapers, and other forms. Large ovate bifaces of quartzite were also found (Mason and Irwin 1960; MacDonald 1971).

The Brohm site on Thunder Bay yielded seven typical Plainview points and a series of large blades made of jaspery taconite. Two large triangular points and a long, round-based point with ripple flaking were also found along with a bifacially chipped drill (similar in form to Archaic and Woodland forms), several large knives or "daggers," and some square and ovoid thin bifaces. Fan-shaped, snub-nosed end scrapers almost identical to those from the Plainview site and Folsom points similar to those found at the Lindenmeier site were recovered, as well as other forms of ripple-flaked end scrapers, large side scrapers, and flake knives. Large, pointed or "pick-like" choppers and ovoid choppers completed the tool kit. 


\section{Transitional and Early Archaic}

There has been no clearly defined transition from the Late Paleo-Indian to the Archaic period in the Northeast. Since the region was subject to major shifts in environment, it was occupied and abandoned or at least subjected to extremely low population density as ecological conditions altered. "The Northeastern picture suggests rather a displacement of Plano industries on ly weakly represented at best, with Archaic industries from the South" (MacDonald 1971:38). As deciduous forests moved northward, already adapted Archaic cultures moved, or at least their influence moved, northward with the advancing forest line. There has been some suggestion of very late Plano to Early Archaic development at the Canadian Shield at about 7000 years ago as evidenced by the presence of generalized lanceolate forms in the Shield Early Archaic.

There has been a absence in the Northeast of we11-dated Archaic cultures older than 5000 years ago. Recent discoveries made on Staten Island, New York, have produced the first Early Archaic expressions similar to those found in the Southeast. (A11 reported by Ritchie and Funk 1973:38.)

The Hollowell site revealed an Early Archaic assemblage previously unknown in the Northeast: 30 projectile points, most with bifurcated bases, conforming closely with the Kanawha type dated at 8210 B.P. at the St. Albans site, and an Eva Notched type. Also found were several large, ovate choppers, biface knives, small flake scrapers (some end and some side), utilized flakes, hammerstones, abrading stones, an adze fragment, and two bifacially chipped celts with ground bits.

Ward's Point revealed Kanawha Stemmed, Kirk Stemmed, and Lecroy BifurcatedBase points, a biface knife, retouched flake side and end scrapers, a small retouched tool with three graving spurs, a dril1 fragment, about 75 choppers, anvilstones, abrading stones, and a possible bannerstone fragment. Two charcoal samples have been dated at between 7310 and 8300 B.P.

Richmond Hill has produced small corner-notched, ground-based Palmer points, Kirk Corner-Notched points, what appears to be a Hardaway point, retouched flake end and side scrapers, and choppers. A hearth sample yielded a date of 9410 B.P.

The Middle Archaic period is also hazy. However, late Middle to Late Archaic cultures in New York are well represented and will be discussed here for comparison. Diagnostics for the Lamoka culture include beveled adzes and certain bone and antler items: celts, adzes, chisels, worked beaver incisors, bone awls, antler flakers, drills, anvilstones, and hammerstones. Fishing was of primary importance as well as the hunting of white-tailed deer. The Lamoka culture is firmly dated at 4500 B.P. Sites are located on small lakes, which are in close relationship to present day topography. Paleoecological data suggest conditions for support of the Lamoka lifeway existed at about 6000 B.P. Sheep Rock Shelter in Pennsylvania shows it was already established by 9000 B.P. where al1 faunal remains are modern. Archaic groups were apparently visiting the lower Hudson Valley between 6000 and 7000 B.P. as shown by the chronology at Sylvan Lake Shelter. A modern biome existed, but the bifurcated base and other early southeastern point types are lacking, and the lower level assemblages seem to be linked with northeastern developments of the Middle and Late Archaic periods (Ritchie and Funk 1973:38-41). 
The Llano Tradition

Introduction

Early man's presence in North America has always best been known from sites on the Great Plains. Clovis sites, the oldest of the Big Game Hunting traditions have been well documented and well dated in this region with evidence as far back as 12,000 years ago. The early discovery of Plains Paleo-Indian adaptations and good conditions for preservation of faunal and other materials have caused this tradition to be better understood on the Plains than in eastern regions, where similar lithic traditions are at least as old if not older than to the west. Big Game Hunters or Paleo-Indians are characterized by distinctive lithic assemblages and most classically by the presence of stylized lanceolate projectile points and certain types of flake tools. Representative point types and accompanying cultural traditions are: Clovis (Early PaleoIndian or Llano); Folsom and its related forms--Plainview and Midland (Middle Paleo-Indian); and Agate Basin, Eden, Scottsbluf6, Cody, and Angostura (Late Paleo-Indian or Plano). Paleo-Indians occupied the High Plains from at least 12,000 B.P. to approximately 6000 years ago in some areas. Clovis points and most other Paleo-Indian points have a wide distribution throughout the High Plains, as well as into the desert regions of New Mexico and Arizona, where paleoclimatic conditions were substantially moister and cooler than at the present time (Fig. 57).

Clovis sites are primarily kill sites, such as Naco (Haury 1953), Domebo (Leonhardy 1966), and Murray Springs (Haynes and Hemmings 1968), where mammoth were hunted. Portions of disarticulated skeletons along with numbers of stone and bone tools have been found. Unfortunately, the lack of occupation camps has skewed the proportions and types of tools in tool kits, so that comprehensive analysis of tool kits has been difficult. Several stratified campsites have been discovered, however, and these have done much to clarify the picture of these early cultures. Lindenmeier and Blackwater Draw sites are prominent in this list.

Based especially on the Hell Gap sequence, Plainview, a projectile point found in rather limited distribution seems to be a transitional form between clovis and Middle Paleo-Indian Folsom points, both in style of projectile point and prey species exploited--bison. Plainview points are similar in flaking and form, but are not a classic Clovis point. They are basally thinned, but do not carry the extent of fluting (Irwin 1971:47). At Hell Gap, the level below Folsom-related materials dates to at least 10,500 B.P.

Stratified, long occupied sites such as Blackwater Draw, Lindenmeier, and Hel1 Gap have supported each other and helped clarify the Paleo-Indian evolutionary sequence. The point sequence at Hel1 Gap goes from pre-Folsom/Plainview (?) through later Plano styles. Also at Hell Gap, Folsom points are followed by Midland points which resemble Folsom points, except they are unfluted. The suggestion has been made that a Midland point is a Folsom point which is too thin to flute. However, Midland points have been found in sites where no Folsom points were found. Folsom points have been dated at Lindenmeier at 10,830 B.P. Agate Basin points follow Midland points and are dated at about 10,000 years or possibly a little younger. Agate Basin points have a wide distribution far into Canada and along the Hudson Bay (Irwin 1971). 


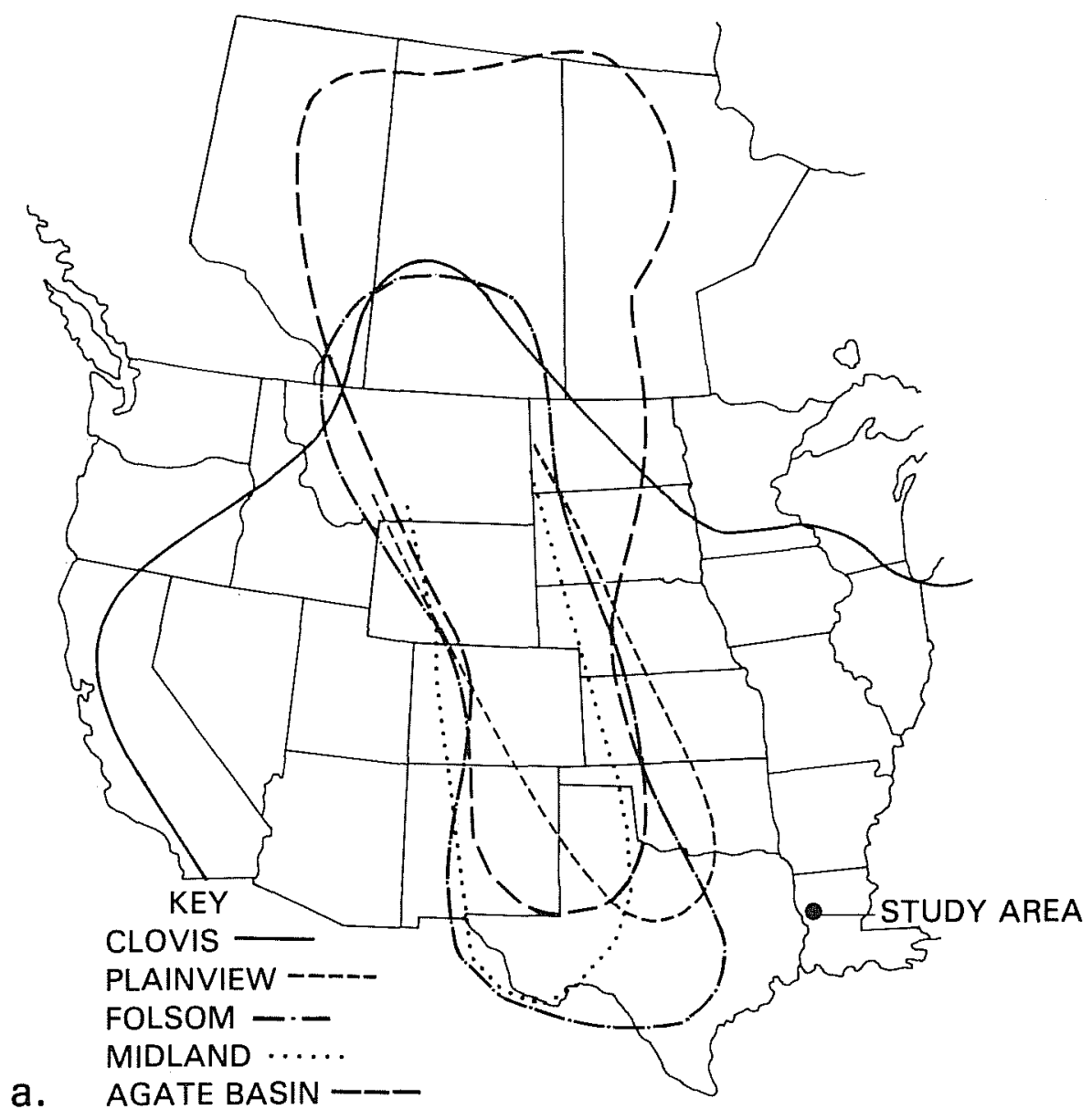

a. AGATE BASIN - -

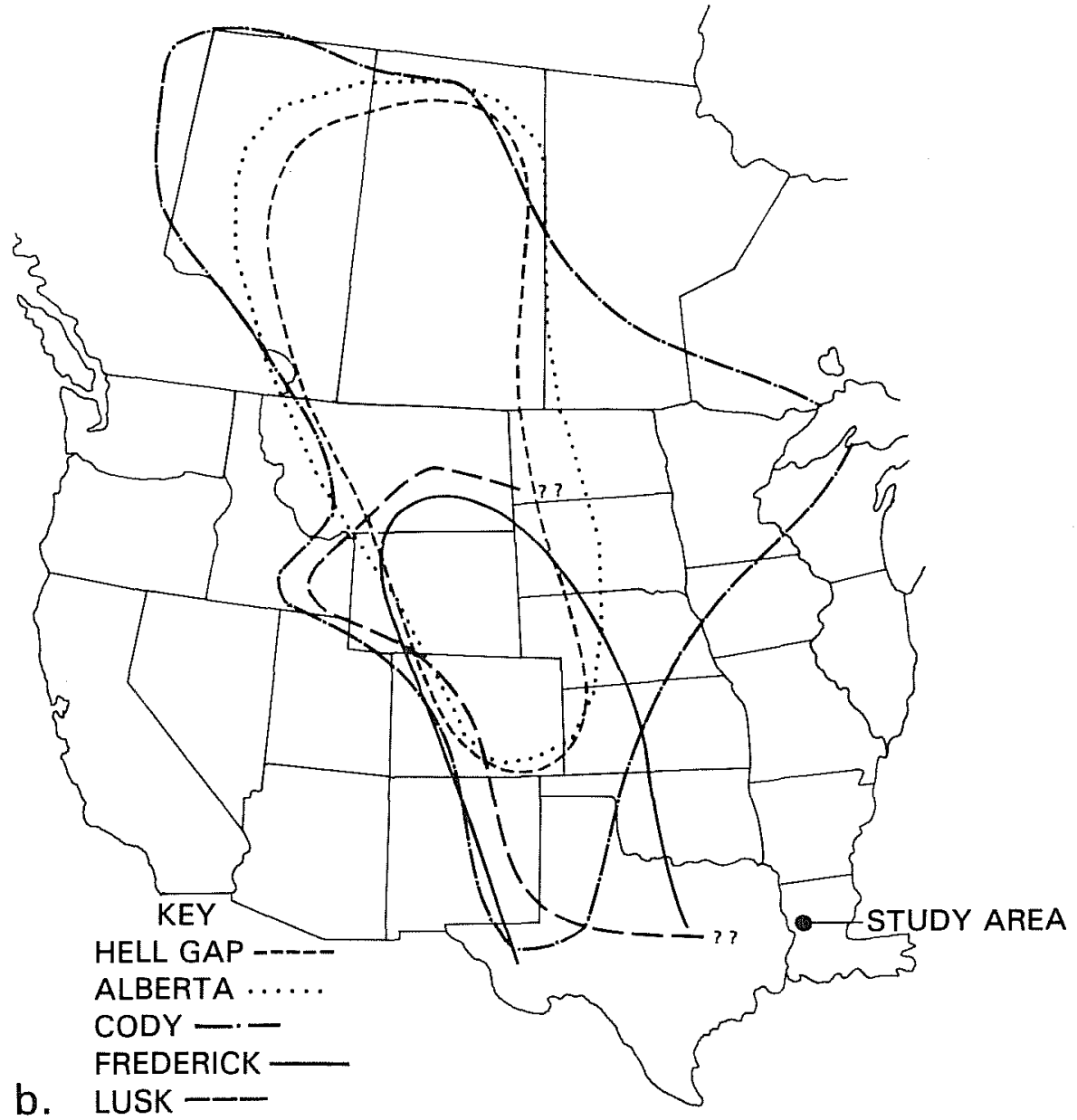

Figure 57. Geographic Distribution of the Paleo-Indian Llano Tradition. a, 10,000-11,500 B.P.; b, 8000-10,000 (after Irwin 1971). 
Agate Basin is followed by the Alberta complex. This complex is widespread to the north, but limited in the south. The Alberta complex is followed by the Cody complex, one of the most spectacular in terms of flintworking skill. This complex is also found far into Canada and extensive evidence for trade in flint is indicated. We have already mentioned distributions of Plano industries in the Northeast and Southeast. These distributions mark the northward and eastward movement of the Plano cultures. Another Late Paleo-Indian point which has long been involved in controversy is the Angostura. It is the last of the Late Paleo-Indian types and is often assigned to an Early Archaic horizon in some areas such as Texas. A date from the Greene site of 7930 B.P., however, (Monseth 1967) suggests it represents the last known Paleo-Indian hunters of the Plains. Following the Plano complexes are the Archaic traditions, tools gradually change with point styles moving toward notches. Different tool types and major changes in economy and settlement patterns transpire.

The origin of the earliest Plains cultures has long been of fascination to archaeologists. Many have commented on the similarity of certain aspects of the Clovis assemblage with 01d World, Paleolithic cultures, thus proposing direct 01d World origins for the Clovis hunters. Vance Haynes (1978) feels that the complex of traits suggests $01 \mathrm{~d}$ lorld origins; tool assemblages contain bifaces, scrapers, burins, blades, flake knives, etc. The bone technology of Clovis cultures includes bevel-based cylindrical points and foreshafts. Grave goods exhibit red ochre. Noting such Siberian sites as Malta, Afontova Gora II, Kokorevo, and Kostienki 1-5, he proposes origins from the Siberian Paleolithic during a time when the Arctic steppe biome existed on the Beringian Platform.

Two separate, but contemporary traditions, were in existence in Beringia before 11,000 B.P.: Diuktai, with leaf-shaped bifaces, wedge-shaped cores, and microblades; the other tradition contained flake tools, some bifaces, cylindrical bone points, and no microblades. "It is reasonable to postulate that clovis developed from the one without microblades" (Haynes 1978:119).

Although an arid grassland today, the High Plains in former times was spotted with ponds, lakes, and marshes. In an effort to recreate the paleoecology of the Llano Estacado, Hester and Wendorf (1975) 1aunched an interdisciplinary study and collected pollen, faunal, and archaeological data in order to develop a chronology for the Southern High Plains. Based on the results of their studies, they have divided the period from approximately 20,000 years ago into climatic episodes.

The first episode, the Tahoka pluvial, began sometime around 20,000 years ago and lasted at least unti1 13,500 B.P. and perhaps as long ago as 11,500 B.P. During this period, extensive woodlands or forests were contemporary with lakes and ponds both in the LI ano area and in adjacent areas to the east. In the Llano area at this time were boreal forests dominated by pine and spruce and extending at least as far south as the southern edge of the Llano area and probably beyond. Summer temperatures were at least $10^{\circ} \mathrm{F}$ cooler than today, with winters no colder than today. This period was interrupted approximately midway by a brief drying trend with a short term expansion of grasses at the expense of forests, but then returned quick1y to moister conditions. 
The Tahoka pluvial was followed by a markedly dry episode--the Scharbauer interval. This dry period correlates (Haynes 1975) with the Two Creeks interstadial. Clearly, forests could not stand these dry conditions, and trees and forests of the Tahoka pluvial were no longer present. There is some indication that shortly after the onset of the Scharbauer interval, there was an increase in pine pollen and other indicators of cooler, moister times. This Blackwater subpluvial (?) would be approximately contemporary with Clovis-related materials and would suggest dates which cluster closely between 11,500 and 11,200 years ago. Folsom and Midland points appear after the Blackwater subpluvial (?) and toward the end of the Scharbauer interval.

The next climatic episode, the Lubbock subpluvial, is well validated by pollen and invertebrate fauna. An abrupt increase in pine and spruce pollen almost certainly reflects major changes in the vegetation in the Llano area and probably a reinvasion of boreal woodlands or forests over much of the High Plains landscape. Distributions of snails during this period indicate cool summer temperatures depressed by at least $10^{\circ} \mathrm{F}$. An exact time range for the Lubbock subpluvial has not yet been established, but an average of four solid dates suggests a maximum range of 10,000 to 11,000 B.P., and possibly 10,300 to 10,500 B.P. as a more restricted range. Cultural materials associated with the Lubbock subpluvial are primarily Folsom related. Point finds indicate Midland and Folsom points succeeded Clovis points during the time interval immediately following the Blackwater subpluvial (?) and certainly replacing them by the time of the maximum aridity of the Scharbauer interval. Folsom points endured without Midland points into a period of major environmental changes represented by the Lubbock subpluvial. Finally, during the latter part of this period characterized by extensive boreal forests in the LTano area, or immediately thereafter, Folsom points were replaced by Agate Basin points, the first of a series of related projectile point types representing around 1000 years between 10,000 and 11,000 B.P.

Wendorf (1975:274) proposes that the forest expansion during the Lubbock subpluvial significantly reduced the mobility of bison that were concentrated in the few open park areas during this period, and the folsom groups who hunted them would have been similarly restricted. A test of this hypothesis would determine if there were lower frequencies of lithic materials from distant sources rather than those located in more open grasslands. Hester and Grady's (1977) study of Clovis and Folsom settlement patterns in the Llano area supports Wendorf's hypothesis. Wendorf suggests, in addition, that the isolation of the Lubbock subpluvial could have been responsible for the explosion of Plano point styles and, with the retreat of the forest at the end of the Lubbock subpluvial a sudden widespread dispersal of previously isolated types throughout the region.

The Yellow House interval following the Lubbock subpluvial was, again, a period of increasing aridity and a decline of woodland elements. Another subpluvial around 8000 years ago is suggested by increases in pine pollen around this time. There are no direct archaeological associations, but this is evidently a period of a continuation of Plano point styles.

This Yellow House interval with its possibly short lived subpluvial(s) continued, evidently accompanied by Plano adaptations until the Altithermal at 6000 B.P., after which rising temperatures and aridity heralded new adaptations. 
Paleo-Indian Phases

Most of the artifactual material from the Plains region in the Paleo-Indian period comes from kill sites. With occupation data so limited, rather than describe the complex in general and recount artifact assemblages from several representative sites, al1 artifactual data will be incorporated into a description of Paleo-Indian tool complexes. Occupation data from Clovis sites come from Blackwater Draw (Hester and Wendorf 1975) and, data from Folsom sites from Blackwater Draw and Lindenmeier. The Hell Gap site is utilized to specify components of the Plano tool assemblages where extensive data on each complex is available (see Table 26).

TABLE 26. PLAINS CHRONOLOGY FOR THE PALEO-INDIAN PERIOD

Phase

Post-Paleo-Indian phase

Plano phase

Folsom phase

Clovis phase
Subphase

Lusk subphase

Frederick subphase

Cody subphase

Alberta subphase

Hel1 Gap subphase

Agate Basin subphase

Midland subphase

Folsom subphase

Plainview subphase

Blackwater subphase
Date Radiocarbon B.P.

8000
$8500-8000$
$9000-8500$
$10,000-9000$
$10,000-9500$
$10,500-10,000$
$10,750-10,500$
$11,000-10,750$
$11,200-11,000$
$12,000-11,200$

SOURCE: Adapted from Irwin (1971).

Clovis Phase--Identifying characteristics of the Llano complex have been described in some detail earlier so the following is a description of the complex in its Plains manifestation. Most tools of the Llano complex are manufactured on large flakes. Projectile points evidencing rather crude flake scars are primarily percussion flaked, and frequently exhibit impact fractures and resharpening. Some points are made on flakes, but most are produced from bifacial preforms. Tools include side scrapers, end scrapers (spurred), concave scrapers (spokeshaves), unifacial knives, flake knives, gravers, burins, and various combinations thereof. Some of the tools are made on large primary flakes, some on bifacial thinning flakes, and others on bladelike flakes. clovis blades are large and triangular in transverse section. Blade cores are rare, but in many cases these may in fact represent piéces esquillées. In addition to the lithic tools, bone tools also make up a large part of the assemblage. Bone awls, punches, scrapers, fleshers, points, cylindrical points, foreshafts, beads, and a shaft wrench have been recovered from Clovis sites. Haynes (1978) recounts caches of tools and raw materials associated with Clovis peoples. Hunter species were primarily mammoth, although in some instances associations with bison, horse, camel, bear, tapir, and rabbit have been noted. 
Folsom Phase--The Folsom complex is more specialized. In comparing Clovis and Folsom phases at Blackwater Draw, of the 23 tool types identified as in the Folsom occupation, only nine types occur with more than $1 \%$ frequency, while the earlier Clovis phase had 15 types with more than $7 \%$. During the Folsom phase less reliance was on bone tools and more on lithics. Flake scrapers, flake knives, and projectile points occur with greater frequency during the Folsom phase. Folsom people also rely less on core struck blades. Differences in choice of stone are evident as well. Although both industries depend on the highest quality cryptocrystallines, clovis people relied predominantly on Alibates chert and less on Edward chert, while Folsom people reversed this preference. Folsom artifacts tend to be smaller than clovis artifacts with less variation.

Plano Phase--Flaking techniques and tool types are derived from the Folsom phase. Plano industries were characterized by fluorescence in projectile point types. Parallel flaking on projectiles and some other tools are the prominent technological feature. Bison are still the primary prey. Each Hell Gap horizon will be briefly described below.

Goshen Subphase (Plainview)--This subphase is characterized by lanceolate projectile points with parallel to slightly convex or concave sides and concave bases, basally thinned. Scrapers include simple, convex-edged side scrapers and other side scraper forms; end scrapers with a lateral spur on beak; and smal1 numbers of other end scraper types; spur perforators on small flakes; and utilized flakes. Bifaces include numerous biface pieces representing early stages in the manufacture of points and a single large, tear-shaped bifacial knife. This industry contains the highest incidence of true blades of any Paleo-Indian complex at Hell Gap. No radiocarbon date exists, but the stratigraphic position indicates a pre-Folsom culture about 11,000 B.P.

Folsom Subphase--Al ready described.

Midland Subphase--Midland points superficially resemble Folsom points-lanceolate with concave bases, but without flutes. Numerous convex side scrapers, double side scrapers, and certain other side scraper forms were recovered. Also in the scraper category were beaked and angle-edged end scrapers, spur perforators, and utilized flakes. Bifaces included denticulate forms of bifaces and another, single example of a teardrop-shaped bifacial knife. All are characteristic of the Folsom assemblage with the exception of the relatively large number of side scrapers. Dates based on radiocarbon samples are 10,440 and 10,740 B.P.

Agate Basin Subphase--The Agate Basin projectile point differs considerably from earlier periods. There is an emphasis on extreme thinness; long, slender, and lanceolate with convex edges reaching maximum width at the midpoint. The scraper category includes a large variety of single- and double-edged side scrapers, notched flakes, spur perforators, simple retouched flakes, utilized flakes, and end scrapers--principally of the beaked and angle-edged forms, but less prominent than previously. Also, interestingly, new tools appear: bone tools such as an eyed needle and a notched flattened implement. The biface category includes a very large form of a thin bifacial knife. 
He11 Gap Subphase--The Hell Gap projectile point seems to be a stylistic development out of earlier forms through a shift in proportions and treatment of the lower hafting section. Proportions of other tools are reminiscent of the Agate Basin complex. Scrapers include various forms of side scrapers (side scrapers predominate) and end scrapers of angle-edged or beaked varieties (although in smaller numbers), spur perforators, utilized flakes, retouched flakes, and notched pieces. A most interesting innovation is the asymmetrical, one-shouldered bifacial knife, the "Hell Gap Knife." Dates are approximately 9500 to 10,000 B.P.

Alberta Subphase--Projectile points may represent, in part, a continuation of stylistic trends already noted above in the preceding period. There is a trend to constrict the lower section of the point and delineate a definable stem. Scrapers demonstrate a shift toward the increasing importance of end scrapers over side scrapers, and there is evidence of the growing popularity of the thin "raclette" scraper. No bifaces were recovered. Dates range between 8600 and 9000 B.P.

Cody Subphase--Both Scottsblufor and Eden points are customarily found together, both characterized by a long, narrow lanceolate outline with a relatively short stem set off from the blade by well-defined shoulders. The accompanying tool kit is distinguished by the growing importance of new types of end scrapers such as ground bit end scrapers; end scrapers on large, flat flakes; asymmetrical end scrapers; end scrapers retouched over the entirety of the ventral surface; and early forms of end scrapers (beaked and angleedged varieties) no longer common. Side scrapers, although varied, are of secondary importance. Raclette scrapers continue to be important as are notched flakes, spur perforators, denticulates, and utilized and retouched flakes. Bifaces include several stemmed and shouldered bifacial pieces similar to what has been called Scottsbluff Type II. Also found is another bifacial knife form, which is asymmetrical and single shouldered, but distinct from the cody knife form. The cody subphase is dated to 8650 B.P.

Frederick Phase--This represents a radical shift from earlier occupations, especially in typology and character of the faunal remains. Projectile points seem unrelated to the slowly evolving tradition represented by the Agate Basin to Cody transition. Points have a lanceolate outline with concave base and no distinguishable shoulder or stem. The diagnostic is a specialized technique of oblique-paralleled pressure flaking. Scrapers include raclette scrapers; several types of side scrapers, although these are minimally important; spur perforators; notched flakes; and utilized and retouched flakes. The predominant scraper class is the end scraper, particularly the ventraliy retouched, asymmetrical, and ground bit forms. Diagnostic bifaces are bifacial knives made on or similar in form to the Frederick projectile point.

Settlement Technologies

Judge (1973), in his analysis of many aspects of Paleo-Indian cultures in New Mexico, has described the generalized Paleo-Indian pattern for site selection as being based upon (1) the proximity to a water source; (2) short travel time to hunting areas, which is usually a broad open area unobstructed by sharp topographical relief; and (3) an overlook or overview area. Within the generalized 
pattern of the Paleo-Indian period, however, differences in site location occur. The Clovis period was represented in the survey by only one site and as a result was useless for comparative purposes. Folsom and cody cultures were well represented and evidenced a distinctive trend. Folsom sites are located farther from present permanent water sources such as rivers and large streams and close to what were playa lakes in Folsom times. Playas were not only a source of water for man, but also for large grazing animals such as bison. For Folsom hunters water sources and hunting areas were practically the same. Folsom times must have been much moister to allow for this site patterning. Later cody cultures were located next to permanent water sources and much farther from hunting areas.

Judge also suggests another developmental trend among Paleo-Indian technologies in New Mexico. Factor analysis of various categories of the transverse scraper (categories based upon the degree of lateral modification) suggested that variation between cultures in categories of this tool type could best be explained by the change from a generalized to a highly specialized artifact form. By analyzing wear patterns as well as retouch, Judge describes Clovis transverse scrapers as being included in a generalized group of end and side scrapers with the transverse scraper defined by certain characteristic lateral and end modification. Folsom peoples produced the transverse scraper as a unique category or type with consistent use patterns. Cody peoples, on the other hand, produced functionally specialized transverse scrapers. Judge proposes the tool specialization as a general trend of specialization in other elements of technology through time.

\section{Archaic Complexes}

The Archaic period began much Tater on the Plains where the Big Game Hunting traditions of Paleo-Indian cultures continued until approximately $6000 \mathrm{~B}$.P. and when climatic changes forced its demise. The transitional and Early Archaic period is poorly represented archaeologically. Due, no doubt, to the heat and aridity of the Altithermal from 5000 to 6000 B.P., few sites can be dated to this period. The Plains would have been an extremely inhospitable place, and the transition from Paleo-Indian to Archaic 1 ifeways would have occurred more easily along southern and eastern margins of the Plains. Paleo-Indian to Archaic transitions are well documented in Texas and the Southeast where conditions other than parched grassland existed. Only after 5000 B.P. do Archaic sites again appear on the High Plains.

Although basically a local development from the Big Game Hunting tradition, the Plains Archaic was strongly influenced by diffusion from the Eastern Woodlands area, where the Archaic 1 ifeway had been well established for thousands of years (Willey 1966). There was a general continuation of Paleo-Indian tool types, but with new additions. The McKean phase of Wyoming is a typical early Plains Archaic adaptation. Typical tools include the McKean point, a lanceolate point with an indented base, resembling Paleo-Indian styles but much more crudely fashioned, and other typical stemmed and notched Archaic types similar to the East and Desert forms. Other tools include milling stones and handstones. Occupation camps, cave sites, and bison kill sites are representative types. Faunal remains of bison, deer, birds, reptiles, and mussels have been recovered. 
The Cumberland Tradition

Introduction

The southeastern culture area comprises all territory east of central Texas and south of the Ohio River. Archaeological research on early man in the Southeast is limited, but recent efforts at cultural resource management in conjunction with water management and conservation endeavors are yielding new information on Paleo-Indian lifeways. Research has primarily centered on the highly elaborated cultures of Woodland and Mississippian peoples, while neglecting interdisciplinary studies of early man's activities. This has been partly due to the scattered surface nature of early man sites, and partly due to the natural fascination of more fluorescent cultures.

Man's early entry into the Southeast is demonstrated by Clovis points and other variations of the fluted lanceolate point scattered across the area. The finds are predominantly along the major river valleys, especially the Mississippi and the Tennessee. Fluted points are found as far south as Florida (Bullen 1969; Gagliano 1977). Marine adaptations, at least by later Paleo-Indian times, were no doubt in effect, although much of the evidence has been lost along submerged coastal shelves. Gagliano (1977) has surveyed cultural resources along the western Gulf Coastal regions and located "pre-projectile point" possibilities such as evidence of pebble tool (Lively complex) industries on Avery Island off the Louisiana coast (Gagliano 1977:173). Some other tantalizing evidence of much earlier occupations on the Florida coast are noted. Several occurrences of mammoth bones exhibiting butchering marks ostensibly made, while the bone was sti1l green have been reported (Bullen, Webb, and Waller 1970:205; Nei11 1971:68), but no tools or other human associations have been found. A sloth bone was recovered along the Florida coast associated with a Lerma-like three-inch spear point with an estimated age of 12,000 to 14,000 years based upon similar type occurrences elsewhere. No incontrovertible evidence, however, has surfaced. Discovery of Paleo-Indian occupation along coastal areas is most dependent upon understanding the patterns of developing stream banks, beaches, margins of estuaries, and important ecotones. Earliest evidence of man in the central Gulf area is at Avery Island (Gagliano 1977), a protruding salt dome evidencing Late Paleo-Indian point types such as San Patrice, Lerma, and other lanceolate type points along with prepared cores, bladelets, flake end scrapers, flake side scrapers, and bifacial knives. Two other sites off the coast of Louisiana, the Bayou Grand Louis site and the Palmer site, yielded Paleo-Indian points such as Cumberland, Angostura, Meserve, San Patrice (Bayou Grand Louis), Plainview, and San Patrice (Palmer), along with unifacial scrapers, bladelets, and gougelike tools (Gagliano 1977). The Fish Creek site in Florida located in the eastern Gulf region produced Suwannee points (Gagliano 1977). Paleo-Indians along the southeastern coastal regions chose deltaic beaches, salt domes, and natural levees as occupation locations, because these sites would have been rich in resources such as plants, animals, fish, fowl, fruit, nuts, and berries.

Southeastern variants of the fluted point tradition include the Cumberland, quad, Suwannee, Hardaway, and Dalton. Cumberland points appear predominantly in the Tennessee and Ohio Valley regions. They are relatively long, like the clovis, but are frequently widest at the midsection and most often bear long Folsomlike flutes (Willey 1966). The Cumberland point is often called the eastern 
version of the Folsom point due to its contemporaneity and stylistic similarity. Folsom points are extremely rare in the East with only a few noted occurrences. At the Williamson site (McCary 1951), a Paleo-Indian workshop-occupation site in Virginia, Folsom or Folsom-like points occur along with a fluted local variant (pentagonally shaped points) as part of a typical Paleo-Indian tool assemblage of end scrapers (many spurred or eared), side scrapers, large fluted bifacial knives, gravers, and several crudely made bifacial knives. Willey (1966) suggests Cumberland points are in complementary distribution with Middle and Late Paleo-Indian industries of the Plains with Plains varieties occurring as far east as the Great Lakes region and north of the Ohio River, but becoming progressively less frequent further to the southeast. Evidence of Plains types is rare in the Southeast where Cumberland, Suwannee, and Dalton points become the dominant types during this time frame.

Gardner (1974), in his typologies of Flint Run complex projectile points, has proposed a three stage evolutionary model for classifying the varieties of fluted points found in the East, which parallels the three stages of development proposed for the Plains. Plains point complexes are represented by the Clovis root complex during Early Paleo-Indian times, giving rise to Folsom at Middle Paleo-Indian, and Plano during the Late Paleo-Indian. The late phase represents the beginning of regional variations. Gardner's stages are Clovis, Middle Paleo-Indian, and Dalton. In this sequence, there is some variation in point size (primarily length) from East to West, differences attributable to such factors as variations in raw materials, individual knappers, resharpening, and distance from the site. When tested along certain "diagnostic" variables (Gardner and Verrey 1979) and applied to eastern sites already discussed, principally length-width and width-thickness ratio, they are eminently classifiable into the Clovis and Middle Paleo-Indian typologies. The Shoop site is verified as an early, probably one time habitation camp with Clovis points; while the williamson site has two components, Clovis and the visually recognizable Paleo-Indian. Gardner's scheme is pan-continental and aside from being extremely workable in classifying points of all localities, goes a long way in explaining both the universality of the clovis point, and the complementary distributions of Middle Paleo-Indian (Cumberland-Folsom points), and Plano (Scottsbluff, Eden, Meserve, Agate Basin, Dalton, and Hardaway) points. In this scheme, Clovis points represent the pan-continental base; the Middle Paleo-Indian Folsom point, a continental-wide offspring of clovis; and Late Paleo-Indian types as a period of regional diversification. Following this line of development to its logical conclusion, it is interesting to note the similarities of all regional cultures in the developmental sequence of notched and bifurcated base points.

Due to the similarity of style and technology across fluted point variations, as well as the similarity of component tools which comprise Paleo-Indian assemblages, many have suggested a similar Big Game Hunting adaptation for all carriers of the fluted point tradition. Gardner, however, suggests that these carriers of a basically Upper Paleolithic technology quickly became locally adapted to the hunting of plentiful smaller game such as deer, and although they were carriers of the Tithic traditions of Big Game Hunters, they may not have been carriers of their adaptive strategies. Evidence in the Northeast appears to indicate exploitation of smaller game such as the caribou. Although larger Pleistocene fauna were certainly available to early inhabitants of the Southeast, there has been no evidence of their exploitation other than the 
presence of a proven Paleo-Indian tool kit containing hunting tools such as end scrapers, side scrapers, knives, and other bone and hide-working tools. Historically, there does seem to be some symbolic element in the association of a highly stylized point typology and the predominant exploitation of a single prey species, for example, the Solutrean Laurel leaf and the reindeer, the Clovis fluted point and the mammoth, the Folsom point and the bison, etc. Whether transference of style and technology automatically results in transference of adaptive strategy and possibly other symbolic units of the culture holds significance for analysis of archaeological materials. The old question of whether temporal or spatial distribution of a tool type (principally projectile points) can be taken to represent the distribution of a particular culture (i.e., entire cultural configurations) including that culture's adaptive strategies is still debated (Weir 1975). Whether similar subsistence patterns and other symbols accompany the similar style and technology of the fluted point complex waits to be discovered in the acid soils of the East where buried sites are most difficult to discover. Because of the condition of the soils and the surface nature of the finds, associations of faunal specimens and the earliest PaleoIndian points have been missing. Late Paleo-Indian manifestations such as the Dalton, however, were exploiting white-tailed deer, while retaining to a great extent a classic Paleo-Indian tool kit. In addition, it is highly probable that Early Paleo-Indians were foragers as well as hunters. Nutshells, and possible nutstones, have been recovered from the williamson site (Benthall and McCary 1973). Paleoenvironmental recreations suggest this based on the nature of flora and fauna in the Southeast during the hypothesized period of PaleoIndian occupation.

Figure 58 illustrates proposed vegetation regions at late glacial period. Florida evidenced deciduous forest. The rest of the Southeast exhibited a type of pine savanna until approximately 11,000 years ago when an oak-hickory forest became established.

Carbone's (1976) recreation of paleoenvironmental conditions at the Thunderbird site in Virginia divides the ending glacial and subsequent approaching boreal periods into three episodes: Late Glacial (up to approximately 10,500 years ago), Preboreal (10,500-9300), and the Boreal (9300-8700). The Late Glacial was characterized by increased precipitation, lower temperatures, and a relatively open (pine savanna) boreal landscape. Average temperature was at least five degrees lower than at present, but with increased albedo and increased precipitation principally in the form of more extensive winter snowfal1. Gardner (1974) suggests this period correlates with the Clovis occupation of the Thunderbird site, apparently the first human occupation of the Shenandoah valley.

The Preboreal, an interim episode between Late Glacial and Boreal, experienced an expansion of forests, both coniferous and deciduous species, and a reduction of open habitats, the oncoming of a closed canopy forest environment. There appears to be an abrupt boundary between these two periods characterized by very rapid climatic change. Gardner (1974) and Verrey (1980) feel this corresponds approximately to Middle Paleo-Indian and Dalton subphases at the Thunderbird site. 


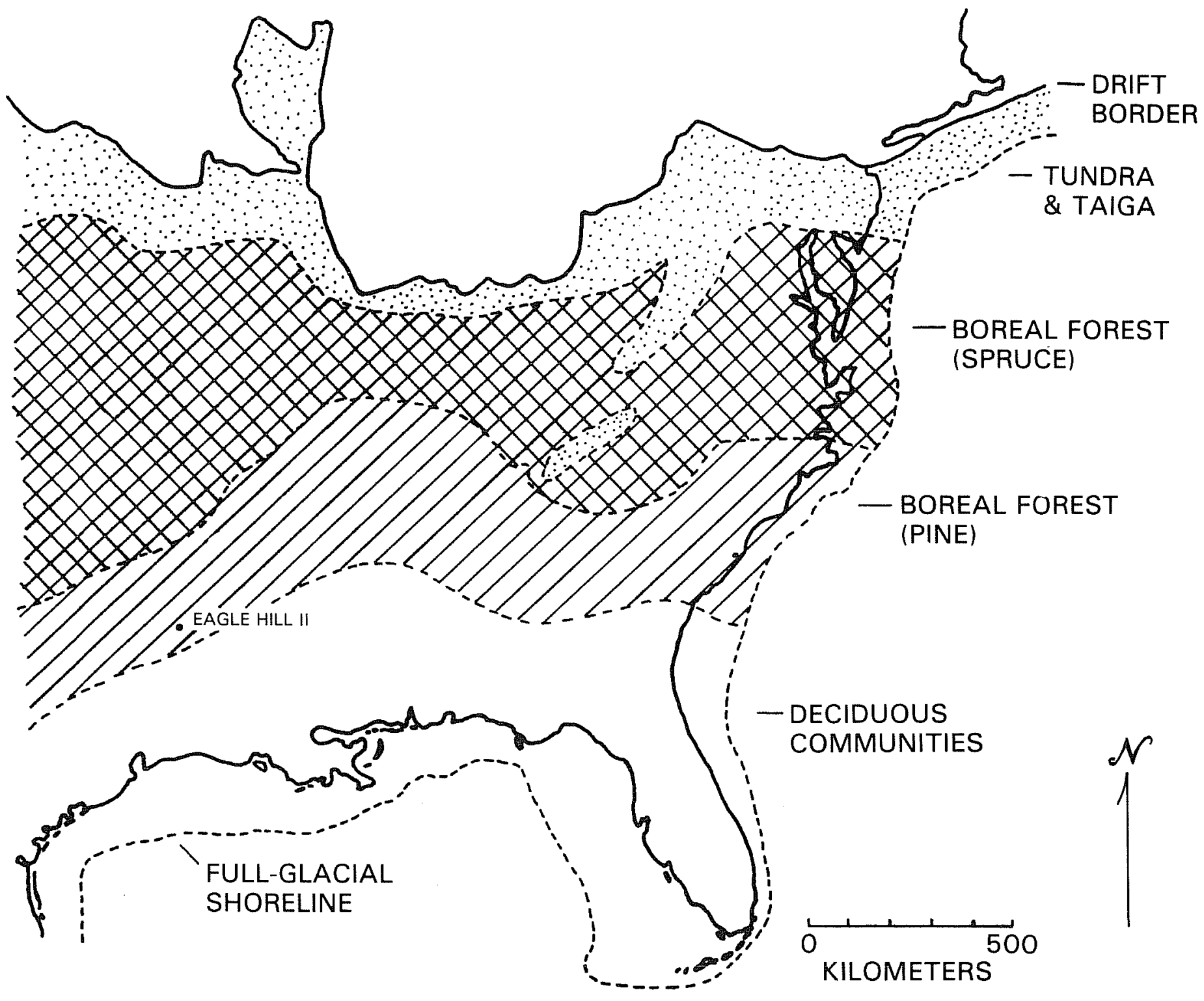

Figure 58. Vegetation Regions of the Southeast. From Whitehead (1982).

$\stackrel{\simeq}{\Xi}$ 
Characterized by a "continental" environment, the Boreal showed increases in average temperature with warmer summers and milder winters (the relatively "flat" temperature curve necessary for the maintenance of deciduous communities). This episode correlates with the notched point (Early Archaic) phases at the Thunderbird and Fifty sites (Gardner 1974; Verrey 1980). Both Verrey and Carbone suggest a decline in Clovis occupations (which had been along the major river valleys) as the boreal forests spread into Virginia at the beginning of the Preborea1.

Succeeding populations would be more limited and probably found specialty areas either rich in food or lithic resources. The upland pattern of Dalton sites could represent this situation. The Early Archaic correlates well with the Boreal episode. Changes in adaptation may be seen by the addition of more bifacial tools and the shift to notched projectile points as evidenced at the Thunderbird, Eva (Lewis and Lewis 1961), and Rose Island sites (Chapman 1975). Evidence at these and other Archaic sites in the Southeast seem to indicate that the major change in adaptation came with the Early to Middle Archaic transition rather than from Late Paleo-Indian to Early Archaic. Aside from a change in projectile point styles and the addition of a few more bifacial tools, the assemblages are remarkably similar. The Late Paleo-Indian to the Early Archaic appears to be more of a continuum. Following the Early Archaic, great environmental changes occurred that led to the Atlantic climatic episode and accompanying Middle and Late Archaic occupations (Verrey 1980:14). Middle Archaic cultures exhibit a significant switch to the exploitation of shellfish and other aquatic species and evidence a heavy foraging adaptation. The Eva site demonstrates this with the decrease in deer and the huge increase in shellfish. Specialized tools such as conoidal pestles, types of bone fishhooks, and large chert pounders were used to exploit aquatic resources.

\section{Paleo-Indian Complexes}

The classic Paleo-Indian tool kit as described earlier applies to the Southeast as well. Classic southeastern sites such as Williamson and Quad are indicative of the Enterline Chert industry, although there are some new aspects. Bladelets occur along coastal sites at least during the Late Paleo-Indian period and possibly earlier. There also appears to be an increase in blades, core tools, choppers, and chisels. However, this may be due to the nature of the types of sites sampled and not to a "real" increase.

\section{Representative Sites and Tool Kits}

Williamson--The Williamson site (McCary 1951) is located on the Appomattox River, Virginia. It is a camp-workshop that was probably occupied many times during the Early Paleo-Indian period. Thirty-three fluted points were found along with 174 snub-nosed end scrapers (predominantly of the eared or spurred variety), 24 side scrapers, 16 fluted basal fragments of bifacial knives, and a smal1 number of gravers. McCary (1951) compares the site to the western Folsom site (see Gardner's hypothesis stated earlier). The large fluted bifacial knives are finely manufactured, while the six small bifacial varieties are crudely made using percussion only. Soday (1972) describes the Williamson 
material as almost conforming to the Enterline Chert industry, but containing rougher fluted points, a snub-nosed blade (?), end scrapers, (many with graving spurs), side scrapers, bifacial flake knives, and possibly a few gravers. Scrapers in the Enterline Chert industry are characterized by having the bulb (often somewhat pronounced) at the tip of the scraper (bit), rather than at the distal end of the flake (Witthoft 1952).

We11s Creek Crater--We11s Creek Crater (Dragoo 1965) in Steward County, Tennessee, is a surface, quarry-workshop-occupation site. No stratigraphic information is available, but over 5000 Paleo-Indian tools have been recovered. The site yields the first direct evidence of large bifacial cores with ground margins and of large prepared platform cores designed to yield large preform flakes for unifacial tools. These cores are unique to fluted sites in the East. The flaking technique is similar to the Levallois technique. Lithic materials consist of nearby Fort Payne chert which is of hiqh quality. Bifacial tools include a somewhat unusual disc scraper, biface knives, drills, preforms, and fluted points (nontyped). Other bifaces were a beaked side scraper and core tools. The unifaces included a great assortment of multifunctional tools: end scrapers--many on true blades, others on flakes--a large number spurred. Also recovered were unifacial flake end scrapers, free form end and side scrapers, unifacial knives, blade knives, flake knives, and unifacial side scrapers, some from polyhedral cores, some from bifacial cores. The flake scrapers were predominantly primary flakes which had been modified (unifacial core/flake or core fragment scrapers). Spokeshave scrapers were common, as were unifacial gravers, chisels, and utilized flakes. An interesting collection of large tools included unifacial choppers, large beaked flake scrapers, and picks. Core tools were in evidence as well. Tools made on percussion flaked polyhedral cores were large core spokeshaves, core denticulate-beaked tools, core scraper planes, and tabular core beaked tools. The basic set of tools includes, in addition to the fluted points, end scrapers (usually with spurs), a variety of side scrapers on blades and elongated flakes, bifacial and unifacial knives, spokeshaves, sharp-pointed and chisel-edged gravers, drills, utilized flakes, and an assortment of large cutting, scraping, and chopping tools rare or absent at most eastern and western sites.

Quad--The Quad site (Soday 1972; Cambron and Hulse 1960) is a surface site containing artifacts dating to both the Paleo-Indian and later Archaic periods. Paleo-Indian material was easily distinguishable from later industries by raw material, tool types, technology, and artifact patination. Paleo-Indian material was primarily of heavily patinated blue gray chert, ryolite, or green jasper. The site is remarkable for its similarity to the Enterline Chert industry first identified at the Shoop site in eastern Pennsylvania. Artifacts included fluted points, thin and well ground (some typologically like clovis and Folsom points), and end scrapers, many which were keeled and spurred. Two types of these end scrapers existed. One type was a long pointed scraper with both sides retouched and of the typical Enterline style with the bulb of percussion at the bit of the tool. The second type was made of short thick flakes often as broad as long. Both types were identified at the Shoop site as were other large scrapers, such as side scrapers that appeared to be notched (spokeshaves?) and manufactured on lamellar flakes. Gravers were in unusually high proportion at Quad, many with multiple (up to five) tips. Flake knives were found along with many combination tools such as knife-gravers, scraperknives, and knife-graver-spokeshaves. A few drills and crude choppers averaging 
four to five inches in length were also present in addition to what appears to be a fluted bifacial knife.

Thunderbird--The Thunderbird site (Gardner 1972, 1974) is part of the Flint Run complex found in the Shenandoah Valley. The Flint Run complex is composed of at least six types of sites: quarry, quarry reduction, base, base camp maintenance, outlying hunting sites, and isolated point finds. Thunderbird is a base camp maintenance site. It is a stratified site dating from Paleo-Indian through Early Archaic. Sites like Thunderbird and other base camps such as the Wi11iams site (Gardner 1974, 1976) function in the same manner as the seasonal base camps exploiting rich seasonal resources as is known ethnographically among hunters and gatherers. In addition to the classic Paleo-Indian tools such as found at Williamson, crude bifaces and large blank flakes were recovered, evidently carried from the nearby quarry to the maintenance site. Materials represent the entire Paleo-Indian period through the Early Archaic. The primary change in artifact types is found in projectile point types moving from clovis points, through Middle Paleo-Indian and Dalton points, into the notched and bifurcated point styles. The following chronology for the site is given: clovis ca. 11,500-11,000 B.P., Middle Paleo-Indian ca. 11,000-10,500 B.P., Dalton ca. 10,500-10,000 B.P., Palmer ca. 10,000-9500 B.P., Kirk ca. 9500-9000 B.P., and Warren ca. 9000-8000 B.P.

Hardaway--The Hardaway site (Coe 1971) is another site marking the transition from Late Paleo-Indian to Early Archaic in North Carolina. The excavated sequence begins with the Hardaway blade (one like Ouad) and Hardaway-Dalton points and proceeds through Palmer Side and Corner Notched styles, Kirk Stemmed, Kirk Serrated, Stanley Stemmed, and finally the bifurcates. The Palmer period was associated with a particular type of end scraper, which has long been associated with the Paleo-Indian points in the East, but whose terminal period was not previously known. At the Hardaway site, there appears to have been a primary association with only the Palmer period. Through time the use of hafted end scrapers increased considerably, while large heavy side scrapers decreased. The Kirk occupation signaled primarily a change in projectile point forms. These peoples continued to use scraper types that were known for the earlier periods, but there was a definite shift in emphasis to the cruder forms of end scrapers and the thin blade type of side scraper. Production of quarry blades began in Kirk times and increased in frequency through time. Chronology for the Hardaway site based on similar point styles from other areas such as the St. Albans site, the Habron Site, and Sheep Rock Shelter is (all dates B.P.): Hardaway 10,000, Palmer Side-Notched ca. 9900, Kirk Corner-Notched ca. 8930, St. Albans bifurcates ca. 8825, LeCroy bifurcates ca. 8250.

Stanfield-Worley--Stanfield-Worley Bluff Shelter (DeJarnette, Kurjack, and Cambron 1962) is a stratified multicomponent site in Colbert County, Alabama. The lowest cultural zone contained numerous uniface tools, side and end scrapers, gravers, and spokeshaves as well as two major types of projectile points: Dalton-Meserve and the side-notched, basally ground Big Sandy points. Radiocarbon dates of 9640 and 8920 were secured for this zone. This association raises the possibility that Dalton represents a transitional Paleo-Indian tradition in the Southeast (MacDonald 1971). Goodyear (1982), who extensively researched the Dalton cultures and chronologies, feels that the association of Dacton and Big Sandy points may not be valid. Although these two point styles 
were separated from overlying levels by wind-blown deposits, he feels there is the distinct possibility of mixing below this deposit and argues for an earlier date for Dalton points $(10,500-9900$ B.P.). Dalton points represent Late PaleoIndian, while Big Sandy points were of an Early Archaic tradition.

Brand--The Brand site (Goodyear 1974) is a Dalton site located in northeast Arkansas. The site was a satellite camp which existed for specialized hunting and gathering. The Dalton tool kit mirrors those of the earlier Paleo-Indian assemblages with unifaces such as classic end scrapers (many spurred) and side scrapers, gravers on flakes, true blades, retouched blades, bipolar cores, and piéces esquillées. An important addition, however, is a bifacially chipped stone adze that has been ground on the side margins to prevent haft damage in much the same manner that Dalton points are ground. In addition, there appears to be evidence that the Dalton point was used as a knife as well. The Brand site analysis shows that the different "types" of Dalton points may be merely the result of successive resharpening, rather than intentional differences in design (experimental resharpening was done by Goodyear).

There have been problems with assigning the Dalton point a time period. Many assign Dalton points to Paleo-Indian status based upon the technical and stylistic similarity of the points and other tools with the classic PaleoIndian point and tool traditions. Also, Dalton is considered a southeastern variety of the Meserve point which is considered a Late Paleo-Indian point style on the Plains. Others consider Dalton points to be Early Archaic based on dates from such sites as Stanfield-Worley and Graham Cave but, as mentioned earlier, Goodyear questions the dating of Dalton as an Early Archaic point because of radiocarbon dates from such places as Stanfield-Worley. In addition, at Ice House Bottoms (Chapman 1973) and the Rose Island site (Chapman 1975) with Early Archaic components, none exhibit Dalton points. Goodyear (1974) assigns the interval of 10,500-9900 B.P. to the Dalton complex. Dalton peoples appear to have switched settlement patterns from earlier Paleo-Indian groups. They occupied uplands, caves, and rockshelters, in addition to river floodplains. The Dalton adze must be a highly significant tool in understanding the Dalton lifeway due to its frequent occurrence throughout sites. Goodyear (1974) suggests that the presence of the Dalton adze could provide clues to functional differences between Dalton sites. He compares it to the Clear Fork gouge, a long-lived tool in the Late Paleo-Indian through Archaic periods in central Texas. Both apparently have something to do with exploiting an upland habitat.

In the Southeast, there is a wel1-documented transition from the Paleo-Indian to the Archaic. The largest jump, however, does not appear to occur from the Paleo-Indian to the Early Archaic, but rather from the Early to Middle Archaic. There appear to be continuities in tool types from Late Paleo-Indian to Early Archaic as evidenced by such sites as Eva and a series of Archaic sites dug by Jefferson Chapman in Tennessee. There is a documented point transitional (technostylistic) change from the Paleo-Indian Dalton to the side- and then, corner-notched point types, such as Palmer and Kirk. Other than this change, the rest of the tool kit remains essentially unchanged in the Early Archaic.

Eva--The Eva site components represent a gradual continuum due to adaptation to varying ecological conditions, to internal development, and to influence from 
other cultural traditions (Lewis and Lewis 1961:13). Stratum Five or the earliest stage represents approximately 8000 to 6000 years ago. During the earliest occupation of the site, $10 \%$ more deer and mammals in general were exploited. This horizon is also characterized by more projectile points, blades, and choppers. Characteristic trapezoidal uniface scrapers, many with graving spurs, ovoid uniface scrapers, turtleback scrapers, circular biface scrapers, drills, anvilstones, nutstones, and large ovoid choppers occur. The Middle Archaic, however, evidenced a greatly increased exploitation of shel1fish, an increase in bone fishhooks, and' a decline in deer, either from a decline in deer populations or a preferred aquatic resource base. Early Archaic projectile points associated with the lowest levels are Eva and Kirk Serrated.

Rose Island--Rose Island (Chapman 1975) is a well-documented site having over 40,000 artifacts associated with the Early Archaic. Early Archaic assemblages at Rose Island span over 1100 years and are representative of four, perhaps five phases. Except for changes in the projectile point styles, 1ittle change is apparent in the assemblages over time. The Palmer phase is indefinite, based on radiocarbon dates from other sites; little time separates Palmer from Kirk material. Two radiocarbon dates for Kirk Corner-Notched points suggest an average date of 9270 B.P. Bipolar flaking, piéces esquillées, and associated pitted cobbles are present and continue to be present throughout the rest of the Early Archaic. Anvilstones occur and continue throughout as well. 0ther tools include end scrapers, side scrapers, a dri11, a perforator, a graver, utilized flakes, hammerstones, and a possible grinding stone. There is the possible production of blades. A great similarity with earlier Paleo-Indian assemblages is apparent. The next stage, St. Albans, marks the beginning of the bifurcated base point. Ground stone increases as celts and a distinctive chopper/scraper occurs. Stages in biface manufacture also appear. Radiocarbon dates for the St. Albans period average 8770 years ago. Lecroy points (ca. 8300) follow with a bifurcated base evolving into a bifurcated stem. The remainder of the assemblage remains unchanged. The Kanawha phase is the last phase of the bifurcated projectile point tradition, but is poorly represented at the site. A bipolar technique is still apparent, but the number of other tool types is sma11--comprised of one graver, a biface, and two side scrapers. A date of 8200 B.P. is estimated.

\section{Lithic Period Sites in Texas}

The Eagle Hill II project area is at the junction between the Cumberland and the Llano tradition areas, and the archaeological literature is in two rather regionalized segments, Texas and Louisiana. Texas poses a mosaic of widely differing environmental zones. Because of its location on the plains/forest ecotone it provides an appropriate setting for studying both environmental and cultural factors involved in cultural change and diffusion.

Northwest Texas is in the High Plains region and is characterized as mixed grassland prairies and mesquite plains. Archaeological and cultural sequences are basically those described for the Plains. Although Paleo-Indian materials are found scattered throughout the state, the heaviest concentrations are probabiy to be found in this region. 
Central Texas is marked by karst topography and is dissected by many rivers, streams, and broad uplands, which span the distance between streams. Typical plant association is a scrub forest of Mexican cedar, Texas oak, bear grass, and lecheguilla. It is rich in faunal resources with 57 species of mammals known from the region today. At all times, this area would have provided abundant resources for prehistoric man, especially along floodplains with rich resources of oak, hackberry, pecan, and other riverine floral and faunal species.

Closely related to the culture areas of the Southeast and lower Mississippi Valley, east Texas has the environmental characteristics of the mesic forests of the Southeast. Paleo-Indian evidence is scarce in east Texas, while the later cultures, with a wel1-developed forest efficiency adaptation, are well defined. Shafer (1975) considers Paleo-Indian lithic assemblages in east Texas to be a broader based hunting and gathering oriented strategy. By considering total assemblages and environment and not simply the presence of lanceolate points and the absence of milling stones, an unbiased assessment of adaptive tactics could be determined. Artifact components of early lithic assemblages in east Texas include several varieties of bifaces such as lanceolate projectile points (Plainview, Angostura, Scottsbluff. Meserve, San Patrice, and others), gouges, dri11s, choppers, hammerstones, grooved "Waco Sinkers," but no bones of extinct megafauna (Shafer 1975).

East Texas is extremely sensitive to climatic change with the grassland shifting east to west with fluctuating precipitation. As a continuation of the High Plains, at times, Plains hunters (primarily Plano peoples) moved into the forested areas of east Texas and southwestern Louisiana. Figure 59 lists and plots some of the lithic period sites in Texas.

\section{Paleo-Indian Complexes}

Clovis points are commonly found throughout Texas, but only one unequivocal site, the Miami site (Sellards 1938) has been reported. Cultures with Folsom points are better represented. In addition to scattered finds, several sites with Folsom points have been investigated. Among these are the Lubbock Lake site (Black 1974), the Adair-Steadman site (Tunne1 1975), and Bonfire Shelter (Dibble and Lorraine 1968). An occupation site, the Lubbock Lake site, established along an old river channel, yielded Folsom points along with bones of bison and bifacial knives, drills, beaked end scrapers, side scrapers (one notched or concave), gravers, denticulates, and utilized flakes. Adair-Steadman is a surface site producing Folsom points, preforms, channel flakes, "typical" end and side scrapers, gravers, and drills. Bonfire Shelter is a site of repeated bison jumps with three excavated bone beds. In the second or Bone Bed II, bison bones are found in association with a Folsom point. Midland points are likewise found. The indication of cooler and moister climate during Folsom times appears to be substantiated throughout Texas, with even the southern Pecos area of the state (a desertiike environment today) supporting pine forests during Folsom times.

The Late Paleo-Indian period in Texas is more complex. The Plainview point, excavated below the Folsom period at Hell Gap, appears in the Late Paleo-Indian period in Texas. Plainview sites are bison kill sites and date to 9200-8800 B.P. 


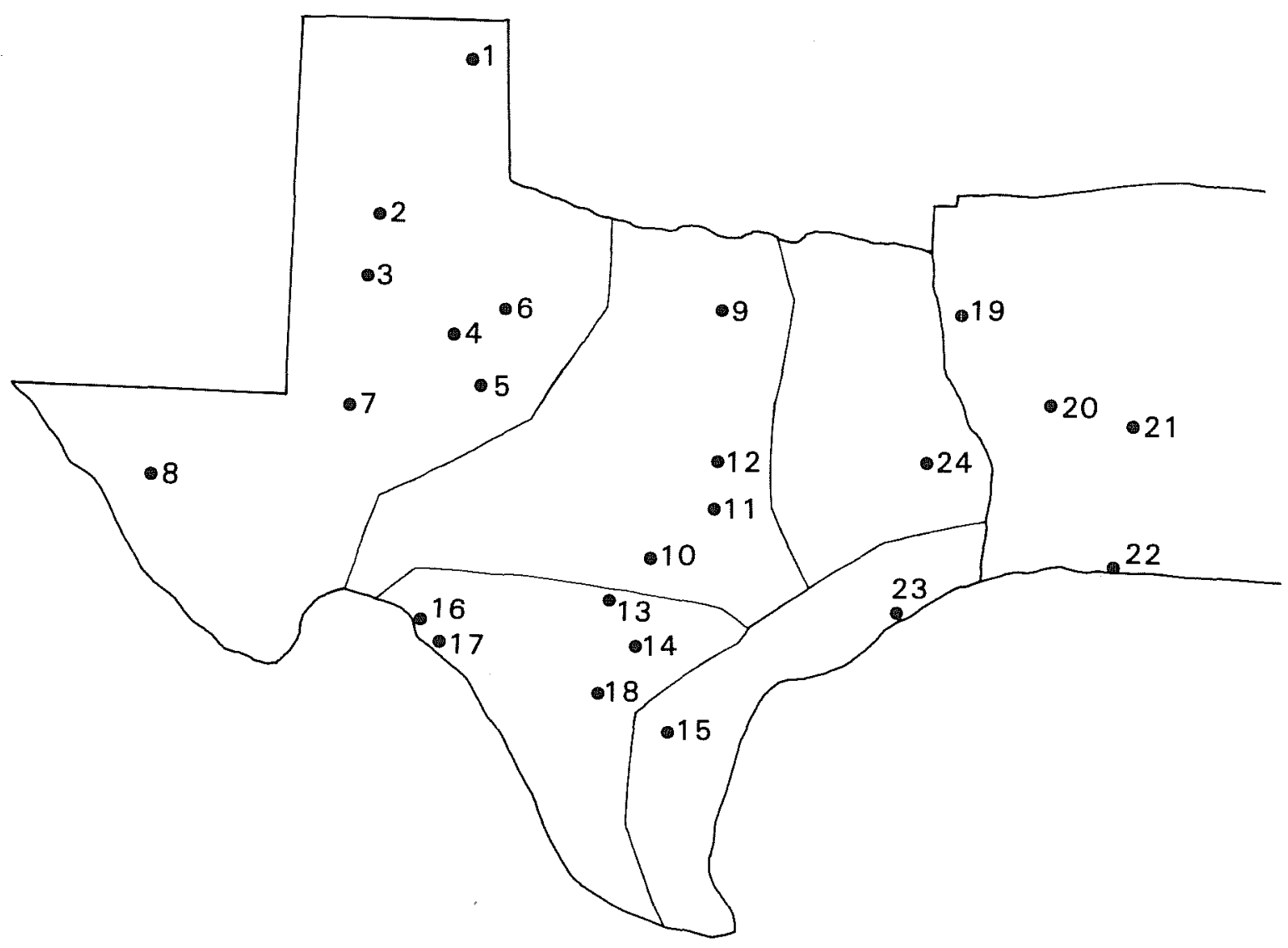

1. Lipscomb

2. Plainview

3. Lubbock Lake

4. Steadman

5. Lone Wolf Creek

6. Biedleman Ranch

7. Kidland (Scharbauer)

8. Chispa Creek (Van Horn)

9. Lewisville

10. Levi

11. Stillhouse Hollow

12. Horn Shelter
13. Friesenhahn Cave

14. Salado Creek

15. Berclair Terrace

16. Bonfire Shelter

17. Devil's Mouth

18. San Miguel Creek

19. John Pierce

20. Eagle Hill II

21. Whatley

22. Avery Island

23. Beaumont Beach

24. Wolfshead

Figure 59. Lithic Period Sites in Texas and Louisiana. 
Like the Midland-Folsom problem, Plainview points, except for a more random flaking pattern, are practically mirrored by the Golondrina point. Golondrina points have been found in buried contexts without Plainview elements and are associated with certain other tools such as the clear Fork gouge (bifacial variety). Clear Fork gouges are found in two forms, a bifacial variety found in Paleo-Indian assemblages, and a unifacial form found in Early and Middle Archaic assemblages. They are similar to the "Dalton Adzes" reported by Morse and Goodyear (1973).

The Cody complex is represented in Texas by surface finds of Scottsblufb points, primarily in the Panhandle Plains, east Texas, and along the coast. As described for the southeastern coast along the Gulf of Mexico, coastal regions in Texas reveal Late Paleo-Indian occupations. No doubt, Early Paleo-Indian sites are present along the now submerged margins of the continental shelf. The present coastline is now approximately 50 miles inland from the coastlines of Clovis and Folsom times.

The most common Late Paleo-Indian point in Texas is the Angostura point. This form is somewhat different, however, from its High Plains manifestations.

\section{Archaic Complexes}

The transitional period from Paleo-Indian to Early Archaic, the "Pre-Archaic" (Sollberger and Hester 1972), is represented by a series of distinctive tools: unifacial Clear Fork gouges, Guadalupe gouges, triangular dart points, Bell points, and the smaller Gower point. Other components of the Pre-Archaic assemblage are a continuation of typical Paleo-Indian scraper types, gravers, and drills. Although the transition period may have been more abrupt in western and southern portions of the state, continuity from Late Paleo-Indian to Early Archaic appears to be more the case. Gouges were present in Paleo-Indian times. Stylistic elements are somewhat changed, but component tool types remain essentially the same.

Archaic sequences for the Plains and the southeastern forested regions are basically those described earlier for the "parent" regions. Central Texas, however, has a unique culture chronology because of its ecotonal situation between these two areas. Weir (1975), in his discussion of the central Texas Archaic, describes the earliest Archaic period (the San Geronimo phase) as being a sparse period during the Hypsithermal when resources would have been scarce. Groups of hunter/gatherers would have been smaller and wider ranging, in order to maximize scarce resources. Tools are essentially a continuation of the Pre-Archaic/Late Paleo-Indian. This Early Archaic pattern matches what has been noted for other transitions over the areas previously discussed. With the Middle Archaic, Clear Fork (5000-4000 B.P.) and Round Rock (4200-2600 B.P.) phases, however, a significant change occurs. The burned rock middens occur, although elements of the San Geronimo tool kit continue, with heavier emphas is on new resources. Weir, pointing to an increase of oak forests in previously open areas, postulated heavy dependence upon acorn and associated whitetailed deer beginning in the clear Fork phase and flourishing into a forest efficient adaptation in the Round Rock phase. The Clear Fork gouge ceases to be used at the end of the Clear Fork phase. 


\section{Lithic Period Sites in Louisiana}

Previous archaeological investigation in Louisiana has been extremely limited with primary consideration, as in the Southeast in general, placed on Late Archaic and later cultures.

Scattered finds of Paleo-Indian points have been found almost exclusively in Tertiary and Quaternary uplands. Much of Louisiana is geologically too new to have been in existence during Paleo-Indian times. Gagliano (1977) has reported coastal finds of Late Paleo-Indian San Patrice and Lerma points along with bladelets, scrapers, and bifacial knives. Between 8000 and 9000 B.P., portions of Louisiana became an extension of the Plains with resultant influxes of Plano hunters. The Angastura and Meserve points, Plains equivalent of Dalton, are found along with eastern types such as Quad, Dalton, and the more 1ocalized San Patrice points. Webb, Shiner, and Roberts (1971) describe the San Patrice point as a late survival of the fluted, concave base points typical of Early Paleo-Indian styles. They fee 1 it represents a Late Paleo-Indian manifestation centered in northwestern Louisiana which lasted no later than 8000 years ago. Two types of site locations have been described for the San Patrice culture, the margins of upland terraces overlooking streams and along small streams dissecting uplands, but away from major rivers and lakes. The San Patrice complex is basically a small tool complex and a unifacial industry manufactured of local materials (Webb, Shiner, and Roberts 1971). The artifact assemblage associated with San Patrice points at the John Pearce site (Webb, Shiner, and Roberts 1971) includes side-notched scrapers (Albany spokeshaves), side and end scrapers, lanceolate projectile points, small numbers of burins, drills, cores, gravers, and retouched flakes.

Another Paleo-Indian point found in Louisiana, the Pelican point, is a pentagonal point reminiscent of the fluted pentagonal points from the Reagan and Williamson sites of the Northeast.

The Whatley site (Thomas and Campbel1 1978) in north central Louisiana contained a Paleo-Indian level with San Patrice and Meserve points (Fig. 59). It also contained several end scrapers of the Enterline types (one spurred), three gravers, a concave scraper, a unifacial knife, a wedge, and six notched and denticulate scrapers.

The Early Archaic in Louisiana is not well known. The Whatley site produced an Early Archaic level not significantly different from the Late Paleo-Indian level below. Paleo-Indian types continued with the addition of grooved axes, mortars, nutstones, and manos. Middle Archaic cultures in Louisiana are best represented by shel1 middens indicating the great change to a more aquatic or riverine orientation in Archaic cultures.

Paleo-Indian Projectile Point Chronology in Western Louisiana (Wallace)

One of the most engaging questions in Louisiana archaeology is the late persistence of the Paleo-Indian transition. The Eagle Hill excavations shed a tiny ray of light on a large and complex problem. Llano tradition occupations 
of Louisiana have long been recognized in both the Gulf Coastal Plains and the upland hills (Gagliano and Gregory 1965; Gagliano 1967b) primarily from projectile points found during survey and surface finds in private collections. Virtually all these finds are in the northwestern uplands of the state. Before recent work on the Mississippi Delta (Gagliano 1977) none had come from the younger coastal 1and. Most of the data obtained from surveys has been of little use, however, because of the imprecise dating of the finds. Of particular interest are Plano points originally called Yuma, but later renamed Scottsbluff (Davis 1953; Wormington 1957). No Plano sites, however, have been excavated. There is also a question as to whether the Scottsbluff points of east Texas and northwestern Louisiana are technological relatives of the classic High Plains forms or only morphologically similar pieces.

The persistence of the Paleo-Indian technology is exhibited by morphology and associations of San Patrice points in northwest Louisiana. San Patrice occupations have been dated as early as 8000 B.P. and as late as 6000 B.P. If the San Patrice occupation is not Plano then it must surely be an Archaic culture. However, morphological and associational analyses have shown a greater resemblance to Plano than to Archaic (Webb, Shiner, and Roberts 1971). Generally, the San Patrice occupation has been designated terminal Paleo-Indian.

Observation shows morphological similarity between San Patrice and other PaleoIndian assemblages. The San Patrice point type is comprised of three varieties: Hope, Gooduin, and St. John. San Patrice Hope and Meserve points both have concave stems, edge ground concave bases, alternately beveled blades, and basal thinning by removal of short, flutelike longitudinal flakes. San Patrice points are shorter and relatively wider than Meserve points. Meserve points, however, appear more often to be reworked. The shoulders of San Patrice points are more prominent, while the shoulders of Meserve points are poorly defined. San Patrice point stems have small but distinct ears at 45 degree angles, while Meserve point ears are less well defined. Finally, beveling on a San Patrice point is generally on the left, while beveling of a Meserve point is on the right (Davis and Davis 1960).

San Patrice points were originally noted at the typesite in Louisiana (Webb 1948) and are also known from the Wolfshead (Duffield 1963), John Pearce (Webb, Shiner, and Roberts 1971), Whatley (Thomas and Campbel1 1978), 16 GR 58 (Morehead and Guderjan n.d.), and Big Brushy (Guderjan and Morehead n.d.) sites, and at an unconfirmed Texas excavation (Morehead and Guderjan n.d.). Additionally, the San Patrice point can be found throughout Texas, Oklahoma, Louisiana, and Arkansas. This complex has also been found in the Forrest city area of eastern Arkansas (Webb, Shiner, and Roberts 1971). Occurrences elsewhere in Texas and as far north as Missouri show that the San Patrice assemblage ranges between the Meserve type to the west and the Dalton type to the east.

Several attempts have been made to establish the San Patrice assemblage as transitional. At Wolfshead, San Patrice and lanceolate, concave base points have been shown to precede side-notched and expanding stem corner-notched dart points (Duffield 1963), but tool complexes and associations cannot be accurately determined for the San Patrice assemblage, because the projectile points do not exactly fit Webb's 1948 definition. There is, however, a resemblance to Paisano and Meserve points (Suhm, Krieger, and Jelks 1954; Davis and Davis 1960; Ford and Webb 1956) which indicates a strong relationship between 
San Patrice and Meserve points. At Wolfshead, the San Patrice culture has been assigned to the transition, because it possesses traits of both the PaleoIndian and Archaic 1ifestyles. Typological, distributional, and associational data support this but, due to excavation methodology, the assemblage was not isolated.

At the John Pearce site (Webb, Shiner, and Roberts 1971) dated to 8000-6000 B.P., the San Patrice assemblage is assigned to the Paleo-Indian period, because grinding and milling tools do not appear in association with the tool assemblage. Technological and morphological observations such as edge angles on scrapers, etc., prove no relationship to Plains Paleo-Indian material, but they do indicate that the technology at the site is closer to Plano than previously thought (Irwin and Wormington 1970), because beaks, spurs on flakes, burins, and drills are common as in plains technology.

A related and perhaps equally pivotal problem is the undated, questionably affiliated Scottsbluff-like component in the area. It is not entirely illogical that Plano cultures might have moved into the region as the Hypsithermal dessicated the Plains after 8000 years ago. A highly resolved examination of High Plains and Louisiana/east Texas specimens is certainly warranted. Opinions vary as to whether the morphologically similar types are technologically the same (Goodyear, personal communication) or different (Den is Stanford, personal communication).

The present chronology is so $i 11$ defined that San Patrice and Scottsblufo points could occupy any time slot in the post-Clovis period in any order. Other problems revolve around a scarcity of artifacts such as projectile points, the location of Paleo-Indian sites due to the geological age of alluvial floodplains in Louisiana, sketchy reports of most investigations, lack of stratigraphically wel1-controlled excavations, and the inconclusiveness of projectile point chronologies.

Lithic sites can be expected to be rare in Louisiana due to erosion-sedimentation, i.e., degradation of Pleistocene soils and aggradation of Holocene alluvial sediments. The alluvial valleys and delta plain of the Mississippi River date to no older than 3500 to 4000 B.P. (Saucier 1974). Most of the evidence of early man will be deflated and buried beneath the marshes of Louisiana or wi11 be on the continental shelf covered by the Gulf of Mexico (Gagliano 1977). The more northern upland reaches of the state hold a great deal of potential, because of the presence of Pleistocene terraces and rare depositional curiosities such as Peason Ridge (see geomorphic report, this volume).

Most current data from the Paleo-Indian period results from surface artifacts retained in private collections. The San Patrice typesite has never been reported in detail (Webb 1948). Although the results of the Avery Island excavations are tantalizing, the mechanized excavation (Gagliano 1967a, 1970), for whatever reason, resulted in no great stratigraphic control, and the context of the archaeological finds can only be generally ascertained. The Whatley site (Thomas and Campbe11 1978) near Alexandria and the John Pearce site (Webb, Shiner, and Roberts 1971) near Shreveport are the only thoroughly reported sites. The Whatley site is unfortunately undated. The John Pearce site dates suggest the late occurrence of San Patrice points. The excavation 
of 16 GR 58 near Pollock is still in preparation. Interestingly enough, grinding and milling tools were found in levels with San Patrice points at 16 GR 58 (Morehead and Guderjan n.d.).

The Archaic tradition is defined as a generalized hunting and gathering economy and by stemmed projectile points. The Paleo-Indian period is defined solely upon a "specialized" hunting strategy and accompanying fluted points. Most sites indicate that Early Archaic stage peoples with such an economy appeared after the more specialized Paleo-Indian adaptations, but this evidence does not deny the possibility that this specialized economy was contemporary with the Archaic stage, nor does it imply that hunting was all the Paleo-Indians ever did to survive. Most clovis sites represent big game or megafauna hunting activities, although the presumed consistent association of big game or herd animals has never been discovered, with one possible exception, or demonstrated east of 0klahoma. It has never been confirmed except in the environmentally similar grass prairies of the Plains regions of Arizona, New Mexico, Texas, Oklahoma, Colorado, and Wyoming, but then neither does the fluted point disappear with the extinction of these animals.

Fluted points persist even into a time when stemmed points predominate. They persist in California for some time after A.D. 1. Technologically similar points with basal thinning, but not true fluting, can be found in many areas such as in the Northeast on some Copena ceremonial points, in northern Alberta, and even in southern Texas (Bryan 1977). The concept of the Desert Archaic, for example, has been questioned, because of the associations of flaked stone tools with fluted points. This assemblage was regarded as being used by Desert Archaic people, but was assumed to have been made by Paleo-Indians. Even the wide distribution of Paleo-Indian points is represented by fluted and unfluted points. In the West, clovis points are fluted, while in the East and Southeast these points are unfluted.

Paleo-Indian Technology and Demography in Northwestern Louisiana (Gunn)

Paleo-Indians, because of the substantial time depth of their occupation and the relative sparseness of their populations, have provided North American archaeologists with only infrequent insights into their daily lives. Where archaeological remains of Ice Age Americans have been located, lavish attention is paid to the thinly scattered shadows of distant lifeways. The result of this effort is an emerging picture of settlement patterns, subsistence, and technological preferences, and in some cases cultural forms which suggest burial customs and other more intangible and elusive habits. While these conjectural reconstructions of Paleo-Indian lifeways cannot be considered conclusive, they do provide the prospective researcher with a body of knowledge from which rather direct questions can be asked of prospective data sets prior to their recovery in archaeological excavation and analysis. This ability to anticipate results and prestructure research designs is particulariy important in regions where little previous work has been done such as in west central Louisiana. In the following pages, a demographic model is developed for Paleo-Indians of Louisiana which draws upon the theory of Paleo-Indian lifeways as is currently understood by specialists in the field and upon the knowledge of archaeologists in surrounding areas such as east Texas, the Plains to the west, and of Louisiana and Arkansas 
archaeologists to the east. The model explains some of the cultural changes that occurred during the Paleo-Indian period as a result of demographic succession, as well as culture changes, which probably reflect the influence of environmental change.

A study of Paleo-Indian point types from Louisiana taken from museum and private collections by Gagliano and Gregory (1965) provides some insights into the cultural chronology of the late Pleistocene of Louisiana. Four point types appeared as important components of the review. The quantities and characteristics of these types are presented in Table 27. Several lines of evidence place Pelican and San Patrice points later than Clovis points as a local variant of the Dalton/Meserve phenomenon. The Scottsblufb point, on the other hand, is well recognized and as yet is undated. Opinions differ as to its placement in the local chronology and whether it is a legitimate relative of the High Plains Scottsblufo type.

\section{TABLE 27. PRIMARY PALEO-INDIAN POINT TYPES IN LOUISIANA}

\begin{tabular}{|c|c|c|}
\hline Style & Frequency & Comments \\
\hline Clovis & 18 & $\begin{array}{l}\text { Texas flints and Arkansas novaculites, widely } \\
\text { distributed, uplands along Red River, percus- } \\
\text { sion flaked }\end{array}$ \\
\hline Scottsblubo & 64 & $\begin{array}{l}\text { Texas flint }(77 \%) \text { and Texas locus, northwest } \\
\text { Louisiana, percussion flaked }\end{array}$ \\
\hline Pelican & 17 & Local material, good workmanship \\
\hline San Patrice & 72 & Local, grainy chert \\
\hline
\end{tabular}

A careful examination of the materials and craftmanship observed by Gagliano and Gregory (1965) shows a notable and usual progression from exotic to local cherts as we11 as a general increase in frequency with the exception of the Pelican type. Webb, Shiner, and Roberts (1971:20-23) report a remarkably Upper Paleolithic tool assemblage at the John Pearce site, a predominantly San Patrice point locality near Shreveport, Louisiana. Their analysis of debitage indicates an almost wholly flake-oriented 1ithic technology (Webb, Shiner, and Roberts 1971:25, 33). They report a late date (8000-6000 B.P.), for San Patrice, which is contemporary with the Plains Scottsbluff type. This contemporaneity, among other things, directly questions the opinion that the Louisiana Scottsblufo point is late and equivalent to the Plains point. For the sake of argument, the position that the Louisiana Scottsbluff point is earlier than the San patrice point will be examined in the following section. 
Theory of Paleo-Indian Culture, Demography, and Culture Change for Western Louisiana

In the next section the rather typical Paleo-Indian lithics from Eagle Hill II will be described. The occupation floor on which they were found was dated by thermoluminescence to about 10,000 B.P. While it can be said that the date and tools fit comfortably into the late Folsom/Dalton periods defined from neighboring areas, there is reason to believe that western Louisiana and its immediate vicinity may offer the possibility of a late surviving Paleo-Indian tradition.

As yet there is little theory and only a modest amount of excavated and collected artifactual material through which one can view the context of the Eagle Hill finds. The following few paragraphs attempt to synthesize a plausible model of Paleo-Indian cultural development which explains known facts.

Irrespective of whether the bearers of "Clovis Culture" some 11,000 to 12,000 years ago were of western or eastern hemispheric origin the wide spread homogeneity of their technology marks them as a pioneer culture with the attendant attributes of high mobility. The full implications of this mobility, argued for decades (Martin 1973), has only recently become evident. Goodyear (1979) has begun to build a model of Early Paleo-Indian technology that accounts for heavy reliance on cryptocrystalline tools through mobility based on his experience with experimental replication of Clovis technology, extensive experience in the Southeast, and recent ethnographic studies by Binford (1978).

Goodyear's "logistical model," in essence, arques that the highly mobile PaleoIndians frequently encountered opportunities to collect food under very unpredictable conditions. Perhaps the most unpredictable and critical of factors is the unlikely availability of good quality material from which to make tools, coincident with the encounter of an unexpected but preferred subsistence opportunity. This set of circumstances encouraged late Pleistocene hunters to manufacture tools from the most manageable and predictable of materials, specifically cryptocrystalline stones. Goodyear (1979) further argues that frequent retouching and recycling is a logical outgrowth of this logistical system since preferred sources of the requisite material are only visited on scheduled occasions as a subsidiary activity of the annual subsistence round. Evidence for this is found in the ethnographic record among Eskimo cultures and Australian aboriginal groups.

Goodyear explains the movement away from exotic, fine-grained raw material to the exploitation of coarser, local cherts typical of southeastern cultural sequences as cultural change in the face of varying demographic conditions. As best as can be determined by the movement of flints away from their source, the Paleo-Indians must have traveled over territories at least 100 miles in 1 ateral extent. As the land became more densely settled and movement restricted, a broader range of resources was exploited. This broader based, spatially restricted economy enhanced the predictability of resources, reduced accessibility to distant quarries of select material, and generally encouraged the use of locally available if not as workable material. This trend was no doubt 
encouraged by the fading necessity to reshape and recover critical tools at points distant from raw materials.

While there may be alternative models, Goodyear's ideas provide a logical and plausible explanation of the elaborate treatment of fine-grained flints by Ice Age hunters and is readily seen as a complementary scheme to the bone quarrying ideas suggested by Bonnichsen, E. Johnson, Frison, and others. Bone quarrying is also thought to be an expedient measure by highly mobile hunters.

I might suggest, at this point, a refinement of the Goodyear (1979) model in view of the Gagliano and Gregory (1965) data discussed earlier. Let us assume, for the moment, that the order of occurrence of the points in time is sequential, and that they are correctly ordered as in Figure 60. Only the Scottsblubo specimens are in question as to order. Technological parallels drawn between Folsom preforms and parallel-flaked Scottsblufb points suggest a tenuous, but possible position between the clovis point and the very Late Paleo-Indian San Patrice manifestation.

The technological trajectory outlined by this order is logical enough. Clovis and Scottsbluff points are both percussion flaked. Exotic flints grade into local cherts, workmanship begins at a high level with clovis and Scottsblufó points and suffers a de-emphasis with the San Patrice point. The frequencies of the Pelican type, however, are a matter of some incongruence. Workmanship on the Pelican point is reported (Gagliano and Gregory 1965) as being "good" as one might expect in a culture transitional from one making Scottsbluf́ points. On the other hand, the material used for the Pelican points are local as might be foreseen for a type transitional to the San Patrice type. The frequency of Pelican points, however, are remarkabiy much lower than either of the adjacent categories.

High

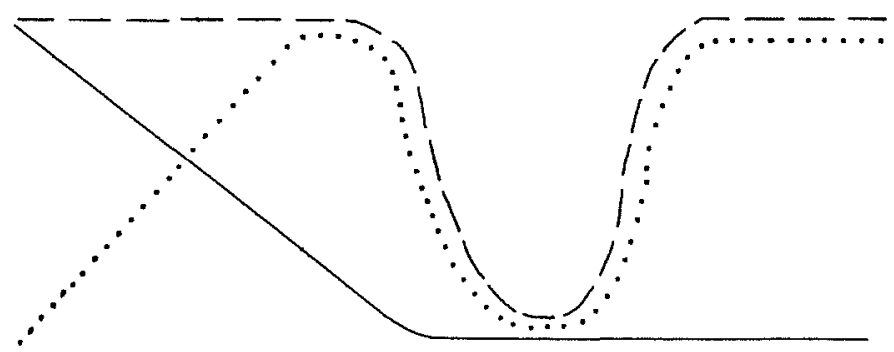

.... Population (number of
points) - - Stylistic Integrity Mobility

Low

$$
\text { Clovis Scottsbluff Pelican San Patrice }
$$

Figure 60. Relationship between Demography, Climate, and Cultural Change in the Louisiana Area Based on Point Frequencies and Patterns of Manufacture.

Without being overly optimistic in the absence of any sound chronometric data, it could be suggested that this low frequency of Pelican points intermediate between Scottsblubf and San Patrice represents a period of population decline and/or a short, intermediate period. If such is the case, a trajectory of 
culture change in the Louisiana area as in Figure 60 may be suggested. Of primary interest is the fact that influences favoring cultural change are, in part, demographic and, in part, environmental. If "stylistic integrity" is defined by the duration and coherence of a cuiture as measured by a common pattern of procurement and manufacture, then we might assume that clovis and Scottsbluff points with their common penchant for exotic material, percussion flaking, and general lanceolate form are a stylistic unit. Stylistic integrity then breaks down for a time and reappears as the we11-represented San Patrice style. The Pelican series is represented as being low in stylistic integrity, because it shares the pattern of styles preceding and following it.

We might infer, in the case of clovis and Scottsblubs points, that the differences between the styles is a function of demography. As the sparse Clovis hunters became more numerous their subsistence and technology changed to meet shifting demographic conditions. The subsequent radical change in style, however, possibly punctuated by an intermediate population decline, need not be explained in terms of culture responding to demography. More likely, an explanation might be sought in the realm of environmental or culturally induced change.

The find of an apparently Folsom-related component at Eagle Hill suggests that there was a Plains-related bridge, most likely between Clovis and Scottsbluff times. From Eagle Hill it is known that the Folsom phenomenon existed at 10,000 B.P. Nothing is known about the Scottsbluff component except that it existed on the Plains after 8000 B.P. and was very wide spread. The scarcity of chronological data as indicated by a possible 1000-1500 year gap either way from Eagle $\mathrm{Hill}$ serves to emphasize the great amount of work to be done in western Louisiana and east Texas before a meaningful early man chronology is manifested.

\section{Conclusions}

In summary, with respect to Paleo-Indian tool kits and complexes, the Clovis tool kit appears to support a homogeneous, wide-ranging root culture with a very generalized technological base for a wide variety of adaptations. The variety of stone and bone implements was probably quite flexible in allowing clovis hunters to exploit either the open environment of the Plains or the less hospitable environment of the far Northeast or the richer, more forested, "different" environment of the Southeast.

Folsom cultures, on the other hand, as indicated by differences in numbers and percentages of utilization of particular tool types, was a more highly specialized tool kit in terms of both stone and bone implements. In addition, Folsom tool types vary less than Clovis types. Judge's (1973) work with transverse scrapers indicates increasing specialization from Clovis to Folsom industries. To what extent the Middle Paleo-Indian industries of the East conform to this pattern is somewhat unclear due to the nature of Middle Paleo-Indian finds. But if Gardner and Verrey's (1979) classification scheme for Early and Middle Paleo-Indian points is valid, there is reason to assume that other elements of the technology are similar as well. A high degree of standardization is suggested for Middle Paleo-Indian times regardless of prey species. 
Folsom peoples appear to have been highly specialized in an optimum environment. They had more freedom in site selection due to the greater availability of water and were highly specialized in bison exploitation. Judge (1973) suggests that the Folsom point consistency in size and shape was due to the practice of hafting them onto a bison rib foreshaft. This proposition appears to be consistent with the highly specialized nature of their technology and adaptation. Although it is probable that all of the Paleo-Indian hunters concentrated on other foods as well, it seems imminently more likely that Late Paleo-Indian peoples would have exploited a wider range of resources than Folsom peoples.

With the exception of some minor fluctuations such as the moist period associated with the Cochrane Readvance ca. 8000 B.P., Late Paleo-Indian cultures existed primarily in a time of increasing aridity. They would have been forced to choose less advantageous site locations to maximize certain resources: Plano peoples, the decreased water supplies on the Plains; Dalton peoples, the decreased areas of open land as forests continually encroached upon the landscape. Tool kits change little from components of earlier traditions except for an explosion of point types. In the Plains, tool kits show an increasing trend toward specialization with a continuing dependence on bison as a resource. Dalton peoples in the East were similarly highly specialized in the exploitation of white-tailed deer, although theirs and probably all eastern adaptations were always dependent upon a wider variety of forest resources. The Dalton tool kit continues, in most respects, the tradition of Middle Paleo-Indian assemblages, but adds the classic adze, possibly a good example of a high degree of increasing specialization in the exploitation of a changing environment.

The surprising find in this study has been the degree of continuity between Late Paleo-Indian/Early Archaic assemblages, not only in the East where it might be expected given an early trend toward developing a forest efficient adaptation, but in the Plains and Texas as we11. The biggest change appears to occur from the Early to Middle Archaic periods. In the Southeast, a substantial change is noted with the appearance of shellfish and artifacts. Little is known in the Northeast of the transition to Early Archaic and from Early to Middle Archaic. What evidence does exist (Staten Island sites) shows remarkable continuity from Paleo-Indian tool types to earliest Archaic assemblages with the greatest changes occurring in the Middle Archaic Lamoka culture, which was highly dependent upon aquatic resources. In the Plains and Texas, "PreArchaic" and earlier Archaic assemblages continue with major components of Paleo-Indian tool kits. Bifacial Clear Fork gouges appear in the Late PaleoIndian period and possibly earlier and switch to a unifacial form in the Early to Middle Archaic stages. Early assemblages along the Plains/eastern Woodland ecotones show continuation of trends in proportion and types of tools from Late Paleo-Indian through Early Archaic with only minor changes, primarily in point styles which are either significant in foretelling changes in adaptive strategies or not. Major changes in assemblages occur in Middle Archaic times with the addition of more ground stone tools, an increase in large tools such as core tools and large choppers, and an increase in bifacial tools in general.

One final note about the latter change is offered. Given what is known about Paleo-Indian groups, namely their mobility and their need and use of high quality cryptocrystalline materials (Goodyear 1982) as a portable, dependable, and 
flexible technology, the change from unifacially oriented to bifacially oriented assemblages is not at all surprising, nor is the decline in "fine workmanship," and the use of more exotic materials. As their mobility was gradually reduced by major changes in climate, topography, plant and animal resources, and population pressures, the need for a more portable technology diminished. Another aspect of a portable technology of high quality materials is that it is naturally a conservative one. Materials were procured at great expense. Unifacial industries are, by definition, more conservative than bifacial ones. Finer workmanship, when continually reworking and resharpening tools, is conservative as well. Not only would the increase in bifaces, larger core tools, and choppers indicate a heavier dependence on plant resources, but also the decreasing need to conserve lithic materials. No doubt, it was a highly dynamic, multicausal system which was obtained during the change from unifacial to bifacial technologies. But loss of extensive mobility and of concomitant dependence upon high quality stone and not simply the need to exploit plant foods could have influenced Archaic peoples' choice of materials and technologies. Rather than a purely culturally biased "aesthetic" choice, their livelihood simply no longer depended upon it.

\section{TOOLS AND TECHNOLOGY}

At Eagle $\mathrm{Hill}$ the inventory of formal tools and recognizable large flakes used as tools is sma11, but certainly existent. The sequence (Table 28 and Fig. 61) from the Paleo-Indian to the Historic period is standard in its composition. A relatively high frequency of tools gives way to less specialized forms until the Classic period of lower Mississippi Valley culture during the first millennium A.D. After the Classic period, formal tools again diminish.

The Paleo-Indian inventory consists of scrapers, burins, and beaks which appear only infrequently later. Gravers, notches, retouched flakes, and points are present thoughout most of the period.

The OP 4.12 episode is the most distinctive assemblage. It is comprised of virtually no formal tools, but large edge-altered flakes are present. OP 4.12 is succeeded by an erosional episode that represents a significant gap in the archaeological record.

Each occupation plane (OP) after the erosional lapse is marked by a character of its own. OP 3.11 contains a few tools. However, it is mostly distinguished by a large number of edge-altered pieces. Also, bipolar cores appear in $O P 3.11$ and the subsequent Plane. OP 3.11 has the highest index of occupation. Perhaps the large numbers of flakes indicate a brief interval of domestic activity on Peason Ridge. OP 2.13 is much like OP 3.11 except for a drastic reduction in the number of edge-altered pieces. Finally, OP 1.13 is marked by a reduction in frequency of occupation and a singularly unspectacular accumulation of 1 ithic scatter.

Table 29 indicates the total number of classes of tools and their ratios with the number of platformed flakes. The number of platformed flakes represents the total amount of lithic-related activity at the site. A flake/tool ratio shows the relative emphasis on formal tools relative to less formal utilization of lithics. A thorough inspection of the Eagle Hill lithics leads one to 
TABLE 28. FNS OF TOOLS RECOVERED FROM OCCUPATION PLANES AT 16 SA 50

\begin{tabular}{|c|c|c|c|c|c|c|}
\hline Artifact & 4.17 & 4.12 & $\begin{array}{c}\text { Substratum } \\
3.11\end{array}$ & 2.13 & 1.13 & Total \\
\hline Points & 3429 & & & 2965 & 1236 & 3 \\
\hline End scraper & 2439 & & 3087 & & & 2 \\
\hline Denticulate & & & 1853 & & & 2 \\
\hline Side scraper & $\begin{array}{l}3365 \\
2737 \\
\end{array}$ & & & & & 2 \\
\hline Notch & $\begin{array}{l}2708 \\
2737 \\
3318 \\
3399 \\
3426\end{array}$ & & $\begin{array}{l}1765 \\
1846\end{array}$ & 7322 & & 6 \\
\hline Graver & $\begin{array}{l}2838 \\
3388\end{array}$ & & 1520 & $\begin{array}{l}1754 \\
1374 \\
1365\end{array}$ & & 6 \\
\hline Beak & $\begin{array}{l}2685 \\
2779 \\
2858 \\
3403\end{array}$ & & $\begin{array}{l}1756 \\
1779\end{array}$ & 1379 & & 7 \\
\hline Burin/Spa 71 & $\begin{array}{l}2876 \\
3370 \\
2554\end{array}$ & & & 1367 & & 4 \\
\hline $\begin{array}{l}\text { Retouched } \\
\text { Flake }\end{array}$ & $\begin{array}{l}3218 \\
3220\end{array}$ & $\begin{array}{l}2091 \\
3154\end{array}$ & $\begin{array}{l}1617 \\
1784\end{array}$ & $\begin{array}{l}7171 \\
1221\end{array}$ & $\begin{array}{l}918 \\
955 \\
800\end{array}$ & 11 \\
\hline $\begin{array}{l}\text { Biface Frag- } \\
\text { ment }\end{array}$ & 2814 & $\begin{array}{l}2182 \\
2204 \\
\end{array}$ & & 1201 & & 4 \\
\hline $\begin{array}{l}\text { Edge-Al tered } \\
\text { Pieces }\end{array}$ & $\begin{array}{l}2706 \\
2708 \\
2718 \\
2731 \\
2861 \\
2858 \\
3016 \\
3285 \\
3372 \\
3384 \\
3403\end{array}$ & $\begin{array}{l}1954 \\
1958 \\
2037 \\
2034 \\
2114 \\
2179 \\
2180 \\
2215 \\
3181 \\
3205 \\
3210\end{array}$ & $\begin{array}{l}1510 \\
1550 \\
1591 \\
1716 \\
1726 \\
1734 \\
1742 \\
1749 \\
1767 \\
1773 \\
1782 \\
1807 \\
1817 \\
1832 \\
1844 \\
1849 \\
3041 \\
3074 \\
3105\end{array}$ & $\begin{array}{l}1317 \\
2982\end{array}$ & $\begin{array}{l}836 \\
818 \\
800\end{array}$ & 46 \\
\hline $\begin{array}{l}\text { Blade/ } \\
\text { Bladelets }\end{array}$ & $\begin{array}{l}2439 \\
3403\end{array}$ & $\begin{array}{l}2029 \\
2194\end{array}$ & $\begin{array}{l}0221 \\
1715 \\
1720 \\
\end{array}$ & & $\begin{array}{r}744 \\
61\end{array}$ & 9 \\
\hline Flake Core & $\begin{array}{l}2718 \\
3318\end{array}$ & & 7798 & & & 3 \\
\hline $\begin{array}{l}\text { Microblade } \\
\text { Core }\end{array}$ & 2714 & & & & 1043 & 2 \\
\hline Bipolar Core & & & $\begin{array}{l}1515 \\
1810 \\
1812 \\
\end{array}$ & $\begin{array}{l}1170 \\
1466\end{array}$ & & 5 \\
\hline TOTAL & & & & & & 112 \\
\hline
\end{tabular}




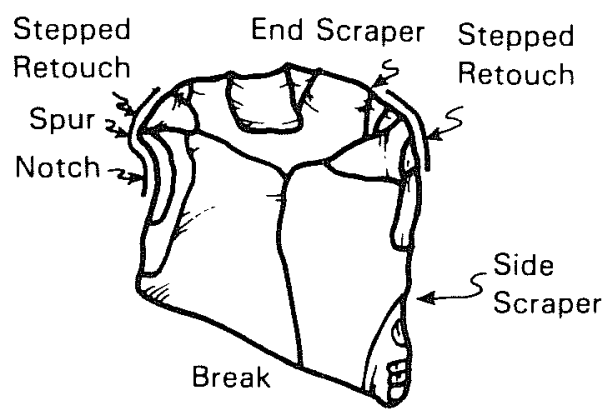

End Scraper

FN 2439

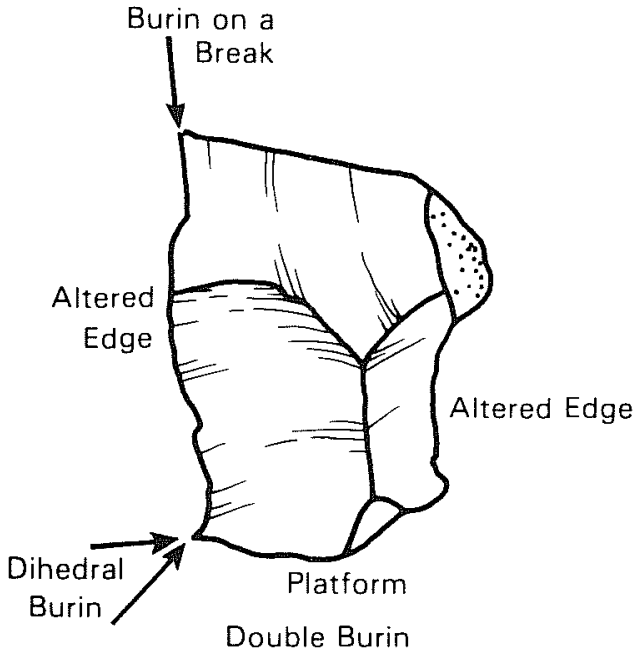

FN 2876

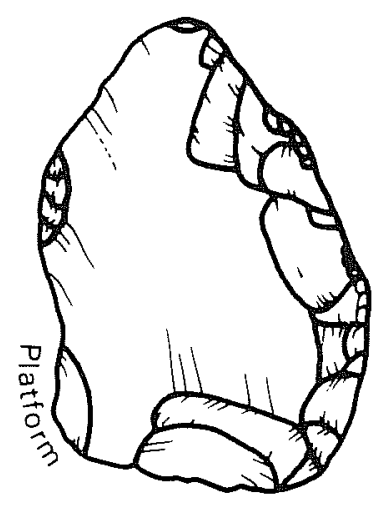

Side Scraper on Inner Face of Bifacing Flake

FN 3365

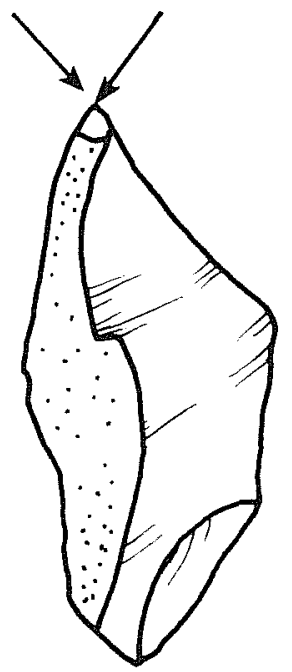

Dihedral Burin

FN 2554

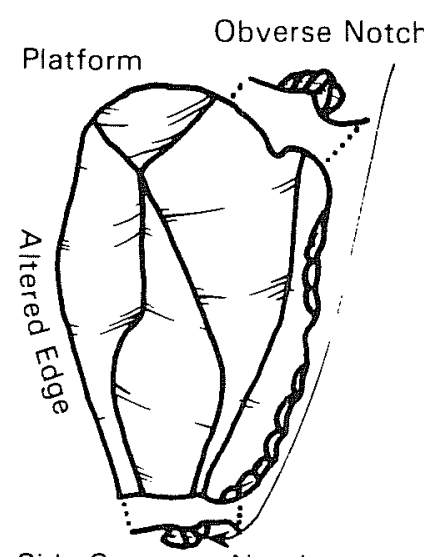

Side Scraper - Notches on a Flake

FN 2737

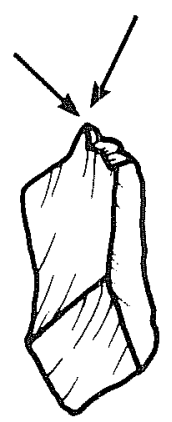

Dihedral Burin

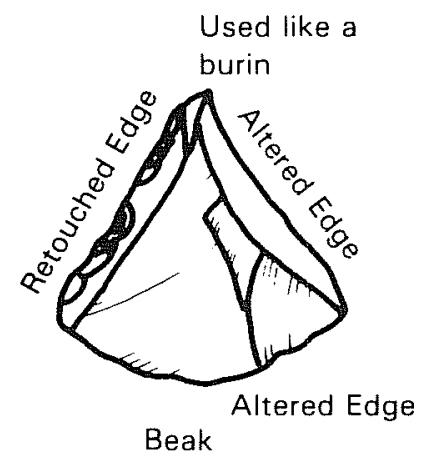

FN 2779

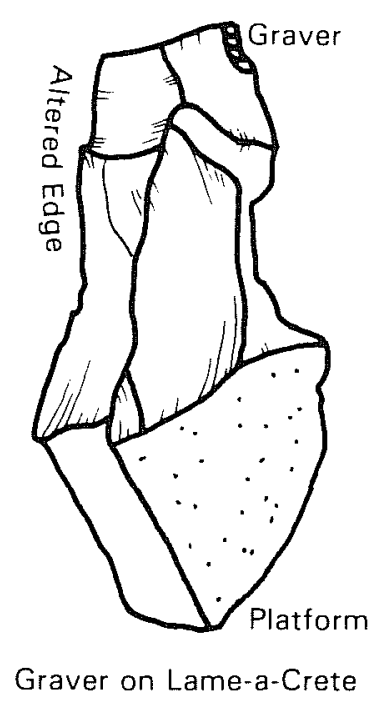

FN 3388

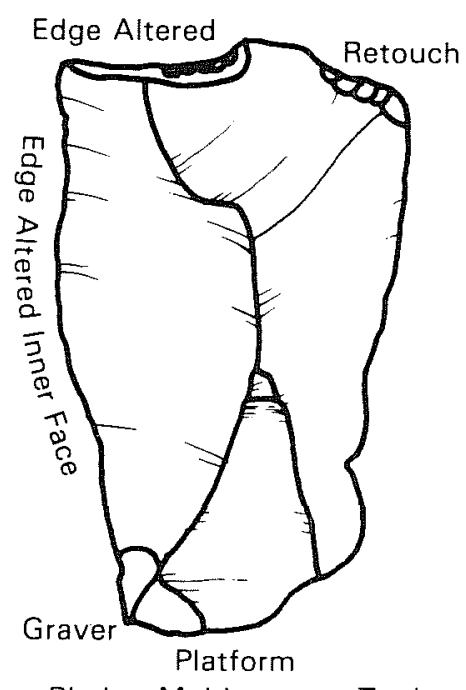

Blade - Multipurpose Tool FN 3403

Figure 61. Paleo-Indian Lithics from op 4.17. 
TABLE 29. FLAKE TO TOOL RATIOS

\begin{tabular}{|c|c|c|c|c|c|c|c|}
\hline Substratum & $\begin{array}{l}\text { Artifact } \\
\text { Formal } \\
\text { Tools }\end{array}$ & $\begin{array}{l}\text { Edge-altered } \\
\text { Flakes }\end{array}$ & Cores & $\begin{array}{l}\text { Total Plat- } \\
\text { formed Flakes }\end{array}$ & $\begin{array}{l}\text { Tool/Platformed } \\
\text { Flake Ratio }\end{array}$ & $\begin{array}{l}\text { Edge-Altered } \\
\text { Flake/Plat- } \\
\text { formed Flake } \\
\text { Ratio }\end{array}$ & $\begin{array}{l}\text { Al1 Tools/Plat- } \\
\text { formed Flake } \\
\text { Ratio }\end{array}$ \\
\hline 1.13 & 4 & 3 & 1 & 139 & .029 & .022 & .058 \\
\hline 1.21 & 1 & - & - & - & - & - & - \\
\hline 2.13 & 11 & 2 & 2 & 265 & .042 & .008 & .057 \\
\hline 2.14 & 1 & & & & & & \\
\hline 2.31 & 2 & & & & & & \\
\hline 3.11 & 9 & 19 & 4 & 300 & .030 & .063 & .107 \\
\hline 3.12 & 1 & & & & & & \\
\hline 3.21 & 2 & & & & & & \\
\hline 4.12 & 4 & 11 & - & 274 & .015 & .040 & .055 \\
\hline 4.17 & 21 & 11 & 4 & 141 & .149 & .078 & .255 \\
\hline
\end{tabular}


believe that any piece of silicious material carried to Peason Ridge was probably brought to be used. Large cores and core fragments, for instance, are universally edge altered. As Table 29 shows, when tool and toollike pieces are compared to platformed flakes, the Paleo-Indian assemblage is comprised of a ful1 $25 \%$ of tools in contrast to 5 to $10 \%$ in later assemblages.

Some of the tools recovered are illustrated in Figures 61 to 63 and Plates 8 and 9. Aside from the sheer presence of formal tools on the Paleo-Indian floor (Fig. 61), the points provide some insight into the cultural chronology of the site.

Lanceolate--The lanceolate (Plate 8 ) is made of a very tough, purple quartzite. The workmanship is clearly Paleo-Indian. Attempts at fluting failed, but it has the flat appearance of Paleo-Indian lanceolates. The artisan used the "diving flake" technique, which may have been more applicable to this unusual material. The shape, edge preparation, and flaking are reminiscent of Texas Folsom points (Thomas R. Hester, personal communication). The associated scrapers, burins, beaks, gravers, etc. (Fig. 61), al1 point to a classic PaleoIndian assemblage.

Corner Notch 1--A corner-notched point with a long blade and well-defined barbs was found in OP 3.12 of the Eagle Hill control column (Fig. 63,e; Plate 9,h). Typologically it is most like a Williams point (Suhm, Krieger, and Jelks 1962:259). It was apparently associated with the materials deflated onto the IB/IIB interface. The point could very well represent a Subboreal period occupation (2500 B.P.) on Peason Ridge which was subsequently deflated during the Roman Empire Climatic Optimum (see section on climate of the Gulf Coastal Plain, page 74). An engraver on a blade (Fig. 63,f; Plate 9,g) and a complete, al though crudely made biface (Fig. 63,d; Plate 9) were found in the same level.

No points were directly associated with OP 3.11 , but there were tools (Fig. 62). The small bipolar cores of this period may indicate the use of microlithic alternatives to points.

Corner Notch 2--A second corner-notched point similar to Edgewood was found in the OP 2.31 intermediate level in E3019-N999 (Fig. 63,b; Plate 9,e,f). It is made on pebble chert (note the pebble cortex on the reverse side). A couple of sherds from this level also appear to be similar to a style of the Marksville period. While two sherds of questionable integrity and a possible Edgewood point singly do not argue for a Marksville period occupation, in combination they do pose the possibility.

Smal1 Gary--A smal1 Gary point was found in the occupation plane immediately below OP 2.13 (Fig. 63, C and Plate 9,d). It suggests that by A.D. $935 \pm 70$, local attempts were clearly underway to adopt bow and arrow technology. A more detailed discussion of the matter follows. The strangest biface in the site assemblage (Plate 9,c) is reminiscent of the barbed points succeeding the smal1 Gary as arrow points. However, it is thick and poorly made, perhaps prototypical. 


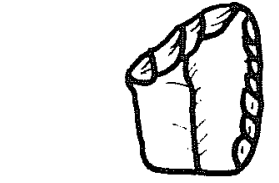

Small Scraper

OP 3.11 - FN 3082

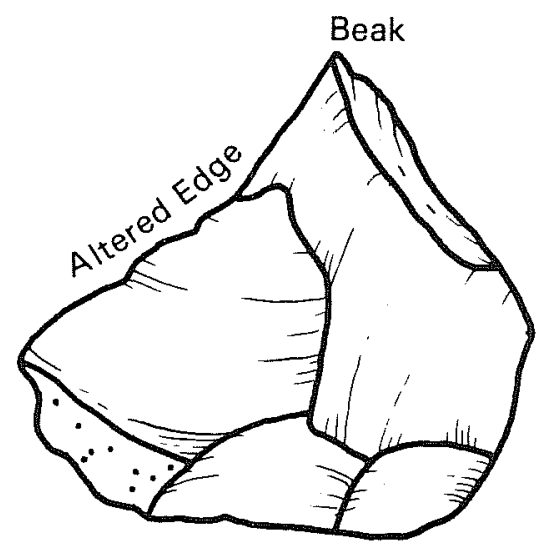

Beak on Bifacing Flake OP 3.11 FN 1756

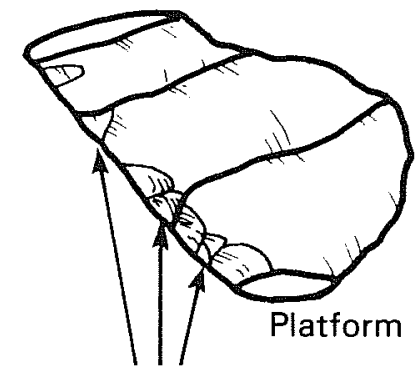

Old Denticulate Teeth

OP 3.11 - FN 1853

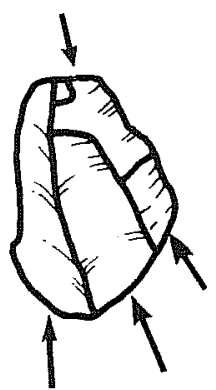

Bipolar Core

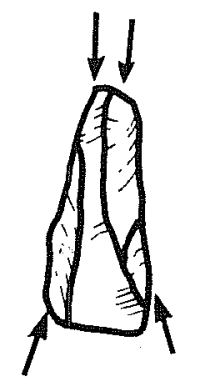

Bipolar Core

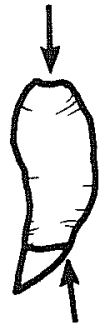

Bipolar Core

OP 3.11 - FN 1812

OP 3.11 - FN 1515

Figure 62. Tools, Resharpening Flake, and Bipolar Cores from op 3.11, A.D. 820 . 


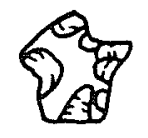

a obverse

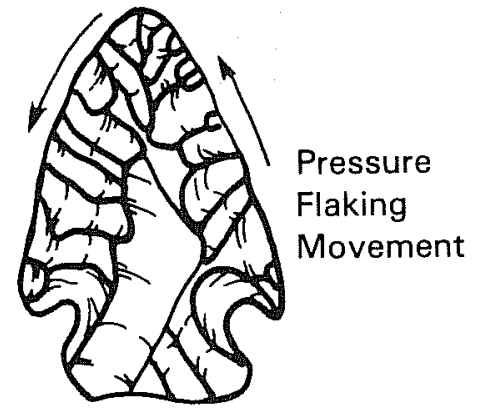

FN 1431

b obverse
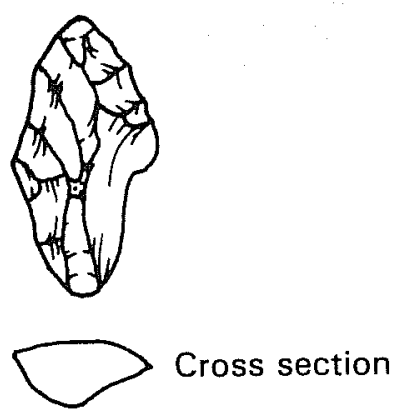

FN 193

c obverse

$$
\stackrel{ }{1 \mathrm{~cm}}
$$

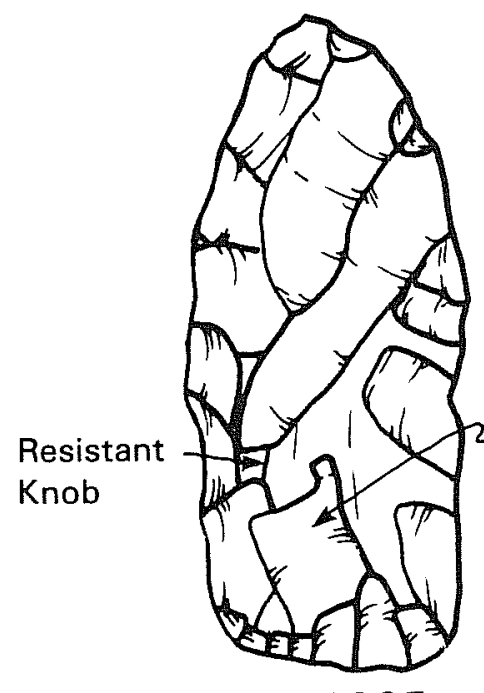

FN 1905

d obverse
End Flake

Attempt to

Remove knob,

Failed with

Hinge Fracture

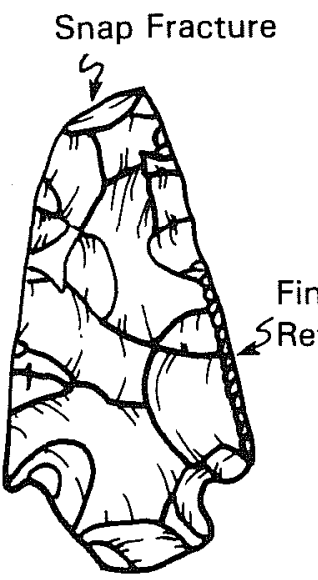

FN 242

e obverse

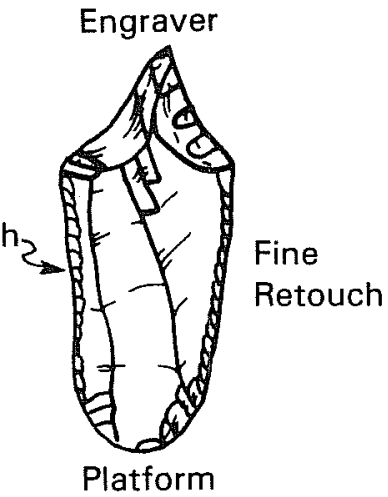

FN 1855

$f$ engraver

Figure 63. Late Holocene Artifacts. 

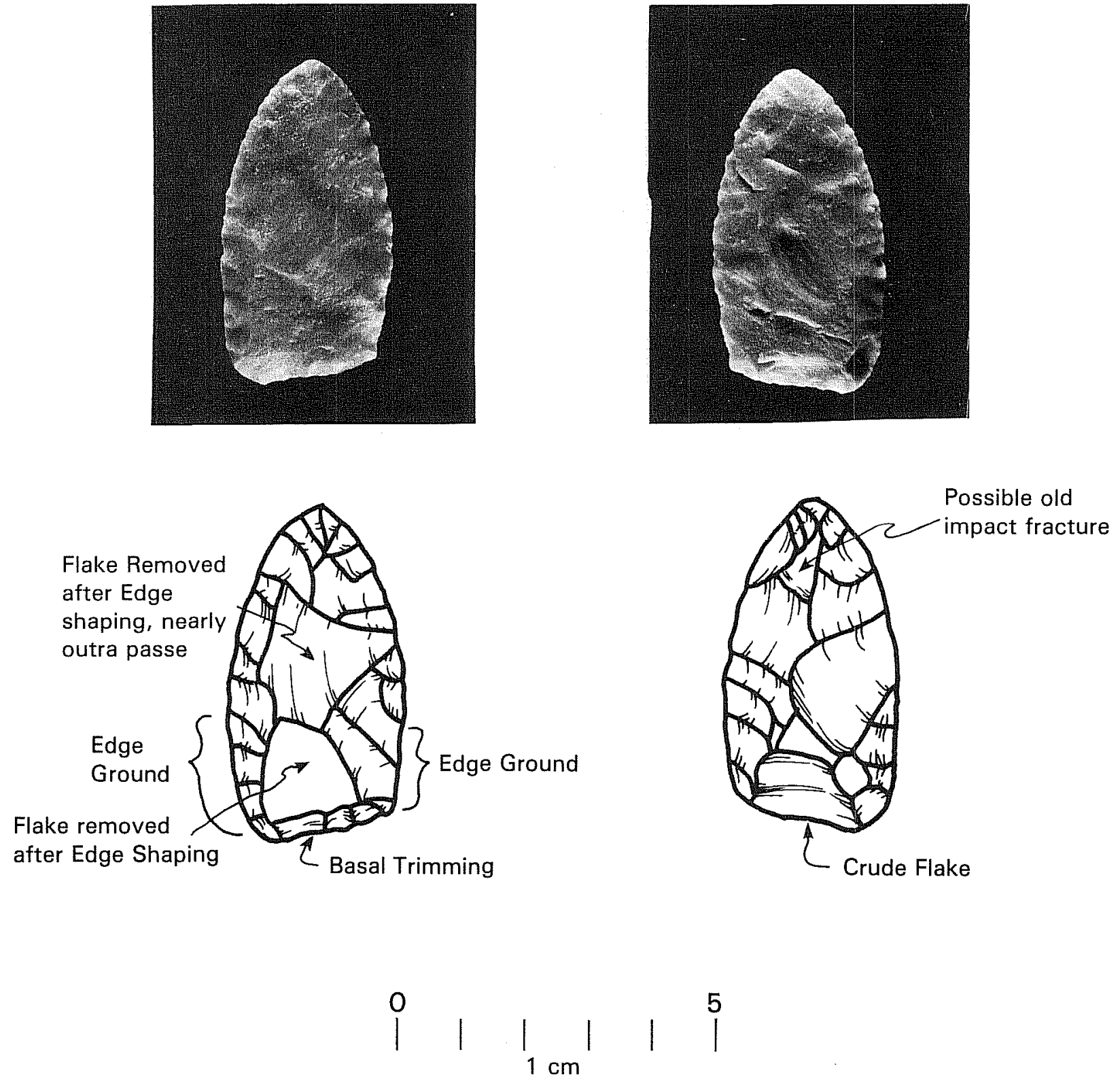

\section{EAGLE HILL (16SA50)}

FN 3429

Material: Purple quartzite, material makes it difficult to see scar spines for drawing. No fissure lines so cannot determine sequence of flaking in a 11 cases.

Workmanship: Generally very good. Basal trimming may be a different grade of workmanship. Point may have been broken near present base and reworked into shorter point by thinning and trimming base. Very slight possibility of impact fracture remnant near tip.

P1ate 8. Paleo-Indian Lanceolate (Folsom-like) from OP 4.17. 


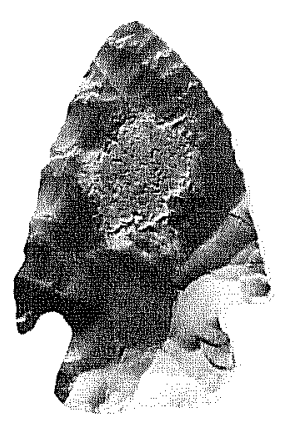

e reverse

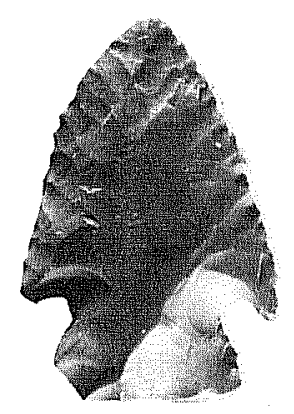

f obverse

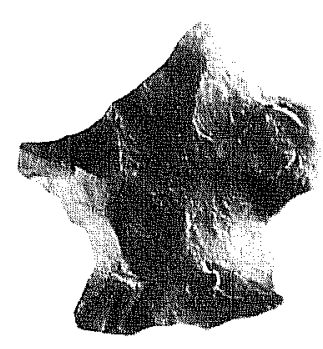

b reverse

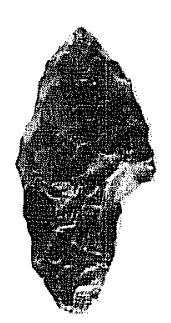

d obverse

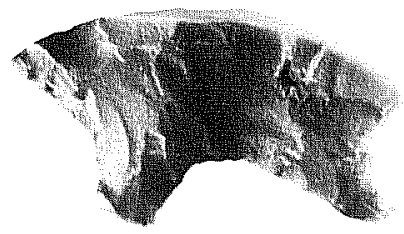

a

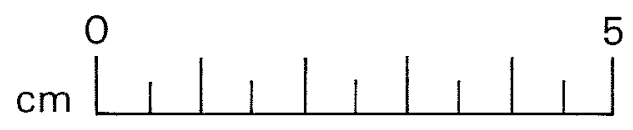

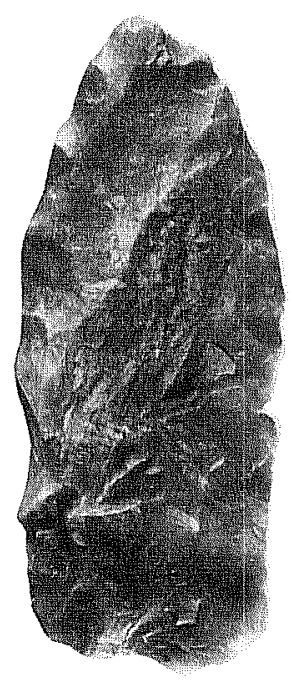

i reverse

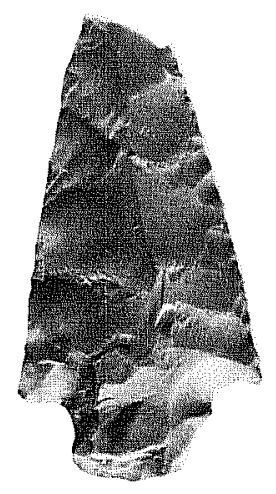

h reverse

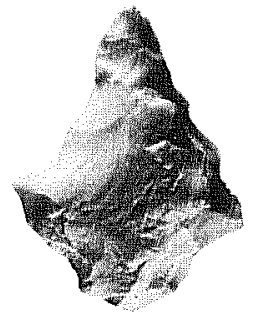

c

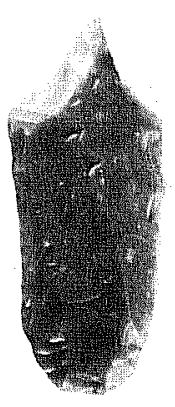

g

Plate 9. Late Holocene Artifacts. 
Friley--A Friley arrow point was found in the 1.21 intermediate level (Fig. 63,a; Plate $9, b)$. It indicates that by the time represented by the intermediate level OP 1.21, the inhabitants of Peason Ridge had made the technological transition to a refined bow and arrow technology. Unfortunately, OP 1.21 is not securely dated, perhaps after A.D. 935.

Base Fragment--The basal fragment of a possible Cuney point (Suhm, Krieger, and Jelks 1962:271) was found in OP 1.13. The Cuney arrow point (Plate 9,a) is associated with Caddoans of the Hasinai branch during the Historic period and dates between A.D. 1600 and 1800. If the dating is valid, OP 1.13 completes a very long if not full occupational history for Peason Ridge.

As was spelled out in the previous section we had hoped to define the tool kit using the resharpening and reshaping flakes of tools brought into and carried away from Peason Ridge. As the denticulate resharpening flake (Fig. 62) shows, we were able to do this to some degree by visual inspection. However, we were able to augment the tool sample only marginally with the unaided eye. Microscopic studies are discussed in the section Interpretation of Occupation Plane Patterns (page 316).

Most of the points were found in the exact top of the mound, especially in the control column. This is one of the first indications of the "dry feet phenomenon." As an escape from the surrounding swampy terrain, the site was probably most attractive during wet periods.

Biface and Core Flake Technology

Several authors (Weir 1975; Gunn and Mahula 1977; Carter 1978) have suggested that there is an environmental relationship between biface flaking and core flaking technologies. It is thought that core flake technology is related more to the Plains, while bifacing is a Woodland-preferred technology. To my knowledge these are speculations for which there is no direct supporting evidence. The matter is complicated by the fact that there is no such thing as a purely bifacial technology or a pure core flaking technology. As a rule, assemblages contain elements of both.

A logical analysis of the two industries suggests that the presumed relationship is not altogether unlikely. The stone tools of mobile Plains groups probably serve as butchering tools and for related tasks. Core flaking and particularly blade core technology provides light tools and efficient use of raw materials that occasionally have to be carried. Woodland groups, on the other hand, make additional demands on their tool repertoire. Tasks related to woodworking, horticulture, etc., require more robust implements. Furthermore, Woodland groups are usually settled at least part of the year so that a heavier tool assemblage does not hamper nomadic movements. Larger and more robust tools are more easily made by bifacing. Therefore, woodland groups preferring bifacing is not illogical.

As has been discussed in the perspective of long-term, Holocene climatic change, the west Louisiana/east Texas region is located in the unstable ecotone between the Plains and Woodlands. The location of Eagle Hill in this zone 
indicates that it could be important in resolving the core-biface ecology problem. Both Hester (1971a:113) and Gunn and Mahu1a (1977:252) have observed shifts between the two technologies in south and central Texas. More recently Thoms, Montgomery, and Portnoy (1981) have attempted to test the model in south Texas with mixed results. In any case, no connections between technological shift and ecological change have been securely established or well defined.

It is also worth noting that any such analysis at Eagle Hill is likely to be one-sided relative to ecological changes. If our surmise is correct that the site would only have been occupied during wetter and cooler periods, it follows that the assemblages from the occupation planes should a 11 be a product of Woodland style occupations. Thus, the following analysis does not provide conclusive proof of the techno-ecological relationships. It does provide a body of data on which future comparative research can be based.

Determining the bifacing component of an assemblage is a complicated problem, because the early stages (Fig. 64) yield biface flakes indistinguishable from core flakes. As the biface is thinned, the flakes begin to take on the characteristics of the typical bifacing flake. The most careful examination of a flake assemblage can only show the proportion of characteristic bifacing flakes to the corelike pieces produced early in the bifacing process and the true core flake specimens. Our counts of bifacing and nonbifacing flakes are an attempt to index the frequency of characteristic bifacing flakes against an indeterminate population of the other two types of flakes. The flake terminology illustrated in Figure 65 wi 11 be used in the following discussion. A comparison of Figures 64 and 65 shows the relatively arbitrary nature of most flake analyses.

Of the 1119 one-centimeter provenienced specimens identified as platformed flakes, 417 were determined to be bifacing flakes. Bifacing flakes were judged quite conservatively so that questionable specimens fell into the nonbifacing category. Attributes considered to be indicative of bifacing flakes were platforms with triangular profiles, strongly lipped platforms, ground platforms, and the broad, very flat slightly curved inner face and nonsalient bulbs of percussion (Table 30 ).

TABLE 30. FREQUENCIES OF PLATFORMED BIFACING TO NONBIFACING FLAKES BY OCCUPATION PLANES

\begin{tabular}{|c|c|c|c|c|c|}
\hline Substratum & $\begin{array}{l}\text { Total } \\
\text { FNs* }\end{array}$ & $\begin{array}{l}\text { Platformed } \\
\text { Bifacing } \\
\text { Flakes }\end{array}$ & $\begin{array}{l}\text { Platformed } \\
\text { Nonbifacing } \\
\text { Flakes }\end{array}$ & $\begin{array}{l}\text { Bifacing } \div \\
\text { Nonbifacing } \\
\text { Flakes }\end{array}$ & $\begin{array}{l}\text { Total Plat- } \\
\text { form Flakes }\end{array}$ \\
\hline $\begin{array}{l}1.13 \\
2.13 \\
3.11 \\
4.12 \\
4.17\end{array}$ & $\begin{array}{l}184 \\
326 \\
385 \\
345 \\
185\end{array}$ & $\begin{array}{r}32(23 \%) \\
100(38 \%) \\
118(39 \%) \\
99(36 \%) \\
68(48 \%)\end{array}$ & $\begin{array}{r}107(77 \%) \\
165(62 \%) \\
182(61 \%) \\
175(64 \%) \\
73(52 \%)\end{array}$ & $\begin{array}{l}0.30 \\
0.61 \\
0.66 \\
0.57 \\
0.93\end{array}$ & $\begin{array}{l}139 \\
265 \\
300 \\
274 \\
141\end{array}$ \\
\hline TOTAL & 1425 & $417(37 \%)$ & $702(63 \%)$ & 0.59 & 1119 \\
\hline
\end{tabular}

* total number of pieces identifiable as tools, cores, or platformed flakes 


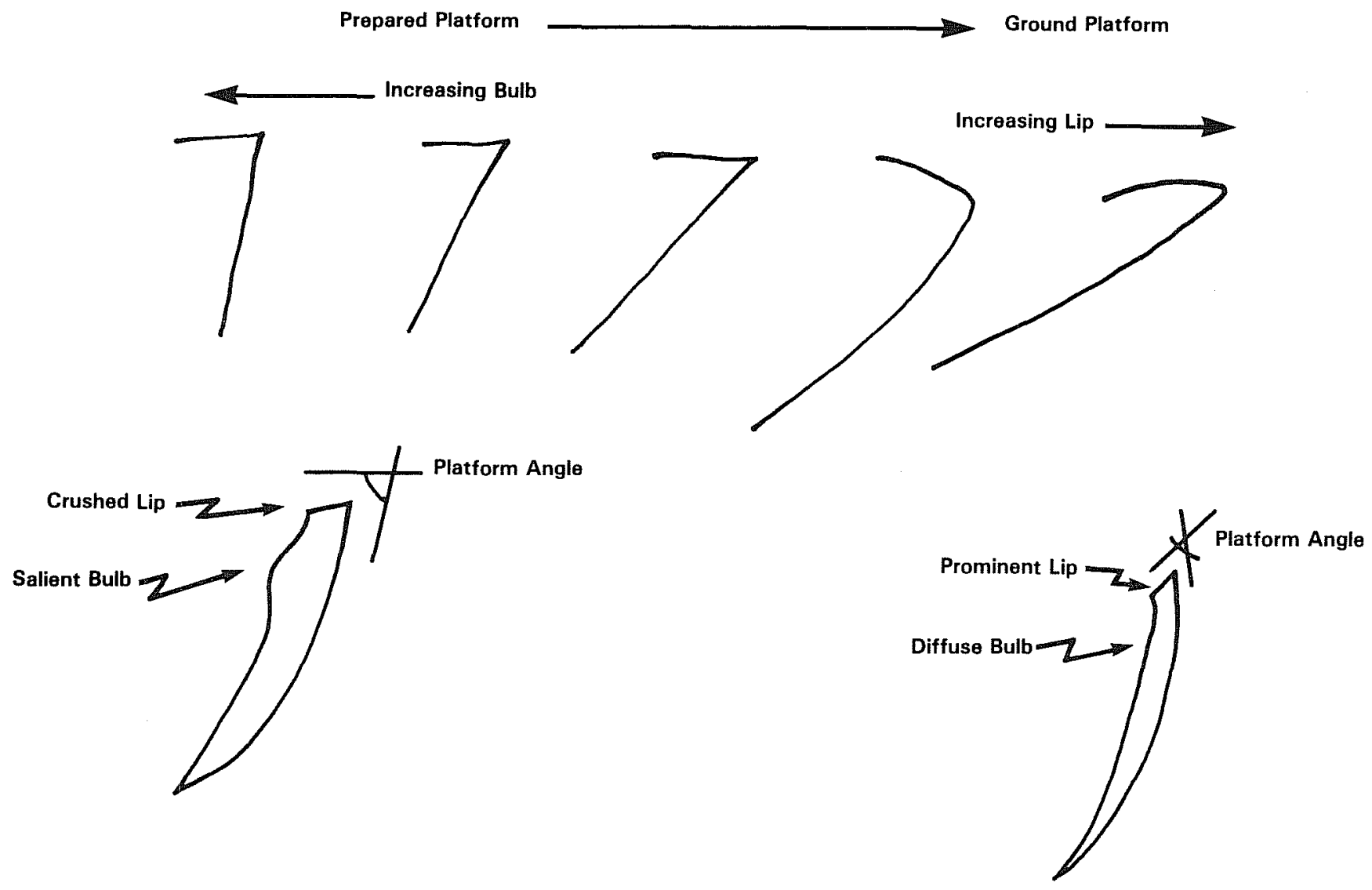

Figure 64. Progressive Bifacialization of Flakes in the Biface Producing Process. As the platform angle decreases, the platform must be ground to insure long flakes. The bulb becomes progressively more diffuse. 


\section{FLAKE TERMINOLOGY}
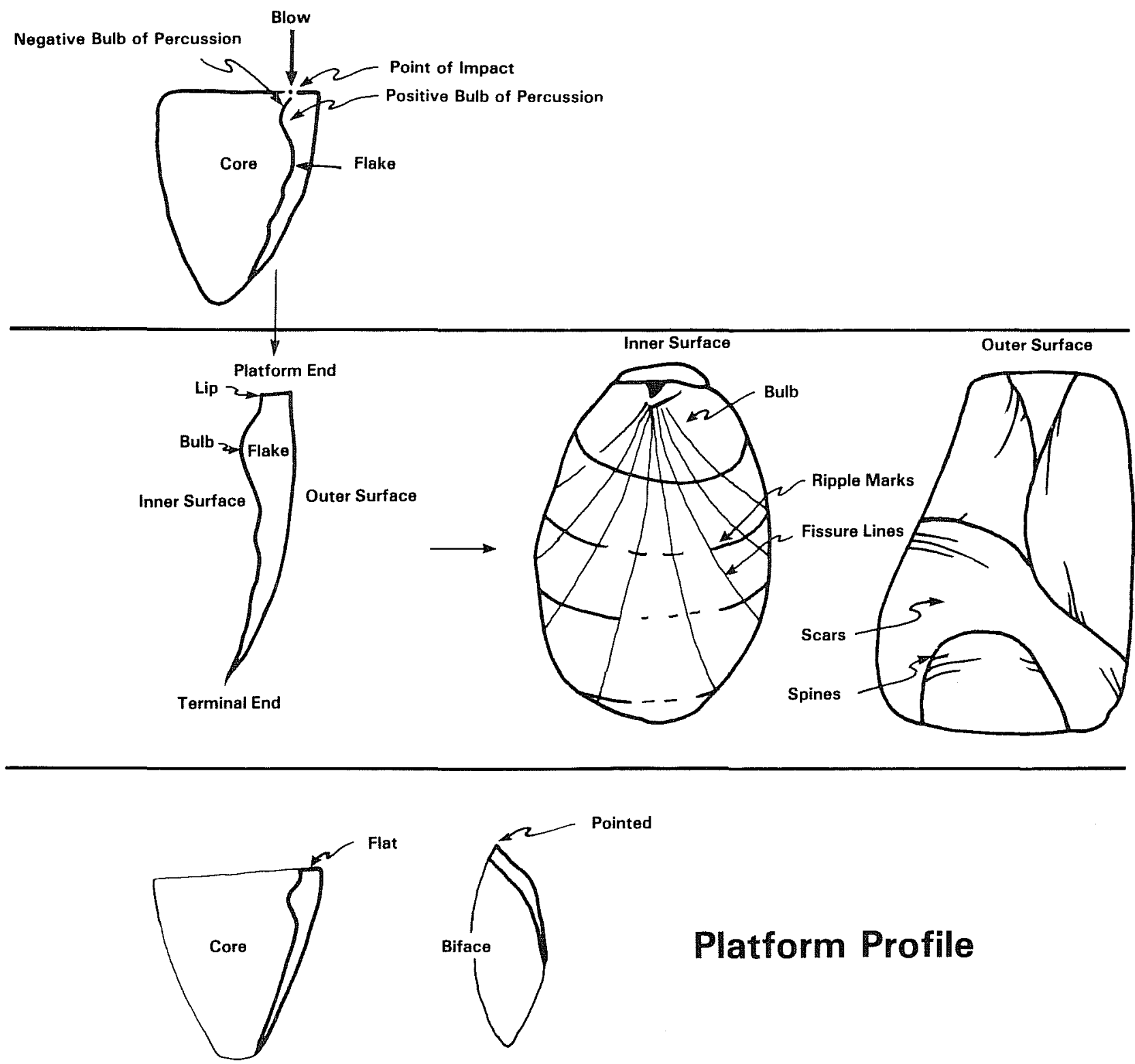

\section{Platform Profile}

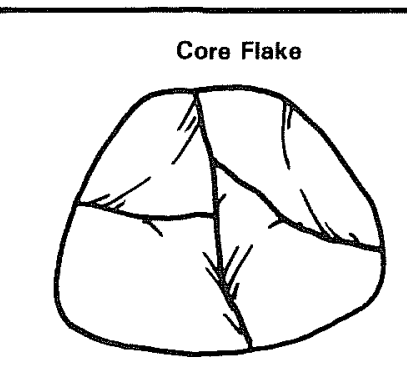

Blade

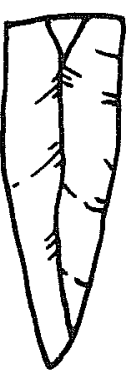

Flakes \& Blades

Figure 65. Flake Terminology. 
As Table 30 shows, the middle three occupation planes are marked by a consistent relationship between bifacing and nonbifacing flakes. The lowest occupation associated with the Paleo-Indian component is different. It contains a very high proportion of bifacing flakes. The uppermost occupation plane is notable for a decided dip in the popularity of bifacing. This is a common characteristic of Late Prehistoric phase sites in Texas. It is contemporary with OP 1.13, but an explanation remains to be developed. In general, bow and arrow technology of the last millennium seems to have fostered an impoverished lithic technology, perhaps caused by low quantitative demands imposed by arrowhead making.

The frequencies of bifacing and nonbifacing flakes are shown by squares in Figure 66. In the right half of the illustration, the squares dominated by bifacing are shaded.

Frequency of Habitation at Eagle Hill

Given that site function at Eagle Hill was probably consistent throughout time, the numbers of blows struck during the flaking process (platformed flakes) is a likely index of human activity at the site. This criterion indicates almost equal amounts of activity during Paleo-Indian occupation and the latest occupation. Likewise, approximately 250-300 platformed flakes in the middle three occupation planes indicate almost equal occupation frequencies during those times. The middle three occupation planes were utilized about twice as intensively as the early and late components.

\section{Points as a Functional System}

American archaeologists have traditionally examined points from a morphological perspective. The primary intention has been to use points as diagnostic time markers, thus supplementing chronological information on site context. During the last decade several attempts have been made to reduce morphological types to systematic measurements in order to compare types numerically. Such an approach has the advantage of examining large numbers of points simultaneously and thus, escaping to some extent, the biasing interest of point typologists in exempiary specimens (Luchterhand 1970; Gunn and Prewitt 1975).

Benfer and Benfer (1981) examine the Gunn and Prewitt article in some detail. They suggest that the angular measurements used by Gunn and Prewitt are not heuristic and should be replaced by linear measures. Prewitt has been working on such a scheme for some time and now has a nonangular set of measures for several hundred points from the central Texas area.

The following analysis is directed toward two objectives. The first is to generate a "point space" for east Texas and Louisiana which will serve as an analytical background for the points excavated at Eagle Hi11 (16 SA 50) during the summer of 1980. "Point space" is more easily demonstrated than explained (see example at end of the Gunn and Prewitt article). A similar space was generated using principle components analysis by Gunn and Prewitt (1975). As a test of the Benfers' suggestion only linear measures will be used. 


\section{EAGLE HILL (16SA50)}

Occupation Floor Analysis - $1 \mathrm{~m}$ Study

TOPIC: Bifacing and Non-Bifacing Flakes

Occupation

Occupation
Fioor

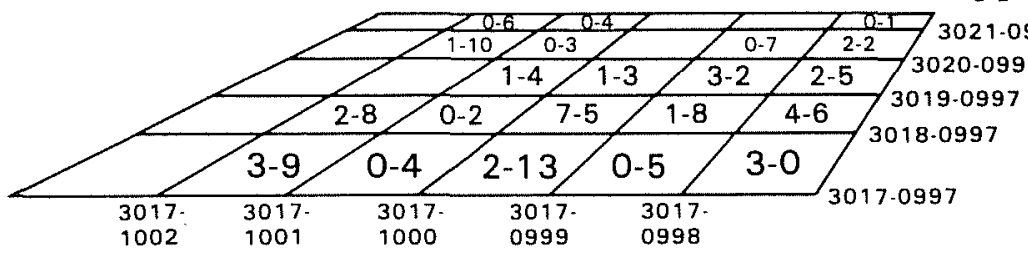

1.13

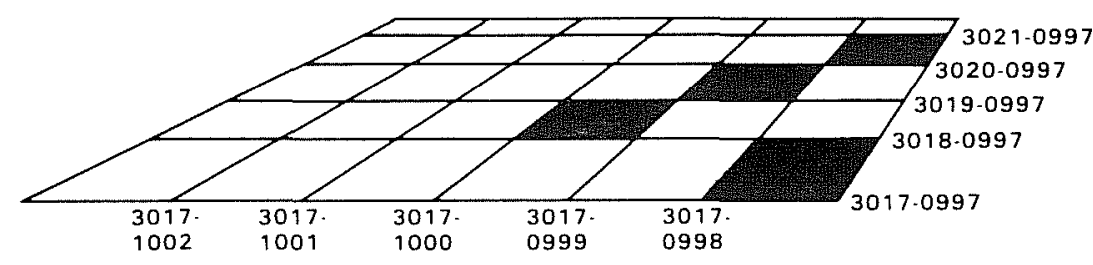

$\underbrace{}_{2-1}$

2.13
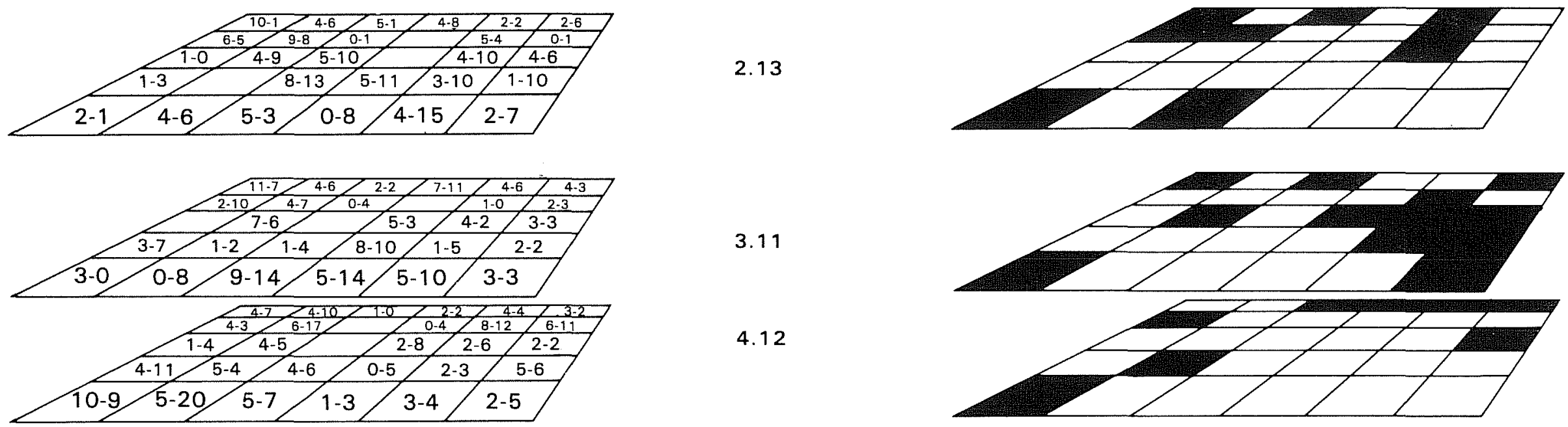

3.11

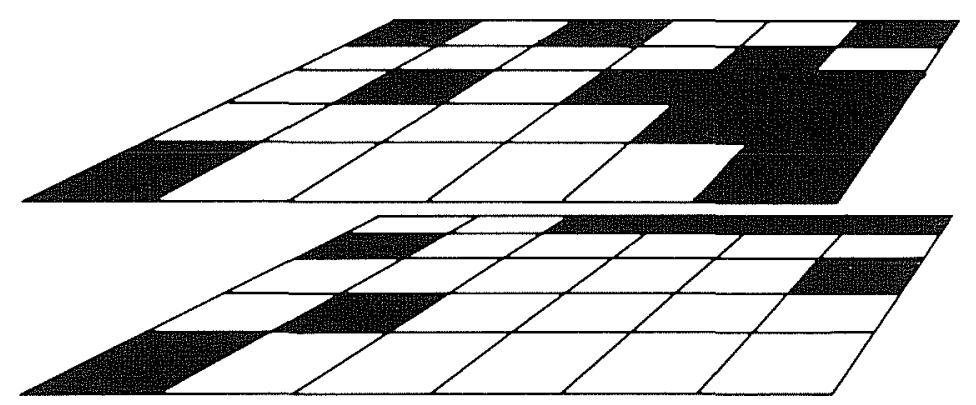

4.12

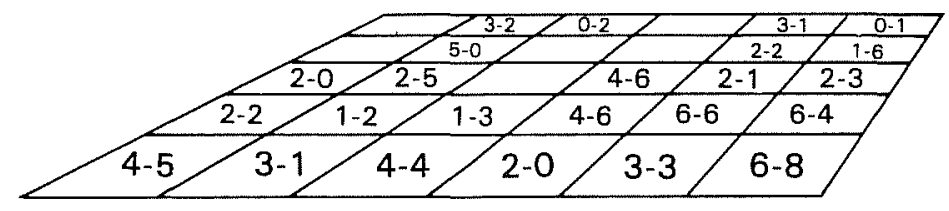

4.17

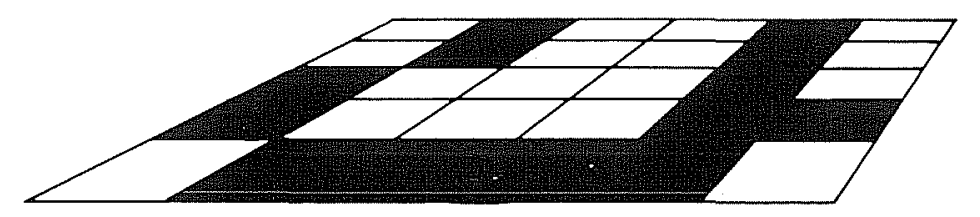

Figure 66. Bifacing Loci on the Occupation Planes. 
The second objective is to develop a functiunal model for point morphology. In the past, measurements have been taken on points with no thought given to the rationale for those measurements, except that they describe the morphology of the point. In this effort measurement will be justified by function rather than morphology. The first task is the development of a functional model for the universe of point forms.

\section{Principles of Leverage and Prehistoric American Points}

One of the first formally noted characteristics of the universe in which we live concerned leverage. Archimedes boasted, that given a place to stand, he could move the world with a lever. While our plans for this study are not so grandiose, it is not unreasonable to expect that some functional insight into the relative shapes of various points could be gained by examining their relationships in the context of the laws of leverage.

Normally levers are classified into three general types depending upon where the fulcrum engages the lever. The fulcrum of a first class lever is located near the middle or between the load which the lever is bearing and the source of energy which moves the load, perhaps a human. A crowbar is a good example. A second class lever locates the load between the fulcrum and the source of effort such as in a wheelbarrow. Finally, a third class lever locates the fulcrum and the effort at one end and the load at the other. A fishing pole or human forearm are examples of third class levers.

The relationship between the effort arm and the load arm of the lever can be described mathematically. The lever is said to be in equilibrium when the product of the length of the effort arm and the energy applied to it are equal to the product of the load and the length of the load arm. An equilibrium situation would be a seesaw with a box of sand suspended from one end. When a man applies enough downward force to hold the seesaw level, the leverages are in equilibrium.

Let us assume that the shapes of points are a system of leverages which perform functions for the user. It would be ideal if points were a simple lever like the seesaw. A preliminary examination of the problem, however, suggests that points are comprised of a complex system of leverages.

Points are generally thought to be hafted at the base into some sort of device, either a foreshaft or the shaft of a spear, dart, or arrow. Together the shaft and point are a third class lever. Singly, however, the relationship becomes that of a first class lever. The point does not sit on the end of the shaft as it would in a third class lever, but rather the shaft projects around the point so the two can be bound together. There is a fulcrum somewhere on the base, but here it will be treated as the interface between basal modification and the blade of the point. Basal modification includes edge grinding on lanceolates and on side corner and basal notches, tangs, etc.

Reflecting on the discussion of levers, the base is the effort arm of a point and the blade--the load arm. Such an identification allows an examination of various point styles in the context of the equilibrium potential of the various point forms. Figure 67 illustrates some well-known point styles consistently divided at the blade-haft interface. Lanceolates have a prominent effort 


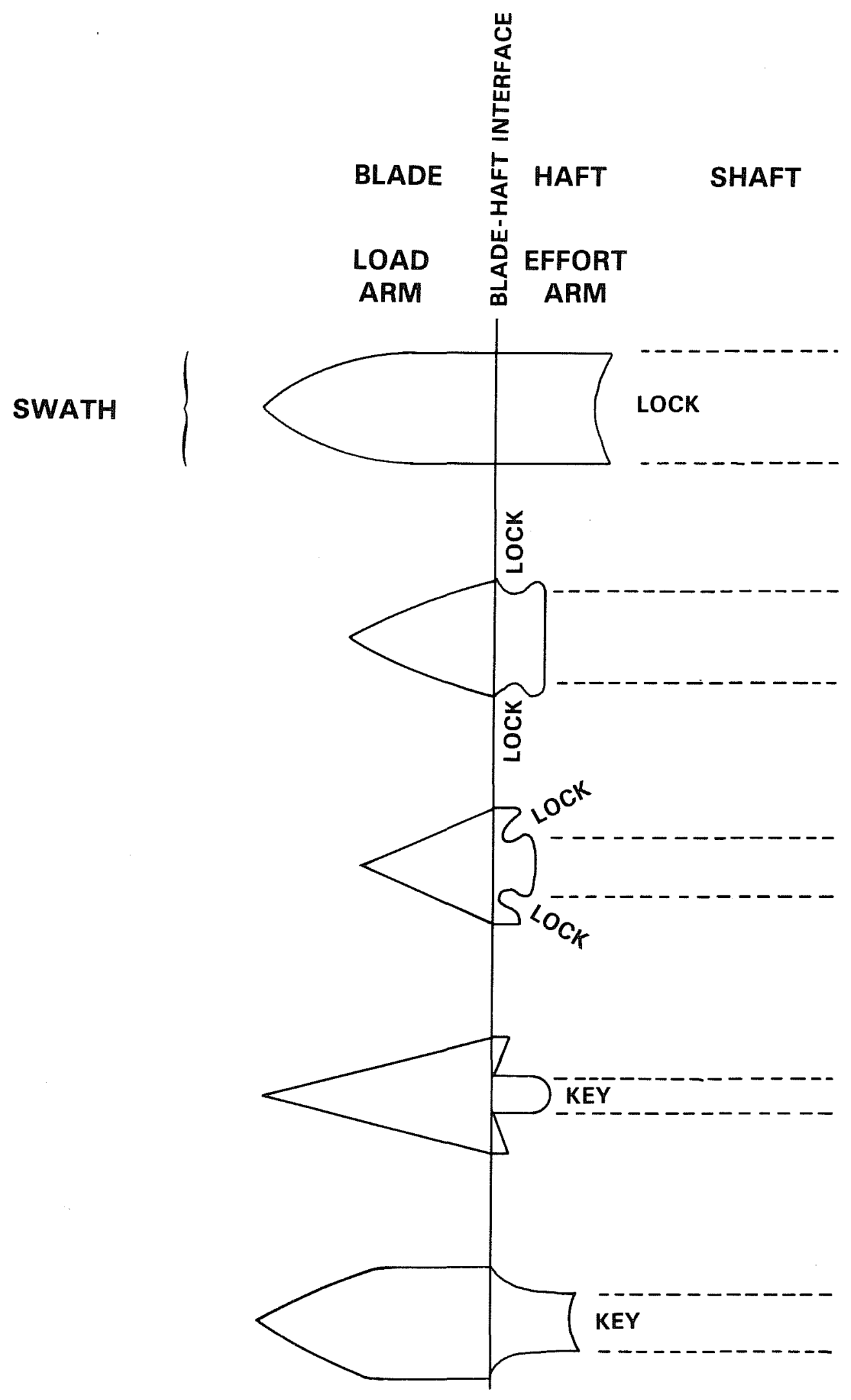

Moments: product of force by its arm length

Principle of Moments: equilibrium is established when the sum of the moments acting in one direction equal the sum of the moments acting in the other direction.

Figure 67. Range of Variation in the Point Morphology Universe. 
arm, perhaps one-fourth to one-third the length of the load arm. In contrast, notches have the effort arm embedded in the load arm, and it is proportionally so short that it is almost vestigial. The specimen illustrated is modeled after a Catahoula point (Thomas and Campbe11 1977:175,e) with the base being one-fourth the length of the blade.

Function has either been inferred from wear patterns or conjectured from various morphological types. For instance, it is thought that the Paleo-Indian lanceolates were mounted in a foreshaft and used both as thrusting spears and sheath knives. Such a multiplicity of functions would certainly conform to Goodyear's (1981) logistic model for the Paleo-Indian. It would also imply a variety of lateral as well as frontal stresses on the tool and would suggest a stronger effort arm.

On the other hand, corner notched and barbed types of points occur later, probably under conditions of less mobility and more tool specialization. They are probably dart points. Function, as a specialized dart point implies less lateral stress, is supported by the shortening of the effort arm.

This perspective leads to some rather interesting conclusions about the San Patrice points recovered by Webb, Shiner, and Roberts (1971) from the John Pearce site. San Patrice Hope has a robust base and a small blade while the San Patrice St. Johns has a small base and a large blade. One might suspect that the Hope variety is a knife and the S.t. Johns variety a projectile. Notably the Hope variety specimens are resharpened to a nub, while the St. Johns variety appears to have been relatively untouched by such activities. This would be an interesting hypothesis to test with edge wear analysis.

Man and His Point

It would be very useful if, in additon to the leverage principal outlined above, there was some consistent function that crosscut all styles of points. As we have seen, the base varies with function and also with style, hafting technique, etc. The triangular shape of the blade is something of a constant, although it seems to vary not only with style, but also with resharpening and reshaping of the tip and edges.

In central Texas there is an interesting sequence of styles. The Late Archaic ends with a slim, stemmed point called Darl. During the early part of the Late Prehistoric and with the introduction of the bow and arrow, arrow points became a diminutive version of the Darl (Scallorn). After a period of time, the Scallorn is replaced by yet another form of arrow point, Perdiz. It is worth noting (Fig. 68) that in this sequence the maximum blade width is first reduced from that of the Dare to that of the Scallorn and again expanded to that of the Dare. A similar sequence probably occurs in Louisiana with the typical Gary, sma11 Gary, and various arrow points such as Colbert, Alba, Agee, etc., al1 with exaggerated barbs.

This interesting observation led to the surmise that perhaps the blade width of projectiles and knives were a constant. It seems reasonable to expect that the human body has remained relatively constant over the late Quaternary, and that the strength of the human body as a delivery system imposed the ultimate limit on the penetrating characteristics of points. If we assume that hunters want to 


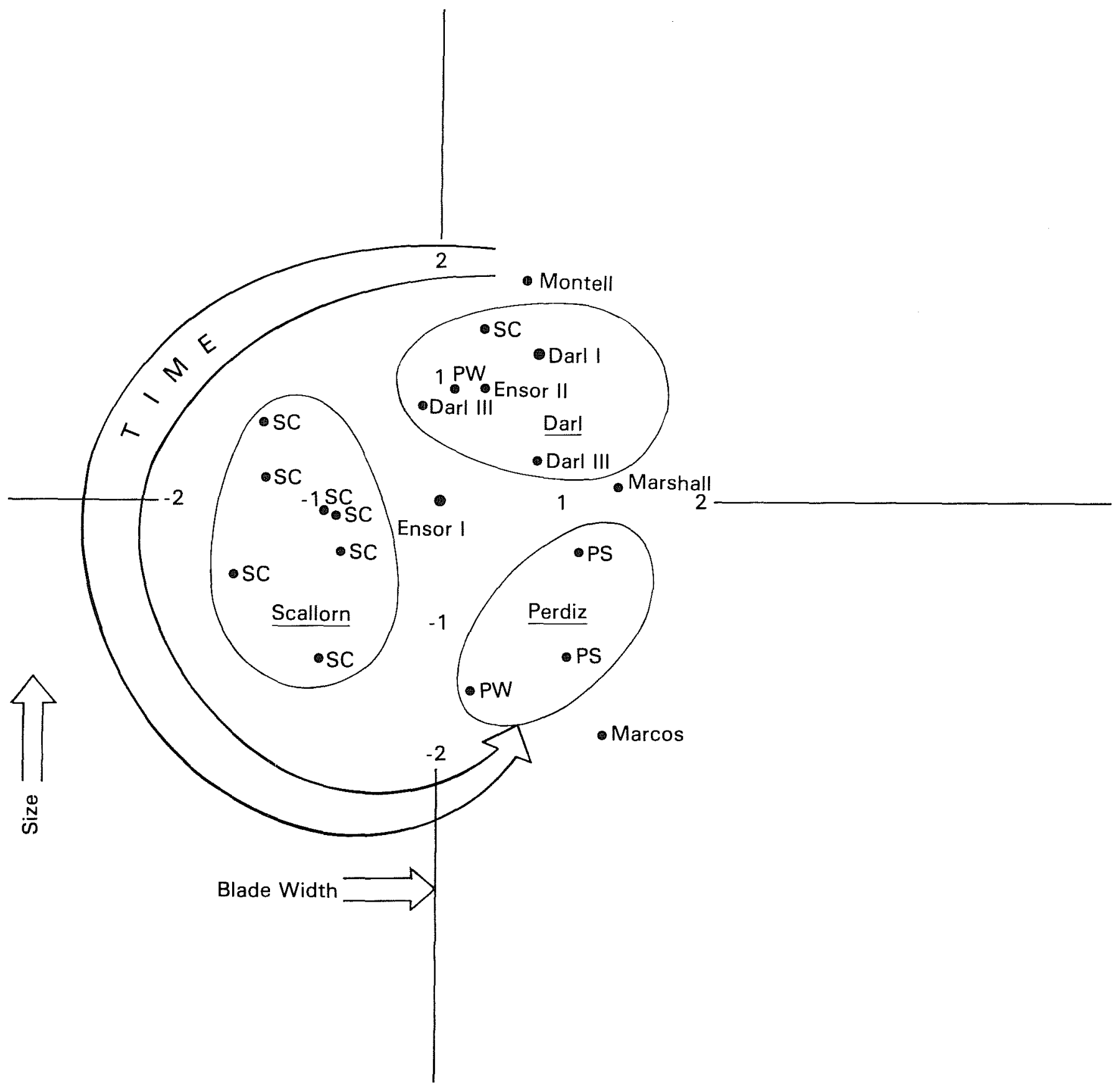

Figure 68. Through Time, the Darl-Scallorn-Perdiz Sequence Marked by a General Reduction in size. Note that maximum blade width recovers to its former proportion in the case of the Perdiz points. Specimens are from the Loeve-Fox site (Prewitt 1974). 
achieve as much damage with the impact of a missile as possible and within the limits of their strength, then we have a possible constant in the maximum blade width. The change from darts to arrows provides interesting insights into the equilibrium between man and his point. With the introduction of the bow, the lithic technology at hand was simply adapted to the end of the arrow by making it smaller. This, however, had the unfortunate effect of reducing the ultimate possible damage by penetration. In effect, the narrower blade width "wasted" a portion of the human potential. The solution was to broaden the maximum blade width by exaggerating the barbs.

For purposes of discussion we might call the maximum blade width "swath." In Figure 67 the points have been drawn so that the swath is constant. If we assume that the neck width is equal to the attached shaft, the shaft progresses from a large spearlike shaft for the Paleo-Indian specimen to dart-proportion shafts for the notched varieties to arrow shaft size for the Perdiz specimen. This provides something for the argument that swath is in equilibrium with human energy potential. It assumes that the force delivered by the bow and arrow and by the atlatl and dart are of similar magnitude. This remains to be determined.

Another test of the swath idea is to be found in measurements of various attributes of points. If swath is in equilibrium with human energy potential, then one would expect it to remain relatively constant across time and space while other attributes varied in response to changing function and style. Six attributes were measured on 49 points selected from site reports across central Texas to southwest Mississippi. The attributes will be discussed in detail later. It is sufficient to note that maximum blade width exhibited the lowest coefficient of variation of any attribute. For this analysis then, maximum blade width will be an indicator of the equilibrium of the economic and technological system rather than a technologically and stylistically controlled variable.

One further examination of Figure 67 shows that there are two basic styles of bases. They are designated as styles simply because there is no explanation as to why one would be functionally more advantageous than the other. The style illustrated in the upper three specimens provides a series of notches for hafting which will be referred to as the "lock" system. The other provides a tang which apparently interfaces with the shaft. This will be called the "key" system. The above concepts will be used in the following analysis of variability in point forms.

The curvature of the base will not be used. This diverges from standard typological dogma. In all of the analyses that have been run, the base depth has behaved as an almost completely independent variable. It is not related to the other attributes which apparently define the physical and stylistic system of points. Why curvature of the base should be so divergent is an interesting matter. It could be idiosyncratic, measured improperly, functionally insignificant, or all of the above. The matter warrants future consideration.

\section{Attribute Analysis}

In the previous section, a projectile point model was developed which indicated that the blade to haft ratio should vary with function. This section will 
examine in part that model in a population of points selected from western Louisiana and adjoining regions. The idea of swath and hafting style will also be examined. Al1 attributes are measured in millimeters taken only from complete or reconstructable specimens (Fig. 69). These attributes divide into three groups.

Swath--Swath is measured by maximum blade width, BLWD. Maximum blade width is measured perpendicularly to the axis of the tool wherever it may occur. It frequently corresponds to the blade-haft interface, although not always.

Blade--The length of the blade is measured by one attribute, blade length $(B L L G)$. It is measured from the blade-haft interface to the tip parallel to the axis of the piece. Tips frequently have to be reconstructed.

Haft--Four measurements were required to describe the complex features of this part of a projectile point.

1. Base width (BSWD) measures the line drawn across the lowest part of the base from one extremity to the other. Convexity and concavity are ignored. The line is usually not perpendicular to the axis of the tool, but should not diverge from the perpendicular more than ten degrees.

2. Haft length (HALG) measures a line drawn from the right or left side of the piece to the interface of the blade and haft. It is parallel to the axis of the tool.

3. Neck width (NKWD) measures a line drawn across the narrowest dimension of the base. It need not be perpendicular to the axis of the piece, but should not vary more than ten degrees. It will usually cross between notches or lateral concavities. If the sides of the base are parallel, draw the line halfway up. If the base is a contracting stem, draw the line across the highest width before the base expands into the blade.

4. Neck length (NKLG) measures a line drawn from the right side of the base to the neck. The line should be parallel to the axis of the piece.

The sample selections were made so as to insure a relatively full range of morphological types in the area between central Texas and southwest Mississippi. That is not to say that a11 types are represented. If additional specimens were added to the sample, they would probably fit into this sample's range of variation.

Since the objective of the analysis was to examine the range of functional variation it seemed reasonable to utilize a method which would remove size from consideration. If a principle components analysis is performed, the first unrotated eigenvector normally extracts the variance generated by the size of the objects analyzed. The remaining vectors will then represent the shape of the objects. The unrotated principle components analysis for this sample is shown in Table 31. 

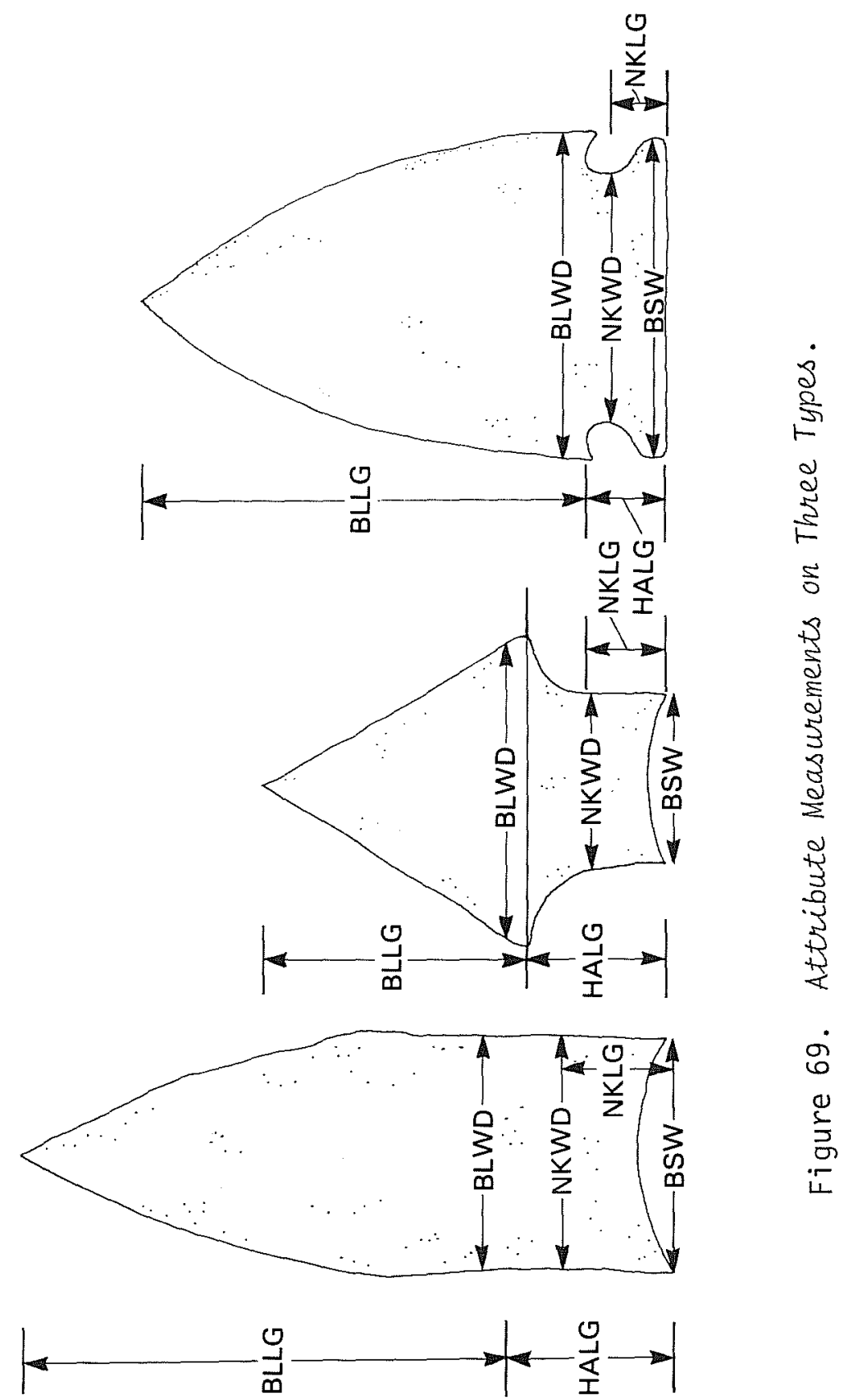
As would be expected, Factor I loads for all of the variables. The loadings are very high and all positive, which is a sure indication of a size factor.

Factors II and III account for much smaller percentages of the total variation in the sample. Factors whose variation are accounted for in Factor III are usually suspect of being composed of random noise in the data. However, with small numbers of variables as in this study, smal1 eigenvalues (less than 1.0) are frequently meaningful. As we shall see, Factor III makes perfectly good sense.

TABLE 31. UNROTATED PRINCIPLE COMPONENTS ANALYSIS OF MID-SOUTHERN PROJECTILE POINTS

$\begin{array}{lcll}\text { Variable } & \begin{array}{c}\text { Factor I } \\ \text { Size }\end{array} & \begin{array}{l}\text { Factor II } \\ \text { Leverage }\end{array} & \begin{array}{l}\text { Factor III } \\ \text { Haft Style }\end{array} \text { Communality }\end{array}$

\begin{tabular}{lrrrr} 
Blade Width & .88 & $-.31^{\star}$ & $.05 /$ & .87 \\
Blade Length & .76 & $-.45^{\star}$ & $.41^{\star}$ & .95 \\
Base Width & .86 & $-.08 /$ & $-.46^{\star}$ & .96 \\
Haft Length & .86 & $.33^{\star}$ & $.16 /$ & .87 \\
Neck Width & .93 & $-.01 /$ & $-.28 /$ & .94 \\
Neck Length & .76 & $.55^{\star}$ & $.21 /$ & .92 \\
Eigenvalue & 4.27 & .73 & .53 & \\
\% Variance & .71 & .12 & .09 & .92 \\
\hline
\end{tabular}

Note: $n=49, *=$ high loading, $/=$ no loading

As is shown in Table 31, Factor I accounts for the size of the points in the sample and effectively removes the size factor from further consideration. Seventy-one percent of the variance is bound up in size. The remaining variance in Factors II and III describes that portion of the measurements which outline shape. Factor I I indicates that blade length increases as the neck and haft length decrease. This is clearly the leverage factor expected. About $12 \%$ of the variance is concerned with leverage.

The third factor is primarily concerned with a relationship between blade length and base width. The meaning of this relationship is not immediately apparent but will become so. The relationship between the "lock" and "key" type hafts is one of narrowing base width. This for some reason is associated with the blade length. Key-type hafts are associated with longer, probably slimmer blades. It may be that key-type hafts are exclusively associated with projectile function. Testing that idea, however, is beyond the scope of this study, because it requires wear and fracture pattern analysis.

Exactly what the components analysis means in terms of specific specimens can be seen in Table 32 and Figure 70. In Table 32, loadings from Table 31 are used in conjunction with the means and standard deviations of six variables to 
TABLE 32. VALUES USED TO CALCULATE FACTOR SCORES PLOTTED IN FIGURE 68

Factor scores for Factor II

$$
\text { ([Measurement- } \bar{x}] / \mathrm{s} \text { ) }
$$

1 ([B1 ade width-19.5]/7.7)

2 ([B1ade 1ength-32.6]/17.6)

3 ([Base width-13.2]/6.8)

4 ([Haft length-8.5]/4.0)

5 ([Neck width-11.8]/6.2)

6 ([Neck length-5.7]/2.4)

Score $=$ sum of 1 ines $1-6$

Factor scores for Factor III

* 1oading $=$

$*(-.31)=$

* $(-.45)=$

* $(-.08)=$

$*(.33)=$

$*(-.01)=$

$*(.55)$
([Measurement- $\bar{x}] / s)$

([Blade width-19.5]/7.7)

([B1ade length-32.6]/17.6)

([Base width-13.2]/6.8)

([Haft length-8.5]/4.0)

([Neck width-11.8]/6.2)

([Neck length-5.7]/2.4)

Score $=$ sum of 1 ines $1-6$
* loading $=$

$*(.05)=$

$*(.41)=$

$*(-.46)=$

$*(.16)=$

$*(-.28)=$

$*(.21)=$

Note: $(\bar{x}=$ mean, $s=$ standard deviation, $*=$ multiply, $/=$ divide $)$ 


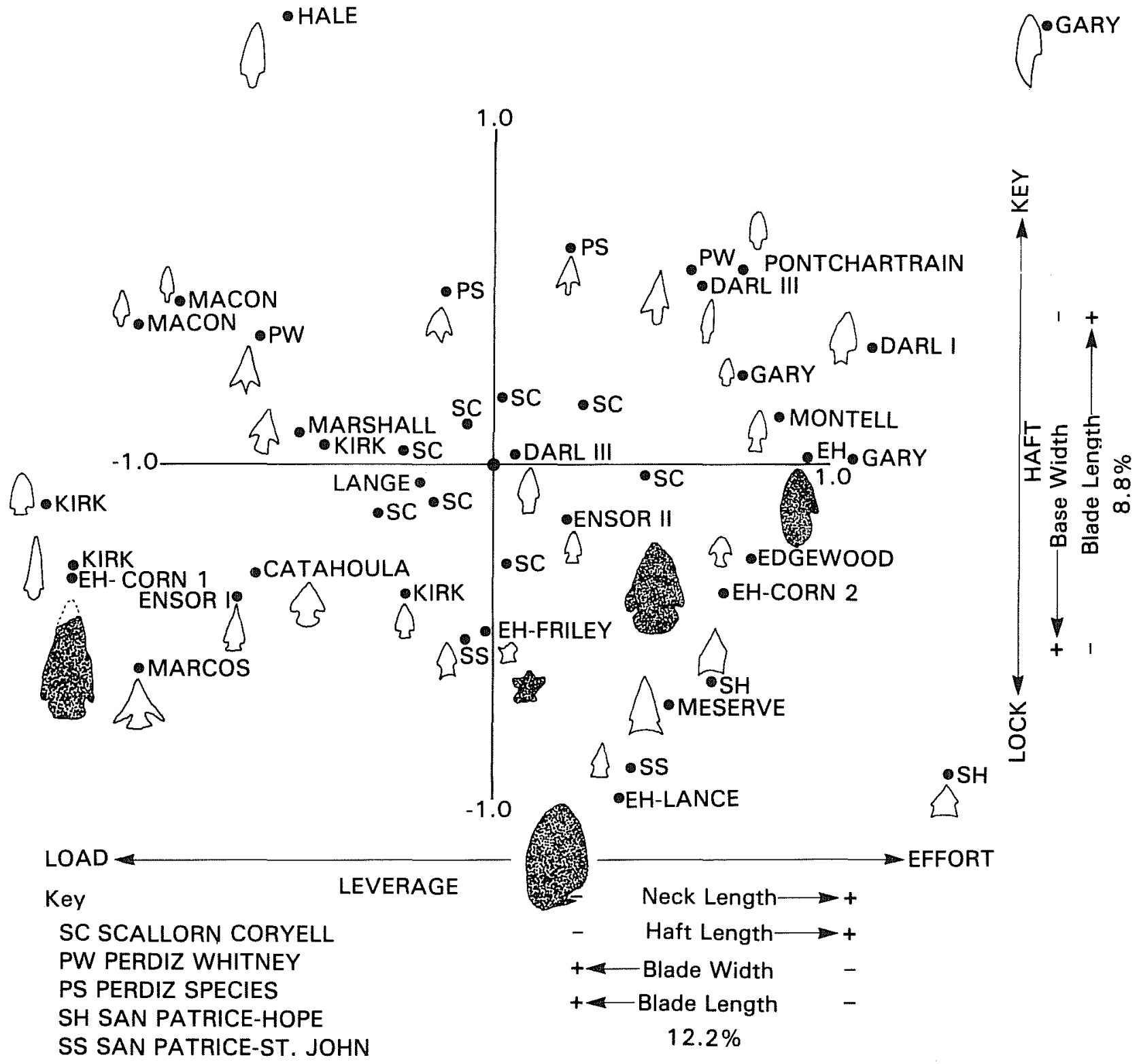

Figure 70. Factor Score Plot for the Mid-Southern Point Morphology Sample. 
calculate a component score for each specimen on each component. Anyone interested in the location of their own specimens in the point space can do so by plugging in observations from the point to be plotted.

The score obtained from Factor II in Table 32 will be the horizontal coordinate in Figure 70. The score obtained from Factor III is the vertical coordinate in Figure 70 .

Figure 70 displays the individual specimens in accordance with the relationships discussed for Factors II and III. The leverage component is displayed horizontally. Note that the points (1anceolates, Edgewood, Friley, Darl, Pontchartrain) to the right in Figure 70 have large hafts (effort arms) and smal1 blades (load arms). In contrast, those to the left (Kirk, Macon, Hale) have small hafts and large blades. Examining the distribution vertically shows that those at the bottom have "lock" style hafts (Friley, Kirk, Marcos), while those at the top have "key" style hafts (Pontchartrain, Perdiz, Macon, Hale).

The "point space" represents the range of variation that native American peoples of the late Quaternary allowed in their mind in search of a workable combination of attributes for points. Also, the shear physics of point form is probabiy involved. From a temporal point of view, it might be suggested that these stylistic and technological wanderings began in the lower right hand corner of the plot and generally proceeded left and upward in that order. The five points from Eagle Hill that are plotted are, with one exception, all located in the lower right corner, al though they represent over 10,000 years of prehistory. One might suspect that people who came to Peason Ridge were technologically and stylistically conservative. Whether this conservatism was functionally compelled by resources available on the ridge or because the region was stylistically out of the mainstream of the Mississippi Valley cannot be determined at this time. However, Gagliano (1967b) working from a completely different point of view concluded that the uplands flanking the lower Mississippi Valley were culturally stagnant between Paleo-Indian and Plaquemine times. It could very well be that the old ways of doing things, the Ice Age ways, survived a very long time on Peason Ridge.

\section{CERAMIC PERIOD}

\section{Sedentary Period Analysis (Gunn)}

We have adopted Muller's (1978) term "sedentary" for the last 3000 years, because the lower Mississippi River valley and related river systems in the MidSouth exhibited sedentary traits such as those during the Poverty Point period without the occurrence of ceramics. The absence of ceramics seems to cloud the literature on the lower Mississippi Valley and obscure obvious gestures toward civilization such as the Poverty Point mound complex, trade networks, and a general 01mec-like character and contemporaneity (Gibson 1974).

The discussion of the lithic period began on a continental scale, because the issues in that early period are continental issues. For instance, what caused the differentiation of Paleolithic culture in the New World, and how do conditions and cultural residues at Eagle $\mathrm{Hill}$ shed light on those problems? 
The sedentary period raises a new set of issues at another scale. When life resumed on Peason Ridge after the Hypsithermal, the world of culture issues had shrunk. There is no longer a question about whether influences came from the Plains or from the Greater Southeast. Cultural pulses were coming from the river valleys where settlers were shifting to a cultivation of domestic plants for food or at least thinking about it. Direct evidence for earlier domestication is lacking. Perhaps like the Egyptians who were known to be tardy in their interest in agriculture, the inhabitants of the lower Mississippi Valley found their environment rich enough to delay the extra labor of farming. Peason Ridge was again holding deposits against erosion, presumably because of moister conditions and verdant vegetation. Peason Ridge again attracted human attention and the interaction of occupation refuse; moisture and plants collected and held the residues of the new kind of visitor. Occasionally pottery was brought to Eagle Hill.

I use the word "pulses" when referring to cultural influences from the alluvial valleys, because there seems to be an up and down frequency to the occupation. There is Tittle evidence for a Tchefuncte-Marksville period at the site. By contrast, the Coles Creek-Plaquemine period must have been a virtual clamor of human activity by normal Peason Ridge standards. If there ever was a domestic turn to occupation at Eagle Hill, it was during the Coles Creek period. The visitations continue throughout Plaquemine and then drop off. When Caddoan hunters visit the site, they again leave only sparse remains to witness their passage.

This peculiar trend of occupation seems to parallel what is generally thought of as population trends in the Southeast (Haag 1965). Perhaps people only came to Peason Ridge when resources of the alluvial valleys were being totally utilized. K1inger (1977) has outlined most of the current subsistence strategies and how they apply to the Mississippi Valley in the Arkansas region. He suggests that rather than merely exploiting the catchment around a camp, gatherers and hunters move on a vector which minimizes distance to all necessary resources. One route to travel, which takes a task group to nuts and white-tailed deer, is better than two trips, one for each resource. Seasonal vectors were only deflected to Peason Ridge when locales more accessible to the alluvium settlers were overexploited or restricted due to territorial boundaries. If such was the case, indicators of habitation frequencies on Peason Ridge may be very sensitive barometers of population density in the Sabine and Red River valleys.

This thought led to the following examination of the problem of population density variation in the lower Mississippi Valley. If population density and erosional/depositional episodes at Eagle Hill could be accounted for by a common forcing variable(s), it would provide a strong explanation for the cultural and sedimentological chronology of the late Holocene at the site.

Global Climate and Culture Chronology in the Lower Mississippi Valley

Since the 1930s, some researchers have thought that prehistoric cultural change in the lower Mississippi Valley was heavily influenced by environmental conditions (Gagliano 1967b). The perceived sources of influence were mostly alluvial 
such as changes in meander belts and stream channels. More recently, Saucier (1974) has shown that human occupation of the Delta is also heavily influenced by gross shifts in the distributary pattern of delta channels.

In addition to environmentally induced culture change, archaeologists have perceived strong cultural influences from the upper Mississippi River valley system, the eastern or western Gulf Coastal Plain, and from along the Gulf Coast. In some cases, the local cultures appear to have been overrun by these influences while in others, they appear to have maintained their cultural integrity (Haag 1965; Gagliano 1967b).

Archaeologists perceived the effects of environment and external cultural influence in the lower Mississippi Valley (LMV) in an essentially local context without attributing ultimate causal mechanisms to the events which transpired. In this article, a more distant perspective on the progress of culture change in the lower Mississippi Valley will be developed. In the last decade, a considerable amount of information on global synchronous climatic changes and, in particular, climatic change in the Southeast has appeared. Likewise, culture change models have become increasingly sophisticated. According to Butzer (1980) most models, especially of more complex cultures, were evolutionary. However, as his analysis of the ups and downs of riverine Egyptian civilization shows, an ecological explanation for cyclic evolution and retrogression of civilized societies can be quite convincing. Two articles have been prepared on culture and climatic change in North America and Mesoamerica with favorable results (Gunn and Adams 1981; Folan et al. 1982).

\section{Cultural Geography}

There are several cultural areas in North America pertinent to the progress of culture change in the lower Mississippi Valley. The key factors are restricted areas which are overtly or latently rich in resources. The alluvial floodplain and delta of the lower Mississippi Valley are particularly productive environments due to juxtaposition of alluvial soils, wetlands, forests, uplands, etc. The upper Mississippi River valley river system is an aggregate of potentially rich areas linked by river communication such as American Bottoms at St. Louis and the Middle Ohio Valley around Cincinnati. This complex will be referred to as the upper Mississippi Valley sphere (UMV). Another water-linked system is the Gulf Coast, the Gulf Coast sphere. The Tennessee River valley is also an exceptionally stable and rich resource zone of very long duration.

\section{Climatic Model}

Rather complex models of climatic change have been developed (Gunn and Adams 1981 ; Folan et al. 1982; Gunn and Mahula 1977; Gunn et al. 1982) for the Southeast. While those models serve as background, a relatively simple system will serve this discussion. In an analysis of modern western hemisphere weather records of the 20th century (Sanchez and Kutzbach 1974) bands of temperature and moisture shift southward with global cooling. These quasi-stable bands divide the United States approximately into thirds. The two southern bands conform to the upper Mississippi Valley and the lower Mississippi Valley spheres. As will be explained later, the issue of climatic stability is 
probably more directly related to this discussion than to temperature and moisture. However, moisture is the stabilizing agent in the atmospheric system. Climatic stability from year to year should be directly related to the Sanchez and Kutzbach analysis. The system is driven by the global energy budget, the sum of the heat reserved in the atmosphere, oceans, and land masses of the world, and is directly controlled by the jet stream which moves north as the energy budget increases and south as the world cools. Thus, during hot periods the "stability band" is over the upper Mississippi Valley sphere; during warm periods it is over the lower Mississippi Valley sphere and during cool times it is over the Gulf. Regions to the north of the stability band grow progressively less stable and more susceptible to perturbation as the band recedes southward.

During the late Holocene (5000-500 B.P.), a number of mutually reinforcing climatic chronologies have appeared which suggest marked movements in the global energy budget. As Table 33 shows there have been two warmer intervals and three colder periods. The level of the energy budget and the locations of the stability bands is also indicated. The table is adapted from glacial and tree 1 ine chronology (Denton and Karlén 1973), sea levels (Stapor and Tanner 1977), biosilicas (Robinson 1978), and mathematical projections (Gunn 1982a, 1982b).

TABLE 33. ENERGY BUDGET LEVELS FOR THE LATE HOLOCENE Years A.D./B.C. Years B.P. Energy Budget Location of Stability Band

$\begin{array}{rrll}1880 & 100 & \text { cool } & \text { Gulf } \\ 1250 & 700 & \text { warm } & \text { Lower Mississippi } \\ 900 & 1100 & \text { coot } & \text { Gulf } \\ 500 & 1500 & \text { hot } & \text { Upper Mississippi } \\ 500 & 2500 & \text { cool } & \text { Gulf } \\ 2000 & 4000 & & \end{array}$

\section{Cultural Model}

Butzer (1980) argues that although evolutionary models can explain developmental aspects of culture change, they cannot explain oscillation, especially of periods of retrogressive cultural change. It is the ecological aspect of culture change that accounts for the majority of the retrogressive movements.

Cleland (1976) has developed a subsistence adaptation model, the Focal-Diffuse Model, which has the interesting property of being conceptualiy reversible relative to cultural change. It is, in essence, a reworking of Caldwell's Primary Forest Efficiency concept which is evolutionary in principle. The Focal-Diffuse 
Model, however, is defined as a continuum with focal economies (those depending upon few resources) at one end and diffuse economies (those dependent on a wide range of resources) at the other. Since the focal and diffuse economies directly imply certain social structures (under conditions of circumscribed resources, Carneiro 1970), it can serve both as an adaptive model and a cultural evolution model. A focal economy implies trade, ceremonialism, and/or legitimate force to organize and maintain the flow of specialized products in the economy. Such economies are usually associated with large populations and positive feedback demographic systems. Diffuse economies are usually organized with relatively equal access to resources by everyone. Populations are small and mobility acts as a negative feedback mechanism to population growth (Sahlins 1972).

Adaptively, the diffuse economy is very flexible. Since a wide range of resources is exploited, the diffuse economy is unaffected by climatic change. Focal economies, on the other hand, are likely to be disrupted by climatic change, since organization has to be restructured. If excessive adaptive demands are placed on the focal economy, it is likely to move toward the diffuse end of the spectrum making necessary social adjustments such as population reduction and loss of sedentary status.

\section{Cultural Chronology}

Examination of the 1 ast 5000 years has been selected for several reasons. Previous to 5000 years ago, cultures of the lower Mississippi valley, with the possible exception of Dalton, are not especially visible to the archaeological record. This has to do with the alluvial floodplain developing after that date, but may also be related to a need for more work before anything definitive or even preliminary can be said. Also, after 5000 years B.P., native American cultures seem to have adapted to the lower Mississippi Valley and begun a general developmental, if not monotonic, move toward large populations and a complex society. The cultural periods are relatively well defined and serve as readily available units of analysis for a preliminary examination of the validity of the above model. Table 34 shows the cultural chronology, related cultural "power balance," and climatic information. This table is preliminary as a further 1 iterature search may well change some aspects of the entries. In general, the model seems to function as proposed, although there are some points that require more precise definition.

During the earliest cool period, Poverty Point is divided into two stages. Gagliano defined the Bayou Jasmine phase from a site in the delta, which dates to about 3700 years ago and contained many Poverty Point type clay objects and fiber-tempered sherds. This suggests that Poverty Point probably stems from some sort of circum-Gulf cultural phenomenon. Clay objects have been found along the Gulf Coast from Florida to Texas, and fiber-tempered pottery is known very early from Georgia, all in aquatically oriented situations. The later phase of Poverty Point appears as a movement from the delta-adapted, earlier phase into the alluvial floodplain.

Tchefuncte is a period of far ranging expansion up the alluvial valleys into east Texas and Arkansas. The expansion appears to be that of the local Poverty Point culture mobilized under its own power. It is noted as a donor system, although this does not imply that it penetrated the upper Mississippi Valley. This should be investigated. 
TABLE 34. LOWER MISSISSIPPI VALLEY CULTURAL CHRONOLOGY

\begin{tabular}{lllll}
$\begin{array}{l}\text { Influenced (ing) } \\
\text { Sphere }\end{array}$ & LMV Mode & Period Name & $\begin{array}{l}\text { Mid-Period } \\
\text { Date }\end{array}$ & $\begin{array}{l}\text { Global Climate/ } \\
\text { Stability Band }\end{array}$ \\
\hline East & Receiver & Moundville & 700 & cool/Gulf/LJAII \\
West & Donor & Plaquemine & 800 & warm/LMV/MCO \\
Upper Valley & Donor & Coles Creek & 1100 & warm/LMV/MCO \\
Gulf & Receiver & Troyville & 1500 & cool/Gulf/LIAI \\
Upper Valley & Receiver & Marksville & 1800 & hot/UMV/RECO \\
Upper Valley & Donor & Tchefuncte & 2100 & warm/LMV/RECO \\
Gulf & Receiver & Poverty Point & 2700 & cool/Gulf/Subboreal \\
Gulf & Receiver & Early Poverty & 3700 & cool/Gulf/Subboreal
\end{tabular}

The Marksville period is during the later, cooler part of the Roman Empire Climatic Optimum (RECO) and is a period when the lower Mississippi Valley was overrun by Hopewellian influence. The settlement pattern structure is suggestive of a conquered polity with an implanted, ruling elite. This may be stretching the evidence, but there is no doubt some sort of northern influence conforms to the model's projection.

Troyville falls during the earliest phase of the Little Ice Age. Adverse climate apparently destroyed the cultural hegemony on the north (Struever and Vickery 1973), and there is evidence that Troyville may be another imposed elite, perhaps from the coast of Mexico (Haag 1965). Again, the model correctly retrodicts the source of cultural influence.

Coles Creek and Plaquemine fall in the Medieval Climatic Optimum (MCO). Coles Creek marks the beginning of a period of local development which culminates in an aggressive donor surge to the west during the Plaquemine. Mississippian cultures to the north and Plaquemine seem to be in a stalemate as there is no intercourse between the two areas except at the borders (Haag 1965).

At the end of the Medieval Climatic Optimum, the Mississippian cultures to the north lose their coherence resulting in the complete collapse of Cahokia (Fowler 1952). The lower Mississippi Valley sphere loses the imperviousness it enjoyed during the Medieval Climatic Optimum and receives a cultural donation from the Moundville culture to the east. 


\section{Conclusions}

With the exception of the Moundville intrusion at the end of the sequence, the model seems to explain the sequence of cultural influences in the lower Mississippi Valley. The Moundville influence may be an expectable Gulf source or, if it is from the eastern sphere per se, it may reflect a successful adaptation to oscillating climate, which asserts itself when less resilient neighbors fall prey to climatic change.

It is interesting to ask why the model works as well as it does. Perhaps the lower Mississippi Valley is a basic transportation link for coastal goods when the volatile northern cultures are in a position to demand maritime products. The location amplifies the effects. Also, the resource richness of the delta region may have attracted more powerful culture purveyors.

It is often the case that models which work well at abstract levels of examination are less effective when closely scrutinized. This model is posed as an hypothesis for further testing. It does seem to complement similar climate/ culture models developed for other areas of North America and Mesoamerica. Naturally the usual questions relative to climatic determinisms will eventually be raised. Climatic explanations of culture change (Gunn and Adams 1981) are only partial explanations. North America does have some of the most turbulent weather in the world: tornadoes, for instance, are an almost characteristically North American phenomenon. Tropical storms and hurricanes are not exclusive to North America, but they are not frequent in most parts of the world. They are directly controlled by the energy budget (Wendland 1977) and vastly affect moisture in the Southeast. Therefore, it is safe to suggest that North American cultures may be more susceptible to climatic change parameters than most of the rest of the world.

\section{Ceramic Analysis (Brown)}

A significant portion of 16 SA 50 showed signs of occupation during later periods. In a11, 162 sherds were collected. The following section is an analysis of the distribution of the sherds, their potential significance as cultural and temporal indicators, and some of their macroscopic technological properties. A later section is devoted to a more detailed physical and chemical analysis of both ceramics and lithics from the site.

A1 1 but one of the 162 sherds from 16 SA 50 were from the excavations in Area $A$, the truncated low natural mound that was the target of the most concentrated excavation effort. Area $B$, the smaller natural rise to the northeast and across the small drainage from Area $A$, yielded only one sherd. This single sherd was a fragment of a turpentine collecting cup of the type used before the turn of the century. It was found in Area $B$ near the surface of the excavation unit in substratum 1.11. A single badly eroded fragment of fired clay from the bottom of this excavation unit was originally suspected of being a sherd, but subsequent examination showed it to be very poorly fired and morphologically indistinct from the fired clay balls in Area A. This was the only clay ball found in Area B. 
Three of the 161 sherds recovered from Area A are also fragments of Historic period turpentine collecting cups. Although there was a turpentine crew camp several kilometers to the northeast (James Grafton, personal communication), there was no indication of historic settlement or long term activity in the immediate area of 16 SA 50. Recent military shell casing fragments were the only other historic materials found. The three historic sherds from Area A were all found near the ground surface, two from substratum 1.12 and the third from 1.13 .

The 158 prehistoric sherds from the site were found just beneath the natural surface down to the upper portion of substratum 4.12. No ceramics were found in any of the levels beneath this. Figure 71 shows the vertical distribution of ceramics from each substratum and their percentage of frequency relative to the total ceramic sample.

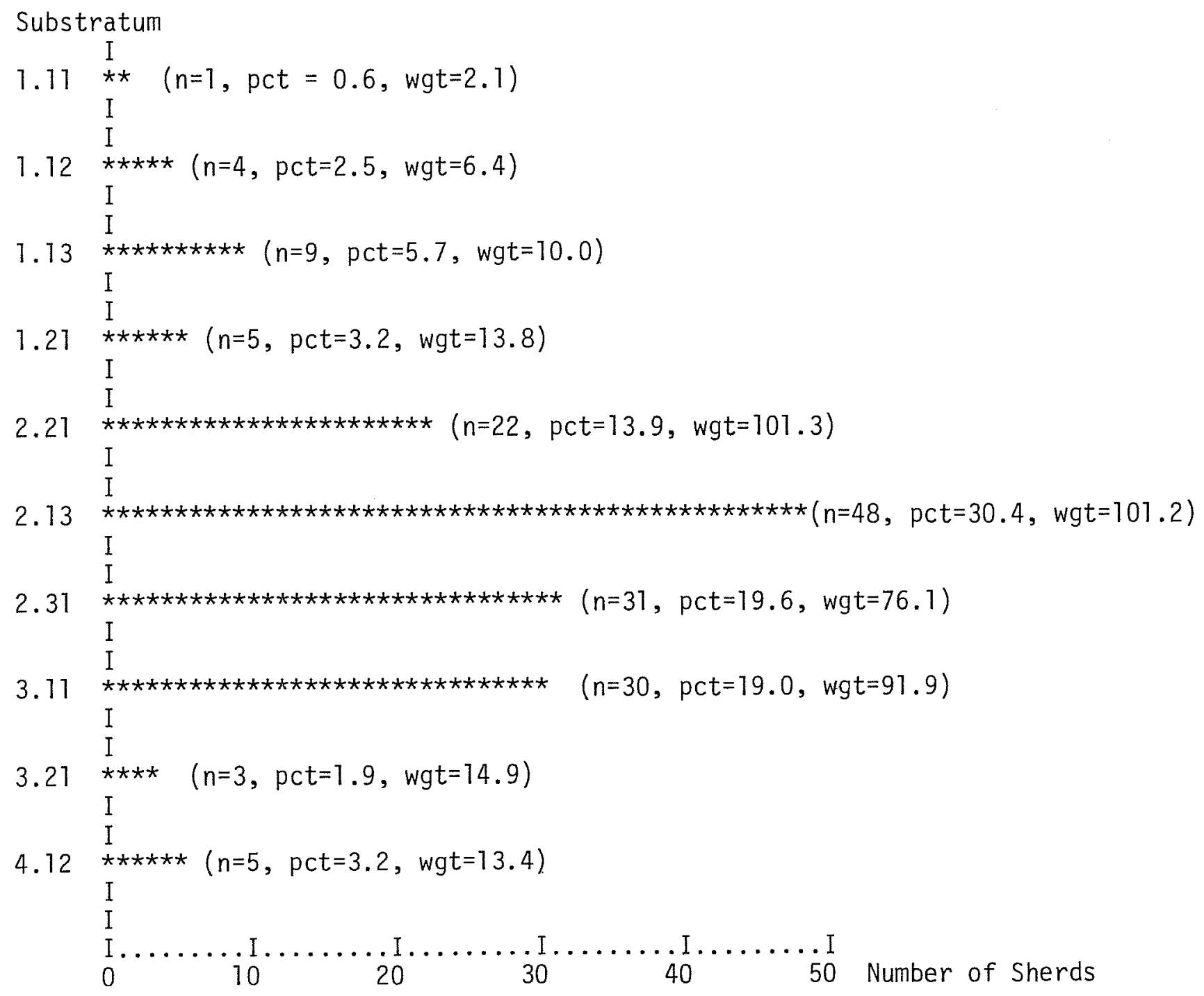

Figure 71. Vertical Distribution of Ceramics at 16 SA 50. 
Note that most of the sherds were found between substratum 2.21 and 3.11 with small numbers occurring above and below. The relative paucity of ceramics below substratum 3.17 and the potential for vertical displacement of artifacts at the site suggest that these sherds may have been displaced downward from higher levels, a hypothesis supported by the occurrence of apparent preceramic artifacts in the lower levels. On the other hand, it is not at all clear whether the small ceramic sample in the upper substrata is due to upward displacement of artifacts into a sterile area by bioturbation or due to more recent occupations at the site by peoples who had little use for ceramics.

\section{Typology}

Thirty-six of the 158 prehistoric sherds were decorated. Two basic styles of decoration were present at the site: incision and punctation. The two styles were not combined on a single sherd, although such combinations are not uncommon in many parts of Louisiana and east Texas (Price and Heartfield 1977). No engraved ceramics of any type were recovered from the site. Engraved ceramics, which are generally regarded as being associated with Caddoan cultures in this region, are found to the northeast on the Red River near Natchitoches and to the northwest along the Sabine River. The total ceramic sample is too small to make conclusive statements regarding the relationship between the ceramic period occupants and their Caddoan neighbors. The ceramics do suggest that the site was occupied earlier than the Caddoan development in the area or that the inhabitants might be related to coastal or inland Gulf Coastal Plain groups to the south.

Parallel Incised--This is the largest single group of decorated sherds--19 specimens. On all of the sherds, which are sufficiently large to make the determination, line orientation is horizontal. There are four decorated sherds; two other rims are plain. Depth, spacing, and definition (squareness of the incision) of the incised lines varies. All sherds, but one, have regular spacing. This single, irregularly incised sherd was found with a small cache of Tithics (a rodent burrow?) on OP 2.13 of N3020 E1000; it is distinctly different from the other decorated sherds from the site. Several other sherds among the parallel-incised specimens clearly do not match other sherds in the decorated sample. On the basis of differences in line depth and spacing as well as morphological properties, there are at least five different vessels represented by these 19 sherds and perhaps a few more than this. Most of these sherds, however, may have come from the same one or two vessels, while the other three or four vessels are represented by only one or two specimens. Only two sherds in this group match one another; these are from adjacent substrata (2.13 and 2.31) in the same unit (N3018 E1002). The greater part of the paralled-incised sherds, forming only one or two vessels, are of a distinctive red paste with numerous small bits of clay grit showing in both the interior and exterior vessel walls; only a few sma11 fragments of this paste are obvious among the undecorated sherds.

Horizontal parallel-incised motifs are quite common throughout the Caddo and lower Mississippi Valley area, often typed as varieties of Coles creek Incised (Phillips 1970) or Davis Incised (Suhm and Jelks 1962). Along the coast the 
similarities between coles creek and Goose creek Incised have been noted (Aten and Bollich 1969). Because of the general lack of other Caddoan or coastal culture indicators at the site, it is probably best to view the specimens from the site as examples of coles creek. In comparison to the defined variants of coles creek, the majority of these sherds come closest to the definition of Coles Creek hardy (Phillips 1970:73), with some significant deviations. The most notable is that the incisions are neatly made with a blunt or slightly rounded tool. Two of these hardy-like sherds are illustrated in Figure 72,a,b. The USL test excavations identified coles creek hardy as being present, but it is unclear how close these specimens are to the defined variety.

Of the two remaining parallel-incised sherds, one has slightly overhanging lines (Fig. 72,C) and resembles more the classic Coles creek variety of Coles creek Incised than other sherds at 16 SA 50 (Phillips 1970:70). The other has closely spaced, extremely shallow incisions probably made on a dry paste. This latter specimen cannot be definitely typed, but is close to the mott variety of coles creek Incised (Phillips 1970:75). Both of these two latter varieties occur earlier in time than the Coles Creek hardy in the lower Mississippi Valley culture area; Coles creek and mott varieties both date to the Coles Creek period between A.D 750 and A.D. 1000 in the lower Mississippi Valley area. The possible Coles Creek variety sherd, occurring in OP 3.11 is one of the deeper para11e1incised specimens. The possible mott variety sherd, dated to the latter portion of the Coles Creek period, occurs in the next higher level at OP 2.31. The hardy variety is assigned to the early Mississippian period and could date between A.D. 1000 and A.D. 1200. A1though the hardy-1ike sherds are scattered, the median of the distribution is in OP 2.31.

Horizontal Incised with Pendant Diagonals--These five sherds have diagonal incisions below (perhaps above in some cases) parallel, horizontally incised 1ines. Spacing and method of incision are similar, but at least two vessels present are based on paste differences. Also apparent from a consideration of general paste characteristics is the fact that neither of these two vessels could be part of the other decorated vessels. All of the sherds are badly eroded. One sherd has an abraded exterior surface on which the design is barely visible. The remaining four sherds, all apparently from the same vessel, have slightly abraded exterior surfaces and are extremely friable. Unlike any of the other incised sherds, these four sherds from a single vessel show broad, shallow incisions with small brush marks along their length. They also show a clearly displaced distribution; three are from the northern row of one-meter units in Area $A$ in OP 4.12, while the fourth occurs in the same row in substratum 2.31. Only a few other undecorated sherds might be part of this vessel, but none can be clearly associated with it. It is possible that it represents the earliest vessel made at or brought to the site, and that a single sherd was displaced upward from the floor where it was discarded. One of these sherds is illustrated in Figure 72,e.

A designation of Mazique Incised mazique (Phi11ips 1970:129) affords the best description of these sherds. They might equally well fit Alligator Incised alligator (Phillips 1970:39), especially since the larger group was incised on a relatively wet paste, a characteristic of alligator. The former, however, is probably the better choice given the rarity of the latter in the region. 

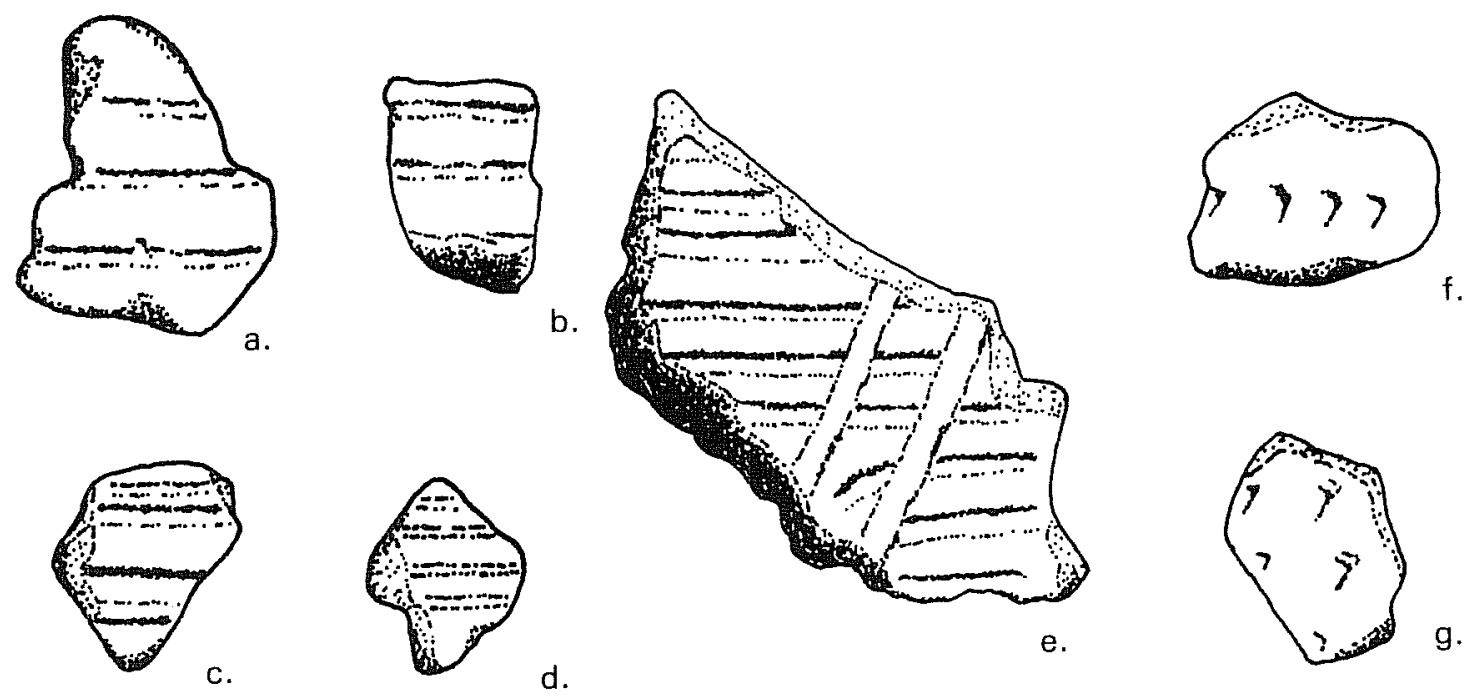

Figure 72. Eagle Hill Ceramics. a-d, parallel incised; e, crosshatch incised; f-g, punctate. 
Mazique Incised mazique is dated to the early Coles Creek period, while alligator variety of Alligator Incised is earlier, occurring in the Baytown period between A.C. 300 and A.D. 750.

Crosshatch Incised--These three sherds are distinquished by crossed diagonal incised lines on a thin and apparently we11-fired vessel wal1. Two and perhaps three of these sherds come from the same vessel, although they do not fit together. Because of their thinness and their distinctive tan pastes, it is apparent that they do not match any of the other decorated vessels, although they may match a number of similar undecorated sherds. In contrast to all the other incised sherds from the site, these three have distinctly wide, shallow, and rounded incisions. The largest is illustrated in Figure 72 ,e.

These unusual sherds cannot by typed. The broad U-shaped, diagonal 1 ines show some similarities to the lower Mississippi Valley type Sanson Incised (Phillips 1970), but 16 SA 50 is far from the Tensas Basin where the type was first defined and to which it seems to be generally restricted. In addition, Sanson Incised does not appear to have diagonal lines trailed across opposing diagonals as these specimens do. The time range for these sherds is undetermined.

Punctate--Nine small sherds have fingernail punctate designs. None is large enough to allow examination of patterning, but they do appear to be oriented diagonal1y. Although there is some variation in thickness, color, and punctate size, the differences are not distinctive enough to indicate more than one vesse1. All have very similar paste characteristics. If they are from the same vessel they show a scattered vertical distribution occurring from OP 1.13 down to OP 3.11. No more than two specimens are in any one leve1. The horizontal distribution is also rather scattered. Specimens occur in four onemeter squares along the N1002 row, three along the N1001 row, and a single specimen located in E3017 N998. Two of these sherds are illustrated in Figure $72, f, g$.

A sherd of Evansuille Punctated wilkinson (Phil1ips 1970:81) was identified from the USL test excavation. This identification could well fit these nine small sherds. On the other hand, they could fit equally well into the definition of Evansuille Punctated evansville (Phi11ips 1970:78-79). Unfortunately, there are no clear means of distinguishing between the two. The former is dated to the Plaquemine phase of the Mississippi period (post A.D. 1000), while the latter is found from late Marksville and Coles Creek (approximately A.D. 100-A.D. 1000).

Plain Types--Although on the whole it is difficult to identify Plains ceramic types, one sherd at the site shows some similarities to Williams plain, a type which occurs with some frequency in 0klahoma, but only rarely south along the Red River (Prentice Thomas, personal communication). This single sherd is in many ways the most unusual specimen at the site. Large, thick (11.8 mm), and heavy ( 55.9 grams), it contains large, rounded particles of clay grit. It is the basal corner of what may have been a very large vessel. It was found in OP 2.21 in E3021 N1000.

First defined in Oklahoma (Bell and Baerreis 1951), williams Plain has been identified in east Texas (Webb et al. 1969) and along the Red River at the 
Hanna site (Thomas, Campbell, and Ahler 1977). Because of the suggested early occurrence of this type in OKlahoma and the presence of only a single specimen among the ceramic collection from 16 SA 50 it is perhaps better to refer to this sherd as coarse-tempered plain rather than to identify it as an example of williams Plain. It seems more likely that it represents some functional difference in ceramic use rather than cultural or temporal differences.

\section{Vertical Distribution of Ceramic Types}

Table 35 shows the vertical distribution of ceramic types from 16 SA 50. The sample size is too small and the distribution too scattered to demonstrate any valid statistical differences, and it is clear from simple observation that there is some vertical mixing. In fact, given the simple and only minimally diagnostic motifs found at the site, it would not be surprising to find all these types coexisting at the same time within the same group.

The radiocarbon dates from the ceramic levels at the site generally conform with the typological identifications suggested above. As noted elsewhere in this report, charcoal from OP 3.11 has been dated to A.D. 820 with a one sigma deviation of 70 years (uncorrected), a date which suggests an occupation of the site during the middle Coles Creek period. This agrees well with the presence of Mazique Incised and the tentative coles creek variety sherd. OP 2.13 has been dated to A.D. $935 \pm 80$ years, a date falling near the end of the coles Creek period. With a two sigma deviation this date could either overlap the earlier one, or fall well into the succeeding Plaquemine period. The remaining Coles Creek specimens, with a median of OP 2.13, would fit nicely into this time period. Depending upon the variety chosen, the Evansville Punctated could fit either period. On the whole, the dates generaliy confirm the typological and technological evidence, which suggests multiple, but not widely spaced occupations.

TABLE 35. DISTRIBUTION OF CERAMIC TYPES

\begin{tabular}{|c|c|c|c|c|c|c|}
\hline $\begin{array}{l}\text { Occupation } \\
\text { Planes }\end{array}$ & $\begin{array}{l}\text { Coles } \\
\text { creek }\end{array}$ & $\begin{array}{l}\text { Mazique } \\
\text { Incised }\end{array}$ & $\begin{array}{l}\text { Crosshatch } \\
\text { Incised }\end{array}$ & $\begin{array}{l}\text { Evansuille } \\
\text { Punctated }\end{array}$ & $\begin{array}{l}\text { Coarse- } \\
\text { Tempered }\end{array}$ & TOTAL \\
\hline $\begin{array}{l}1.11 \\
1.12 \\
1.13 \\
1.21 \\
2.21 \\
2.13 \\
2.31 \\
3.11 \\
3.21 \\
4.21\end{array}$ & $\begin{array}{l}1 \\
1 \\
1 \\
4 \\
5 \\
4 \\
1 \\
1 \\
1\end{array}$ & $\begin{array}{l}1 \\
1 \\
1 \\
2\end{array}$ & 1 & $\begin{array}{l}1 \\
2 \\
1 \\
1 \\
2 \\
2\end{array}$ & 1 & $\begin{array}{l}1 \\
1 \\
2 \\
4 \\
7 \\
6 \\
9 \\
3 \\
1 \\
3\end{array}$ \\
\hline TOTAL & 19 & 5 & 3 & 9 & 1 & 37 \\
\hline MEDIAN & 2.13 & 2.31 & 2.31 & 2.13 & 2.21 & 2.13 \\
\hline
\end{tabular}


Manufacture

There is a moderate amount of technological variation within the ceramic sample, but it is not distinctively different from other collections in the general area. This is especially noted in details of manufacture. The technological characteristics of the sample would be undistinguished in many Caddoan or 1ower Mississippi Valley sites. There is, however, some technological variability between the two major culture areas as well as some technological change through time in the area. In addition to these concerns with culture history, there is the potential for asking behavioral questions of the data. For these reasons, manufacture and raw material-related variables were recorded. Those which relate to source materials and chemicals will be dealt with in a later section of this report. This section presents a summary of the information gained from an analysis of the manufacture-related variables.

Clay Preparation--Although the selection and preparation of clay raw materials will be discussed later, this section will examine some of the macroscopic properties of the ceramics. Clay selection and preparation are not random behaviors since not al1 clays are equally serviceable for ceramic manufacture. Factors affecting the procurement and treatment of raw materials are guided by cultural bias as well as functional necessity. Every ceramic item must fulfi11 its intended purpose, but within such limitations, there are numerous ways to utilize the raw material resources of a given locality.

In the Eagle Hill environ sands and sandy clays are common, so it is no surprise that many of the vessels would have a sandy paste rather than a silty one. The surface texture of 16 SA 50 sherds was classified as either predominantly silty, sandy, or coarse sandy: 93 were sandy, 61 were silty, and only four had a coarse sandy texture. Table 36 shows the vertical distribution of these textural characteristics. Sandy textures clearly predominate in substrata 2.13 and 2.31. Below substrata 2.31 , silty texture reaches its highest percentages. The chisquare of a combined version of this table (in order to increase expected cell values) was 2.1903 , a value not significant at the 0.05 level.

Aside from decorative technique, the identification of temper is probably the most commonly recorded technological variable in the region. Change in tempering material has both cultural and temporal significance. Sand-tempered pottery occurs southward toward the coast. There is a developmental trend showing change through time in the use of various tempering agents. There are, however, occasional difficulties in the identification of visible, aplastic materials as being natural or intentional. These difficulties were noted in the USL draft report on the test excavations (Serve110 n.d.).

Shepard (1965:161-162) discusses some of the problems involved in making the distinction between naturally occurring and intentionally added, nonplastic inclusions. The problem is compounded in the 16 SA 50 environ because of numerous varieties of sandy and fine sandy clays. These difficulties are especially apparent at 16 SA 50 where the more obviously, intentionally added nonplastics such as fiber, bone, or shell are totally absent. Nonplastic materials at the site are restricted to sand and particles of previously dried or fired clay. Although a few of the sandy paste sherds have a distribution of coarse-grained sand particles, which suggests that they are not naturally occurring 
clays (Shepard 1965:161-162), this cannot be clearly ascertained for the majority of the sandy paste and clay grit sherds.

TABLE 36. TEXTURE BY SUBSTRATUM

$\begin{array}{ccccc}\text { Substratum } & \text { Silty } & \text { Fine Sandy } & \text { Coarse Sandy } & \text { Total } \\ 1.00^{*} & 8 & 11 & 0 & 19 \\ 2.21 & 8 & 12 & 2 & 22 \\ 2.13 & 16 & 31 & 1 & 48 \\ 2.31 & 10 & 20 & 1 & 31 \\ 3.11 & 14 & 16 & 0 & 30 \\ 4.00^{*} & 2 & 1 & 0 & 3\end{array}$

$\begin{array}{lllll}\text { TOTAL } & 61 & 93 & 4 & 158\end{array}$

*1.00 is the combined total of levels 1.11 through 1.21 , and 4.00 is the combined total of levels 3.21 and 4.12 .

To confront this question, the author gathered clay samples from the surrounding area and attempted ceramic vessel manufacture. Even with carefully controlled drying, none of the vessels survived to be fired. All developed fatal cracks in the drying stage, even when a small amount of sand tempering was added. A later mineralogical analysis of the raw clays and others collected from the area showed them to be composed almost entirely of montmorillonite clay minerals, expandable lattice clays not suitable for ceramic manufacture in their pure state. Although such a finding does not preclude the possibility of suitable ceramic clays nearby, it suggests that intentionally added, nonplastic materials should be expected.

Sherds were coded for the presence or absence of sand and/or clay grit in both the vessel wall and core. The two tempers were not mutually exclusive; some sherds had both sand and clay grit. Others had no recognizable temper. In all, 114 sherds had clay grit present, and 50 had coarse particles of sand. Because of the difficulty in distinguishing between finer sandy clays and ceramics tempered exclusively with the fine sand, these were excluded. The correlation, Spearman's R, between clay grit and sand temper is -.1541 , significant to $p=.003$ indicating a weak tendency for both not to occur on the same sherd. Table 37 shows the correlations between apparent temper and other distributional and technological variables. As is obvious, there is essentially no correlation with directional variables and low to moderate correlations with many of the other technological variables. 
TABLE 37. TECHNOLOGICAL CORRELATIONS

\begin{tabular}{|c|c|c|}
\hline & Sand Temper & Grit Temper \\
\hline East & $p=\begin{array}{r}.0238 \\
.329\end{array}$ & $p=\begin{array}{r}.0284 \\
.299\end{array}$ \\
\hline North & $\begin{array}{l}-.0856 \\
p=.056\end{array}$ & $\begin{array}{c}.0582 \\
p=.739\end{array}$ \\
\hline Substratum & $\begin{array}{l}-.0649 \\
p=.114\end{array}$ & $\begin{array}{c}.2141 \\
p=.001\end{array}$ \\
\hline Decoration & $\begin{array}{l}-.2074 \\
p=.001\end{array}$ & $\begin{array}{c}.2365 \\
p=.001\end{array}$ \\
\hline Finish & $\begin{array}{l}-.1416 \\
p=.005\end{array}$ & $\begin{array}{c}.1991 \\
p=.001\end{array}$ \\
\hline Size & $p=\begin{array}{r}.0494 \\
.179\end{array}$ & $p=\begin{array}{l}.2317 \\
.001\end{array}$ \\
\hline Color & $p=\begin{array}{r}.0194 \\
.360\end{array}$ & $\begin{array}{r}-. .1153 \\
p=.017\end{array}$ \\
\hline Oxidation & $p=.2033$ & $\begin{array}{r}-.1909 \\
p=.001\end{array}$ \\
\hline
\end{tabular}

Some of the associated observations are so, for obvious reasons. A few deserve explanation. Decoration is the presence or absence of some decorative element. Therefore, the correlation indicates that more of the decorated sherds were grit tempered. Finish is an ordinal variable describing uneven, smooth, or burnished exterior surfaces; grit-tempered sherds in the sample are more likely to have a more carefully finished surface than the sand-tempered sherds. Color is a simple categorical, nominal variable dividing sherds into those with black or gray exterior surfaces versus those with red, tan, or buff exterior surfaces. In this case, the grit-tempered sherds show a slight tendency to be black or gray. Oxidation or core color, indicates that the grit-tempered sherds are less likely to have fully oxidized cores.

Perhaps the most interesting statistic of temper at the site is its vertical distribution. Tables 38 and 39 show the distribution of sand and clay grit by combined substrata above and below OP 2.13 (combinations made to achieve a valid statistical sample size). These clearly show increasing amounts of clay grit temper with depth (that is, a decrease in obvious clay grit through time). There is a distinct increase in sand temper only in OP 2.13.

On the whole, it is clear that the use of sand and grit temper is only partially related. While it is obvious that the use of grit temper diminishes through time, sand temper reaches a peak in the middle ceramic occupation. 
This is in apparent contrast to the developmental sequence of temper in nearby east Texas, where sandy paste pottery is replaced by grog-tempered pottery with the onset of Coles Creek and early Caddoan assemblages (Shafer 1975:251).

TABLE 38. SAND TEMPER BY COMBINED SUBSTRATA

\begin{tabular}{lccc} 
Substratum & \multicolumn{2}{c}{ Sand Temper } \\
Absent & Present & Total \\
Above 2.13 & 30 & 11 & 41 \\
Substratum 2.13 & 26 & 22 & 48 \\
Below 2.13 & 52 & 17 & 69 \\
\hline TOTAL & Raw chi square $=6.473$ & $\mathrm{p}=.039$ \\
\hline
\end{tabular}

TABLE 39. GRIT TEMPER BY COMBINED SUBSTRATA

\begin{tabular}{lccc} 
Substratum & \multicolumn{2}{c}{ Grit } & Temper \\
Above 2.13 & 17 & 24 & Total \\
Substratum 2.13 & 16 & 32 & 41 \\
Below 2.13 & 11 & 58 & 48 \\
TOTAL & 44 & 114 & 69 \\
\hline & Raw chi square $=9.399$ & $p=.009$
\end{tabular}

Shafer (1975) associates sandy paste pottery in this region with the Woodland tradition and Marksville ceramics. Sandy paste pottery is found locally in Sabine Parish at the Coral Snake Mound (Jensen 1968). Unfortunately, al though the dating of the Marksville manifestations may be considered secure, other Marksville components in Louisiana and Mississippi are not so unquestionable and may continue much later in time (Phil1ips 1970:959-960). Despite its ultimate decline in the Caddo area, sandy paste pottery survives until historic times to the south along the coast (Shafer 1975; Patterson 1979). In the lower Mississippi Valley area, Phillips (1970:54-55) is dubious of the value of sandy paste as a diagnostic indicator, but is reluctant to dispense with his sandy paste variety of plainware, the thomas variety of Baytown Plain. 
Whether one argues for or against intentional tempering it makes sense that ceramic pastes will be environmentally dependent to a large degree. The clays available in any given region dictate the ultimate paste characteristics. However, such environmental limitations do not fully explain changes through time at a particular site or broad areal changes as mentioned above for east Texas.

Any hypothesis concerning these changes in paste through time raises questions about locus of manufacture and whether the inclusions were intentional or not. These questions will be discussed in more detail later, but several suggestions can be made. The change in sand content in the paste may indicate a shift in the locus of ceramic manufacture either between the immediate site environ and some other locale or between two external manufacturing loci if none are made locally. Two mutually exclusive hypotheses can be formed concerning the clay grit. In the first of these, the decrease in the intentional addition of sherd fragments might indicate a return to a more nomadic lifestyle where sherd fragments are not readily available. The second hypothesis, which fits well with Weaver's (1963) suggestion that the clay temper is an accidental inclusion, states that with increasing sedentarism and a growing complexity of social organization, a specialist class of potters arises which manufactures ceramics with more care and precision than before. This latter hypothesis is more in keeping with the regional development of the Coles Creek and early Mississippian cultures, but such an extension into the project area cannot be made uncritically.

\section{Vessel Construction and Firing}

Because of the generally small size of the sherds, it is extremely difficult to determine manufacturing details. Although the ceramics were probably made by coiling, it is not clearly discernible in the sample. A few rectangularshaped, horizontally oriented sherds are present in any case. However, exterior and interior scraping has essentially obliterated coil marks.

While the interiors of almost every sherd show some degree of scraping or smoothing, the exterior surface of every unabraded sherd shows a moderate degree of smoothing and, in cases, even burnishing. Of 141 sherds that had exterior surfaces unabraded by postdepositional processes, 50 were classified as uneven, 82 were smoothed, another nine were burnished. Table 40 shows the vertical distribution of these finishes. Although the chi-square for this table is not significant to the 0.05 level, there is a slight trend for unevenly smoothed surfaces to become more prevalent through time. The chi-square from a condensed version of the table is also not significant at the 0.05 level, but Kendall's Tau for the two ordinal variables is 0.1889 , significant to $p=.019$, indicating that such a trend does exist.

Most of the sherds collected from 16 SA 50 are not highly oxidized; they were either fired at a low temperature or in a reducing atmosphere. Since most of the sherds from the site (115 of 144) have a tan or red core, the former seems the more likely. There is essentially no correlation between exterior color and core color, as Table 41 indicates. Neither color nor oxidation shows any strong correlation with locational variables, but there are some slight correlations with other technological variables. In the strongest of these, 
black or gray sherds tend to have grit temper while, as noted in Table 41 , oxidation shows some correlation with temper. Grit-tempered sherds are less likely to have fully oxidized cores. This latter correlation is potentially a factor of recognition, since higher firing may tend to obscure particles of obvious grit by fully oxidizing them.

TABLE 40. VARIATION IN FINISH CHARACTERISTICS WITH DEPTH

Substratum

Uneven

Smoothed

Burnished

Total

$1.00 *$

10

6

1

17

2.21

7

13

1

21

2.13

15

22

3

40

2.31

10

18

0

28

3.11

6

18

4

28

$4.00 *$

2

5

0

7

TOTAL

50

82

9

141

$\star 1.00$ is the combined total of levels 1.11 through 1.21 , and 4.00 is the combined totals of levels 3.21 and 4.12 .

TABLE 47. EXTERIOR COLOR AND CORE COLOR

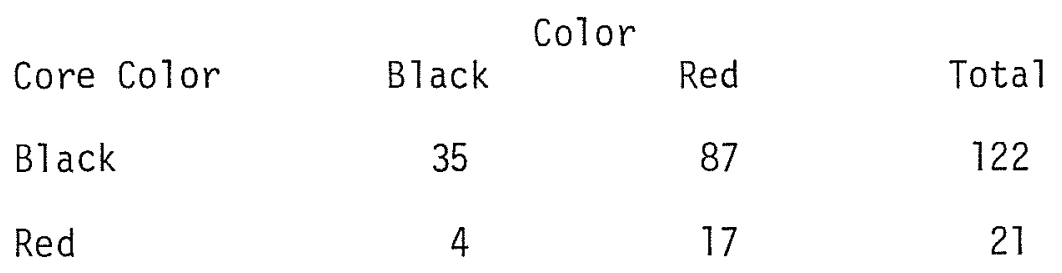

TOTAL

$\begin{array}{cl}39 & 104 \\ \text { Raw chi square } & =.4239 \quad p=.515\end{array}$

For the most part, sherd size is too small to allow vessel form identification. Identifiable body parts are rare; the sample contains only six rim sherds and two basal fragments. Most of the sherds have a broad curvature suggesting larger vessels. Tightly curved sherds, as in bottle necks or miniature vessels, are absent. Also, no carinated bowl fragments were encountered. One large sherd has a hint of recurve and may be from a vessel with an outflaring neck. The single sherd of williams Plain is from the basal edge of a large, outflaring, flat-based vessel. 
Ceramic Distribution

Initially, the vertical distribution of ceramics at 16 SA 50 was examined. Subsequent analyses of technological variables have emphasized differences in vertical distribution supporting the assertion that, despite bioturbational activities, the cultural remains at the site retain some part of their original stratification. This assertion is additionally supported by the sequence of radiocarbon dates which apparently verifies the typological conclusions concerning the temporal placement of cultures occupying the site.

The most obvious conclusion, which can be drawn from the vertical ceramic distribution, is that the central portion of the low natural mound known as Area A saw several occupations of approximately equal intensity. These occupations took place within a relatively restricted period of time separated by brief intervals lasting from a score of years to a century. It appears that there are definitely two such separate occupations centered at OP 2.13 and OP 3.11 , although there may have been others contained in the intervening levels. The frequency of ceramics in these levels suggests that they derive from relatively long, sedentary occupation at the site. Such a sedentary occupation would require that the surrounding area be relatively wet, perhaps more so than at present.

Toward the upper portion of the site, there appears to be a discrete occupation plane visible in level 1.13. The evidence is not clear, but the levels above may have been displaced upward from the original level. The paucity of ceramics in this cultural substratum suggests a shorter period of occupation than in the cultural levels below. This may be an indication of a less populated upland area, perhaps during a slightly drier period. An interesting parallel to this later occupation can be drawn from the inland sites of the upper Texas coasts where pottery declines in the Late Prehistoric period (Patterson 1979: 109).

The horizontal distribution of ceramics at the site is shown in Figure 73 and Tables 42 and 43 . The strata shown represent the specially excavated substrata, plus the two intermediate strata, which have the highest frequency of ceramics, 2.21 and 2.37; the other levels either have too few specimens or are clearly disturbed. Substrata 1.11 and 1.12, for example, have sherds in on $7 y$ one unit each, while in substrata 3.21 and 4.12 , where sherds have apparently been displaced downward from substrata 3.17 , the distribution parallels that of OP 3.11 almost exactly.

Little can be said about the upper high-resolution substrata, 1.13, because of the scarcity of ceramics. Only nine sherds were recovered from five units (Fig. 73). The distribution does not appear to be random; there is a marked concentration along the west wall. This concentration borders on both activity areas identified on this occupation plane (see 0ccupation Plane Analysis, page 324). An examination of the sherds from this substratum shows most to be extremely sma11. None appear to be from the same vessel.

Although level 2.21 was not a targeted occupation plane, it is 111 ustrated (Table 42) because of the abundance of ceramics. Most of these ceramics may have originally come from the occupation plane identified as 2.12 in the 
EAGLE HILL (16SA50)

Occupation Floor Analysis - 1m Study
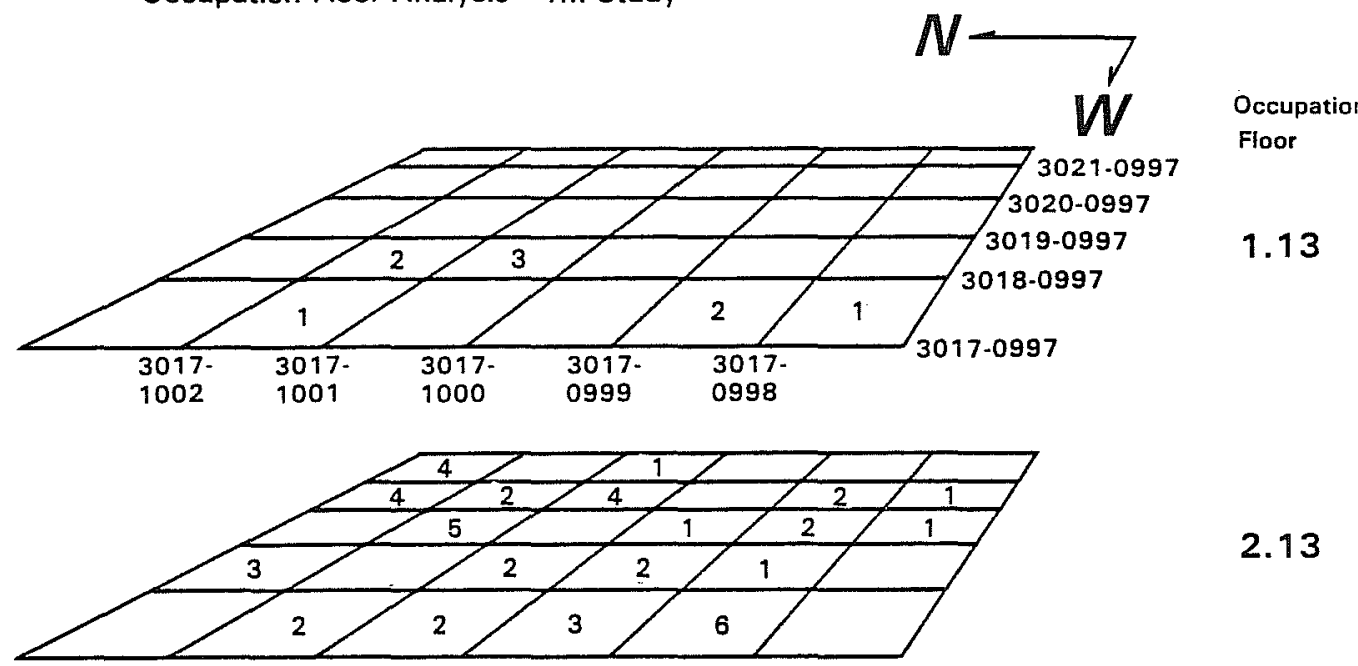

2.13

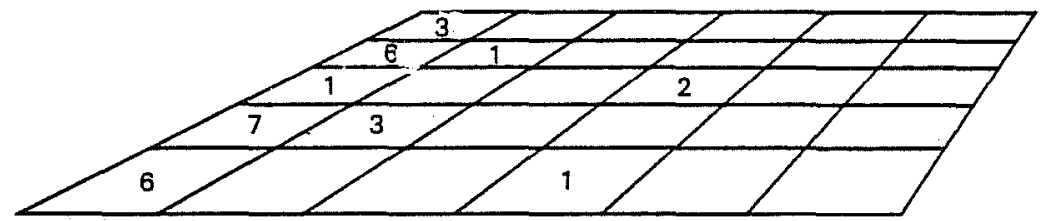

3.11

Figure 73. Distribution of Ceramics on Occupation Planes. 
May 1980 test excavations, but there may be some mixing from other levels. In this level, 21 sherds were recovered from eight units. Most of the sherds in this unit are smal1 with the notable exception of the large williams Plain sherd, the largest at the site weighed $55.9 \mathrm{~g}$. Two groups of three and two groups of two sherds may have come from four vessels, while the rest do not match any others. The distribution of sherds in this level generally matches the high density distribution of ceramics in OP 2.13, indicating possible vertical displacement, but there is no clear evidence either way.

TABLE 42. CERAMIC DISTRIBUTION IN OP 2.21

East

\begin{tabular}{lcccccc}
\hline North & 3017 & 3018 & 3019 & 3020 & 3021 & Total \\
\hline 1002 & & & 2 & 2 & 4 \\
1001 & 3 & 6 & & 1 & 9 \\
1000 & 4 & 1 & & 5 \\
999 & 2 & & & & 2 \\
998 & & & & 3 & 21 \\
987 & 9 & 0 & 7 & 2 & 3 \\
\hline TOTAL & & & & & 0 \\
\hline
\end{tabular}

TABLE 43. CERAMIC DISTRIBUTION IN OP 2.31

East

\begin{tabular}{lcccccc}
\hline North & 3017 & 3018 & 3019 & 3020 & 3121 & Total \\
\hline 1002 & 2 & 5 & 2 & 2 & 6 & 17 \\
1001 & & & 1 & & 3 & 4 \\
1000 & 7 & & 1 & & & 8 \\
999 & & 2 & & & 2 \\
998 & & & & & & 0 \\
997 & 9 & 5 & 6 & 2 & 9 & 31 \\
\hline TOTAL & & & & & & 0 \\
\hline
\end{tabular}


The distribution of ceramics in OP 2.13 appears to be spread more eventy across the excavation unit than in any other substratum. There is a slight tendency for material to form a linear cluster running from the southwest of the excavation unit to the northeast corner. This clustering trend would be even more pronounced if it were determined that the material from OP 2.21 were displaced upward from this level. Several vessels are represented in the sample; ten sherds are from a thin, orange-colored, very sandy paste vessel; while seven others are from a wel1-smoothed, partially sandy reddish brown vessel which has broken into tiny fragments. There are also two groups of three and five groups of two sherds which may have come from seven other vessels. The most concentrated area of ceramics in this substratum is around the suggested trash dump (labelled activity area 3) and near the possible hearths (associated with activity areas 1 and 4 ).

Thirty-one sherds were found in ten units of level 2.31, al1 clustered toward the north of the excavation. Five sherds are possibly from a single, very thin, clay grit-tempered, blackish gray vessel. Three groups of three and one group each of four and two sherds are possibly from two other vessels. The distribution of sherds in this level parallels that of OP 3.11 and may represent upward displacement from that occupation plane.

The lowest targeted occupation plane, which clearly contains a ceramic occupation, is OP 3.11 with 30 sherds in nine units. Most occur along the north wal1 of the excavation. The distribution of sherds in 2.31 and in both 3.21 and 4.12 are similar and may possibly represent vertical displacements. Five groups of two and two groups of four sherds from this level may be from seven distinct vessels.

\section{Conclusions}

In the final analysis there appears to be at least two distinct long term ceramic occupations at the site. The exact length of occupation cannot readily be ascertained, but a seasonal base camp utilized for a number of months is suggested. Both of these occupations must have occurred during a wetter period when population expansion in the river valleys led to settlement in the uplands, and a spring at or near the site made it a desirable location. Such a conjunction of climate should have been the case during the ninth and tenth centuries A.D., the radiocarbon dates for these levels.

The cultural affiliation of the 16 SA 50 occupants is not clear, but it is suggested they may have been closer to the lower Mississippi Valley Coles Creek tradition than the Caddoan tradition. There is not much evidence either way. In any case, the relatively high percentage of decorated sherds and the predominance of motifs common to both cultural areas at this time indicates there were at least minimal participants in a broader tradition, perhaps as a splinter group from one of the major floodplain ceremonial centers. Alternatively, the lack of any high quality, decorated ceramics may indicate that the inhabitants of $16 \mathrm{SA} 50$ were not in direct contact with mainstream, river valley occupations. 
Whether or not they were an independent band or a group tied politically and/or religiously to a ceremonial center, the presence of a wide variety of paste types in the ceramic sample suggests mobility and possibly trade at some major floodplain center. The flow of trade goods into the site might be expected to peak with high ceramic concentrations.

In contrast to the long term occupations, levels which are the remains of shorter occupations such as OP 1.13 should show a high percentage of nonlocal ceramics and nonlocal materials. Although no date is available for the latest occupation at the site, it is probably relatively recent, perhaps occurring during the late Mississippian period. The lack of ceramics as well as other artifactual evidence suggests a small group of hunters in a temporary camp. Although the climate may possibly have been drier than during the long term occupations, it may have still been wet enough for a spring to have existed at the site. Such a spring (which may have been active only during the rainy season in relatively wet periods, suggesting spring and fall occupations) seems the best explanation for the choice of this location as a campsite throughout the history of its use. 


\section{BACKGROUND: ANALYSIS OF OCCUPATION PLANES AT ONE-METER RESOLUTION}

\section{A. THE BACKGROUND-FOREGROUND CONCEPT (Gunn)}

Over the last quarter century the capability to generate data from archaeological sites has grown at a phenomenal rate. If Eagle Hill had been excavated in 1950 , it would probably have warranted a 10-page report with six projectile points and a dozen or so sherds illustrated. Since that time archaeological interests have expanded in at least two directions. The first set of sources of new data is the multidisciplinary input from geologists, pedologists, biologists, etc. In other words, the context as well as the content of a site is an item of major interest. The second vast new source of information is the various technological facilities developed since World War II that extract both site content and context from the most unexpected sources. Various radioactive isotope laboratories date sites by numerous means. Soils can be analyzed for an infinity of data bits. Even the artifacts can be analyzed for age and chemical content.

In spite of massive efforts to keep pace with the influx of methods and concepts, most archaeologists are restrained from utilizing a full or even sizable range of the techniques available. There are a number of legitimate reasons. It is rightly thought that it is scientifically $i 11$ conceived to simply use methods without a specific problem in mind. In this project we would not have tried to date lithics using thermoluminescence if the old reliable radiocarbon analysis had not been denied us as a source of dates. In other words, concepts and needs often come more slowly and painfully than methods inherited from other disciplines. Expense, expertise, availability, personal contacts, etc., are often additional considerations.

On the other hand, archaeologists are generally anxious to develop the concepts and try the new technologies. One might further suggest that the Eagle Hill site is an exemplar of the whys and wherefores for expanding archaeological horizons into the invisible past. Using the methods of the 1950s Eagle Hill is a practically invisible site. Yet in the conceptual framework of late 20th-century archaeology, it is an important site. It is a part of a system of locations between which prehistoric and historic people of western Louisiana and east Texas wove the fabric of their existence. The fact that they did not leave fancy pottery or multitudes of arrowheads is not material to the problem. What is important is understanding the whole system of movements and activities. If people visited Eagle Hill, it is important to that overall concept of human 1ife and process.

Eagle Hill is intriguing because the lines of evidence are faint and the archaeological evidence of prehistoric visits nearly invisible. The water supply on the ridge is unreliable, which dictates temporary habitation. So, who is going to carry fancy pottery to a temporary camp high on a hill?

This is the point where modern analytical technology comes into the picture. In 1950 we might have dusted our hands off and walked away from Eagle Hill. We would have wondered what these faint residues of human occupation meant, and there would have been little we could do about it. In 1980 we are in a position to frame ideas about prehistoric 1 ife in the locale and ask if there are methods, which would reveal the lines of ancient movement (even though they are not 
readily perceivable by the naked eye). Using recently developed analytical technologies a single, unassuming flake can tell the chipping habits of its makers, what it was used on, when it was made, where it came from, and why it was discarded. Add a pinch of nearby soil and its relationship to camp facilities can be determined. Add its brother (or sister) flakes and its relationship to other similar and different tasks can be surmised. Properly treated, out of this welter of information the form (if not the face) of ancient man emerges.

This and the following section summarize the numerical gymnastics that were necessary before reliable interpretations of what was found at Eagle Hill could be made. We used some, but certainly not even a small portion of the recently developed scientific technology to attempt a discovery of man's passage on Peason Ridge. This discovery is not an easy task and requires both forethought and effort. It should be made clear from the first that we are in search of the processes and patterns of prehistory, not its finite detail. In the time available to us we were able to intensively collect and analyze data from five occupation planes measuring $5 \times 6 \mathrm{~m}$. Approximately 100,000 observations were made on this $150 \mathrm{~m}^{3}$ space. We probably excavated one-third of the discernible occupation planes with the greatest of care and about one-half of the site, which had not been destroyed. Thus 600,000 observations would be required to examine the whole site as thoroughly. How large the site was at various times we cannot know. It was certainly large, and most of it has probably been destroyed by natural and human agents. Because of these missing data points, even our best efforts can show only a part of the total reality of Eagle Hill. However, there is no direct relationship beiween reliable interpretation of cultural process and the size of a site. Reliable interpretation is relative to the space required to execute the activities common at the site. It only requires a window to culture to observe process. A full reconstruction is not a practical or even desirable objective.

The immediate problem of this and the next section is a concept for managing 100,000 data points in a way that will provide a reliable window to cultural processes. We have two basic kinds of data. The first is observations on one-meter squares: the number of flakes, the amount of charcoal, etc. The second is observations which are located to the centimeter: tools, platformed flakes, sherds, etc. To make maximum use of both kinds of information an idea was needed that would comfortably knit the two types of data into a single picture.

Providing reliable interpretations is a matter of properly fitting the resolution of recovery to the scale of activities. The unit of analysis for this study is the occupation floor or, more specifically, the occupation plane as it was defined in the section on excavation methods. The occupation plane (OP) is a window, hopefully positioned and sized, to avail a good view of culture process. The preliminary field excursion, control column analysis, etc., were all executed to assist in the proper positioning of the window. Once properly positioned, we can expect to see that people had room to go about their daily camp activities. The rush of the summer's excavation was intended to assure this. Naturally we have to justify our reasons for excavation of a room size unit rather than a city block. We assume we are dealing with a particular segment of a particular mode of $1 \mathrm{ife}$. The segment is camp activities, and the mode of $1 \mathrm{ife}$ is hunting and/or collecting. As a consequence, we suspect that a culturally meaningful 
area will be rather small. Within that small area, however, artifacts need to be recovered with some care. Duties performed in camp afford insight into field activities. A broken arrowhead on a floor, for instance, tells us that rehafting was done at camp at day's end rather than in the field.

In general, we depend on the one-meter resolution data to delimit the features, which structure the camp: fireplaces, rubbish heaps and, certainly not least important, barren places where people sat. A one-meter square is about the amount of space a static human body rests and works in, so it is a good basic subunit for an occupation plane. A $6 \times 5 \mathrm{~m}$ occupation plane is a reasonable amount of space for some portion of a camp.

The one-centimeter data defines locations of activities within and between background units. We would, for instance, want the discarded base of a projectile point located to the centimeter. As is illustrated in Figure 74, when the one-centimeter and one-meter data are analyzed together, they provide the backdrop or "background" and the detail or "foregound" necessary to provide a clear indication of cultural activities. "Cultural" is emphasized because the likelihood of following prehistoric movements of an individual are slim in most sites. It has been done under special conditions; however, in slow depositional situations the odds are that we are watching a lineage of fathers and sons, who hunted the same range, built fires in well-remembered places, and upon each return performed duties, which were indicated by their cultural heritage.

The Background-Foregound concept is a means of managing data resolution so that the analyst is not overwhelmed by detail or the analysis so superficial as to miss important patterns. Some analysis of one-meter resolution data was performed in the previous section along with the discussion of formal tools. The following articles complete analysis of specific artifacts at one-meter resolution and provide a general analysis of all one-meter data.

\section{B. ANALYSIS OF BACKGROUND DATA}

The next several subsections of this report examine the one-meter resolution data set from several perspectives. All the variables in the one-meter resolution data set are listed in Table 44. Each of the following subsections provides an analysis of some portion of that data. Also provided is an analysis of all the variables in the one-meter resolution data set together. The larger data set is resident on an SPSS (Statistical Package for the Social Sciences) file. When a subset is desired for a project it is extracted onto a smaller file or analyzed directly from the main file depending upon considerations of convenience and economy. The variables listed in Table 44 are defined in subsequent subsections. 


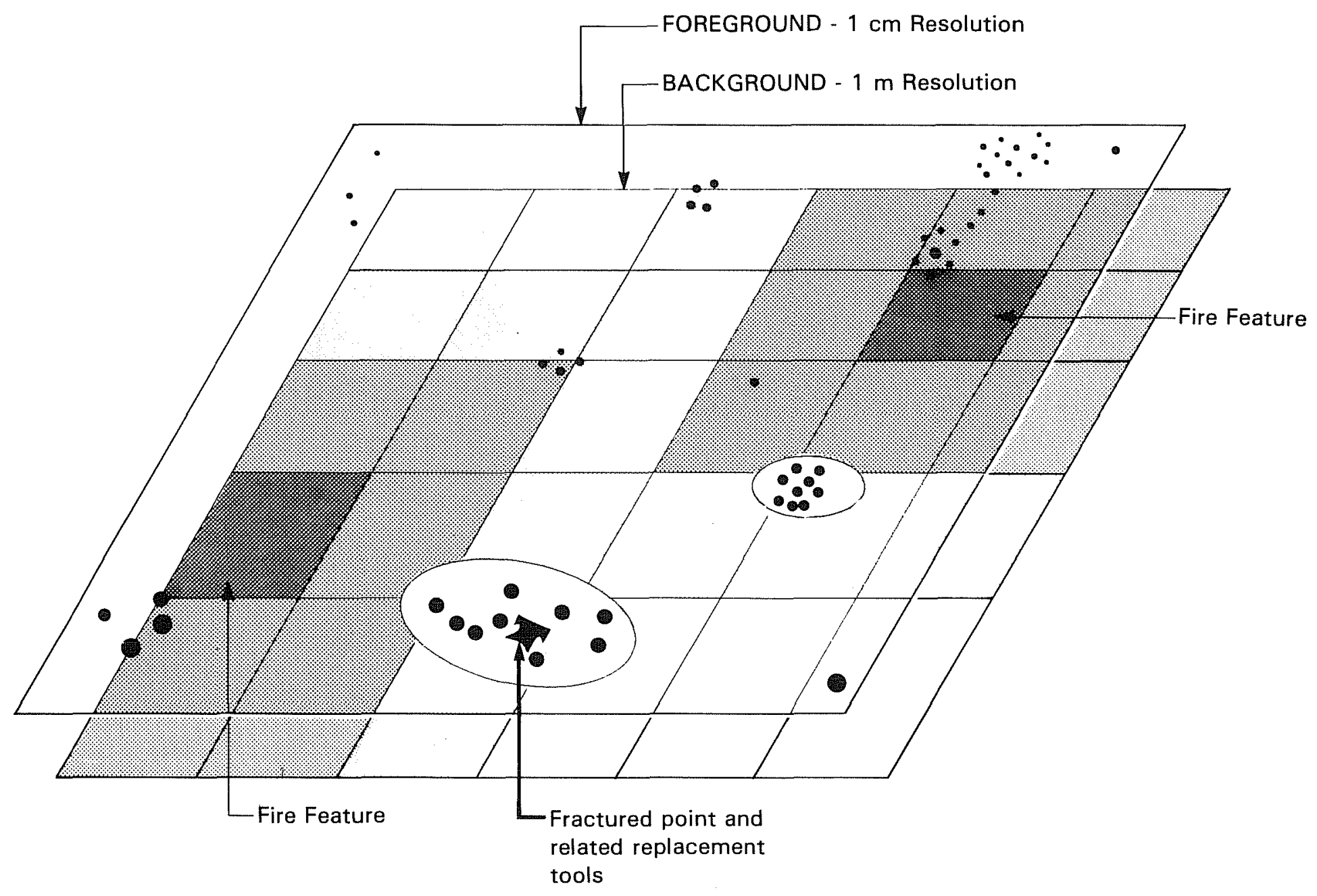

Figure 74. Background-Foreground Site Evaluation Strategy. 
1. Physical Units (Subfile Name = EHOCPU, see Excavation Methods)

\begin{tabular}{ccccl}
$\begin{array}{c}\text { System } \\
\text { Variable \# }\end{array}$ & $\begin{array}{c}\text { Format } \\
\text { Variable \# }\end{array}$ & $\begin{array}{c}\text { Column } \\
\text { \#S }\end{array}$ & Pneumonic & \multicolumn{1}{c}{ Variable Name } \\
\hline 1 & 1 & $1-2$ & FM & Format Number (02) \\
2 & 2 & $3-7$ & FN & Field Number \\
3 & 3 & $8-11$ & SS & Substratum (3 decima1) \\
4 & 4 & $12-17$ & EA & East Coordinate (2 decima) $)$ \\
5 & 5 & $18-23$ & NO & North Coordinate (2 decima1) \\
- & 6 & $24-26$ & - & Unit Size (cm) \\
6 & 7 & $27-28$ & LTHK & Level Thickness \\
- & 8 & 29 & - & Level Type \\
7 & 9 & $30-35$ & TDP & Transit Depth \\
8 & 10 & $36-38$ & HU & Hue (number) \\
9 & 11 & 39 & HU & Hue (Alpha; 1=y, 2=yr, 3=r) \\
10 & 12 & $40-41$ & VAL & Value \\
11 & 13 & $42-43$ & CHR & Chroma \\
12 & 14 & 44 & CV & Constant Volume $(1=$ no, 2=yes) \\
13 & 15 & $45-46$ & TX & Texture \\
14 & 16 & 47 & CON & Consolidation \\
15 & 17 & 48 & CHAR & Charcoal Flecks \\
16 & 18 & 49 & SAD & Shells, Aquatic \\
17 & 19 & 50 & SLND & Shells, Land \\
18 & 20 & 51 & CHP & Chipped Stone \\
19 & 21 & 52 & EXT & Extent of Excavation \\
20 & 22 & $53-54$ & REC & Recorder \\
21 & 23 & 55 & MAP & Mapped \\
- & 24 & 56 & - & Tagged \\
22 & 25 & 57 & DEP & Depth to Top \\
23 & 26 & $58-59$ & IN & Inspector \# \\
24 & 27 & $60-61$ & DA & Day \\
25 & 28 & $62-63$ & MO & Month \\
26 & 29 & $64-65$ & YR & Year
\end{tabular}

2. One-Meter Resolution (Subfile Name = EHOCIM, see Laboratory Methods

$\begin{array}{rrrll}- & 1 & 1-2 & \text { FM } & \text { Format Number (05) } \\ - & 2 & 3-7 & \text { FN } & \text { Field Number } \\ - & 3 & 8-10 & \text { SS } & \text { Substratum/Occupation Plane } \\ - & 4 & 11-14 & \text { EA } & \text { East Coordinate (meters) } \\ - & 5 & 15-18 & \text { NO } & \text { North Coordinate (meters) } \\ 27 & 6 & 19-20 & \text { EXC } & \text { Excavator \# } \\ 28 & 7 & 21-23 & \text { CLCT } & \text { Clay Bal1 Count } \\ 29 & 8 & 24-25 & \text { SHCT } & \text { Sherd Count } \\ 30 & 9 & 26-28 & \text { PFCT } & \text { Platformed Flake Count } \\ 31 & 10 & 29-30 & \text { PFFC } & \text { Platformed Flake Fired Count } \\ 32 & 11 & 31-32 & \text { NFFC } & \text { Nonplatformed Flake Fired Count } \\ 33 & 12 & 33-34 & \text { FNCT } & \text { Field Number Count } \\ 34 & 13 & 35-38 & \text { CWT } & \text { Clay Ball Weight } \\ 35 & 14 & 39-41 & \text { SHWT } & \text { Sherd Weight } \\ 36 & 15 & 42-44 & \text { PFPW } & \text { Platformed Flake Plotted Weight } \\ 37 & 16 & 45-47 & \text { PFSW } & \text { Platformed Flake Screens Weight } \\ 38 & 17 & 48-51 & \text { NFPW } & \text { Nonplatformed Flake Plotted Weight } \\ 39 & 18 & 52-55 & \text { NFSW } & \text { Nonplatformed Flake Screens Weight } \\ 40 & 19 & 56-59 & \text { PBLW } & \text { Pebble/Granule Weight } \\ 41 & 20 & 60-63 & \text { LMTW } & \text { Limonite/Hematite Weight } \\ 42 & 21 & 64-67 & \text { Cl4WT } & \text { Carbon/Charcoal Weight } \\ 43 & 22 & 68-70 & \text { RESWT } & \text { Resin Weight }\end{array}$

3. One-Meter Wear Analysis (Subfile Name = EHOCWR, see Wear Analysis)

$\begin{array}{rrrrl}- & 1 & 1-2 & \text { FM } & \text { Format (06) } \\ - & 2 & 3-7 & \text { FN } & \text { Field Number } \\ - & 3 & 8-10 & \text { SS } & \text { Substratum/Occupation Plane } \\ - & 4 & 11-14 & \text { EA } & \text { East Coordinate (meters) } \\ - & 5 & 15-18 & \text { NO } & \text { North Coordinate (meters) } \\ 44 & 6 & 19 & \text { SS } & \text { Soft Scraping } \\ 45 & 7 & 20 & \text { SC } & \text { Soft Cutting } \\ 46 & 8 & 21 & \text { SB } & \text { Soft Boring }\end{array}$


Table 44. (cont.)

$\begin{array}{lrrll}47 & 9 & 22 & \text { SP } & \text { Soft Perforation } \\ 48 & 10 & 23 & \text { SM } & \text { Soft Multiple Use (Scraping/Cutting) } \\ 49 & 11 & 24 & \text { SI } & \text { Soft Indeterminate } \\ 50 & 12 & 25 & \text { HS } & \text { Hard Scraping } \\ 51 & 13 & 26 & \text { HC } & \text { Hard Cutting } \\ 52 & 14 & 27 & \text { HB } & \text { Hard Boring } \\ 53 & 15 & 28 & \text { HP } & \text { Hard Perforating } \\ 54 & 16 & 29 & \text { HM } & \text { Hard Multiple Use (Scraping/Cutting) } \\ 55 & 17 & 30 & \text { HI } & \text { Hard Indeterminate } \\ 56 & 18 & 31-34 & \text { SUWD } & \text { Sum of Widths (mm) } \\ 57 & 19 & 35-36 & \text { NF } & \text { Number Of Pieces }\end{array}$

4. X-Ray Fluorescence Analysis (Subfile Name $=E H O C X L$, see XRF Data)

$\begin{array}{rrrll}- & 1 & 1-2 & \text { FM } & \text { Format Number (08) } \\ - & 2 & 3-7 & \text { FN } & \text { Field Number } \\ - & 3 & 8-10 & \text { SS } & \text { Substratum/Occupation Plane } \\ 58 & 4 & 11-13 & \text { FE } & \text { Iron } \\ 59 & 5 & 14-16 & \text { AL } & \text { Aluminum } \\ 60 & 6 & 17-19 & \text { SI } & \text { Silicon } \\ 61 & 7 & 20-22 & \text { TI } & \text { Titanium } \\ 62 & 8 & 23-25 & \text { K } & \text { Potassium } \\ 63 & 9 & 26-28 & \text { CA } & \text { Calcium } \\ 64 & 10 & 29-31 & \text { MG } & \text { Magnesium } \\ 65 & 11 & 32-34 & \text { MN } & \text { Manganese } \\ 66 & 12 & 35-37 & \text { GE } & \text { Germanium } \\ 67 & 13 & 38-40 & \text { RB } & \text { Rubidium } \\ 68 & 14 & 41-43 & \text { SR } & \text { Strontium } \\ 69 & 15 & 44-46 & \text { W } & \text { Wolfram } \\ 70 & 16 & 47-49 & \text { P } & \text { Phosphorus } \\ 71 & 17 & 50-52 & \text { CR } & \text { Chromium } \\ 72 & 18 & 53-55 & \text { NI } & \text { Nickel } \\ 73 & 19 & 56-58 & \text { ZR } & \text { Zirconium } \\ 74 & 20 & 59-61 & \text { CU } & \text { Copper } \\ 75 & 21 & 62-64 & \text { PB } & \text { Lead } \\ 76 & 22 & 65-67 & \text { ZN } & \text { Zinc } \\ 77 & 23 & 68-70 & \text { NA } & \text { Sodium } \\ 78 & 24 & 71-73 & \text { BA } & \text { Barium } \\ 79 & 25 & 74-76 & \text { GA } & \text { Galiium } \\ 80 & 26 & 77-79 & \text { SE } & \text { Selenium } \\ & & & & \end{array}$

5. Lithic Material Types (Subfile Name = EHOCIM, see Lithic Materials)

$\begin{array}{rrrll}- & 1 & 1-2 & \text { FM } & \text { Format Number (11) } \\ - & 2 & 3-7 & \text { FN } & \text { Field Number (FN) } \\ - & 3 & 8-10 & \text { SS } & \text { Substratum/Occupation Plane } \\ 81 & 4 & 11-12 & \text { DT } & \text { Dark Tan } \\ 82 & 5 & 13-14 & \text { DTM } & \text { Dark Tan Mottled } \\ 83 & 6 & 15-16 & \text { LTN } & \text { Light Tan } \\ 84 & 7 & 17-18 & \text { LTM } & \text { Light Tan Mottled } \\ 85 & 8 & 19-20 & \text { LTO } & \text { Light Tan Opaque } \\ 86 & 9 & 21-22 & \text { WO } & \text { White Opaque } \\ 87 & 10 & 23-24 & \text { BO } & \text { Brown Opaque } \\ 88 & 11 & 25-26 & \text { GO } & \text { Gray Opaque } \\ 89 & 12 & 27-28 & \text { CH } & \text { Chalcedony } \\ 90 & 13 & 29-30 & \text { BL } & \text { Black } \\ 91 & 14 & 31-32 & \text { BLM } & \text { Black Mottled } \\ 92 & 15 & 33-34 & \text { PW } & \text { Petrified Wood } \\ 93 & 16 & 35-36 & \text { BFG } & \text { Black Fine Grained } \\ 94 & 17 & 37-38 & \text { RY } & \text { Red Yellow } \\ 95 & 18 & 39-40 & \text { CO } & \text { Caramel Opaque } \\ 96 & 19 & 41-42 & \text { WQ } & \text { White Quartzite } \\ 97 & 20 & 43-44 & \text { YQ } & \text { Yellow Quartzite } \\ 98 & 21 & 45-46 & \text { PQ } & \text { Purple Quartzite } \\ 99 & 22 & 47-48 & \text { RO } & \text { Red Opaque } \\ 100 & 23 & 49-50 & \text { COR } & \text { Cortex } \\ 101 & 24 & 51-52 & \text { LIM } & \text { Limestone } \\ 102 & 25 & 53-54 & \text { POT } & \text { Potlidding } \\ 103 & 26 & 55-56 & \text { HC } & \text { Heat Crazed } \\ 104 & 27 & 57-58 & \text { HS } & \text { Heat Sheen } \\ 105 & 28 & 59-60 & \text { TRC } & \text { Translucent } \\ 106 & 29 & 61-62 & \text { OTH } & \text { Other } \\ & & & & \\ & 15 & & & \end{array}$


Baked Clay Balls (Sherwood)

One of the unusual artifacts recovered during the excavation of the Eagle Hill site was the large number of baked clay balls that appeared throughout the site in all levels. It was thought that distribution patterns might emerge to indicate the presence of hearths (their very presence implied some kind of firing), so the balls were plotted on unit maps. It was also thought that the clay balls were related to pottery making. A description of the clay balls, their location in relation to other artifacts (particularly charcoal), and their possible origin will be discussed in this section.

The soil at Eagle $\mathrm{Hill}$ consists of sandy loam in the surface and upper layers. Sediment composition becomes finer toward the bottom of excavation because of translocated pedogenic clays. The deepest level examined is a culturally sterile Miocene lake bed with high clay content. Clay balls were recovered from all levels of the site, regardless of the proportion of clay to sand in the soil (Fig. 75) except, of course, the Miocene lake bed. All balls showed evidence of baking. Colors varied from light brown and reddish yellow to bright red (7.5 YR 6/4, 7.5 YR 6/8, and 2.4 R 5/8; respectively on the Munse 1 Color scale). Many showed signs of blackening on one side, while some of the smaller lumps were completely black. In a few cases, balls were found that were black on one side and red on the other. These variations in color can be explained by the conditions under which the clay was fired. Clay that is exposed to oxygen during firing will assume a yellow color and, where the fire is hotter or upon repeated firing, will turn red. However, if clay is fired under conditions where oxygen does not come in contact with the clay, the clay will turn gray or black due to atmospheric reduction of carbon.

Table 45 shows the amount of clay balls by weight and number recovered from the excavated occupation planes. Frequencies of clay ball recovery may have been affected by different methods of screening used as the excavation progressed. During excavation of OP 1.13, artifacts were washed through $1 / 8$-inch screen by pouring buckets of water over the matrix. Some clay may have dissolved in this process. In later screening, material was dry screened, which probably increased the recovery rate of the clay. As excavation continued into lower levels, it became increasingly difficult to dry screen material, because of the growing clay content of the soil.

TABLE 45. TABULATION OF CLAY BALLS, WEIGHT, AVERAGE SIZE, AND PERCENTAGES OF TOTAL

\begin{tabular}{lrrrrr} 
Substratum & Number & $\begin{array}{c}\text { Percentage } \\
\text { of Total } \\
\text { No. }\end{array}$ & $\begin{array}{c}\text { Total Weight } \\
\text { (grams) }\end{array}$ & $\begin{array}{c}\text { Average Weight } \\
\text { (grams) }\end{array}$ & $\begin{array}{r}\text { Percentage } \\
\text { of Total } \\
\text { Weight }\end{array}$ \\
\hline 1.13 & 165 & 8.9 & 75.6 & 0.46 & 4.9 \\
2.13 & 1105 & 59.9 & 781.2 & 0.71 & 50.7 \\
3.11 & 538 & 29.1 & 633.5 & 1.18 & 41.2 \\
4.12 & 20 & 1.1 & 42.5 & 2.13 & 2.8 \\
$4.15,4.16$ & 17 & 1.0 & 5.9 & 0.35 & 0.4 \\
\hline TOTAL & 1845 & 100.0 & 1538.7 & 0.83 & 100.0 \\
\hline
\end{tabular}


EAGLE HILL (16SA50) Occupation Plene Analysis - $1 \mathrm{~m}$ Study

TOPIC: Distribution Clay Balls

N

Occupation

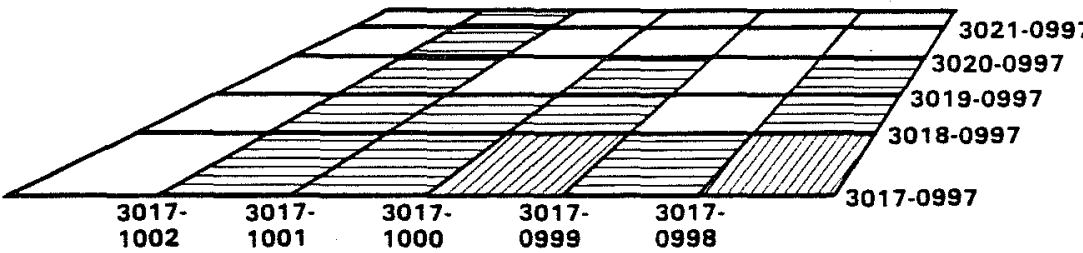

Plane

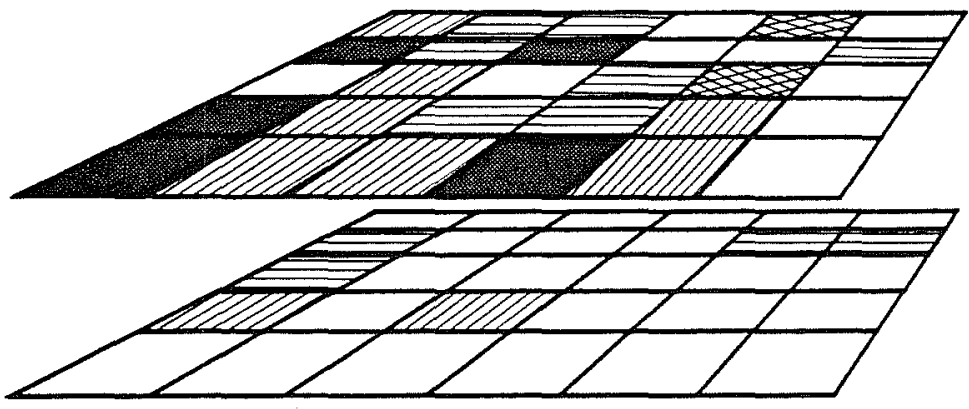

Key

[I] $=0-10$

$10-25$

$=25-50$

= over 50

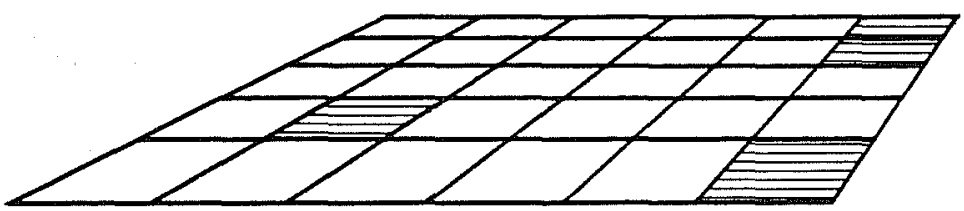

$4.15-4.16$

Figure 75. Clay Ball Distribution. 
In the lower levels (OP 4.12-4.17), it was necessary to soak excavated material in ammonium hydroxide so that it would pass through the screens, or screen it under high water pressure. This process may have dissolved less coherent clay balls. To test this, clay balls were soaked in ammonium hydroxide overnight. The less solid clay balls did dissolve.

Water screening technique probably contributed to some unknown reduction in clay ball recovery. Excavator experience may also have been a factor early, al though the crew had previously completed two levels (OP 1.11 and OP 1.12) before excavating $O P$ 1.13. The effect of ammonium hydroxide, as determined by the above experiment, would have reduced the frequency of recovery in OP 4.12 and below.

None of the clay balls recovered shows any signs of hand molding. They are irregular in shape and range in size from four millimeters to three centimeters. In many cases, the size of the larger balls seems to be the result of two or three balls compacted together. This conclusion is drawn from what appear to be seam-lines on the surface of the balls (Fig. 76,C). In addition, where balls are cut in two, layers of different colored clays are revealed (Fig. 76,b) At least two balls have a thin layer of sand trapped between two layers of clay (Fig. 76,f), and several have what appear to be charcoal particles imbedded in them. One shows stratification of different colored clays interspersed with thin layers of black material (Fig. 76,e). The cross section of one ball shows a bright red interior with a lateral fissure across the interior and another short fissure perpendicular from the top partway into the interior. The area around the lateral fissure is yellow and is pierced with a series of small holes (Fig. 76,g).

The fact that two or more lumps of clay may have been pressed together to form a larger lump suggests that some animal, or perhaps man, may have stepped on them when they were on the surface, thus forming one large ball.

There are two additional balls that exhibited unusual features and deserve mention. One, upon opening, showed a small circle of dark clay in a yellow clay setting, taking up $1 / 4$ of the surface area. There is another small dark spot at the lower end of the cut surface (Fig. 76,a). The other and most unusual clay ball has a small flint chip buried in it. The outside of the ball is grayish yellow in color. The top half of its interior is yellow with the bottom half shaded from gray to black. The chip is positioned at an angle $45^{\circ}$ from the bottom of the ball, which is flattened and smooth. Microscopic examination shows evidence that the bottom may have been abraded (Fig. 76,d). D. R. Lewis (personal communication) found a cache of clay balls in a site on Lake Houston, which contained small flints and colored stones. No determination of the use or meaning of these objects has yet been made, if indeed they are handmade and not a natural deposition of clay around an object.

Fourteen balls recovered from OP 3.11 and weighing a total of 10.3 grams were composed partly of clay and partly of charcoal. The cores are charcoal with a clay coating. It is possible that the clay was accidently stuck to the wood with which it came in contact. Later, when the wood burned, the clay hardened and continued to adhere to the charcoal (Fig. 77). 


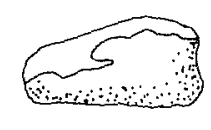

b

a
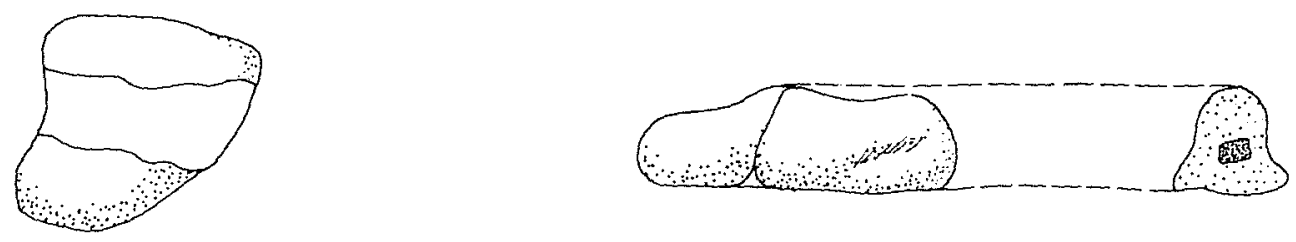

d

c
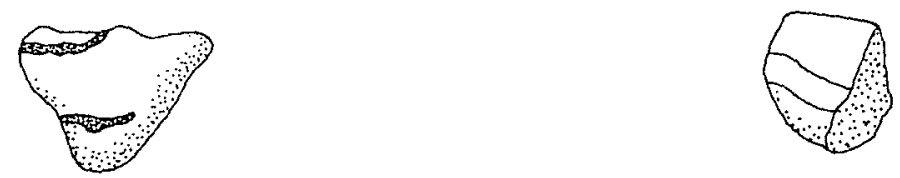

f

e

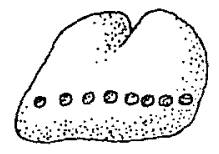

9

Figure 76. Clay Balls Found at Eagle Hill. 
EAGLE HILL (16SA50)

Occupation Plane Analysis - $1 \mathrm{~m}$ Study
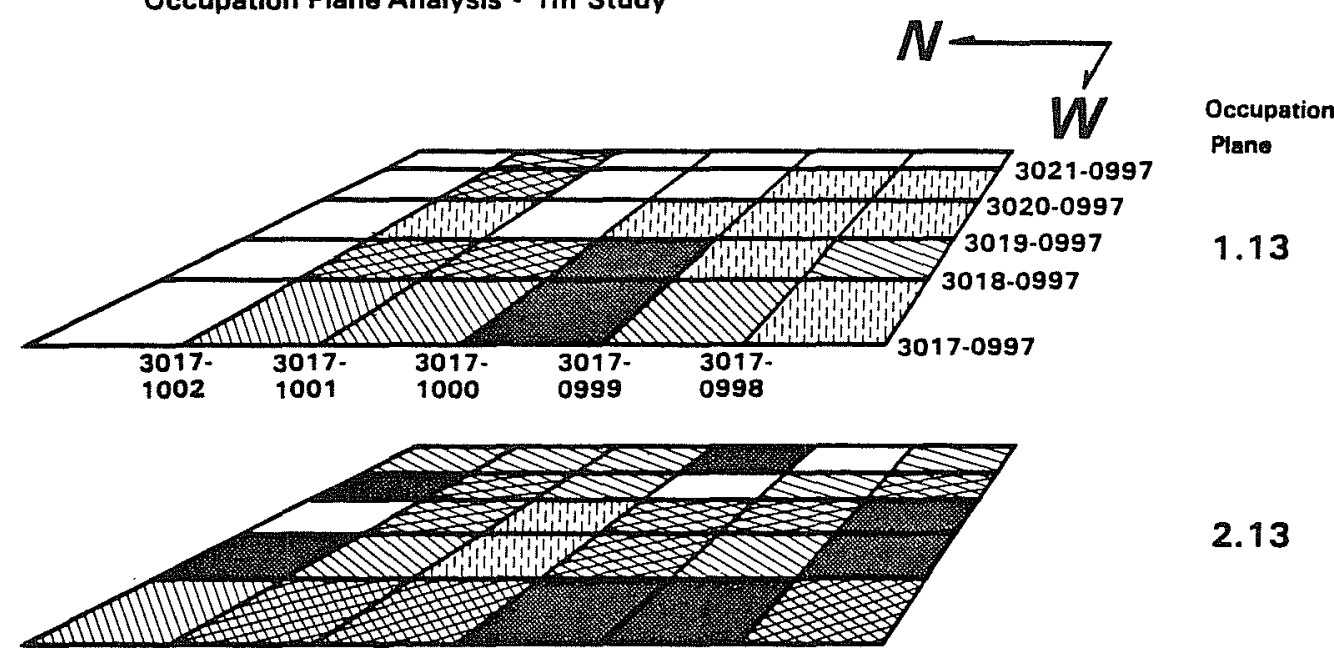

2.13
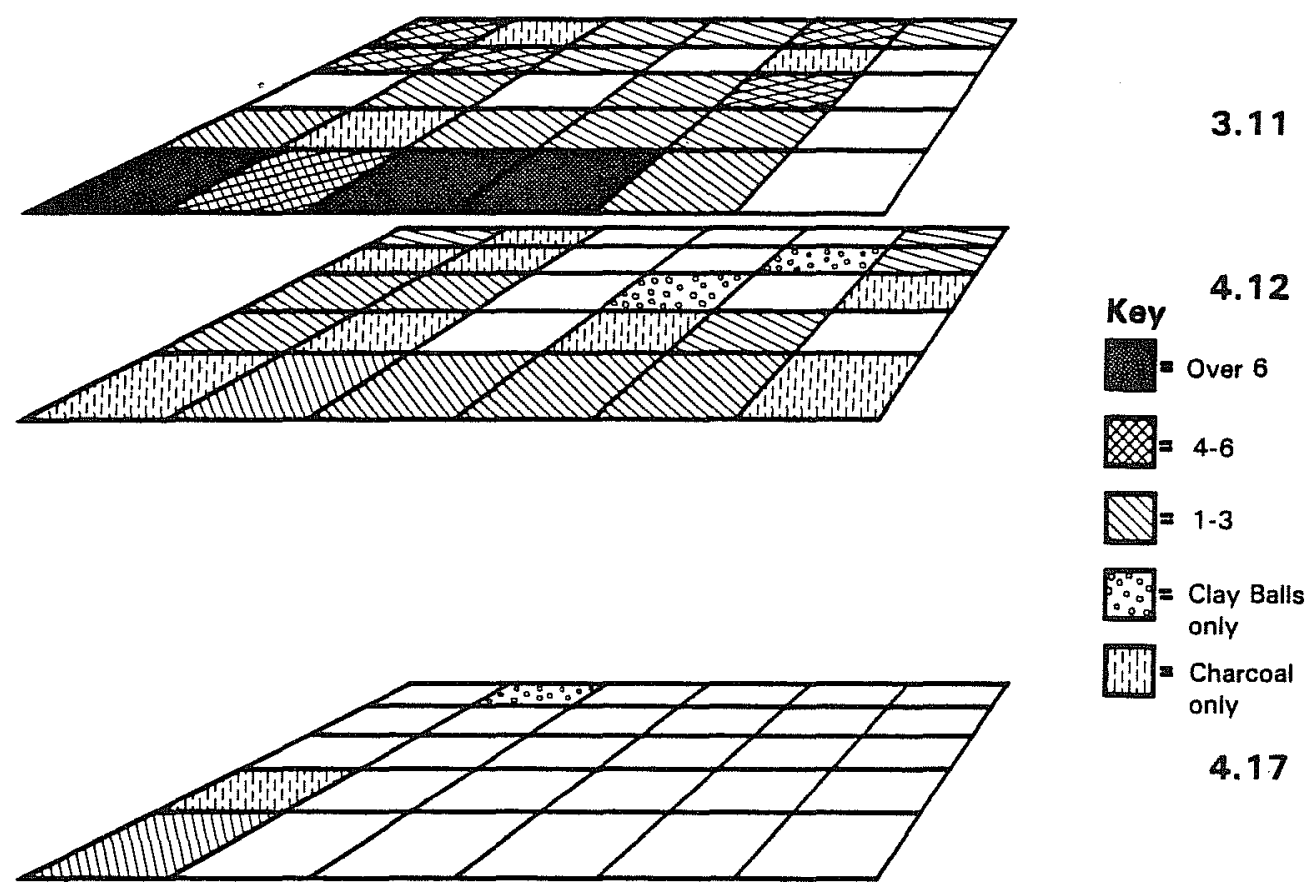

\subsection{1}

4.17

Figure 77. Clay Ball Plots Found with Charcoal. 
With the aid of a magnifying glass, visual inspection of a random sample of clay balls from the different strata showed that the clay of these balls contained grains of foreign material. Grains are present at a ratio of $1 \%$ to $2 \%$ of the bulk of the clay with the majority in the $1 \%$ range. The estimates were made by use of the Comparison Chart for Estimating Percentage of Composition (Terry and Chilingar 1955:229-234). Exact composition of clay was determined by laboratory tests.

Clay balls have been found at many sites throughout the United States, usually in areas deficient in natural stone. Large quantities of clay balls or "Poverty Point" objects have been recovered from sites in Louisiana and along the Gulf coast. They have been formed into cylindrical, spherical, and other shapes (Ford and Webb 1956:36-49). Similar clay balls have been recovered from sites in California (Heizer 1937:34-50); Charles Towne, South Carolina (South 1970:3-16); and along the Gulf coast from Florida to Louisiana (Gagliano 1977). Most of the objects (definitely hand molded) have been tentatively identified as cooking stones. Other clay balls, also man-made, but with a distinctive shape, known as "Kersey Clay 0bjects" have been recovered from sites in Missouri (Williams 1974:79-80). None of the clay balls recovered at Eagle Hill can be so identified, however, as they are small and do not show signs of hand molding or decoration.

There have been numerous reports of clay balls in Texas sites. Clay balls from Loyola Beach bear signs of human manufacture (Hester 1971:100-102). Hester hypothesizes that these clay balls may have been used as stone substitutes in hearths or may have been baked and used in containers as cooking stones. Another explanation for the formation of clay balls is offered by Corbin (1963:29) who suggests that they may be the result of open hearths built on the surface in areas where there is a large amount of clay in the soil. Black (1978:28-29) suggests that clay balls may also result from brush fires on top of clay surfaces. In Victoria and Goliad Counties, Texas (Fox, Black, and James 1979:56), concentrations of burned sandstone and clay were found that appear to be remnants of hearths, although no charcoal was found in direct association with these features.

None of these explain the presence of clay balls at Eagle Hill. Charcoal in association with clay balls in many areas of the excavation may account for the fact that the clay was baked, but it does not explain the origin of the clay since, as stated previously, the upper soil horizons are sandy.

We have also considered the possibility that the clay balls may have been the residue from pottery making. Potters may have discarded pieces of clay unsuitable for use or may have trimmed the pottery in the manufacturing process. Some sherds were found during excavation, but there is no other evidence (hearths) to indicate that pottery making was taking place in the area. Tests were performed to determine if the pottery sherds were made from clays at the site.

An explanation for the clay balls is suggested by the presence of crayfish on Peason Ridge. The depth of crayfish burrows varies from a few inches to as much as eight to ten feet depending upon the level of the water table. Crayfish must burrow down to the water level to keep their gills wet. The 
crayfish carries the mud from his burrow and deposits it at the entrance to the chimney. Chimneys may range in height from six to eighteen inches (Pennak

1953:456). Such chimneys were observed on the Eagle Hill site. Since the clay content of the soil increased with depth, particularly in the Miocene lake bed, it is probable that the clay found in the soil was carried to the surface by the crayfish activity over a long period of time and eventually deposited at different levels as the soil zones accumulated. This would account for the presence of the clay balls in the sandy soil. Probably the clay was baked as a result of successive ground fires, which swept the area over the years. Manmade fires may also have been responsible. The fact that clay balls and charcoal were found in the same areas supports this hypothesis.

\section{Lithics (Jo11y)}

Excavations at Eagle $\mathrm{Hill}$ yielded a diverse assemblage of artifactual materials noteworthy for its scarcity of formalized tools. This lack of traditionally accepted temporal/cultural indicators necessitated more intensive analysis of some of the ancillary aspects of the cultural remains. In this study, the lithic raw materials as represented in the debitage assemblage are examined and presented in relation to a model for subsistence and lithic procurement.

The Method

An initial analysis of the debitage was conducted in conjunction with the edge wear analysis. Examination of approximately 1500 one-centimeter provenienced flakes provided a broad base of observations from which 21 material type categories were established (see Table 46). Delineation of these categories was judgmental and qualitative as defined by the following six attributes:

(1) Color, (2) Fracture, (3) Cortex (Presence/Absence), (4) Grain, (5) Translucence, and (6) Mottling (Presence/Absence).

The final analysis consisted of raw counts of the material in each category for each one-meter unit from each occupation plane. The counts were made on all of the platform flakes of the units recovered by troweling. Counts of platformed flakes are thought to be an accurate representation of the amount of on-site flaking, the number of blows struck during lithic-related activities.

The total data set consisted of approximately 4600 flakes. Preliminary data analyses were directed toward establishing the integrity of the 21 1ithic categories. In light of the fact that the categories were defined qualitatively, there was a question as to whether they were, in fact, discrete categories. Six material types (CT, DTM, LT, LTM, LTO, WO), due to conspicuous similarities, in particular, were targeted for a statistical analysis of their possible interrelation. A stepwise regression was performed on the frequencies of the six types by one-meter units as a means of isolating overlapping material type categories. It was assumed that material types originating from similar sources were likely to be found next to each other in the same squares and thus highly correlated. The simple correlations presented in Table 47 show that LTO and LTN exhibit the highest positive correlation (0.47), and LTN and WQ slightly lower (0.39). These relationships are examined more closely in the 
Dark Tan Chert (DT)

Dark Tan Mottled (DTM)

Light $\operatorname{Tan}(\mathrm{LT})$

Light Tan Mottled (LTM)

Light Tan Opaque (LTO)

White Opaque (wo)

Brown Opaque (Bro)

Gray Opaque (GO)

Chatcedony $(\mathrm{CH})$

B 1 ack（BL)
Brown to dark tan

Irregular to smooth fracture

Occasional cortex, Cortex inclusions

Medium to fine grained

Edge translucent

No mottiing

Some large pieces, probably local

Brown to dark tan

Smooth to irregular fracture

Occasional cortex

Medium to fine grained

Edge translucent to opaque

Tan to white mottling

Tan to light tan

Smooth fracture

Occasional cortex

Medium to fine grained

Translucent to edge transiucent

No mottling

Largest category, probably local

Tan to right tan

Generally smooth fracture

Occasional cortex

Medium to fine grained

Edge translucent to opaque

Light tan to white mottling

Tan to light tan

Irregular to smooth fracture

No cortex

Medium to coarse grained

Opaque

White

Generally smooth

No cortex

Medium to coarse grained

Opaque

Brown

Generally smooth

No cortex

Medium grained

Opaque

Gray

Smooth fracture

No cortex

Medium to very fine grained

Opaque to edge translucent

Possibly river cobble, exotic

White

Smooth fracture

Occasional cortex

Medium to very fine grained

Opaque to translucent

Black

Irregular to smooth fracture

Occasional cortex

Medium to fine grained

Opaque to edge translucent

More of this burned than any other type, thermal alteration? 
TABLE 46. (continued)

Black Mottled (BLM)

Black Fine Grained (BFG)

Red/Yellow (RY)

Carmel Opaque (CO)

White Quartzite (WQ)

Yellow Quartzite (YQ)

Purple Quartzite (PQ)

Red Opaque (RO)

Limestone (LI)

Translucent Cortex (TrC)
Black

Smooth fracture

No cortex

Medium to fine grained

Opaque to edge translucent

White to gray mottling

Black

Smooth fracture

Very fine grained

Edge translucent

No cortex

Two pieces in site, Flint, Arkansas or Oklahoma

Brown to light tan

General1y smooth fracture

No cortex

Medium to coarse grained

Edge translucent to opaque

Very little in site despite local source

Yellow to red

Smooth fracture

No cortex

Fine to very fine grained

Edge translucent to translucent

Might be thermally altered L.T.

Carme1

Smooth fracture

Occasional cortex (river cobble)

Fine to very fine grained

Opaque

Could be thermally altered Gray Opaque

White to gray

Irregular fracture

No cortex

Coarse grained

Edge translucent

Yellow

Irregular fracture

No cortex

Coarse grained

Edge translucent

Purple

Irregular fracture

No cortex

Coarse grained

Edge translucent

Red

Smooth fracture

No cortex

Fine to very fine grained

Opaque

Tools from this material, not many flakes

Exotic

White to gray

Irregular fracture

No cortex

Coarse grained

Opaque

White

Smooth fracture

At least $50 \%$ cortex

Fine grained (chert component)

Translucent (chert component)

Could be waste from chalcedony 
regression analysis (Table 48) where $L T O$, with an $R^{2}$ value of 0.22 , accounts for $22 \%$ of the variance in the category LTN, and WQ accounting for an additional $6 \%$. The influence of the remaining categories is minimal, less than $1 \%$. With the exception of these three categories, the relationships are below statistical significance and present no serious threat to the integrity of the categories. While the analysis suggests that categories LTO, LTN, and WO could be combined, their correlation could also be due simply to high frequency. While this needs to be kept in mind during the following analysis, the categories were kept separate in case the additional resolution should prove valuable in later analyses. This established, the data base was considered complete.

TABLE 47. CORRELATION BETWEEN SELECTED CHERT TYPES

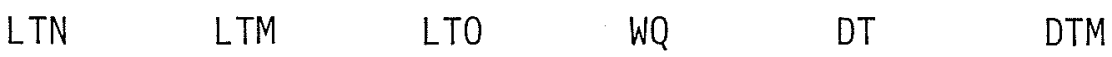

\begin{tabular}{|c|c|c|c|c|c|}
\hline LTN & 1.00 & & & & \\
\hline LTM & .06 & 1.00 & & & \\
\hline LTO & .47 & .10 & 1.00 & & \\
\hline WQ & .39 & .11 & .31 & 1.00 & \\
\hline DT &.$\overline{.25}$ & .15 & .40 & .37 & 1.00 \\
\hline DTM & .27 & .13 & .77 & $\overline{.22}$ & .24 \\
\hline
\end{tabular}

Note: By unit from a11 occupation planes, $n=4600$

TABLE 48. REGRESSION ANALYSIS

Dependent Variable LTN Summary Table

\begin{tabular}{lcccc} 
Variable & R Square & R Square Change & B & Beta \\
\hline LT0 & .23 & .23 & 1.15 & .39 \\
WQ & .29 & .07 & 1.09 & .26 \\
DTM & .30 & .01 & .52 & .10 \\
DT & .30 & .001 & -.72 & -.03 \\
LTM & .30 & .0004 & -.15 & -.02 \\
(Constant) & & & &
\end{tabular}

Table 49 shows the raw counts of platformed flakes for each of the occupation planes by material type.

The Mode1

One of the basic problems in developing a meaningful analysis of the lithic raw materials at Eagle $\mathrm{Hill}$ has been the lack of information on lithic source areas, both local and exotic. In order to circumvent this problem and deal 
TABLE 49. FREQUENCIES OF LITHIC MATERIAL CATEGORIES FOR EAGLE HILL OCCUPATION PLANES

\begin{tabular}{|c|c|c|c|c|c|c|c|c|c|c|c|c|c|c|c|c|c|c|c|c|c|c|c|c|c|c|}
\hline & \multicolumn{19}{|c|}{ LITHIC CATEGORIES } & \multicolumn{7}{|c|}{ SPECIAL OBSERVATIONS } \\
\hline & DT & $\underline{D T M}$ & LTN & $\underline{\text { LTM }}$ & $\underline{\text { LTO }}$ & $\underline{\text { WQ }}$ & $\underline{B O}$ & $\underline{\mathrm{GO}}$ & $\underline{\mathrm{CH}}$ & $\underline{B L}$ & $\underline{B L M}$ & $\underline{\mathrm{PW}}$ & $\underline{B F G}$ & $\underline{\mathrm{RY}}$ & $\underline{\mathrm{CO}}$ & WQ & $\underline{\text { YQ }}$ & $\underline{P Q}$ & $\underline{\mathrm{R} 0}$ & $\underline{\mathrm{COR}}$ & $\underline{\operatorname{LIM}}$ & $\underline{\mathrm{POT}}$ & $\underline{H C}$ & $\underline{\mathrm{HS}}$ & $\underline{\text { TRC }}$ & OTHER \\
\hline OP 1.13 & 24 & 20 & 193 & 14 & 37 & 34 & 9 & 16 & 69 & 12 & 8 & 1 & 0 & 28 & 6 & 0 & 0 & 0 & 0 & 1 & 1 & 0 & 0 & 2 & 17 & 2 \\
\hline OP 2.13 & 48 & 10 & 395 & 122 & 88 & 41 & 5 & 35 & 186 & 32 & 85 & 0 & 0 & 76 & 13 & 0 & 0 & 1 & 3 & 0 & 1 & 0 & 10 & 1 & 48 & 10 \\
\hline OP 3.11 & 42 & 24 & 308 & 222 & 80 & 41 & 0 & 22 & 152 & 16 & 21 & 15 & 2 & 63 & 19 & 1 & 1 & 1 & 1 & 11 & 10 & 0 & 5 & 4 & 41 & 11 \\
\hline OP 4.12 & 84 & 23 & 303 & 208 & 85 & 50 & 3 & 21 & 139 & 19 & 23 & 0 & 0 & 36 & 11 & 0 & 1 & 0 & 1 & 9 & 1 & 0 & 0 & 2 & 50 & 2 \\
\hline OP 4.17 & 90 & 25 & 178 & 103 & 72 & 31 & 7 & 9 & 75 & 10 & 2 & 0 & 0 & 16 & 4 & 0 & 0 & 0 & 0 & 11 & 1 & 0 & 2 & 1 & 35 & 0 \\
\hline Total & 288 & 102 & 1377 & 669 & 362 & 197 & 24 & 103 & 621 & 89 & 139 & 16 & 2 & 219 & 53 & 1 & 2 & 2 & 5 & 32 & 14 & 0 & 17 & 10 & 191 & 25 \\
\hline
\end{tabular}

Note: Refer to Table 44 for definitions to category abbreviations 
with the materials in a useful and direct manner, a model representing the relationship between subsistence and lithic procurement was developed.

The model itself is a meld of two concepts. The basic theoretical underpinnings can be traced to the German economist, Von Thünen, whose 1826 work (Der Isolierte Staat) presents the concept of 'diminishing returns with distance'. More recently, anthropologists have utilized this perspective in analyzing land use and subsistence. Chisom's (1968) study of rural German settlements, Lee's (1969) study of the !Kung and, most recently, Vita-Finzi and Higgs' (1970) 'site catchment' model, have shown the applicability of this concept as an analytical tool. The other half of our conceptualization of this model is drawn from Willey and Phillips (1958). In their analysis of a geography of culture, they present the concepts of locale, region, and area as cultural units. Application to this analysis of these terms is, as we shall see, slightly different.

The model (Fig. 78) divides theoretical space into "use spheres." Adapting Willey and Phillips' (1958) terminology, they are labeled the "locale," the "region," and the "area." Considering the model from the view of economics of 'diminishing returns with distance', note that the "use spheres" delineate points on a continuum sphere encompassing the site specific subsistence pattern, the "region" encompassing the annual/multiseasonal subsistence pattern, and the "area" encompassing the overall subsistence pattern, including trade networks. Also note that in theoretical space these use spheres are round and concentric, with all resources distributed, skewed toward the center (Fig. 78). Skewed means more frequent at the center and less frequent at the periphery. In practical (real) space, however, these spheres are distorted by topographic variablity and unequal resource distribution (Fig. 79).

Applying this model specifically to lithics, two concepts are implicit.

1. Lithic source areas are discrete.

2. Lithic materials, unlike food resources, are depleted through attrition rather than consumption.

From these assumptions we infer that resources relatively removed from the site will show a lower frequency of material than closer resources. By comparing the changes in frequencies of lithic resource utilization, changes from level to level, and possibly of general resource utilization, shifts in source areas, regions, and locales, etc., may be observed.

\section{Application of the Model}

Of primary interest in this study of lithic raw materials is the nature of the relationship between the debitage assemblage, the human subsistence patterns, and the procurement of lithics. The model can be drawn upon to develop a set of terms which define a presumed spatial grouping of chert resources. One must assume a seasonally occupied site with materials being carried into it. A nonseasonal occupation would call for a somewhat different formulation. That all materials are equally usable is also assumed. 


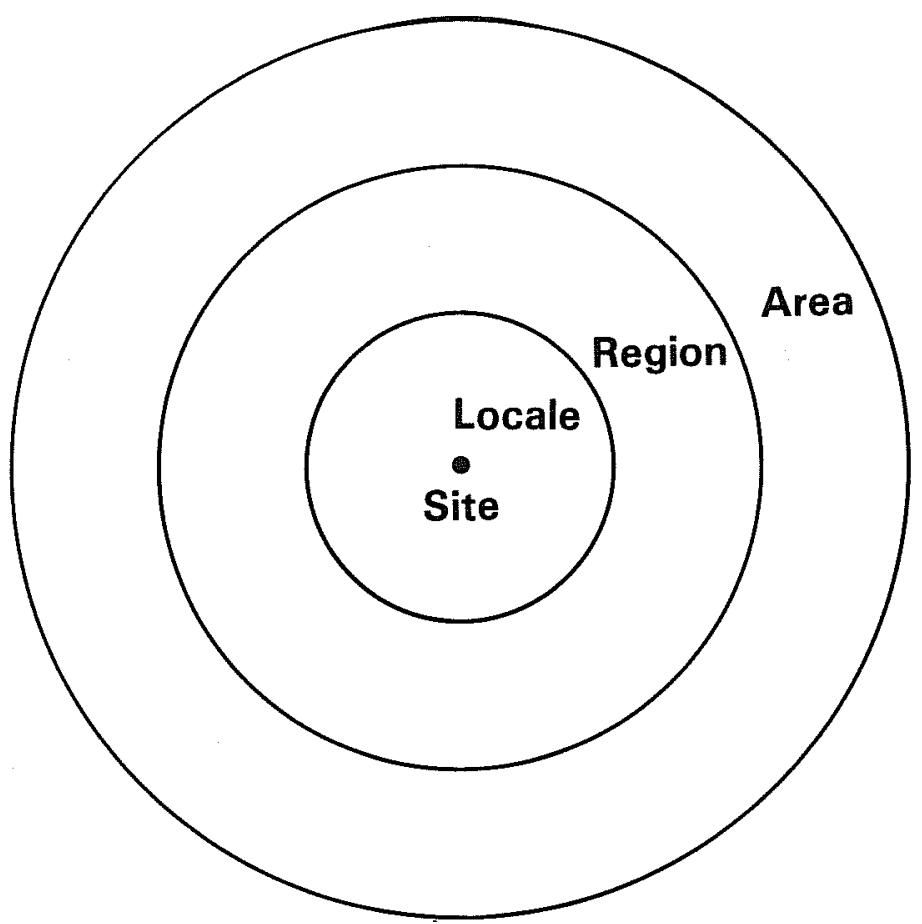

Figure 78. In Theoretical Space, use Spheres are Round and Concentric.

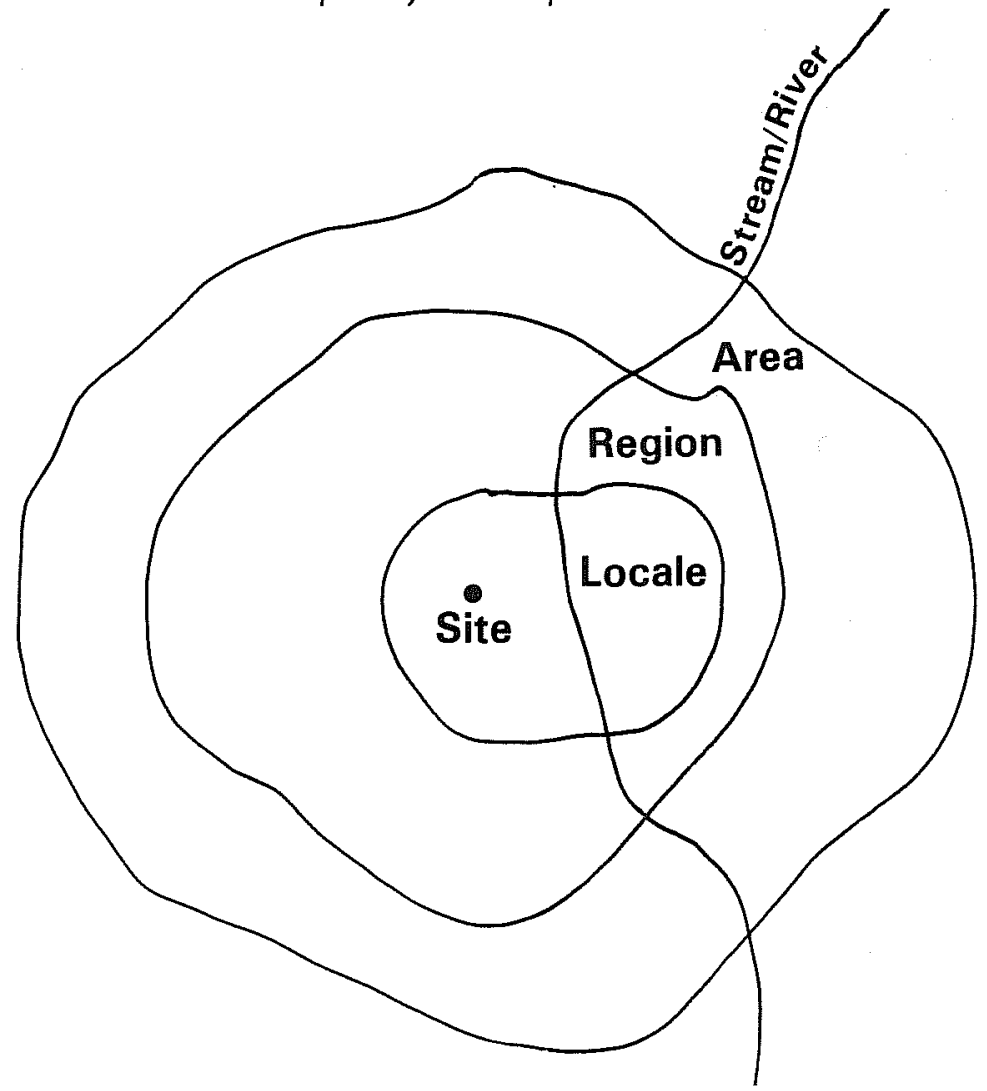

Figure 79. In Practice, use Spheres may be Distorted by Local Topography and Resource Distribution. 
1. Local

2. Regional

3. Exotic
Resources within the general site specific resource pattern (collected). Represented by high relative frequencies.

Resources within the annual/multiseasonal general resource pattern (collected). Represented by lower relative frequencies.

Resources outside the general resource pattern (traded). Represented by extremely low relative frequencies.

Certain changes should be evident with regard to these groups as assemblages change through time. Local resources should present a fairly stable frequency throughout time, while regional and exotic materials show greater change due to fluctuating cultural, environmental, and demographic conditions. This can be seen in Figure 80 where the hypothesized local materials show a fairly stable frequency compared to Figure 81 which shows relatively greater change in the regional materials. Figure 82 shows the presence of exotic materials which peak in 0ccupation Plane 3.11. The increased variation in the regional/exotic materials provides us with an insight into changing patterns of resource utilization, while materials in the margins of the use spheres provide a more sensitive indicator of change in subsistence emphasis than do the readily available local materials.

In order to provide insight into these subsistence changes, one must return to the model for a moment. Lithic source areas are discrete and distributed in decreasing frequency away from the source or, in other words, a type of skewed distribution. The materials should be represented at the site in inverse proportion to their distance. The mean relative frequencies of local and regional materials (11.28\% and $1.9 \%$ ) have arbitrarily been assigned as cutoff points for local/regional/exotic materials (Table 50). That is, materials below the respective means are considered to have moved to the next use sphere outwards. Although the spatial relationship of sources to site is the same, the functional relationship of the material types rises and degrades with time (Fig. 83). Note, for instance, that Light Tan chert (LT) is a constant feature of local material through all the Eagle Hill occupation planes. Black chert (BL), however, changes in functional importance becoming more important in the economy with time. In Figure 83, a-e these distributions have been mapped onto the theoretical space. The following section discusses the implications of these distributions.

\section{Interpretation and Integration}

Examining the theoretical/empirical distributions for the different occupation planes yields some interesting patterns. In OP 4.17 (Fig. 83) there is a complete absence of exotic materials. Considering that this level is most probably Paleo-Indian, this is a rather unexpected conclusion. Paleo-Indian sites are generally notorious for the presence of exotic materials. Resolution of this problem falls within the realm of a re-examination of tool use and material selection, both of which are outside the scope of this report. From 


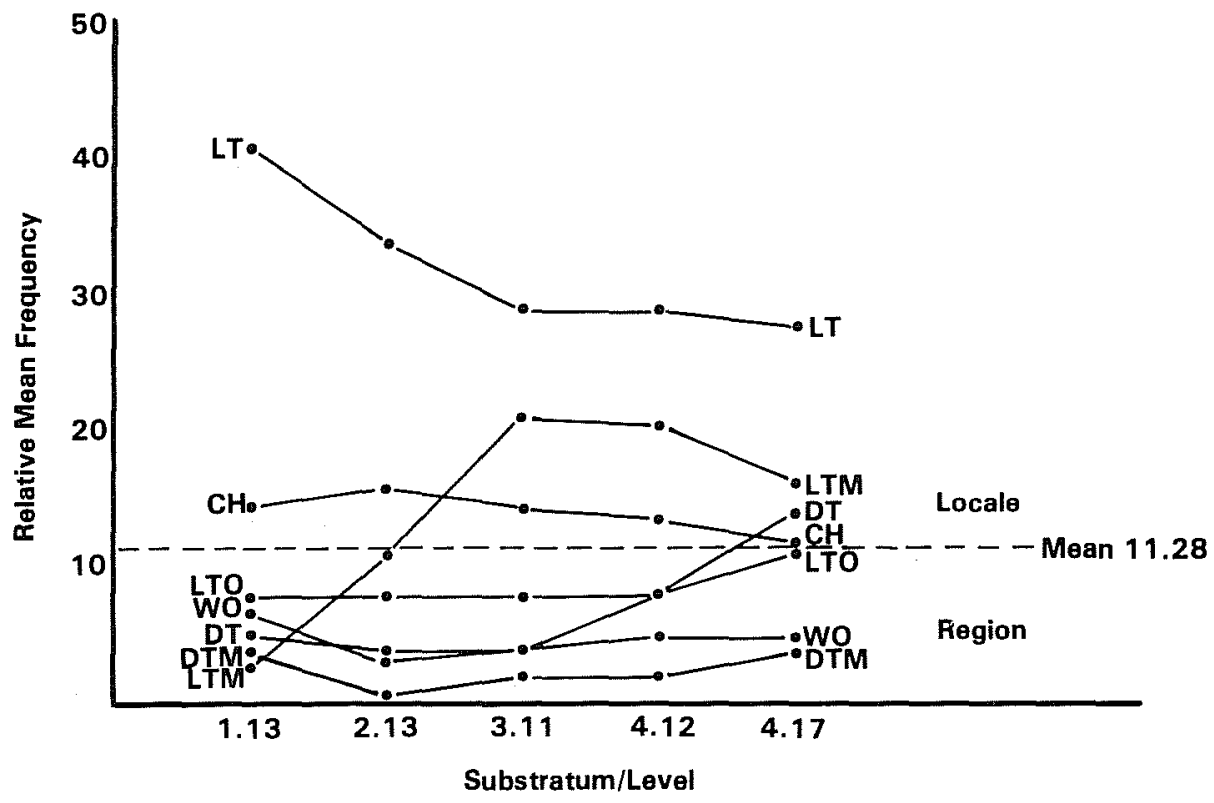

Figure 80. Relative Mean Frequency of Hypothesized Local Materials.

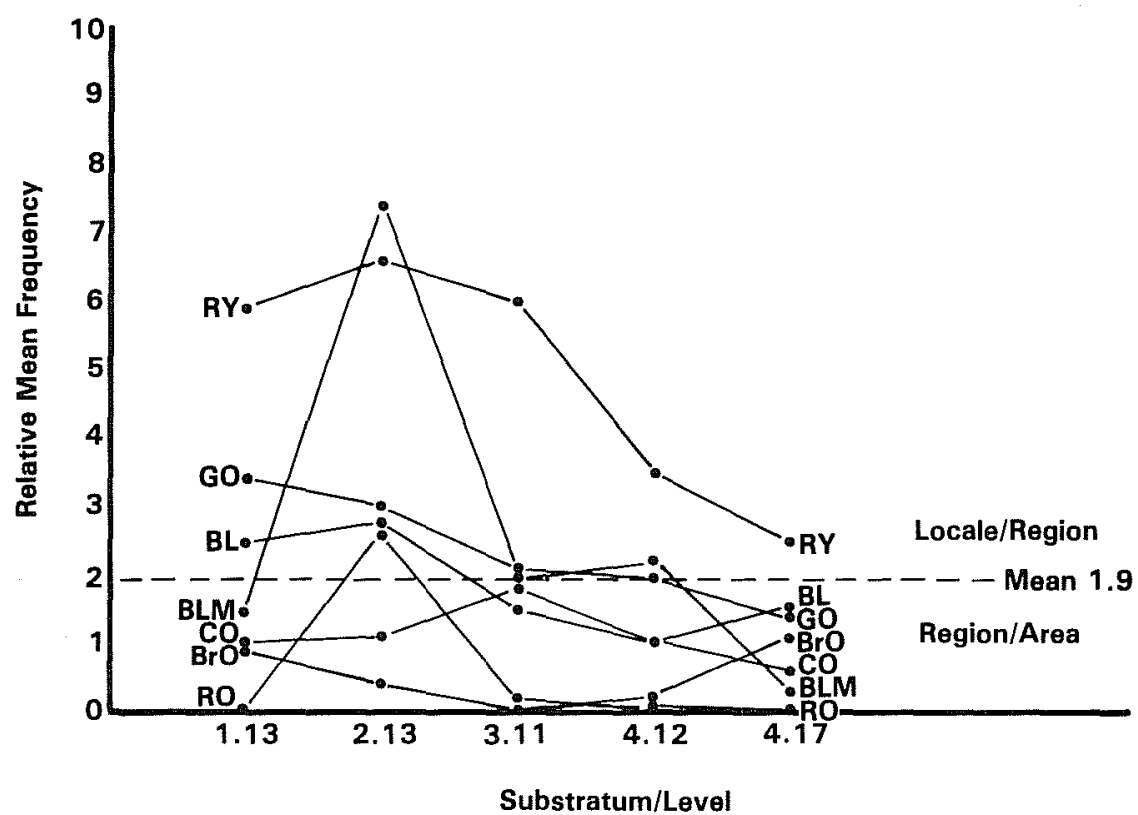

Figure 81. Relative Mean Frequency of Hypothesized Local Materials.

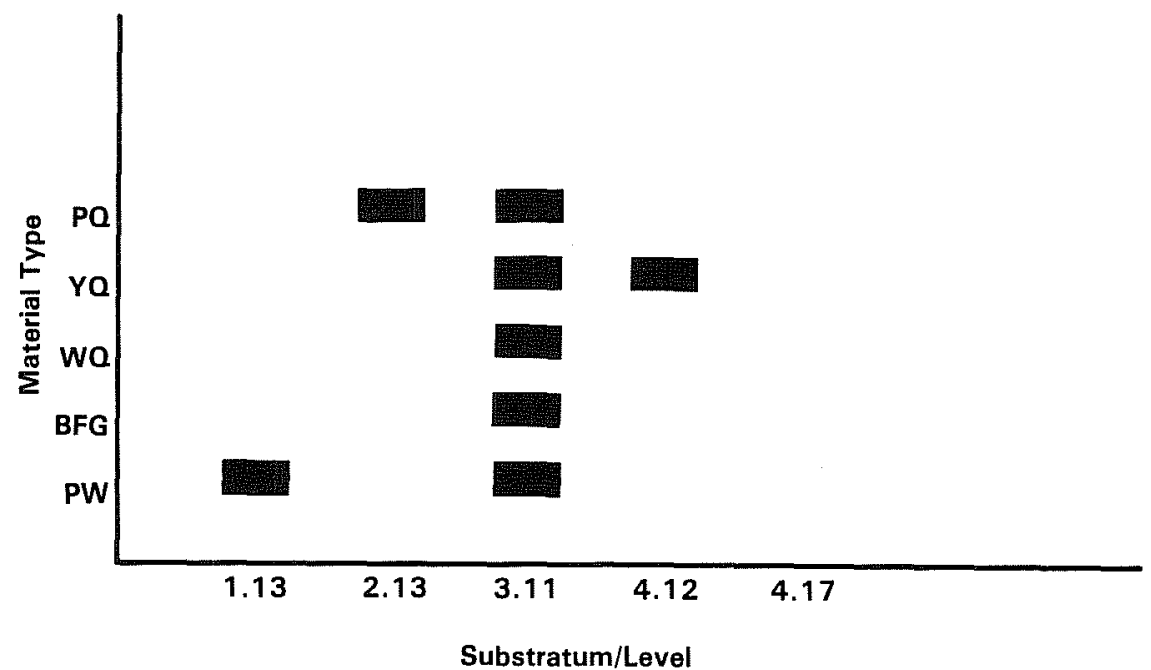

Figure 82. Presence and/ar Absence of Hypothesized Exotic Materials. 
b. 2.13
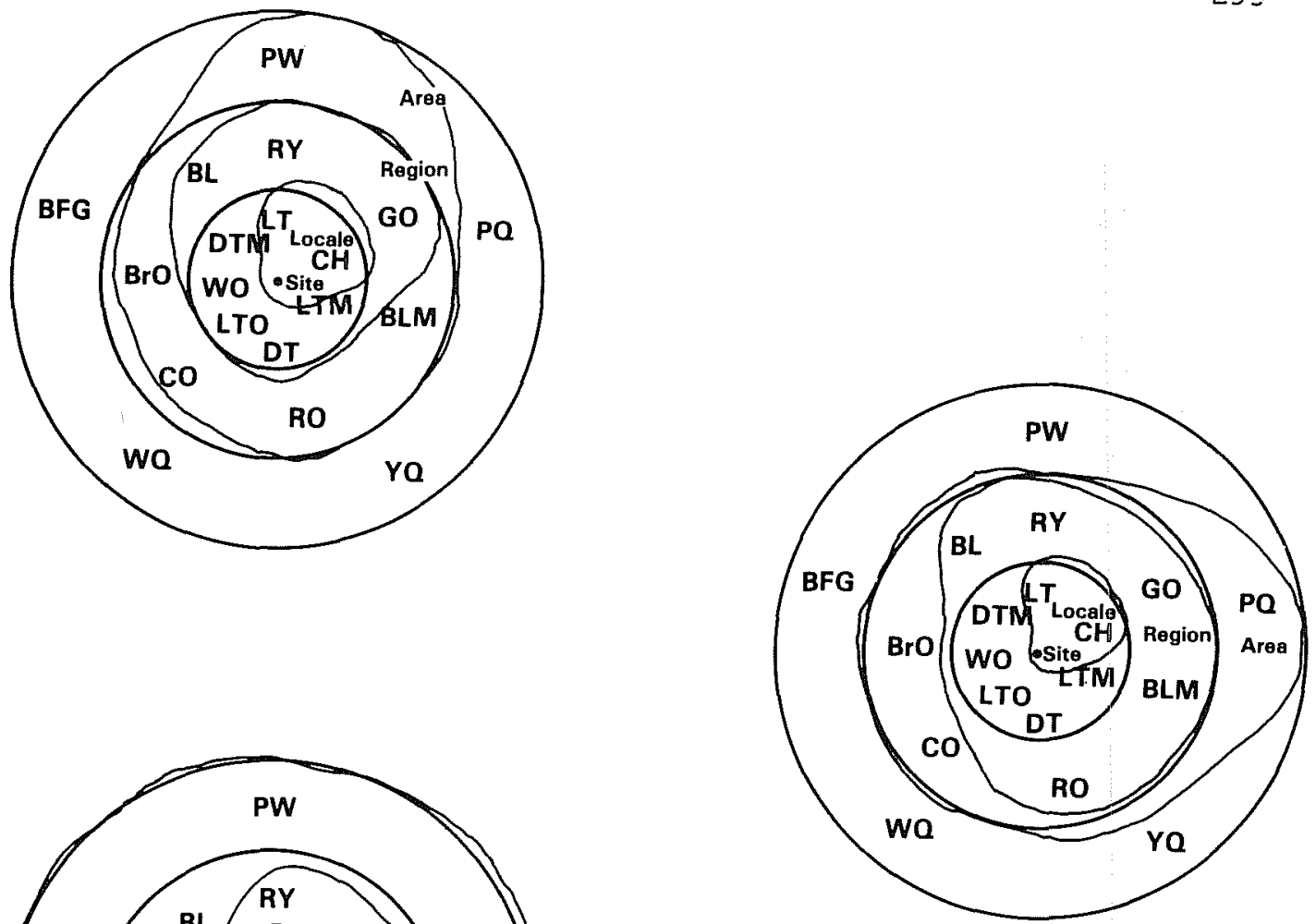

c. 3.11

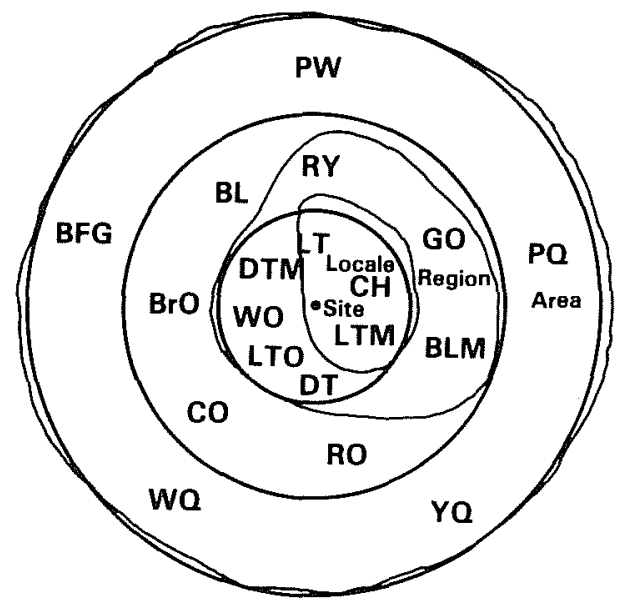

d. 4.12

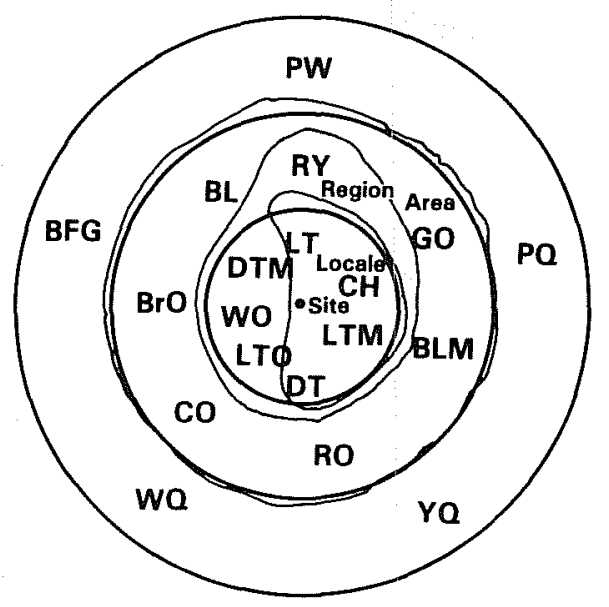

e. 4.17

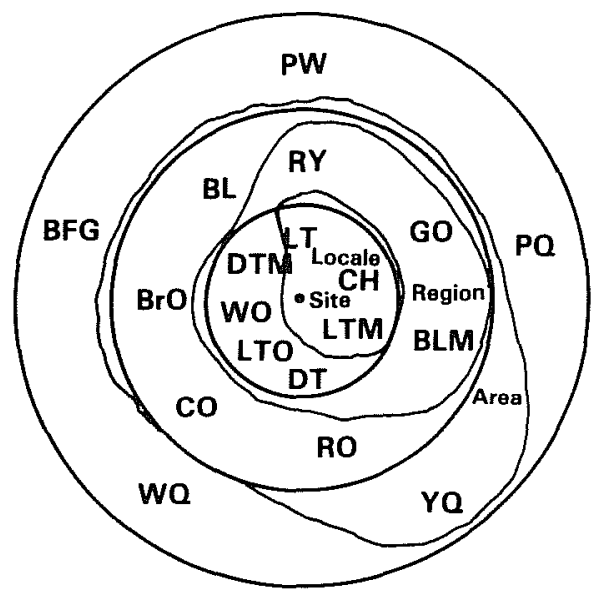

Figure 83. Lithic Material Distributions on the Occupation Planes at Eagle Hill. 
the previous evidence it is noted that the occupation on this level was very localized and that late Paleo-Indian patterns are often thought to be drawn on more localized material sources.

0ccupation Plane 3.11, in contrast, presents a tota17y different picture. The high predominance of exotics seems to indicate a broader based, more farranging subsistence pattern. If we posit this level as Archaic, the pattern is consistent with utilization of a variety of ecotones during drier periods. Evidence from pollen (Sheehan, page 161) seems to substantiate this as a relatively dry episode.

Occupation Planes 1.13 and 4.12 present essentially the same picture of a relatively balanced system. This similarity may be related to similar climatic episodes. Close examination of pollen may provide substantive evidence.

OP 2.11 shows a greater regional emphasis that may represent a transitional pattern from the far-ranging OP 3.11 to the balanced OP 1.13.

\section{Conclusions}

This analysis attempted to provide insights into the general nature of Eagle $\mathrm{Hill}$ and into the specific influence of the subsistence pattern on the lithic material assemblage. Although the constraints of time and money prevented a more exhaustive search for source materials, it is hoped that this theoretical approach has presented a perspective that is both practical and provocative.

TABLE 50. MATERIAL CATEGORIES BY SPATIAL MODEL

\begin{tabular}{|c|c|c|c|c|c|}
\hline $\begin{array}{c}\text { Substratum } \\
\text { Leve }]\end{array}$ & Local & Local/Region & Region & Region/Area & Area \\
\hline 1.13 & $\mathrm{LT}, \mathrm{CH}$ & $\mathrm{BL}, \mathrm{GO}, \mathrm{RY}$ & $\begin{array}{l}\text { LT0, W0, } \\
\text { DT, DTM, } \\
\text { LTM }\end{array}$ & $\mathrm{BLM}, \mathrm{CO}, \mathrm{BrO}$ & PW \\
\hline 2.13 & $\mathrm{LT}, \mathrm{CH}$ & $\begin{array}{l}\mathrm{BLM}, \mathrm{GL}, \mathrm{BL}, \\
\mathrm{RO}, \mathrm{RY}\end{array}$ & $\begin{array}{l}\text { LT0, w0, } \\
\text { DT, DTM, } \\
\text { LTM }\end{array}$ & $\mathrm{CO}, \mathrm{BrO}$ & $\mathrm{PQ}$ \\
\hline 3.11 & $\begin{array}{l}\text { LT, CH, } \\
\text { LTM }\end{array}$ & $\mathrm{RY}, \mathrm{CO}, \mathrm{BLM}$ & $\begin{array}{l}\text { LTO, WO, } \\
\text { DT, DTM }\end{array}$ & $\begin{array}{l}\mathrm{BL}, \mathrm{CO}, \mathrm{BrO}, \\
\mathrm{RO}\end{array}$ & $\begin{array}{l}P Q, Y Q, \\
W Q, B F G, \\
P W\end{array}$ \\
\hline 4.12 & $\begin{array}{l}\text { LT, CH, } \\
\text { LTM }\end{array}$ & $R Y, G O, B L M$ & $\begin{array}{l}\text { LTO, WO, } \\
\text { DT, DTM }\end{array}$ & $\begin{array}{l}\mathrm{BL}, \mathrm{CO}, \mathrm{BrO}, \\
\mathrm{RO}\end{array}$ & YQ \\
\hline
\end{tabular}

Note: See Table 46 for category definitions 
General Analysis of Occupation Plane Background Data

The analysis of background data proceeded in four steps. First, the variables appropriate for analysis were selected from the larger one-meter resolution data set. Second, the essential patterns of the selected data set were reduced by principal components. Component scores were calculated and plotted on maps of the occupation planes. Fourth, the scores were clustered, and the clusters interpreted in conjunction with the maps.

\section{Data Selection}

The general one-meter resolution data set consists of 106 observations on 134 squares from five occupation planes. We selected a set of variables which describe the cultural, sedimentological, and geochemical character of the entire occupation plane environment. The two major requirements of the data set are that it be as free of missing data as possible and that the variables be as nearly universally distributed as possible within the sample. Since rapid X-ray fluorescence tests were only made on 98 of the 134 units, this immediately limits the overall sample to 98 units. Nevertheless, the effects were not entirely undesirable since the loss of a few samples alleviated spatial autocorrelation to some degree. Some variables with blanket distributions, such as soil texture are not adversely affected by the sample-size reduction. Other variables which tend to occur in "hot spots," such as wear patterns on flakes are adversely affected.

of the 106 observations on units, some are not interval scales and therefore not useful to the analysis. These include such observations as the date of excavation, unit size (always one meter), etc. Some variables occur only rarely and so were analyzed qualitatively. The selected data set consisted of 41 variables. These 41 variables describe six aspects of the site milieu: location, sediments, XRF elements, cultural indicators, lithic material types, and wear analysis (Table 51).

Location--In this analysis, occupation plane serves as an indicator of time. The east and north coordinates were included to pick up general directional trends in the data. Although there were no east-west trends, there was a north-south trend related to sediment color and preservation of clay materials. The nature of this phenomenon is discussed in Factor IV.

Sediments--The color and texture of the soil were observed in the southwest corner of each square at the time the constant volume sample was taken. This continuous grid of observations, the weight of pebbles and granules, and limonite-hematite weight from units serve to describe the weathering and textural character of the sedimentary environment of the site. In this study, including these variables is not so much to describe the sediments per se, but rather to control for any effects of postdepositional soil genesis. As we shall see, the effects are significant and need to be considered as part of the cultural interpretation. For instance, the north end of the excavation appears to have been much more oxidized than the south, and the oxidation affected the preservation of clay artifacts. 
TABLE 51. VARIABLES USED IN THE BACKGROUND ANALYSIS

Location

SS Occupation Plane

EA East Coordinate

NO North Coordinate

Sediments

HUE Hue

VAL Value

CHRO Chroma

TX Texture

PBLW Pebble Weight

LMTW Limonite/Hematite Weight

Cultural Indicators

PFCT Platformed Flake Count

PFW Platformed Flake Weight

NPFW Nonplatformed Flake Weight

CWT Clay Ball Weight

SHWT Sherd Weight

C14WT Carbon Weight

RESW Resin Weight

Wear

SOFT Soft Wear

HARD Hard Wear

MNWD Mean Width
Elements

$\begin{array}{ll}\text { FE } & \text { Iron } \\ \text { AL } & \text { Aluminum } \\ \text { SI } & \text { Silicon } \\ \text { TI } & \text { Titanium } \\ \text { K } & \text { Potassium } \\ \text { CA } & \text { Calcium } \\ \text { MN } & \text { Manganese } \\ \text { RB } & \text { Rubidium } \\ \text { SR } & \text { Strontium } \\ \text { W } & \text { Wolfram }\end{array}$

Lithic Material Types

DT Dark Tan

DTM Dark Tan Mottled

LTN Light Tan

LTM Light Tan Mottled

LTO Light Tan Opaque

wo White Opaque

BO Brown Opaque

GO Gray Opaque

$\mathrm{CH}$ Chalcedony

BL Black

BLM Black Mottled

RY Red Yellow

Elements--More or less universal1y occurring elements determined by rapid X-ray fluorescence were included in the analysis. They describe the general geochemical composition of the sediments, translocation of elements downward through the soil zones and, hopefully, cultural concentration of elements. Correlated occurrences of elements presumably represent chemical compounds.

Cultural Indicators--Various indicators such as lithics, clay, and carbon materials were used as general indicators of human utilization of occupation planes. Resin was included as a separate variable in the belief that it might be more an indicator of the natural organic composition of the soil due to the burning of tree roots.

Lithic Material Types--The frequency of lithic types as observed on platformed flakes was analyzed in those cases where sample distributions were relatively universal. The types are discussed extensively in section III. 
Wear--The frequency of wear on lithics of soft and hard material was summed for each square. Also, the mean width of worn flakes per square was analyzed.

Principal Components Analysis

Christenson and Read (1977) demonstrated that the use of principal components analysis in tandem with cluster analysis has several useful effects. The principal components analysis simplifies the data and opens up relationships to interpretation. Cluster analysis clarifies the groupings of cases in the data set and allows direct reference to tangible evidence, at least in the archaeological data used in this study. In this section, a principal components analysis of the data described above will be interpreted. A cluster analysis of the factor scores generated from the principal components analysis will also be presented.

A principal components analysis was performed on the 98 case/41 variable data matrix using the SPSS factor program (type = PAI, Nie et al. 1975). Five factors were judged to be important by making several runs and observing the loading sizes in the unrotated factor matrix. The five factors were rotated to varimax criteria, and factor scores calculated using SPSS procedures. Table 52 shows the variables loading on the five factors in order of their loading strength.

\section{Interpretation}

Factor I: CULTURE--Most of the loading for Factor I pertains to the cultural materials. Lithics (both frequencies and materials), sherds, and carbon are positively correlated with Factor I. The two noncultural elements loading on Factor I are iron and limonite-hematite concretions. Iron is negatively correlated with the component, while the iron-oxides are positive. This suggests the interesting possibility that human occupation catalyzes iron into iron-oxide concretions.

It is also interesting to note that the sherd weights load on the cultural component, but not the clay balls. During the whole project there has been a persistent question as to whether the clay balls were of natural, quasi-natural (crayfish and human activity), or human origin. The association of clay balls with noncultural phenomena suggest that they may be, at least in part, of natural origin. Clay artifacts are also differentially preserved depending upon their location in the site (see Factor IV).

Factor II: ELEMENTS--Virtually all of the variance provided by the geochemical analysis loads on the same factor. In part, this indicates that the measurements of elemental composition of the sediments are related to each other as an artifact of measurement technique. This is not uncommon and requires that the elements be pretreated as a data set in order to make full use of them. Such an analysis is performed in the section on XRF (page 156). Iron, in contrast to the extreme coherence between other elements, appears on several factors. Iron is extremely active in the soil-human system, while the other elements may be only part of the soil-sediment background. Manganese also shows some tendency to break off from the other elements and relate, logically enough, to the color of the soil. 
TABLE 52. PRIMARY LOADINGS AND VARIABLES FOR PRINCIPAL COMPONENTS ANALYSIS OF EAGLE HILL BACKGROUND DATA

Factor I--CULTURE

.84 Platformed Flake Count

.82 Carbon Weight

.73 Chalcedony

.72 Resin

.72 Red Yellow

.66 Light Tan

.63 Limonite Weight

.55 Black

.48 Light Tan Mottled

.44 Light Tan Opaque

.38 Sherd Weight

-.38 Iron

.35 Black Mottled

Factor I I I--TIME

.81 0ccupation Plane

.74 Nonplatformed Flake Weight

.72 Platformed Flake Weight

-.72 Soil Texture

.69 Iron

.60 Light Tan Mottled

.56 Dark Tan

.39 Limonite Weight

. 38 Platformed Flake Count

. 37 Strontium

. 30 Chroma
Factor II--ELEMENTS

.96 Titanium

.95 Silicon

.94 Aluminum

.81 Calcium

.75 Rubidium

.74 Manganese

.69 Potassium

-.45 Iron

.43 Wol fram

Factor IV--CLAY

.85 Value

.83 Hue

.65 Chroma

-.47 Clay Ball Weight

-.46 Sherd Weight

-.45 North

.30 Manganese

Factor V--WEAR

.81 Hard Wear

.77 Soft Wear

.51 Mean Width

.41 Light Tan

. 40 Brown Opaque

. 39 Light Tan Opaque

. 32 Dark Tan

Factor III: TIME--0ccupation planes load on Factor III so it represents variables that age through time. Since the numbers assigned to the occupation planes are small at the top and become larger toward the bottom, positive association with the time factor indicates increasing frequency toward older sediments. For instance, platformed flake counts increase with time, or toward the bottom of the site. Several lithic material types are more frequent toward the bottom of the site. In other words, they were popular early and became less so later. There is also a general decrease in the total weight of flakes per unit with time.

Several of the sediment variables change with time as could reasonably be expected. Soil texture becomes fine toward the bottom, which is normal for an old soil. Finer particles leach toward the bottom leaving coarser material at the top. As was shown in the physical environments section (page 126), some of this may be due to large aeolian particles being deposited in the upper colluvium. 
Iron increases toward the bottom of the section. There is either downward migration of iron, or older sediments are a richer source of iron. Strontium also increases toward the lower sediments.

Finally, the chroma of the soil increases with time. The lower sediments are more saturated or brilliant in color. Mottling and general reddening of the lower strata is caused by longer exposure to oxidation, which turns soils red.

Factor IV: CLAY--The north-south coordinate of the site grid is associated with Factor IV. Since the relationship is negative, as are clay ball weights and sherd weights, clay balls and sherds are more frequent in the north of the excavation. Color acts in the opposite direction. The soils become redder and darker to the south, yellower and lighter to the north. The colors are more saturated to the south. Since the relationships are opposite what would be expected if human habitation were the causal agent in soil coloration, the controlling factor must be natural. Since both clay balls and sherds are associated, one apparently natural and the other unnatural, the overall effect must be differential preservation of clay artifacts. Since the soils are redder to the south, the implication is that oxidation encourages the dissolution of sherds.

Manganese loads on the clay factor. It is probably the coloring agent that darkens the value to the south.

Factor V: WEAR--Both hard and soft wear load on Factor V. The most popular types of chert used also load for wear. There is probably an element of bias since all of the cherts are opaque and of darker color. Dark, opaque cherts facilitate the observation of wear patterns.

Factor Scores

The factors discussed above show the trend and communalities resident in the one-meter resolution data of the site as a whole. Naturally, our interests turn now to how those trends appear on a unit by unit basis. Factor scoring is a means of reducing the original 41 variables to five new variables represented by the factors just discussed. Factor scores were calculated for the data using SPSS procedures (Nie et al. 1975). A FORTRAN program written for this project was then used to map the results of this scoring onto a visual display (Fig. 84). Each row of squares represents an occupation plane, and each square is a map of the Area A excavation. Dashes (-) indicate missing data; an XRF sample was not run. Each number between zero and nine represents a one-meter unit. Fours and fives represent $\frac{1}{2}$ standard deviation below and above the mean and so are nothing out of the ordinary. Zeros represent two or more standard deviations below the mean; nines indicate two or more standard deviations above the mean. Both are therefore extraordinarily low and high, respectively.

As an interpretive example, note that the last column of squares is the WEAR factor (Factor $V$ ). The bottom square shows the distribution of wear on Occupation Plane 4.17. The lower left unit of that square is marked by a nine indicating that an unusually large number of worn flakes were found in that square. It is also the unit in which the Paleo-Indian lanceolate was found. The association of a Paleo-Indian point with utilized flakes is a matter of 
EAGLE HILL BACKGROUND STUDY, COMPONENT SCORES OCCUPATION PLANE 1130.
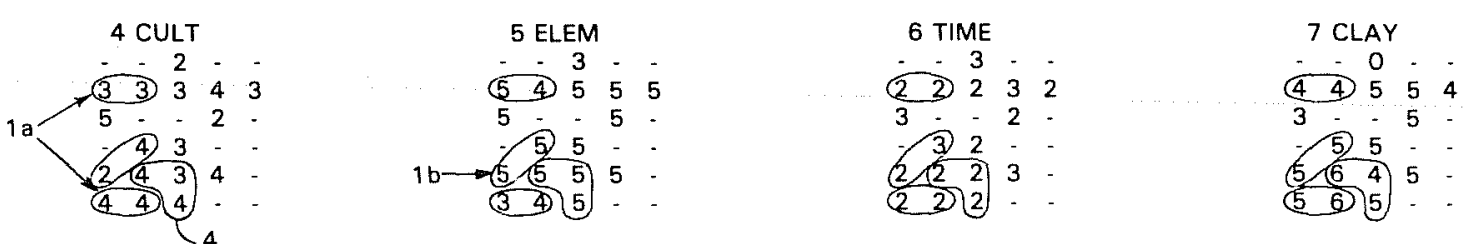

8 WEAR

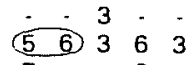
$7 \div 2$ $\begin{array}{rr}8 & 5 \\ 7 & 4 \\ (46) & 7\end{array}$

OCCUPATION PLANE 2130.
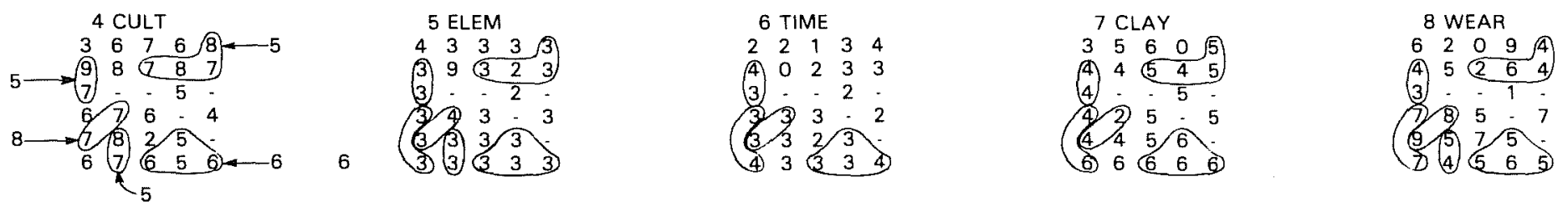

OCCUPATION PLANE 3110.
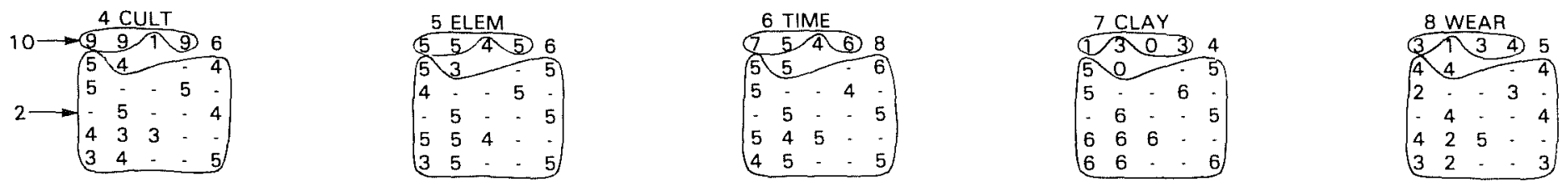

OCCUPATION PLANE 4120.
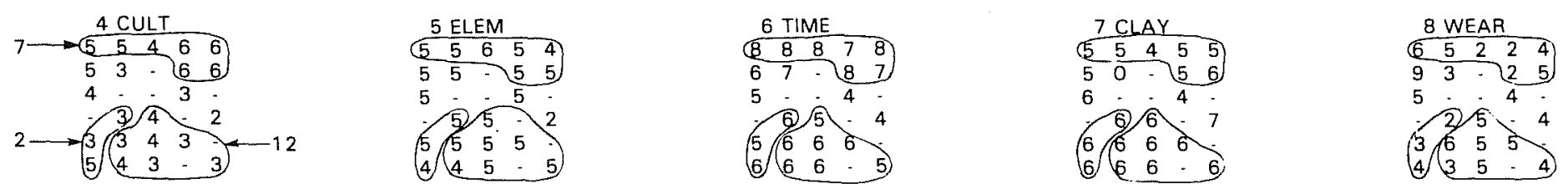

OCCUPATION PLANE 4170
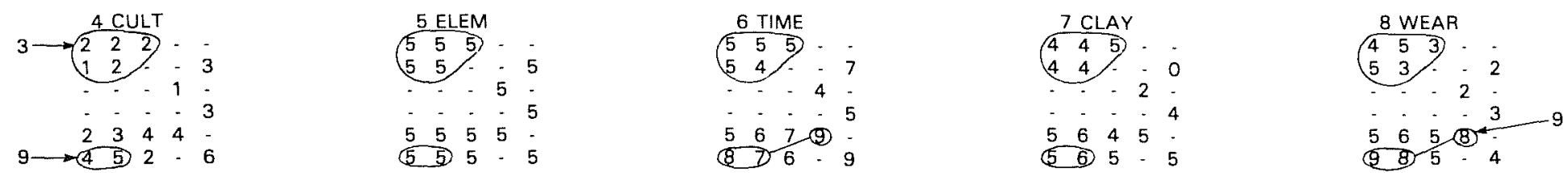

SYMBOLS $0<-2.1$ SD, $1=1.51$ TO -2.00 SD, $2=-1.50$ TO -1.01 SD, $3=-.51$ TO -1.00 SD; $4=-.01$ TO -.50 SD, $5=.0$ TO .5 SD, $6=.50$ TO $.99 \mathrm{SD}, 7=1.0$ TO $1.49 \mathrm{SD}, 8=1.50$ TO $1.99 \mathrm{SD}, 9=>2.00 \mathrm{SD}-=$ NO DATA

Figure 84. Factor Scores for Five Components on Five Occupation Planes. Each number represents a one-meter excavation unit. Numbers with arrows are clusters. 
some interest and suggests that perhaps a tool kit was left in that and the adjacent squares rather than just an isolated point. The work flakes may have been used in conjunction with the point or perhaps in a rehafting effort. Toward the opposite side of the excavation, the relative frequency of work lithics falls far below normal, as is marked by the twos.

Casual inspection of Figure 84 highlights several "hot spots," as related to the various factors. A full and systematic analysis of the distributions, however, requires that we turn to cluster analysis to make sense of a large number of values.

\section{Cluster Analysis}

To assist in the interpretation of patterns on the occupation floors, a cluster analys is (BMDP2M) was performed on the 98 sets of factor scores. Twelve clusters resulted along with the seven apparently deviant squares (marked with "D"s). Each cluster and subcluster were assigned a numerical and letter designation and plotted on the perspective map of the site (Fig. 85). The clusters are also outlined and marked on the factor score map (Fig. 84).

\section{OP 1.13}

Cluster 1a: Humic Zone--Clusters $1 \mathrm{a}$ and $1 \mathrm{~b}$ are located almost exclusively in $O P$ 1.13. Cluster $7 a$ consists of six scattered squares. Culturally they are low in the more general cultural indicators, but high in use and wear. Clearly the most characteristic aspect of Cluster $1 a$ is the fact that it rates very low on the TIME component. This implies that it is low in lithics, popular early in the long history of the site, low in unit flake weights, low in the downward migrating elements (iron and strontium), and low in iron-oxides.

Cluster 1b: Use Spot--Cluster 1b differs 1ittle from Cluster 1a, except it is very high in use-wear as compared to any location in the site. This use-wear "hot spot" is congruent with a similar feature in the occupation plane below, OP 2.13. It is difficult to determine if the feature is a pile of occupation debris on OP 2.13 or a pit dug from OP 1.13. No differentiation of the soil was observed in the field. The fact that Cluster 8 , the manifestation of the feature in $O P 2.13$, is isolated, while Cluster $1 \mathrm{~b}$ appears to be a part of both Cluster la and OP 1.13, suggests that it is a pit dug from the apparent Caddoan leve1. As we shall see, conflicting evidence appears on OP 2.13.

Cluster 4: Seating--Cluster 4 resembles Clusters $1 a$ and $1 b$, except that it lacks the wear "hot spots" observed in the other two clusters. Cluster 4 squares interdigitate between the units with wear and contain a normal amount of inorganic soil elements. In a11, it is a picture of seating, rather than a discard pile or fireplace.

Cluster 8: Debris--Cluster 8 is the cultural debris and wear "hot spot" in the upper soil horizon. It is located in OP 2.13 under the similar Cluster ib as in OP 1.13. It seems to be a part of a wider spread pattern of cultural accumulation and work tools in the southwest corner of OP 2.13. One must 


\section{EAGLE HILL (16SA50)}

Occupation Plane Analysis - 1m Study

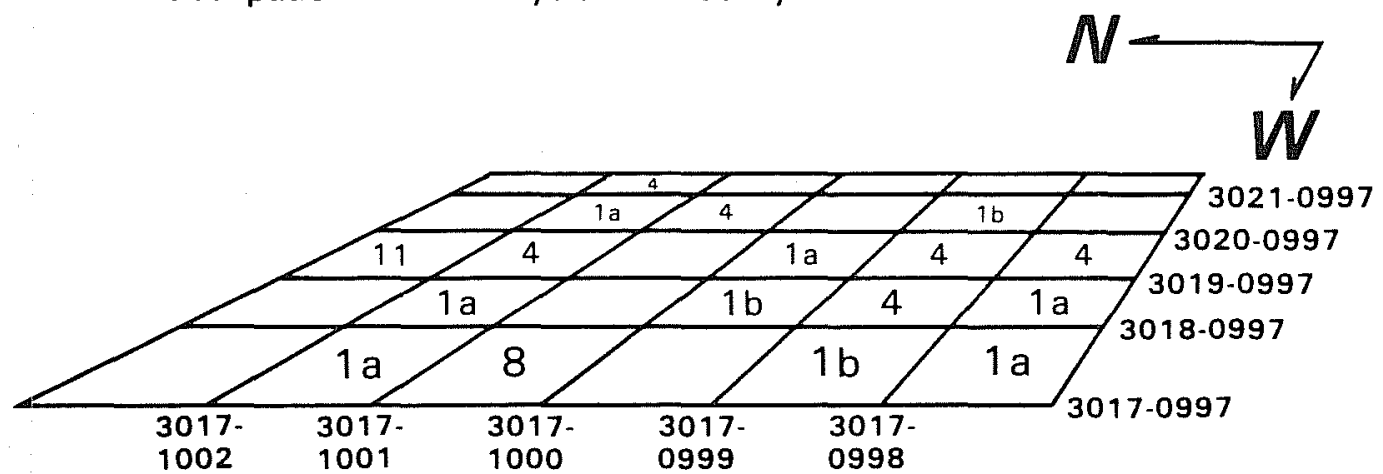

Occupation

Plane
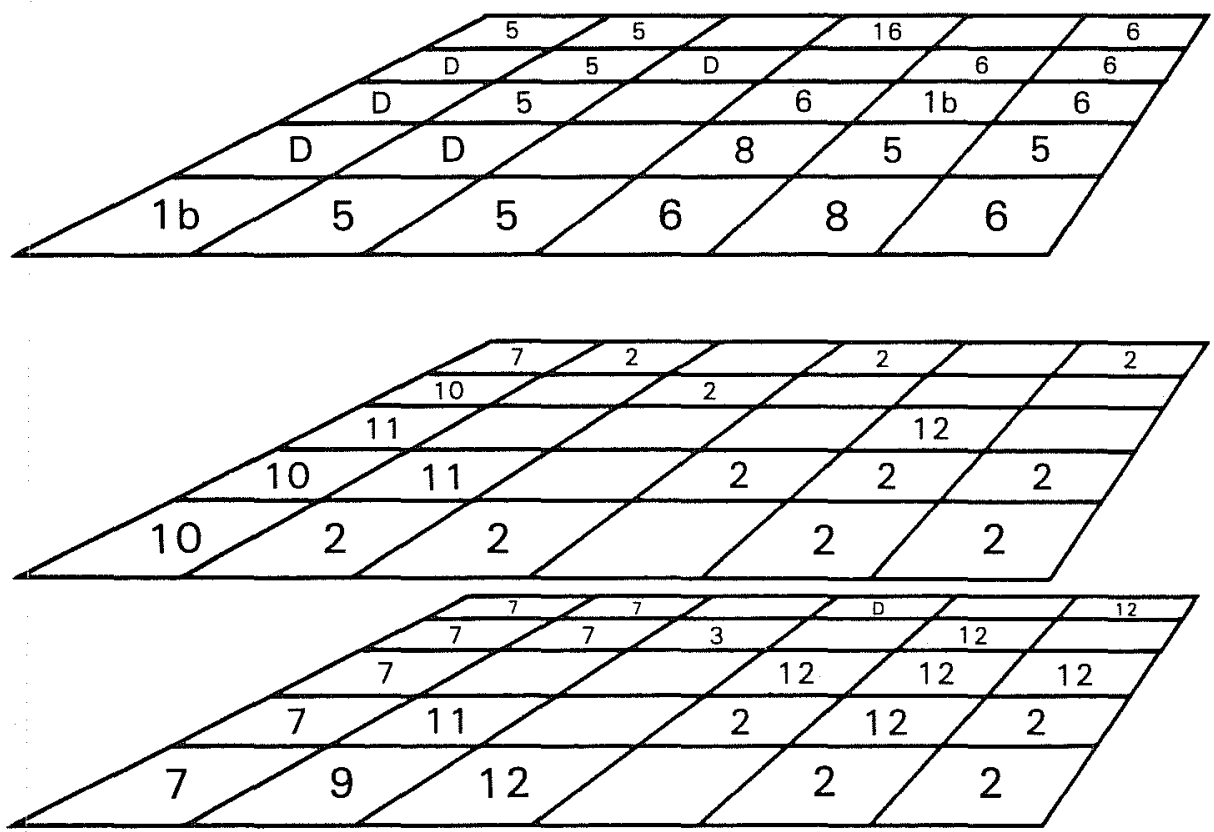

4.12

4.17

Figure 85. Clusters 1-12 and Deviant (D) units. 
assume that the material similarly discovered in OP 1.13 is the top of a pile that either protruded or mixed upward into OP 1.13. In addition to high cultural ratings, Cluster 8 is low in inorganic soil constituents. This is probably due to human activity turning the soil more organic. It is also low in clay items, a curiosity in a rich ceramic horizon. The overall picture is of a "trash dump."

Cluster 5: Clay--Cluster 5 is like Cluster 8 except that it contains clay objects. As will be seen in later analyses, carbon plays a major role. It is also above average in clay item content. Since the clay balls and carbon indicate probable fire activity, Cluster 5 is a likely prospect for hearths. This supposition is supported by low inorganic soil constituency on the ELEMENT factor.

Cluster 6: Wear--Cluster 6 resembles Clusters 8 and 5, except that the overall cultural indicators decrease, notably carbon. There is more wear and a notable increase in clay items. The indications are of a rather open work surface toward the southeast corner of the occupation plane.

OP 2.13

Aside from the three clusters just described, OP 2.13 is notable for all of the deviant squares except one. There are four contiguous deviant squares in the north central part of the floor, a 11 marked by extreme values of one type or another. One has the highest ELEMENT values and the lowest TIME values of any square in the site. Something peculiar was going on in the north side of OP 2.13, which deserves detailed qualitative analysis later.

\section{OP 3.11}

For the most part, OP 3.11 is comprised of two clusters. Cluster 2 can be projected over the southern five-sixths of the floor with only occasional exceptions. It is the largest contiguous area encompassed by any cluster. Cluster 10 follows the north edge of the excavation.

Cluster 2: CLAY--The inorganic composition of the soil increases, which reflects the position of OP 3.11 in Soil Horizon B. Cluster 2 at the south end is marked by a general low cultural item yield and an abundance of clay balls. Use-wear is generally low. TIME related variables climb past the mean for the first time indicating translocation of soil constituents and the first appearance of the "old" cultural ways, those important in the earlier period of the site.

Cluster 10: CULTURE--The northern tier of squares shows the beginnings of the translocated materials, which will culminate in the next level down. The northern edge of the unit is marked by a massive concentration of cultural materials very much in contrast to the southern sector. The focus of occupation appears to be to the north in OP 3.11 ; the excavation caught the southern edge of the main activity area. In spite of all the cultural activity, there is a marked absence of use-wear on lithics. 
The overall picture for OP 3.11 is that of the southern margin of an intensively utilized activity area.

OP 4.12

OP 4.12 is the first targeted floor in the lower colluvium (soil Zone II). It is perhaps most interesting sedimentologically for the massive concentration of translocatable elements, especially along the north edge. Apparently the influx of movable elements from the upper colluvium deposited in the last 1000 years is just arriving at the contact between the two soil horizons. Use-wear on lithics is more frequent than in the plane above, but the artifact yield is more varied and generaliy low. The floor seems to be structured in a manner similar to OP 3.11 with open work surfaces to the south and artifact concentrations to the north. The focus of activity appears to be more to the east.

Cluster 12: CLAY--Cluster 12 comprises the southeast quadrant of the OP 4.12 excavation and is not unlike the same area in the two levels above. Wear tapers off to the east and west, but is relatively prominent in the center. Clay balls are frequent, but other artifacts are rare. The TIME component affected factors of the soils, and cultural systems are now clearly in the "old" sector, well above mean values for old types of cherts, etc.

Cluster 2: CLAY--The units of the western tier resemble the units above enough to cluster with them. The main distinction from Cluster 12 is a drop in the frequency of use-wear.

Cluster 7: CULTURE--The units along the northern edge of the excavation have a higher artifact yield, less clay balls and some wear. They are most similar, however, in their very high relationship to the TIME factor. The units were particularly notable for large, edge-altered flakes constructed from the Dark Tan Mottled materials type. Dark Tan Mottled materials is an important contributor to the TIME component since it was popular earlier.

OP 4.17

Occupation Plane 4.17 is notable for concentrations of wear especially where important artifacts were found in the west margin of the excavated area. The topographic situation during this time period would have been quite different from the one imposed during the upper occupation levels. The site was probably a mound during most of its history. During the earliest occupation, however, the whole ridge appears to have been a flat to gently sloping surface. In other words, there would have been less incentive to concentrate camps on the site to avoid wet spots. It is not surprising, therefore, to find that the artifact yield is consistently low. If there is a locus of activity it is to the west. There may have been a bump in the recently eroded Pleistocene soil to the west of the excavation. This apparent erosional remnant includes the west one-meter tier of the site.

Cluster 9: PALEO-INDIAN POINT--There are two important clusters. Cluster 9 is in the southwest corner. It is very high in worn tools and is associated 
with the Paleo-Indian lanceolate. Cluster 9 and the south edge of the excavation seem to have caught the translocation of downward bound elements in the south side. For some reason, the IB/IIB interface was more permeable to the south.

Cluster 3: PALEO-INDIAN TOOLS--This area in the northwest corner of the excavation was associated with an end scraper on a blade and other formal tools. It is fairly high in WEAR, but very low in general artifact yield.

\section{Conclusions}

Perhaps as much can be told about changes in the topography of the site from the changing occupation patterns as from any other line of evidence. During the Paleo-Indian period, residence seems to have been encouraged by an erosional remnant hardly more than $10 \mathrm{~cm}$ high, a remnant of the well-developed B zone of the Pleistocene soil to the west of the excavation. After this, the locus of activity seems to have shifted to the north. Perhaps the topographic high, which brought people to the exact location of Eagle Hill again and again, was located slightly to the north. It has probably been eroded since it is on the uphill side of the site. The upper levels seem to show a southward shift toward the present location where the final occupation locus indicates that the top of the mound was virtually where it is now.

While the evidence for occupation activity areas outlined above cannot be considered conclusive in itself, it is the first in a series of contributions to an overall understanding of natural and human activity at the site. In the next section the artifact classes, which contributed to this analysis, will be examined in detail. 
V. FOREGROUND: ANALYSIS OF OCCUPATION PLANES AT ONE-CENTIMETER RESOLUTION

A. USE-WEAR ANALYSIS (Gibson, Gunn)

Introduction

Determining stone tool function is a complex process, which must account for characteristics of raw material, its modification, how the tool was used, and what it was used on. Lithic artifacts from Eagle Hill II (16 SA 50) belong to an industry that incorporates varying degrees of raw material modification and use ranging from minimal, such as use of flakes, to extensive, e.g., use of formal tools. Our lithic analysis methodology involved several approaches. We were able to distinguish between different raw materials, artifact forms, and functions by characterizing the raw material types, the attributes of artifact morphology, and the degree, kind, and distribution of edge damage. By this approach we sought to determine whether or not the artifact was utilized, the mode of use, and if it was a fragment of a tool. If it was a fragment, the tool form was inferred. The material(s) the tool was used on (wood, bone, hide, etc.,) was also inferred. Our objective was to identify the artifacts within each of these wear categories and their intrasite distributions. Resources were too limited to implement an experimental program in tool replication and use. Keeley (1980) has shown that such a program is essential to a full understanding of use-wear. Our results can only therefore be considered as a preliminary step toward a thorough elucidation of tool function at Eagle Hil1.

Determining function is a complicated process that must account for characteristics of raw material and modification of that material whether it be intentional or fortuitous. "Modification" is considered here to encompass alterations of the raw material that result from intentional technological efforts and the effects of use-wear. In addition to intentional modifications by humans, we must also consider possible alterations resulting from natural processes such as bioturbation. The identification of wear traces produced by specific materials or ranges of materials was the key to understanding the function of the lithic artifact assemblage. The selection of desirable raw materials for alteration into usable tools is the first step toward a functioning too1. As has been pointed out by other authors (Binford 1978; Goodyear 1982), selection of appropriate materials by prehistoric hunter-gatherers may have been an important logistical problem, particularly for highly mobile groups. Given the nature of the Eagle Hill occupation we have every reason to suspect high mobility. The selection of materials is thought to have been carried out at two levels. The first level is the selection of specific raw materials for the hunting tool kit (bifaces, projectile points, scrapers). In general these were hafted tools that were maintained through retouching, and/or reshaping. In a far-ranging nomadic subsistence-settlement system these raw materials were often not locally available. The second level of selection occurred locally. Lower quality raw material in proximity to sites were collected for nonspecific tasks and for immediate use and discarded.

The hypothesis we proposed to test was that the individuals who utilized the Eagle Hill site programatically selected certain types of rock from the local and regional environments in order to manufacture, maintain, and use them for 
specified kinds of tools. For the hafted tools, which were not manufactured and rarely discarded at the site, retouching, resharpening, and edge-damaged flakes (resulting from use) made of nonlocally available flint should be present. For the expedient, disposable tools made from locally available flint, the whole gamut of manufacturing, maintenance, and use flakes as well as the discarded tools themselves should be present on the site. The Null hypothes is is that there was a random distribution of stone tool forms manufactured from both nonlocally and locally available raw materials in the site area. The hypothesis was tested by statistically comparing the frequency and distribution of the material types, correlated with such 1ithic artifact categories as utilized flakes and flakes that were produced from manufacturing, maintenance, and use.

Given that the excavated portion of the site of Eagle Hill contained both (presumably) locally available and nonlocal flints (see lithic material section, page 180), and that there was a difference in the flake forms that resulted from different activities (e.g., manufacturing, maintenance, and use), it was necessary to construct an analytical methodology, which allowed us to distinguish between different raw materials and flake uses. The lithic raw materials were characterized as occurring in one of five categories, which are shown in Table 53.

Technological and wear attributes for each of the 1519 closely provenienced flakes and tools were observed and coded according to the attributes 1 isted in Table 53. Our intention was to determine through these attributes if certain technologies were used to produce flakes slated for use. The observations determine which edge of the specimen was used, its shape, the edge angle, the aspect of use, and the specific edge damage. Properly done this task requires a stereomicroscope, similar in magnification, program, and equipment to that suggested by Lawrence (1979). Since we lacked the equipment at the time (it has since been acquired) our analysis has to be thought of as a preliminary screening of the use-wear problem at Eagle Hi11. Table 54 shows the states of the attributes.

TABLE 53. VARIABLES OBSERVED ON EAGLE HILL LITHICS

1. Material
2. Color-Material
3. Opaqueness
4. Texture
5roduction Technology
5. Formal Tool Type
6. Technotype
7. Fragmentation
8. Platform Shape
9. Platform Exterior
10. Outline
11. Edge Thickness
12. Feathered Termination
13. Hinge Termination
14. Step Termination
15. Snap Termination
16. Asymmetrical Orientation
17. Axis of Force

Modification

18. Overlapping Flake Scars

19. Nibble Scars

20. Edge Damage

21. Crushed Edge

Wear

22. Probably Soft Use

23. Probably Hard Use

24. Polish

25. Aspects of Use

26. Initiation of Force

27. Wear Analys is Potential

Observation Mode

28. Microscope

29. Magnification 
TABLE 54. ATTRIBUTES OF VARIABLES IN TABLE 53

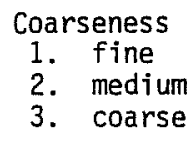

Color-Material

1. dark tan

2. light tan

3. light $\tan$ opaque

4. black

5. petrified wood

6. White chalcedony

7. red-yellow

8. miscellaneous

Opaqueness

1. translucent

2. edge-translucent

3. opaque

\section{Texture}

1. norma1

2. patina

3. weathered

4. heat sheen

5. potlidded

6. cortex

Formal Tool Type

0 . indeterminate

1. edge-altered flake

2. biface tool

3. biface fragment

4. uniface fragment

5. waste

6. maintenance flake

7. other-projectile pt.

Technotype
1. flake
2. blade
3. chunk
4. indeterminate
5. nonartifact

\section{Fragmentation}

1. whole

2. platform end

3. medial

4. terminal end

5. nearly whole

6. indeterminate

\section{Platform Shape}

0 . missing

1. flat

2. curved

3. triangular

4. irregular

Platform Exterior

0 . missing

1. untrimmed

2. slightly trimmed

3. moderately trimmed

4. heavily trimmed
Outline

1. contracting

2. parallel

3. expanding

4. irregular

Edge Thickness

1. thick

2. thin

3. extremely thin

Feather Termination

0 . absent

1. present

2. indeterminate

Hinge Termination

0 . absent

1. present

2. indeterminate

Step Termination

0 . absent

1. present

2. indeterminate

Snap Termination

0 . absent

1. present

2. indeterminate

Asymmetrical Orientation

0 . absent

1. present

2. indeterminate

Axis of Force

000. indeterminate

999. multiple

Overlapping Flake Scars

0 . absent

1. present

2. indeterminate

Nibble Scars

0 . absent

1. present

2. indeterminate

Edge Damage

0 . absent

1. present on terminal end

2. present on lateral edge

3. on platform

4. on lateral and terminal end

5. on lateral edge and platform

6. indeterminate

Crushed Edge

0 . absent

1. present

2. indeterminate
Probably soft use

0 . absent

1. scraping

2. cutting

3. boring

4. perforating

5. multiple use

6. indeterminate

Probably hard use

0 . absent

1. scraping

2. cutting

3. boring

4. perforating

5. multiple use

6. indeterminate

Polish

0 . absent

1. present

2. indeterminate

Aspects of Use

0 . absent

1. one side

2. two sides

3. three sides

4. four sides

5. all sides

6. indeterminate

Initiation of Force

0 . absent

1. point

2. bending

3. indeterminate

Wear Analysis Potential

0 . none

1. good

2. very good

3 . excellent

Microscope

1. stereomi croscope

2. ILM

3. SEM

\section{Magnification}

1. $10 \mathrm{X}$

$215 \mathrm{X}$

3. $20 x$

4. $25 X$

5. $30 \mathrm{X}$

6. $35 \mathrm{X}$

7. $50 \mathrm{X}$

8. $100 \mathrm{X}$

9. $200 \mathrm{X}$

10. $400 \mathrm{X}$ 
Approaches to Use-Wear Analysis

Regardless of which method of use-wear analysis one chooses, al1 of the methods involve judgement, interpretation of artifacts, and familiarity with the uses of stone tools. The analyst must first determine what level of accuracy is needed for functional interpretations. High magnification microwear analysis provides very precise information. Of course if one has specimens from a site like Hinds Cave where macro-organic residue is preserved on tool edges, the methods and techniques of Shafer and Holloway (1977:103-106) are much more precise. However, for the other $99 \%$ of archaeological sites, where such preservation does not exist, high magnification microwear analysis provides the most precise information.

In the past ten years there have been two major approaches to use-wear analys is that link method with technique and equipment with procedure. Edge damage has been emphasized in some lithic studies. Edge damage may be considered as an attribute (or attributes) of an artifact wherein flakes have been removed from stone tools through use and other processes. The technique of analyzing edge damage has been to use low magnification with a stereomicroscope. Ruth Tringham et al. (1974), George Ode11 (1975), and Lawrence (1979) have conducted some of the best edge damage studies. Researchers concentrated on edge damage with a stereomicroscope, because one observes a basically 3-D image. The depth of field is high relative to the specimen size. The problem with stereomicroscopes is magnifications above 100X are necessary for identifying specific microwear polishes (i.e., plants, hide, bone, wood). It is difficult to obtain a sufficient depth of field above $80 \mathrm{X}$ on a stereomicroscope. Microwear polishes can be detected with stereomicroscopes, but they cannot be confidently identified (see Keeley 1980).

In our analyses of the Eagle Hill material we were 1 imited to edge damage analysis at low magnification with a stereomicroscope and, therefore, could only determine if the tool had been used on a hard or soft substance as opposed to identifying hard substances such as wood or hide. Using an incident light, high magnification microscope could have solved this problem, but none was available.

Following the program and attribute definitions described by Lawrence (1979), which are presented above, analysis proceeded in the following manner. We initially replicated Lawrence's experiments in use-wear, although we did not use the Instrom Machine; we found his results to be nearly identical with ours, and decided to adhere to his attribute terminology. The archaeological specimens were examined under low magnification with the stereomicroscope then the presence or absence of the edge and use-wear attributes was recorded (see Lawrence 1979:119). After recording these attributes, we examined them as a group or constellation of resultant characteristics and in most cases were able to identify the specific mode of use and whether or not it was used on a hard or soft material. Sometimes we were able to infer that a specimen was used, but its specific mode of use was ambiguous, because of a lack of certain attributes. These specimens were recorded as indeterminate in the relevant attribute categories.

Throughout our use-wear studies the analyst had information only on the vertical position of the artifacts. The horizontal placement of the specimens was 
unknown to avoid inadvertent biasing of the results. Examples and explanations regarding the meaning of hard and soft use-wear will be discussed in the following section.

\section{B. INTERPRETATION OF OCCUPATION PLANE PATTERNS (Gunn)}

\section{Introduction}

The analysis of relationships between artifacts and features recovered from excavated occupation floors is not new to North American archaeology, although the art is hardly more than a decade old. Examples in the Mid South and adjacent regions include Goodyear's (1974) examination of the Brand site remains from eastern Arkansas, Gunn and Mahula (1977) at the Hop Hill site, and Brose and Scarry (1976) at the Boston Ledges shelter. The European literature contains several exemplary efforts such as those of Leroi-Gourhan (1966) at Pincevent and of de Lumley (1969) at Lazaret Cave and, more recently, Cahen, Kelley, and Van Note (1979). There is also a body of writing on the problem of statistical analysis of horizontal distributions (Whallon 1973, 1974; Gunn and White 1977; Hanson and Goodyear 1976). Virtually all of these analyses were predicated on the recognition of formal tool types such as points and scrapers, features such as fire hearths and huts, and associated materials such as animal claws, mollusks, etc. There are two basic types of information derived from occupation floor analyses. One is the re-creation of camp habits, a sort of momentary snapshot from ancient man's life. The other is the locational association of tools, an attempt to interpret functional association of formal tools. When properly interpreted, both types of data provide substance from which social and cultural inferences of past 1 ifeways can be made.

The analysis of the Eagle $\mathrm{Hill}$ assemblage was particularly challenging for a number of reasons. In the first instance, there were so few formal tools that little pertaining to behavior could be derived from them except as regards their manufacture. Small frequencies thwarted statistical analysis. Also, there was an absence of soil stains and alteration of soil color, which usually mark fire hearths. An examination of the distributions of charcoal fragments and smal1 clay balls thought to be the result of building fires on crayfish castles may suggest the general locations of prehistoric fires. As we shall see, a logical examination of these surrogate features seems to bear out the inference of fires in at least some locations.

To produce an analysis of chert implements, which would suggest functional work areas on the floors, was a more involved process. While there were few formal tools, there was certainly no lack of chipped stone in the form of flakes. From the five occupation planes selected for intensive study, 5535 lithic items were recovered. These included 1519 pieces that were provenienced to the centimeter, and 4016 items that were provenienced to the meter. Given the proliferation of flakes, most of which were not primary reduction specimens, it seemed likely that, although we found few tools, the inhabitants of Eagle Hill brought tools to the site and reshaped and resharpened them there, always being careful to take them away rather than leave them. Workable cryptocrystalline rocks are unevenly distributed on the coastal plain and such a practice is more expectable than surprising. It, therefore, seemed reasonable that any evidence of 
the function of stone tools and of the site would have to be found among the flakes. To this end, the 1519 flakes, which were provenienced to within one centimeter on occupation planes, were examined for evidence of use and wear (see section IV).

The equipment available at the time of the study only allowed us to examine the flakes under low power with a binocular microscope, usually about 30X. Keeley (1980) found that detailed examination of stone tools under much higher magnification coupled with replicative experimentation is necessary to define the exact material on which toois were used. Gibson was, therefore, only able to identify use in a general sort of way, whether lithics were used on hard materials such as wood and bone or soft materials such as hide, meat, and softer plants. From Occupation Planes $1.13,2.13,3.11,4.12$, and $4.16,270$ flakes were found to bear evidence of alteration from use.

\section{Occupation Floor Logic}

Every archaeologist has had the experience of optimistically laboring over a map for hours plotting points and finally facing a bewildering array of " $x$ 's" and "o's" that resist any reasonable interpretation. As was explained in detail (page 14), Eagle Hill was dug in a way that we thought would give us a reasonable chance of recovering the remains of relatively brief intervals of human occupation. In assuring brief intervals, we thought we might capture some of those fleeting insights into personal $1 \mathrm{ife}$, which ancient persons occasionally leave within the arrangements of fire hearths and stone tools. Unfortunately, however, even the most carefully excavated occupation floor is not likely to leave a completely unambiguous record of events. As with virtually all data, interpretation requires a set of logical principles, which can be used to decipher associated arrangements of artifacts.

The logic of our interpretation of occupation floors can be stated briefly. We are interested in activities resulting from repeated, culturally defined events or episodes of activity. A single flake used to slice plant fibers and dropped on an occupation floor is a culturally defined event, marked by the discarded flake. However, it is of little use to us, because we have no particular means of logically assuring ourselves that the flake and its evidence are a part of a systematic set of acts.

On the other hand, several flakes used to scrape a shaft and then discarded within a few centimeters of each other represent an activity episode. The same wear pattern appearing on several flakes close together, in contrast to other similar associations with different wear, gives us some confidence that we are dealing with a coherent episode. This confidence is bolstered if that episode is in logical relationship to other evidences of camp activity. For instance, it would be logical for the flakes to be beside a fire hearth rather than in it. If illogical associations occur (such as flakes in fireplaces), then we must assume that we are dealing with more than one camp located in the same position and be duly wary of overinterpretation.

Beyond the episode is the more usual unit of analysis, the concentration. Concentrations are sets of episodes usually in a logical relationship to a fire 
feature. They are also found isolated within and without fire features. Definition of a concentration depends upon spatial discreteness. Definition of an episode, on the other hand, depends on functional discreteness. Together episodes and concentrations comprise a relatively powerful logic for defining spatio-functional activity sets.

A final element of logical inference relates to the location of fireplaces. Apparently, the stains and structure common to fireplaces has leached away at Eagle Hill. Most such stains and colors relate to carbon and iron-oxides in the soll. We have seen that both are mobile in the Eagle Hill sediments (see section IVB). However, we were able to locate isolated bits of charcoal. Those over one centimeter in their longest dimension are plotted on Figures 86-90. We also found burned pieces of flint, both potlidded and firecrazed, and we assume they are indicative of fire.

Finally, the assumption is made that the clay balls, which appear on every floor, were a product of humans building fires on the remains of crayfish castles. This process hardened the clay thus resisting severe leaching. Also, some of the clay is hardened and red on one side and black on the other. There may well have been clay-lined fire pits on occasion at Eagle Hill. It is highly likely that natural fires swept through the Eagle Hill site. Whether they would have provided the more than $700^{\circ} \mathrm{F}$ necessary to harden clay is a matter that requires study. For now we will assume not.

\section{Statistical Analysis}

In addition to the logic of spatial relationships, there is a battery of statistical-numerical analysis techniques, which can assist in the characterization of occupation floor patterns. The method used in this analysis centers on the concept of "adjacencies," which is calculated in a manner similar to "nearest neighbor" analysis. That is, the Euclidean distance between each pair of artifacts is determined from coordinates observed in the field to one-centimeter resolution. Distance between items, however, is managed according to a cross-cultural theory of work space (Gunn and White 1977).

The population of tool types is segregated into two cells, one within and one without a criterion distance. The criterion distance is arbitrarily set based on an idea of how far apart tools are likely to be discarded if they are utilized at the same task. The criterion value for this study was set at $1.6 \mathrm{~m}$. Natural1y, there are problems with any criterion distance, and whatever is used must be justified in terms of purpose. Consider for instance, two different tasks: rehafting a foreshaft with a broken point and dressing a deerskin. If the individual who is doing the rehafting is sitting and does not move during the operation, most of the tools used and discarded should be found one meter or so apart. On the other hand, tools used in dressing a large deerskin would be discarded at the periphery of the stretched skin. Some of them will be more than a meter apart. If adjacencies are determined on the one meter criterion, all of the tools associated with the rehafting episode will be correctly determined to be adjacent and, therefore, utilized in the same tasks. Adjacencies for the skinning episode will be somewhat deflated by the fact that the task area was larger than the criterion value. 
For this study, the distance was set at $1.6 \mathrm{~m}$ because it seemed 1ikely that most of the activities would be confined to tasks involving sma11, individualsized spaces. This distance is approximately the range of a comfortable arm's reach in either direction. It is probably better to set the criterion too small and miss a few real adjacencies than to set it too large and falsely inflate the adjacency index.

Alternative settings of the criterion follow alternative research objectives and assumptions. For instance, a research project might be directed toward the behavior of family-sized units. Yellen's (1977) study suggests that a criterion distance of five to six meters would be sufficient to discover such associations. The criterion distance could be similarly adjusted for village-sized units, etc. The cross-cultural and ethnographic background studies for the adjacency technique are described in Gunn and White (1977).

An adjacency analysis was calculated for each occupation plane (Tables 55-59). The tables contain an adjacency transition matrix. Each row of the matrix contains an "A" and a "B" vector for each tool-wear type. A row is calculated for all types in the analysis. The "A" vector contains all of the adjacencies between types which fall within the criterion distance. The "B" vector holds the frequency of items which fall outside that distance. At the bottom of each table, an adjacency probability matrix is provided. The adjacency probabilities are calculated as: $P=A /(A+B)(1-[1 / A])(1-[1 / B])$. Multiplication by the reciprocals weights "p" in favor of larger sample sizes. If one cell is empty, the cell in which adjacencies occur is simply weighted for sample size. This is the second stage in developing the adjacency technique. The first was presented in Gunn and White (1977).

The advantage of the adjacency concept is the ability to externally and internally examine concentrations of tool types. Hanson and Goodyear (1976) developed a technique for determining tool associations. Concentrations of tools, which can be spatially defined, are associated with the same task, the "shared tool" technique. However, it is probable that spatially defined concentrations are likely to contain the product of more than one task. Of course, one is committed to determine the criterion distance. This is not wholly disadvantageous since it forces an explicit theoretical position to be defined to justify the criterion.

There are certain interpretive aids to understanding the adjacency probability matrix. The diagonal probabilities are the adjacencies between tool types and other tools of the same type. The off-diagonal statistics are between differing tool types. If the adjacencies on the diagonal are high, it suggests that the assemblage is homogeneous, a large number of the same tools being used in the same places.

Homogeneity of tool adjacencies is caused by one of two conditions. Lengthy tasks are being performed that consume large numbers of tools. Alternatively, it may be that generalized tools are being used for many different tasks, which explains the homogeneity. Which condition is effective in a given instance can be determined by the frequency structure of the assemblage, i.e., frequency of tools compared with number of tool types. A table will be given with each occupation plane with this information as well. 
TABLE 55. EAGLE HILL TOOL-WEAR PATTERN DISTRIBUTIONS

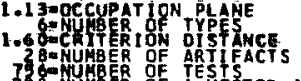

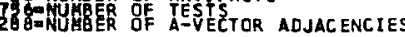

ADJACENCY MATRIXIVECTORS ARE MARKOV TRANSITIONAL \& REFLEXIVE

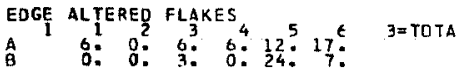

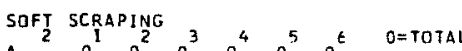

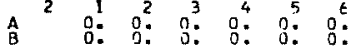

SOFT CUTTING 3 , 5 E $3=$ TOTAL

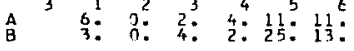

HARD SCRAPING

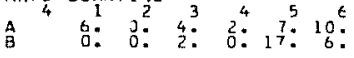

HARD CIITTING ${ }_{5}^{3}{ }^{4}, 5$ E $1 z=$ TOTAL

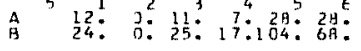

HARD CUTTINC E STPAPINO

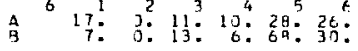

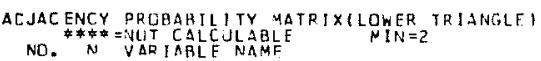

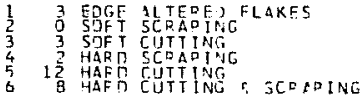

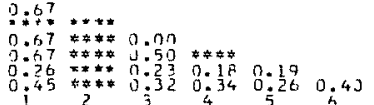

EABLE 58. EAGLE HILL TOOL-WEAR PATTERN DISTRIBUTIONS

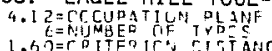

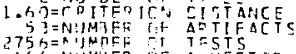

G4 4 ENUWHER IF A-VECTCR ACJACTACTLS

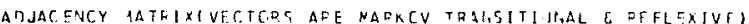

EOGE ALTEDOR FLAKF

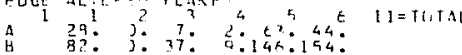

SGAT SCFAMIN

SOF CUTT 1 M.:

4 T. $2^{2} 3^{7}: 10^{5} 0^{6}$

HAFO STEADING

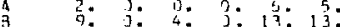

HAPD CUTT IAT,

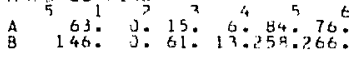

HAPO CUTT ING $E$ STFAOIN:

A $154:$ ?

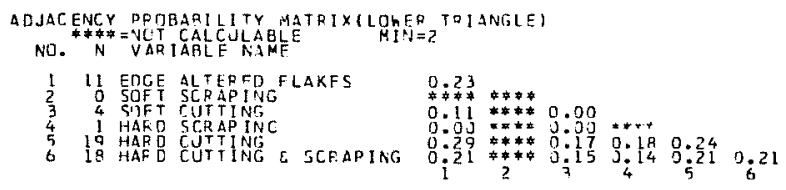

TABLE 57 EAGLE HILL TOOL-WEAR PATTERN DISTRIBUTIONS

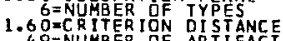

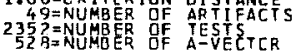

ACJACENCY YATRIXIVECTORS ARE HARKCY TRANSITIONAL C REFLEXIVEI

TÁBLE 59. EAGLE HILL TOOL-WEAR PATTERN DISTRIBUTIONS

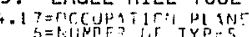

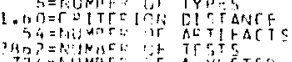

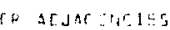

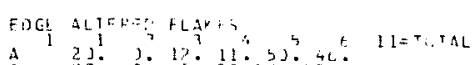

SOFT SAR!

i3 $3:$ i: $\because:$ i: $\quad 3$ ก

Seftertily

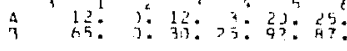

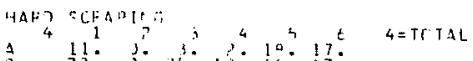

MAFI CUTT ITS

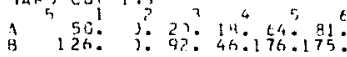

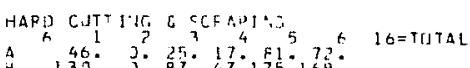

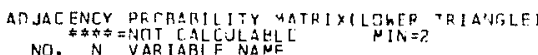

$\frac{1}{2} \quad 11$ EDGE ALTFDEDFLTKES

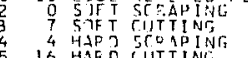

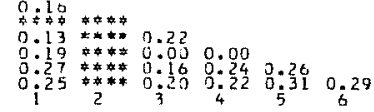

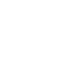

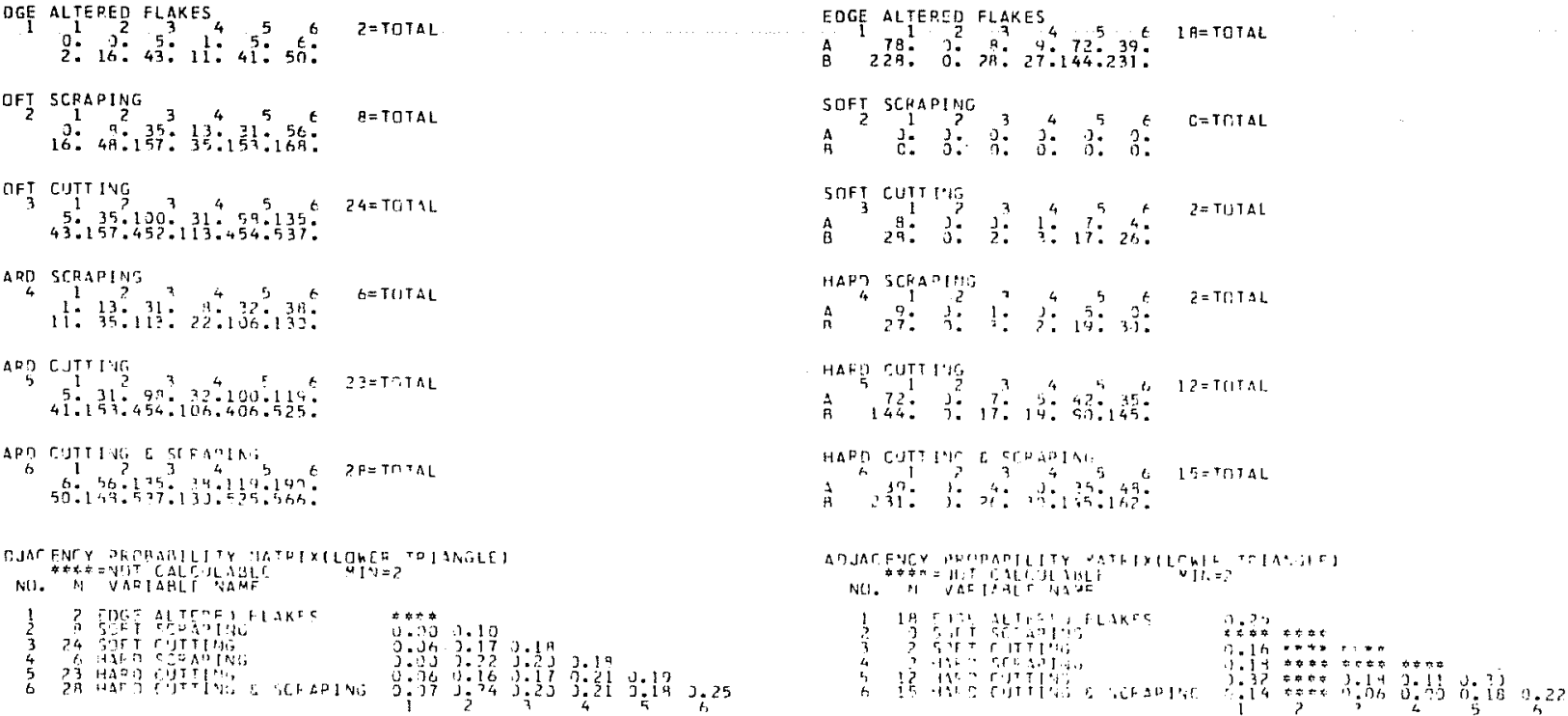

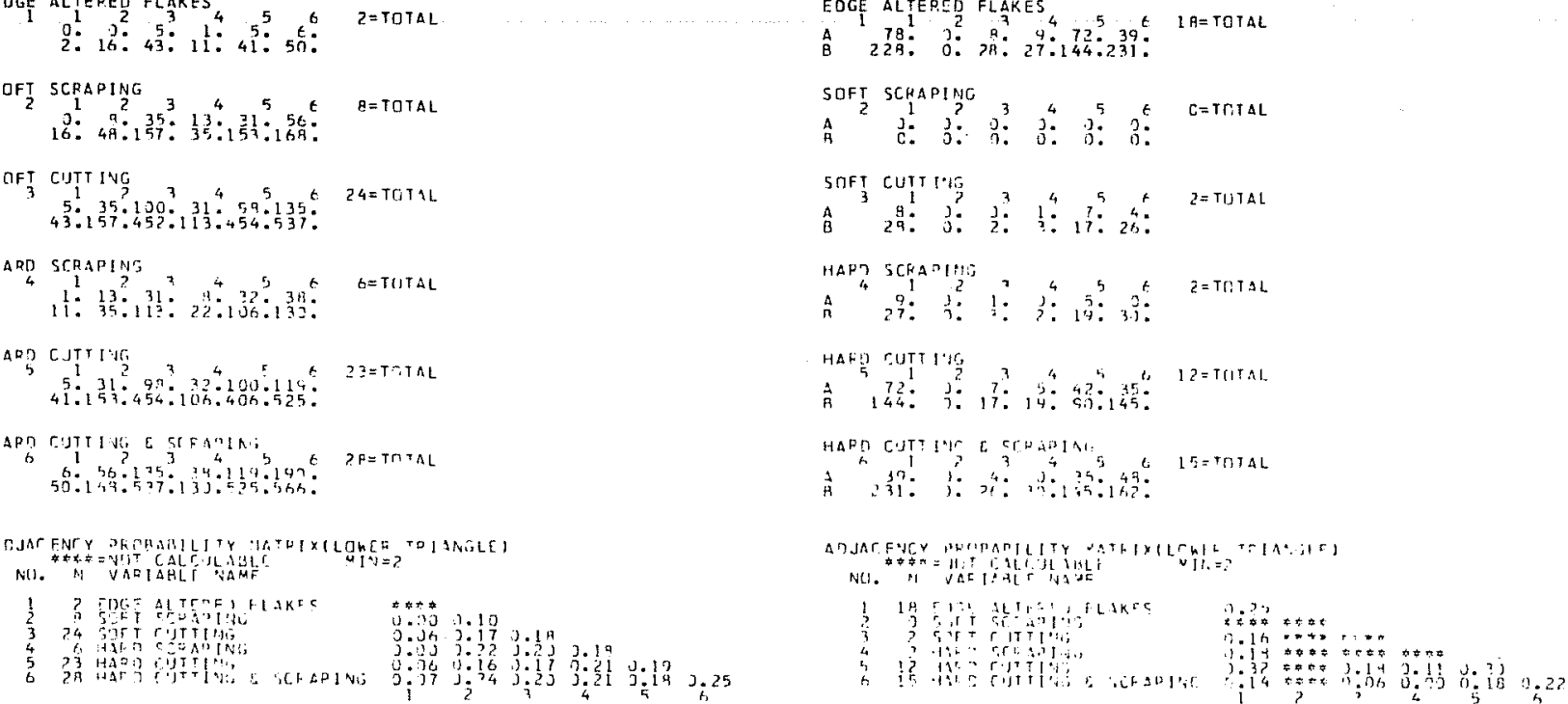

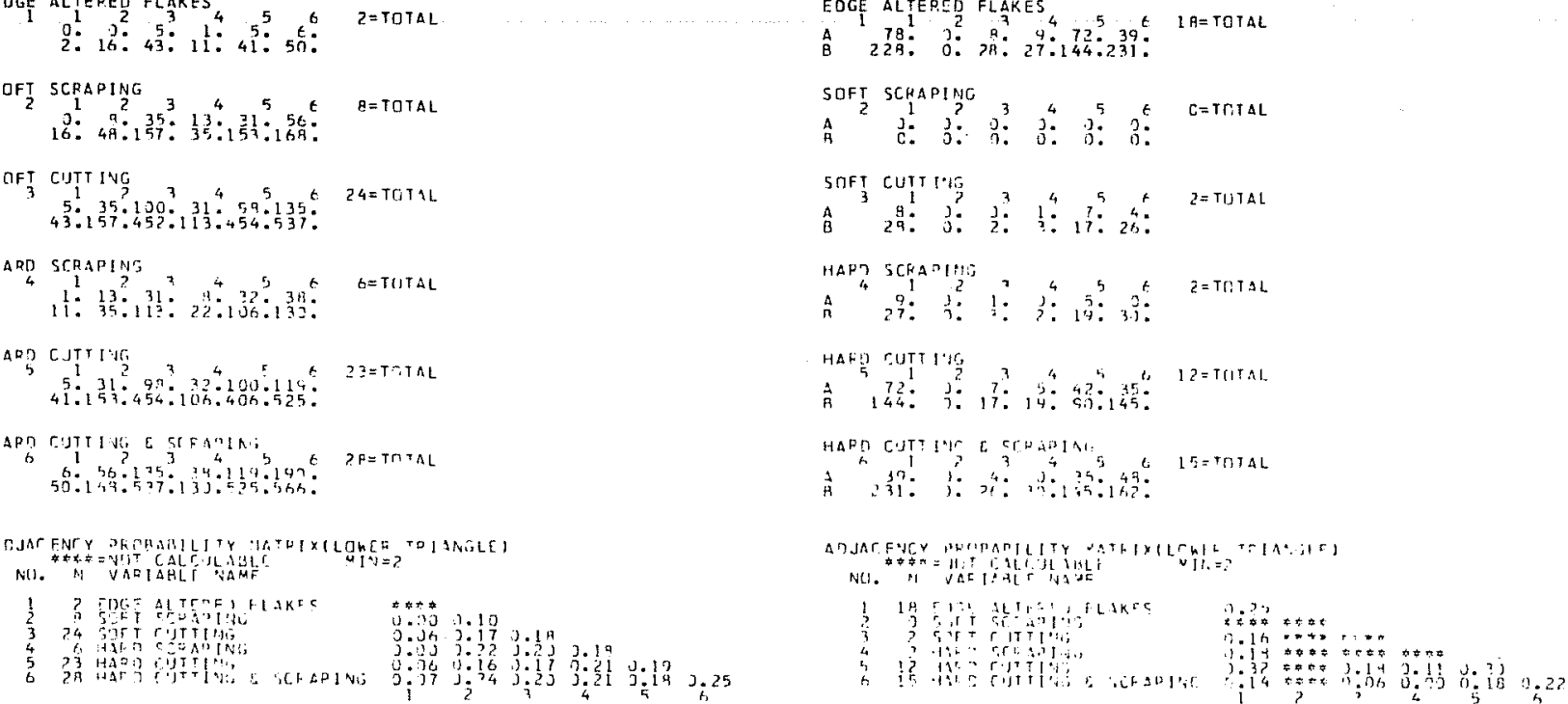

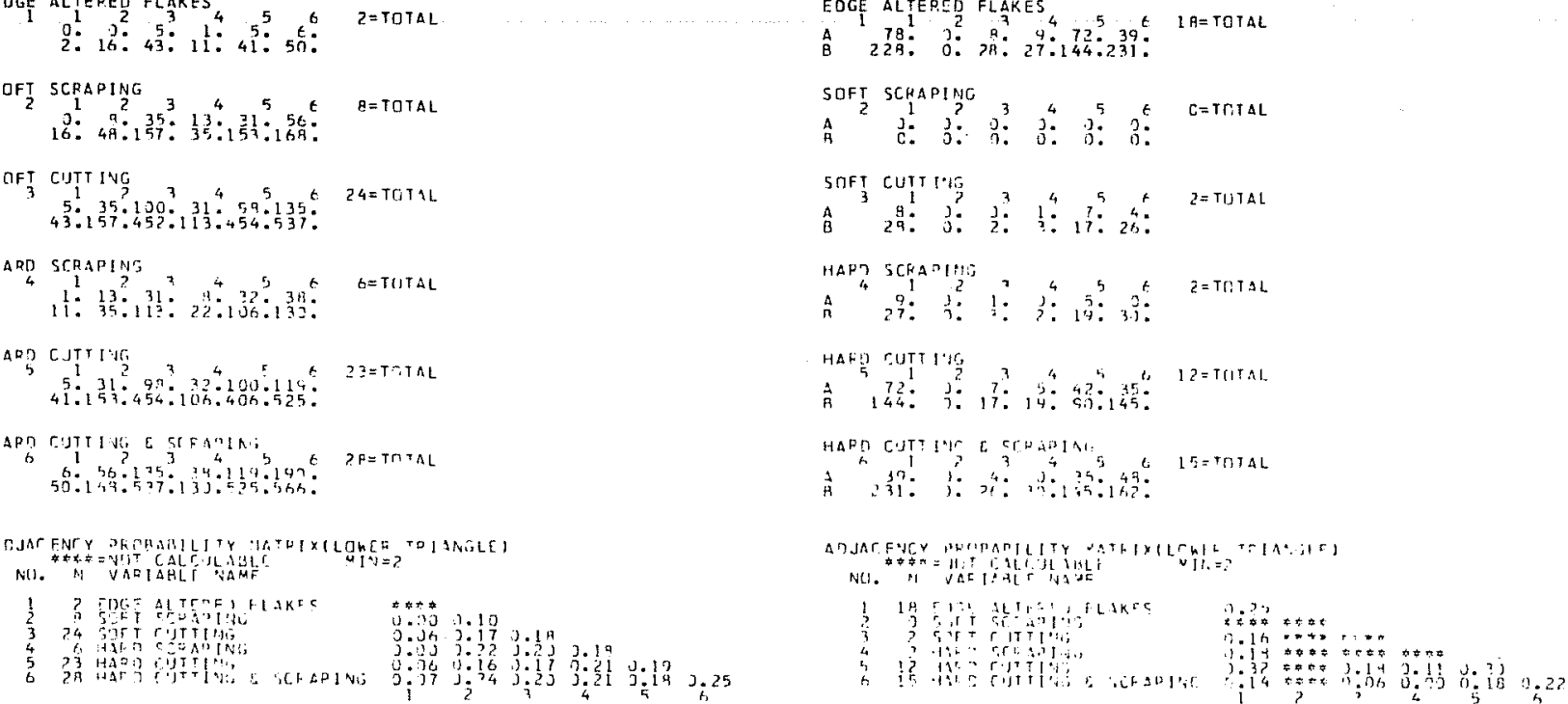

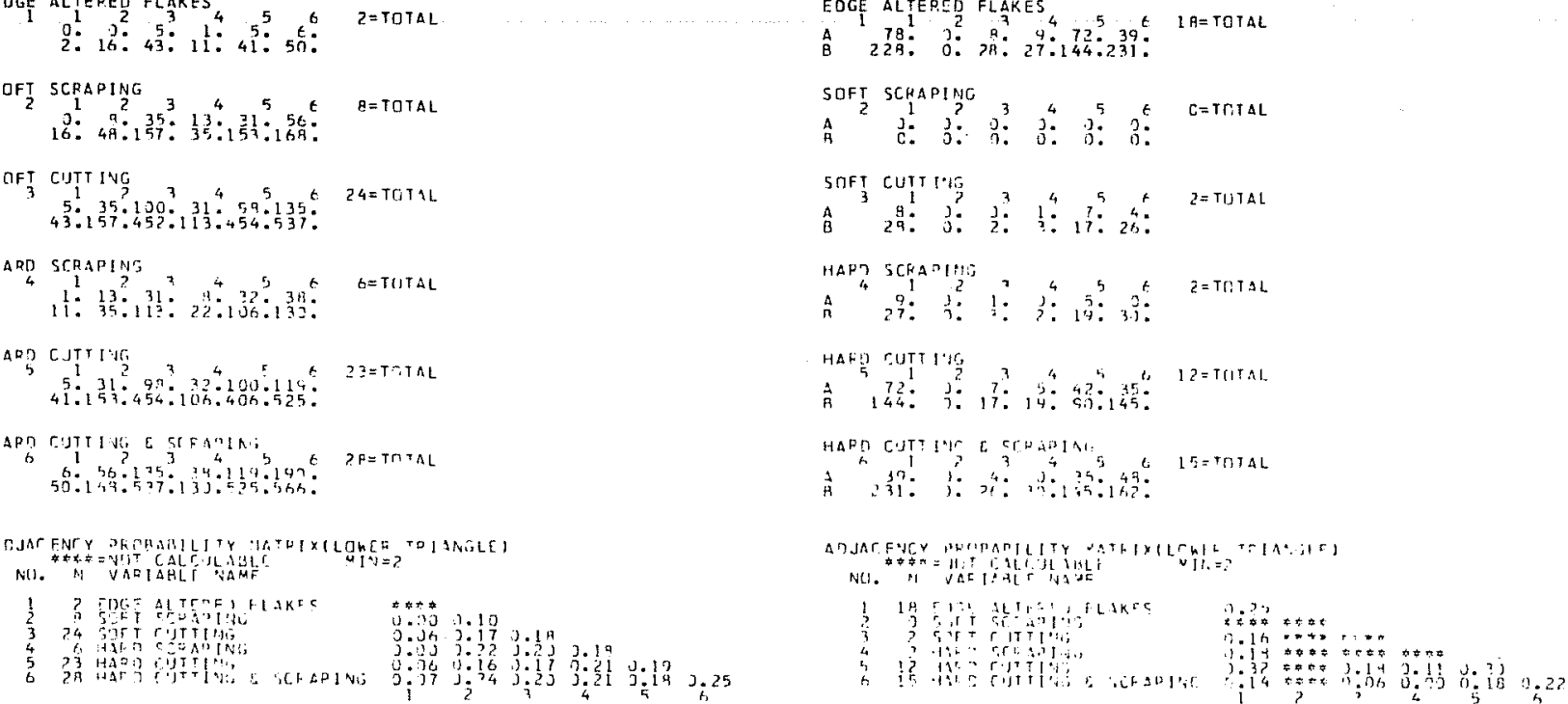

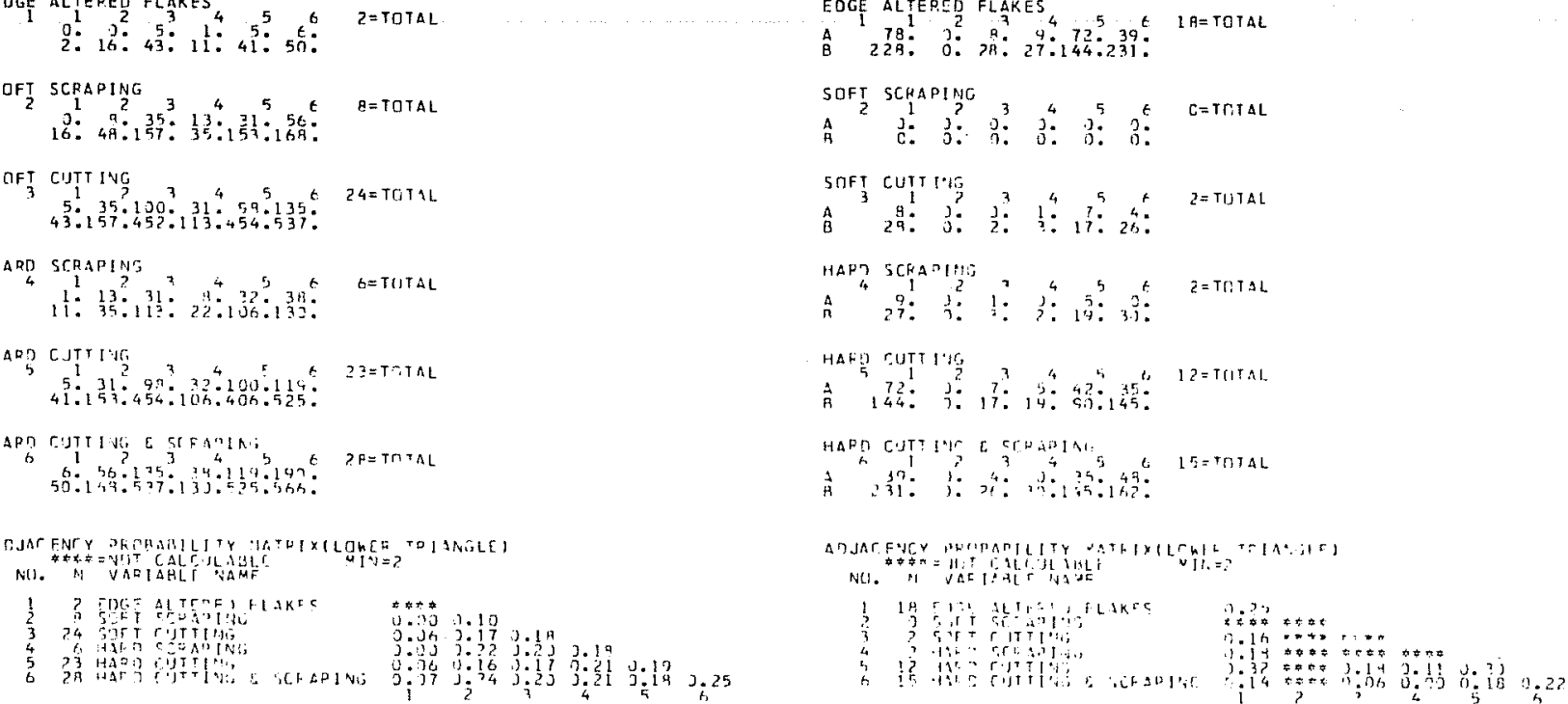

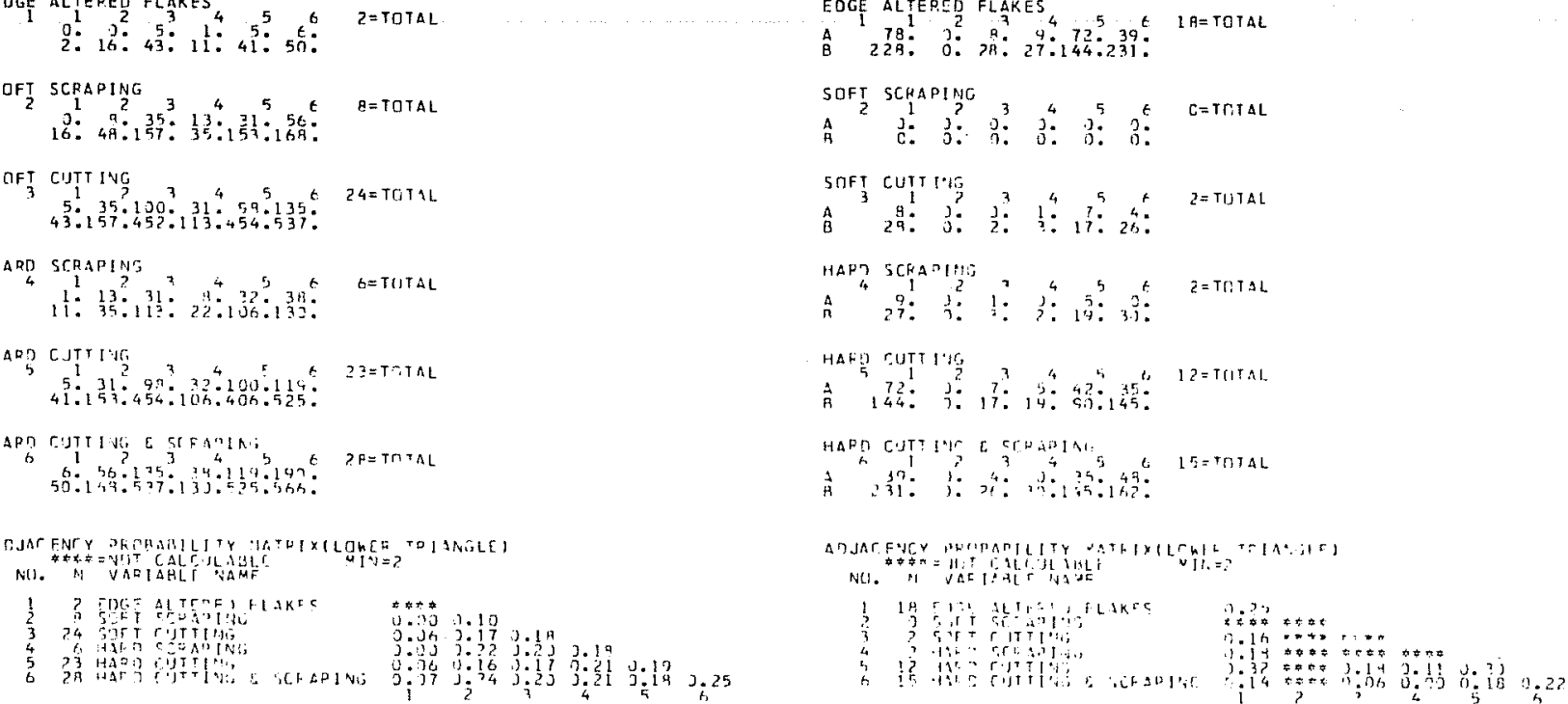

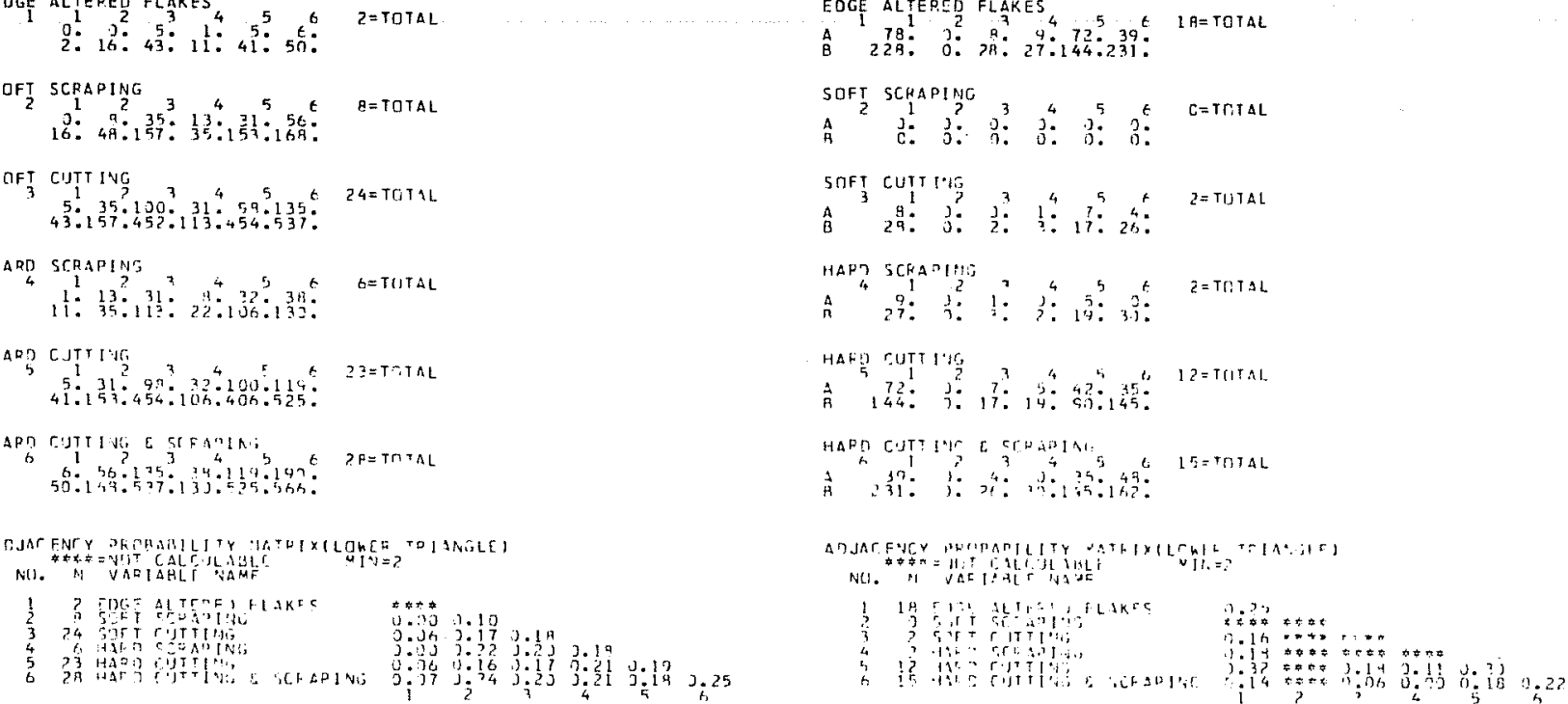

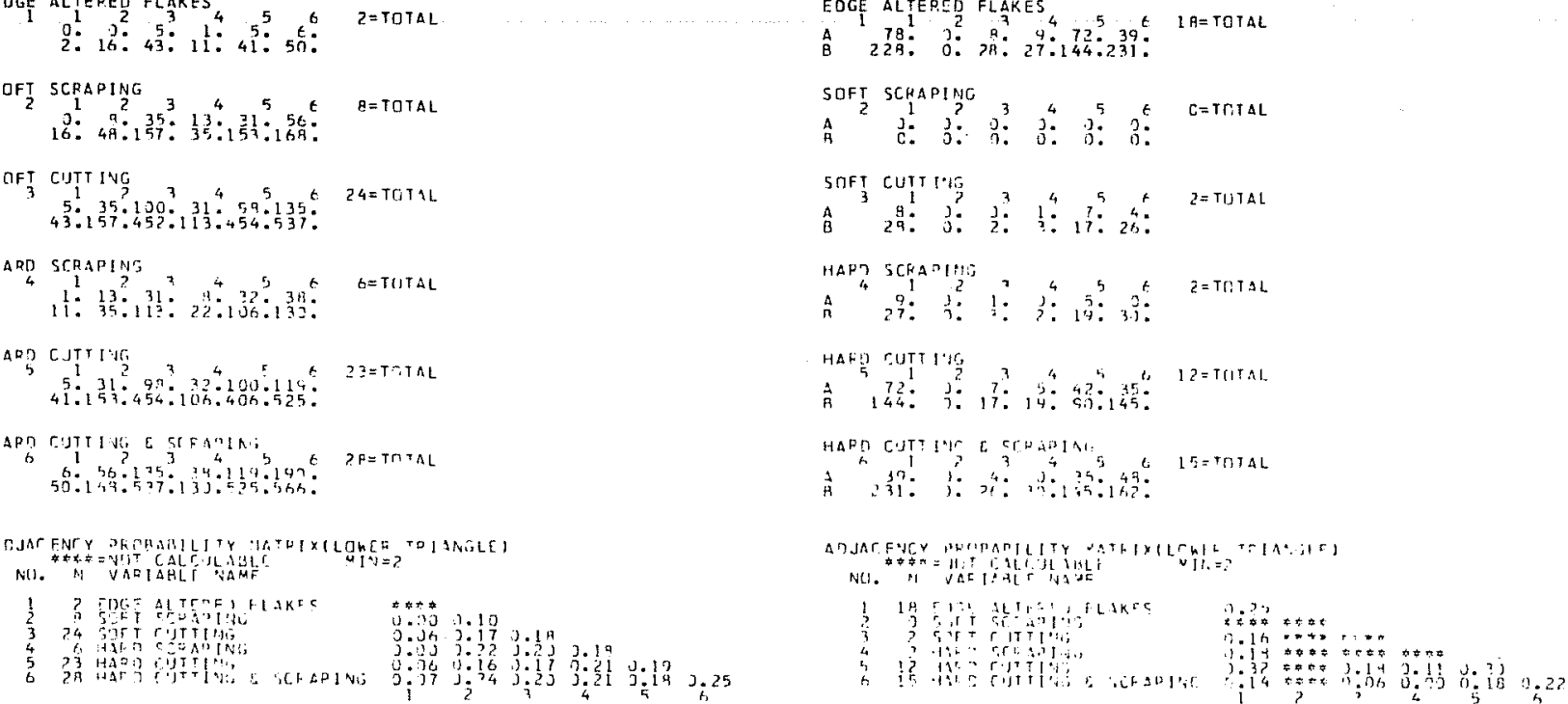

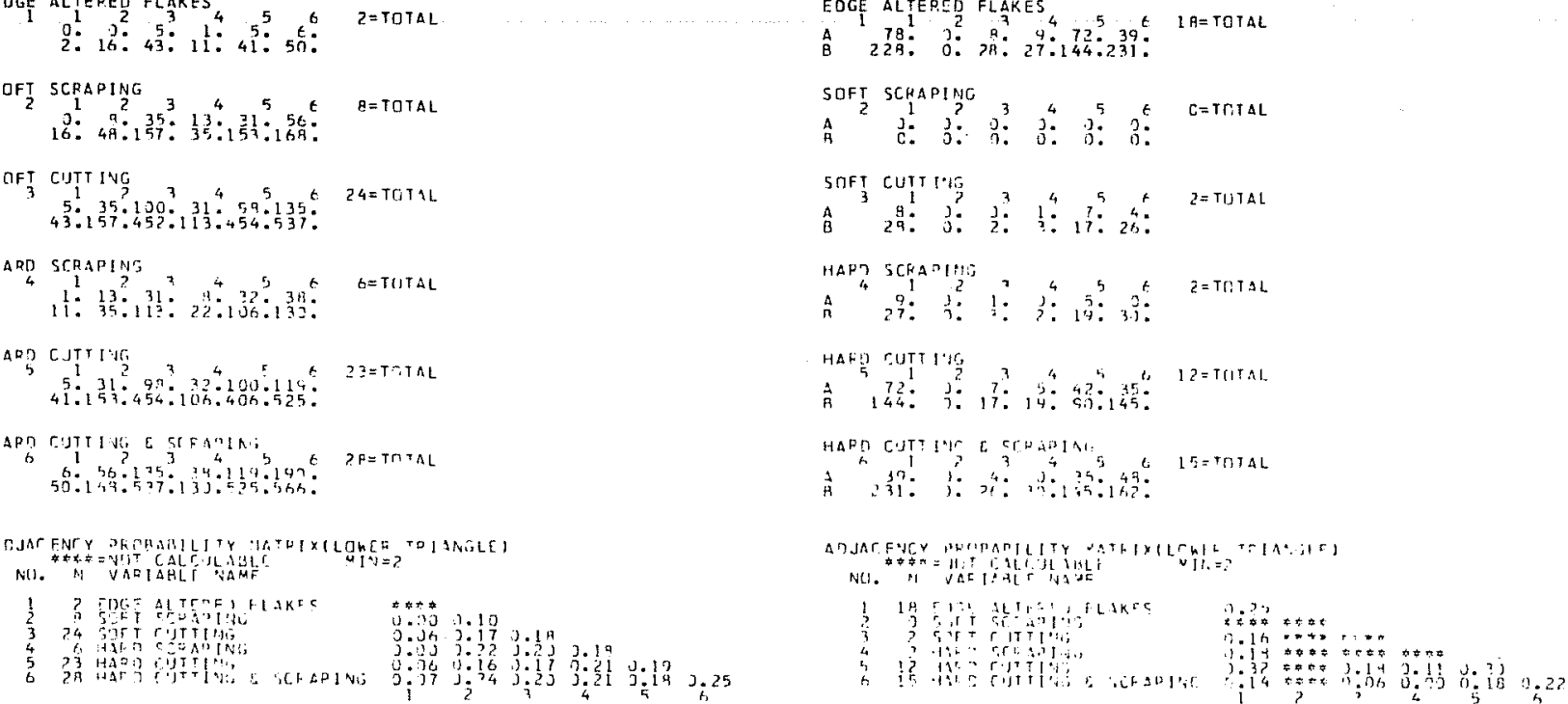

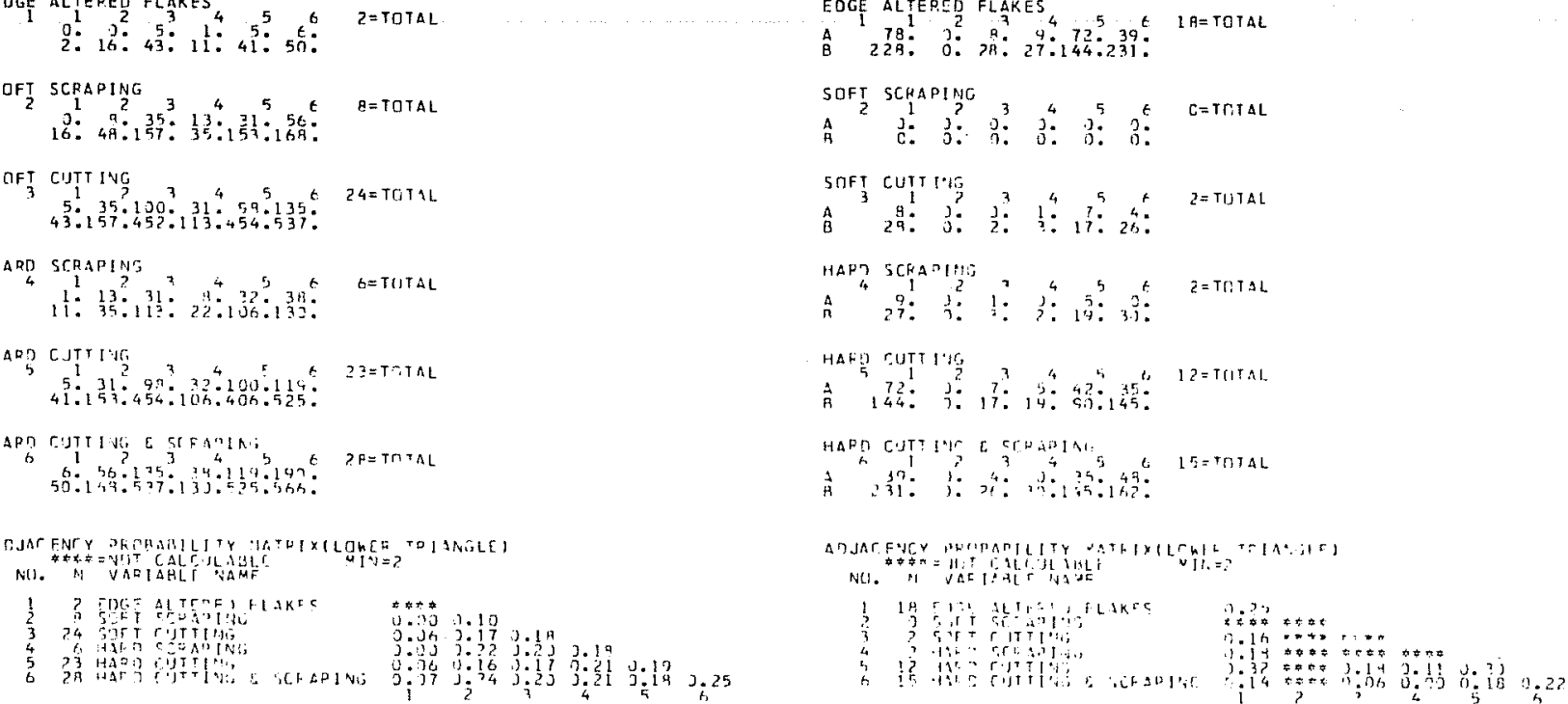

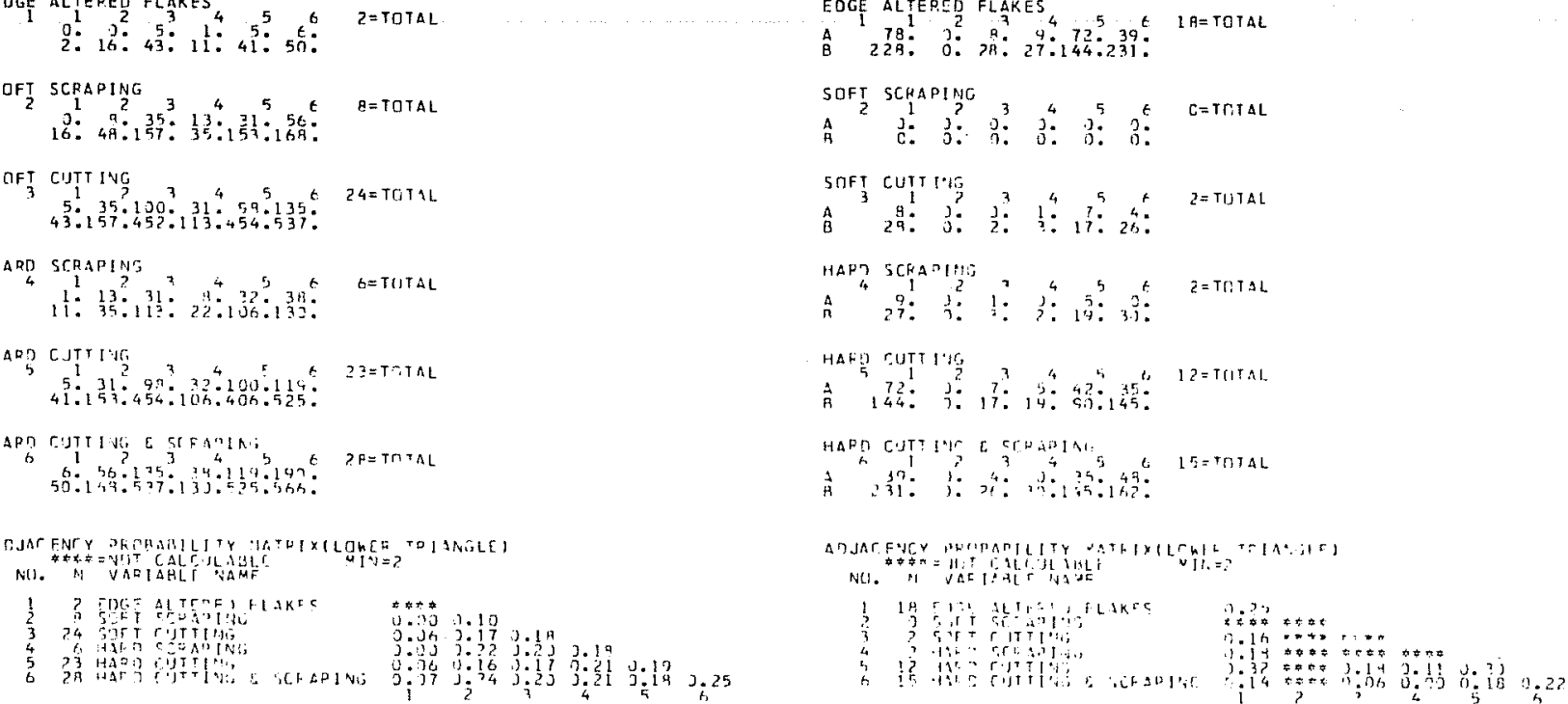

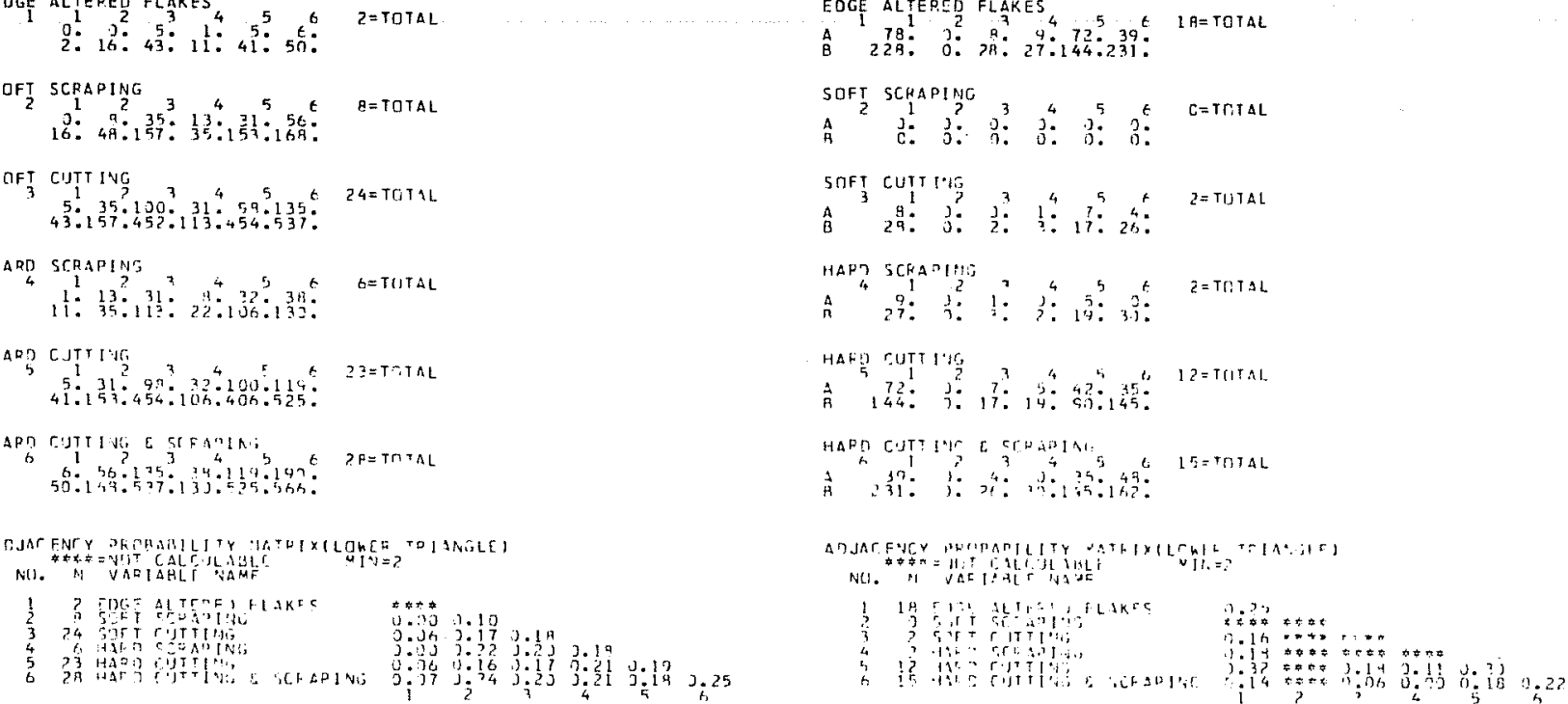


If the larger values are off the diagonal, then the tool assemblage must be specialized and diverse. This should be reflected in moderate frequencies of a wide range of tool categories.

The tool categories consist of edge-altered flakes, soft and hard scraping, soft and hard cutting, hard scraping and cutting, and indeterminate. That indeterminate is a valid wear category is suggested by the fact that indeterminant patterns sometimes cluster together and appear more frequently on some floors than others. Whatever causes the indeterminate observation was systematic in nature.

\section{Tool Typology}

The formal tool types were not numerically analyzed as part of the adjacency analysis. However, they are plotted on the wear pattern maps. In many cases, they appeared with wear patterns in apparently meaningful relationships and contributed materially to deciphering the use episodes and tool concentrations. The tool types and their symbols are listed in Table 60.

TABLE 60. FORMAL TOOL TYPES FOUND AT EAGLE HILL AND SYMBOLS USED IN MAPS Type Map Symbor

$\begin{array}{ll}1 \text { Point } & P \\ 2 \text { End Scraper } & \text { SE } \\ 3 \text { Side Scraper } & \text { SS } \\ 4 \text { Denticulate } & \text { D } \\ 5 \text { Notch } & \mathrm{N} \\ 6 \text { Graver } & \mathrm{G} \\ 7 \text { Beak } & \mathrm{BK} \\ 8 \text { Burin/burin spal1 } & \mathrm{BR} \\ 9 \text { Retouched flake } & \mathrm{R} \\ 10 \text { Biface fragment } & \mathrm{BF} \\ 11 \text { Edge-altered flake } & \mathrm{E} \\ 12 \text { Blade/Bladelet } & \mathrm{B} \\ 13 \text { Flake core } & \mathrm{CF} \\ 14 \text { Microblade core } & \mathrm{CM} \\ 15 \text { Bipolar core } & \mathrm{CB}\end{array}$

\section{Vertical Perspective}

Before attempting to examine the maps of the occupation planes, it was necessary to define the range of variations and frequencies in 251 worn items. Table 61 , a shows the states the variables, hard and soft, can take and the frequencies in which they appear on the same and/or different tools. Any one flake can have both hard and soft wear and, thanks to the direction of the wear relative to the edge on which it appears, it is possible to determine if it was hard and/or soft. The hard and soft wear patterns can resemble scraping (2), cutting (3), or boring 
(5). Slightly more complicated is the matter of absence of wear (1) or indeterminate wear (6). Note that most of the pieces that showed hard wear were also found to have associated indeterminate soft wear, $197(78.5 \%$, see total, indeterminant soft wear) in a11. This is to be expected since any act involving hard wear will also produce marginal soft wear or may be associated with limited soft wear tasks. Consider, for instance, the carpenter who trims a delicate edge in the midst of hard planing a flat surface.

As a whole the population of worn flakes is dominated by hard use. Only 17 items $(6.8 \%)$ show a total absence of hard use. It is quite possible that soft use is under-represented in the sample, because it is more likely to be missed under low magnification: Note that soft use is usually associated with indeterminate hard wear (19.8\%) rather than soft wear alone (7.8\%). Conversely, virtually all hard worn pieces have some sort of soft wear $(98.2 \%)$.

TABLE 61. USE-WEAR AT EAGLE HILL

a. Contingency table for all hard and soft wear at Eagle Hill

\begin{tabular}{ccccc}
\multicolumn{5}{c}{ SOFT WEAR } \\
1 & 2 & 3 & 6 & \\
Absent & Scraping & Cutting & Indeterminate & Total
\end{tabular}

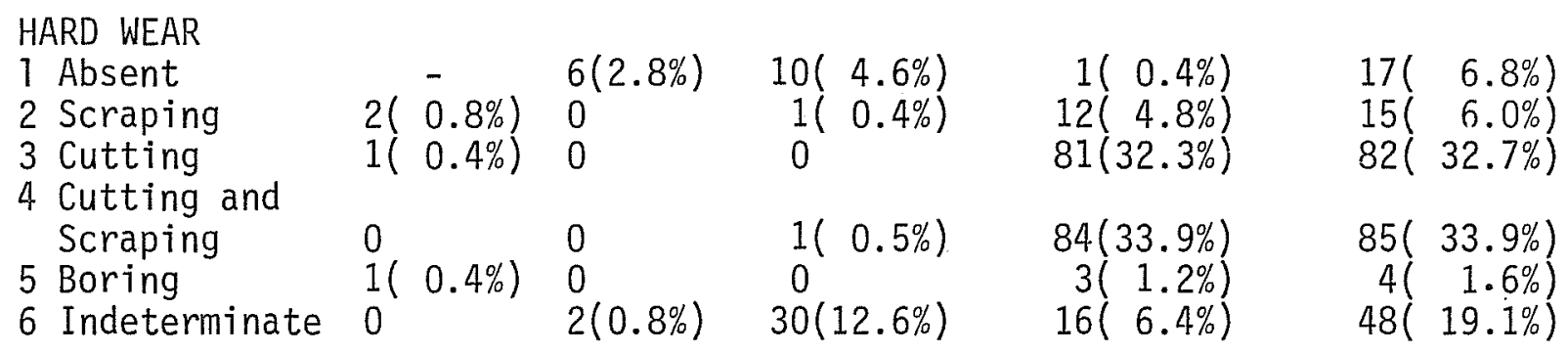

TOTAL

$4(1.6 \%) \quad 8(3.2 \%) \quad 42(16.7 \%) \quad 197(78.5 \%)$

$251(100.0 \%)$

b. Frequencies of wear on 0ccupation Planes

$O P$ SS SC HS HC HM HB II Contigency $V$ P Coefficients

\begin{tabular}{rrrrrrrrrrr}
\hline 1.13 & 0 & 3 & 2 & 12 & 8 & 0 & 0 & .71 & 1.00 & $<.001$ \\
2.13 & 8 & 25 & 6 & 23 & 28 & 1 & 6 & .73 & .61 & $<.001$ \\
3.11 & 0 & 2 & 2 & 12 & 15 & 0 & 4 & .48 & .54 & .017 \\
4.12 & 0 & 4 & 1 & 19 & 18 & 2 & 0 & .77 & .86 & $<.001$ \\
4.17 & 0 & 8 & 4 & 16 & 16 & 1 & 6 & .54 & .46 & .008 \\
\hline
\end{tabular}


For purposes of analysis it is desirable to reduce the number of types of wear as much as possible. Examination of Table 61 shows that some of the cells are empty and can be disregarded. Some of them can be combined since their meanings are ultimately the same. For instance, soft wear with hard wear absent, and soft wear with indeterminate hard wear are, for practical purposes, the same. Table 62 shows the combinations of hard and soft codes from Table 61.

\section{TABLE 62. TYPES OF WEAR SUGGESTED BY HARD AND SOFT STATES}

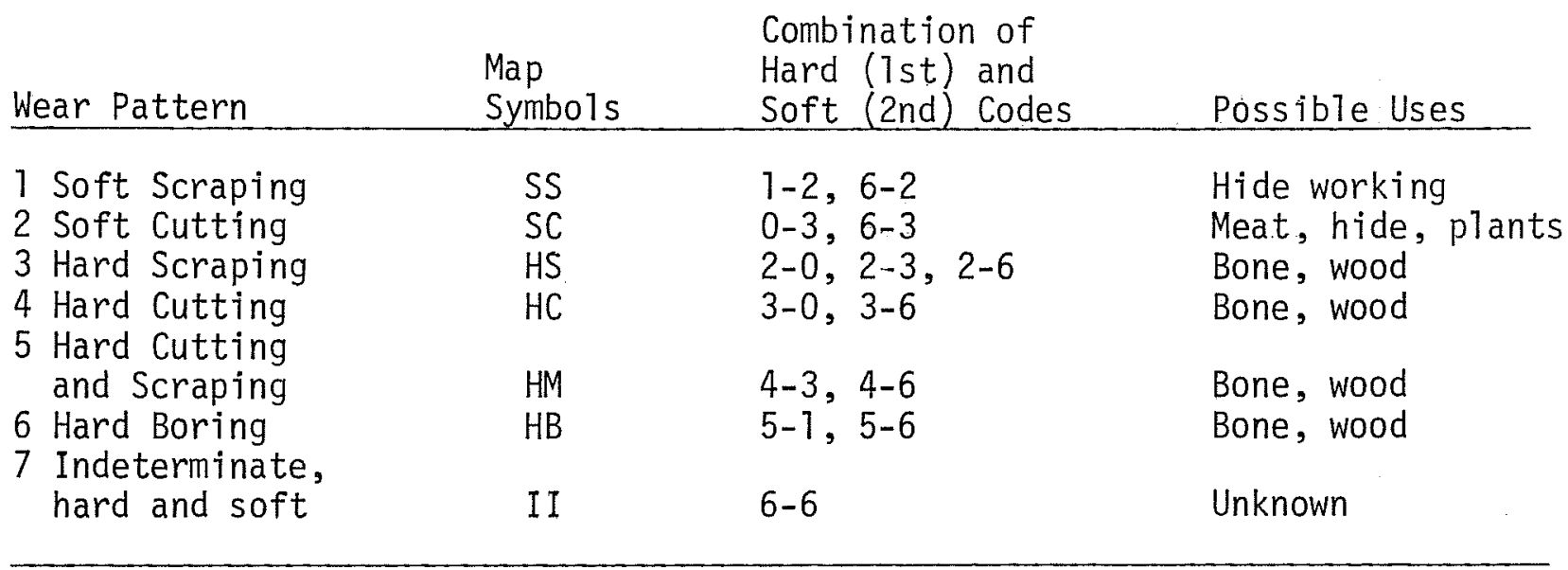

Table 63 shows the frequency and percentages of determinate hard and soft scraping in the various occupation planes. Most show about the same ratio of hard to soft wear. The exception is OP 2.13, which contains a much higher incidence of soft wear. Every indicator of occupation activity suggests that OP 2.13 is peculiar, an interval of much-increased occupation activity at Eagle $\mathrm{Hi}$ 11. The incidence of charcoal and pottery fragments is very high in OP 2.13 as well as sizable numbers of lithics. Casual inspection of the following maps affirms the aberrant nature of oP 2.13. This complex of artifacts along with more soft wear suggests that Eagle Hill assumed more of a base camp status during OP 2.13 times than it knew before or after. Hard use is probably a product of . manufacture and refurbishment of "weapons, perhaps during idle hours while waiting for game. Soft use from preparation of meat and hides or plants during the OP 2.13 occupation seems a likely indication of a well-rounded complement of activities by both sexes (White, Burton, Brudner, and Gunn 1975).

TABLE 63. HARD AND SOFT USE THROUGH THE OCCUPATION PLANES

\begin{tabular}{llrr} 
Occupation Plane & Hard & Soft & Total \\
\hline 1.13 & $22(88 \%)$ & $3(12 \%)$ & 25 \\
2.13 & $57(63 \%)$ & $33(37 \%)$ & 90 \\
3.11 & $29(94 \%)$ & $2(6 \%)$ & 31 \\
4.12 & 38 & $4(10 \%)$ & 42 \\
4.17 & $36(82 \%)$ & $8(18 \%)$ & 44 \\
\hline TOTAL & $182(76 \%)$ & $50(24 \%)$ & 232 \\
\hline
\end{tabular}


Table $61, \mathrm{~b}$ shows the frequencies of a11 categories of utilization by occupation planes.

\section{Overal1 Occupation Control}

As was indicated in the background study, topography of the immediate area of the site seems to have encouraged occupation. The focus of Paleo-Indian occupation to the west was controlled by the advantage offered by a slight rise in the ground. Later the slope downhill and southeast of this rise formed a basin in the impervious, Pleistocene-weathered clay which acted as an aquifer for overlying vegetation. This fostered a more or less consistent topographic high in the vicinity of the site and promoted occupation during the Holocene.

In addition to the moderate relief, there is evidence that large trees with tap roots a half-meter in diameter grew in the site. Skimpy pollen evidence (page 161), inference from the general southeastern vegetational succession (page 104), and local history indicate that the trees present during the late Holocene were longleaf pines. One tree was located at the western edge of the Servel10 test pit in unit E3017-3018 N1000. The other is along the northeast edge of the excavation in unit E3021 N1001. Since these are the only places in the $30-\mathrm{m}$ excavation with tap roots, the locations of trees must have been constant or limited to one generation. Human intervention may have acted to prevent the growth of additional trees past the first generation.

The two trees in the excavation were apparently dead and burned by the time logging began early in this century, or stumping would have destroyed what was left of the site. The tree northeast of the excavation contained decayed wood, while the one on the west contained only charcoal. Occupation during the historic Caddo period seems to have focused around the western tree; it may have been alive as late as A.D. 1700-1800. A massive stump hole centering three meters west of the excavation marks the location of the 20th-century tree. In hindsight, it might have been interesting to date the trees. When we were selecting carbon for dating we went to the utmost effort to avoid burned trees

Occupation P1ane 1.13

Occupation Plane 1.13 (Fig. 86) is dated to the last millennium and possibly to historic Caddoan as indicated by a smal1 point base fragment. It encompasses three artifact concentrations with 34 items (tool forms and wear patterns).

Logical Analysis

Two areas seem to bear the marks of fireplaces. Concentration 1 is located south of what was probably a large, longleaf pine judging by the tap root extending down through the levels. Also, there are burned pieces of flint among the charcoal and clay balls. This suggests multiple occupation events. Their placement was guided by the presence of the western tree. Perhaps it was used 

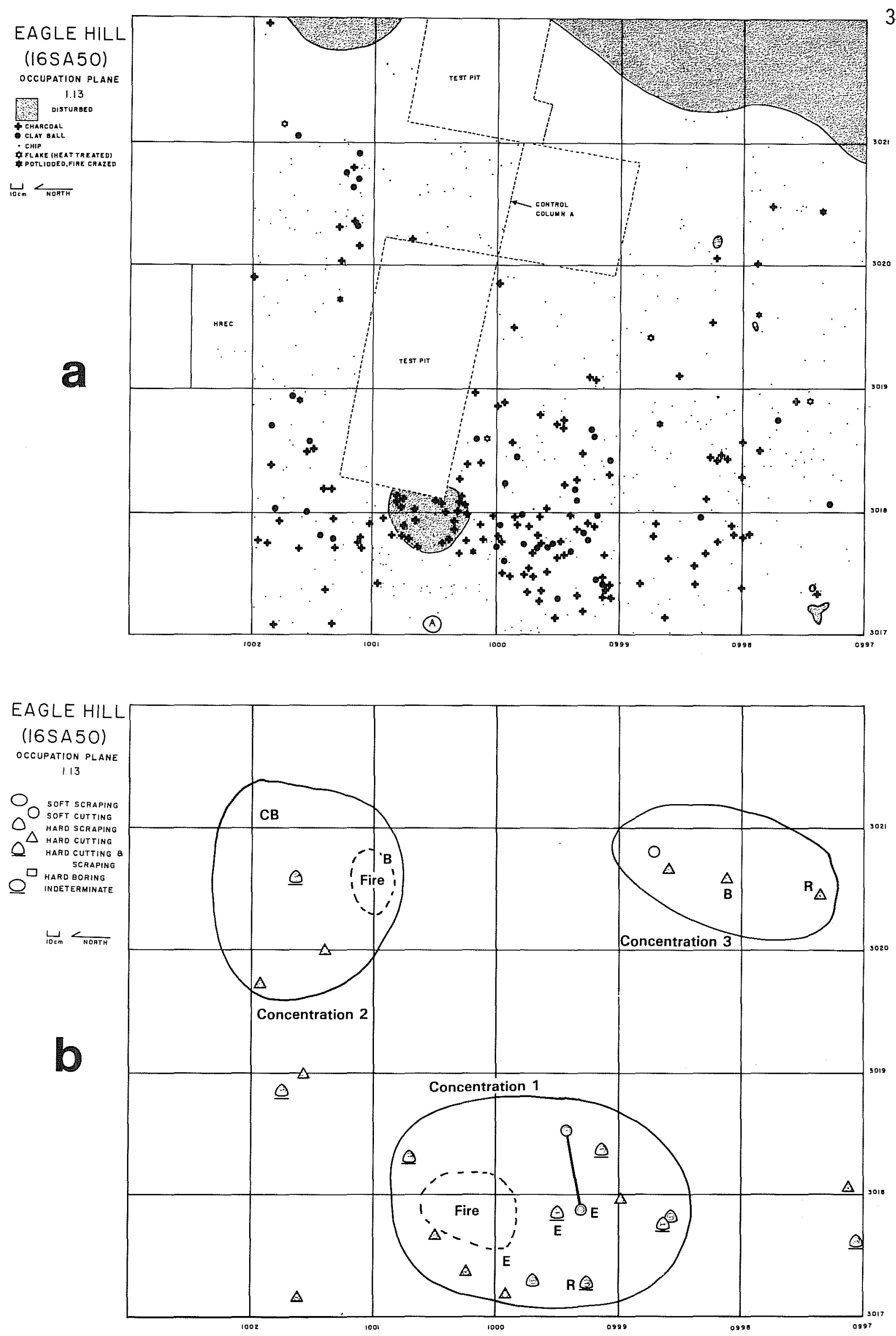

Figure 86. Map of Occupation Plane 1.13. a, fire-related artifacts and chips from Occupation Plane 1.13 (see Table 60 and 62 ); b, wear patterns and forma 7 tonlc (cee Tahte fn) 
to shield the fire from a northerly wind, an indication of cool season occupation. Most of the wear is hard cutting. A11 of the soft cutting on the floor is located east of the fire, which suggests a single incident within the scope of the excavation.

Less can be said about Concentration 2. Charcoal and clay balls mark the location of a fireplace. An episode of hard cutting is recorded east of the fire. Concentration 3 is a hard cutting episode, although there is one soft cutting implement. A single burned chip and charcoal fragment to the south mark a possible fire. The concentration may have been truncated by the roadcut to the east.

\section{Concentration Content}

The concentrations are spatially discrete and easily analyzable because of the small number of artifacts. However, in following floors, the problem becomes more difficult due to increasing numbers of items. It is necessary to tabulate artifacts to discover patterns of association. Table 64 provides data on frequencies within the concentrations.

TABLE 64. FREQUENCIES OF WEAR CATEGORIES IN OP 1.13 BY CONCENTRATION

Wear type

\begin{tabular}{llll}
\hline SS & 0 & 0 & 0 \\
SC & 2 & 0 & 1 \\
HS & 2 & 0 & 0 \\
HC & 4 & 2 & 3 \\
HS and C & 5 & 1 & 0 \\
II & 0 & 0 & 0 \\
Boring & 0 & 0 & 0 \\
\hline TOTAL & 13 & 3 & 4 \\
\hline
\end{tabular}

Adjacencies

Adjacencies on OP 1.13 are interesting because of the floors simplicity. There is only one concentration with a complex assortment of tools. The other two are dominated by hard cutting. The adjacency probability matrix (Table 55) shows that there are substantial variations in the degree of association between toolwear categories. The edge-altered pieces are all in the main concentration, and the strength of the adjacency between edge-altered pieces and other categories are all appropriately high. 
The relatively low adjacency index between mutual occurrences of hard cutting on the same flakes sounds a note of interpretive caution. Hard cutting is present in all three clusters (many pieces are within the criterion distance). The low 0.19 adjacency index reflects the effect of differential frequencies between concentrations. Ideally, categories should be evenly distributed throughout all concentrations. This methodological liability would disappear if distances between concentrations were discounted. This is the next logical step in developing the adjacency methodology.

An element of interpretive logic that was utilized to define use episodes was that, if tools of a single use pattern (scraping or cutting) were mixed with items of mixed wear such as scraping and cutting, the mixed items were considered as the pure form. On OP 1.13 hard scraping and cutting are associated more often with hard scraping.

\section{Occupation Plane 2.13}

Op 2.13 (Fig. 87) is dated to A.D. $935 \pm 80$ years (UGa-3703). Contemporary ceramics indicate a late Coles Creek occupation. Artifacts are so frequent on the floor that selecting concentrations is problematical. However, there appear to be six concentrations with 106 items (tool forms and wear patterns).

\section{Logical Analysis}

It is apparent from several indicators that OP 2.13 was a heavily occupied floor. The complexity of the occupation pattern is practically an order of magnitude greater than any other judging by the number of logically discernible features. This is not to mention the general jumble of artifacts, which do not lend themselves to interpretation within the scope of the logic we are using to make interpretations. The clutter is probably too great to allow any sure interpretations without the assistance of numerical analysis. However, a few suggestions may ultimately help to unravel the confusion. As was mentioned before, there is a marked increase in soft wear in OP 2.13 suggesting that a broader range of activities accompanied the increased frequency of activity.

Concentration la contains practically every variety of material: clay bal1s, charcoal, a 17 types of wear, and burned flint. There is no apparent pattern to it although there is an unusual frequency of soft cutting. It may well be an accumulation of trash (see section IV for related problem, page 307). Concentration 1b may be associated with Concentration la or it could be an independent episode of soft cutting. Concentration $1 c$ is a large area relatively free of charcoal and clay bal7s. The complement of used flakes is $71 \%(18)$ hard use in contrast to the usual high incidence of soft use on the floor. The incidence of soft cutting and scraping next to the fire counterpoised by hard utilization away from fire is congruent with standard, cross-cultural division of labor schemes (White, Burton, Brudner, and Gunn 1975). 

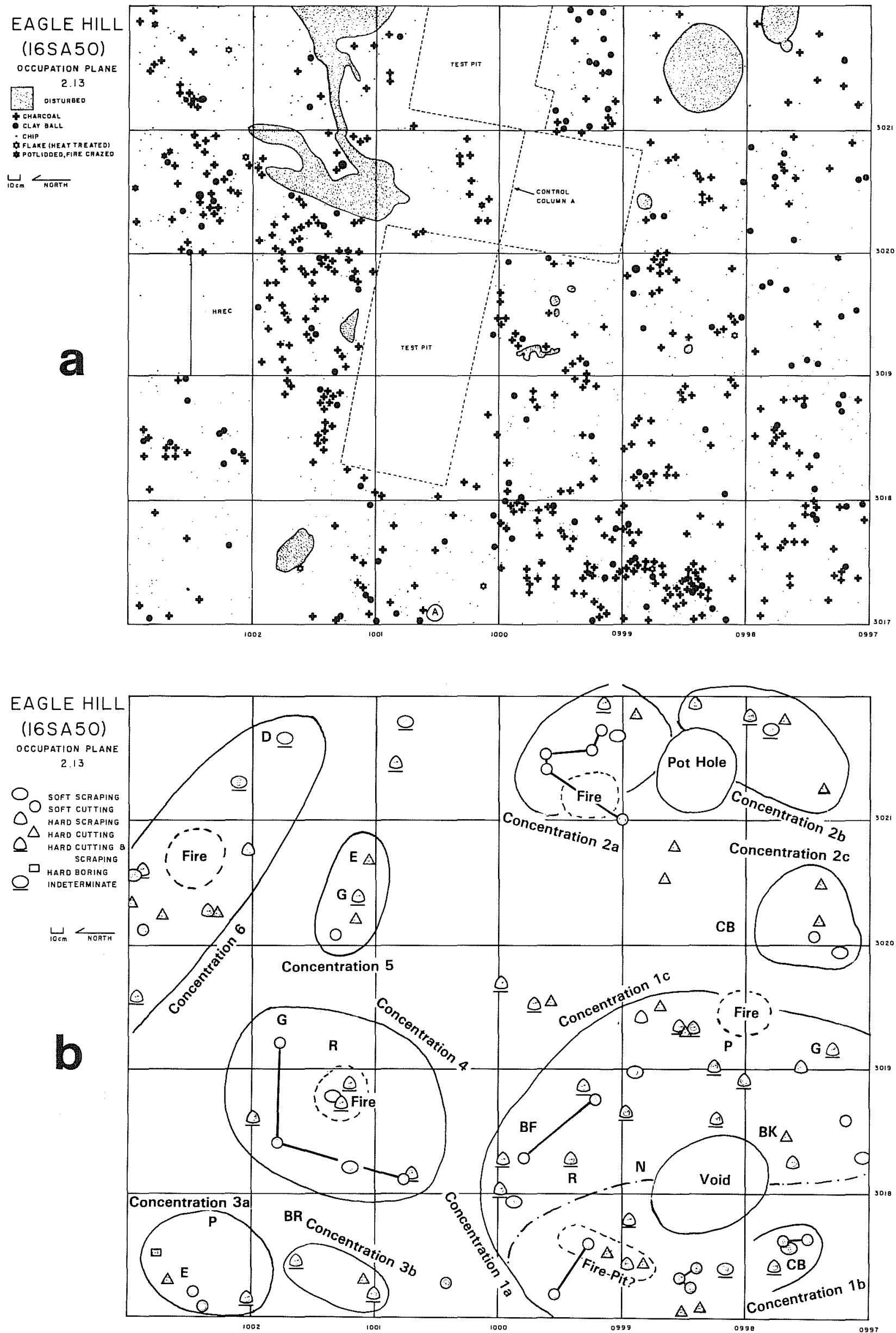

Figure 87. Map of Occupation Plane 2.13. a, fire-related artifacts and chips from Occupation Plane 2.13 (see Tables 60 and 62 ); b, wear patterns and formal tools (see Table 60) 
Concentration $2 \mathrm{a}$ is a charcoal and clay ball feature with a locus of soft cutting on its northeast side. Concentration $2 b$ is an episode of hard cutting without an associated fire feature. The distributional pattern is not like the Concentration la-7c complex, but the wear composition is similar. Concentration $2 b$ and 5 are composed of hard cutting implements. It would be interesting to know if they represent work on the same material. If so, their location may result from a tendency for certain activities to be barred from the campfire. One can envision someone wielding a long shaft while rehafting the point being politely called away from the fire. Concentration $2 \mathrm{c}$ is a mixed hard and soft utilization cluster.

Concentration $3 a$ is a charcoal and clay ball feature with an associated, varied tool kit. Wear is dominated by soft cutting. A boring wear pattern is noted next to a projectile point. Concentration $3 b$ is composed of hard use. Note that the burin spall is associated with the hard cutting pieces.

Concentration 5 is an episode of hard cutting. Charcoal and clay balls are so frequent that a single fireplace cannot be reasonably outlined.

Concentration 6 is a charcoal and clay ball feature associated with cutting. A great majority of it is hard cutting and more like the hard cutting episodes above and below rather than on the rest of OP 2.13.

\section{Concentration Content}

It appears that three out of six concentrations are composed of contrasting sets of hard and soft wear with soft wear more closely associated with the fire. This is a logical set of associations whether women were present or not. However, the marked contrast to the rest of the occupation planes in terms of wear and distributional patterns assuredly indicates a change in site status, possibly involving visits by nuclear families rather than specialized task groups.

This inference is further supported by the fact that soft scraping appears only on OP 2.13. It probably implies hide scraping and provides another indication of lengthier camps at the site. Soft scraping is distributed rather uniformly among the concentrations containing larger and more varied numbers of tools.

The concentration of charcoal and clay balls in the northeastern sector of the floor is so scattered that specific campfires cannot be located. However, Concentrations 4 and 6 may be another dyadic pair of subconcentrations. Inspection of Table 65 clearly shows the rhythm of alternation between hard and soft use-wear patterns.

The number of artifacts in the concentrations are larger than in any other occupation plane. Yellen (1977) found that larger numbers of implements scattered over a greater area is a direct indicator of length of occupation. We might surmise that the length of stay during the OP 2.13 period was two to three times longer than during periods before and after. This estimate would roughly agree with the artifact frequencies. 
TABLE 65. FREQUENCIES OF WEAR CATEGORIES IN OP 2.13 BY CONCENTRATION

\begin{tabular}{|c|c|c|c|c|c|c|c|c|c|c|c|}
\hline & & & & Cono & $\operatorname{tra}$ & & & & & & \\
\hline Wear type & la & $1 b$ & 1c & $2 a$ & $2 b$ & $2 c$ & $3 a$ & $3 b$ & 4 & 5 & 6 \\
\hline $\begin{array}{l}\text { SS } \\
\text { SC } \\
\text { HS } \\
\text { HC } \\
\text { HS and C } \\
\text { II } \\
\text { B }\end{array}$ & $\begin{array}{l}1 \\
6 \\
1 \\
4 \\
4 \\
1 \\
0\end{array}$ & $\begin{array}{l}1 \\
2 \\
0 \\
0 \\
1 \\
0 \\
0\end{array}$ & $\begin{array}{l}2 \\
2 \\
3 \\
3 \\
9 \\
0 \\
0\end{array}$ & $\begin{array}{l}1 \\
4 \\
0 \\
1 \\
1 \\
0 \\
0\end{array}$ & $\begin{array}{l}0 \\
0 \\
1 \\
2 \\
1 \\
1 \\
0\end{array}$ & $\begin{array}{l}1 \\
1 \\
0 \\
2 \\
0 \\
0 \\
0\end{array}$ & $\begin{array}{l}0 \\
2 \\
0 \\
1 \\
1 \\
0 \\
1\end{array}$ & $\begin{array}{l}0 \\
0 \\
0 \\
1 \\
2 \\
0 \\
0\end{array}$ & $\begin{array}{l}2 \\
3 \\
0 \\
0 \\
4 \\
0 \\
0\end{array}$ & $\begin{array}{l}0 \\
1 \\
0 \\
2 \\
1 \\
0 \\
0\end{array}$ & $\begin{array}{l}0 \\
1 \\
1 \\
3 \\
3 \\
3 \\
0\end{array}$ \\
\hline TOTAL & 17 & 4 & 19 & 7 & 5 & 4 & 5 & 3 & 9 & 4 & 11 \\
\hline
\end{tabular}

In addition to the large number of flakes with observable wear patterns, there are a large number of formal tools compared with the other late Holocene levels. OP 2.13 shares, in two instances, a bipolar core trait with OP 3.11. Since bipolar cores are unique to these two floors, they may indicate cultural continuity over the 100 or more year period of occupation.

\section{Occupation Plane 3.11}

OP 3.11 (Fig. 88) is dated to A.D. $820 \pm 70$ years by radiocarbon (UGa-3704) and is associated with coles creek ceramics. There are three concentrations containing 71 items (tool forms and wear patterns). OP 3.11 is particularly notable for a high frequency of edge-altered flakes. In fact, "E-pairs" (edgealtered flakes with another tool associated) occur all over the floor.

\section{Logical Analysis}

Concentration 1 is a charcoal and clay feature surrounded by tools and wear patterns. The wear patterns are predominantly hard cutting. Two such objects are located in the fire feature suggesting multiple occupation. It is on the southwest side of the western tree. Tools are consistently paired with edgeal tered flakes.

Concentration 2 contains three episodes. Concentration $2 a$ is very peculiar. A11 of the wear patterns are of the double indeterminate variety. This subconcentration suggests that there is more to the "II" observation than just an inability to assign the use to soft or hard. Some task was being performed which generated an obscure wear pattern. There appears to be no immediate fire feature. Concentration $2 \mathrm{~b}$ is clearly a hard cutting subconcentration. It is associated with one or two fires indicating an episode or episodes in a frequented spot. Concentration $2 \mathrm{c}$ is also against a fire and is purely hard scraping and cutting. Concentration 3, like Concentration 2c, is completely devoted to hard multiple use, perhaps associated with a fire. 

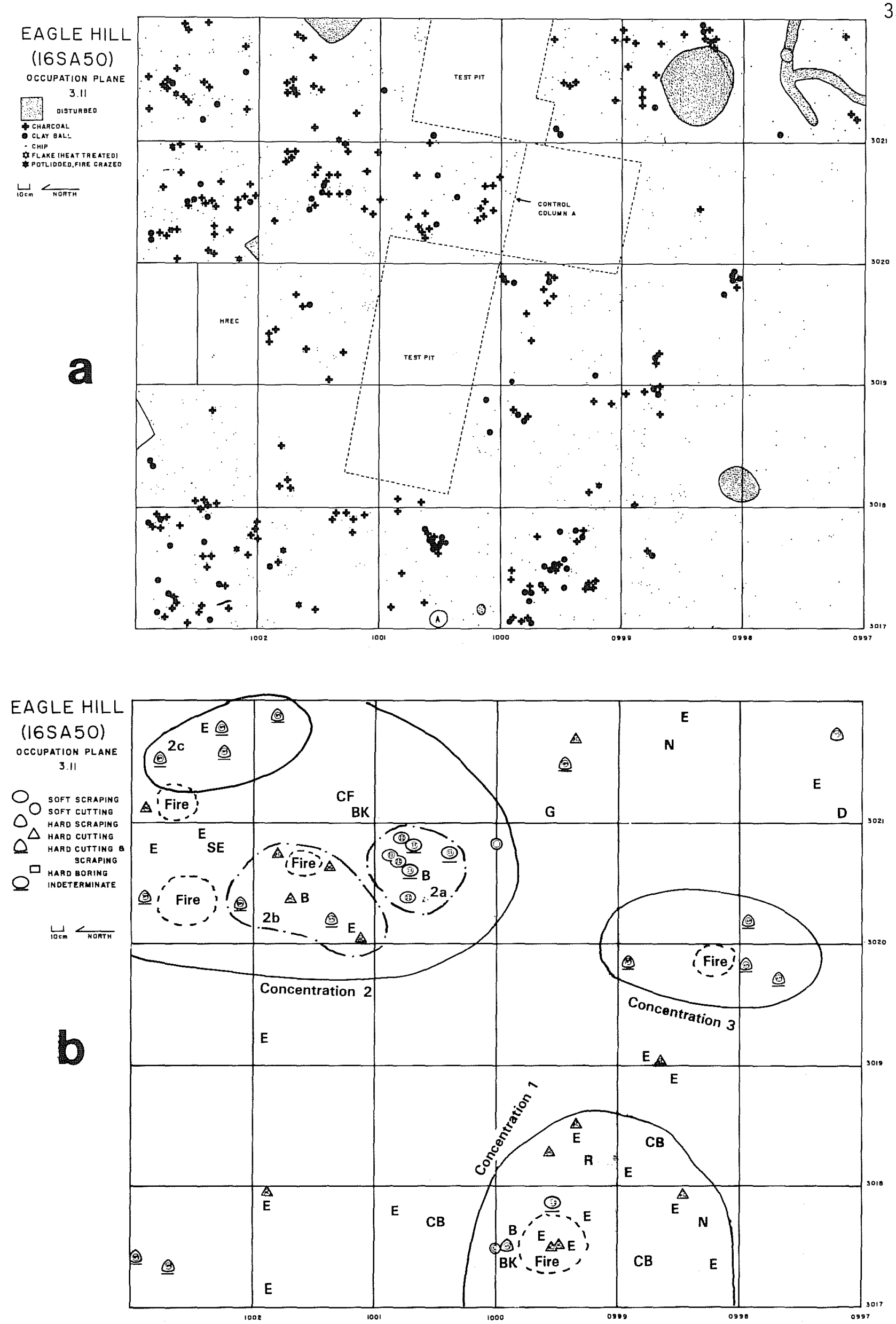

Figure 88. Map of Occupation Plane 3.11. a, fire-related artifacts and chips from Occupation Plane 3.11 (see Tables 60 and 62); b, wear patterns and formal tonle (ces Tahle fol). 


\section{Concentration Content}

OP 3.11 is Tike OP 1.13 in its sparse occupation and high frequency of edgealtered flakes. It is more like OP 2.13 in its high frequency of formal tools and particularly the two bipolar cores located in Concentration 1. Unlike OP 2.13, there are no dyadic spatial relationships between soft and hard use patterns.

The peculiar pattern of pairs of tools, at least one of which is an edge-altered flake, appears frequently across the floor. The pairs appear both within and without the concentrations. The variety of tools in the "E-pairs" is diverse. However, one of them, in many cases, is a scraping implement. It could be that the cultural norm and a function peculiar to the time dictates that a tool kit should consist of a scraping implement and an edge-altered flake for cutting. Concentration 2 appears to be highly internally structured, but all three subclusters are related to hard materials. I assume that the "II" wear category involves hard, destructive usage. This requires further investigation for confirmation.

Consistent with other concentrations, the only soft cutting implement on the floor is located near a fire in Concentration 1. Table 66 shows the wear composition of the concentrations.

TABLE 66. FREQUENCIES OF WEAR CATEGORIES IN OP 3.11 BY CONCENTRATION

\begin{tabular}{lllcll} 
Wear type & 1 & $2 \mathrm{a}$ & $\begin{array}{c}\text { Concentration } \\
2 \mathrm{~b}\end{array}$ & $2 \mathrm{c}$ & 3 \\
\hline SS & 0 & 0 & 0 & 0 & 0 \\
SC & 7 & 0 & 0 & 0 & 0 \\
HS & 1 & 0 & 0 & 0 & 0 \\
HC & 5 & 0 & 4 & 0 & 0 \\
HS and C & 0 & 0 & 2 & 4 & 4 \\
II & 0 & 7 & 0 & 0 & 0 \\
B & 0 & 0 & 0 & 0 & 0 \\
\hline TOTAL & 7 & 7 & 6 & 4 & 4 \\
\hline
\end{tabular}

Adjacencies

Hard cutting provides the highest adjacency probabilities, both with itself and edge-altered flakes. Note that the wear patterns are frequently identified from a formal tool both of which are in the adjacency analys is if the tool happens to be an edge-altered piece. In those cases, the adjacency analys is is also acting to associate wear patterns with a tool type. Edge-altered flakes are also frequently located next to each other. Contrary to previous experience, pure hard cutting is not so strongly juxtaposed with hard scraping and cutting. Examination of the concentrations confirms that hard cutting and hard multiple use are located in separate areas. 
It is difficult to escape the conclusion that OP 3.11 pertains to highly specialized activities being approached through a highly specialized technology. Higher resolution analysis of the wear patterns to determine exactly what the tools were being used on would be necessary to find the particular aspect of the environment with which the technology was interfacing.

\section{Occupation Plane 4.12}

OP 4.12 (Fig. 89) dates to the late early Holocene based on the upland sedimentation-erosion cycle delineated in the section on climatic change in the Southeast (page 104). It is composed of six concentrations and 53 items (tool forms and wear patterns). It is notable for the virtual absence of formal tools.

\section{Logical Analysis}

Concentration 1 is a single episode judging by the absence of chert in the charcoal and clay ball feature. A line of hard scraping and cutting flakes flanks the fire feature. Outside this is another circle composed exclusively of hard cutting. Concentration 2 is a hard cutting episode. There may have been a fire feature in its southeast corner. Concentration 3 is a cluster of hard cutting and scraping wear patterns. Charcoal and clay balls suggest a fire hearth to the north or northeast. Concentration 4 is a fragment of a possible activity area in the southeast corner of the excavation. It is a concentration composed of a fire feature with hard cutting around it. Concentration 5 is a fire feature indicated by a burned flake and a clay ball surrounded by implements of hard scraping and cutting. Concentration 6 is a concentration of hard cutting and scraping wear patterns with a fire at the southwestern end.

\section{Concentration Content}

There is little to argue that OP 4.12 is not a hard cutting floor with occasional variations toward hard scraping and cutting. Soft cutting implements are distributed more or less uniformly over the floor and two of the four boring patterns recovered appear at this leve1. Formal tools are not absent but are remarkably infrequent. Table 67 summarizes the wear pattern frequencies. 

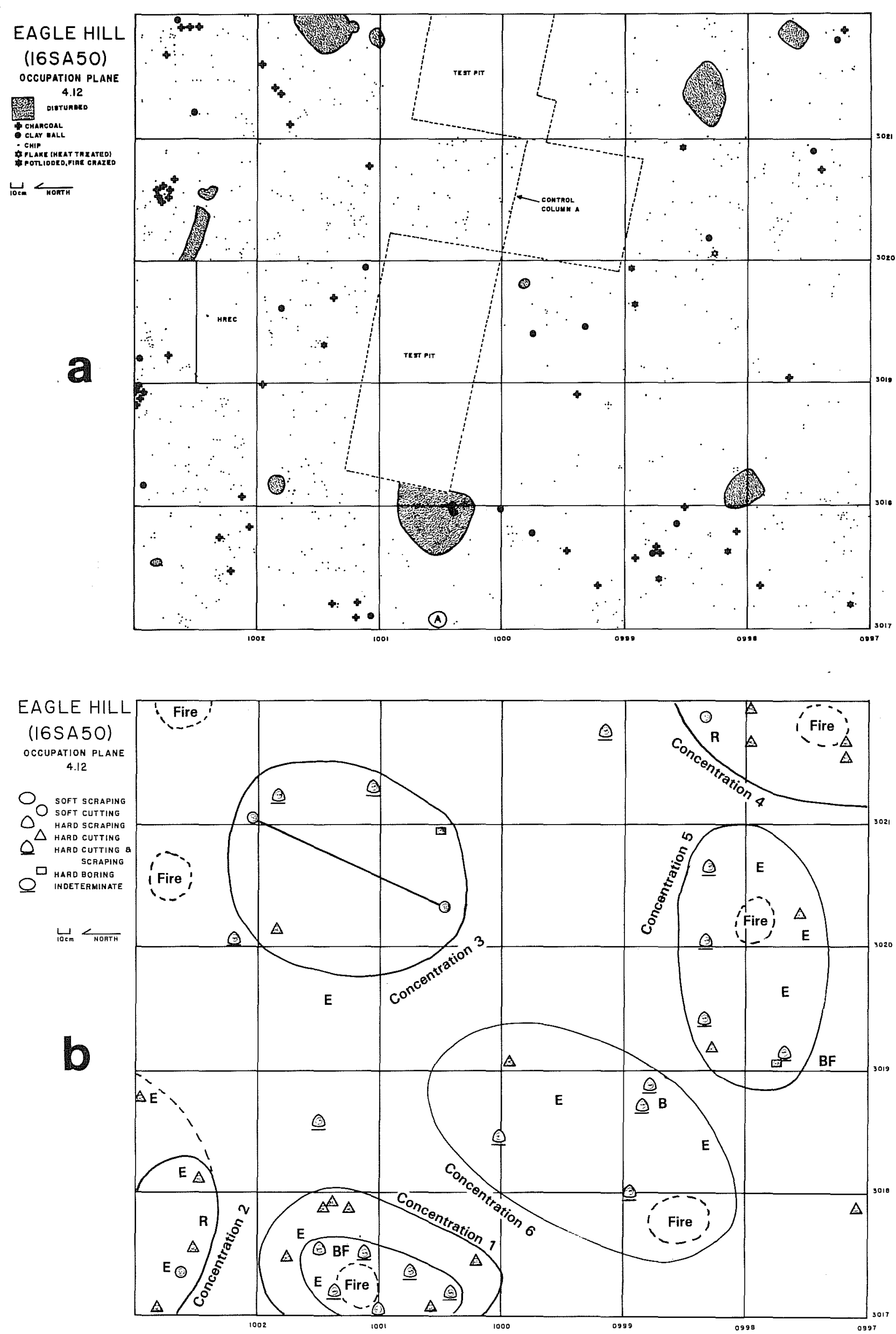

Figure 89. Map of Occupation plane 4.12. a, fire-related artifacts and chips from Occupation Plane 4.12 (see Tables 60 and 62 ); b, wear patterns and formal tools (see Table 60). 
TABLE 67. FREQUENCIES OF WEAR CATEGORIES BY CONCENTRATIONS ON OP 4.12

\begin{tabular}{lllllll} 
& \multicolumn{7}{c}{ Concentration } & 5 & 6 \\
Wear type & 1 & 2 & 3 & 4 & & 0 \\
\hline SS & 0 & 0 & 0 & 0 & 0 & 0 \\
SC & 0 & 1 & 2 & 1 & 0 & 0 \\
HS & 1 & 0 & 0 & 0 & 0 & 0 \\
HC & 6 & 3 & 1 & 4 & 2 & 4 \\
HS and C & 5 & 0 & 3 & 0 & 4 & 0 \\
II & 0 & 0 & 0 & 0 & 0 & 0 \\
B & 0 & 0 & 1 & 0 & 1 & 5 \\
\hline TOTAL & 12 & 4 & 7 & 5 & 7 & 5 \\
\hline
\end{tabular}

As is shown in Table 68, the tendency for soft scraping to occur in pairs is very high, so high in fact that it is hard to resist the temptation to draw lines between the pairs and see what results. I yielded to the temptation and connected pairs and higher sets by line. The distances between pairs of implements fall into two distinct categories. One is between 500-1000 mm. The other is between $100-200 \mathrm{~mm}$. A11 of the close sets fall on OP 2.13, while the wide sets occur on al1 floors except OP 3.11 (Table 69). OP 3.11 has only one soft cutting implement.

TABLE 68. OCCURRENCE OF SOFT CUTTING IN SETS

$\begin{array}{llll}\text { Substratum Singles } & \text { Pairs } & \text { Quadruples/ } \\ \text { Quintuples }\end{array}$

\begin{tabular}{lllll}
\hline 1.13 & 1 & 1 & 0 & 0 \\
2.13 & 4 & $4\left(B^{*}\right)$ & 2 & 1 \\
3.11 & 1 & 0 & 0 & 0 \\
4.12 & 2 & $1(B)$ & 0 & 0 \\
4.17 & 2 & 1 & $1(B)$ & 0 \\
\hline TOTALS & 10 & 7 & 3 & 1 \\
\hline B & & & \\
$*$ Borer & & & \\
\hline
\end{tabular}


TABLE 69. DISTANCES BETWEEN SETS OF SOFT CUTTING IMPLEMENTS

Close sets $\quad$ D $\quad \bar{x}$ s

\begin{tabular}{|c|c|c|c|}
\hline $\begin{array}{l}2.13 \\
2.13 \\
2.13 \\
2.13 \\
2.13 \\
2.13\end{array}$ & $\begin{array}{l}143 \\
143 \\
171 \\
200 \\
200 \\
229\end{array}$ & 181 & 35 \\
\hline Far Sets & $D$ & $\bar{x}$ & $\mathrm{~S}$ \\
\hline $\begin{array}{l}1.13 \\
2.13 \\
2.13 \\
2.13 \\
2.13 \\
2.13 \\
2.13 \\
2.13 \\
4.12 \\
4.17 \\
4.17 \\
4.17\end{array}$ & $\begin{array}{c}686 \\
543 \\
1058 \\
771 \\
771 \\
771 \\
1029 \\
942^{\star} \\
1714 \\
942 \\
571 \\
486\end{array}$ & $\begin{array}{r}1714 \\
666\end{array}$ & $\begin{array}{r}0 \\
195\end{array}$ \\
\hline
\end{tabular}

NOTE: a11 measurements in $\mathrm{mm}, \mathrm{D}=$ distance

* = distance between two close sets

Since there are so few examples to work with it would be dangerous to draw farranging conclusions from the soft cutting sets. However, a few suggestions can be made. First, the close sets fall within the span of a hand and are probably "piles" of discarded tools used in similar or the same task. This could be checked by high resolution microwear analysis. The fact that the "piles" occur on OP 2.13 and not other floors indicate longer sittings, perhaps by a specialist rather than the single use episodes represented on the other floors. This is consistent with the general, specialized character of OP 2.13.

The far sets all fall within a range larger than 400-500 $\mathrm{mm}$ and 1 ess than $1500 \mathrm{~mm}$. These distances I take to be wider than a sitting person's body, but within comfortable arm's reach. Mean distances fall exactly within the range one would expect tools to fall if they were being placed on either side of a sitting person with minimal outward movement of the arms, between 600-700 mm. The distance between pairs on OP 4.12 is outside the usual distance, but still within a comfortable arm's reach. 
Natural7y, to know the mechanisms, which caused the paired sets of soft cutting implements, would be very interesting. About half the soft cutting implements appear as singles and half of sets. It is possible that a task was being performed, which commonly, but not always, required the expenditure of more than one flake because of the dulling of the edge. I would further suggest that this task involved cutting first with the right hand and then with the left. With an equal number of cuts per hand the statistical changes are, over time, that discarded tools would appear as pairs placed on opposite sides of the artisan's sitting position. Adovasio (personal communication) knows of net making techniques that might require such movements.

Once we are informed of the artisan's body orientation by a soft cutting set, other relationships can be contemplated. Bores, for instance, appear to the front and right indicating a tool used exclusively in the right hand, perhaps set forward and in view, because of intermittent re-use rather than discard. The majority of the hard use tools appear to the front and right of the presumed, seated user. The analysis could be carried much further, but not without more intimate knowledge of their specific uses.

Other intriguing potentials present themselves. For instance, from the larger mean distances between pairs, Coles Creek people were of a larger body size than Paleo-Indians. This is counter to the findings of a recent article (Frayer 1981) on the different body styles of bowmen and spearmen, which indicates that spearmen would be larger and more linear. However, pygmies are elephant hunters and have specialized techniques to overcome the handicap of body size and turn it to advantage. It is also counter to dogma on hunter and horticultural nutritional affects on stature. With such a small sample we can at best ask questions of future data.

The absence of the soft cutting complex on OP 3.11 is a novelty. It may be that the "E-pairs" are a complementary trait that replaced them.

\section{Adjacencies}

The OP 4.12 concentrations are rather consistent in composition. Edge-altered pieces are associated with hard usage (Table 58), especially with hard cutting. The various types of hard usage cluster together. Soft cutting tends not to be associated with one or the other types of hard usage.

\section{Occupational Plane 4.17}

OP 4.17 (Fig. 90), is dated to the late Pleistocene based on the typological character of the assemblage including a Folsom-like point and by thermoluminescence dating. It is composed of seven concentrations composed of 54 items (tool forms and wear patterns). It is notable for the presence of formal tool types in addition to the usual array of wear patterns. Burned flint provides the only indication of fires. 

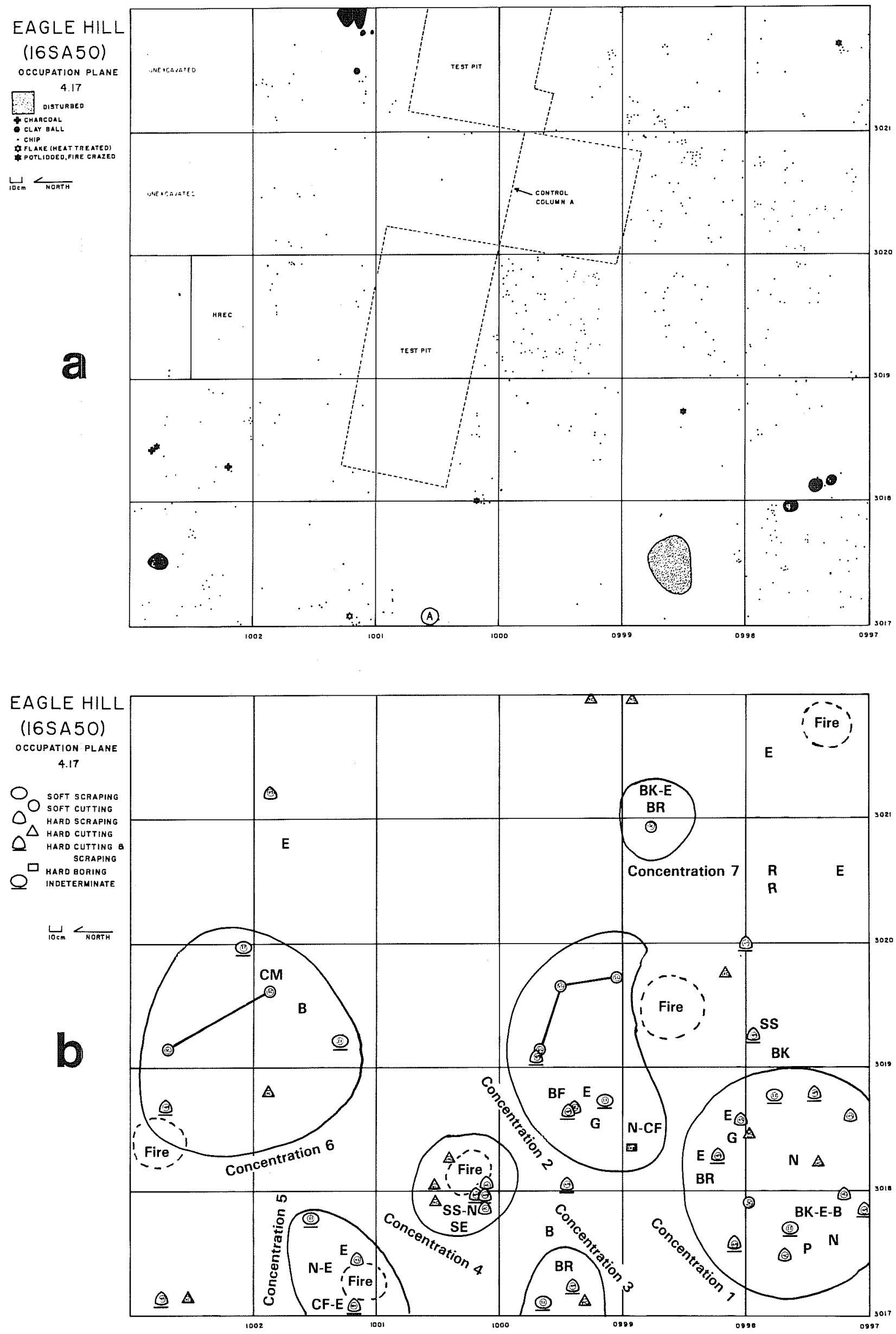

Figure 90. Map of Occupation Plane 4.17. a, fire-related artifacts and chips from Occupation Plane 4.17 (see Tables 60 and 62 ); b, wear patterns and forma 7 
Logical Analysis

Concentration 1 is circular and is composed of a complement of hard use and a diverse, formal tool kit including the Paleo-Indian point, beak, notch, burin, and graver. The combination suggests a complete, abandoned tool kit. Wear is confined, for the most part, to hard cutting and scraping. Concentration 2 is composed of diverse wear patterns reminiscent of Concentration 1 , but with a soft cutting element. It also contains a biface fragment. It appears to be associated with a fire. Concentration 3 is a hard scraping and cutting episode accompanied by a burin. Concentration 4 is a decidedly hard use episode associated with an end scraper and side scraper. If a burned flake in the middle is interpreted as indicating a fire, the tools cluster very closely on it. Perhaps fire was used in whatever craft was being practiced. Concentration 5 is another closely packed, fire-indicated concentration. Concentration 6 includes more soft cutting and more distance from the fire. Concentration 7 is a beak, burin, and soft cutting cluster (Table 70).

It is worth noting that square E3018 N0998 is void of chips and tools except for the burned fragment, while the surrounding squares are rich in artifacts and wear patterns. Its not unlikely that there was a fire in unit E3018 N0998, and Concentratjons 1,2, and 7 were the individual work areas around it.

\section{Concentration Content}

As with the other levels excepting OP 2.13 the emphasis in the Paleo-Indian period technology is clearly on the working of hard materials. Most of the soft cutting examples appear in sets as described in the OP 4.12 section. For the first time concentrations can be defined almost as much on the basis of formal tools as wear pattern.

TABLE 70. FREQUENCIES OF WEAR CATEGORIES BY CONCENTRATIONS ON OP 4.17

\begin{tabular}{llllllll} 
Wear type & 1 & 2 & 3 & 4 & 5 & 6 & 7 \\
\hline SS & 0 & 0 & 0 & 0 & 0 & 0 & 0 \\
SC & 1 & 3 & 0 & 0 & 0 & 2 & 1 \\
HS & 2 & 0 & 0 & 0 & 1 & 0 & 0 \\
HC & 4 & 1 & 1 & 5 & 0 & 1 & 0 \\
HS and C & 4 & 2 & 1 & 2 & 1 & 1 & 0 \\
II & 2 & 1 & 1 & 0 & 0 & 2 & 0 \\
Boring & 0 & 1 & 0 & 0 & 0 & 0 & 0 \\
\hline \multirow{2}{*}{ TOTALS } & 13 & 8 & 3 & 7 & 2 & 6 & 1 \\
\hline
\end{tabular}


Concentration 1 contains almost every variety of standard Paleolithic tool aside from the normally missing soft scraping; it also contains a full range of wear categories. Concentrations 2 and 6 resemble Concentration 1 except that Concentration 6 lacks the formal tools. Concentrations 2 and 6 show the soft cutting complex and thus mark the beginning of its long history. Concentrations 3, 4, and 5 appear to be specialized hard cutting and scraping loci. A11 of them contain formal tools. Concentration 4 has the notable presence of a well-made end scraper on a blade and a side scraper. Both Concentrations 4 and 5 have a peculiarly close proximity between fire and tools. Perhaps wood or bone was being charred and scraped.

\section{Adjacencies}

The adjacencies (Table 59) show that hard cutting tends to be proximate to edgealtered pieces, while hard cutting and hard scraping form a separate group. Soft scraping clusters with itself and nothing else. This is due to the fact that the soft cutting pieces are located at the periphery of the area of a dense implement concentration.

\section{Conclusions}

The adjacencies on inspection indicate the structure of the assemblages change through time. During the Paleo-Indian period there was a cleavage between the soft cutting component of the assemblage and the harder elements. As was pointed out earlier, this was a product of the soft cutting operation being located peripheral to the rest of the activity area. During the following two occupations studied, there seems to be little proclivity to separate functions, at least so far as appears from the methods used in this analysis show.

With OP 2.13, specializations return with hard and soft scraping gravitating away from hard or soft cutting. After OP 2.13, emphas is again returns to a less specialized tool kit with a definite bent toward the use of edge-altered flakes. The interest in edge-altered flakes before and after OP 2.13, along with other continuities, indicates that the upper occupation planes were occupied by the same or similar peoples. The lower archaeological zone appears to have seen a great deal of technological change from bottom to top.

An investigation of the locus of concentrations through time shows that the occupation was prone to move from southwest to northeast or generally north. This movement appears to correspond with changes in location of the high point of the mound.

Early in this presentation it was suggested that the various types of wear might be associated with categories of plant and animal tissue processing. On a somewhat more adventurous note, when the categories of application hypothesized in Table 62 are applied to the map, some interesting observations appear. For instance, the softer plant/animal tissue processing is peripheral to the rest of the activities in the Paleo-Indian camp. The next two occupations are given over almost completely to wood and bone. However, there is a hint of soft tissue processing in OP 3.11 next to the main fire. In OP 2.13 this characteristic changes radically with wood and bone being forced to the periphery 
and soft tissue processing dominating the center of the camp. After OP 2.13, the situation returns to that of the one before with soft tissue related activities limited to a spot by the fire and wood and/or bone work al1 around.

While it would be inappropriate to draw far reaching conclusions from so small a sample of activity areas, it would be equally inappropriate to not point out that this pattern of change could be the basis of future research in the westcentral Louisiana and east Texas activity area analysis. The events in the upper, Tate Holocene levels are easy enough explained. In an analysis of the division of labor in 185 primitive societies, White, Burton, Brudner, and Gunn (1975) showed that there is an overwhelming tendency cross-culturally for males to work with hard materials in the early stages of the processing trajectory. Females on the other hand tend to work with soft materials and manufacturing processes toward the end of the production trajectory. OP 4.12 interpreted from the point of view of this model, indicates a progressive increase in female influence at the site until the final level. If we assume that the female-accompanied visits are a product of increasing population pressure in the alluvial floodplains, the progression fits the general accepted scheme for demographic change in the Southeast.

The Paleo-Indian camp presents a substantially different picture from the others. There seem to be a wide range of activities, which sometimes dictate soft tissue processing near the fire, sometimes away. Each concentration suggests a radically different task. Some appear to be rather domestic activities while others indicate the proximate use of tools and fire. Further study of this very interesting circumstance would yield a wealth of information on obscure Paleo-Indian lifeways.

The Net Hypothesis

The idea that nets were being manufactured and/or repaired at Eagle Hill was not an anticipated discovery. However, there are potentially interesting explanations for such an activity, which interface comfortably with what we know of the environment of the site. The first possible explanation is that the nets were being constructed as carrying devices for nuts and other plant products. Since we suppose that the site was actively utilized only as a substation when alluvial valley populations were so large as to force exploitation of the uplands, the necessity of transporting collected foodstuffs back to the alluvial base camps is apparent.

The second possible explanation is the nets were being used to capture migratory birds. During rainy springs and falls, the flat surface of Peason Ridge takes on a swampy character if the precipitation rate is faster than the infiltration rate. During periods when climate sponsored such conditions consistently from year to year, the swamps might well become acceptable habitats for migratory birds. Spring rains would be necessary to foster the vegetation necessary to hold site deposits so such a hunting and sedimentation relationship would be completely consistent with what is known of the site sedimentological history. This includes the gleying of clays in the lower soil horizon.

Except for OP 3.11 , the soft cutting complex is present on al1 floors. The OP 2.13 floor, however, is qualitatively different in its expression of the soft cutting complex. OP 2.13 suggests long sittings in which a substantial amount of cutting was done. The other floors show evidence for only brief sittings. 
VI. CONCLUSIONS (Gunn)

\section{A. CONTEXT}

Macroenvironmentally the Eagle Hill II (16 SA 50) site is located on the northern margin of the Kisache Wold. Geomorphic forms in the immediate one kilometer or so area of the site are dominated by hilltop sands and colluvium off the hill crests. Within five kilometers the alluvial component of the landscape rises to about $20 \%$.

The site is located on the lower margin of the colluvium next to exposed Miocene clays. Water collected and stored by the sands and colluvial surfaces at the site provide a rich upland environment. The site is located midway along a colluvium-clay interface between, for humans, two nutritionally unproductive sand bodies, which provide the most efficient access to the colluvium-clay ecotone.

Studies of the colluvium indicate erosion and deposition activity during the late Quaternary. A very old soil was eroded to the B zone during the late Pleistocene. Subsequent, similar erosional episodes indicate that the erosion of the Pleistocene soil may have been associated with a warm Two Creeks interstadial. The presence of a Folsom-aged cultural industry at the end of the erosional period supports the Two Creeks erosional hypothesis.

Following the Two Creeks interstadial erosion, there was a period of colluvial deposition which appears to span the Valders substage, Preboreal interstadial, and Cochburn-Cochrane stadial intervals. Since we have no evidence of a break in deposition during the Preboreal, we must assume that conditions were moist enough, particularly in the spring, to maintain vegetation necessary to hold the sediments in place.

Following the early Holocene deposition is a long period of little or no deposition and perhaps erosion. The materials deflated onto this erosion surface appear to be of early late Holocene (after 4500 B.P., Subborea 1) vintage as judged by the archaeological diagnostics. Apparently springs were dry enough during the Roman Empire Climatic Optimum (500 B.C.-A.D. 500) to cause a net erosional balance. Deposition was resumed at the beginning of the Little Ice Age (senso lato; after 1400 B.P. or A.D. 600) and continued to the present century.

Thanks to its delicately balanced hydrology, Peason Ridge and the Eagle Hill II site in particular appear to be sensitive indicators of climatic change. This sensitivity is no doubt magnified by its location near the North American Prairie-Forest ecotone. From what is known about southeastern climatic change during the late Quaternary, it is interesting to note that the Two Creeks interstadial produced an erosional episode equivalent to the Hypsithermal and the Roman Empire Climatic Optimum. By contrast the Preboreal and Medieval Climatic Optimum (A.D. 900-1200) did not produce detectable erosional events.

Dry springs and summers with rushes of precipitation in the form of thunderstorms are most likely to produce erosion. However, whether the climatic genesis of all of the erosional episodes is due to the same combination of seasonal precipitation remains to be determined. In any event, the magnitude 
and timing of erosional events on Peason Ridge is important to the general scheme of southeastern paleoclimatology.

There are reasons to think that the character of the early Little Ice Age (A.D. 600-900) deposits is, in part, due to human intervention. The great frequency of charcoal and the high incidence of human artifacts suggest the possibility that humans contributed substantially to the rapid accumulation of deposits during that interval. Furthermore, the contributions may have come from several sources. We might add to the "dry feet phenomenon, "the idea that the site was in part a desirable place to live, because it was slightly higher than the surrounding terrain and a refuge from inclement weather. The fact that the neutron activation shows the clay balls to be from the Miocene clays, but not those directly under the site, suggest the clay balls were of human origin, perhaps carried to the site unintentionally from nearby, exposed deposits to line fireplaces.

In summary, the Eagle Hill site presents a sedimentological record that bears logical relationships to what is known of global paleoclimatology, southeastern alluvial chronology, and upland erosion. It appears to be essentially intact, although as might be expected, there is a certain amount of mixing due to anima 1 and human intervention in the depositional processes.

\section{B. CULTURE HISTORY}

The culture history of the site parallels the sedimentological history, except for the apparent deflated cultural manifestation that is associated with the early late Holocene. In the southeastern cultural chronology the period would have been associated with the Late Archaic.

Preserved human occupation of the site begins in the late Pleistocene with a Folsom-related culture. The industry is not only marked by a Folsom-like point, but it also has many other Folsom characteristics of the Texas region. Tools are predominantly unifacial and diminutive as in the Plains traditions and unlike the more robust and bifacial eastern variant in the Paleo-Indian. The assemblage does not contain any of the highly specialized scrapers characteristic of later Plains Paleo-Indian assemblages.

The geomorphic situation on Peason Ridge was different from the present in the late Pleistocene. There was no colluvium to act as an aquifer for nut-bearing trees as there was later. Certainly the impermeable clays were there. Judge (1973) has noted the tendency for Folsom sites to be located far from present day water sources in the Southwest. This probably indicates much wetter conditions than present. If it was wetter in western Louisiana, Peason Ridge may have been swampy.

The wear patterns found on the Folsom occupation floor are not unlike those determined for later periods, particularly as relates to the soft cutting sets. In the event that soft cutting was used in net making, and assuming that there were no nuts to be carried in nets, the idea that Folsom people may have been there to catch aquatic birds from swamps is not unappealing. 
Neither, for that matter, is the possibility that they were hunting bison, deer, or other large animals that prefer open habitats. A great deal is contingent on the presence or absence of seasonal swamps.

The fact that Folsom visitors to the site left tools about is highly unusual with respect to later occupations. It is not unlikely that leaving tools reflects an expectation to return to a spot on a predictable basis. The contrast is most striking between the Folsom level and the Early Archaic occupation, where no tools are left. It is generally thought in the Midsouth that the Early Archaic sported the same tool kit as the Paleo-Indians, except the point styles changed. Apparently by the early stages of the Hypsithermal people were less sure of their intentions to return to Peason Ridge.

The Early Archaic component supports the same range of use-wear evidence as the Paleo-Indian level. Formal tools, however, are massively absent. The colluvial aquifer was in place by the early Holocene so occupants of the site may have visited the site for plant resources and hunting.

The Late Archaic seems to be deflated onto the interface between the early and late Holocene deposits. Since we were not interested in disturbed material, the interface was removed as an intermediate level at one meter resolution. There was, however, a Late Archaic point associated with the interface. Also, Servel10 (n.d.) reports that the debitage from the interface zone clusters with the late rather than early Holocene deposits.

A number of concurrent processes are evident in the late Holocene. Technologically, there is a shift from dart to arrow points, through a series of transformations that appear to be related to a rather lengthy period of adjustment to the new weapons system. There are few hunting weapons, however, and the bulk of the assemblage is comprised of simple cutting and scraping implements.

There is a clear trajectory of change in the frequencies of use-wear categories. The late Holocene begins with a sparsely cluttered camp that is dominated by hard wear. In about a century this pattern shifts to one in which soft cutting is strongly represented. As in other levels the soft cutting is organized in pairs separated by the width of a sitting person's body. However, rather than being single specimens there are triples. This is taken to mean that whatever was being done to produce the peculiar arrangements of soft cutting wear patterns was now done in sittings three times as long as in the smaller, more ephemeral camps. If the task was net making, three times more nets were being constructed or repaired. Since this period corresponds with maximum populations in the alluvial valleys in the Southeast, the increased activity was probably a product of overexploitation of the alluvial floodplains and consequent appeal to upland habitats for relief.

With the onset of the main stage of the Little Ice Age (A.D. 1250), populations declined in the Southeast and Eagle Hill returned to its former status as an occasionally visited, outlying camp.

Visual and neutron activation studies of ceramics and cherts found at the site indicate multiple sources of materials in both instances. These studies appear in a more detailed format in Brown (1981). Future research should be directed 
toward the prospect that Eagle Hill was part of the settlement patterns in the Sabine and/or Red Rivers during the various prehistoric periods.

\section{RECOMMENDATIONS}

Area $B$ was determined to be a very low productivity archaeological deposit and geomorphically equivalent to the upper soil zone in Area A after thorough testing and augering. In my judgement, it would be fruitless to pursue any further work in Area B.

The Area A excavation removed about $90 \%$ of the available deposits in the uppermost levels. Remaining deposits are progressively larger toward the bottom of the site, because the road cut and stumping operation destroyed the upper leve1s. As nearly as we can determine, given that our estimates are handicapped by the low incidence of artifacts in the site, it seems likely that about $50-60 \%$ of the in situ sediments from the lower part of the site have been removed by the efforts of the project. It would add to our knowledge of the Paleo-Indian occupation to recover the remainder of the site. However, the risk of a return season being unproductive is so great due to the proximity of the stumping operation that I cannot recommend further work in Area A.

While there is no reason for National Register status for the site, it is customary to preserve portions of important sites for future reference. The long chronology and unique upland locale of Eagle Hill will make it an important reference point in the future of west-central Louisiana archaeology. While the essential archaeological value of the site has been recovered, both the site and the surrounding habitat are in danger of being destroyed by the erosive effects of the water directed into the saddle by the road coming from the airstrip. In the opinion of one of our geologists, the road is responsible for the erosion of the site. It is possibly clay-lined and certainly compacted to the point that it prevents downward percolation of surface water. Thus, sediments, which have withstood 10,000 years, are suddenly eroding away. It seems likely that both the site and the habitat could be saved by terraces perpendicular to the road, which would disperse the running water and let it soak into the sandy sediments to the side of the road as it has done for 10,000 years. 


\section{REFERENCES CITED}

Adam, D. P.

1975 Ice-Ages and the Thermal Equilibrium of the Earth, II. Quaternary Research 5(2):161-171.

Adovasio, J. M., J. D. Gunn, J. Donahue, and R. Stuckenrath

1978 Meadowcroft Rockshelter, 1977: An Overview. American Antiquity $43(4): 632-651$.

Ange 11, J. K. and J. Korshover

1978 Global Temperature Variation, Surface-100-MB: An Update Into 1977. Monthly Weather Review 106(6):755-770.

Anderson, H. V.

1960 Geology of Sabine Parish. Louisiana Geological Survey, Bulletin 34.

Andrews, J. T. and G. H. Miller

1978 Was There a Late-Wisconsinan Laurentide Ice-Sheet? American Quaternary Association: Abstracts 157. University of A1berta, Edmonton, Alberta.

Aten, L. E. and C. N. Bollich

1969 A Preliminary Report on the Development of a Ceramic Chronology for the Sabine Lake Area of Texas and Louisiana. Bulletin of the Texas Archeological Society 40:241-258.

Baker, V. R. and M. M. Penteado-Orellana

1977 Adjustment to Quaternary Climatic Change by the Colorado River in Central Texas. Journal of Geology 85:395-422.

Ballard, R. D. and E. Uchupi

1970 Morphology and Quaternary History of the Continental Shelf of the Gulf Coast of the United States. Bulletin of Marine Science 20(3):547-559.

Be11, R. E. and D. A. Baerreis

1951 A Survey of Oklahoma Archeology. Bulletin of the Texas Archeological and Paleontological Society 22:7-100.

Benfer, R. A. and A. N. Benfer

1981 Automatic Classification of Inspectional Categories: Multivarate Theories of Archaeological Data. American Antiquity 46(2):381-396. 
Bense, J.

1982 Areal Site Location Data. In A Study of Late Quaternary Environments and Early Man Along the Tombigbee River, ATabama and Mississippi, edited by G. R. Muto and J. Gunn:4-1 to 4-23. Benham Group, Oklahoma City, Oklahoma (in press).

Bentha11, J. L. and B. C. McCary

1973 The Williamson Site, A New Approach. Archaeology of Eastern North American, Eastern States Archaeological Federation 1(1).

Berger, W. H.

1969 Ecologic Patterns of Living Planktonic Foraminifera. Deep Sea Research 16:1-24.

Bernabo, J. and T. Webb III

1977 Changing Patterns in the Holocene Pollen Record of Northeastern North America - A Mapped Summary. Quaternary Research 8(1):64-96.

Bernard, H. A. and R. J. LeBTanc

1965 Resume of the Quaternary Geology of the Northwest Gulf of Mexico Province. In The Quaternary of the United States, edited by H. E. Wright, Jr. and D. G. Frey:137-185. Princeton University Press, Princeton.

Binford, L. R.

1970 Archaeology at Hatchery West. Memoirs of the Society for American Archaeology 24.

1978 Nunamiut Ethnoarchaeology. Academic Press, New York.

Black, C. C.

1974 History and Prehistory of the Lubbock Lake Site. The Museum Journal XV.

BTack, S. L.

1978 Archaeological Investigations at the Banquete Bend Site (41 NU 63), Nueces County, Texas. Center for Archaeological Research. The university of Texas at San Antonio, Archaeological Survey Report 63.

Blakeman, C. H., Jr.

1975a Archaeological Investigations in the Upper Tombigbee Valley, 1974 Season. Department of Anthropology, Mississippi State University. 
Blakeman, C. H., Jr., (continued)

1975b A Cultural Resource Survey of the Aberdeen Lock and Dam and Canal Section Areas of the Tennessee-Tombigbee Waterway: 1975. Department of Anthropology, Mississippi State University.

Blasing, T. J.

1975 Methods for Analysing Climatic Variations in the North Pacific Sector and Western North America for the Last Few Centuries. Ph.D. dissertation, University of Wisconsin. University Microfilms, Ann Arbor.

Borns, H. W., Jr.

1966 The Geography of Paleo-Indian Occupation in Nova Scotia. Quaternaria 8:49-57.

Brakenridge, G. R.

1980 Widespread Episodes of Stream Erosion during the Holocene and Their Climatic Cause. Nature 283:655-656.

Braun, E. L.

1950 Deciduous Forests of North America. Macmillan, New York.

Brose, D. S. and G. W. Percy

1978 Mississippian Settlement Patterns. Fort Walton Settlement Patterns: 81-108.

Brose, D. S. and J. F. Scarry

1976 The Boston Ledges Shelter: Comparative Spatial Analyses of Early Late Woodland Occupations in Summit County, Ohio. Midcontinental Journal of Archaeology 1(2):179-228.

Brown, C. A.

1972 Wildflowers of Louisiana and Adjoining States. Louisiana State University Press, Baton Rouge, Louisiana.

Brown, D. 0.

1981 Variability in Raw Material Resource Utilization at a Late Quaternary Archaeological Site in Upland West Central Louisiana. M.A. thesis, The University of Texas at Austin.

1983 The Berger Bluff Site (41 GD 30A): Excavations in the Upper Deposits, 1979. Center for Archaeological Research, The university of Texas at San Antonio, Archaeological Survey Report 115. 
Brown, J. A.

1975 Deep-Site Excavation Strategy as a Sampling Problem. In Sampling in Archaeology, edited by J.W. Muel1er:155-169. University of Arizona Press.

Broyles, B. J.

1971 Second Preliminary Report: The St. Albans Site, Kanawha County, West Virginia. West Virginia Geological and Economic Survey, Report of Archeological Investigations 3.

Bryan, A. L.

1977 Developmental Stages and Technological Traditions. In Amerinds and Their Paleoenvironments in Northeastern North America, edited by W. S. Newman and B. Sa7wen:355-368. Annals of the New York Academy of Sciences 288.

Bryant, V. M., Jr.

1977 A 16,000 Year Pollen Record of Vegetational Change in Central Texas. Palynology 1:143-156.

Bryson, R. A.

1966 Air Masses, Streamlines, and the Boreal Forest. Geographical Bulletin 8(3):228-269.

Bryson, R. A., D. A. Baerreis, and W. M. Wendland

1968 The Character of Late Glacial and Postglacial Climatic Changes. In Pleistocene and Recent Environments of the Central Great Plains, edited by W. Dort, Jr. and J. K. Jones, Jr.:53-74. Department of Geology, University of Kansas Press, Special Publication 3.

Bryson, R. A. and T. J. Murray

1977 Climates of Hunger: Mankind and the Changing weather. University of Wisconsin Press, Madison.

Bryson, R. A. and W. M. Wendland

1967 Tentative Climatic Patterns for Some Late Glacial and Postglacial Episodes in Central North America. In Life, Land, and water, edited by $W$. Mayer-Oakes:271-298. University of Manitoba Press, Winnipeg.

Budyko, M. I.

1974 Climate and Life. Academic Press, New York. 
Bullen, R. P.

1969 A Clovis Fluted Point from the Santa Fe River, Florida. Florida Anthropologist $22: 36$.

Bullen, R. P., D. S. Webb, and B. I. Waller

1970 A Worked Mammoth Bone from Florida. American Antiquity 35:203205.

Butzer, K.

1964 Environment and Archaeology. University of Chicago Press.

1980 Civilizations: Organisms or Systems? American Scientist 68(5): 517-523.

Byers, D. S.

1954 Bu11 Brook - A Fluted Point in Ipswich, Massachusetts. American Antiquity 19(4):343-351.

1966 The Debert Archaeological Project: The Position of Debert with Respect to the Paleo-Indian Tradition. Quaternaria 8:33-47.

Cable, J. S. and R. Most

1979 The Bipolar Technique, Error or Adaptation? Paper presented at The Society for American Archaeology.

Cahen, D., L. H. Keeley, and F. L. Van Noten

1979 Stone Tools, Tool Kits, and Human Behavior in Prehistory. Current Anthropology 20(4):661-683.

Cambron, J. W. and D. C. Hulse

1960 An Excavation on the Quad Site. Tennessee Archaeologist 16(1): 14-269.

Carbone, V. A.

1976 Environment and Prehistory in the Shenandoah Valley. Ph.D. dissertation, Catholic University of America, Washington, D.C.

Carneiro, R. L.

1970 Theory of the Origin of the State. Traditional Theories of State Origins are Considered and Rejected in Favor of a New Ecological Hypothesis. Science 169:733-738. 
Carr, K. W.

1974 The Fifty Site; A Stratified Early Archaic Processing Station. In The Flint Run Paleo-Indian Complex: A Preliminary Report, 19711973 Seasons, edited by W. M. Gardner. Department of Anthropology, Catholic University of America, Occasional Publication 1.

Carter, G. F.

1978 The American Paleolithic. In Early Man in America from a CircumPacific Perspective, edited by A. Bryon:10-19. Department of Anthropology, University of Alberta, Archaeologic Research International, Occasional Papers 1. Edmonton, Alberta.

Chapman, J. B.

1973 The Icehouse Bottom Site 40 MR 23. Department of Anthropology, University of Tennessee, Report of Investigations 13.

1975 The Rose Island Site. Department of Anthropology, University of Tennessee, Report of Investigations 14.

Chisom, M.

1968 Rural Settlement and Land Use. 2nd edition. Hutchinson, London. Christenson, L. and D. W. Read

1977 Numerical Taxonomy, R-Mode Factor Analys is and Archaeological Classification. American Antiquity 42(2):163-179.

Churcher, C. S.

1978 Did the North American Mammoth Migrate? Canadian Journal of Anthropology 1(1):103-105.

Cleland, C. E.

1976 The Focal Diffuse Mode1: An Evolutionary Perspective on the Prehistoric Cultural Adaptations of the Eastern United States. Midcontinent Journal of Archaeology 1(1):59-76.

\section{CLIMAP}

1976 The Surface of the Ice-Age Earth. Science 191(4232):1131-1137. Coe, J. L.

1964 The Formative Cultures of the Carolina Piedmont. Transactions of the American Philosophical Society New Series 54(2):5. 
Corbin, J. E.

1963 Archaeological Materials from the Northern Shore of Corpus Christi Bay, Texas. Bulletin of the Texas Archeological Society 34:5-30.

Cornwa11, I. W.

1958 Soils for the Archaeologist. Macmi1lan, New York.

Crabtree, D. and R. B. Butter

1964 Notes on Experiment in Flintknapping: Heat Treatment of Silica Materials. Tebiwa 7(1)1-6.

Curray, J. R.

1960 Sediments and History of Holocene Transgression, Continental Shelf, Northwest Gulf of Mexico. In Recent Sediments, Northwest Gulf of Mexico, edited by F. P. Shepard, F. B. Phleger, and

T. H. van Andel. Scripps Institution of Oceanography, American Association of Petroleum Geologist, Tulsa.

1965 Late Quaternary History, Continental Shelves of the United States. In The Quaternary of the United States, edited by H. E. Wright, Jr. and D. G. Frey:723-736. University of Princeton Press, Princeton.

Cwynar, L. D., E. Burden, and J. H. McAndrews

1979 An Inexpensive Sieving Method for Concentrating Pollen and Spores from Fine-Grained Sediments. Canadian Journal of Earth Sciences 16:1115-1120.

Dahl in, B. A.

1980 Climate and Ancient Maya Civilization: A Trial Balloon. Paper read at the 1980 Conference of the American Anthropological Association.

Davis, E. M.

1953 Recent Data From Two Paleo-Indian Sites on Medicine Creek, Nebraska. American Antiquity 18:380-386.

Davis, M. B.

1969 Palynology and Environmental History During the Quaternary Period. American Scientist 3:317-322.

Davis, W. A. and E. M. Davis

1960 The Jake Martin Site: An Archaic Site in the Ferrell's Bridge Reservoir Area, Northeastern Texas. Department of Anthropology, The University of Texas at Austin, Archeology Series 3. 
DeJarnette, D. L., B. Kurjack, and J. W. Cambron

1962 Stanfield-Worley Bluff Shelter Excavations. Journal of Alabama Archaeology 8(1 and 2):1-24.

Delcourt, H. R.

1979 Late Quaternary Vegetation History of the Eastern Highland Rim and Adjacent Cumberland PTateau of Tennessee. Ecological Monographs $49(3): 255-280$.

Delcourt, P. A.

1980 Goshen Springs: Late Quaternary Vegetation Record for Southern A1abama. Ecology 61:371-386.

Delcourt, P. A. and H. R. Delcourt

1979 Late Pleistocene and Holocene Distributional History of the Deciduous Forest in the Southeastern United States. Veroff. Geobot. Inst. 68:79-107.

Delcourt, P. A., H. R. Delcourt, R. C. Brister, and L. Lackey

1980 Quaternary Vegetation History of the Mississippi Embayment. Quaternary Research 13:111-132.

Denton, G. and W. Karlén

1973 Holocene Climatic Variations - Their Patterns and Possible Cause. Quaternary Research 3(2):155-205.

Dibble, D. S. and D. Lorraine

1968 Bonfire Shelter: A Stratified Bison Kill Site. Val Verde County, Texas. Texas Memorial Museum, Miscellaneous Papers 1.

Dixon, W. J. and M. B. Brown

1977 Biomedical Computer Programs P-Series. University of California Press, Berkeley.

Dragoo, D. W.

1965 Investigations at a Paleo-Indian Site in Stewart County, Tennessee. Eastern States Archaeological Federation, Bulletin 24:12-13.

1973 Wel1s Creek - An Early Man Site in Stewart County, Tennessee. Archaeology of Eastern North America 1(1):1-56. 
Dragoo, D. W. (continued)

1976 Some Aspects of Eastern North American Prehistory, A Review 1975. American Antiquity 47:3-27.

Dreimanis, A.

1977 Late Wisconsin Glacial Retreat in Their Great Lakes Region, North America. In Amerinds, and The ir Paleoenvironments in Northeastern North America, edited by $W$. Newman and B. Salwen:70-89. Annals of the New York Academy of Sciences 288.

Duffield, L. F.

1963 The Wolfshead Site: An Archaic-Neo-American Site in San Augustine County, Texas. Bulletin of the Texas Archeological Society $34: 83-141$.

Dzerdzeevskij, B. L.

1968 Circulation Mechanisms in the Hemisphere in the 20th Century

(Translation by R. Goedecke), edited by B. F. Berryman.

Institute of Geography, Soviet Academy of Sciences, Moscow.

Eddy, J. A.

1977a The Case of the Missing Sunspots. Scientific American 236(5):80-92.

1977b Historical Evidence for the Existence of the Solar Cycle. In The Solar output and Its Variation, edited by 0. R. White:51-72. Colorado Association University Press, Boulder.

Eidt, R. C.

1977 Detection and Examination of Anthrosols by Phosphate Analysis. Science 197:1327-1333.

Ewing, M., D. B. Ericson, and B. C. Heezen

1958 Sediments and Topography of the Gulf of Mexico. In Habitat of Oil, edited by L. G. Weeks:995-1053. American Association of Petroleum Geologists, Tulsa.

Faegri, K. and J. Iversen

1974 Textbook of Pollen Analysis. Munksgaard, Copenhagen.

Fitting, J. E.

1968 Environmental Potential and The Post-Glacial Readaptation in Eastern North America. American Antiquity 33(4):441-444. 
Fitting, J. E. (continued)

1969 Settlement Analysis in the Great Lakes Region. Southwestern Journal of Anthropology 25(4):360-377.

1974 Climatic Change and Cultural Frontiers in Eastern North America. The Michigan Archaeologist 21(1):25-39.

Fitting, J. E., J. DeVissher, and E. J. Wahla

1966 The Paleo-Indian Occupation of Holcombe Beach. Museum of Anthropology, University of Michigan, Anthropological Papers 27.

Fladmark, K. R.

1979 Routes: Alternate Migration Corridors for Early Man in North America. American Antiquity 44(1):55-69.

Fleetwood, A. R.

1969 Geological Investigation of the Ouachita River Area, Lower Mississippi Valley. U. S. Army Corps of Engineers, Waterways Experiment Station, Technical Report S-69-2. Vicksburg.

Flint, R. F. and B. J. Skinner

1974 Physical Geology. 2nd edition. Wiley and Sons, New York.

Folan, W. J., J. Gunn, J. D. Eaton, and R. Patch

1982 Paleoclimatological Patterning in Southern Mesoamerica; The Political and Socioeconomic Prehistory and History of the Lowland Maya. Manuscript accepted for publication by Journal of Field Archaeology. On file; The University of Texas at San Antonio.

Ford, J. A. and C. H. Webb

1956 Poverty Point: A Late Archaic Site in Louisiana. American Museum of Natural History. Anthropological Paper 46:136.

Fowler, M. L.

1952 The Clear Lake Site: A Hopewellian Occupation. Hopewellian Communities in Illinois, edited by $T$. Deuel. Illinois State Museum Scientific Papers 5.

Fox, A. A., S. L. Black, and S. R. James

1979 Intensive Survey and Testing of Archaeological Sites on Coleto Creek, Victoria and Goliad Counties, Texas. Center for Archaeological Research. The University of Texas at San Antonio, Archaeological Survey Report 67. 
Frayer, D. W.

1987 Body Size, Weapon Use, and Natural Selection in the European Upper Paleolithic and Mesolithic. American Anthropologist 83(1):57-73.

Frazier, M.

1982 Paleontology Study. In A Study of Late Quaternary Environments and Early Man Along the Tombigbee River, Alabama and Mississippi, edited by G. R. Muto and J. Gunn:C-91. Benham Group, OkTahoma City, Oklahoma (in press).

Frye, J. C.

1961 Fluvial Deposition and the Glacial Cycle. Journal of Geology 69:600-603.

Frye, J. C. and A. B. Leonard

1963 Pleistocene Geology of Red River Basin, Texas. Bureau of Economic Geology. The University of Texas at Austin, Report of Investigations 49.

Funk, R. E., G. R. Walters, and W. F. Ehlers

1969 A Radiocarbon Date for Early Man from the Dutchess Quarry Cave. New York State Archaeological Association Bulletin 46:19-21.

Gagliano, S. M.

1967a Occupation Sequence at Avery Island. Louisiana State University, Coastal Studies Series 22.

1967b Late Archaic-Early Formative Relationships in Southern Louisiana. Proceedings of the 23rd Southeastern Archaeological Conference, Bulletin 6.

1970 Archaeological and Geological Studies at Avery Island. Coasta1 Studies Institute, Louisiana State University, Baton Rouge.

1977 Prehistoric Cultural Resource Potential (Vol. 1). In Cultural Resources Evaluation of the Northern Gulf of Mexico Continental Shelf. National Park Service (Cultural Resource Management Studies). United States Department of the Interior.

Gagliano, S. M. and H. F. Gregory

1965 A Preliminary Survey of Paleo-Indian Points from Louisiana. Louisiana Studies $4(1): 62-77$. 
Gagliano, S. M. and B. G. Thom

1967 Deweyville Terrace, Gulf and Atlantic Coasts. Coastal Studies Institute, Louisiana State University, Technical Report 39. Baton Rouge.

Galloway, W. E.

1977 Catahoula Formation of the Texas Coastal Plain. Bureau of Economic Geology. The University of Texas at Austin, Report of Investigations 87.

Gardner, W. M.

1972 Some Thoughts Concerning Paleo-Indian in the Eastern Woodlands, Including a Proposed Model Based on Excavations at the Thunderbird Site. Paper presented at the Eastern States Archaeological Federation.

1976 Paleo-Indian to Early Archaic: Continuity and Change in Eastern North America During the Late Pleistocene and Early Holocene. Proceedings of the 9th International congress of Prehistoric and Protohistoric Science. Universite de Nice, France.

Gardner, W. M., (ed.)

1974 The Flint Run Paleo-Indian Complex: A Preliminary Report, 1971-1973 Seasons. Archeology Laboratory, Department of Anthropology, Catholic University of America, Occasional Publication 1. Washington, D.C.

Gardner, W. M. and R. A. Verrey

1979 Typology and Chronology of Fluted Points from the Flint Run Area. Pennsylvania Archaeologist 49(1):13-46.

Gates, W. L.

1972 Modeling the Ice-Age C1imate. Science 191:1138-1144.

Gerstle, A., T. C. Kelly, and C. Assad

1978 The Fort Sam Houston Project: An Archaeological and Historical Assessment. Center for Archaeological Research, The University of Texas at San Antonio, Archaeological Survey Report 40.

Gibson, J. L.

1974 Poverty Point: The First American Children. Archaeology 27(2):97-105.

Goodyear, A. C.

1974 The Brand Site: A Techno-Functional Study of a Dalton Site, Northeast Arkansas. Arkansas Archaeological Survey Research Series 7. 
Goodyear, A. C. (continued)

1979 A Hypothesis for the Use of Cryptocrystalline Raw Materials Among Paleo-Indian Groups of North America. Institute of Anthropology, University of South Carolina, Research Manuscript Series 157.

1982 The Chronological Position of the Dalton Horizon in the Southeastern United States. American Antiquity 42(2):382-395.

Graham, R. W., C. V. Haynes, D. L. Johnson, and M. Kay

1981 Kimmswick: A Clovis-Mastodon Association in Eastern Missouri. Science 213(4):1115-1117.

Greenman, N.M. and R. J. LeBlanc

1956 Recent Marine Sediments and Environments of the Northwest Gulf of Mexico. American Association of Petroleum Geologists Bulletin 40:813-847.

Griffin, J. B.

1978 Eastern United States. In Chronologies in New World Archaeology, edited by R. E. Taylor and C. W. Meighan:51-70. Academic Press, New York.

Gross, J. I.

1974 Some Patterns of Chipping Activity of the Thunderbird Clovis Component. In The Flint Run Paleo-Indian Complex: A Preliminary Report, 1971-1973 Seasons. Archeology Laboratory, Department of Anthropology, Catholic University of America, Occasional Publication 1. Washington, D.C.

Guderjan, T. H. and J. R. Morehead

n.d. $\quad 16$ VN 24: A Stratified Multiple Component Site on Big Bushy Creek. Fort Polk Archaeological Survey, A. F. Servello (assembler). Fort Worth Coe District.

Gunn, J.

1982a Aero-Solar Independence: A Theory of Temperature-Moisture Response to External Forcing Variables. In a Study of Late Quaternary Environments and Early Man Along the Tombigbee River, Alabama and Mississippi, edited by G. R. Muto and J. Gunn:B-56 to B-77. Benham Group, OkTahoma City, Oklahoma (in press).

1982b Projecting the Model: Prehistoric Environments. In a Study of Late Quaternary Environments and Early Man Along the Tombigbee River, Alabama and Mississippi, edited by G. R. Muto and J. Gunn: 2-26 to 2-31. Benham Group, Ok1ahoma City, Ok1ahoma (in press). 
Gunn, J. (continued)

1982c An Estimate of Global Energy Budget for the Late Quaternary. In A Study of Late Quaternary Environments and Early Man Along the Tombigbee River, Alabama and Mississippi, edited by G. R. Muto and J. Gunn:B-82 to B-100. Benham Group, Ok1ahoma City, Ok1ahoma (in press).

1982d Analysis of Modern Climatic Data. In A Study of Late Quaternary Environments and Early Man Along the Tombigbee River, Alabama and Mississippi, edited by G. R. Muto and J. Gunn:B-197 to B-211. Benham Group, Oklahoma City, Oklahoma (in press).

1982e General Coastal Seasonal Dynamics Climatic Model. Manuscript on file; Center for Archaeological Research, The University of Texas at San Antonio.

Gunn, J. and R. E. W. Adams

1981 Climatic Change, Culture, and Civilization in North America. World Archaeology 13(1):87-100.

Gunn, J. and E. R. Prewitt

1975 Automatic Classification Projectile Points from West Texas. Plains Anthropologist 20(68):139-149.

Gunn, J., T. R. Hester, R. Jones, R. L. Robinson, and R. A. Mahula

1979 Climatic Change in Southern Texas. Appendix VII in Archaeological Investigations at Choke Canyon Reservoir, South Texas: The Phase I Findings, by G. D. Ha11, S. L. B1ack, and C. Graves:578-596. Center for Archaeological Research. The University of Texas at San Antonio, Choke Canyon Series 5.

Gunn, J., R. A. Mahula, and J. B. Sollberger

1976 The Sollberger Distribution. La Tierra 3(4):2-8.

Gunn, J. and R. A. Mahula (eds.)

1977 Hop Hi11: Culture and Climatic Change in Central Texas. Center for Archaeological Research, The University of Texas at San Antonio, Special Report 5.

Gunn, J. and R. R. Stuckenrath

1982 A Calculated C-14 Correction Curve for the Late Quaternary. Manuscript in preparation. 
Gunn, J. and D. R. White

1977 Clustering of Technological Types (Non-Linear Technospatial Analysis, (Resolv 4). In Hop Hill: Culture and Climatic Change in Central Texas, edited by J. Gunn and R. A. Mahula:209-221. Center for Archaeological Research. The University of Texas at San Antonio, Special Report 5.

Haag, W. G.

1965 Louisiana in North American Prehistory. Louisiana Studies Institute, Northwestern State College, Louisiana Studies 4(4):279-323.

Hanson, G. T. and A. C. Goodyear III

1976 The Shared-Tool Method of Spatial Analysis: Applications at the Brand Site. Institute of Archaeology and Anthropology, University of South Carolina, Columbia.

Haury, E. W.

1953 Artifacts with Mammoth Remains, Naco, Arizona. In Discovery of the Naco Mammoth and the Associated Projectile Points. American Antiquity 19(1):1-14.

Haynes, V. C.

1975 Pleistocene and Recent Stratigraphy. In Late Pleistocene Environments of the Southern High Plains, edited by W. Dort, Jr. and J. K. Jones, Jr.:77-99. Department of Geology, University of Kansas Press, Special Publication 3.

1978 The Clovis Culture. Canadian Journal of Anthropology 1(1):115-121.

Haynes, C. V. and E. T. Hemmings

1968 Mammoth Bone Shaft Wrench from Murray Springs, Arizona. Science 159:186-187.

Heizer, R. F.

1937 Baked Clay Objects of the Lower Sacramento Valley, California. American Antiquity 3(1):34-50.

Hester, J. J. and J. Grady

1977 Paleo-Indian Social Patterns on the Llano Estacado. The Museum Journal XVII. Texas Tech University, Lubbock. 
Hester, J. J. and F. Wendorf

1975 Paleoarchaeology of the Llano Estacado. In Late Pleistocene Environments of the Southern High Plains, edited by F. Wendorf and J. J. Hester:290. Fort Burgwin Research Center, Rancho de Taos, New Mexico.

Hester, T. R.

1971 Loyola Beach: An Example of Aboriginal Adaptation to the Maritime Environment of the Lower Texas Coast. Florida Anthropologist $24(3): 91-106$.

Irwin, H. T.

1971 Developments in Early Man Studies in Western North America. Arctic Anthropology 8(2):42-67.

Irwin, H. T. and H. M. Wormington

1970 Paleo-Indian Tool Types in the Great Plains. American Antiquity $35(1): 24-34$.

Jennings, J. D. (ed.)

1978 Ancient Native Americans. W. H. Freeman, San Francisco. Jensen, H. P.

1968 Coral Snake Mound, 16 SA 48. Bulletin of the Texas Archeological Society 39:9-44.

Johnston, J.

1972 Econometric Methods. McGraw Hill.

Jones, R.

1979 Recent Crop Yields and Climate in Texas. Working Papers Number 5, The Impact of Climatic Change Project. Research by the Center for Archaeological Research, The University of Texas at San Antonio for the Ewing Halsell Foundation of San Antonio and the United States Bureau of Reclamation.

Judge, W. J.

1973 Paleo-Indian Occupation of the Central Rio Grande Valley in New Mexico. University of New Mexico Press, Albuquerque. 
Katz, P. R.

1976 A Technological Analysis of the Kansas City Hopewell Chipped Stone Industry. Ph.D. dissertation, University of Kansas.

Keeley, L. H.

1980 Experimental Determination of Stone Tool Use. University of Chicago Press.

Kennett, J. P. and P. Huddleston

1972 Late Pleistocene Paleoclimatology, Foraminiferal Biostratigraphy and Tephrochronology, Western Gulf of Mexico. Quaternary Research 2(1):38-69.

King, J. E. and W. H. Allen, Jr.

1977 A Holocene Vegetation Record from the Mississippi River Valley, Southeastern Missouri. Quaternary Research 8:307-323.

K1 inger, T. C. (assembler)

1977 Contract Archaeology in the Lower Mississippi Valley of Arkansas. Miscellaneous Papers, Arkansas Archaeological Survey, Research Report 12. Fayetteville.

Kraft, H. C.

1973 The Plenge Site: A Paleo-Indian Occupation Site in New Jersey. Archaeology of Eastern North America 1(1):56-118.

Krumbein, W. C. and L. L. SToss

1963 Stratigraphy and Sedimentation. W. H. Freeman.

Kuchler, A. W.

1964 Potential Natural Vegetation of the Conterminous United States. American Geographical Society, Special Publication 36.

Kuk1a, G. J.

1975 Missing Link Between Milankovitch and Climate. Nature 253(5493):600-603.

Lamb, H. H. and A. Woodroffe

1970 Atmospheric Circulation During the Last Ice Age. Quaternary Research 1(1):29-58. 
Lawrence, R. A.

1979 Experimental Evidence for the Significance of Attributes Used in Edge-Damage Analysis. In Lithic Use-Wear Analysis, edited by B. Hayden:113-121. Academic Press, New York.

Lee, R. B.

1969 !Kung Bushman Subsistence: An Input-Output Analysis. In Environment and Cultural Behavior, edited by A. Vayda:47-79. Natural History Press.

Leonhardy, F. C.

1966 Domebo: A Paleo-Indian Mammoth Kill in the Prairie Plains. Contributors of the Museum of the Great Plains 1.

Leroi-Gourhan, A.

1966 Habitation Magdalenieene No. 1 de Pincevent. Pres Monteran (Seine-Et-Marne), Gallia Prehistorie IX(2).

Levert, C. F., Jr.

1959 Lower Catahoula Equivalents of Louisiana. Unpublished thesis, Louisiana State University.

Lewis, T. N. and M. K. Lewis

1961 Eva: An Archaic Site. University of Tennessee Press.

Long, R. J.

1977 McFaddin Beach. The Pattillo Series of Natural History and Anthropology 1.

Luchterhand, $K$.

1970 Early Archaic Projectile Points and Hunting Patterns in the Lower Illinois Valley. Illinois Archaeological Survey Monograph 2.

de Lumley, H. (ed)

1969 Une Cabane Acheuleenne Dans La Grotte Du Lazaret. Centre National de la Recherche Scientifique, Memoirs de la Societe Prehistorique Francaise 7.

Lundelius, E. L., Jr.

1974 The Last Fifteen Thousand Years of Faunal Change in North America. In History and Prehistory of the Lubbock Lake Site. The Museum Journal XV:141-160. 
MacDonald, G. F.

1968 Debert: A Paleo-Indian Site in Central Nova Scotia. National Museum of Canada, Anthropology Papers 11(16).

1971 A Review of Research on Paleo-Indian in Eastern North America. Arctic Anthropology 8(2):32-41.

MacNeish, R. S.

1952 A Possible Early Site in the Thunder Bay District. Annual Report of the National Museum for the Fiscal year 1950-1951. Bulletin 126.

1971 Early Man in the Andes. In Early Man in America. Readings from Scientific American, edited by R. S. MacNeish:143-154. W. H. Freeman, San Francisco.

Mahula, R. A.

1982a Rise and Fall of Sea Levels in the Gulf of Mexico. In A Study of Late Quaternary Environments Along the Tombigbee River, Alabama and Mississippi, edited by G. R. Muto and J. Gunn:B-186 to B-196. Benham Group, Oklahoma City, Oklahoma (in press).

1982b Late Quaternary of the Gulf of Mexico: 25,000 B.P.-Present. In A Study of Late Quaternary Environments and Early Man Along the Tombigbee River, A7abama and Mississippi, edited by G. R. Muto and J. Gunn:B-157 to B-173. Benham Group, Ok1ahoma City, Ok1ahoma (in press).

Malmgren, B. and J. P. Kennett

1976 Principal Component Analys is of Quaternary Planktic Foraminifera in the Gulf of Mexico: Paleoclimatic Applications. Marine Micropaleontology $1: 299-306$.

Manabe, S. and D. G. Hahn

1977 Simulation of the Tropical Climate of an Ice Age. Journal of Geophysical Research 82(27):3889-3911.

Martin, P. S.

1973 The Discovery of America. Science 179(4077):969-974.

Mason, R. J. and C. Irwin

1960 An Eden-Scottsbluff Burial in Northeastern Wisconsin. American Antiquity 26:43-59. 
Mayer-Oakes, W. J.

1951 Starved Rock Archaic, A Pre-Pottery Horizon from Northern I11inois. American Antiquity 16(4):313-324.

McCary, B. C.

1951 A Workshop of Early Man in Dinwidde County, Virginia. American Antiquity 17(1):9-17.

McFarlan, E., Jr.

1961 Radiocarbon Dating of Late Quaternary Deposits, South Louisiana. Geological Society of America, Bulletin 72(1):129-158.

McGuff, P. R.

1978 Prehistoric Archaeological Investigations at Palmetto Bend Reservoir: Phase 1, Jackson County, Texas. Texas Archeological Survey, The University of Texas at Austin, Research Report 58.

McMillan, B.

1976 Rodgers Shelter - A Record of Cultural and Environmental Change. In Prehistoric Man and His Environment, A Case Study in the Ozark Highlands, edited by W. R. Woods and R. B. McMi11an:111-122. Academic Press.

Milankovitch, M.

1930 Handbuch der Klimatologie. Verlag Brontrager, Berlin.

Mitche11, J. M., C.W. Stockton, and D. M. Meko

1979 Evidence of a 22-Year Rhythm of Drought in the Western United States Related to the Hale Solar Cycle Since the 17th Century. In Solar-Terrestrial Influences on Weather and Climate, edited by B. McCormac and Seliga. D. Reidel Publishing Co., Holland.

Monseth, A.

1967 The Betty Green Site, A Late Paleo-Indian Site in Eastern Wyoming. M.A. thesis, University of Pennsylvania.

Morehead, J. R. and T. H. Guderjan

n.d. An Overview of the Prehistory of Louisiana. In Fort Polk Archaeological Survey, assembler A. F. Servello. Submitted to the Fort Worth Coe District. 
Morse, D. F.

1971 Recent Indication of Dalton Settlement Patterns in Northeast Arkansas. Southeastern Archaeological Conjerence, Bulletin $13: 5-10$.

Morse, D. F. and A. Goodyear

1973 The Significance of the Da1ton Adz in Northeast Arkansas. Plains Anthropologist 18:316-322.

Muller, J. D.

1978 The Southeast. In Ancient Native Americans, edited by J. D. Jennings:281-326. W. H. Freeman and Company, San Francisco.

Muto, G. R. and J. Gunn (eds.)

1982 A Study of Late Quaternary Environments and Early Man Along the Tombigbee River, Alabama and Mississippi. Benham Group, OKlahoma City, Ok1ahoma (in press).

Namias, J.

1976 Negative Ocean Air Feedback Systems Over the North Pacific in the Transition from Warm to Cold Seasons. Monthly Weather Review $104(9): 1107-1121$.

Nei11, W. T.

1958 A Stratified Early Site at Silver Springs, Florida. The Florida Anthropologist 11(2):33-49.

Neumann, A. C.

1958 The Configuration and Sediments of Stetson Bank, Northwestern Gulf of Mexico. Texas A\&M University, Department of Oceanography and Meteorology, Project 24.

Newman, W. S. and B. Salwen

1977 Amerinds and Their Paleoenvironments in Northeastern North America. Annals of the New York Academy of Sciences 288.

Nials, F.

1982 The Geosciences Perspective. In A Study of Late Quaternary Environments and Early Man Along the Tombigbee River, Alabama and Mississippi, edited by G. R. Muto and J. Gunn:3-1 to 3-77. Benham Group, Oklahoma City, Oklahoma (in press). 
Nie, N. H., C. H. Hull, J. G. Jenkins, K. Steinbrenner, and D. H. Brent

1975 Statistical Package for the Social Sciences. Second Edition. McGraw-Hi11, New York.

Nriagu, J. 0.

1976 Phosphate-Clay Relations in Soils and Sediments. Canadian Journal of Earth Sciences 13:717-736.

Ode11, G.

1975 Micro-Wear in Perspective: A Sympathetic Response to Lawrence H. Kelley. World Archaeology 7:226-240.

01 sen, R. A.

1975 Rate of Dissolution of Phosphate from Minerals and Soils. Proceedings: Soil Science Society of America 39:634-639.

Orton, R. B.

1964 The Climate of Texas and Adjacent Gulf waters. United States Department of Commerce, Washington, D.C.

Otvos, E. G.

1975 Southern Limits of Pleistocene Loess, Mississippi Valley. Southern Geology $17(1)$.

Patrick, W. H., Jr. and R. A. Khatid

1974 Phosphate Release and Sorption by Soils and Sediments: Effect of Aerobic and Anaerobic Conditions. Science 186:53-55.

Patterson, L. W.

1979 A Review of the Prehistory of the Upper Texas Coast. Bulletin of the Texas Archeological Society 50:103-124.

Pennak, R. W.

1953 Freshwater Invertebrates of the United States. Ronald Press, New York.

Phillips, P.

1970 Archaeological Survey in the Lower Yazoo, Mississippi, 1949-1955. Papers of the Peabody Museum of Archaeology and Ethnology 60. Harvard University, Cambridge, Massachusetts.

Poag, C. W.

1973 Late Quaternary Sea Levels in the Gulf of Mexico. American Association of Petroleum Geologists, Bulletin 57:1836-1873. 
Prewitt, E.

1974 Archeological Investigations at the Loeve-Fox Site, Williamson County, Texas. Texas Archeological Survey, Research Report $49: 1-147$.

Price, G. R. D. and L. Heartfield

1977 Archaeological Test Excavation of Site 16 ou 15 Located on the Monroe to Sancy Bayou Levees, Quachita Parish, Louisiana. The Research Institute, College of Pure and Applied Sciences, Northeast Louisiana University, Monroe, Louisiana.

Reagan, M., R. Rowlett, E. Garrison, W. Dort, Jr., V. Bryant, and C. Johannsen

1978 Flake Tools Stratified Below Paleo-Indian Artifacts. Science 200:1272-1275.

Reiter, E. R.

1961 Jet Stream Meteorology. University of Chicago Press, Chicago.

Ritchie, W. A.

1953 A Probable Paleo-Indian Site in Vermont. American Antiquity $18(3): 249-258$.

1969 The Archaeology of New York State. Revised 2nd Edition. Natural History Press, New York.

Ritchie, W. A. and R. E. Funk

1973 Aboriginal Settlement Patterns in the Northeast. The University of New York, New York.

Robinson, R.

1978 Biosilica and Climatic Change at 41 GD 21 and 41 GD $21 \mathrm{~A}$. Appendix IV in Archaeological Investigations of Two Prehistoric Sites on the Coleto Creek Drainage, Goliad County, Texas, by D. E. Fox:83-102. Center for Archaeological Research, The University of Texas at San Antonio, Archaeological Survey Report 69.

Rodgers, C. L.

1968 The Habron Site, Warren County, Virginia. Quarterly Bulletin of the Archaeological Society of Virginia 23(2):90-98.

Sahlins, M.

1972 Stone Age Economics. Aldine, Chicago.

Saltzman, B. and A. D. Verneker

1975 A Solution for the Northern Hemisphere Ciimatic Zonation During a G1acial Maximum. Quaternary Research 5(3):307-320. 
Sanchez, W. A. and J. E. Kutzbach

1974 Climate of the American Tropics and Subtropics in the 1960s and Possible Comparisons with Climatic Variations in the Last Millennium. Quaternary Research 4(2):128-135.

Saucier, R. T.

1967 Geological Investigations of the Boeuf-Tensas Basin, Lower Mississippi Valley. U.S. Army Corps of Engineers, waterways Experiment Station, Technical Report 3:757. Vicksburg.

1974 Quaternary Geology of the Lower Mississippi Valley. Arkansas Archaeological Survey, Research Series 6.

1978 Sand Dunes and Related Eolian Features of the Lower Mississippi River Alluvial Valley. Geoscience and Man XIX:23-40.

Saucier, R. T. and A. R. Fleetwood

1970 Origin and Chronologic Significance of Late Quaternary Terraces, Ouachita River, Arkansas and Louisiana. Geological Society of America, Bulletin 81:869-890.

Schiffer, M. B.

1975 The Effects of Occupation Span on Site Content. In The Cache River Archaeological Project, edited by M. B. Schiffer and J. H. House:283-288. Arkansas Archaeological Survey, Research Series 8.

Schumm, S. A.

1965 Quaternary Paleohydrology. In The Quaternary of the United States, edited by H. E. Wright, Jr. and D. G. Frey:783-794. Princeton University Press, Princeton.

Scurlock, J. D.

1961 The Culpepper Site, A Late Fulton Aspect Site in Northeastern Texas. Bulletin of the Texas Archeological Society 32:285-316.

Sellards, E.

1938 Artifacts Associated with Fossil Elephant. Geological Society of America, Bulletin 49:999-1009.

Semenov, S. A.

1964 Prehistoric Technology. (Translated by M. W. Thompson.) Cory, Adams and Mackay, London. 
Serve110, F.

n.d. Attribute Analyses and Cultural Resources Management at Fort Polk. Report prepared for Interagency Archeological Services, Atlanta.

Shackley, M. L.

1975 Archaeological Sediments: A Survey of Analytical Methods. Ha1sted, New York.

Shafer, H. J.

1975 Comments on Wood1and Cultures of East Texas. Bulletin of the Texas Archeological Society 46:249-254.

Shafer, H. J. and R. G. Holloway

1977 Organic Residue Analysis and Stone Tool Function from Hinds Cave, Val Verde County, Texas: A Progress Statement. In Archeological and Botanical Studies at Hinds Cave, Val Verde County, Texas, by H. J. Shafer and V. Bryant, Jr.:103-128. Anthropology Laboratory, Texas A\&M University, Special Series 1.

Shepard, A. 0.

1965 Ceramics for the Archaeologist. Carnegie Institution of washington, Publication 609(5).

Sjoberg, P., N. E. Smeck, and E. C. A. Runge

1971 Phosphorus Availability and Redistribution in Relation to Profile Development in an I11inois Landscape Segment. Proceedings, Soil Science Society of America 35:952-959.

Smeck, N. E.

1933 Phosphorus: An Indicator of Pedogenic Weathering Processes. Soil Science 115:199-206.

Snowden, J. 0., Jr. and R. R. Priddy

1968 Geology of the Mississippi Loess. Bulletin of the Mississippi Geological Survey 3.

Sollberger, J. B. and T. R. Hester

1972 The Strohacker Site: A Review of the Pre-Archaic Manifestation in Texas. Plains Anthropologist 17(58):326-344.

Sorenson, C. J., R. Mandel, and J. Wall is

1976 Change in Bioclimate Inferred from Paleosols and Paleohydrologic Evidence in East Central Texas. Journal of Biogeography 3:141-149. 
South, S.

1970 Baked Clay Objects from the Site of the 1670 Settlement at Charles Towne, South Carolina. Institute of Archaeology and Anthropology, The University of South Carolina, Notebook 2(1): 3-16.

Stalker, A. M.

1977 Indications of Wisconsin and Earlier Man from the Southwest Canadian Prairies. In Amerinds and Their Paleoenvironments in Northeastern North America, by W. S. Newman and B. Salwen: 179-136. Annals of the New York Academy of Sciences 288.

Stapor, F. W. and W. F. Tanner

1977 Late Holocene Mean Sea Level Data from St. Vincent Island and the Shape of the Late Holocene Mean Sea Level Curve. In Coastal Sedimentology, edited by W. F. Tanner:35-68. Geology Department, Florida State University.

Struever, S. and K. D. Vickery

1973 The Beginnings of Cultivation in the Midwest-Riverine Area of the United States. American Anthropologist 75(5):1197-1220.

Strahler, A. N.

1969 Introduction to Physical Geography. 3rd edition. John Wiley \& Sons, Inc., New York.

Stuckenrath, R. S.

1965 The Debert Archaeological Project: Radiocarbon Dating. Paper presented at the VII Congress of the International Association for Quaternary Research.

Suhm, D. A. and E. B. Jelks

1962 Handbook of Texas Archeology: Type Descriptions. Texas Archeological Society Special Publication 1 and Texas Memorial Museum Bulletin 4. Austin.

Suhm, D. A., A. D. Krieger, and E. B. Jelks

1954 An Introductory Handbook of Texas Archeology. Bulletin of the Texas Archeological Society 25.

Terry, R. D. and G. V. Chilingar

1955 Summary of "Concerning Some Additional Aids in Studying Sedimentary Formations," by M. S. Shuetsov:229-234. Journal of Sedimentary Petrography 25(3). 
Thom, B. G.

1967 Coastal and Fluvial Landforms, Horry and Marion Counties, South Carolina. Coastal Studies Institute, Louisiana State University, Technical Report 4.

Tunne11, C. D.

1975 Fluted Projectile Point Production as Revealed by Lithic Specimens from the Adair-Steadman Site in Northwest Texas. Texas Historical Commission, office of the State Archeologist, Special Report 18.

Van Noten, F., D. Cahen, and L. Keeley

1980 A Paleolithic Campsite in Belgium. Scientific American 242(4): 48-55.

Verrey, R. A.

1980 Lithic Analysis of Areas 1A and 1B of the Thunderbird Site. Dissertation proposal, Catholic University of America, Washington, D.C.

Viellard, P., Y. Tardy, and D. Nahon

1979 Stability Fields of Clays and Aluminum Phosphates: Paragenes is in Lateritic Weathering of Argillaceous Phosphatic Sediments. American Mineralogist 64:626-634.

Vita-Finzi, C. 0. and E. S. Higgs

1974 The Rudacil Site, Its Relationship to the Thunderbird Site. In The Flint Run Paleo-Indian Complex: A Preliminary Report, 19711973 Seasons, edited by W. M. Gardner:130-137. Archeology Laboratory, Department of Anthropology. The Catholic University of America, Occasional Publication 1. Washington, D.C.

Weaver, E. C.

1963 Technological Analysis of Prehistoric Mississippi Ceramic Materials: A Preliminary Report. American Antiquity 29(1): 49-56.

Webb, C. H.

1948 Evidence of Pre-Pottery Cultures in Louisiana. American Antiquity 13:227-232.

Webb, C. H., J. Shiner, and E. Roberts

1971 The John Pearce Site (16 CD 56): A San Patrice Site in Caddo Parish, Louisiana. Bulletin of the Texas Archeological Society $42: 1-49$. 
Webb, C. H., F. E. Murphey, W. G. Ellis, and H. R. Green

1969 The Resch Site 41 HS 16, Harrison County, Texas. Bulletin of the Texas Archeological Society 40:3-106.

Webb, W. S.

1951 The Parrish Village Site. The University of Kentucky, Reports in Anthropology 7(6):407-461.

Weir, F. A.

1975 The Central Texas Archaic. Unpub7ished Ph.D. dissertation, Washington State University, Pul1man.

Welch, R. N.

1942 Geology of Vernon Parish. Department of Conservation, Louisiana Geological Survey, Geological Bulletin 22. Baton Rouge.

Wend 7 and, W. M.

1977 Tropical Storm Frequencies Related to Sea Surface Temperatures. Journal of Applied Meteorology 16:477-481.

Wendorf, F.

1975 Summary and Conclusions in Pleistocene Environment of the Southern High Plains. Fort Burgwin Research Center, Taos, New Mexico.

What1on, R., Jr.

1973 Spatial Analysis of Occupation Floors I: Application of Dimensional Analysis of Variance. American Antiquity 38(3): 266-278.

1974 Spatial Analysis of Occupation Floors II: The Application of Nearest Neighbor Analysis. American Antiquity 39(1):16-34.

White, D., M. Burton, L. Brudner, and J. Gunn

1975 Implicational Structures in the Sexual Division of Labor. School of Social Sciences, University of California-Irvine, working papers 83.

Whitehead, D. R.

1982 Late Quaternary Vegetation in the Southeast. In A Study of Late Quaternary Environments and Early Man Along the Tombigbee River, Alabama and Mississippi, edited by G. R. Muto and J. Gunn: B-266 to B-279. Benham Group, Ok1ahoma City, Oklahoma (in press). 
Whitehead, D. R. and M. Sheehan

1982 Postglacial Vegetational and Environmental Changes Near Columbus, Mississippi, Preliminary Results. In A Study of Late Quaternary Environments and Early Man Along the Tombigbee River, Alabama and Mississippi, edited by G. R. Muto and J. Gunn:B-280 to B-306. Benham Group, Oklahoma City, Oklahoma (in press).

Wigley, T. M. L., P. D. Jones, and P. M. Kelly

1980 Scenario for a Warm, High- $\mathrm{CO}_{2}$ World. Nature 283:17-21.

Willey, G. R.

1966 An Introduction to American Archaeology. Prentice-Ha11, Inc., Englewood Cliffs, New Jersey.

Willey, G. R. and P. Phillips

1958 Method and Theory in American Archaeology. University of Chicago Press.

Williams, J. R.

1974 The Baytown Phases in the Cairo Lowlands of Southeast Missouri. Missouri Archaeologist 36.

Wilmsen, E. N.

1970 Lithic Analysis and Cultural Inference: A Paleo-Indian Case. Anthropological Papers of the University of Arizona 6. Tucson.

Witthoff, $\mathrm{J}$.

1952 A Paleo-Indian Site in Eastern Pennsylvania an Early Hunting Culture. Proceedings of the American Philosophical Society $96: 464-495$.

Wood, R. W. and R. B. McMil1an (eds.)

1976 Prehistoric Man and His Environments. Academic Press, New York.

Woodward, T. P. and A. J. Gueno

1940 The Sand and Gravel Deposits of Louisiana. Louisiana Geological Survey, Bulletin 19.

Wormington, H. M.

1957 Ancient Man in North America. Denver Museum of Natural History, Popular Series 4.

Yellen, J. E.

1977 Archaeological Approaches to the Present. Academic Press, New York. 
APPENDIX A:

pH AND PHOSPHATE TESTING PROCEDURE 
$\mathrm{pH}$ Paper Test

The first series of $\mathrm{pH}$ paper tests were performed on one soil sample from each core from the contact of the interface of sand and bedrock clay. The second series of tests were run on the samples in each core above and below the contact zone samples.

Lab procedure for both series consisted of taking one half gram of each soil sample crushed and placed in a test tube with $5 \mathrm{ml}$ of distilled water. Two drops of a saturated solution of potassium chloride and distilled water were added and the samples stirred and allowed to settle. The potassium chloride solution aids in particle settling and acid release from clay particles. $\mathrm{pH}$ 4.0-7.0 paper was placed in the water and immediately removed and graded with the color chart provided with the paper. The results were recorded on previously formatted code sheets.

\section{pH Meter Test}

The second series of $\mathrm{pH}$ tests were performed on selected soil core columns using a Corning Model $20 \mathrm{pH}$ meter calibrated to $\mathrm{pH} 7.0$ at $26^{\circ} \mathrm{C}$ between each five sample tests. Each sample was prepared by crushing $5 \mathrm{~g}$ of soil and mixing this with $25 \mathrm{ml}$ of distilled water in a 30-ml beaker. After sediment had settled, the $\mathrm{pH}$ level was measured with the meter and recorded on specially prepared code sheets. The meter probe was placed in the solution above the sediment and rinsed and dried between each test. Each sample was then buffered by mixing with two drops of the same saturated potassium chloride solution used in the paper test and allowed to settle. A second reading was taken with the meter and these $\mathrm{pH}$ levels recorded. Noted was a substantial increase of acid content after the potassium chloride solution was added and the $\mathrm{pH}$ level measured.

\section{Phosphate Spot Test}

The first series of phosphate spot tests were performed on several selected core columns using one gram of soil. The soil was placed on filter paper and two drops of reagent " $A$ " added and allowed to react for 30 seconds. Two drops of reagent " $B$ " were added. A judgmental evaluation of phosphate content was made at the end of a two-minute interval according to intensity of blue on the filter paper. Grading was done on a one to six scale on a comparative basis between all the samples tested. A second series of phosphate spot tests were run identical with the first, except that grading was limited to a value of one or two. The results of the first series of tests were made compatible with the second test by changing values between one and three to a value of one and values between four and six to a value of two. This regrading was done because tests in the first and second series were run on different days, and test results are not valid after five minutes due to phosphates in the air. The two series also had to be made statistically compatible. 
Reagent " $\mathrm{A}$ " was prepared in a $100-\mathrm{ml}$ beaker by dissolving 5.0 grams of ammonium molybdate in approximately $50 \mathrm{ml}$ of distilled water and slowly adding $30 \mathrm{ml}$ of $6 \mathrm{~N}$ hydrochloride acid. The mixture was then poured into a graduated 100-m1 cylinder and brought to $100 \mathrm{ml}$ with distilled water. The solution was poured into a 250-ml bottle and stored when not in use.

Reagent "B" was prepared in a $100-\mathrm{ml}$ beaker by dissolving 0.5 grams of granular ascorbic acid in $35 \mathrm{ml}$ of gently swirled distilled water. The solution was poured into an unused graduated $100-\mathrm{ml}$ cylinder and brought to $100 \mathrm{ml}$ with distilled water. The solution was poured into a 250-m7 bottle and kept capped when not in use. Solution reagent "B" must be remade daily as ascorbic acid neutralizes in 24 hours. 

APPENDIX B:

SITE FORMS 
EAGLE HILL (16 SA 50) PROJECT

Center for Archaeological Research-UTSA

PHYSICAL UNIT RECORD

Data Recovery and Prompting Device

$\underline{0} 2$ Record Number

$$
\text { _. }-2 \text { FN \# }
$$

\section{$1 \quad 1-2$}

Before Excavation

$\begin{array}{rr}3 & 9-13 \\ 4 & 14-19 \\ 5 & 20-25\end{array}$

$6 \quad 26-28$

$\begin{array}{ll}7 & 29-30\end{array}$

$8 \quad 31$

9 32-34

$\begin{array}{ll}10 & 35-37\end{array}$

$11 \quad 38-40$

$12 \quad 41$

$13 \quad 42-43$

$14 \quad 44-45$

$15 \quad 46$

$16 \quad 47-48$

$$
\begin{aligned}
& \text { _. } \ldots \text { Substratum (m) } \\
& \text { East (m) SW corner } \\
& \text { - North (m) SW corner } \\
& \begin{array}{l}
\text { Size of unit }(\mathrm{cm}) \\
-- \text { Level thickness }(\mathrm{cm}) \text { average }
\end{array} \\
& \text { - Level type (1=natura 1, 2=arbitrary, 3=cultural) } \\
& \text { _ _ STope (dip, degrees, 0=horizontal) } \\
& \text { - - Direction of slope (strike, degrees) } \\
& -- \text { - Hue (number) } \\
& \begin{aligned}
& \text { - Hue (alpha: 1=y, 2=yr, } 3=r, 4=g, 5=n, 6=b g, \\
&- \text { Value } \\
& \text { - } \text { Chroma } \\
& \text { - Constant Volume Sample ( } 1=\text { absent, } 2=\text { present) } \\
& \text { - Texture }
\end{aligned}
\end{aligned}
$$$$
\begin{aligned}
& 1=c 1 \text { ay } \\
& 4=\text { sandy clay loam } \\
& 7=\text { sandy loam } \\
& 10=1 \text { oamy sandy }
\end{aligned}
$$$$
17 \quad 49
$$$$
\text { After Excavation }
$$$$
1850
$$$$
19 \quad 51
$$$$
20 \quad 52
$$$$
21 \quad 53
$$$$
22 \quad 54
$$$$
\begin{array}{rr}
23 & 55-56 \\
24 & 57 \\
25 & 58 \\
26 & 59
\end{array}
$$$$
27 \text { 60-61 }
$$$$
28 \text { 62-63 }
$$$$
29 \text { 64-65 }
$$$$
30 \quad 66-67
$$

$$
\begin{array}{ll}
2=\text { sandy clay } & 3=\text { silty clay } \\
5=\text { clay loam } & 6=\text { silty clay loam } \\
8=1 \text { loam } & 9=\text { silt loam } \\
11=\text { silt } & 12=\text { sand }
\end{array}
$$

$$
\begin{aligned}
& \text { _ Consolidation (1=usual, 2=unusual) } \\
& \text { Charcoal flecks ( } 1=\text { absent, } 2=\text { present) } \\
& \text { - Aquatic she11 frags (1=absent, } 2=\text { present) } \\
& \text { - Land snail frags (1=absent, 2=present) } \\
& \text { - Chipped stone (1=absent, } 2=\text { present) } \\
& \text { - Extent of excavation ( } 0=n 0,1=N, 2=E, 3=S, 4=W \text { ) } \\
& \text { - Recorder } \\
& \text { - Mapped (1=no, 2=yes) } \\
& \text { - Tagged (1=no, 2=yes) } \\
& \text { - Depth to Top on Transit Shot Format (1=no, 2=yes) } \\
& \text { _- Inspected (Inspector \#) } \\
& - \text { Day } \\
& \text { - Month } \\
& \text { - - Year }
\end{aligned}
$$

Excavator Photo \#s

Excavator Photo \#s

Excavator Photo \#s Excavator Photo \#s

Figure 91. Physical unit Record. 
EAGLE HILL (16 SA 50) PROJECT

Center for Archaeological Research-UTSA

CULTURE UNIT RECORD

Data Recovery and Prompting Device

$\begin{array}{rr}1 & 1-8 \\ 2 & 9-13 \\ 3 & 14-18 \\ & \\ 4 & 19-24 \\ 5 & 25-30 \\ 6 & 31-36 \\ 7 & 37-38 \\ 8 & 39-42 \\ 9 & 43-46 \\ 10 & 47-50 \\ 11 & 51-53 \\ 12 & 54-56 \\ & \\ 13 & 57-61 \\ 14 & 62-64 \\ 15 & 65 \\ & \\ 16 & 66-67 \\ 17 & 68-69 \\ 18 & 70-71 \\ 19 & 72-73\end{array}$

03

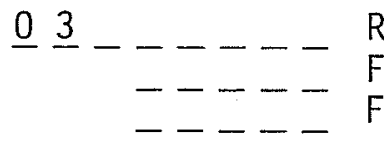

$---\cdot--$
$----\cdot]$
$--\ldots$

East (m) from SW corner

North (m) from SW corner

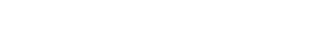

$-\ldots$
$-\cdot---$
$-\cdot---$
---
$-\ldots$

Datum

$\mathrm{E} / \mathrm{W}$ Length

N/S Length

Thickness

Direction, downhi11, $\mathrm{E}$ of $\mathrm{N}$

Slope ( 0 =horizonta 1, 90=down)

$-\ldots-$
$-\ldots$
-

Occupation plane (rel. microstr.)

Cultural Type

- Artifact labeled ( $1=N A, 2=0 n$ artifact, 3=on bag,4=on artifact and bag)

- Inspected (Inspector \#)

- Day

- Month

- Year

\section{Recorder}

\section{Excavator}

\footnotetext{
Cultura1 Types (May 80)

8 Charcoal

10 Fire Feature

11 Reddened Rock

12 Reddened Stone Conc.

13 Burned Limestone

14. Burned Sandstone

15 Boiled Stone

20 Storage Feature

30 Hammerstone

31 Chip

32 Chunk

33 Core

34 Core fragment

35 Core, edge altered

36 Flake, decortification

37 Flake

38 Flake, pressure

39 Flake, retouched

40 Flake, edge altered

41 Flake, bif. thinning

42 Flake concentration

43 Heat spal1
}

50 Blade

51 Blade fragment

52 Blade, retouched

53 Blade, edge altered

60 Quarry blank

61 Chopper

62 Biface

63 Biface fragment

70 Point

90 Scraper

91 Scraper, side

92 Scraper, end

93 Scraper, side and end

94 Scraper, ovate

95 Scraper, circular

96 Scraper, thumbnail

97 Scraper, misc.

110 Perforator

120 Engraver

130 Burin

131 Burin Spal1

140 Notch
150 Denticulate

160 Gouge

170 Metate

171 Metate fragment

172 Mano

173 Mano fragment

180 Ceramics

190 Clay objects

200 Glass

300 Rock

301 Sandstone

302 Limestone

310 Nodule

311 Nodule concentration

400 She11, mussel

401 She11, snail

500 Bone

501 Post-skeletal

510 Skin

520 Turtle Carapace

600 Meta 1

999 Other

Figure 92. Culture unit Record. 


\section{UNIT MAPPING RECORD}

$\begin{array}{ll}1 & 1-2 \\ 2 & 3-8 \\ 3 & 9-10\end{array}$

$4 \quad 11-15$

$5 \quad 16-18$

$6 \quad 19-20$

$\begin{array}{ll}7 & 21-23\end{array}$

$8 \quad 24-25$

$9 \quad 26-27$

$10 \quad 28-29$

$\square=5 \mathrm{~mm}^{2}=$
1 Record \#

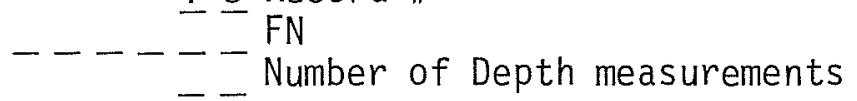

$\begin{aligned} & \text { Substratum } \\ --\cdot & - \\ & -- \text { Plan photo number } \\ & -- \text { Inspected (Inspector \#) } \\ & - \text { Scale }(5 \mathrm{~mm}=\mathrm{x})\end{aligned}$

Coder
- Day

$\overline{8} \underline{0}$ Year

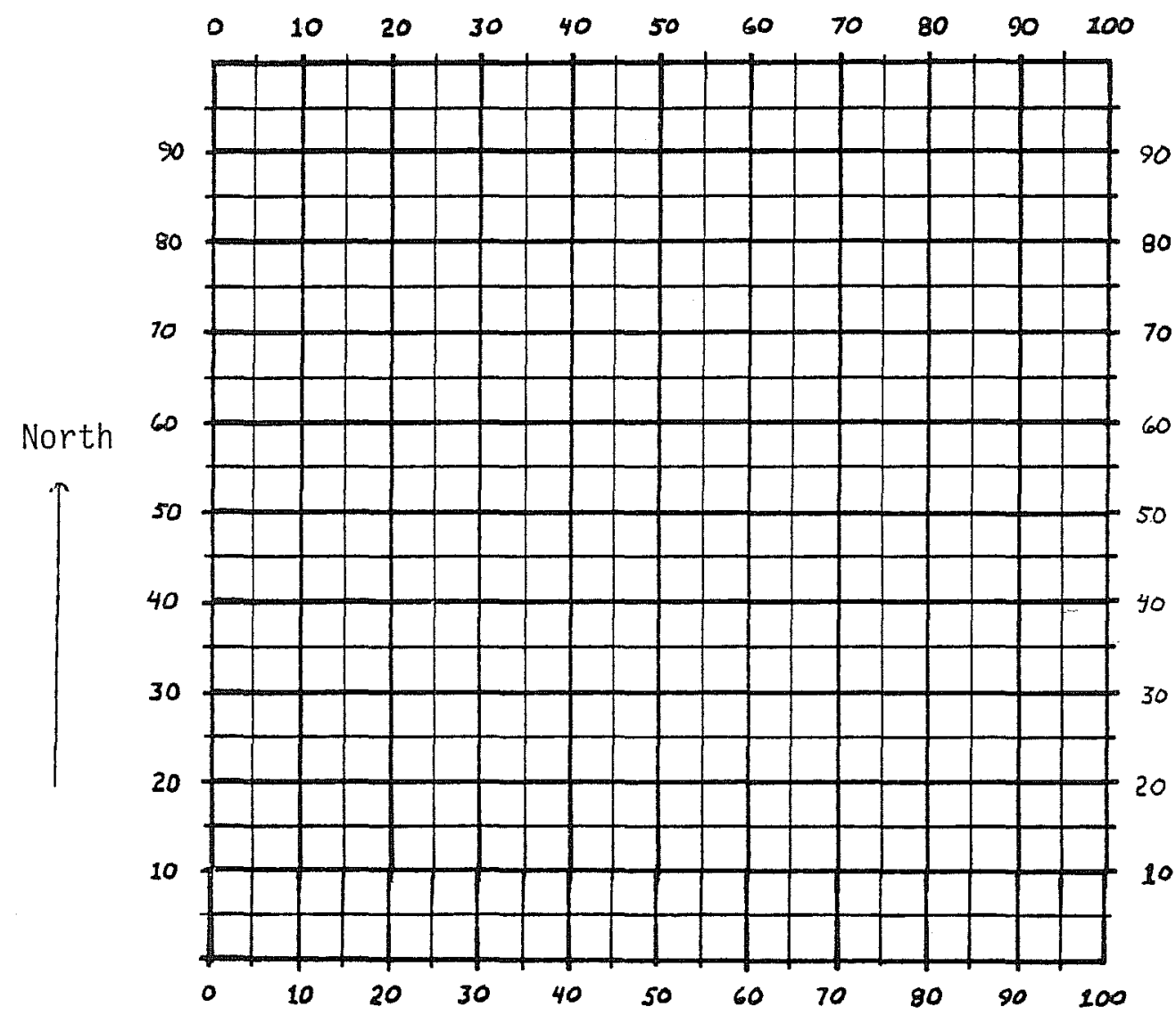

East

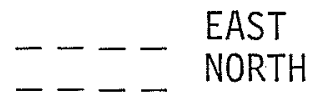

Measurer

Recorder

Figure 93. Unit Mapping Record. 
EAGLE HILL (16 SA 50) PROJECT

Center for Archaeological Research-UTSA

\section{pH-PO4 Soil Chemistry Format}

$\begin{array}{llll}\text { Format } & \text { Sample } & \begin{array}{l}\text { Sample } \\ \text { Segment }\end{array} & \begin{array}{c}\text { pH/KC } \\ \text { Paper }\end{array} \\ 1 \underline{0} & ----- & -- & -- \\ 1 \underline{0} & ------ & -- & -- \\ 1 \underline{0} & ------ & -- & -- \\ 1 \underline{0} & ------ & -- & -- \\ 1 \underline{0} & ------ & -- & -- \\ 1 \underline{0} & ------ & -- & -- \\ 1 \underline{0} & ------ & -- & -- \\ 1 \underline{0} & ------ & -- & -- \\ 1 \underline{0} & ------ & -- & -- \\ 1 \underline{0} & ------ & -- & -- \\ 1 \underline{0} & ------ & -- & -- \\ 1 \underline{0} & ------ & -- & -- \\ 1 \underline{0} & ------ & -- & -- \\ 1 \underline{0} & ------ & -- & --\end{array}$

Figure 94. Soil Chemistry Record.

$\mathrm{PO} 4 \mathrm{PO} 4$

Spot Colorimeter Day/Month/Yr

$-\quad---$

$-\quad---$

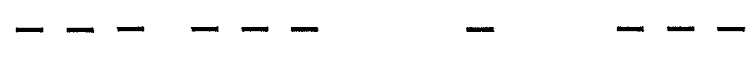

$-----$

$-\quad-\quad-$

$----$

$----$

$--\cdots$

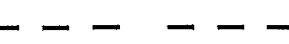

$----$

$-----$

$-\cdots-\cdots$

$-----$

$----\cdots$

$---\cdots$

$--\cdots$ $-1-\ldots$

$-1-1$

$-1-1$

$-1-\ldots$

$-1-18 \underline{0}$

$-1-1 / \underline{0}$

$-1--18 \underline{0}$

-1 - 19

$-1-{ }^{1} \underline{8} \underline{0}$

$-{ }^{\prime}--18 \underline{0}$

$-1--18 \underline{0}$

$-1-1 / \underline{8}$

$-{ }^{\prime}-1 / \underline{0} \underline{0}$

$-1,-18 \underline{0}$ 
EAGLE HILL (16 SA 50) PROJECT

Center for Archaeological Research-UTSA

TRANSIT SHOT FORMAT-01

$$
\begin{aligned}
& \text { - - - - FN\# - }
\end{aligned}
$$

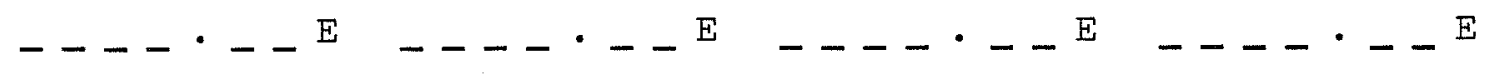

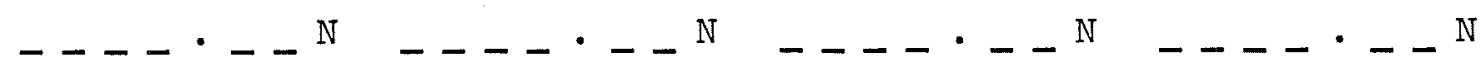

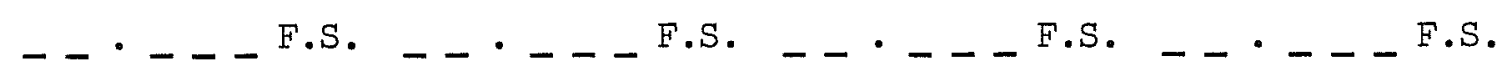

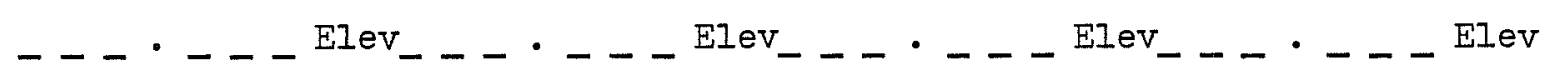

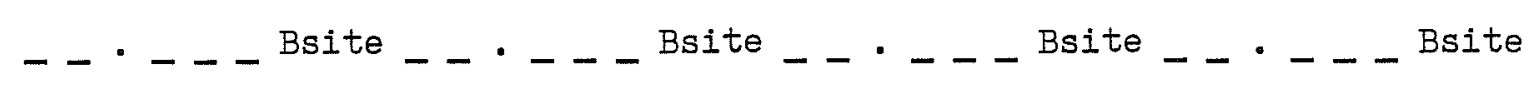

$$
\begin{aligned}
& \text { - Vdat _ - Vdat _ Vdat _ - Vdat } \\
& \text { _ _ _ _ _ Dis _ _ _ _ _ Dis _ _ _ } \ldots \text { _ Dis _ _ _ } \ldots \text { _ Dis } \\
& \text { _Grid _ Grid _ Grid _ Grid } \\
& \text { _ _ - _ Azim _ _ _ _ Azim _ _ _ _ Azim _ _ _ _ Azim } \\
& \text { - Tstat _ _ Tstat _ Tstat _ - Tstat } \\
& \text { _- Format _ _ Format _ Format _ Format } \\
& \text { - } \text { - Area _ _ Area _ Area _ Area } \\
& \text { - - Coder } \quad \text { - Coder } \quad \text { - Coder }
\end{aligned}
$$

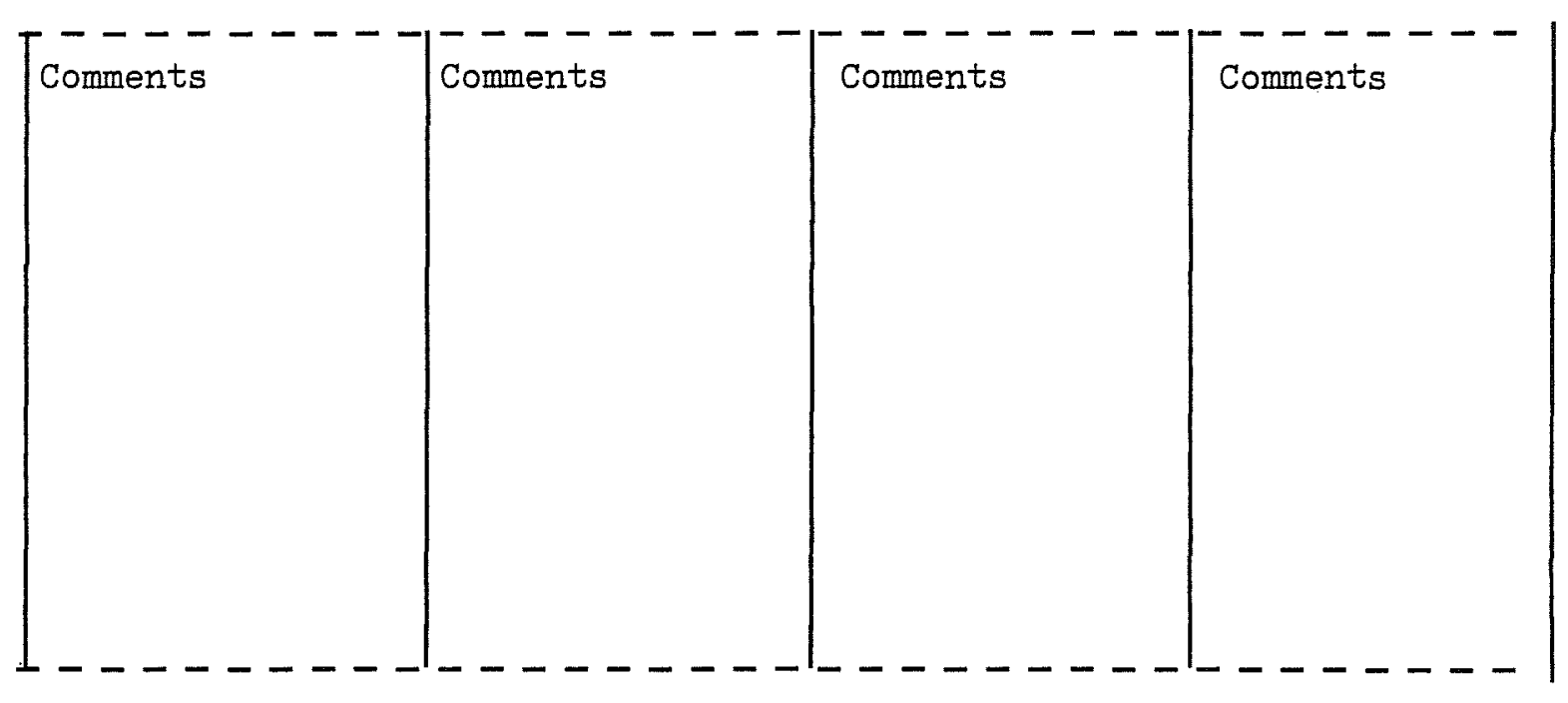

Figure 95. Transit Shot Record. 
EAGLE HILL (16 SA 50) PROJECT

Substratum Plan Map - Forward Perspective

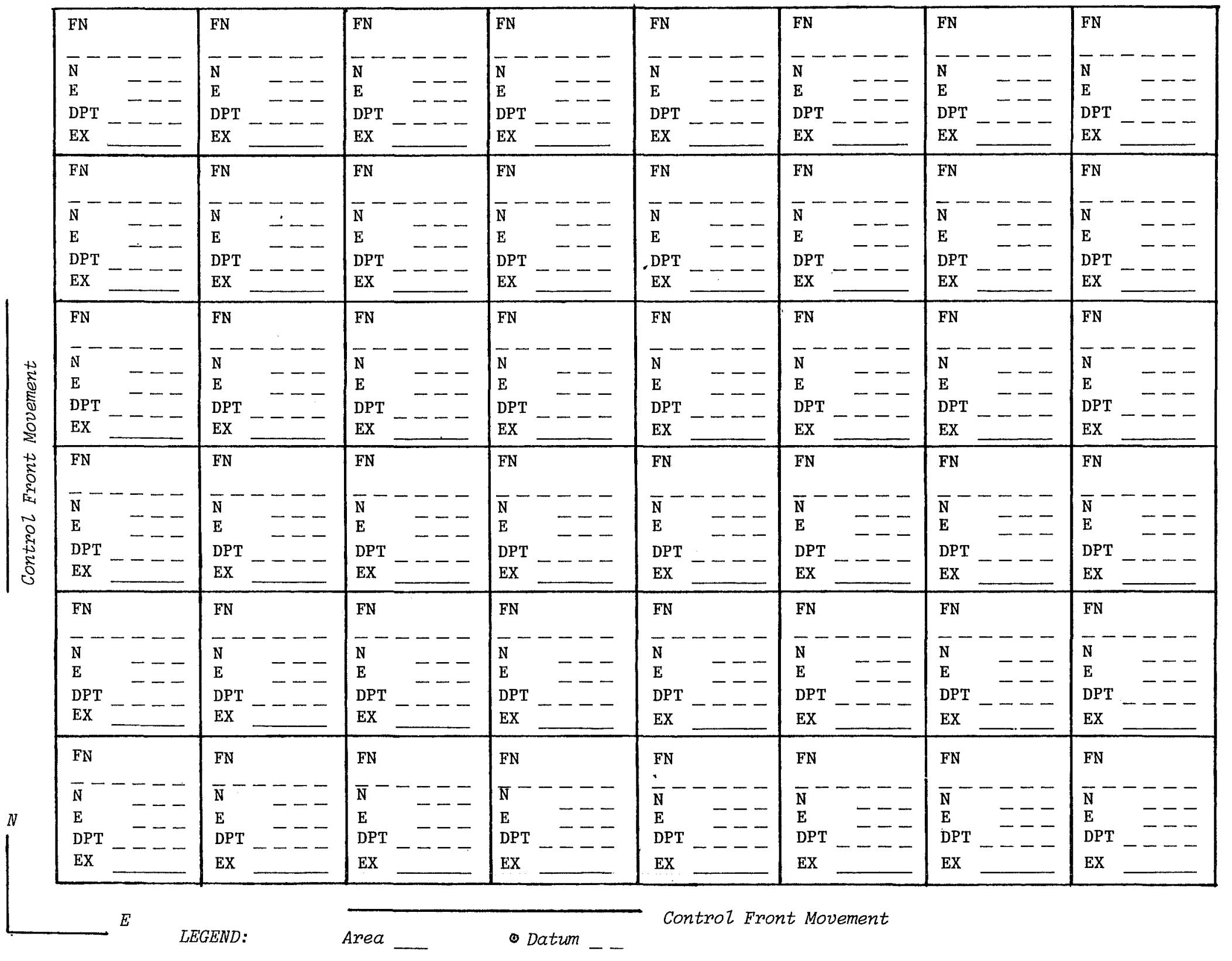

Figure 96. Substratum Plan Map. 
FN ASSIGNIENT INVENTORY

FN Block

$---------$

$---------$

$--------$

$--------$

$---------$

$--------$

$--------$

$--------$

$--------$

$--------$

$---------$

$---------$
Category*

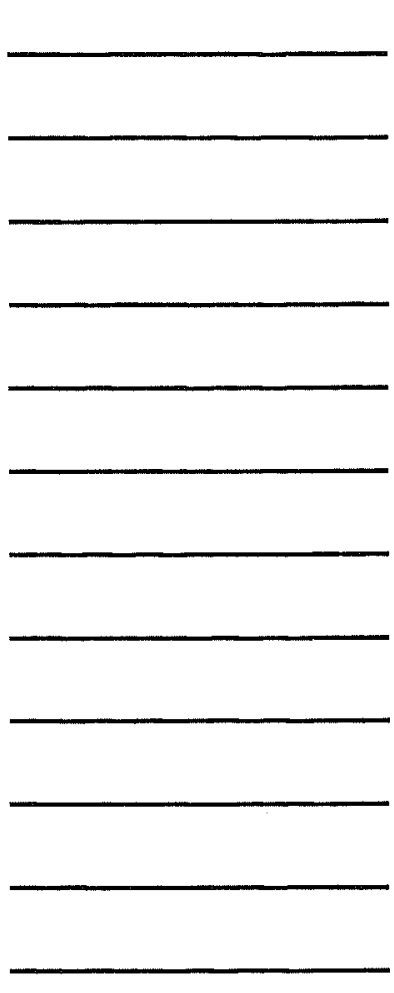

Area

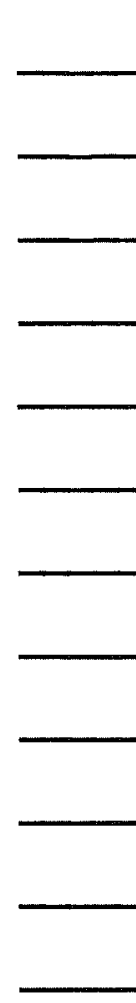

Day/Month/Year

Coder

$1-1$

$--1--1$

$-$

$-1 \ldots$

$-$

$-1 \ldots-1 \ldots$

$--$

$-1 \ldots-1 \ldots$

$-$

- 1 - 1 -

$-1,-1 \ldots$

$-1,-1 \ldots$

$-1,-1 \ldots$

$-1 \ldots-1 \ldots$

$-1 \ldots$

$-1, \ldots$

$-1 \ldots$
$-$

$--$

$-$

$--$

$-$

$-$

$--$

$-$

*core, physical, cultural

Figure 97. FN Assignment Inventory. 
EAGLE HILL

THE UNIVERSITY OF TEXAS AT SAN ANTONIO

CENTER FOR ARCHAEOLOGICAL RESEARCH

FIELD NOTES

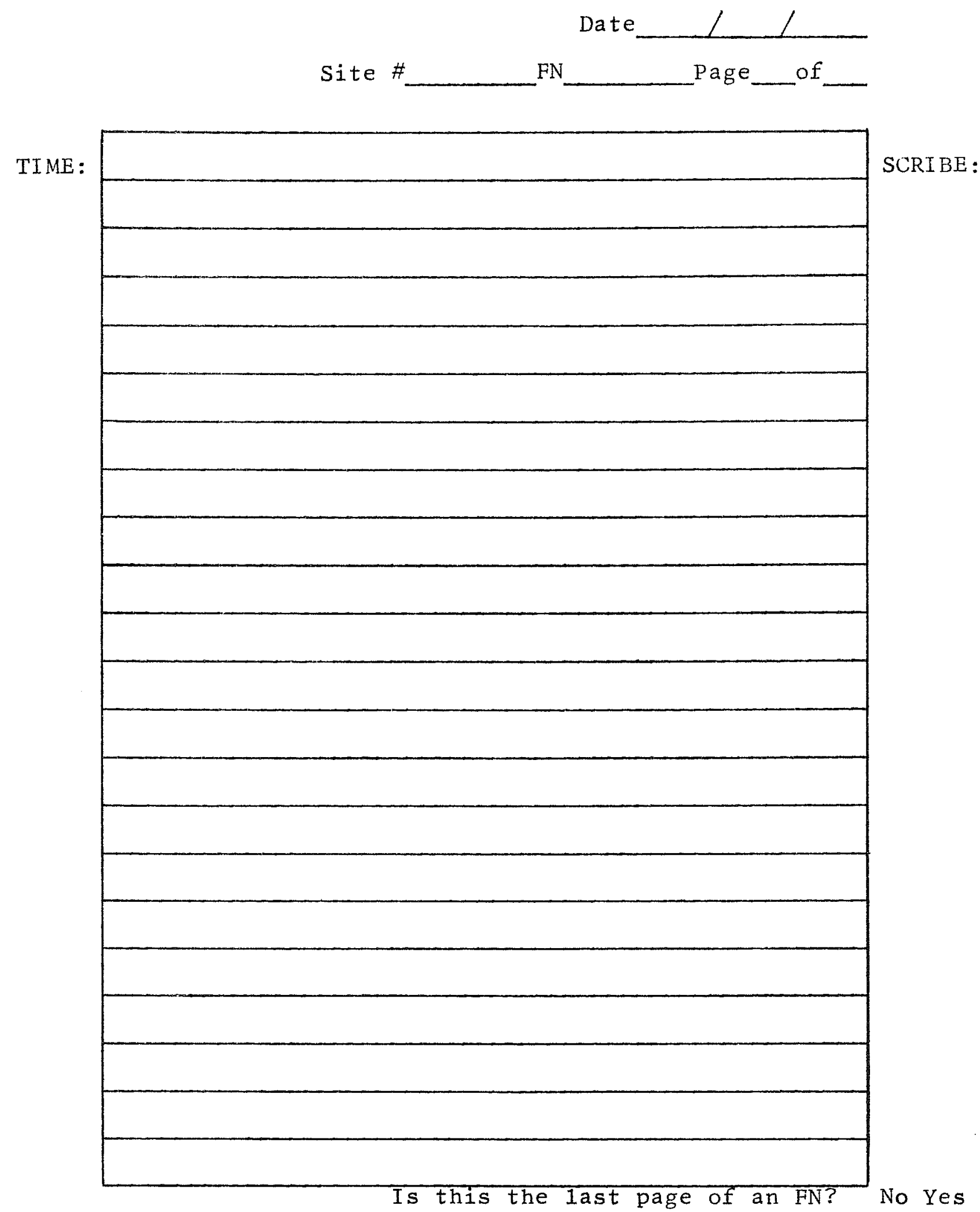

Figure 98. Field Notes. 

APPENDIX $\mathrm{C}$ :

FLAKE CONCENTRATIONS

by

Joan Sherwood 


\section{FLAKE CONCENTRATIONS}

During excavation of the Eagle Hill site (16 SA 50), a total of 92 flake concentrations was identified in a 11 occupation planes and levels (Table 71). A concentration is defined as a group of flakes, chips, pebbles, or other materials lying in close juxtaposition. Some concentrations also contained clay balls. The explanation for these features is not certain. Among the possibilities under consideration are human caches, rodent activity, and erosion.

TABLE 71. NUMBER OF CONCENTRATIONS BY OCCUPATION PLANE

Level

1.73

2.13

3.11

4.12

4.15

4.16

4.17

5.11 (Pleistocene soil)
Number of concentrations

0

1

10

24

24

7

25

Many of the lithics in the concentrations were found lying horizontally. This would seem to be the normal position for any casually discarded or dropped object--gravity would tend to force it into that position. However, a significant frequency of concentrations contained flakes that were vertical.

The fact that these concentrations occur in levels where there are often less chips and flakes in single context would seem to indicate that some action had taken place to gather them.

The possibility of rodent activity, perhaps of packrats collecting and piling objects in their burrows, may have been the cause of these concentrations, but there is no evidence of rodent disturbances in areas where concentrations were uncovered. Furthermore, it would seem that rodent activity would have been responsible for a more varied collection of lithic materials that comprised the concentrations.

Man-made concentration, either accidental or intentional, has also been considered, but ruled out for several reasons. In the first place, the results of tool making or retouching activities would have resulted in debitage spread over a wider area and would tend to consist of material of a more uniform nature than found in Eagle Hill concentrations. Tool making activities would have resulted in a large number of pressure flakes whereas these concentrations contained a mixture of different types: edge-altered flakes, blade fragments, chips, clay balls, and pebbles. All this material seemed to have been mingled indiscriminately. Likewise, any deliberate arrangement of lithics and other materials into piles seems improbable because the piles would not have remained in place, but would have been scattered by subsequent activity in the area. 
The most likely explanation seems to be that of deposition by the action of natural forces such as the runoff from heavy rains. A strong stream of water could have moved these sma 17 pieces along until one piece was obstructed and more accumulated behind it. This action would also explain the many flakes and chips found standing upright since the force of the water would have turned them vertically. Other pieces lodged beside them would have kept them in that position. Dirt washed along by the same water flow would have covered them and protected them from later disturbance.

Occupation Planes 4.12 and 4.15 have the greatest frequency of concentrations, but these are not the levels where the largest number of flakes and chips were found. Below the substrata is where lithics were found in the greatest abundance. The water-action explanation seemed most reasonable with ultimate stabilization by sediments filling the gaps between flakes. For this reason, flake concentrations were eliminated from the systematic analysis of material. 